\title{
RICIAN NOISE CORRECTED MULTI-COMPONENT ANALYSIS OF THE MR DIFFUSION SIGNAL DECAY FOR HUMAN BRAIN IN VIVO
}

by

Marzieh Nezamzadeh, B.Sc. M.Sc.

\author{
A thesis submitted to the \\ Faculty of Graduate Studies and Research \\ in partial fulfilment of the requirements \\ for the degree of \\ Doctor of Philosophy
}

Ottawa-Carleton Institute for Physics

Department of Physics, Carleton University

Ottawa, Ontario, Canada

June, 2006

C Copyright 2006, Marzieh Nezamzadeh 


$\begin{array}{ll}\begin{array}{l}\text { Library and } \\ \text { Archives Canada }\end{array} & \begin{array}{l}\text { Bibliothèque et } \\ \text { Archives Canada }\end{array} \\ \begin{array}{l}\text { Published Heritage } \\ \text { Branch }\end{array} & \begin{array}{l}\text { Direction du } \\ \text { Patrimoine de l'édition }\end{array} \\ \begin{array}{l}\text { 395 Wellington Street } \\ \text { Ottawa ON K1A ON4 }\end{array} & \begin{array}{l}\text { 395, rue Wellington } \\ \text { Ottawa ON K1A ON4 } \\ \text { Canada }\end{array}\end{array}$

Your file Votre référence ISBN: 978-0-494-18228-4 Our file Notre référence ISBN: 978-0-494-18228-4

NOTICE:

The author has granted a nonexclusive license allowing Library and Archives Canada to reproduce, publish, archive, preserve, conserve, communicate to the public by telecommunication or on the Internet, loan, distribute and sell theses worldwide, for commercial or noncommercial purposes, in microform, paper, electronic and/or any other formats.

The author retains copyright ownership and moral rights in this thesis. Neither the thesis nor substantial extracts from it may be printed or otherwise reproduced without the author's permission.
AVIS:

L'auteur a accordé une licence non exclusive permettant à la Bibliothèque et Archives Canada de reproduire, publier, archiver, sauvegarder, conserver, transmettre au public par télécommunication ou par l'Internet, prêter, distribuer et vendre des thèses partout dans le monde, à des fins commerciales ou autres, sur support microforme, papier, électronique et/ou autres formats.

L'auteur conserve la propriété du droit d'auteur et des droits moraux qui protège cette thèse. $\mathrm{Ni}$ la thèse ni des extraits substantiels de celle-ci ne doivent être imprimés ou autrement reproduits sans son autorisation.
In compliance with the Canadian

Privacy Act some supporting forms may have been removed from this thesis.

While these forms may be included in the document page count, their removal does not represent any loss of content from the thesis.
Conformément à la loi canadienne sur la protection de la vie privée, quelques formulaires secondaires ont été enlevés de cette thèse.

Bien que ces formulaires aient inclus dans la pagination, il n'y aura aucun contenu manquant.

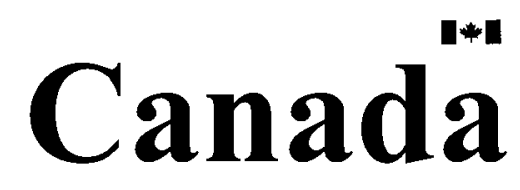




\section{IN THE NAME OF GOD}

\section{MOST GRACIOUS AND MOST MERCIFUL}

This thesis is dedicated to my parents;

Effat-sadat Shafiee

Solayman Nezamzadeh 


\section{Abstract}

The diffusion of water in the white matter of human brain tissue in vivo was studied in this thesis. To provide a better understanding of diffusion behavior related to brain tissue microstructure, an analysis based on restricted diffusion and exchange behavior in the tissue is required. The diffusion signal decays were accurately measured over an extended range of b-values up to $12,500 \mathrm{~s} / \mathrm{mm}^{2}$ and short diffusion times between $19.9 \mathrm{~ms}$ and $53.8 \mathrm{~ms}$ in healthy human brain. In order to get reliable information from the decays, even when the SNR is small, a few Rician noise correction bias methods were investigated. A new method of correcting for the Rician noise bias presented in this work performs better than existing methods when the SNR is small.

To analyze the diffusion decays for water in brain tissue, an analysis code, which is based on the non-negative least squares $(N N L S)$ algorithm, was developed for this thesis. For all diffusion times studied there was a diffusion coefficient of approximately $(0.93 \pm 0.003) \times 10^{-3}(80 \pm 1 \%) \mathrm{mm}^{2} / \mathrm{s}$ and another of about $(0.06 \pm 0.002) \times 10^{-3}(19 \pm 1 \%)$ $\mathrm{mm}^{2} / \mathrm{s}$. A small contribution $(1 \%)$ at about $1 \times 10^{-2} \mathrm{~mm}^{2} / \mathrm{s}$ was also detected. The decays show small diffusion time dependence for the slow diffusion coefficient which has not previously been reported.

The results from the experiments of the studied regions in human brain are comparable with analytical results of intracellular restricted diffusion behavior and water exchange between intracellular and extracellular spaces in tissue obtained using the Tanner planar equation combined with Karger diffusion exchange theory for a human model. Using the theoretical predictions with the experimental results for the studied regions, tissue parameters such as average cell size and exchange time were roughly estimated. 


\section{Acknowledgements}

This thesis could not be carried out without the kind assistance and support of many individuals and organizations.

I am indebted to my supervisor, Ian Cameron, not only for his scientific guidance and the support that he has provided throughout, but also for making our lab a happy environment with healthy competition.

I would like to thank the Radiologists and Technicians in the Department of Radiology at The Ottawa Hospital for their flexibility in schedules despite a very limited time for research.

I wish to thank the research team in our MRI lab; the graduate students, post-docs, co-op students and other individuals who made my stay more memorable. Their honest yet considerate comments to this work have helped much in improving its quality; my thanks to Andra Smith, Claire Foottit, Dominik Royko, Elena Olariu, Ewan Hill, Fakhereh Mirrashed and Sorina Truica. I appreciate the valuable feedback and advice from my Ph.D. committee members; Paul Johns and Thomas Brabec.

There are many professors at Carleton University I would like to express my gratitude. My special thanks to Paul Johns, Giles Santyr, Peter Raaphorst, Dean Karlen and Dave Rogers.

I would like to thank Albert Cross, Gregory Cron, Julia Wallace and Nikolai Romanenko for kindly sharing their knowledge during the period of their post doctorate at Physics Dept. of Carleton University. I would also thank my fellow graduate students at the university. 
I would like to thank Christine Monaghan, Sheila Thayer and Marija Gojmerac for their constant help in the graduate administrating office.

The financial support provided by The Ottawa General Hospital studentship is greatly appreciated.

Thanks from my deepest heart to my family; my parents, my sisters and brothers, for their unconditional love, support and encouragement always provided to me. 


\section{Contents}

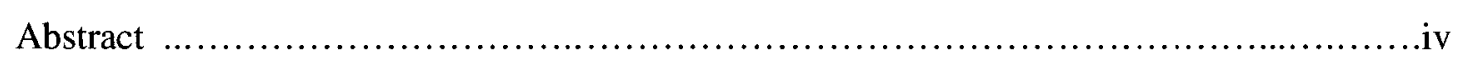

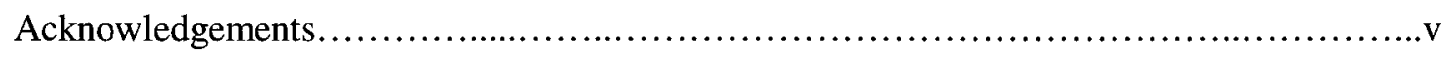

List of Tables ...................................................................

List of Figures............................................................. xiv

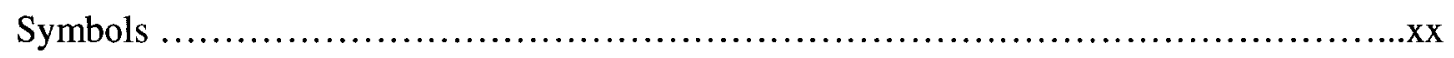

1 Introduction 1

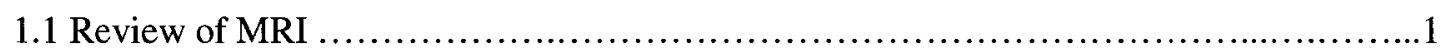

1.2 Review of Diffusion in MR .....................................................

1.3 Clinical Applications.................................................... 10

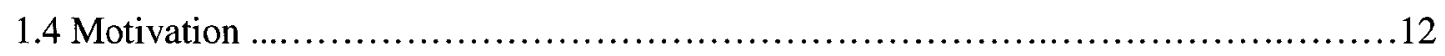

1.5 Thesis Outline and Significance........................................... 13

2 Background $\quad 16$

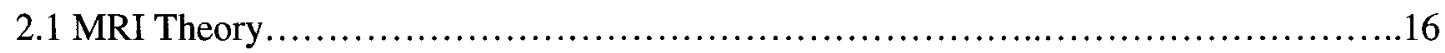

2.1.1 Spin states.............................................................. 16

2.1.2 Boltzmann distribution.............................................. 19

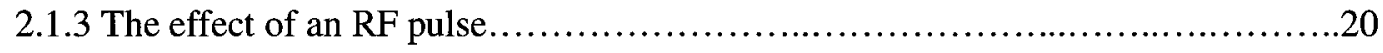

2.1.4 MR relaxation........................................................ 28

2.1.5 The spin echo pulse sequence ...........................................

vii 
2.1.6 The gradient echo pulse sequence ......................................... 31

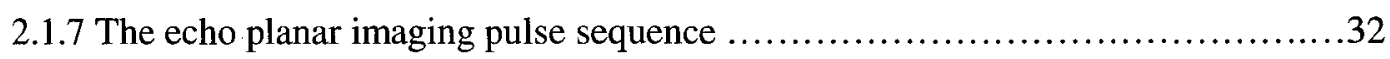

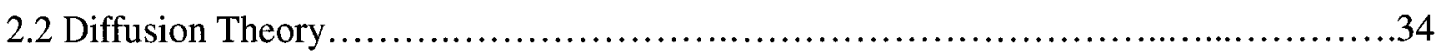

2.2.1 Diffusion equation for free diffusion.................................... 34

2.2.2 Diffusion equation for restricted diffusion.................................. 36

2.2.3 Diffusion time..................................................................

2.2.4 Anisotropic diffusion and the diffusion tensor...................................39

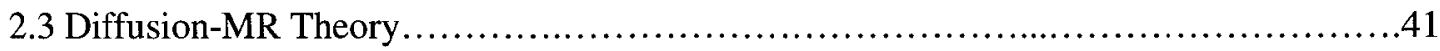

2.3.1 Attenuation of the MR signal due to diffusion ..................................43

2.3.1.1 MR signal attenuation due to restricted diffusion.......................46

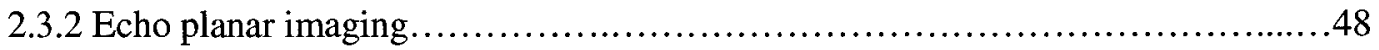

2.3.3 Diffusion time in MR diffusion measurements................................49

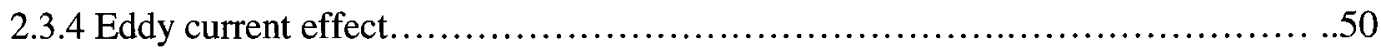

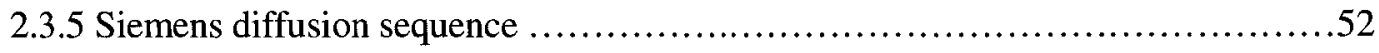

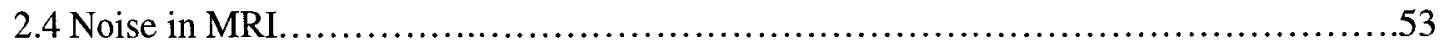

2.4.1 The sources of noise in MRI............................................53

2.4.2 Gaussian, Rician and Rayleigh distributions..............................56

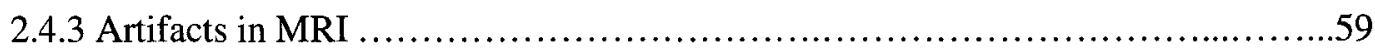

2.4.3.1 Ghost artifacts ................................................. 59

2.4.3.2 N/2 ghost artifacts ...........................................60

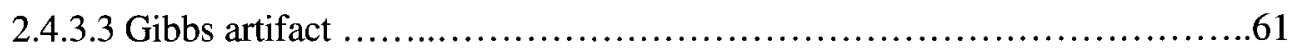

2.4.3.4 Motion artifacts...............................................63

2.4.3.5 Chemical shift artifacts .........................................64

2.4.3.6 $\mathrm{B}_{0}$ inhomogeneity artifacts .......................................64

viii 


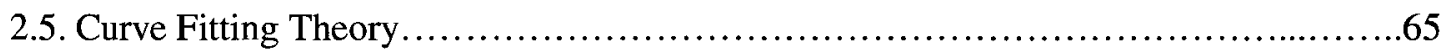

2.5.1 Least Squares........................................................67

2.5.2 Non-Negative Least Squares...........................................68

2.5.3 Non-Negative Least Squares with regularization .......................... 72

2.5.4 Comparing $N N L S$ with other standard curve fitting methods......................74

2.5.5 NNLS literature review.................................................... 75

2.6 Literature Review............................................................

3 Experiments $\quad 8$

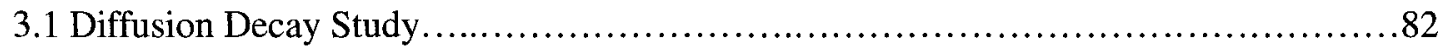

3.2 Diffusion Time Dependence Study ........................................... 84

3.2.1 Primary experiments..................................................... 84

3.2.2 Supplemental experiments for noise correction and eddy current effects..........87

4 Noise Correction $\quad 89$

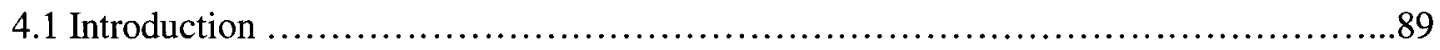

4.2 The Rician Distribution Function and its Moments..................................92

4.2.1 The low SNR limit of the Rician distribution:

The Rayleigh distribution function and the moments..........................95

4.2.2 The high SNR limit of the Rician distribution..............................97

4.2.3 The noise bias of the Rician distribution ...................................... 97

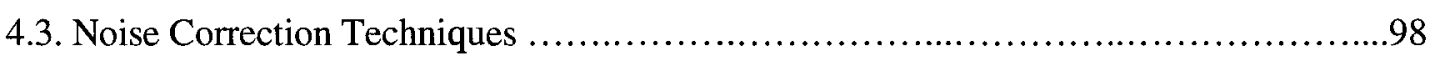

4.3.1 Signal averaging of the complex MR signal................................99

4.3.1.1 Complex averaging simulations ................................99

4.3.1.2 Phantom studies of complex averaging ............................ 103

4.3.2 Review of post-processing noise correction techniques from the literature.........105

4.3.3 A new Rician noise correction technique....................................113 
4.4 Noise Estimation from the Image Background

4.4.1. The PDF of the background

4.4.2 Noise estimation from the image background without the phantom. .125

4.4.3 Noise estimation from the image background with the phantom. .134

4.5 Validation of the Noise Bias Correction Procedure .143

4.5.1 Signal strength estimation from the phantom image 144

4.5.2 Noise bias correction technique using the head coil phantom. 147

4.6 Discussion of the Noise Correction .151

4.7 Conclusion. 155

5 Diffusion Decay Measurements in Human Brain in vivo and Analysis with NNLS 157

5.1 Introduction. 157

5.2 Materials and Methods 158

5.3 Data Analysis with $N N L S$. .161

5.4 Using NNLS for the Analysis of the Simulated Data.... 162

5.5 The Diffusion Measurements for a Water Phantom and Analysis by NNLS 172

5.6 Using $N N L S$ for $\mathrm{T}_{2}$ relaxation analysis in HXe experiments 176

5.7 The Data Analysis of the Diffusion Decay in Human Brain by NNLS-diff .180

5.8 Discussion 183

5.9 Conclusion .187

6 Diffusion Time Dependence

6.1 Introduction 189

6.2 Materials and Methods .193

6.2.1 The TRSE sequence .193

6.2.2 The $b$-factor and $\tau_{\mathrm{D}}$ evaluation for the TRSE sequence. .196 
6.2.3 Optimizing the diffusion pulse sequence for short $\tau_{\mathrm{D}}$ and high $\mathrm{b}$-values

6.3 Experimental Procedure and Sequence Validation...............................207

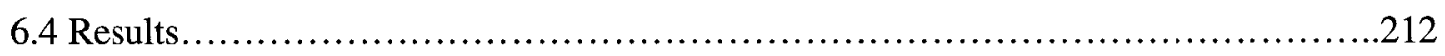

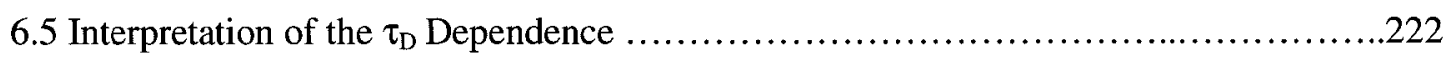

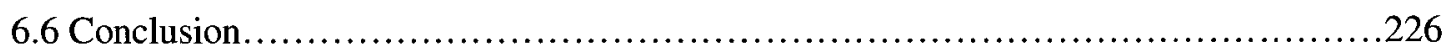

7 Discussion and Conclusions $\quad 227$

7.1. Brain Tissue Structure........................................................227

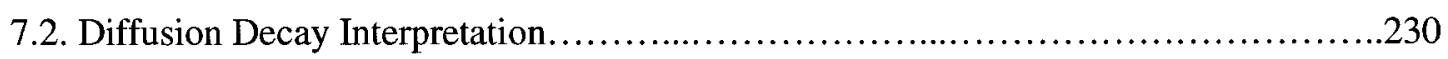

7.2.1. Compartmentalization of tissue structure..............................231

7.2.2. Intracellular restricted diffusion .....................................232

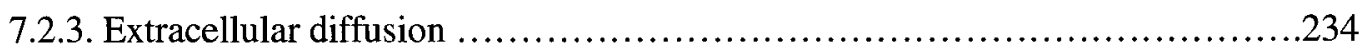

7.2.4. Exchange between compartments........................................236

7.2.5. $\mathrm{T}_{2}$ relaxation, anisotropy and blood flow effects..........................242

7.3. Rician Noise Effects on the Diffusion Decay................................. 243

7.4. Diffusion Time Dependence Interpretation...................................244

7.4.1. The intracellular diffusion restriction.................................246

7.4.2. The two-site diffusion exchange theory .................................249

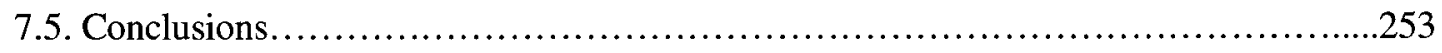

$\begin{array}{ll}\text { Appendix I The NNLS Flow Charts } & 255\end{array}$

Appendix II The b-factor and $\tau_{\mathrm{D}}$ for the TRSE Sequence 258

II.1 b-value Calculation.......................................................... 258

II.2 Diffusion Time Calculation..............................................264

$\begin{array}{ll}\text { References } & 266\end{array}$

$\mathrm{xi}$ 


\section{List of Tables}

1.1 NMR sensitivities for nuclei of biomedical interest.......................................

3.1. The $16 \mathrm{~b}$-values $\left(\mathrm{s} / \mathrm{mm}^{2}\right)$ applied for each diffusion time sequence..................... 86

4.1 The estimated mean value of Rician noise corrected PDFs by various approaches over a range

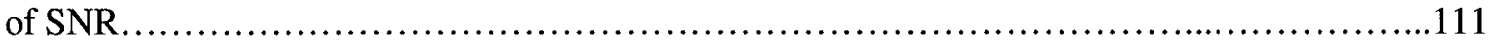

4.2 The mean value of the PDF when $\mathrm{NCl}$ is used for noise bias correction compared to GP correction over a range of SNR........................................................ 115

4.3. Study of $\hat{\mathrm{A}}_{\mathrm{NC} 2}$ as a function of $\mathrm{m}$, the number of correction terms in the expansion, for $\mathrm{NC} 2$ noise bias correction using the mean from the original Rician PDF........................ 117

4.4 The results of simulations for NC2 correction versus the number of terms used in the expansion and the number of averages of neighboring pixels for SNR between 0.0 and

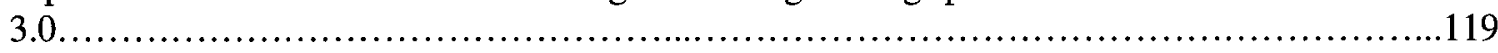

4.5 The parameter values of fit to the simulated decay biased with Rician noise and corrected with NC2 as shown in figure 4.11 .

4.6. The noise $\sigma$ estimated with the various estimators for $b=0,2600$ and $5000 \mathrm{~s} / \mathrm{mm}^{2}$ for 16 ROI

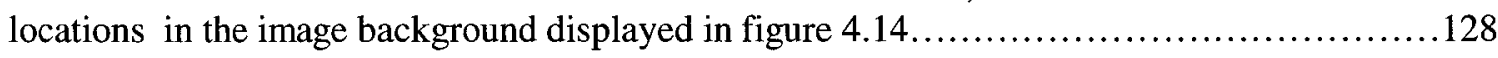

4.7 The noise $\sigma$ estimated from the background of the images without phantom; a. averaged over 16 ROI locations for each b-value, b. averaged over 16 b-values for each ROI location ...132

4.8 The noise $\sigma$ estimated with the various estimators vs. b-value for 0 to $5000 \mathrm{~s} / \mathrm{mm}^{2}$ in 8 ROI locations in the image background with the phantom displayed in figure $4.18 \ldots \ldots \ldots \ldots \ldots \ldots . . .135$

4.9 The noise $\sigma$ estimated in the image background of the water phantom; a. averaged over 8 ROI locations for each $b$-value, $b$. averaged over $16 \mathrm{~b}$-values for each ROI location. 138

xii 
4.10 The mean values and their standard deviations vs. the ROI index. The diameter of the ROI and the number of pixels in the ROI are also given in the table.

4.11 Comparison of the three estimators averaged over both ROI locations and b-values. .155

4.12 Comparison of the Rician theoretical ratio with the measured ratio with and without the phantom.....

5.1 The statistics of the simulated data of a continuous spectrum analyzed by NNLS with the three regularizers

5.2 The quantitative results of diffusion measurements on human brain. .182

6.1 The results of eddy current detection in the $g_{2} g_{3}$ sequences .206

6.2 The results of diffusion in pure water phantom using the $g_{2} g_{3}$ sequences analyzed by $N N L S$ diff after noise bias correction.

6.3.a-f The results of diffusion in human brain tissue for the diffusion times between 19.9 to 53.8 $\mathrm{ms}$ from top to the bottom respectively of 8 human subjects analyzed using $N N L S$-diff.

6.4 The diffusion data of human brain tissue vs. diffusion time after removing the points which exceed $\pm \sigma$ of the averaged data over 8 human subjects.

7.1 The evaluated intracellular restricted diffusion coefficient vs. cell size for the Tanner-planar equation

7.2a The results of Karger exchange theory with $\mathrm{f}_{\mathrm{i}}=0.8, \mathrm{D}_{\mathrm{i}}=0.001 \mathrm{~mm}^{2} / \mathrm{s}$ and $\mathrm{D}_{\mathrm{e}}=0.003 \mathrm{~mm}^{2} / \mathrm{s}$. .240

7.2b The results of Karger exchange theory with $\mathrm{f}_{\mathrm{i}}=0.2, \mathrm{D}_{\mathrm{i}}=0.001 \mathrm{~mm}^{2} / \mathrm{s}$ and $\mathrm{D}_{\mathrm{e}}=0.003 \mathrm{~mm}^{2} / \mathrm{s}$. .240

$7.3 \mathrm{D}_{\mathrm{a}}\left(10^{-3} \mathrm{~mm}^{2} / \mathrm{s}\right)$ values calculated using the Tanner-planar equation vs. diffusion time for restricted diffusion in a square box over lengths, a, consistent with the range of axon size for human brain .248 


\section{List of Figures}

2.1 Precession of magnetic moment $\mu$ about a static magnetic field $\mathbf{B}$ in the classical view

2.2 The energy states diagram for a proton spin system. Transitions between two Zeeman energy levels can occur by absorption of electromagnetic energy of frequency $\omega_{0}$. .18

2.3 a. Free precession of the expectation value of spin vector $\langle\mathrm{S}\rangle$ for a non-stationary mixture of spin-up and spin-down proton states in a constant magnetic field. b. Motion of the expectation value of $\left\langle S>\right.$ from its initial position under the influence of a $B_{1}$ magnetic field (i.e. $R F$ ) at the resonant frequency spiraling down along $\mathrm{z}$ in the laboratory system....

$2.4 \mathrm{a}$. The spin echo timing sequence. $\mathrm{b}$. The associated diagram of the effect of $90^{\circ}$ and $180^{\circ} \mathrm{RF}$ pulses on the spin system magnetization during spin echo generation.

2.5 a. The echo planar imaging (EPI) sequence with a train of readout (RO) and phase encoding (PE) gradient echoes centered around one spin echo. b. the associated k-space trajectory...... .33

2.6 The schematic diagram of the freely diffusion and the restricted diffusion of a particle in a sphere with radius $\mathrm{R}$ in terms of the variation of the time scale....

2.7 The hypothetical surface of constant mean-squared displacement in a. an isotropic medium representing a diffusion sphere, $b$. an anisotropic medium representing a diffusion ellipsoid......40

2.8 The Stejskal-Tanner diffusion pulse sequence. The rectangular gradient pulse of duration $\delta$ and magnitude $g$ is inserted into a spin echo sequence sensitizing the system to diffusion so that the signal will be attenuated due to the diffusion process. .42

$2.9 \mathrm{a}$. The timing diagram of the TRSE sequence. $\mathrm{b}$. The build up and decay of eddy currents due to switching of the gradients.

2.10 The probability density functions of the magnitude image versus SNR .58

2.11 a. The zigzag pattern of $\mathrm{k}$-space for an EPI sequence due to a time delay. $\mathrm{b}$. A typical image showing an N/2 ghosting artifact 
4.1. Diffusion decay measurements of a water phantom over an extended range of b-values and fitted with least squares.

4.2 A simulated bi-exponential diffusion decay with Rician noise bias compared with the actual decay . .91

4.3 The convergence of the Rician distribution to a Gaussian in the high SNR limit and to a Rayleigh distribution at the very low limit of SNR.

4.4 A simulated diffusion decay averaged over 10 measurements for complex averaging compared with magnitude averaging .... 100

4.5 Simulated diffusion decays using signal averaging of the magnitude and of the complex data for different amounts of averaging..... 102

4.6 The diffusion decay of the water phantom comparing complex averaging with magnitude averaging for 32 measurements spatially averaged over 50 pixels.

4.7 The PDFs of the simulated $\mathrm{M}_{\mathrm{j}}$ and $\mathrm{M}_{\mathrm{j}}^{2}$ values biased with Rician noise for $\mathrm{SNR}=0.0$ and $\mathrm{SNR}=2.0$ 108

4.8 The $\hat{\mathrm{A}}_{\mathrm{GP}}$ PDF at several SNRs between 0.0 and 3.0 compared to the corresponding Rician noise PDF.

4.9 The PDFs for the $\hat{\mathrm{A}}_{\mathrm{GP}}, \hat{\mathrm{A}}_{\mathrm{MS} 2}$ and $\hat{\mathrm{A}}$ Rician noise bias correction schemes simulated for

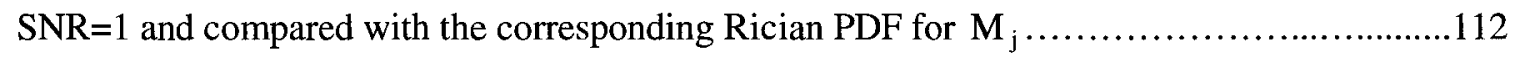

4.10 PDFs when NC1 correction is compared with the original PDFs versus SNR.

4.11 Simulated bi-exponential diffusion decay with Rician noise bias and data corrected with $\mathrm{NC} 2$.

4.12 The histogram of the magnitude data from the image background of the MR diffusion measurement compared with the Rayleigh density function. 124

4.13. The histogram of the magnitude data from the image background of the MR diffusion measurement compared with a Hanning filtered Rayleigh density function. 124

4.14 The 16 ROIs selected in the background image for MR diffusion measurements to study the noise $\sigma$ estimates. 128

4.15 Values of the background noise estimates without the phantom are shown vs. ROI location for selected b-values 
4.16 The noise $\sigma$ estimates averaged over ROI locations vs. b-value in the background image without phantom.

4.17 The noise $\sigma$ estimates averaged over b-values vs. ROI location in the background image without phantom.

4.18 The 8 ROIs selected in the image background for MR diffusion measurements of the water phantom to study the noise $\sigma$ estimates

4.19 Values of the background noise estimates with the water phantom are shown vs. ROI locations for selected $b$-values

4.20 The noise $\sigma$ estimates vs. b-value in the image background of the water phantom over the 8 larger ROIs.

4.21 The noise $\sigma$ estimates averaged over b-values vs. ROI location in the image background of the water phantom

4.22 The noise $\sigma$ estimates vs. b-value in the image background of the water phantom over the 8 smaller ROI locations.

4.23 Simulated Rayleigh PDF filtered by Hanning filter using equation (4.41).

4.24 Simulated Rayleigh PDF filtered by Hanning filter using equation (4.42)

4.25 The 16 circular ROIs selected in the phantom image of the MR diffusion measurements to study the signal and noise uniformity vs. ROI size.

4.26 The mean values vs. the ROI index for the $1^{\text {st }}$ and the $2^{\text {nd }}$ set of measurements on the water phantom .146

4.27 The standard deviation (std) of the mean vs. the ROI index for the $1^{\text {st }}$ and the $2^{\text {nd }}$ set of measurements on the water phantom 146

4.28 The phantom image of the MR diffusion measurement with the ROI selected in the optimum location of the image and the background.... 147

4.29 The diffusion MR signal for a water phantom biased with Rician noise and corrected with NC2 noise correction using $\hat{\sigma}(<M>)$ a. on a logarithmic scale fit with least squares, $b$. on a linear scale. 148

4.30 The diffusion MR signal for a water phantom biased with Rician noise and corrected with NC2 noise correction using $\hat{\sigma}(\operatorname{std}<M>)$ a. on a logarithmic scale fit with least squares, $b$. on a linear scale. 149 
4.31 The diffusion MR signal for a water phantom biased with Rician noise and corrected with NC2 noise correction using $\hat{\sigma}\left(<\mathrm{M}^{2}>\right)$ a. on a logarithmic scale fit with least squares, $b$. on a linear scale.

5.1 MR images showing the region of brain studied. Left: this represents the slice selected above the ventricles. Right: the ROI's selected in the white matter and in the background noise. 159

5.2 a. Assumed discrete spectrum of diffusion coefficients, b. Generated diffusion decay curve with simulated noisy data computed using the diffusion coefficient distribution shown in a.....163

5.3 a. Assumed continuous spectrum of diffusion coefficients, b. Generated diffusion decay curve with simulated noisy data computed using the diffusion coefficient distribution shown in a.....164

5.4 a. Discrete spectrum of diffusion coefficients obtained using NNLS analysis of simulated diffusion data, $b$. True discrete spectrum. 166

5.5 The spectrum of diffusion coefficients found using the discrete form of $N N L S$ for a continuous distribution of diffusion coefficients.

5.6 The spectrum of diffusion coefficients obtained using $N N L S$ with a regularizer to minimize the power of the spectrum. 169

5.7 Spectrum of the diffusion coefficients obtained using $N N L S$ compared with the true solution for three forms of the regualrizer.

5.8 a. The spectrum for the diffusion coefficient of the water phantom using $N N L S$ analysis with and without the regularizer. $b$. The diffusion decay for the water phantom after noise correction.

5.9 Typical spectra of $\mathrm{T}_{2}$ relaxation time distributions for a CPMG experiment on $0.85 \% \mathrm{EYP}$ emulsions using $N N L S$ showing four main peaks.

5.10 Comparison of the multi-exponential fit to the $\mathrm{T}_{2}$ relaxation measurements for the EYP emulsion using the $N N L S$ method versus the bi-exponential fit using non-linear least squares...178

5.11 A typical best fit of $\mathrm{T}_{2}$ relaxation for the two emulsions by multi-exponential analysis using the $N N L S$ algorithm.

5.12 The NNLS results for the two emulsions of short and long $\mathrm{T}_{2}$ relaxation in 4 experiments

5.13 The spectrum of diffusion coefficient distribution for human brain tissue observed by MR diffusion measurements in human in vivo.

5.14 The diffusion decay curve of human brain tissue fitted with $N N L S$-diff results as a triexponential decay with diffusion coefficients of $D_{1}=0.06 \times 10^{-3}(19 \%), D_{2}=0.9 \times 10^{-3}(80 \%)$ and $\mathrm{D}_{3}=1.2 \times 10^{-2}(1 \%) \mathrm{mm}^{2} / \mathrm{s}$. 
6.3 a. The TRSE sequence developed based on keeping two pairs of bipolar diffusion gradients. $b$. The TRSE sequence with one pair of bipolar gradients $\left(g_{1}\right.$ and $\left.g_{3}\right)$, c. Another TRSE sequence

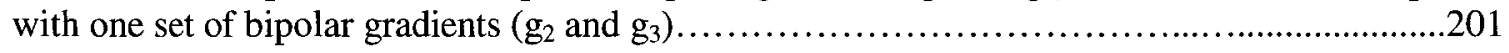

6.4 The typical $g_{2} g_{3}$ diffusion sequence for diffusion times of 6,20 and $54 \mathrm{~ms}$ .202

6.5 Water phantom images measured with Siemens' sequence compared with the $\mathrm{g}_{2} \mathrm{~g}_{3}$ sequence for $\tau_{\mathrm{D}}=54 \mathrm{~ms}$ showing a very small amount of edge magnification. 204

6.6 The subtracted water phantom images of the $b=0$ images from the corresponding maximum $b-$ value images to detect any edge due to eddy current effects.

6.7 The results of the normalized edge magnification detected in the images taken with the $\mathrm{g}_{2} \mathrm{~g}_{3}$ sequences for different diffusion times as well as Siemens' sequence for the investigation of possible eddy current effects .206

6.8 a-f. Typical images of human brain obtained using the $\mathrm{g}_{2} \mathrm{~g}_{3}$ sequences for $\tau_{\mathrm{D}}=19.9$ to $53.8 \mathrm{~ms}$ and their respective $b$-values associated with a $13 \mathrm{mT} / \mathrm{m}$ diffusion sensitizing gradient strength .208

6.9 a-f. Typical images of human brain obtained using the $g_{2} g_{3}$ sequences for $\tau_{D}=19.9$ to $53.8 \mathrm{~ms}$ and their respective maximum b-values associated with the $28.5 \mathrm{mT} / \mathrm{m}$ diffusion sensitizing gradient strength. 209

6.10 A typical human brain image for the $\mathrm{g}_{2} \mathrm{~g}_{3}$ sequence of the slice selected above the ventricles. The ROI's were selected in the white matter of the images and the background for the Rician noise bias correction.

6.11 The results of diffusion of water molecules in human brain tissue analyzed by NNLS-diff using the wide range of 150 diffusion data of the first trials in 8 to 10 subjects and 6 diffusion times.

6.12 The diffusion decays for the water phantom measured with $g_{2} g_{3}$ sequences for $\tau_{D}=19.9$ to $53.8 \mathrm{~ms}$ each measured with $16 \mathrm{~b}$-values up to its maximum possible $\mathrm{b}$-value and analyzed using both $N N L S$-diff and the regular Least Squares after the Rician noise bias correction.

6.13 Typical diffusion decays for human brain tissue vs. $\tau_{\mathrm{D}}$ for one subject from $\tau_{\mathrm{D}}=19.9 \mathrm{~ms}$ to

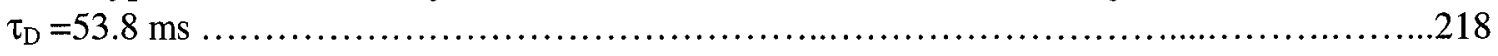

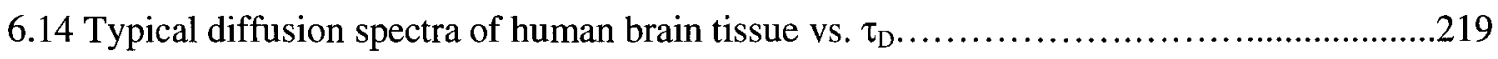


6.15 The diffusion decays of human brain tissue averaged over 8 human subjects vs. diffusion time, all data Rician noise bias corrected and analyzed by $N N L S$-diff multi-exponential analysis.

6.16 The variation of the slow and the fast diffusion coefficients for human brain tissue vs. diffusion time.

7.1 A schematic diagram showing the regions of the brain and water exchange between the two main compartments

7.2 The calculated diffusion signal decays using the Tanner-planar and Karger exchange theory for a healthy human model vs. intracellular residence time.

7.3 The discrete spectrum of diffusion coefficients obtained using NNLS-diff analysis of the experimental diffusion signal decays for human brain $a$. before Rician noise bias correction and $b$. after Rician noise bias correction.

7.4 The normalized diffusion coefficient for restricted diffusion calculated from the TannerPlanar formula with the box length $\mathrm{a}=5 \mu \mathrm{m}$ vs. the dimensionless reduced time, $\xi$.

$7.5 \mathrm{D}_{\mathrm{a}}\left(10^{-3} \mathrm{~mm}^{2} / \mathrm{s}\right)$ calculated using the Tanner-planar equation vs. diffusion time for restricted diffusion in a square box over lengths, a, consistent with the range of axon size for human brain. .248

$7.6 \mathrm{D}_{\mathrm{s}}\left(10^{-3} \mathrm{~mm}^{2} / \mathrm{s}\right)$ values from the $\tau_{\mathrm{D}}$ dependence experiments on human brain in vivo averaged over 8 healthy volunteers.

7.7 Diffusion decays computed using Tanner and Karger theory for $\tau_{\mathrm{i}}=17 \mathrm{~ms}$

7.8 Diffusion decays computed using Tanner and Karger theory for $\tau_{\mathrm{i}}=100 \mathrm{~ms}$.

7.9 The experimental diffusion decays from the diffusion time dependence study on human brain in vivo averaged over 8 volunteers.

I.1. Flow chart for the $N N L S$ algorithm without regularization .256

I.2. Flow chart for the $N N L S$-diff based on the $N N L S$ algorithm with regularization. .257

II.1 The TRSE sequence timing for $b$-value and diffusion time evaluation .252 


\section{Symbols}

MRI

Magnetic Resonance Imaging

DWI Diffusion Weighted Imaging

DTI Diffusion Tensor Imaging

EPI Echo Planar Imaging

TRSE Twice Refocusing Spin Echo

PGSE Pulsed Gradient Spin Echo

SDE Sequence Development Environment

IDEA .Integrated Development Environment and Application ICE Image Calculation Environment SBB Sequence Build Block $\mathrm{T}_{1}$ spin-lattice relaxation time $\mathrm{T}_{2}$ spin-spin relaxation time

D. free diffusion coefficient $\eta$ viscosity $\tau_{\mathrm{D}}$ diffusion time ADC, $\mathrm{D}$ apparent diffusion coefficient $\operatorname{Tr}(\mathrm{D})$ .trace of diffusion tensor $\gamma$. gyromagnetic ratio $\mu$. magnetic moment

XX 
$\omega$. angular frequency

$\omega_{0}$ Larmor frequency

RF. .radio frequency

$\mathrm{B}_{0}$ ..static magnetic field

$\mathrm{B}_{1}$ RF magnetic field

T. .absolute temperature

$\Delta \mathrm{E}$ .absorbed RF energy

$\mathrm{M}_{0}$ bulk nuclear magnetization

H. Hamiltonian NEX. number of averages

TR .repetition time TE. .echo time $\mathrm{k}_{\mathrm{x}}, \mathrm{k}_{\mathrm{x}}$ $\mathrm{k}$-space spatial frequency G. constant magnetic field gradient amplitude $\mathrm{g}(\mathrm{t})$ ..time dependent magnetic field gradient amplitude $<\mathrm{r}^{2}>$ mean squared molecular net displacement $\mathrm{J}(\mathbf{)}$ particle flux $c()$ number of particles per volume $\mathrm{P}\left(\mathrm{r}_{0} \mid \mathrm{r}, \mathrm{t}\right)$ probability distribution function of the particles initially at position $\mathbf{r}_{0}$ and moving to $\mathbf{r}$ in the time interval $t$ f. fraction of the diffusion components b. b-value or b-factor for diffusion weighted imaging S(b) diffusion signal intensity

$\mathrm{xxi}$ 

time interval between dephasing and rephasing gradients in DWI

$\delta$ gradient duration in DWI

$\mathrm{t}_{\mathrm{r}}$ .ramp time of the gradients

Mom. .gradient moment (area)

$\Delta \phi$ ..phase shift due to magnetic field inhomogeneity

$\mathrm{T}_{\mathrm{e}}$ .time between subsequent gradient echoes

FOV field of view

$\Delta \mathrm{r}_{\mathrm{pe}}$ change of position in the phase-encoding direction SNR signal to noise ratio $\mathrm{R}_{\mathrm{e}}$ equivalent series resistance due to dielectric loss $\tau$ loss factor

$\mathrm{L}$ coil inductance

$\mathrm{C}_{\mathrm{d}}$ distributed capacitance

$\mathrm{R}$ coil resistance

$\mathbf{R}_{\mathrm{m}}$ effective resistance due to inductance loss $\rho$ resistivity

$$
\Delta \mathrm{v}
$$
bandwidth of the signal

$\Delta \mathrm{V}$. imaging volume

$\mathrm{P}_{\mathrm{M}}(\mathrm{M} \mid \mathrm{A})$ magnitude distribution function $\mathrm{M}, \mathrm{A}$ measured and true magnitude image signals $\mathrm{I}_{0}(), \mathrm{I}_{1}()$ ..zeroth and first order modified Bessel function of the first kind $\mathrm{A}_{\mathrm{R}}, \mathrm{A}_{\mathrm{I}}$ real and imaginary signals $N N L S$ Non-negative least squares

xxii 

an unknown element of the spectrum of data

$\omega_{l}$ dual vector in $N N L S$

$\mu$ regularizer parameter

$\chi^{2}$ .chi-square of the statistics

$\sigma$ standard deviation ROI .region of interest

$\mathrm{D}_{\mathrm{s}}, \mathrm{D}_{\mathrm{f}}$ slow and fast apparent diffusion coefficient

$\mathrm{D}_{\mathrm{i}}, \mathrm{D}_{\mathrm{e}}$ intracellular and extracellular diffusion coefficient

$f_{i}, f_{e}$ intracellular and extracellular volume fraction

$\mathrm{D}^{\mathrm{a}}{ }_{\mathrm{i}}, \mathrm{D}_{\mathrm{e}}^{\mathrm{a}}$ restricted intracellular and extracellular diffusion coefficient

$\mathrm{D}_{\mathrm{i}}^{\prime}, \mathrm{D}_{\mathrm{e}}^{\prime}$ ..apparent intracellular and extracellular diffusion coefficient after exchange

$\mathrm{f}_{\mathrm{i}}^{\prime}, \mathrm{f}_{\mathrm{e}}$. .apparent intracellular and extracellular volume fraction after exchange

$\lambda$ extracellular tortuosity

$\mathrm{k}_{\mathrm{ie}}, \mathrm{k}_{\mathrm{ei}}$ intracellular to extracellular and extracellular to intracellular exchange rate of water molecules

$\tau_{\mathrm{i}}, \tau_{\mathrm{e}}$ .exchange time of water molecules from intracellular to extracellular and from extracellular to intracellular space

q. q-value $\left(\gamma^{2} g^{2} \delta^{2}\right)$ 


\section{CHAPTER 1}

\section{Introduction}

\subsection{Review of MRI}

The Nuclear Magnetic Resonance (NMR) phenomenon was not clearly established until Bloch et. al. [1] and Purcell et. al. [2] separately measured NMR absorption in bulk materials in 1946. In the decades that followed this discovery NMR was primarily an analytical tool for chemists and physicists probing chemical structure and reaction processes. In 1973, Lauterbur modified a NMR spectrometer to provide spatially encoded signals through linear variation of the magnetic field [3]. He produced the first images of an inhomogeneous object, thereby providing the first demonstration of Magnetic Resonance Imaging (MRI). The development of clinical MRI was reported soon afterwards by Mansfield et. al. in 1976 [4]. After the creation of the first superconducting whole body imager, MRI soon become recognized as a technique for producing images with superior soft tissue contrast. 


\begin{tabular}{|c|c|c|c|c|}
\hline Nucleus & Spin & $\gamma / 2 \pi(\mathrm{MHz} / \mathrm{T})$ & $\begin{array}{c}\text { Natural } \\
\text { abundance }(\%)\end{array}$ & $\begin{array}{c}\text { Relative sensitivity } \\
\text { at constant field }\end{array}$ \\
\hline${ }^{1} \mathrm{H}$ & $\frac{1}{2}$ & 42.57 & 99.98 & 1 \\
${ }^{2} \mathrm{H}$ & 1 & 6.54 & 0.015 & $2.4 \times 10^{-6}$ \\
${ }^{13} \mathrm{C}$ & $\frac{1}{2}$ & 10.71 & 1.108 & $2.5 \times 10^{-4}$ \\
${ }^{14} \mathrm{~N}$ & 1 & 3.08 & 99.63 & $1.9 \times 10^{-3}$ \\
${ }^{19} \mathrm{~F}$ & $\frac{1}{2}$ & 40.05 & 100 & $8.5 \times 10^{-1}$ \\
${ }^{23} \mathrm{Na}$ & $\frac{3}{2}$ & 11.26 & 100 & $1.3 \times 10^{-1}$ \\
${ }^{31} \mathrm{P}$ & $\frac{1}{2}$ & 17.23 & 100 & $8.3 \times 10^{-2}$ \\
${ }^{33} \mathrm{~S}$ & $\frac{3}{2}$ & 3.27 & 0.76 & $3.3 \times 10^{-5}$ \\
${ }^{35} \mathrm{Cl}$ & $\frac{3}{2}$ & 4.17 & 75.53 & $6.3 \times 10^{-3}$ \\
${ }^{39} \mathrm{~K}$ & $\frac{3}{2}$ & 1.99 & 93.1 & $1 \times 10^{-3}$ \\
& & & & \\
\hline
\end{tabular}

Table 1.1. NMR sensitivities for nuclei of biomedical interest [5].

There are a few factors which affect the suitability of a given nucleus for NMR measurements. These include the percentage of atoms having NMR visible nuclei, the amount of signal given off by the nucleus and the density of the nucleus in the material being studied. In Table 1.1 nuclei which are of biological significance are listed along with their spin number, resonance frequency, natural abundance and relative sensitivity [5]. Natural abundance is a critical factor in determining the suitability of isotopes for magnetic resonance (MR) measurements and it is specified as the percentage of the total number of a given nucleus that occurs as the isotope being considered. Most MR imagers are designed to observe the hydrogen nucleus, ${ }^{1} \mathrm{H}$, because of its relative abundance in the body and the relative strength of the ${ }^{1} \mathrm{H} \mathrm{MR}$ signal. 
An MR image represents the relative response of specific nuclei to absorbed radio frequency (RF) energy. Thus, an MR image is often a tomographic map of the distribution of ${ }^{1} \mathrm{H}$ nuclei in the imaged sample. However, MR image contrast is also influenced by other physical properties such as $T_{1}$ and $T_{2}$ relaxation. The loss of energy absorbed from the RF electromagnetic radiation by the ${ }^{1} \mathrm{H}$ nuclei to their surroundings is characterized by $\mathrm{T}_{1}$ relaxation. This is normally called the spin-lattice or longitudinal relaxation time. $T_{2}$, the spin-spin or transverse relaxation time, characterizes the signal decay associated with energy exchange within the nuclear spin system. The relative appearance of normal versus pathologic tissue can be modified by altering the acquisition protocol parameters to emphasize different anatomical features, assuring exceptional tissue contrast across a wide range of tissue types.

Although early MRI hardware and software greatly benefited from existing computed tomography (CT) scanner technology, MR differs from CT in many ways. MR imaging offers the unique ability to acquire images in any orientation without repositioning the patient. Besides, MR can provide chemical information not measurable with conventional radiography or ultrasonography.

MRI uses a combination of a static magnetic field, imposed local variations of the magnetic field (gradients) to encode spatial information and RF pulses, applied through an RF pulse generation system to generate an MR signal from the tissue. In order to reconstruct an MR image, it is necessary to encode the emitted signal such that its components can be related to the spatial position of the nuclei which contribute to them. The gradient fields are used in the selection of an image slice in one direction and then to spatially encode the MR signal emanating from that slice in the two other orthogonal directions, the so called frequency and phase encoding directions. This combination of gradients is the basis for the two-dimensional Fourier Transform (2D-FT) techniques [4] commonly used in MR. 
In MR imaging, pulse programs, or pulse sequences, control the relative contribution of proton density, $T_{1}$ and $T_{2}$ relaxation, diffusion, etc. by adjusting the RF pulses, gradients and timing of data acquisition. Thus, two functional parts of a typical MR pulse sequence are spin preparation, where the image contrast is set up, and signal detection. Improvements in MR imaging hardware and software have allowed fast MR imaging modalities such as EPI (Echo Planar Imaging), TSE (Turbo Spin Echo) and GRASE (Gradient and Spin Echo) to be developed with scan times which can be less than a second in duration, in some cases.

Clinical MRI technology demonstrates a great deal of maturity but still continues to evolve and improve. The diagnostic utility of MRI in neurology is well established. Additionally, some specific clinical application areas of MRI, including MR Angiography (MRA), cardiac imaging, MR spectroscopy (MRS) and Functional MRI (fMRI), have made MRI of tremendous interest for research studies. MRI has been rapidly accepted because of its versatility, sensitivity and specificity as a diagnostic modality.

\subsection{Review of Diffusion in MR}

During the early development of MRI, diffusion did not play a significant role. The very first measurements of self-diffusion coefficients using MR were done by Hahn in 1953 and further developed by Carr and Purcell in 1961 [6,7]. By eliminating some of the experimental limitations of the basic spin echo technique Tanner et. al.[8] developed the pulsed-gradient spin echo (PGSE) method in 1968 to study restricted self-diffusion in colloidal systems. In the mid-1980's Le Bihan et. al. [9] produced the first diffusion weighted MR images by introducing encoding of molecular diffusion effects into MRI with bipolar magnetic field gradient pulses as introduced to NMR pulse sequence by Stejskal and Tanner in 1965 [10]. Since the early 1990's diffusion weighted 
imaging (DWI) has advanced to play an important role both in clinical MRI and in research. Although diffusion is not strictly an MR parameter, it is nevertheless an important tissue characteristic that can be measured using MR techniques.

Self-diffusion refers to the random translational motion of molecules or ions (i.e. Brownian motion) driven by the thermal energy of the system. Since reacting species must collide before they can react, diffusion is responsible for all chemical reactions as the most fundamental form of transport. Translational diffusion is also related to molecular size by the Stokes-Einstein equation:

$$
\mathrm{D}=\mathrm{kT} / \mathrm{f},
$$

where $\mathrm{k}$ is Boltzmann's constant, $\mathrm{T}$ is the temperature, $\mathrm{D}$ is the self-diffusion coefficient of the freely diffusing molecules in the liquid and $\mathrm{f}$ is the friction coefficient which, for the simple case of a spherical particle, can be given by:

$$
\mathbf{f}=6 \pi \eta r_{s}
$$

where $r_{s}$ is the effective hydrodynamic radius (i.e. Stokes' radius) and $\eta$ is the viscosity of the solution. Diffusion consequently can provide information on the interactions and shape of the diffusing molecule $[11,12]$. The diffusion coefficient for water molecules in tissues is found to be about one third the self-diffusion coefficient of pure water. This has been attributed to the complex heterogeneous structure of tissue which causes diffusion to deviate considerably from the predictions of the simple Einstein equation:

$$
<\mathrm{r}^{2}>=6 \mathrm{D} \tau_{\mathrm{D}},
$$

where $\left\langle\mathrm{r}^{2}\right\rangle$ is the mean squared molecular displacement and $\tau_{\mathrm{D}}$ is the diffusion time.

The success of diffusion MRI is deeply rooted in the powerful concept that during their random displacement, molecules probe tissue structure on the microscopic scale well beyond the usual image resolution. During typical diffusion times of about $50 \mathrm{~ms}$, water molecules in brain tissue, for example, move over distances of the order of $10 \mu \mathrm{m}$ on average, randomly interacting 
with many tissue components such as cell membranes, fibers and macromolecules. The displacement distribution of water molecules observed within a voxel of several $\mathrm{mm}^{3}$ in diffusion MR provides unique information about the structure and geometric organization of the tissue. Diffusion images provide access to both superficial and deep organs at high resolution without interfering with the diffusion process itself as an intrinsic physical process (totally independent of the MR effect or the magnetic field).

The diffusion coefficient in tissue as measured in MRI is often called the "apparent diffusion coefficient" as opposed to the "true" diffusion coefficient or self-diffusion coefficient, D. In this thesis the apparent diffusion coefficient will be designated by $D_{a}$ but it is also frequently called $\mathrm{ADC}$ in the literature. $\mathrm{D}_{\mathrm{a}}$ is often influenced by direction (i.e. anisotropy), tissue structure and cell membranes with impeded permeability to water (i.e. restricted diffusion) and the exchange of water molecules between different tissue compartments. Measurement of diffusion as a function of time can, in principle, yield information about tissue microstructure, permeability, and the molecular environment, and may provide a useful basis for tissue characterization. The PGSE NMR technique is the classic method for measuring the selfdiffusion coefficient with MR.

DWI is now a routine clinical technique for detecting brain pathologies such as the early stages of ischemia $[13,14]$. Changes in $D_{a}$ of water molecules can be used to characterize the status of brain tissue. Many experimental and theoretical studies have been made to interpret the underlying mechanism of the diffusion contrast and its changes during a brain infarct and other pathologies. According to Anderson et. al. [15], the $D_{a}$ decrease of about $40 \%$ during ischemia is mainly caused by the shift of interstitial water into the intracellular space (i.e. cell swelling). The changes in the extracellular volume fraction and its tortuosity factor, $\lambda$, as measured by iontopherosis (i.e. by examining the concentration of ions introduced into the extracellular space 
as a function of time) have not been sufficient to quantify the absolute value of $D_{a}$ and its changes during ischemia $[16,17,18]$. Increased cell membrane permeability may also be a reason for the observed decrease in $D_{a}$.

The study of diffusion in tissues is also complicated by the fact that tissues are normally composed of several different cell populations (e.g. neurons and glial cells). The locally different environments can introduce microscopic and macroscopic anisotropy into the molecular water diffusion. In order to understand diffusion in such a system one has to study various tissue parameters such as intracellular and extracellular diffusion coefficients, the exchange time, extracellular tortuosity, and intracellular diffusion restriction. However, even if all of this information is available, it is still difficult to relate the results of diffusion measurements from different tissue locations to the state of the tissue (i.e. whether it is healthy or not).

Experiments which measure $\mathrm{D}_{\mathrm{a}}$ in vivo are frequently reported as showing monoexponential decays which can be fit to the equation

$$
\ln [\mathrm{A}(\mathrm{b}) / \mathrm{A}(0)]=-\mathrm{bD}_{\mathrm{a}},
$$

where $b$, an important factor in diffusion experiments, is proportional to the square of the diffusion gradient amplitude and the sequence timing. $\mathrm{A}(\mathrm{b})$ is the measured MR signal amplitude as a function of $b$. This can be seen as an advantage, as it implies that over a wide range of experimental conditions the diffusion characteristics in tissue can be described by a single decay constant. However, significant deviations from mono-exponential behavior are observed when the decay is carefully measured over a wide range of $b$ values and/or diffusion times. One possible source of non-exponentiality could be structures that restrict the diffusion of water molecules (e.g. cell membranes). The effects of restricted diffusion on the measured decay change with the diffusion time of the measurement. 
Diffusion studies show that the distance a molecule diffuses in one direction in space may or may not be the same as in some other direction. Clearly, in a pure liquid where there are no hindrances to diffusion or in a sample where the barriers are not coherently oriented, diffusion is the same in all directions and is termed isotropic diffusion. However, if diffusion depends on direction, such as in brain tissue where the axonal membranes act as highly oriented barriers, it is termed anisotropic diffusion. Tissues in which diffusion anisotropy has been reported in vivo include human spinal cord, cat optic nerve, rat trigeminal nerve and the corpus callosum [19-24]. In these tissues the anisotropy is attributed to axonal fibers which are long and narrow. $\mathrm{D}_{\mathrm{a}}$ for diffusion parallel to the axonal direction, $\mathrm{D}_{\text {all }}$ has been reported to be between $0.9 \times 10^{-3} \mathrm{~mm}^{2} / \mathrm{s}$ and $1.3 \times 10^{-3} \mathrm{~mm}^{2} / \mathrm{s}$. For diffusion perpendicular to the axonal direction the $\mathrm{D}_{\mathrm{a}}$ value, called $\mathrm{D}_{\mathrm{a}}$, is reported to be between $0.3 \times 10^{-3} \mathrm{~mm}^{2} / \mathrm{s}$ and $0.5 \times 10^{-3} \mathrm{~mm}^{2} / \mathrm{s}$. For these tissues the anisotropy, defined as $\eta_{\mathrm{a}}=\mathrm{D}_{\mathrm{all}} / \mathrm{D}_{\mathrm{a} \perp}$, is typically between 2.0 and 3.0.

To characterize anisotropic diffusion a single parameter (i.e. D or $\mathrm{D}_{\mathrm{a}}$ ) is no longer enough. Instead the diffusion tensor, $\mathbf{D}$, must be evaluated. $\mathbf{D}$ is a mathematical construct that describes the properties of an ellipsoid. Since $\mathbf{D}$ is a $3 \times 3$ symmetric tensor:

$$
\mathbf{D}=\left[\begin{array}{lll}
D_{x x} & D_{x y} & D_{x z} \\
D_{x y} & D_{y y} & D_{y z} \\
D_{x z} & D_{y z} & D_{z z}
\end{array}\right]
$$

there are six elements of the tensor that need to be determined. This requires a minimum of six measurements. For some situations the variation of image contrast caused by diffusion anisotropy can be used to good advantage but for others it is a problem since it can obscure pathology. However, since the trace of the tensor, $\operatorname{Tr}(\mathbf{D})=\mathrm{D}_{\mathrm{xx}}+\mathrm{D}_{\mathrm{yy}}+\mathrm{D}_{\mathrm{zz}}$, is rotationally invariant, an image of $\operatorname{Tr}(\mathbf{D})$ does not show the effects of anisotropy and can be very useful for displaying pathology in the presence of diffusion anisotropy. Clinically, properties derived from the 
diffusion tensor, like the trace, which reflects overall water content, have been used successfully to evaluate brain ischemia [27-32].

Diffusion anisotropy in white matter, which originates from the specific organization of axonal fibers into bundles [12, 33, 34], can be characterized by diffusion tensor imaging (DTI) as introduced by Basser et. al. [35, 36, 37]. This can provide exquisite details about the tissue microstructure. In DTI, D is evaluated for each voxel from measurements of diffusion in several directions $[24,38]$. The eigenvalues of $\mathbf{D}$, which correspond to the diffusion coefficients along the major and minor axes of the diffusion ellipsoid for the voxel, are then determined by diagonalizing the tensor. From the eigenvalues the anisotropy can be quantified using various parameters such as fractional anisotropy (FA), relative anisotropy (RA), and others [35-37]. One can then generate anisotropy maps that show the spatial variation of the anisotropy which can be directly related to the axonal fiber orientation in the tissue. Many studies have been published dealing with optimization of the MRI sequence for diffusion tensor imaging, the processing and display of DTI data and its potential applications.

Diffusion anisotropy is significantly correlated with age in children [39-41]. This is consistent with increasing neuronal cell density and myelination resulting in reduction in the size of the extracellular space of the brain tissue. Anisotropy parameters have been used to evaluate white matter diseases such as Multiple Sclerosis (MS) [42, 43, 44], age-related changes [45], schizophrenia [46], Alzheimer's disease [47], and epilepsy [48]. The most advanced application of DTI is certainly that of fiber tracking in the brain, which in combination with functional MRI could open a window to the important issue of connectivity [12]. DTI has also been used to demonstrate subtle abnormalities in a variety of brain diseases and is currently becoming part of many routine clinical protocols such as detection of acute stroke, MS, dyslexia, and 
schizophrenia. Other studies have also probed the potential of diffusion tensor MR imaging of brain tumors [49] and migraine [50].

MR fiber tracking or tractography is a technique for studying the orientation of anisotropic tissues by traversing a continuous path of greatest diffusivity in $3 \mathrm{D}$ image space from an initial set of points based on similarities between neighboring voxels in the shape (quantitative anisotropy measures) and orientation (principle eigenvector map) of their diffusion ellipsoid [52]. The study of axonal fiber bundles in neuronal networks is important for the understanding of normal and pathological processes that can affect brain functions. These studies may play an important role in treatment planning for neurosurgery or dose sculpting in radiation therapy by adding this to the diagnostic process available to the oncologist.

Although the vast majority of MR tractography studies have focused on tracking significant white matter tracts in the brain, this technique can also be applied to other anisotropic tissues such as peripheral nerves, the myocardium, ligaments, tendons, and skeletal muscles [53]. In addition to the more immediate clinical impact of evaluation of brain tumors and acute stroke, white matter tractography combined with DTI has also been used to study other things such as adrenoleukodystrophy (a genetic disorder involving the white matter), the adrenal cortex [54], the testes [54], anoxic brain injury in premature infants [55] and infant holoprosencephaly (a congentional brain malformation related to errors in ventricle induction) [56].

\subsection{Clinical Applications}

Diffusion imaging for neuroradiological evaluation of acute stroke has attained significant clinical relevance [57-62]. Diffusion is exquisitely sensitive to cellular geometric features such as cell size (reduced diffusion in cytotoxic edema) and orientation. This leads to such important 
clinical applications as the detection of multiple sclerosis lesions [63], tumors [64] and hippocampal sclerosis in epilepsy [65] as well as the diagnosis of Creutzfeld-Jakob disease [66], schizophrenia [67] and various brain injuries [68]. The kinds of disease that lead to structural damage on the cellular scale can change the restricted diffusion behavior around axons and neurons. Thus $\mathrm{D}_{\mathrm{a}}$, if measured accurately, relates to the degree of cell damage, demyelination and axonal loss [69-72]. Some pathological models have been examined based on the $\mathrm{D}_{\mathrm{a}}$ reduction in tissue, e.g. following the time course in focal and global ischemia [73 -77], cell swelling in status epilepticus in rats [109] and the induction of cortical spreading depression in the rat brain $[79,80,81]$.

Brain connectivity is one of the important potential applications of DTI. It can be used, in combination with functional MRI to establish how activated foci are linked together through networks $[82,83]$. DTI also has promising applications in brain development investigations, helping in neurosurgical planning and the evaluation of white matter disease, e.g. in Krabbe's disease [93], cerebral adrenoleukodystrophy [94], AIDS [95], hypertensive encephalopathy [96], ischemic leukoariosis [97 ] and Alzheimer's disease [47].

Outside the brain diffusion has been more difficult to use successfully because of the occurrence of respiratory motion artifacts and short $\mathrm{T}_{2}$ values of body tissues, which require shorter TE's than in the brain and are thus less sensitive to diffusion gradient pulses. In spite of these complications a potential for tissue characterization has been shown in the extremity muscles $[84,85]$, the spine $[86,87]$, the breast $[88,89]$, the kidney and the liver $[90,91]$. Providing data on heart contractility is a tremendous potential of myocardium DTI [92] but still very challenging to perform in vivo due to heart motion. 


\subsection{Motivation}

The real mechanism of diffusion behavior in human brain tissue and its link to the tissue microstructure is still largely unknown. To provide a better understanding of the overall water diffusion characteristics for a heterogeneous system such as the white matter of the brain a full understanding of the diffusion decay and the various processes that contribute to it is required. Diffusion measurements are conventionally reported in terms of a single global parameter, the apparent diffusion coefficient, often using only 2 or 3 data points to define the diffusion signal attenuation assuming that the signal decay is mono-exponential. However, the diffusion behavior of tissue is affected by a great number of things such as the existence of sub regions (intracellular, interstitial and intravascular), water exchange among the regions, axon orientations, cell wall permeability, volume fractions, extracellular tortuosity and others. Consequently, the diffusion decay in the tissue cannot be expected to be a mono-exponential decay.

To better understand this behavior, restricted diffusion as well as exchange behavior of healthy human brain in vivo was studied for this thesis. Diffusion decays were accurately measured over an extended range of b-values up to $12,500 \mathrm{~s} / \mathrm{mm}^{2}$ for human brain over a range of diffusion times. Previous MR diffusion imaging experiments using high b-values up to 1000 $\mathrm{s} / \mathrm{mm}^{2}$ have shown non mono-exponential diffusion signal decays [100-104], but none of these studies considered the effects of Rician noise bias on the signal decay. To obtain reliable quantitative diffusion information from the signal decays the noise bias must be properly dealt with. In this study, we propose a new more reliable technique for Rician noise bias correction of the diffusion decay. We also take care to avoid other systematic errors such as artifacts and eddy current effects to make sure we are measuring the diffusion decay accurately. 
Performing diffusion weighted MRI experiments on human brain tissue in vivo at very large b-values allows us to sensitize the intracellular water molecules in order to understand the restricted diffusion behavior. Measurements over a range of diffusion times are also useful for better understanding of the exchange behavior.

It should be noted that the real challenge is to find the explanation for changes in the apparent diffusion coefficient that occur in ischemia and other pathologies. The approximately $40 \%$ reduction in $\mathrm{D}_{\mathrm{a}}$ recorded within minutes of an ischemic insult has attracted much attention. Changes in tissue properties that could affect the diffusion behavior include an increase in the extracellular tortuosity and cell swelling. To explain this behavior in pathological situations requires an accurate understanding of the diffusion behavior in healthy human brain in vivo.

It has become clear that the interaction between the intracellular and extracellular compartments is complex with the respective diffusion coefficients, the volume fractions of the compartments, the cell membrane permeability, the compartmental exchange time, and the extracellular tortuosity all playing an important role. The accurate determination of these parameters is essential for verification with theoretical models [105-107].

\subsection{Thesis Outline and Significance}

In this work we study the diffusion signal decay in human brain in vivo for healthy subjects over a very large range of b-values and we determine the diffusion components contributing to the decay after Rician noise bias correction using non-negative least squares analysis. The results show three components in the spectrum of diffusion coefficient data. We also studied the diffusion decay as a function of the diffusion time. The intracellular water signal at large diffusion 
weighting shows characteristics of restricted diffusion as well as exchange between intracellular and extracellular spaces.

Following the introduction of NMR, MRI and diffusion in chapter 1, the background for the whole study is reviewed in chapter 2 of the thesis. This chapter includes MR theory, diffusion theory and their combination followed by the main sources of noise in MR measurements and their effect on diffusion experiments along with a discussion of various artifacts that can affect diffusion measurements. More about noise theory is also presented in later chapters. To study the diffusion signal attenuation curves at high b-values with the hypothesis that the decay is the sum of an unknown number of exponential contributions, for such a heterogeneous system as brain tissue, we needed to develop analysis software which uses the non-negative least squares method rather than the more commonly used non-linear least squares algorithms, such as LevenbergMarquardt technique, for analyzing the diffusion decay curves. The theory of curve fitting methods, with advantages and disadvantages of different methods, is discussed in chapter 2.

A general discussion of the experimental equipment and procedures used for the experiments reported in the thesis is presented in chapter 3. Experimental details for the experiments reported in chapters 4 to 6 are given in the chapters where the respective experiments are discussed.

In chapter 4 , we study the noise in the images and present a new method for correction of the noise bias on the diffusion decay [186]. Obtaining reliable diffusion information from the signal decays is dependent upon this noise correction. A quantitative analysis of the MR-diffusion system with noise estimation in diffusion measurements is included in this chapter. The benefits and limitations of the noise correction schemes are then discussed.

Following the noise correction of the diffusion signal decay, the NNLS-diff, code which was developed for this study, based on the non-negative least squares algorithm, was used for the 
diffusion data analysis of human brain tissue measured for 96 b-values. This program was first tested with simulated data for both the discrete and continuous mode with known data and then validated for some experimental studies such as measurements of diffusion data for a water phantom and $T_{2}$ relaxation data analysis of hyperpolarized xenon experiments. These results are all discussed in chapter 5 .

In order to investigate the effects of diffusion restriction and exchange between the intracellular and extracellular spaces of brain tissue, the diffusion time dependence for human brain was studied $[185,187]$ and discussed in chapter 6 . The 'ep2d-diff' pulse sequence of Siemens was modified to change the diffusion times and diffusion gradient amplitudes for this experiment. The sequence was optimized based on having a range of short diffusion times with high b-values while keeping the bi-polarity of the diffusion gradients in order to avoid eddy current effects as much as possible. Diffusion restriction and exchange behavior for brain tissue is discussed in chapters 6 and 7, based on our diffusion time dependence for 8 healthy human subjects.

Discussion of the whole study is presented in chapter 7. In this chapter, the results are discussed in terms of a number of possibilities and parameters which could affect the diffusion decays. A combination of the restricted diffusion theory of Tanner [8] and the exchange theory of Karger [127] is then applied over a range of different tissue parameter values to compare our experimental results with the analytical results for a human model. 


\section{CHAPTER 2}

\section{Background}

\subsection{MRI Theory}

\subsubsection{Spin states}

Nuclear magnetic resonance (NMR) relies on the magnetic properties of the neutrons and protons which make up atomic nuclei. Just as with electrons, the nuclei possess intrinsic 'spin' associated with their magnetic moment, $\boldsymbol{\mu}$, where the scalar constant of proportionality is known as the gyromagnetic ratio, $\gamma$, and is defined by

$$
\boldsymbol{\mu}=\boldsymbol{S},
$$

where $\mathbf{S}$ is the nuclear spin angular momentum. A simple classical model of the proton as a spinning ball of mass, $\mathrm{m}$, with uniformly distributed charge, e, can be used to show that the gyromagnetic ratio for the proton depends only on the charge and the mass $\left(\frac{\mathrm{e}}{2 \mathrm{~m}}\right)$ of the proton.

The classical equation of motion for a spin in a magnetic field, B, can be derived by setting the torque ( $\boldsymbol{\mu} \times \mathbf{B}$ ) equal to the rate of change of angular momentum 


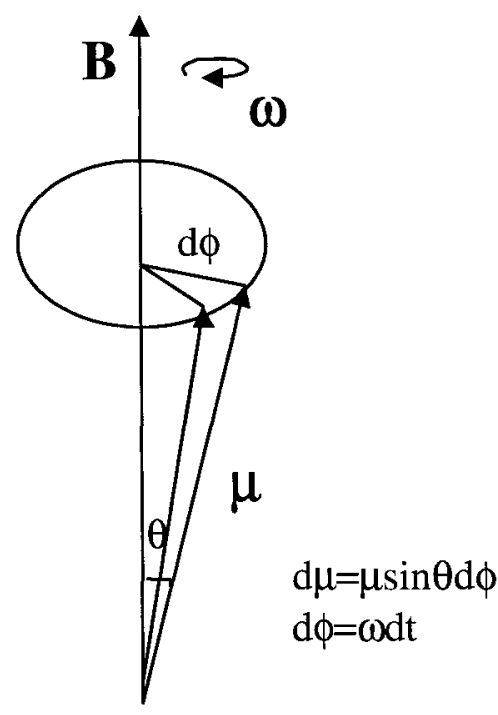

Figure 2.1. Precession of the magnetic moment, $\mu$, about a static magnetic field, $\mathbf{B}$, in the classical view.

$$
\frac{\mathrm{d} \mathbf{S}}{\mathrm{dt}}=\boldsymbol{\mu} \times \mathbf{B}
$$

Therefore

$$
\frac{\mathrm{d} \boldsymbol{\mu}}{\mathrm{dt}}=\boldsymbol{\mu} \times \gamma \mathbf{B}
$$

and

$$
\left|\frac{\mathrm{d} \boldsymbol{\mu}}{\mathrm{dt}}\right|=\gamma \mu \mathrm{B} \sin \theta
$$

From equation (2.3) and figure 2.1, it can be seen that $\mu$ precesses about the magnetic field $\mathbf{B}$ and the angular frequency of the precession is:

$$
\omega=\gamma \mathbf{B}
$$

This is known as the Larmor frequency and the equation is called the Larmor equation. It states that the precessional frequency is directly proportional to the magnetic field [117]. 


\section{Error!}

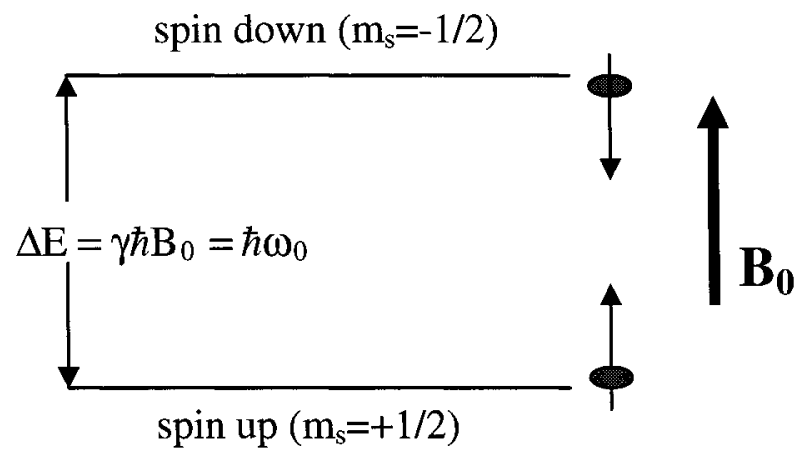

Figure 2.2. The energy states diagram for a proton spin system. Transitions between the two Zeeman energy levels can occur by absorption of electromagnetic energy of frequency $\omega_{0}$.

A quantum mechanical treatment of a spin in a magnetic field is made by setting the Hamiltonian equal to the potential energy of the spin system as follows:

$$
\mathbf{H}=-\boldsymbol{\mu} \bullet \mathbf{B} \text {. }
$$

In a time-independent magnetic field, $\mathrm{B}_{0}$, along the $\mathrm{z}$-axis, the Hamiltonian can be written as

$$
\mathrm{H}=-\gamma \mathrm{B}_{0} \mathrm{~S}_{\mathbf{z}}
$$

and the energy levels for a spin S particle are given by

$$
\mathrm{E}_{\mathrm{m}}=-\gamma \mathrm{B}_{0}\left(\hbar \mathrm{m}_{\mathrm{s}}\right)
$$

where $-\mathrm{S}<\mathrm{m}_{\mathrm{s}}<\mathrm{S}$ and $\mathrm{h}=2 \pi \hbar$ is Planck's constant and $\mathrm{S}$ is the nuclear spin quantum number.

For a spin $1 / 2$ particle such as a proton, only two energy states are allowed $\left(\mathrm{m}_{\mathrm{s}}= \pm 1 / 2\right)$, corresponding to spins parallel $\left(\mid+1 / 2>, E_{+}\right)$and anti parallel $\left(\mid-1 / 2>, E_{-}\right)$to the magnetic field direction as shown in figure 2.2. The two energy states, spin-up and spin-down, with the corresponding wave functions are then: 


$$
\begin{aligned}
& \mathrm{E}_{+}=\hbar \omega_{+}=-\frac{\gamma \hbar \mathrm{B}_{0}}{2}, \Psi_{+}=\Psi_{+} \exp \left(-\mathrm{i} \omega_{+} \mathrm{t}\right) \\
& \mathrm{E}_{-}=\hbar \omega_{-}=+\frac{\gamma \hbar \mathrm{B}_{0}}{2}, \Psi_{-}=\psi_{-} \exp \left(-i \omega_{-} \mathrm{t}\right)
\end{aligned}
$$

and thus the energy separation of the two states, known as the Zeeman splitting, is given by:

$$
\Delta \mathrm{E}=\gamma \hbar \mathrm{B}_{0}=\hbar \omega_{0},
$$

where $\omega_{0}=\omega_{-}-\omega_{+}$, which can be recognized as the classical Larmor precession frequency since $\omega_{0}=\gamma \mathrm{B}_{0}$. For a proton this resonant frequency for energy absorption $\left(v_{0}=\frac{\omega_{0}}{2 \pi}\right)$ is $42.58 \mathrm{~B}_{0}$ $\mathrm{MHz}$ as recorded in table 1.1 of chapter1. A transition from the lower state to the upper state (figure 2.2) occurs by absorption of radio-frequency (RF) energy at the angular frequency $\omega_{0}$ where $\hbar \omega_{0}=\Delta \mathrm{E}$.

\subsubsection{Boltzmann distribution}

In order for a net absorption (or emission ) of energy to occur in the spin system, there must be a difference in population of the two energy levels. The spin system in thermal equilibrium with the lattice is assumed to be distributed amongst the Zeeman energy levels according to the Boltzmann distribution. If there are $n_{+}$and $n$. spins parallel and anti parallel to $B_{0}$ respectively, then the ratio of the populations is written as [116]

$$
\frac{\mathrm{n}_{+}}{\mathrm{n}_{-}}=\exp \left(\frac{\Delta \mathrm{E}}{\mathrm{kT}}\right)=\exp \left(\frac{\gamma \hbar \mathrm{B}_{0}}{\mathrm{kT}}\right)
$$

where $\mathrm{k}$ is Boltzmann's constant and $\mathrm{T}$ is the absolute temperature. The difference in population is evaluated as 


$$
\mathrm{n}_{+}-\mathrm{n}_{-}=\mathrm{N} \frac{1-\exp \left(-\frac{\gamma \hbar \mathrm{B}_{0}}{\mathrm{kT}}\right)}{1+\exp \left(-\frac{\gamma \hbar \mathrm{B}_{0}}{\mathrm{kT}}\right)}=\mathrm{N} \tanh \left(\frac{\gamma \hbar \mathrm{B}_{0}}{2 \mathrm{kT}}\right)
$$

where $\mathrm{N}=\mathrm{n}_{+}+\mathrm{n}_{-}$is the total number of spins. Since the Zeeman energy is very small compared with the quanta of thermal energy (i.e. $\gamma \hbar B_{0}<<k T$ ), the tanh function can be approximated by its argument so that equation (2.12) can be written as:

$$
\frac{\mathrm{n}_{+}-\mathrm{n}_{-}}{\mathrm{N}} \approx \frac{\gamma \hbar \mathrm{B}_{0}}{2 \mathrm{kT}}
$$

This is known as the high temperature approximation. The bulk nuclear magnetization $\mathrm{M}_{0}$, in the high temperature approximation, is given by

$$
\mathrm{M}_{0}=\sum_{\mathrm{i}} \mu_{\mathrm{i}} \approx\left(\mathrm{n}_{+}-\mathrm{n}_{-}\right) \gamma \hbar \mathrm{m}_{\mathrm{s}} \approx \gamma^{2} \hbar^{2} \frac{\mathrm{B}_{0} \mathrm{~N}}{4 \mathrm{kT}}
$$

which shows that $\mathrm{M}_{0}$ is proportional to the external field strength $\left(\mathrm{B}_{0}\right)$ and the square of the magnetic moment $\left(\gamma^{2} \hbar^{2} \mathrm{~m}_{\mathrm{s}}^{2}\right)$.

\subsubsection{The effect of a RF pulse}

Although the angular frequency, $\omega_{0}$, obtained from the Hamiltonian is the same as the classical Larmor frequency, the motion of a quantum mechanical spin in one of the stationary states should not be interpreted as a precession since the probability densities (i.e. $\left\langle\Psi_{i} \mid \Psi_{j}\right\rangle$ ) and also the expectation values of the components of the magnetic moment in a static magnetic field are independent of time and thus $\left\langle\mathrm{S}_{\mathrm{x}}\right\rangle=\left\langle\mathrm{S}_{\mathrm{y}}>=0\right.$ and $\left\langle\mathrm{S}_{\mathrm{z}}>= \pm \frac{1}{2} \hbar\right.$. 

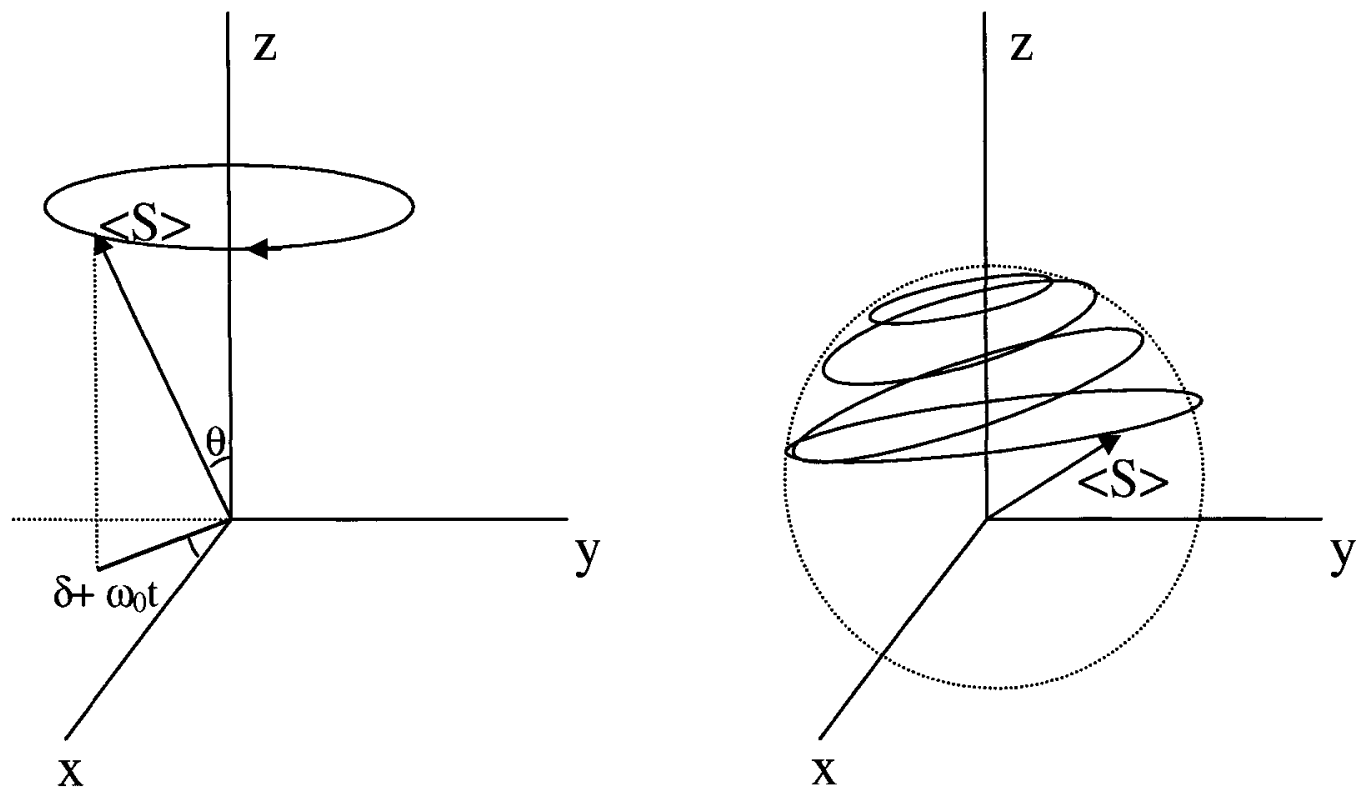

Figure 2.3. a. Free precession of the expectation value of the spin vector $\langle\mathbf{S}\rangle$ for a non-stationary mixture of spin-up and spin-down proton states in a constant magnetic field. b. Motion of the expectation value of $\langle\mathbf{S}\rangle$ from its initial position under the influence of a $\mathbf{B}_{1}$ magnetic field (i.e. $\mathrm{RF})$ at the resonant frequency spiraling down along $\mathrm{z}$ in the laboratory system .

To better understand pulse NMR, it is necessary to follow the motion of spins undergoing time dependent perturbations caused by varying magnetic fields, where the wave functions are a mixture of time dependent spin-up and spin-down states $[116,122]$. For a proton spin such a wave function is given as

$$
\Psi=a \Psi_{+}+b \Psi_{-}=a(t) \psi_{+} e^{-i \omega_{+} t}+b(t) \psi_{-} e^{-i \omega_{-} t}
$$

and the probability density now contains a term of the form:

$$
e^{i\left(\omega_{+}-\omega_{-}\right) t}=e^{-i \omega_{0} t},
$$

which describes a time dependence at the Larmor frequency. 
For a time independent or static magnetic field, $a$ and $b$ will be constant and of the form $\mathrm{a}=\mathrm{Ae} \mathrm{i}^{\mathrm{i} \alpha}$ and $\mathrm{b}=\mathrm{Be}^{\mathrm{i} \beta}$ and we have "free precession" such that the angular momentum, $<\mathrm{S}>$ evolves with time as follows:

$$
\begin{aligned}
& <\mathrm{S}_{\mathrm{X}}>=\frac{\hbar}{2} \sin \theta \cos \left(\delta-\omega_{0} \mathrm{t}\right) \\
& <\mathrm{S}_{\mathrm{y}}>=\frac{\hbar}{2} \sin \theta \sin \left(\delta-\omega_{0} \mathrm{t}\right) \\
& <\mathrm{S}_{\mathrm{z}}>=\frac{\hbar}{2} \cos \theta
\end{aligned}
$$

where $\cos \theta=\mathrm{A}^{2}-\mathrm{B}^{2}$ and $\sin \theta=\frac{1}{2} \mathrm{AB}$ by definition. The $\left\langle\mathrm{S}_{\mathrm{x}}>\right.$ and $<\mathrm{S}_{\mathrm{y}}>$ are evaluated using the ladder operators $S_{+}=S_{x}+i S_{y}$ and $S_{-}=S_{x}-i S_{y}$ which have the effect of raising or lowering the spin state (i.e. $S_{+} \Psi_{+}=\hbar \Psi_{-}, S_{+} \Psi_{-}=0$ and $S_{-} \Psi_{-}=\hbar \Psi_{+}, S_{-} \Psi_{+}=0$, since $E_{+}<E_{-}$). The initial angular offset from the $x$-axis at time $t=0$, as shown in figure $2.3 a$, is $\delta=\beta-\alpha$ and is related to the choice of the constants a and b. A precession of the spin angular momentum, $<\mathbf{S}\rangle$, in the xy plane (i.e. $\sin \theta=1$ ) at the Larmor frequency, i.e. "free precession", can be described through equations (2.17) such that at time $\mathrm{t}=0$ with $\delta=0,<\mathrm{S}_{\mathrm{x}}>=+\frac{1}{2} \hbar,<\mathrm{S}_{\mathrm{y}}>=0$ and clearly $\left\langle\mathrm{S}_{\mathrm{z}}\right\rangle=0$.

A non-stationary state in a time varying magnetic field $\mathbf{B}_{1}(\mathrm{t})$ superimposed on the static field, i.e. $\mathbf{B}(t)=\mathbf{B}_{0}+\mathbf{B}_{1}(t)$, can be explained using the Hamiltonian $\mathrm{H}=\mathrm{H}_{0}+\mathrm{H}_{1}(\mathbf{t})$ where:

$$
\mathrm{H}_{0}=-\boldsymbol{\mu} \cdot \mathbf{B}_{0}=-\gamma \mathrm{B}_{0} \mathrm{~S}_{\mathrm{z}}
$$

and 


$$
H_{1}(t)=-\gamma B_{1}(t) \bullet S
$$

If $\mathbf{B}_{1}$ is resolved into two counter rotating components, it is only the component that rotates in the same sense as the classical Larmor precession (i.e. clockwise) that produces any significant effect. Hence $\mathbf{B}_{1}$ can be written as a rotating field:

$$
\mathbf{B}_{1}(\mathrm{t})=\mathrm{B}_{1}(\mathbf{i} \cos \omega \mathrm{t}-\mathbf{j} \sin \omega \mathrm{t})
$$

and, using equation (2.18),

$$
H_{1}(t)=-\gamma B_{1} S_{x} \cos \omega t+\gamma B_{1} S_{y} \sin \omega t
$$

This can be expressed in terms of ladder operators as defined above:

$$
H_{1}(t)=-\frac{1}{2} \gamma B_{1}\left(e^{i \omega t} S_{+}+e^{-i \omega t} S_{-}\right)
$$

Substituting these into the Schrödinger equation with the general wave function of the nonstationary spin states given by equation (2.15), $a(t)$ and $b(t)$ are found to satisfy $[116,117]$ :

$$
\begin{aligned}
& \frac{d a}{d t}=\frac{i \omega_{1}}{2} b e^{i\left(\omega-\omega_{0}\right) t}, \\
& \frac{d b}{d t}=\frac{i \omega_{1}}{2} a e^{-i\left(\omega-\omega_{0}\right) t},
\end{aligned}
$$

where $\omega_{1}=\gamma B_{1} \ll \omega_{0}=\gamma B_{0}$ and $\omega$ is the RF frequency. If the RF is applied at the resonant frequency, $\omega=\omega_{0}$, these equations simplify to:

$$
\begin{aligned}
& \frac{\mathrm{da}}{\mathrm{dt}}=\frac{\mathrm{i} \omega_{1}}{2} \mathrm{~b}, \\
& \frac{\mathrm{db}}{\mathrm{dt}}=\frac{\mathrm{i} \omega_{1}}{2} \mathrm{a} .
\end{aligned}
$$

It can be verified by substitution back into equation (2.23), that the solution is 


$$
\begin{aligned}
& a(t)=\cos \left(\frac{\omega_{1} t}{2}\right), \\
& b(t)=i \sin \left(\frac{\omega_{1} t}{2}\right),
\end{aligned}
$$

where $|a|^{2}$ and $|b|^{2}$ are the probabilities of finding the spin in the spin-up and spin-down state, respectively. For example, when $\omega_{1} t=\pi$ (i.e. a $180^{\circ}$ RF pulse), $|\mathrm{a}|^{2}=0$ and $|\mathrm{b}|^{2}=1$ which means that the spin has been inverted and a "spin flip" transition has been produced.

It is possible to evaluate the expectation value of the three components of $\langle\mathbf{S}>$ under the influence of an RF pulse by inserting equations (2.24) into the general wave function given by equation (2.15):

$$
\begin{aligned}
& <S_{x}>=\frac{\hbar}{2} \sin \omega_{1} t \sin \omega_{0} t \\
& <S_{y}>=\frac{\hbar}{2} \sin \omega_{1} t \cos \omega_{0} t \\
& <S_{z}>=\frac{\hbar}{2} \cos \omega_{1} t .
\end{aligned}
$$

These equations describe the slow turning over of $\left\langle S_{z}\right\rangle$ and the spiraling down of $\langle\mathbf{S}\rangle$ from its initial position along the z-axis as shown in figure $2.3 \mathrm{~b}$. That is $\langle\mathbf{S}>$, or $<\mu>=\gamma\langle\mathbf{S}\rangle$, obeys the classical equation of motion as shown before in equation (2.3):

$$
\frac{\mathrm{d}<\mu>}{\mathrm{dt}}=<\mu>\times \gamma \mathbf{B}
$$

For a system of $\mathrm{N}$ non-interacting spins, the net macroscopic magnetization, which is given by $\mathbf{M}=\mathrm{N}\langle\mu\rangle$, can be seen to satisfy the classical equation $[115,116]$ 


$$
\frac{\mathrm{d} \mathbf{M}}{\mathrm{dt}}=\mathbf{M} \times \gamma \mathbf{B} .
$$

where $\mathbf{B}=\mathbf{B}_{0}+\mathbf{B}_{1}$.

It can be shown that the time derivative of a vector $\mathbf{M}=M_{x} \mathbf{i}+M_{y} \mathbf{j}+\mathbf{M}_{z} \mathbf{k}$ in the laboratory frame can be related to its time derivative in a coordinate frame rotating about the zaxis with the angular velocity $\omega$ as follows:

$$
\begin{gathered}
\frac{d \mathbf{M}}{\mathrm{dt}}=\left(\mathbf{i} \frac{\mathrm{dM}_{\mathrm{x}}}{\mathrm{dt}}+\mathbf{j} \frac{\mathrm{d} \mathbf{M}_{\mathrm{y}}}{\mathrm{dt}}+\mathbf{k} \frac{\mathrm{dM}_{\mathrm{z}}}{\mathrm{dt}}\right)+\left(\mathbf{M}_{\mathrm{x}} \frac{\mathrm{di}}{\mathrm{dt}}+\mathrm{M}_{\mathrm{y}} \frac{\mathrm{dj}}{\mathrm{dt}}+\mathrm{M}_{\mathrm{z}} \frac{\mathrm{dk}}{\mathrm{dt}}\right), \\
\frac{\mathrm{d} \mathbf{M}}{\mathrm{dt}}=\frac{\partial \mathbf{M}}{\partial \mathrm{t}}+(\omega \times \mathbf{M}),
\end{gathered}
$$

where $\frac{\mathrm{d} \mathbf{i}}{\mathrm{dt}}=(\boldsymbol{\omega} \times \mathbf{i})$ and similarly for $\mathbf{j}$ and $\mathbf{k}$, for a reference frame rotating at $\boldsymbol{\omega}$. Note that in equation 2.28 the notation $\frac{\partial}{\partial t}$ does not mean partial derivative. Instead, it represents the rate of change as observed in the rotating reference frame. Thus, by the combination of equations (2.27) and (2.28) we have:

$$
\frac{\partial \mathbf{M}}{\partial \mathrm{t}}+(\boldsymbol{\omega} \times \mathbf{M})=\gamma \mathbf{M} \times\left(\mathbf{B}_{0}+\mathbf{B}_{1}\right)
$$

For the resonance condition (i.e. $\boldsymbol{\omega}=\gamma \mathbf{B}_{0}$ ), the time derivative of the bulk magnetization in the rotating frame becomes:

$$
\frac{\partial \mathbf{M}}{\partial \mathrm{t}}=\gamma\left(\mathbf{M} \times \mathbf{B}_{1}\right)
$$

Similar to the quantum mechanical proton spin solution given in equation $(2.25), \mathbf{M}$ in the rotating frame is found to be 


$$
\begin{aligned}
& M_{x^{\prime}}=0, \\
& M_{y^{\prime}}=M_{0} \sin \omega_{1} t, \\
& M_{z^{\prime}}=M_{0} \cos \omega_{1} t .
\end{aligned}
$$

In general, if the RF pulse is not at the resonance frequency then equation (2.30) can be written in terms of the "effective" magnetic field, $B_{\text {eff, }}$, as follows:

$$
\frac{\partial \mathbf{M}}{\partial \mathrm{t}}=\gamma\left(\mathbf{M} \times \mathbf{B}_{\mathrm{eff}}\right)
$$

where the magnetization, in this case, precesses about the vector $\mathbf{B}_{\text {eff }}=\mathbf{B}_{1}+\Delta \mathbf{B}_{0}$ and $\Delta \mathbf{B}_{0}$ is a field offset.

While the magnetization precesses about the $\mathbf{B}_{1}$ field, it rotates away from the z-axis toward the xy plane. The angle of rotation, $\alpha$, about the $\mathbf{B}_{1}$ field, the so called "flip angle" or "tip angle", is defined as the angle between $\mathbf{M}$ and the z-axis. An $\alpha$ pulse rotates the magnetization by $\alpha$ degrees from its equilibrium state. For example, a $90^{\circ}$ RF pulse causes the magnetization to flip by $90^{\circ}$ from its equilibrium state into the xy plane, and a $180^{\circ}$ RF pulse causes the magnetization to flip by $180^{\circ}$. The flip angle is a function of the amplitude and the duration of the applied RF pulse. The value of $\alpha$ at the end of an RF pulse is given by

$$
\alpha=\int_{0}^{\tau_{\mathrm{p}}} \omega_{1}(\mathrm{t}) \mathrm{dt}=\int_{0}^{\tau_{\mathrm{p}}} \gamma \mathrm{B}_{1}(\mathrm{t}) \mathrm{dt}
$$

where $\tau_{\mathrm{p}}$ is the pulse duration. In the case of a rectangular pulse

$$
\alpha=\omega_{1} \tau_{p}=\gamma \mathrm{B}_{1} \tau_{\mathrm{p}}
$$


It should be noted that if the area under the $\mathbf{B}_{1}$ field is the same for two different RF pulses, the magnetization will end up in the same orientation after the excitation period even for different RF pulse shapes.

The effect of an RF pulse on the magnetization can be expressed using a rotation operator [167]. For example, a rotation about the $x^{\prime}$ axis (i.e. the $\mathbf{B}_{1}$ field axis in the rotating frame) is given by

$$
\mathrm{R}_{\mathrm{x}^{\prime}}(\alpha)=\left[\begin{array}{ccc}
1 & 0 & 0 \\
0 & \cos \alpha & \sin \alpha \\
0 & -\sin \alpha & \cos \alpha
\end{array}\right]
$$

and the effect of an RF pulse applied to a spin system which is initially at thermal equilibrium, $\mathbf{M}(0)$, is given by

$$
\mathbf{M}(\mathrm{t})=\mathrm{R}_{\mathrm{x}^{\prime}}(\alpha) \mathbf{M}(0)
$$

Using this procedure, the effect of a $90^{\circ}$ pulse can be calculated as:

$$
\begin{gathered}
\mathbf{M}(\mathrm{t})=\mathrm{R}_{\mathrm{x}^{\prime}}\left(\frac{\pi}{2}\right) \mathbf{M}(0)=\left[\begin{array}{c}
0 \\
\mathrm{M}_{\mathrm{z}}^{0} \\
0
\end{array}\right], \\
\text { where } \mathrm{R}_{\mathrm{x}^{\prime}}\left(\frac{\pi}{2}\right)=\left[\begin{array}{ccc}
1 & 0 & 0 \\
0 & 0 & 1 \\
0 & -1 & 0
\end{array}\right] \text { and } \mathbf{M}(0)=\left[\begin{array}{c}
0 \\
0 \\
0 \\
\mathrm{M}^{0}
\end{array}\right]
\end{gathered}
$$




\subsubsection{MR relaxation}

In order to discuss MR sequences, it is necessary to understand the relaxation processes of the spins following the absorption of RF energy since the measured MR signal strength is a function of the relaxation time parameters as well as proton density.

Spin lattice relaxation is the process by which the spin population returns to its equilibrium Boltzmann distribution through an exchange of spin energy with the thermal motions of the molecules (the lattice) of which the nuclear spins are a part. The characteristic time for this process is the so called spin-lattice relaxation time, $T_{1}$. The $z$ component of the bulk magnetization obeys the equation $[116,117]$

$$
\frac{\mathrm{dM}_{\mathrm{z}}}{\mathrm{dt}}=-\frac{1}{\mathrm{~T}_{1}}\left(\mathrm{M}_{\mathrm{z}}-\mathrm{M}_{0}\right)
$$

where $\mathrm{M}_{0}$ is the equilibrium value of $\mathrm{M}$ found by equation (2.14). This process is also sometimes referred to as longitudinal relaxation.

The magnetization in the xy plane decays via $T_{2}$ relaxation through the exchange of energy between nuclei in different spin energy states. This is known as spin-spin relaxation or transverse relaxation. The decay of the transverse component of the magnetization in the rotating frame $M_{x^{\prime} y^{\prime}}$, is given by

$$
\frac{d M_{x^{\prime} y^{\prime}}}{d t}=-\frac{1}{T_{2}} M_{x^{\prime} y^{\prime}} .
$$

The phenomenological equation that governs the behavior of the nuclear magnetization, $M(t)$, in the presence of a magnetic field $\mathbf{B}(t)$ was given by Bloch et. al. [1] in 1946 and is generally written in the rotating frame as: 


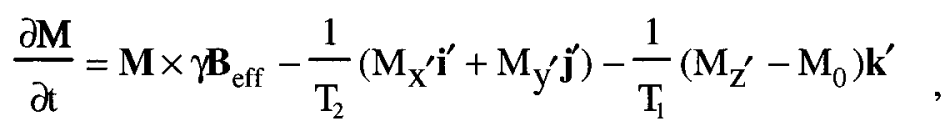

with $\mathbf{B}_{\text {eff }}=\mathbf{B}_{1}+\Delta \mathbf{B}_{0}$ defined as the effective magnetic field as before. The solutions to equations (2.37) and (2.38) are given by:

$$
M_{z}(t)=M_{0}+\left[M_{z}(0)-M_{0}\right] e^{-t / T_{1}}
$$

and

$$
M_{x^{\prime} y^{\prime}}(t)=M_{x^{\prime} y^{\prime}}(0) e^{-t / T_{2}}
$$

respectively.

The application of a $90^{\circ}$ pulse makes the net magnetization precess about $\mathbf{B}_{1}$ (in the rotating frame) toward the xy plane. Based on Faraday's law of electromagnetic induction, a signal in an $\mathrm{RF}$ coil is detected at the end of the excitation pulse and it gradually decreases with time due to the relaxation mechanisms. This signal, the free induction decay (FID), is the most basic form of the transient signal from a spin system after a pulse excitation. The maximum amplitude of a FID signal depends on both the flip angle and the thermal equilibrium value of the net magnetization. The FID signal decay can be characterized by $T_{2}$ if both the sample and the magnetic field are perfectly homogeneous. However, in the presence of $\mathbf{B}_{0}$ inhomogeneity the FID signal decays with a new time constant, $\mathrm{T}_{2}{ }^{*}$, due to the imperfections in the magnetic field and spin-spin relaxation [167].

The relative contribution of processes such as $T_{1}$ and $T_{2}$ relaxation and the proton density can be controlled with the design of the MR pulse sequence by adjusting the RF pulses, the magnetic field gradients and the timing of the data acquisition. 

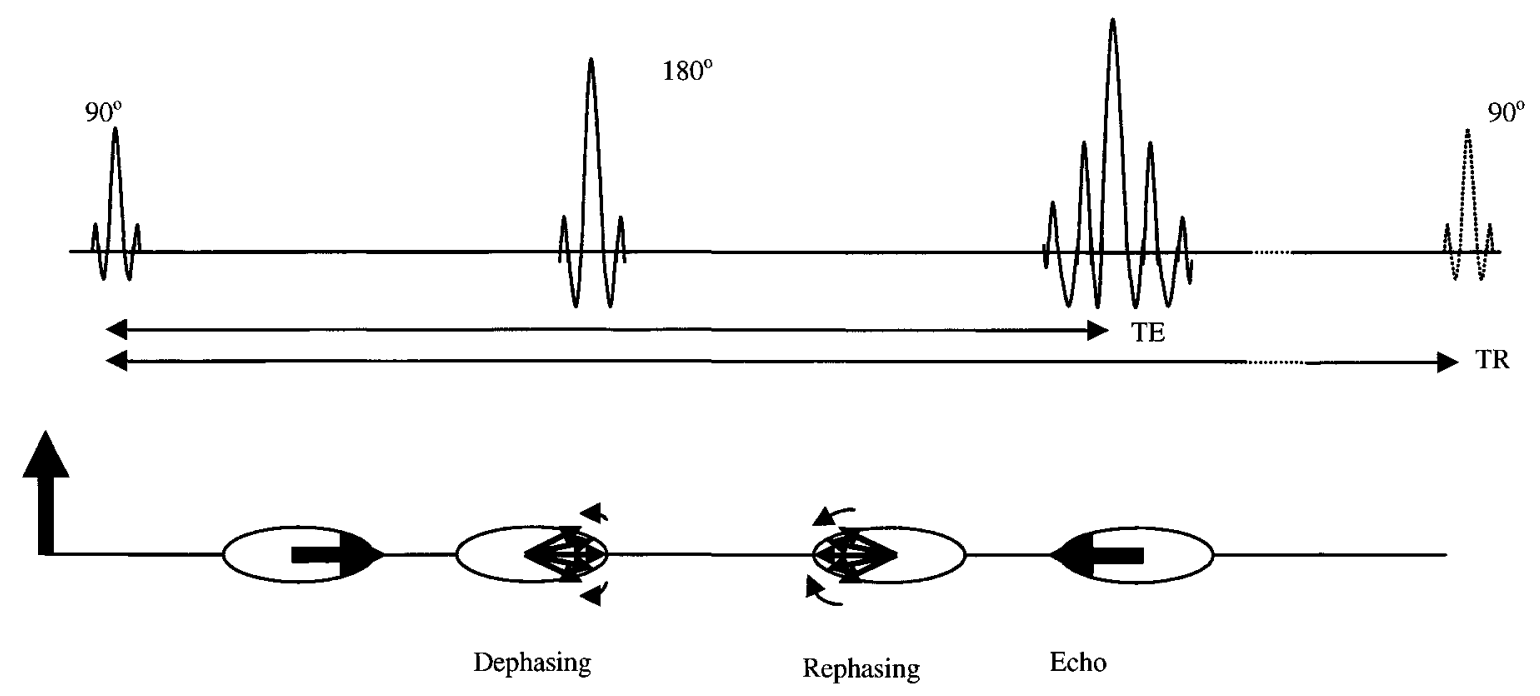

Figure $2.4 \mathrm{a}$. The spin echo sequence timing. $\mathrm{b}$. The associated diagram of the effect of $90^{\circ}$ and $180^{\circ} \mathrm{RF}$ pulses on the magnetization during spin echo generation.

\subsubsection{The Spin Echo pulse sequence}

The combination of a $90^{\circ} \mathrm{RF}$ pulse to flip the magnetization into the horizontal plane and a $180^{\circ}$ refocusing pulse after a time $\frac{\mathrm{TE}}{2}$ gives an RF echo signal at TE, the so called echo time. This is known as a spin echo (SE) pulse sequence. The SE signal can be spatially encoded by applying the transverse readout magnetic field gradient $G_{x}$ during the echo and by repeating the sequence $N_{y}$ times with $N_{y}$ different amplitudes for the phase encoding gradients $G_{y}$. In principle, it is also possible to use the FID following a $90^{\circ}$ RF pulse, however, the FID signal decays with a time constant $\mathrm{T}_{2}^{*}$ due to a combination of spin-spin relaxation and the effect of magnetic field inhomogeneities:

$$
\frac{1}{\mathrm{~T}_{2}^{*}}=\gamma \Delta \mathrm{B}_{0}+\frac{1}{\mathrm{~T}_{2}}
$$


and there is not normally enough time to spatially encode the signal before it has decayed.

Spin-Echo sequences can generate images with $\mathrm{T}_{2}$-weighting by allowing more complete evolution of spin-spin interactions by using a large TE. Typical values for TE and the repetition time, TR, in a $\mathrm{T}_{2}$-weighted SE sequence are $80-100 \mathrm{~ms}$ and $\geq 2000 \mathrm{~ms}$, respectively, depending on the $T_{1}$ and $T_{2}$ values of the tissues being imaged. SE sequences can be used to generate $T_{1}$ and proton density weighted images as well with appropriate choices for the timing parameters. Figures 2.4a and 2.4b show a typical SE sequence along with a diagram showing how the magnetization is affected by the RF pulses during the sequence.

\subsubsection{The Gradient Echo pulse sequence}

A gradient echo (GE) sequence can be generated by applying a gradient in the readout direction for a limited time following the RF excitation pulse followed by another gradient pulse of equal magnitude and duration but opposite polarity. The first gradient pulse causes the spins to precess at different rates leading to dephasing of the spins and signal decay. The second gradient pulse reverses the effect of the first gradient; it rephases the spins and thus produces an echo signal. One significant difference between a gradient echo and a spin echo sequence is the sensitivity to magnetic field inhomogeneities. Unlike the spin echo sequence, gradient echoes do not cancel the effects of magnetic field inhomogeneities, rather this plays a part in the dephasing of the spins.

The gradient echo sequence is compatible with the use of RF pulse flip angles smaller than $90^{\circ}$. This avoids the saturation of the spin system which causes low signal intensity when TR is short relative to $T_{1}$. Due to the time required for restoration of longitudinal magnetization, the flip angle of the RF pulse must be less than $90^{\circ}$ when TR is short and is often selected between $15^{\circ}-$ $45^{\circ}$ to generate an acceptable echo signal with short imaging times. The optimal angle is the Ernst 
angle, $\alpha_{\mathrm{Ennst}}=\mathrm{a} \cos \left(\mathrm{e}^{-\mathrm{TR} / \mathrm{T}_{1}}\right)$. The shorter $\mathrm{TE}$ and $\mathrm{TR}$ of $\mathrm{GE}$ sequences compared with $\mathrm{SE}$ sequences, resulting in a decrease in the total scan time, is one important advantage of GE sequences, depending on the application.

There are a number of reasons, such as improved image quality, reduced motion artifacts, dynamic applications, and "more efficient" MR imaging, which motivated the development of fast imaging sequences. In addition to the effect of TR on the total scan time, there are other factors that can also influence the scan time. These include the number of phase encoding steps, $\mathrm{N}_{\mathrm{y}}$, the number of times each k-space line is measured, NEX, and the "turbo factor", F, which is the number of phase encoding steps measured in each repetition of the sequence. The total scan time, $\mathrm{T}_{\mathrm{s}}$, can be computed from the following formula:

$$
\mathrm{T}_{\mathrm{s}}=\frac{\mathrm{TR} \bullet \mathrm{NEX} \bullet \mathrm{N}_{\mathrm{y}}}{\mathrm{F}}
$$

\subsubsection{The Echo Planar Imaging pulse sequence}

The echo planar imaging (EPI) sequence is the fastest imaging sequence in MR. To image so rapidly requires high-speed trajectories across $k$-space where the spatial frequencies, $k_{x}$ and $k_{y}$ are defined by [121]:

$$
\begin{aligned}
& \mathrm{k}_{\mathrm{x}}=\gamma \int_{0}^{\mathrm{t}} \mathrm{G}_{\mathrm{X}}(\tau) \mathrm{d} \tau, \\
& \mathrm{k}_{\mathrm{y}}=\gamma \int_{0}^{\mathrm{t}} \mathrm{G}_{\mathrm{y}}(\tau) \mathrm{d} \tau
\end{aligned}
$$



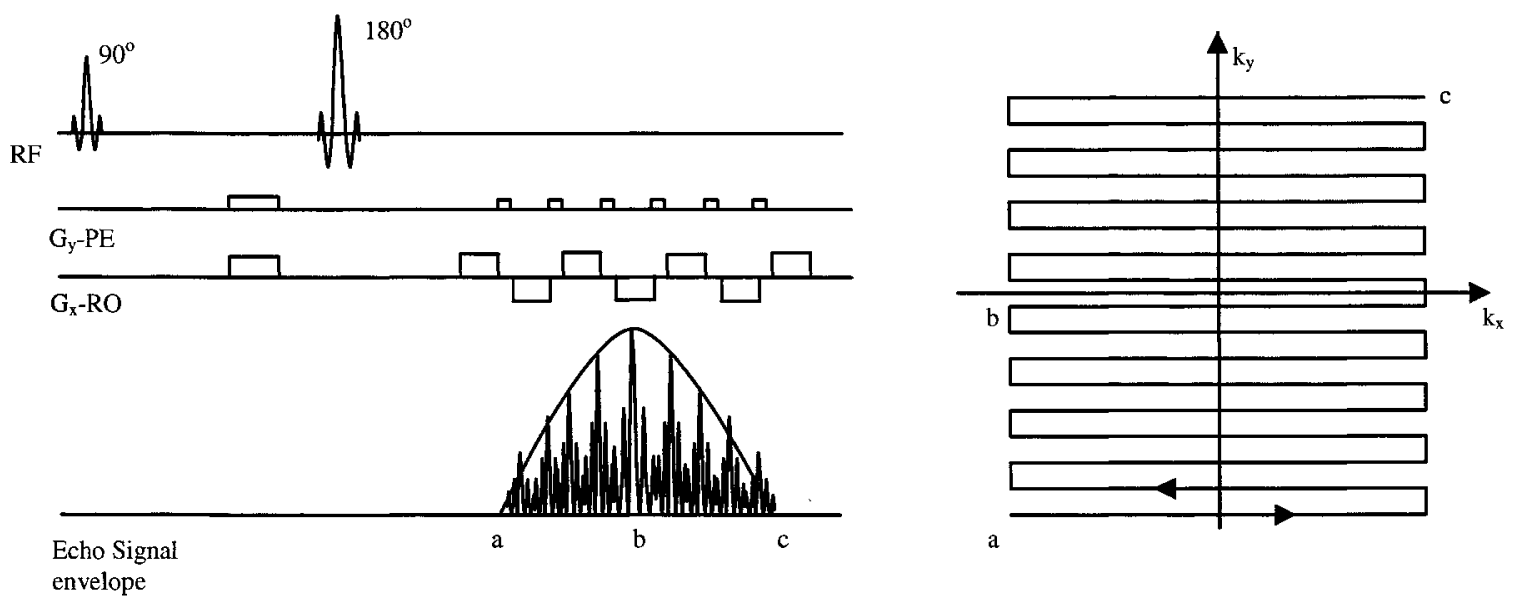

Figure $2.5 \mathrm{a}$. The echo planar imaging (EPI) sequence with a train of readout (RO) and phase encoding (PE) gradient pulses that generate a set of gradient echoes centered around one spin echo. b. the associated k-space trajectory.

and correspond to the frequency and phase encoding gradients, respectively. A typical echo planar pulse sequence and its corresponding k-space trajectory are shown in figure 2.5 . As the figure shows, a spin echo is created by the $90^{\circ}$ and $180^{\circ}$ RF pulses. The rectilinear trajectory through $\mathrm{k}$-space is illustrated in figure $2.5 \mathrm{~b}$. The time points a-c of figure $2.5 \mathrm{a}$ correspond to $\mathrm{k}$ space points a-c in figure $2.5 \mathrm{~b}$. The k-space trajectory and timing are usually designed so that the central phase encoding steps are measured at TE. Despite some limitations of EPI, such as a low signal-to-noise ratio (SNR) and sensitivity to image artifacts (which will be discussed in more detail in section 2.4.3), EPI has developed into a very important MR sequence, particularly when motion is a significant problem. 


\subsection{Diffusion Theory}

\subsubsection{Diffusion equation for free diffusion}

Diffusion is the random process by which the net displacement of particles in fluids due to their random thermal motions occurs [129]. According to Fick's first law of diffusion [133], the flux of the particles, $\mathbf{J}(\mathbf{r}, \mathrm{t})$, is related to the net concentration gradient, $\nabla \mathrm{c}(\mathbf{r}, \mathrm{t})$ by

$$
\mathbf{J}(\mathbf{r}, \mathrm{t})=-\mathrm{D} \nabla \mathrm{c}(\mathbf{r}, \mathrm{t})
$$

where $D$ is the self diffusion coefficient of the particles and $c(r, t)$ is the number of particles per unit volume. Due to conservation of mass, the continuity theorem can be expressed as

$$
\frac{\partial}{\partial \mathrm{t}} \mathrm{c}(\mathbf{r}, \mathrm{t})=-\boldsymbol{\nabla} \cdot \mathbf{J}(\mathbf{r}, \mathrm{t})
$$

where $\frac{\partial}{\partial t} c(\mathbf{r}, t)$ gives the difference between the flux in and flux out at position $\mathbf{r}$ and time $t$. Fick's second law is obtained by inserting equation (2.44) into equation (2.45):

$$
\frac{\partial}{\partial \mathrm{t}} \mathrm{c}(\mathbf{r}, \mathrm{t})=\mathrm{D} \nabla^{2} \mathrm{c}(\mathbf{r}, \mathrm{t})
$$

This is also frequently referred to as the diffusion equation.

The diffusion equation for free diffusion of particles in the absence of a concentration gradient can be developed in terms of $\mathrm{P}\left(\mathbf{r}_{0} \mid \mathbf{r}, \mathbf{t}\right)$, the probability of finding a particle, initially at position $\mathbf{r}_{0}$, at position $\mathbf{r}$ at time $t$, by making the association $\mathrm{P}\left(\mathbf{r}_{0} \mid \mathbf{r}, \mathrm{t}\right) \rightarrow \frac{\mathrm{c}(\mathbf{r}, \mathrm{t})}{\mathrm{N}_{\mathrm{p}}}$ where $\mathrm{N}_{\mathrm{p}}$ is the number of particles in the system. In this case, the diffusion equation can be written as:

$$
\frac{\partial}{\partial \mathrm{t}} \mathrm{P}\left(\mathbf{r}_{0} \mid \mathbf{r}, \mathrm{t}\right)=\mathrm{D} \nabla^{2} \mathrm{P}\left(\mathbf{r}_{0} \mid \mathbf{r}, \mathrm{t}\right)
$$

For three-dimensional diffusion in an isotropic, homogeneous and unbounded system (i.e. 
$\mathrm{P} \rightarrow 0$ as $\mathbf{r} \rightarrow \infty), \mathrm{P}\left(\mathbf{r}_{0} \mid \mathbf{r}, \mathrm{t}\right)$ can be determined using Fourier transforms and is given by [10,11, 122]:

$$
\mathrm{P}\left(\mathbf{r}_{0} \mid \mathbf{r}, \mathrm{t}\right)=\frac{1}{\sqrt{(4 \pi \mathrm{Dt})^{3}}} \exp \left[-\frac{\left(\mathbf{r}-\mathbf{r}_{0}\right)^{2}}{4 \mathrm{Dt}}\right]
$$

This corresponds to a Gaussian distribution of particles, with variance 2Dt, in an infinitely large system where "free diffusion" occurs. It should be noted that $\mathrm{P}\left(\mathbf{r}_{0} \mid \mathbf{r}, \mathrm{t}\right)$ depends only on the net displacement, $\mathbf{r}-\mathbf{r}_{0}$ of the diffusive particle rather than its initial position, $\mathbf{r}_{0}$. This reflects the nature of Brownian motion.

The mean-squared displacement (i.e. the second central moment of the displacement) for particles in such a system is given by:

$$
<\left(\mathbf{r}-\mathbf{r}_{0}\right)^{2}>=\int_{-\infty}^{\infty} \int_{-\infty}^{\infty}\left(\mathbf{r}-\mathbf{r}_{0}\right)^{2} \rho\left(\mathbf{r}_{0}\right) \mathrm{P}\left(\mathbf{r}_{0} \mid \mathbf{r}, \mathrm{t}\right) \mathrm{d} \mathbf{r}_{0} \mathrm{~d} \mathbf{r}
$$

where $\rho\left(\mathbf{r}_{0}\right)$ is the particle density and, by definition, the total particle density is unity (i.e. $\left.\int_{-\infty}^{\infty} \rho\left(\mathbf{r}_{0}\right) d \mathbf{r}_{0}=1\right)$. Evaluation of the integrals in equation (2.49), when $P\left(\mathbf{r}_{0} \mid \mathbf{r}, t\right)$ is given by equation (2.48), is simplified considerably by setting $\mathbf{r}=\mathrm{x} \mathbf{i}+\mathrm{y} \mathbf{j}+\mathrm{z} \mathbf{k}$ and $\mathbf{r}_{0}=\mathrm{x}_{0} \mathbf{i}+\mathrm{y}_{0} \mathbf{j}+\mathrm{z}_{0} \mathbf{k}$. In terms of its Cartesian components $\mathrm{P}\left(\mathbf{r}_{0} \mid \mathbf{r}, \mathrm{t}\right)$ is given by

$$
\mathrm{P}\left(\mathbf{r}_{0} \mid \mathbf{r}, \mathrm{t}\right)=\frac{1}{\sqrt{(4 \pi \mathrm{Dt})^{3}}} \exp \left[-\frac{\left(\mathbf{x}-\mathbf{x}_{0}\right)^{2}}{4 \mathrm{Dt}}\right] \exp \left[-\frac{\left(\mathbf{y}-\mathbf{y}_{0}\right)^{2}}{4 \mathrm{Dt}}\right] \exp \left[-\frac{\left(\mathbf{z}-\mathbf{z}_{0}\right)^{2}}{4 \mathrm{Dt}}\right]
$$

and the mean-squared displacement for "free diffusion" becomes:

$$
<\left(\mathbf{r}-\mathbf{r}_{0}\right)^{2}>=\mathrm{nDt},
$$

where $n=2,4$ or 6 for one, two or three dimensions, respectively. This is well known as Einstein's equation and $t=\tau_{D}$ is called the diffusion time. The equation shows that for "free diffusion", the mean-squared displacement of the diffusing molecules increases linearly with the diffusion time. 


\subsubsection{Diffusion equation for restricted diffusion}

Restricted diffusion refers to the case in which a diffusing species encounters reflecting boundaries that sequester it within a particular compartment [10]. The solution of equation (2.47) becomes much more complicated in this case since the displacement of the particles is now influenced by its boundaries (e.g. diffusion in a sphere or a box) and the probability distribution function of the net displacement is no longer Gaussian. The solutions for many cases of interest can be found in the literature [134,135]. For example, laminar systems consisting of a homogeneous fluid confined between two infinite, parallel barriers separated by distance a, display restricted diffusion $[10,123]$. The motion of particles parallel $(\|)$ to the boundaries is unrestricted, but particles moving perpendicular $(\perp)$ to the boundaries are constrained by the restricting barriers. Tanner et. al. [8] showed that the probability distribution for diffusion inside the layer is a geometry dependent function given by:

$$
\mathrm{P}\left(\mathbf{r}_{0} \mid \mathbf{r}, \mathrm{t}\right)=(4 \pi \mathrm{aDt})^{-1} \exp \left[-\frac{\left(\mathbf{r}-\mathbf{r}_{0}\right)_{\|}^{2}}{4 \mathrm{Dt}}\right] \times\left[1+2 \sum_{\mathrm{n}=1}^{\infty} \exp \left[-\frac{\left(\mathrm{n}^{2} \pi^{2} \mathrm{Dt}\right)}{\mathrm{a}^{2}}\right] \cos \left(\frac{\mathrm{n} \pi \mathrm{r}_{\perp}}{\mathrm{a}}\right) \cos \left(\frac{\mathrm{n} \pi \mathrm{r}_{0 \perp}}{\mathrm{a}}\right)\right]
$$

Another example of restricted diffusion is a homogeneous fluid contained in a spherical cavity of radius $\rho$. The expression for $P\left(\mathbf{r}_{0} \mid \mathbf{r}, t\right)$ for this case is given in the literature $[134,135,136]$.

There are several theoretical models dealing with semi-permeable barriers and numerous MR diffusion experiments on the "apparent diffusion coefficient" $\left(D_{a}\right)$ for inhomogeneous systems like brain tissue. In this case, $\mathrm{D}_{\mathrm{a}}$ can be influenced by the complex interaction between intracellular and extracellular compartments with the respective diffusion coefficients $D_{i}$ and $D_{e}$, the volume fraction of the compartments, $f_{i}$ and $f_{e}$, the cell membrane permeability, $P$, the compartmental exchange times, $\tau_{\mathrm{i}}$ and $\tau_{\mathrm{e}}$, the extracellular tortuosity, $\lambda$, which is a measure of the 
effect on extracellular diffusion of obstacles in the extracellular environment that need to be circumnavigated, and possibly other mechanisms. Restricted diffusion will be discussed in more detail in section 2.3.1 on MR-Diffusion theory.

\subsubsection{Diffusion time}

For a particle confined to the interior of a reflecting sphere of radius $\mathrm{R}$, which is neither transported through the boundary nor relaxed by the contact with the boundary, using equation (2.51) it is possible to define a dimensionless variable as [11]:

$$
\xi=\frac{\mathrm{D} \tau_{\mathrm{D}}}{\mathrm{R}^{2}}
$$

In the case of freely diffusing particles, the determined diffusion coefficient will be independent of the diffusion time, $\tau_{\mathrm{D}}$, and the measured displacement will reflect the true diffusion coefficient, since the mean squared displacement changes linearly with the time. However, the situation is entirely different for the particle confined to a sphere (i.e. restricted diffusion). For very short values of $\tau_{D}$, such that the particle has not diffused far enough to feel the effect of the boundary (i.e. $\xi \ll 1$ ), the measured diffusion coefficient is the same as that for free diffusion. As $\tau_{\mathrm{D}}$ becomes larger (i.e. $\xi \approx 1$ ), a certain fraction of the particles will be affected by the boundary and the measured displacement no longer changes linearly with $\tau_{D}$. In this case the measured diffusion coefficient will certainly be time dependent. At very long diffusion times, the maximum distance that the confined particles can travel is limited by the boundaries. This means that the measured mean squared displacement and diffusion coefficient both become independent of $\tau_{\mathrm{D}}$. Thus, for very short diffusion times, the measured displacement and diffusion coefficient of a particle in a restricted geometry, as observed by the MR signal attenuation using the MR 


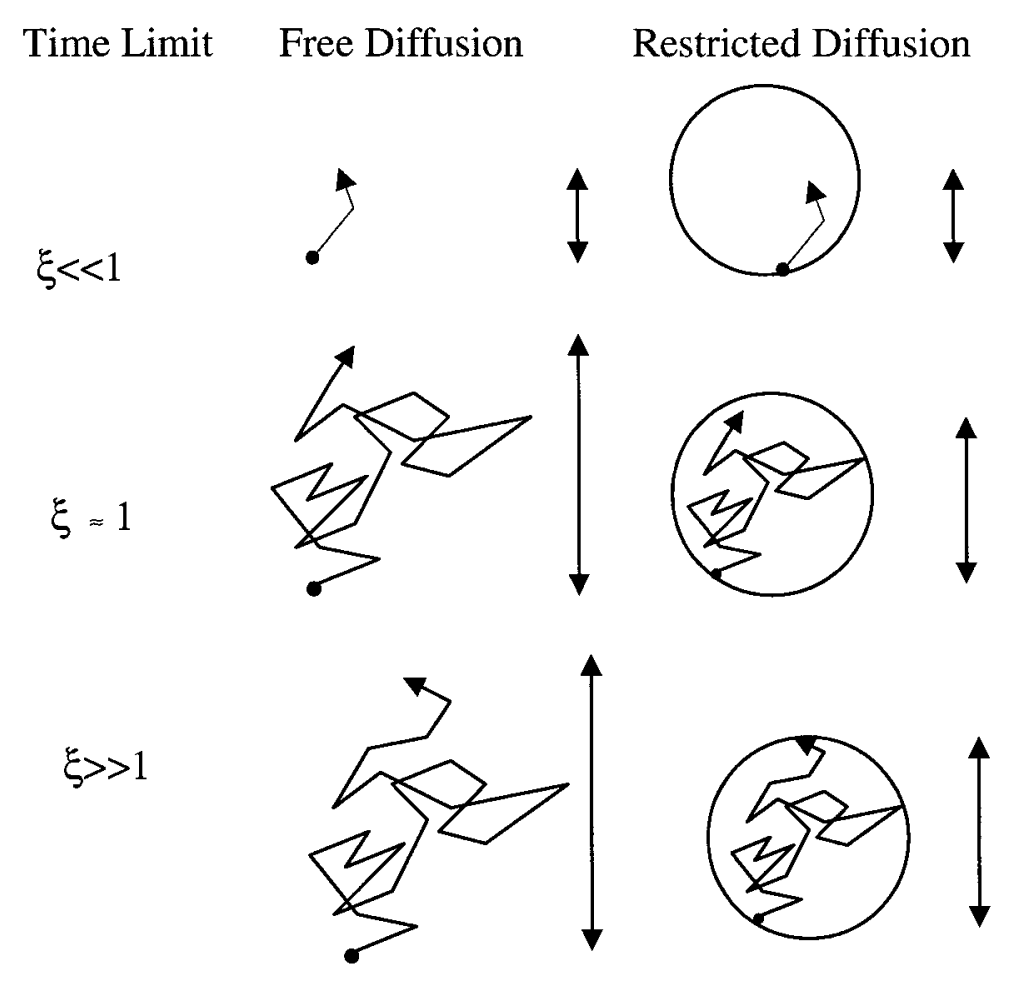

Figure 2.6. The schematic diagram of free diffusion and restricted diffusion for a particle in a sphere with radius $R$ in terms of the variation of the time scale. The net displacement in each case is shown by the length of the arrows. The effects of the restricted diffusion measurements can be related to these three time limits. The apparent diffusion coefficient is a function of diffusion time for the intermediate time limit $(\xi \approx 1)$ when not all of the particles feel the effect of the restricting barrier.

diffusion experiment, is truly sensitive to the diffusion of particles rather than to the shape and dimension of the restricting geometry, which is the case for long values of $\tau_{\mathrm{D}}$. The cases of a freely diffusing particle and a particle confined to the interior of a reflecting sphere are depicted in figure 2.6 for the three diffusion time limits. It should also be noted that the restricting geometry is normally not spherically symmetric (i.e. anisotropic medium) and thus the measured diffusion coefficient will have an orientational dependence. 


\subsubsection{Anisotropic diffusion and the diffusion tensor}

So far, to simplify the description of diffusion, the diffusion process has been assumed to be isotropic. In this case the diffusion can be characterized by the isotropic diffusion coefficient, D, as shown in equation (2.44). In general, however, the characterization of the diffusion process in a homogeneous anisotropic medium requires a second order tensor, $\mathbf{D}$. Thus, the more general expression of equation (2.44) is

$$
\mathbf{J}(\mathbf{r}, \mathrm{t})=-\mathbf{D} \nabla \mathrm{c}(\mathbf{r}, \mathrm{t})
$$

This can be expanded in matrix notation as

$$
\left[J_{X}(\mathbf{r}, t) J_{y}(\mathbf{r}, t) J_{z}(\mathbf{r}, t)\right]^{T}=-\left(\begin{array}{ccc}
D_{x x} & D_{x y} & D_{x z} \\
D_{x y} & D_{y y} & D_{y z} \\
D_{x z} & D_{y z} & D_{z z}
\end{array}\right)\left[\frac{\partial c(r, t)}{\partial x} \frac{\partial c(r, t)}{\partial y} \frac{\partial c(r, t)}{\partial z}\right]^{T}
$$

where $\mathrm{T}$ indicates the transpose. The diffusion equation in this case becomes (see equation (2.46)).

$$
\frac{\partial c(\mathbf{r}, \mathrm{t})}{\partial \mathrm{t}}=\nabla \mathbf{D} \nabla \mathrm{c}(\mathbf{r}, \mathrm{t})
$$

The displacement distribution is now slightly more complicated due to the tensor formulation [24]:

$$
\mathrm{P}\left(\mathbf{r}_{0} \mid \mathbf{r}, \mathbf{t}\right)=\frac{1}{\sqrt{|\mathbf{D}|(4 \pi \mathrm{t})^{3}}} \exp \left[-\frac{\mathbf{r}^{\mathrm{T}} \mathbf{D}^{-1} \mathbf{r}}{4 \mathrm{t}}\right]
$$

where $\mathbf{r}^{\mathrm{T}}$ denotes the transpose of $\mathbf{r}$ and $|\mathbf{D}|$ denotes the determinant of $\mathbf{D}$. As can be seen from this expression, for an anisotropic medium the diffusion tensor, $\mathbf{D}$, which is the matrix of variance and covariance for the distribution is more complicated than $\mathrm{D}$, which is simply the variance for an isotropic medium (see equation (2.48)). Besides, here $|\mathbf{D}|$ appears in the normalization factor 

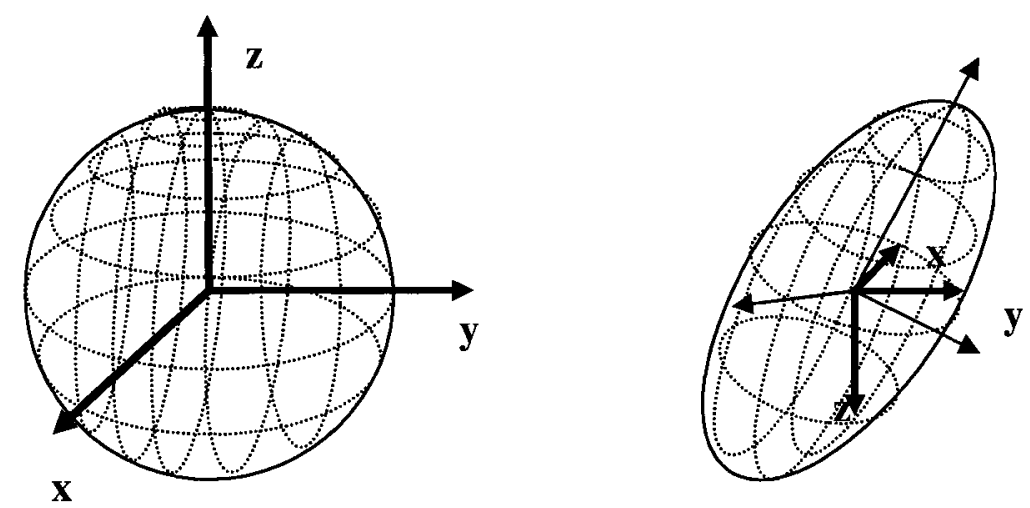

Figure 2.7. The hypothetical surface of constant mean-squared displacement in a. an isotropic medium represented by a diffusion sphere, $b$. an anisotropic medium represented by a diffusion ellipsoid.

of the probability density function instead of $\mathrm{D}^{3}$.

The probability profile for an isotropic medium can be represented by concentric "diffusion spheres", $x^{2}+y^{2}+z^{2}=r^{2}=6 D t$, (figure $2.7 a$ ) by setting the exponent of the displacement distribution in equation (2.48) to a constant $(-3 / 2$ in this case). The surface constructed for anisotropic diffusion by the same process gives the following equation:

$$
\begin{aligned}
& \left(D_{y y} D_{z z}-D_{y z}^{2}\right) x^{2}+2\left(D_{x z} D_{y z}-D_{x y} D_{z z}\right) x y+ \\
& \left(D_{x x} D_{z z}-D_{x z}^{2}\right) y^{2}+2\left(D_{x y} D_{y z}+D_{x z} D_{y y}\right) x z+ \\
& \left(D_{x x} D_{y y}-D_{x y}^{2}\right) z^{2}+2\left(D_{x y} D_{x z}+D_{x x} D_{y z}\right) y z=-4 c|D| t,
\end{aligned}
$$

where $\mathrm{c}$ is a constant. This is the equation of a three dimensional ellipsoid called the "diffusion ellipsoid" (figure 2.7b). To derive equation (2.58) use is made of the fact that $\mathbf{D}$ is a symmetric tensor (i.e. $D_{x y}=D_{y x}, D_{x z}=D_{z x}, D_{y z}=D_{z y}$ ). Thus, for an anisotropic medium at least six independent parameters are required $\left(D_{x x}, D_{y y}, D_{z z}, D_{x y}, D_{x z}, D_{y z}\right)$ to characterize the displacements of the particles in the medium [24]. 


\subsection{Diffusion-MR Theory}

The effect of molecular diffusion on NMR experiments was discovered at a very early stage in the development of the subject $[125,11]$. The relatively poor main field homogeneity available on the early magnets meant that all NMR experiments took place in the presence of a constant magnetic field gradient. The Larmor frequency was thus spatially dependent,

$$
\omega(\mathbf{r})=\gamma \mathrm{B}(\mathbf{r})=\gamma\left(\mathrm{B}_{0}+\mathbf{g} \bullet \mathbf{r}\right)=\omega_{0}+\gamma(\mathbf{g} \bullet \mathbf{r})
$$

where $\mathbf{g}$ is defined as the gradient of the magnetic field component parallel to $\mathbf{B}_{0}$, i.e.

$$
\mathbf{g}=\nabla \mathrm{B}_{\mathrm{z}}=\frac{\partial}{\partial \mathrm{x}} \mathrm{B}_{\mathrm{z}} \mathbf{i}+\frac{\partial}{\partial \mathrm{y}} \mathrm{B}_{\mathrm{z}} \mathbf{j}+\frac{\partial}{\partial \mathrm{z}} \mathrm{B}_{\mathrm{z}} \mathbf{k}
$$

where $\mathbf{i}, \mathbf{j}, \mathbf{k}$ are the unit vectors of the laboratory frame of reference. The phase shift of the spins in the laboratory frame then becomes

$$
\phi(t)=\gamma B_{0} t+\gamma \int_{0}^{t} g \cdot r\left(t^{\prime}\right) d t^{\prime}
$$

where the second term represents the phase shift due to the effects of the gradient. This shows that the degree of dephasing due to $\mathbf{g}$ is not only dependent on the strength of the gradient but also on the displacement of the spin in the direction of the gradient. From this analogy, it can be seen that a well defined magnetic field gradient can be used to label the position of a spin through its Larmor frequency. This provides the basis for measuring diffusion.

The pulsed gradient spin echo (PGSE) sequence, first proposed by Stejskal and Tanner in 1965 [10], is the most commonly used NMR sequence in diffusion experiments. The sequence consists of two equal rectangular gradient pulses of duration $\delta$ inserted into each $\tau=\frac{\mathrm{TE}}{2}$ period of a SE sequence. Although these gradients, which are called diffusion sensitizing gradients, can 

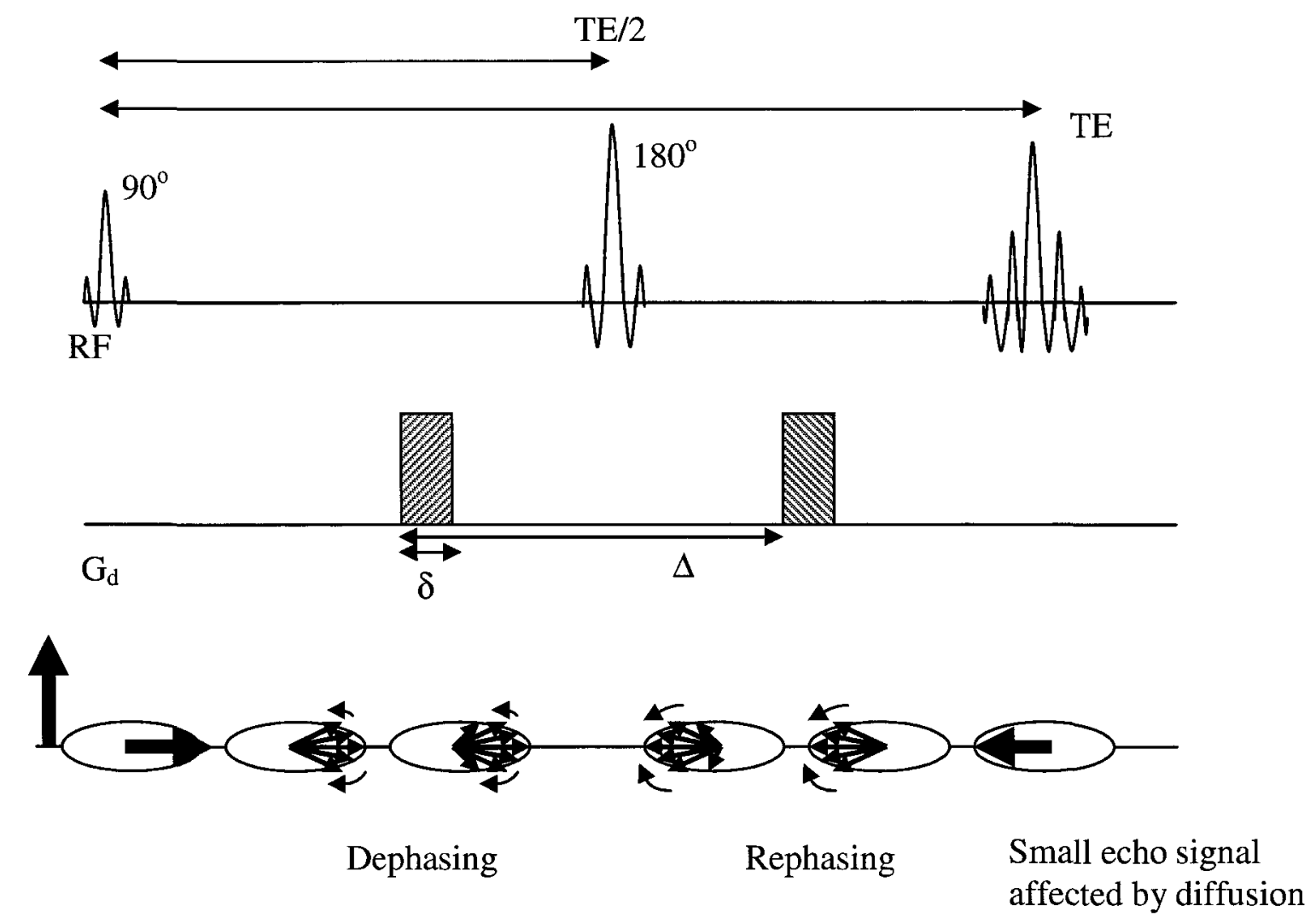

Figure 2.8. The Stejskal-Tanner diffusion pulse sequence. The rectangular gradient pulse of duration $\delta$ and magnitude $g$ is inserted into a spin echo sequence sensitizing the system to diffusion so that the signal will be attenuated due to the diffusion process.

be applied in any direction, it will be assumed for notational simplicity that they are in the $\mathrm{z}$ direction. (i.e. $\mathbf{g}=\frac{\partial}{\partial z} B_{z} \mathbf{k}$ ). As illustrated in figure 2.8, at thermal equilibrium the spin system consists of an ensemble of diffusing spins and the net magnetization is oriented along the z-axis. A $90^{\circ} \mathrm{RF}$ pulse is applied to rotate the macroscopic magnetization from the z-axis into the $\mathrm{xy}$ plane (i.e. perpendicular to the static field). Following the first RF pulse, at time t, a gradient 
pulse of duration $\delta$ and amplitude $g$ is applied. As a result, the spins experience a phase shift given by (see equation (2.61))

$$
\phi(t+\delta)=\gamma B_{0}(t+\delta)+\gamma g \int_{t}^{t+\delta} z\left(t^{\prime}\right) d t^{\prime}
$$

where $\mathrm{g}$ is taken out of the integral since it is a constant amplitude gradient. At time $\frac{\mathrm{TE}}{2}$, a $180^{\circ}$ RF pulse is applied. The effect of this pulse is to reverse the sign of the phase. At time $t+\Delta$, a second gradient pulse with the same amplitude and duration is applied. If the spins have not undergone any translational motion with respect to the z-axis, the effects of the two applied gradient pulses cancel and all spins refocus. However, if the spins have moved (e.g. through the diffusion process), the degree of dephasing due to the applied gradient is proportional to the displacement in the direction of the gradient that occurred in the time period $\Delta$ (i.e. the time between the leading edges of the gradient pulses).

\subsubsection{Attenuation of the MR signal due to diffusion}

It is possible to find the signal attenuation due to diffusion during a MR experiment by solving the Bloch equations with diffusion terms incorporated. The Bloch equation for the macroscopic nuclear magnetization, $\mathbf{M}(\mathbf{r}, \mathbf{t})=\mathbf{M}_{\mathrm{x}} \mathbf{i}+\mathbf{M}_{\mathrm{y}} \mathbf{j}+\mathbf{M}_{\mathrm{z}} \mathbf{k}$, when diffusion is considered, is given by $[125,11]$

$$
\frac{\mathrm{d} \mathbf{M}(\mathbf{r}, \mathrm{t})}{\mathrm{dt}}=\mathbf{M} \times \gamma \mathbf{B}(\mathbf{r}, \mathrm{t})-\frac{1}{\mathrm{~T}_{2}}\left(\mathrm{M}_{\mathrm{X}} \mathbf{i}+\mathrm{M}_{\mathrm{y}} \mathbf{j}\right)-\frac{1}{\mathrm{~T}_{1}}\left(\mathrm{M}_{\mathrm{z}}-\mathrm{M}_{0}\right) \mathbf{k}+\mathrm{D} \nabla^{2} \mathbf{M}
$$

in the laboratory frame. If we consider the static field $\mathbf{B}(\mathbf{r}, \mathbf{t})$ to consist of $\mathbf{B}_{\mathbf{0}}=\mathrm{B}_{0} \mathbf{k}$ and a small gradient $\mathbf{g}$, such that 


$$
\begin{aligned}
& \mathrm{B}_{\mathrm{x}}=0, \mathrm{~B}_{\mathrm{y}}=0, \\
& \mathrm{~B}_{\mathrm{z}}=\mathrm{B}_{0}+(\mathrm{g} \cdot \mathrm{r})=\mathrm{B}_{0}+\mathrm{g}_{\mathrm{x}} \mathrm{x}+\mathrm{g}_{\mathrm{y}} \mathrm{y}+\mathrm{g}_{\mathrm{z}} \mathrm{z}
\end{aligned}
$$

and note that

$$
\mathbf{M} \times \mathbf{B}=\left(M_{y} B_{z}-M_{z} B_{y}\right) \mathbf{i}+\left(M_{z} B_{x}-M_{x} B_{z}\right) \mathbf{j}+\left(M_{x} B_{y}-M_{y} B_{x}\right) \mathbf{k},
$$

equation (2.63), for the transverse magnetization $M_{x y}=M_{x}+i M_{y}$, can be written as

$$
\frac{d M_{x y}}{d t}=-i \omega_{0} M_{x y}-i \gamma(g \cdot r) M_{x y}-\frac{M_{x y}}{T_{2}}+D \nabla^{2} M_{x y}
$$

To facilitate the solution of equation (2.66), the simpler situation where $g=0$ and the effects of diffusion are ignored, will first be considered. In this case, it can be shown that [21]

$$
M_{x y}=M_{x y}^{\prime} \exp \left(-i \omega_{0} t-\frac{t}{T_{2}}\right)
$$

where $\mathbf{M}_{\mathrm{xy}}^{\prime}$, the amplitude of the precessing magnetization, is time independent (see also section 2.1.4). A useful trial solution to equation (2.66) can then be generated by replacing the constant $\mathbf{M}_{\mathrm{xy}}^{\prime}$, by $\mathrm{M}_{\mathrm{xy}}^{\prime}(\mathbf{r}, \mathrm{t})$ in equation (2.67). Substitution of this trial solution into equation (2.66) gives

$$
\frac{\mathrm{dM}_{\mathrm{xy}}^{\prime}}{\mathrm{dt}}=-\mathrm{i} \gamma(\mathbf{g} \cdot \mathbf{r}) \mathrm{M}_{\mathrm{xy}}^{\prime}+\mathrm{D} \nabla^{2} \mathrm{M}_{\mathrm{xy}}^{\prime} .
$$

In the absence of diffusion, the solution to equation (2.68) (a first order ordinary differential equation) is

$$
\mathbf{M}_{x y}^{\prime}(t)=M_{x y}^{\prime}(0) \exp (-i \gamma \mathbf{r} \bullet \mathbf{F})
$$

where 


$$
F(t)=\int_{0}^{t} g\left(t^{\prime}\right) d t^{\prime}
$$

Equation (2.69) can now be used to generate a trial solution for equation (2.68) by replacing the constant $M_{x y}^{\prime}(t=0)$ by the function $M_{x y}^{\prime \prime}(r, t)$. Substitution of this trial solution into equation (2.68) gives, after some simplification:

$$
\frac{\mathrm{d}}{\mathrm{dt}} \mathrm{M}_{\mathrm{xy}}^{\prime \prime}(\mathbf{r}, \mathrm{t})=-\gamma^{2} \mathrm{DF}{ }^{2} \mathrm{M}_{\mathrm{xy}}^{\prime \prime}(\mathbf{r}, \mathrm{t})
$$

and thus

$$
\ln \frac{M_{x y}^{\prime \prime}(t)}{M_{x y}^{\prime \prime}(0)}=-\gamma^{2} D \int_{0}^{t} F^{2}\left(t^{\prime}\right) d t^{\prime}
$$

This can be written in the more convenient form

$$
M_{x y}^{\prime \prime}(t)=M_{x y}^{\prime \prime}(0) e^{-b D}
$$

where the so called "b-factor", also sometimes called the gradient or diffusion weighting factor, is defined as

$$
\mathrm{b}=\gamma^{2} \int_{0}^{\mathrm{t}}\left\{\int_{0}^{\mathrm{t}^{\prime}}\left\{\mathrm{g}\left(\mathrm{t}^{\prime \prime}\right) \mathrm{dt}^{\prime \prime}\right\}^{2} \mathrm{dt}^{\prime}\right.
$$

Thus, the full solution to Bloch's equation with diffusion can be obtained by combining equations (2.67), (2.69) and (2.73) and is given by

$$
M_{x y}^{\prime \prime}(\mathbf{r}, \mathrm{t})=M_{x y}^{\prime \prime}(r, 0) e^{-b D} e^{-t / T_{2}} e^{-i\left(\omega_{0}+\mathbf{r} \bullet \mathbf{F}\right) t}
$$

MR theory in the presence of small magnetic field gradients was initially developed $[1,2]$ to deal with inhomogeneities in the main magnetic field. In this case $\mathbf{g}$ is time independent and $\mathrm{b}$ 
can readily be evaluated to give $b=\frac{1}{3} \gamma^{2} g^{2} t^{3}$. However, in this case, both $b$ and $\mathbf{g}$ are functions of position. With the magnets used in MRI today, these inhomogeneities are very small. Of more importance are the applied gradient fields. These gradients are applied in pulses (i.e. time dependent) and are normally linear, of constant amplitude and independent of position within the bore of the magnet. To evaluate equation (2.74) for specific MR sequences, the applied gradient must be replaced by the "effective field gradient", geff, such that the sign of the effective field gradient is changed every time a $180^{\circ} \mathrm{RF}$ pulse is applied.

The b-factor for the Stejskal-Tanner sequence in the case of free diffusion can be shown to be [10]

$$
\mathrm{b}=\gamma^{2} \mathrm{~g}^{2} \delta^{2}\left(\Delta-\frac{1}{3} \delta\right)
$$

by performing the integration in equation (2.74) from $t=0$ to $t=T E$. The factor $\left(\Delta-\frac{1}{3} \delta\right)$ in diffusion experiments is known as the diffusion time, $\tau_{\mathrm{D}}$. The diffusion echo signal attenuation thus can be written as

$$
\ln \frac{M_{x y}^{\prime \prime}(t)}{M_{x y}^{\prime \prime}(0)}=\ln E=-\gamma^{2} D^{2} \delta^{2}\left(\Delta-\frac{1}{3} \delta\right)
$$

In the limit $\delta \rightarrow 0$, while increasing the gradient strength so as to keep the product g $\delta$ (i.e. the area under the diffusion gradient pulse) finite, the signal attenuation due to diffusion simplifies to

$$
\ln \mathrm{E}=-\gamma^{2} \mathrm{Dg}^{2} \delta^{2} \Delta
$$




\subsubsection{MR signal attenuation due to restricted diffusion}

Although the derivation given in the previous section was performed for free diffusion in isotropic media, it can also be derived generally for anisotropic diffusion using the diffusion tensor D instead of the scalar diffusion coefficient. If the gradients were applied along three Cartesian directions, the signal attenuation due to diffusion would be described as $[11,24]$

$\ln E=-\left[b_{x x} D_{x x}+b_{y y} D_{y y}+b_{z z} D_{z z}+\left(b_{x y}+b_{y x}\right) D_{x y}+\left(b_{x z}+b_{z x}\right) D_{x z}+\left(b_{y z}+b_{z y}\right) D_{y z}\right]$,

where $b_{\mathrm{ij}}$ is now defined by

$$
\mathrm{b}_{\mathrm{ij}}=\gamma^{2} \mathrm{~g}_{\mathrm{i}} \mathrm{g}_{\mathrm{j}} \delta^{2}\left(\Delta-\frac{1}{3} \delta\right)
$$

for a PGSE sequence.

In analyzing the diffusion sequence for restricted diffusion with a non-Gaussian propagation characteristic, the diffusion time dependent "apparent diffusion coefficient" can be defined as the initial slope of the diffusion decay, which will no longer be exponential, in general [111]. The echo signal intensity, $\mathrm{E}\left(\mathbf{q}, \tau_{\mathrm{D}}\right)$, which can be calculated theoretically from a knowledge of the initial spatial concentration distribution, $\rho\left(r_{0}\right)$, and the displacement probability $\mathrm{P}\left(\mathbf{r}_{0} \mid \mathbf{r}, \tau_{\mathrm{D}}\right)$, discussed earlier in section 2.2 .1 , as $[125,10,11]$

$$
\mathrm{E}\left(\mathbf{q}, \tau_{\mathrm{D}}\right)=\int_{-\infty}^{\infty} \rho\left(\mathbf{r}_{0}\right) \int_{-\infty}^{\infty} \mathrm{P}\left(\mathbf{r}_{0} \mid \mathbf{r}, \tau_{\mathrm{D}}\right) \exp \left(\mathrm{i} 2 \pi \mathbf{q}\left(\mathbf{r}-\mathbf{r}_{0}\right)\right) \mathrm{d} \mathbf{r d} \mathbf{r}_{0},
$$

where $\mathbf{q} \equiv(2 \pi)^{-1} \gamma \delta \mathbf{g}$. This equation gives a Fourier relationship between the displacement probability and the diffusion signal attenuation. For example, for molecular diffusion in a box with parallel barriers separated by a distance a, $\mathrm{P}\left(\mathbf{r}_{0} \mid \mathbf{r}, \tau_{\mathrm{D}}\right)$ is defined by equation (2.52). In this case, $\mathrm{E}\left(\mathbf{q}, \tau_{\mathrm{D}}\right)$ can be calculated as $[8]$ 


$$
E\left(\mathbf{q}, \tau_{D}\right)=\frac{2\{1-\cos (2 \pi q a)\}}{(2 \pi q a)^{2}}+4(2 \pi q a)^{2} \sum_{n=1}^{\infty} \exp \left(-\frac{n^{2} \pi^{2} D \tau_{D}}{a^{2}}\right) \frac{1-(-1)^{n} \cos (2 \pi q a)}{\left\{(2 \pi q a)^{2}-(n \pi)^{2}\right\}^{2}}
$$

Once $E$ is determined $D_{a}$ can be evaluated from the initial slope of the $\ln E$ vs. $q^{2}$ decay curve. This definition for $D_{a}$ is motivated by the free diffusion case (where the decay is exponential) from which we get (see equation (2.78))

$$
D_{a}=\lim _{b \rightarrow 0}\left(-\frac{\partial \ln E}{\partial b}\right)=\frac{1}{4 \pi^{2} \tau_{D}} \lim _{q \rightarrow 0}\left(-\frac{\partial \ln E}{\partial q^{2}}\right)
$$

However, to properly use the theoretical models, such as Karger's equations for exchange in a two compartment system, there are other parameters including membrane permeability and exchange time which have to be considered [127].

The investigation of restricted diffusion in biological tissue (e.g. brain tissue) is becoming of great importance. Due to restricted intracellular and extracellular diffusion, it is expected that intracellular water contributes predominantly to the signal at high b-factors or equivalently high q-values. However, the effects of restriction can be detected not only at high b-values but also at short diffusion times. Restriction manifests itself as a difference in signal attenuation for the same b-value at different diffusion times. This is studied for in vivo human brain in this work.

\subsubsection{Echo planar imaging}

As previously discussed, echo planar imaging (EPD) is becoming the standard technique for ultra fast imaging in MR experiments, particularly MR-diffusion studies, despite its being first proposed by Mansfield more than 20 years ago [126, 37,138]. EPI is characterized by a very short readout train of typically 20 to $80 \mathrm{~ms}$, a high acquisition bandwidth in the read direction and a very low bandwidth along the phase-encoding axis. The typical acquisition time is generally 
sufficiently short to freeze out most in vivo motion which is a very important consideration for those interested in diffusion imaging experiments $[35,36]$.

According to Norris [126], single-shot EPI is the only MR technique that yields high quality images which are intrinsically insensitive to macroscopic or bulk motion without compromising on sensitivity. The speed and the motion insensitivity of diffusion weighted EPI (DW-EPI) make this method the best choice for the overwhelming majority of diffusion experiments. In spite of its advantages with respect to avoiding motion artifacts, EPI is susceptible to artifacts such as N/2 ghosting and Gibbs artifacts which will be discussed in the next section on noise theory. The sensitivity of single-shot DW-EPI to magnetic field perturbations resulting from eddy currents and variations in magnetic susceptibility is another limitation of this technique. However, eddy current effects are less problematic on modern high performance MR systems with recently developed DW-EPI pulse sequences $[124,128,158]$.

\subsubsection{Diffusion time in MR diffusion measurements}

The concept of a diffusion time is based on preparing a system in a known initial state, allowing the system to evolve by diffusion for a well defined time, $\tau_{D}$, and then measuring the state of the system at time $\tau_{\mathrm{D}}$ to see the effects that diffusion had on the system. In MR this idealized experiment would be done by using a gradient pulse of amplitude $G$ and duration $\delta$ where $\delta \rightarrow 0$ at time $t=0$ to prepare the initial state and an identical gradient pulse to sample the state of the system after the diffusion time. If no diffusion has occurred between the two gradient pulses then the measured signal at time $\tau_{\mathrm{D}}$ will be the same as the signal at $\mathrm{t}=0$ (ignoring relaxation effects). Otherwise, the signal at $\tau_{\mathrm{D}}$ will be attenuated (see equation (2.73)) by an amount that depends on $\mathrm{b}$ which, for this idealized experiment would be (see equation (2.76)) 


$$
\mathrm{b}=\gamma^{2} \mathrm{~g}^{2} \delta^{2} \Delta
$$

where $\Delta$, the time between the gradient pulses, is equal to the diffusion time.

In practice, $\delta$-function gradient pulses cannot be generated so equation (2.84) is valid only for this idealized experiment. For finite gradient pulses $b$ is given by equation (2.76)

$$
\mathrm{b}=\gamma^{2} \mathrm{~g}^{2} \delta^{2}\left(\Delta-\frac{1}{3} \delta\right)
$$

where the diffusion time, $\tau_{\mathrm{D}}$, is considered to be

$$
\tau_{D}=\frac{b}{\gamma^{2} g^{2} \delta^{2}}=\Delta-\frac{1}{3} \delta
$$

\subsubsection{Eddy current effect}

MR sequences can provide pulsed gradient protocols with rapidly switching input waveforms. However, interaction of the changing magnetic field gradient with surrounding conductive components within the MR system (e.g. magnetic shields, magnet coils) induces eddy currents which generate a time-spatial dependent magnetic field which gets superimposed on the desired gradients [121]. Although DW-EPI is well known to be insensitive to severe motion induced phase artifacts, it can be affected strongly by eddy currents induced by the diffusion gradients resulting in unwanted perturbations to the local magnetic field. The low bandwidth of the EPI sequence in the phase-encoded direction leads to significant distortions caused by phase shifts due to the perturbed local field of the spins $[124,130,131]$.

The resulting distortion of the image can be described in terms of shears, magnifications (or demagnifications) and translations in the phase encoded direction of the distorted image. This is due to phase shifts resulting from the residual field in the readout, phase-encoding and slice selection directions, respectively, as discussed by Horsfield [131]. The magnitude of this phase shift is given by the following equation: 


$$
\Delta \Phi(\mathbf{r}, \mathrm{t})=\gamma \mathrm{T}_{\mathrm{e}}\left[\Delta \mathrm{B}^{\mathrm{Ex}}(\mathbf{r}, \mathrm{t})+\Delta \mathrm{B}^{\mathrm{Ey}}(\mathbf{r}, \mathrm{t})+\Delta \mathrm{B}^{\mathrm{Ez}}(\mathbf{r}, \mathrm{t})\right]
$$

where $T_{e}$ is the time between subsequent gradient echoes in the EPI echo train, and $\Delta B^{E x y z}(\mathbf{r}, t)$ are the magnetic field shifts from the gradients in $\mathrm{x}, \mathrm{y}$ and $\mathrm{z}$ directions due to the eddy current effect. Since the phase shift can be related to the position in the phase-encoded direction, $r_{p e}$, by

$$
\Delta \Phi=\frac{2 \pi \mathrm{r}_{\mathrm{pe}}}{\mathrm{FOV}}
$$

the change in $r_{p e}$, due to the phase shift, $\Delta r_{p e}$, is [131]

$$
\Delta \mathrm{r}_{\mathrm{pe}}=\frac{1}{2 \pi} \gamma \mathrm{T}_{\mathrm{e}} \mathrm{FOV}\left[\Delta \mathrm{B}^{\mathrm{Ex}}(\mathbf{r}, \mathrm{t})+\Delta \mathrm{B} \text { Ey }(\mathbf{r}, \mathbf{t})+\Delta \mathrm{B} E z(\mathbf{r}, \mathbf{t})\right]
$$

In order to minimize eddy currents, several methods are widely employed in MR systems. Design considerations such as physical separation of gradient coils from cryostat components to minimize the electromagnetic coupling of the gradient currents with any conductive parts in the magnet cryostat, shielded gradient design and active eddy current compensation or so called "gradient pre-emphasis", which is accomplished by changing the shape of the field gradient amplitude envelope to compensate for the eddy current induced fields, are commonly used in state-of-the-art high performance MR systems. To undo the effects of the additional gradient fields due to eddy currents, there are also some proposed modifications and algorithms $[124,130,131]$ which may require manual recalibration of sequence timing. In the Siemens sequence described in the following section, the eddy current issue is considered in terms of the design of the pulse sequence with no cost in scanning efficiency or effectiveness compared to the conventional diffusion sequence of Stejskal and Tanner [124]. 

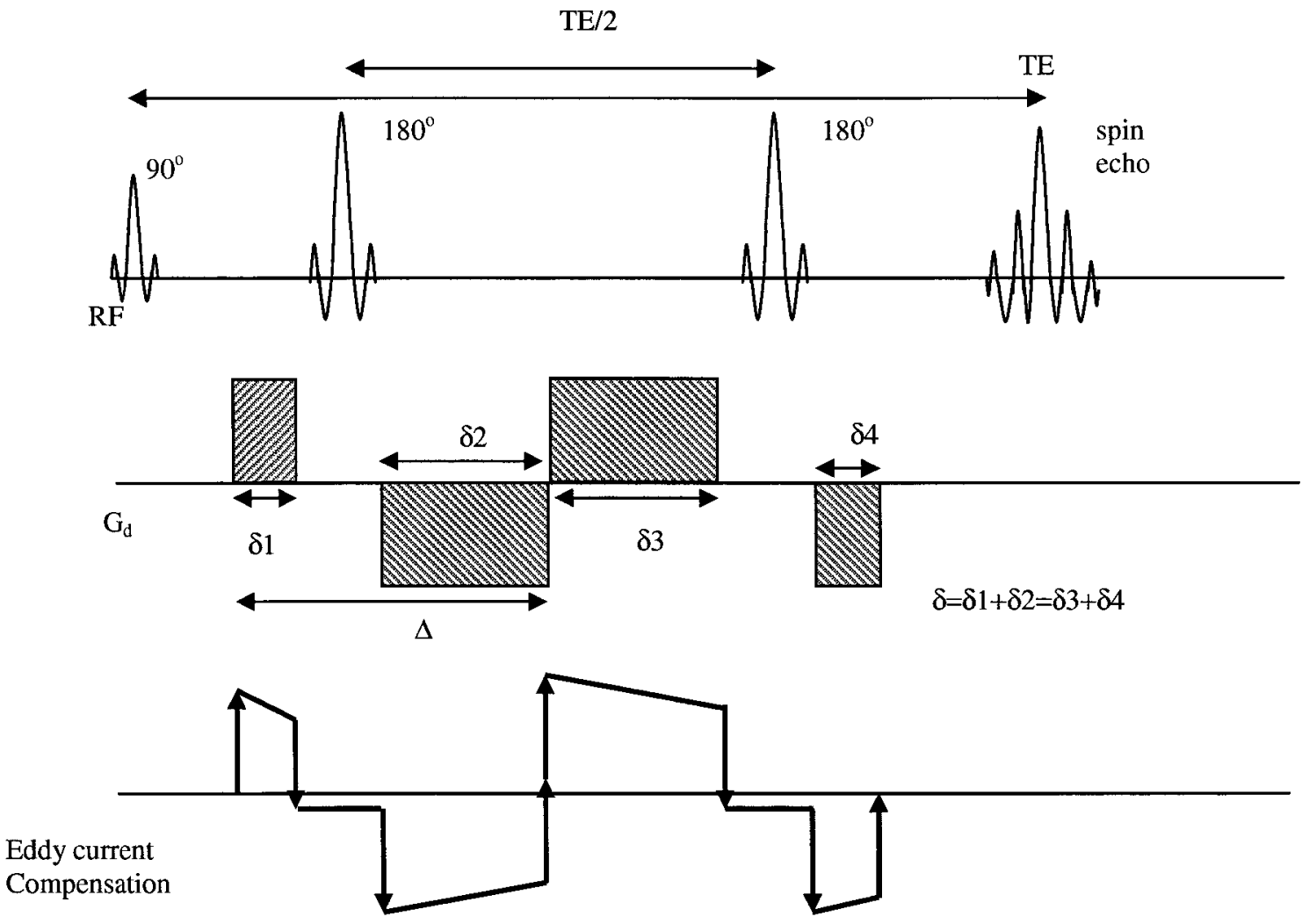

Figure 2.9 a. The timing diagram of the TRSE sequence. b. The build up and decay of eddy currents due to switching of the gradients.

\subsubsection{Siemens diffusion sequence}

In the standard PGSE diffusion sequence of Stejskal-Tanner, a single refocusing $(\pi)$ RF pulse is applied. Using more than one refocusing pulse permits variable intervals between the pulses, requiring only that the spin's alternating defocusing and refocusing times, $\delta_{\mathrm{i}}$, sum equally at the time of the desired spin echo. This flexibility in timing could be an advantage when used for diffusion imaging $[124,132]$.

A SE diffusion sequence with two refocusing pulses forms the twice-refocused spin echo, TRSE, sequence shown in figure 2.9. The TRSE sequence compromises neither the efficiency 
nor the effectiveness of the single-refocusing Stejskal-Tanner Pulsed Gradient SE sequence (PGSE), while reducing eddy current distortion. The bipolar field gradients, $\mathrm{G}_{\mathrm{d}}$, are applied in the TRSE sequence with the RF refocusing pulses splitting each bipolar pair as shown in figure $2.9 \mathrm{a}$ (i.e. $\delta=\delta_{1}+\delta_{2}=\delta_{3}+\delta_{4}$ ). By adjustment of the diffusion gradient duration, eddy current induced time dependent magnetic fields can be cancelled and thus image distortion can be entirely avoided [124]. The graph below the timing diagram of the sequence in figure $2.9 \mathrm{~b}$ shows the build-up and the decay of eddy currents due to gradient switching. The on and off diffusion gradient transitions that generate the eddy currents are shown in bold black arrows. The diffusion gradient durations are evaluated such that the eddy current build-up is nulled prior to readout, as discussed by Reese [124]. The so called "ep2d-diff” sequence on a 1.5 T Siemens Symphony uses the diffusion sensitized spin preparation of the TRSE sequence with a single-shot EPI readout, as proposed by Heid [132], to provide diffusion experiments with better quality and more flexible diffusion gradient timing.

\subsection{Noise in MRI}

\subsubsection{The sources of noise in MRI}

The noise in MR measurements is quite different from that obtained with other imaging modalities such as radiography, computed tomography, positron emission tomography, etc., where the noise is basically related to counting statistics. In fact, the noise in MRI is not dependent on the signal value measured, rather it is primarily dependent on the coil resistance and the volume of the subject that the coil "sees" $[118,119,121]$. 
The noise due to the resistance of RF coils increases with operating frequency, but the strength of the functional dependence varies considerably with the kind of sample being measured. For non-conducting samples, the noise is just thermal noise originating in the resistance of the receiver coil. This is known as electronic noise or Johnson noise. A classical relationship given by Abragam [168] suggests that the noise is proportional to $\omega_{0}^{1 / 2}$ for this case. He also argues that, since the NMR signal itself originates from the emf induced in the receiver coil by the rotating nuclear magnetization (i.e. $\mathrm{M}_{\mathrm{xy}}$ after a $90^{\circ} \mathrm{RF}$ pulse), from Faraday's law of induction the amplitude of the emf generated by this varying magnetic flux $\left(\frac{\mathrm{d} \Phi}{\mathrm{dt}}\right)$ will be proportional to the static magnetic field $\mathrm{B}_{0}$ and hence to the square of the Larmor frequency, i.e.

$$
\operatorname{emf} \propto \frac{\mathrm{d} \Phi}{\mathrm{dt}} \propto \frac{\mathrm{d} M_{x y}}{\mathrm{dt}}=i \omega_{0} M_{x y}(0) \mathrm{e}^{\mathrm{i} \omega_{0} t} \propto \omega_{0} \mathrm{~B}_{0} \propto \omega_{0}^{2}
$$

Using this equation, Abragam shows that the SNR should be proportional to $\omega_{0}^{3 / 2}$. In a more realistic analysis, Hoult and Richards determined that the noise for non-conducting samples is only proportional to $\omega_{0}{ }^{1 / 4}$, so that the SNR increases as $\omega_{0}{ }^{7 / 4}[118]$.

The situation in a conducting sample is considerably more complex since the sample itself becomes a source of noise. The most significant example of such a sample is the human body. In the physical model of Hoult and Lauterbur [119] the noise from this source is proportional to $\omega_{0}$, giving a SNR which increases linearly with frequency. They have shown that the noise is associated not only with receiving coil resistance, $\mathrm{R}$, but also with noise originating from the sample (i.e. the human subject). The "sample noise" is a result of both "dielectric" and "inductive" or "magnetic" losses which can be expressed as an equivalent noise resistance.

Every coil has distributed capacitance associated with its turns. This capacitance is proportional to the coil diameter per unit length. Electric lines of force due to this capacitance 
pass through the sample and result in an equivalent series resistance, $R_{e}$, related to the distributed dielectric loss from the sample. The noise generated by dielectric coupling increases as $R_{e}$ increases [118]. Fortunately, these losses can be minimized by designing the RF transmission coils to produce $\mathbf{E}$ fields which are small.

A far more serious loss mechanism is the one associated with currents induced inside the sample due to inductive coupling with the $\mathbf{B}_{1}$ field of the RF pulses. Hoult and Lauterbur [119] have derived an expression for these inductive losses which shows a significant dependence on physical dimensions and operating frequency. The depth of penetration of the $\mathbf{B}_{1}$ field into the conducting sample is closely related with the inductive loss in the sample and the currents caused by the magnetic conduction within the surface layers of the samples. The inductive loss results in an associated resistance, $\mathrm{R}_{\mathrm{m}}$, which shields the $\mathrm{RF}$ field. Hoult and Lauterbur have derived an expression for $R_{m}$ in series with a solenoidal receiving coil by finding the conductance of the coil and the emf induced around the coil by an alternating $\mathbf{B}_{1}$ field [119].

Considering the main sources of noise in MRI, to be electronic noise and sample losses, the formula for the SNR of MR measurements proposed by Chen and Hoult for a volume $\Delta \mathrm{V}$ inside a conducting sphere of radius $b$ is given by the following equation $[121,118,119,143]$ :

$$
\mathrm{SNR}=\mathrm{M}_{0} \Delta \mathrm{V}\left(\frac{15 \rho}{8 \pi \mathrm{kTb}^{5} \Delta \mathrm{v}}\right)^{1 / 2}
$$

where $\mathrm{M}_{0}$ is the equilibrium magnetization, which is proportional to field strength $\mathrm{B}_{0}, \mathrm{~T}$ is the temperature of the sample (not of the coil), $\rho$ is the resistivity of the coil, $k$ is Boltzmann's constant and $\Delta v$ is the bandwidth of the signal. The ratio of imaging volume, $\Delta \mathrm{V}$, to the total volume of the RF receiver coil and the acquisition bandwidth are two parameters which can be adjusted to improve the SNR of MR measurements for a given magnetic field strength. The others 
are mostly fixed for medical imaging applications. There is no way, for instance, to cool the patient during an MR imaging session.

The SNR can also be presented in terms of the FOV and the acquisition bandwidth, $\Delta v$, (assuming that the number of averages is one) as:

$$
\mathrm{SNR} \propto \Delta \mathrm{X} \Delta \mathrm{Y} \Delta \mathrm{Z}\left(\frac{1}{\Delta \mathrm{v}}\right)^{1 / 2}=\frac{\mathrm{FOV}_{\mathrm{x}}}{\sqrt{\mathrm{N}_{\mathrm{x}}}} \frac{\mathrm{FOV}_{\mathrm{y}}}{\sqrt{\mathrm{N}_{\mathrm{y}}}} \Delta \mathrm{Z}\left(\frac{1}{\Delta \mathrm{v}}\right)^{1 / 2}
$$

where $\frac{F O V_{x}}{\sqrt{N_{x}}}$ and $\frac{F_{y}}{\sqrt{N_{y}}}$ are the spatial resolutions in the $x$ and $y$ directions and $\Delta Z$ is the slice thickness for a transverse image. $\mathrm{N}_{\mathrm{x}}$ and $\mathrm{N}_{\mathrm{y}}$ are the number of times the signal is sampled in the $\mathrm{x}$ and y directions, respectively.

\subsubsection{Gaussian, Rician and Rayleigh distributions}

The noise on the real and imaginary signals measured in MRI is well described by a Gaussian probability density function (PDF) both in k-space and after Fourier transformation where the Gaussian PDF is given by

$$
\mathrm{P}(\mathrm{M} \mid \mathrm{A}, \sigma)=\frac{1}{\sqrt{2 \pi \sigma^{2}}} \exp \left[-\frac{(\mathrm{M}-\mathrm{A})^{2}}{2 \sigma^{2}}\right]
$$

where $M$ is the signal intensity corrupted by noise of variance $\sigma^{2}$ and $A$ is the "true" signal intensity (i.e. the signal induced by the nuclear magnetization).

Although all of the information about the object being imaged is contained in the real and imaginary signals, it is more practical to generate the images from the magnitude of the measured signals. The calculation of pixel magnitudes from the real and imaginary data is a non-linear 
operation under which the Gaussian PDF for the noise is transformed into a Rician distribution for which the PDF is given by $[133,160,161]$ :

$$
\mathrm{P}_{M}(\mathrm{M} \mid \mathrm{A}, \sigma)=\frac{\mathrm{M}}{\sigma^{2}} \exp \left[-\left(\frac{\mathrm{M}^{2}+\mathrm{A}^{2}}{2 \sigma^{2}}\right)\right] \mathrm{I}_{0}\left(\frac{\mathrm{MA}}{\sigma^{2}}\right),
$$

where $I_{0}$ is the zeroth order modified Bessel function of the first kind, $M$ is the measured intensity, $\mathrm{A}$ is the image pixel intensity in the absence of the noise, and $\sigma$ is the standard deviation of the Gaussian noise on the real and imaginary signals. The subscripted $\mathrm{M}$ on the left hand side of equation (2.93) indicates that $\mathrm{P}_{M}(M \mid A, \sigma)$ is the PDF for the magnitude of the MR signal (see also section 4.2). It can be shown for magnitude MR images that, although the Rician PDF reduces to a Gaussian PDF for high SNR, it deviates significantly from a Gaussian when the SNR is low, leading to noise biased results.

The two interesting limits of the Rician distribution are when the SNR, which equals $A / \sigma$ in this case, is very small or very large. The first limit, when $\mathrm{A} / \sigma \rightarrow 0$, corresponds to image regions where only noise is present. In this case the Rician PDF reduces to the Rayleigh PDF given by $[133,160]$

$$
\mathrm{P}_{\mathrm{M}}(\mathrm{M} \mid \sigma)=\frac{\mathrm{M}}{\sigma^{2}} \exp \left(-\frac{\mathrm{M}^{2}}{2 \sigma^{2}}\right)
$$

The "true" noise power, $\sigma^{2}$, can be determined from the magnitude image through the evaluation of either the mean, $\left\langle\mathrm{M}_{\mathrm{bkg}}\right\rangle$, or the variance, $\sigma_{\mathrm{M}}^{2}$, for a region of the image where there is only noise since for a Rayleigh distribution [133-142]:

$$
<\mathrm{M}_{\mathrm{bkg}}>=\int_{0}^{\infty} \frac{\mathrm{M}^{2}}{\sigma^{2}} \exp \left(-\frac{\mathrm{M}^{2}}{2 \sigma^{2}}\right)=\sigma \sqrt{\pi / 2}
$$

and 


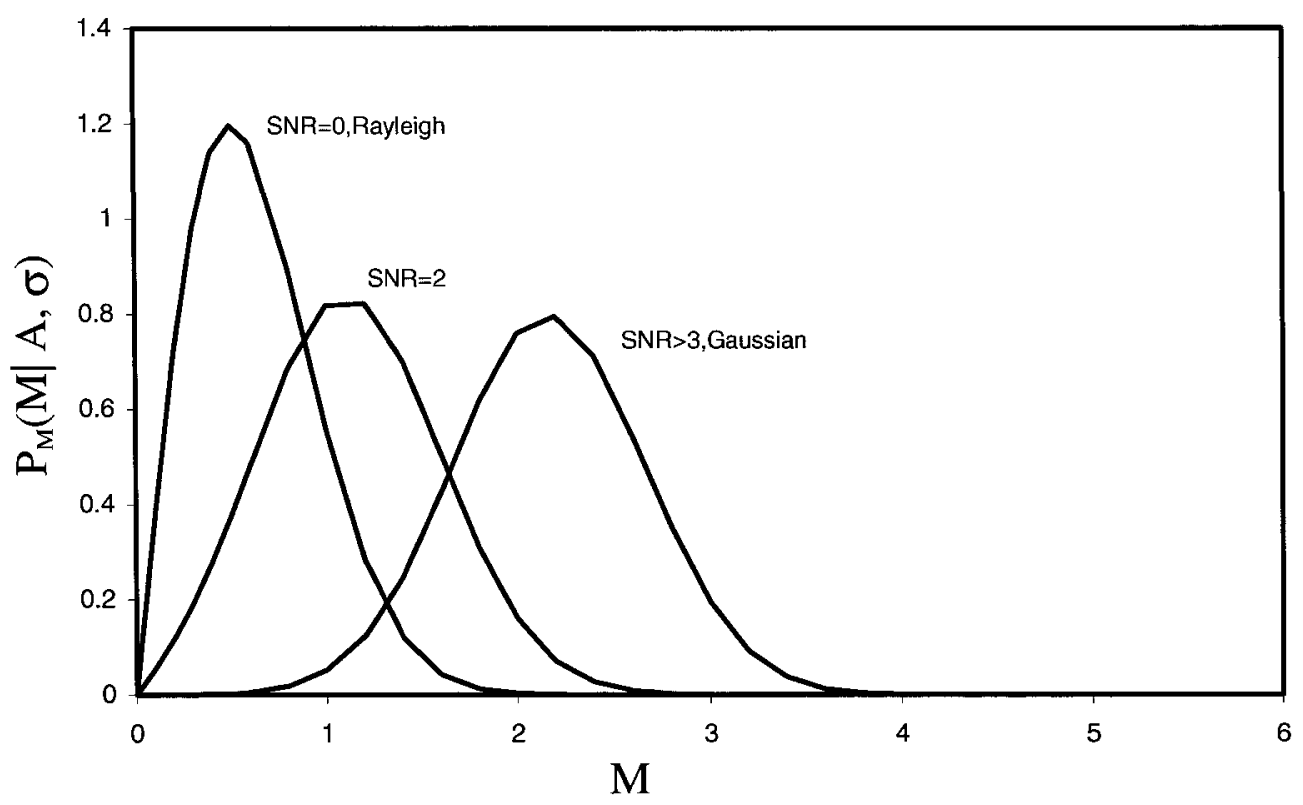

Figure 2.10. The probability density functions of the magnitude image versus SNR.

$$
\sigma_{M}^{2}=(2-\pi / 2) \sigma^{2}
$$

The other interesting limit for the Rician distribution corresponds to large signal intensities such that $\mathrm{A} / \sigma \geq 3$. In this limit the Rician distribution tends to a Gaussian distribution with a mean of $\left(\mathrm{A}^{2}+\sigma^{2}\right)^{1 / 2}$ and a variance equal to $\sigma^{2}[133]$ :

$$
\mathrm{P}_{M}(\mathrm{M} \mid \mathrm{A}, \sigma) \approx \frac{1}{\sqrt{2 \pi \sigma^{2}}} \exp \left[-\left(\mathrm{M}-\sqrt{\mathrm{A}^{2}+\sigma^{2}}\right)^{2} / 2 \sigma^{2}\right]
$$

Although the mean of this PDF is not equal to the "true" image intensity, A, this small deviation is not important when the SNR is large enough $(\geq 3)$. However, this bias cannot be ignored when the SNR is small, as is the case for MR diffusion experiments at very high bvalues. More details about the magnitude image bias due to the Rician noise are given in chapter 4 where several techniques for noise correction are proposed. The Rician PDF is shown in figure 2.10 in the low SNR (Rayleigh), intermediate SNR and high SNR (Gaussian) limits [133-142]. 


\subsubsection{Artifacts in MRI}

Image artifacts are structures in the image which do not correspond to the object being imaged. The purpose of reviewing image artifacts in this work is to describe the most significant artifacts that can affect the measured value of the signal intensity, the noise, or both in MR diffusion measurements. Care must be taken to avoid contributions from artifacts during the post processing and data analysis of the images. The artifacts which are discussed here include "motion" artifacts, ghosting, N/2 artifacts as well as Gibbs ringing or truncation artifacts. There are many other artifacts, such as $B_{0}$ inhomogeneity artifacts, susceptibility artifacts, chemical shift artifacts, etc., which can also distort MR images [121]. These will not be considered further in this thesis.

\subsubsection{Ghost artifacts}

Ghost artifacts manifest themselves as an extra image of the object in the phase encoding direction but offset from the correct location of the image of the object. Ghost artifacts are a result of phase errors in $\mathrm{k}$-space. These phase errors can be generated in a variety of ways depending on the MR experiment, the sequence applied and the anatomy of the object being imaged. Two common sources of phase errors that lead to ghosting artifacts are bulk motion of either the whole object or part of the object (e.g. respiratory motion) and blood flow.

As mentioned above, ghost images generally occur in the phase encoding direction, independent of the direction of the motion. Ghosts arise in this case because the motion modulates the magnetization measured in k-space. When the k-space data are Fourier transformed to generate the MR image these phase modulations in k-space lead to a shift of the object in the 
image domain. Any process that changes the phase or magnitude of the magnetization during data acquisition can cause ghosts in an MR image.

\subsubsection{N/2 ghost artifacts}

EPI sequences are particularly susceptible to ghost artifacts resulting from modulation of the kspace magnetization. A problem specifically related to EPI is that every second line of k-space is read under a negative gradient (i.e. every other line is registered in the opposite direction). This can result in the generation of ghost images known as Nyquist or N/2 ghosts since they are shifted by one-half of the FOV $[121,158]$.

The N/2 ghost artifact arises because the odd and even echoes are acquired under opposite read gradients and the data require reversal prior to image reconstruction. Inaccurate timing of the sampling relative to the switched gradients, temporal asymmetry in the analogue filter and/or inhomogeneities in the static field can cause a modulation of alternate lines in k-space. This leads to ghost images shifted by $\pm N / 2$ pixels in the phase encoding direction. The linear phase shift between odd and even lines in k-space due to the time delay causes a modulation of the k-space signals. This can be seen through the Fourier shift theorem $f(x-\tau) \leftrightarrow e^{-2 \pi i \tau k} F(k)$ to cause a shift of the object position in the image.

One source of non-uniform time sampling that can cause N/2 ghosting is eddy currents which arise every time the gradient is turned on or off. Eddy currents induce an additional magnetic field, which causes a delay in the applied gradient field reaching stability at the desired amplitude. This time delay occurs in every other line with the opposite sign causing the sampling distances in k-space to be unequal. This leads to a zigzag pattern for the k-space trajectory and thus to ghost images shifted by $\pm N / 2$ pixels. A typical N/2 ghost image is shown in figure $2.11 \mathrm{~b}$. 
a.

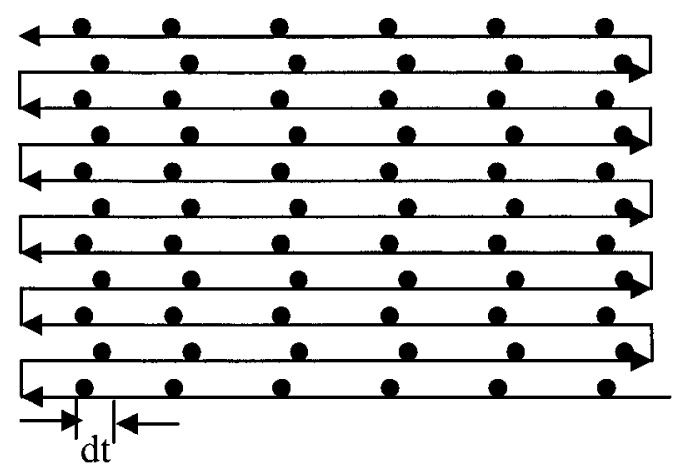

b.

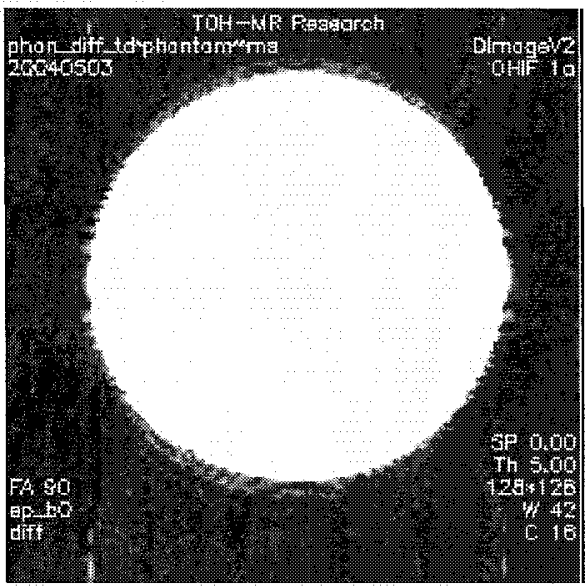

Figure 2.11. a. The zigzag pattern of $\mathrm{k}$-space for an EPI sequence due to a time delay in the signal acquisition. b. A typical image showing N/2 ghosting corresponding to a k-space trajectory similar to that shown in a.

Low pass filtering in the receiver system is usually used in MR experiments in order to reduce high frequency noise and to get a better SNR. However, this operation can also introduce a time delay which is in opposite directions for odd and even lines and this can also become a source of N/2 ghosting [158].

\subsubsection{Gibbs artifact}

Gibbs artifact is caused by sampling a limited band of spatial frequencies in k-space while an object with sharp edges contains some power at all frequencies. This is due to assumptions made for the Fourier transformation process, which are only approximately correct. The Fourier transform strictly applies to functions that are of infinite range. However, it is not normally practical to acquire MR signals over times longer than a few tens of milliseconds. 


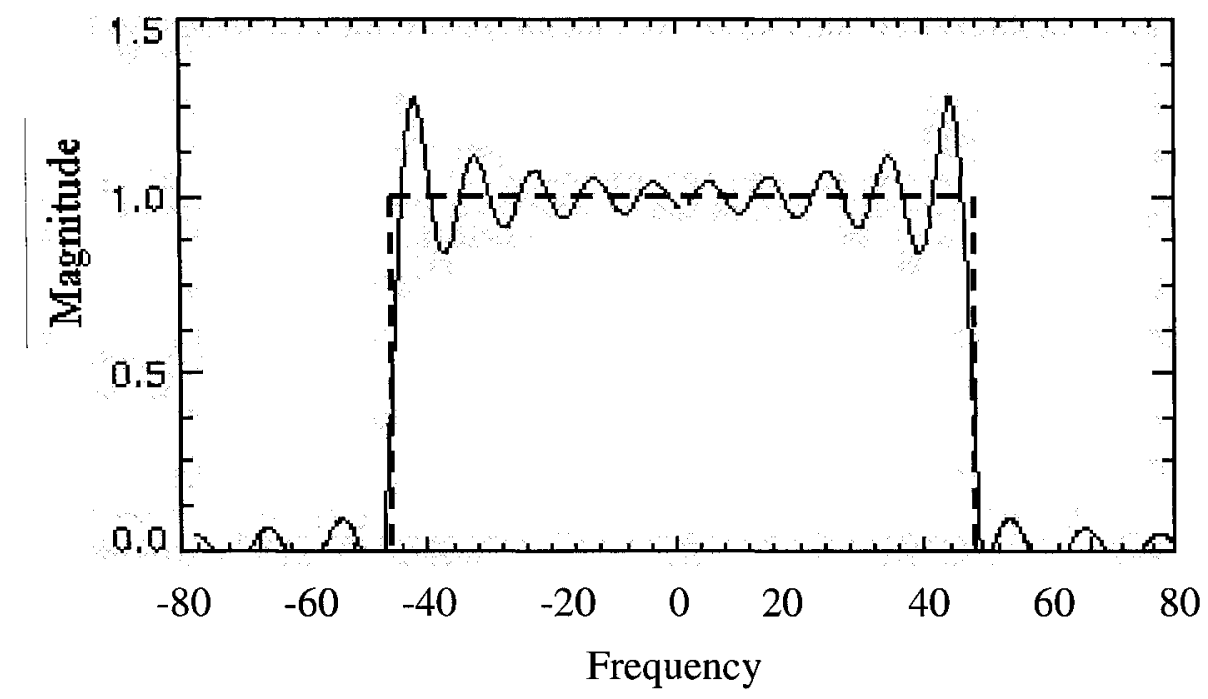

Figure 2.12. The truncation filter resulting in Gibbs ringing (solid line) in comparison with the ideal profile (dashed line).

If the signal magnitude is significantly above the noise level at the beginning and at the end of the sampling window, Fourier transformation of the signal to form the MR image results in a truncation artifact. Truncation or Gibbs artifact can be observed in both the readout and phase encoding directions depending on the signal sampling conditions. For example, if a limited band of spatial frequencies is sampled in order to reduce the total imaging time this artifact can be seen in the frequency encoding direction. Since the image is reconstructed from the limited data, through the inverse Fourier transform, the truncation artifacts manifest as ripples which overshoot in the vicinity of edges, or sharp transitions in signal intensity in the image (figure 2.12). This is known as Gibbs ringing. A low pass spatial-domain filter such as a Hanning filter, which has a cosine roll-off and is set to a value of 1 at the middle, and 0 at the ends, is often used to reduce 
the ringing. The smooth roll-off of the Hanning filter produces less ringing (Gibbs phenomenon) than a square cut-off filter such as truncation. [121, 158].

\subsubsection{Motion artifacts}

There are a variety of sources of motion, which can degrade MR images. The most problematic physiologic motions include blood flow, cerebrospinal fluid (CSF) pulsation, periodic respiratory or cardiac motions, swallowing, gastrointestinal peristalsis and patient motions (especially in the case of pediatric imaging).

Blurring associated with motion is generally slight and occurs in the direction of motion. Ghost images due to the motion, however, can be significant and occur only in the phase encoding direction independent of the motion direction. Motion causes phase shifts which depend on the velocity of the moving structure and also on the configuration of the gradient pulses. The velocity of many anatomic structures changes during the course of several minutes of data acquisition and the phase changes accordingly. Phase shifts also reduce the magnetization in a particular voxel if the spins in that voxel have a distribution of velocities. This is called the intra voxel phase dispersion. It is clear that voxels will have a lower signal intensity if their components have a range of phase angles (i.e. some spins are out of phase with each other). A great many strategies have been developed to correct for motion induced phase shifts, which cause motion artifacts. As mentioned before, the single shot EPI sequence is well known to be insensitive to bulk motion because it is a very fast sequence. This is the main reason why most MR diffusion measurements are done using a diffusion-weighted EPI sequence. 


\subsubsection{Chemical shift artifacts}

The Larmor frequency of a particular spin is determined by the applied magnetic field, $\mathrm{B}_{0}$, and small perturbations to this field from the local environment of the spin. The shift of the resonance frequency due to this local magnetic field from some reference resonance frequency is called the chemical shift. The most commonly observed chemical shift in human MR imaging is between fat and water. Since the position in the MR image is determined by the frequency of the signal, the chemical shift causes a misregistration of the fat signal in the image relative to the water in the frequency encoding direction. This effect is spatially constant. The chemical shift is proportional to the magnetic field strength so that at higher fields the shift, in terms of the number of pixels, is much greater.

\subsubsection{6 $\mathrm{B}_{0}$ inhomogeneity artifacts}

In contrast to chemical shift, $B_{0}$ inhomogeneities introduce a spatially varying off-resonance effect. Magnet imperfection and susceptibility effects due to different materials (e.g. patients having metallic implants) can lead to field inhomogeneities. The resonance frequency of a spin is expected to be $\gamma \mathrm{B}_{0}$, but in the presence of a magnetic field inhomogeneity, $\Delta \mathrm{B}_{0}$, it will become $\gamma\left(\mathrm{B}_{0}+\Delta \mathrm{B}_{0}\right)$. This will cause the signal coming from this location in the object to be shifted from its true location in the image along the frequency encoding direction by $\Delta \mathrm{B}_{0} / \mathrm{G}_{\mathrm{x}} . \Delta \mathrm{B}_{0}$ may either be due to magnetic field imperfections or the difference between the magnetic susceptibilities of two adjacent substances, i.e. $\Delta \mathrm{B}_{0} \propto\left(\chi_{1}-\chi_{2}\right)$. The local image distortion caused by susceptibility artifacts mostly occurs at air-tissue, and bone-tissue interfaces [121]. 


\subsection{Curve Fitting Theory}

The behavior of a system by the process of self diffusion in MR diffusion studies is characterized by exponential decay curves. With homogeneous systems like pure water, the signal attenuation due to diffusion leads to a mono-exponential decay curve. For an inhomogeneous system like human brain tissue, the decay curves are more complex. They may be the sum of two or more exponential functions and hence, for this case, there are many more parameters to be determined in order to obtain the best fit to the curve. These terms could originate, for example, from the effect of different environments or compartments in the tissue like intravascular, intracellular and interstitial regions. The investigation of the behavior of such a system and the accurate determination of the parameters that describe this behavior requires a precise characterization of the distribution of diffusion coefficients in the system $[102,148]$.

The multi-exponential behavior of the diffusion process in an inhomogeneous system can be described by the general integral equation

$$
Y_{k}(b)=\int_{D_{\min }}^{D_{\max }} S(D) \exp (-b D) d D+\varepsilon_{k}(0, \sigma) \quad k=1,2 \ldots m
$$

where the $Y_{k}(b)$ are the measured data at the value of $b, S(D)$ is the unknown weighting factor or the amplitude of the spectral component for the value of the variable diffusion coefficient, $\mathrm{D}$, and $\varepsilon_{\mathrm{k}}$ is the noise component of the measured data for $b$. The exponential terms are the kernel of the integral which can be generalized and applied for any particular system under investigation. As Whittall and MacKay stated, this works well for the analysis of the decay functions for $T_{2}$ relaxation (i.e. $\exp \left(-\mathrm{t} / \mathrm{T}_{2}\right)$ ) for spin-spin relaxation studies $[109,145,146]$. 
In order to solve equation (2.97) using a computer the integral must be converted to a sum of exponential decay terms. This can be done either by introducing the assumption that $S(D)$ is a sum of delta functions [109]:

$$
S(D)=\sum_{j=1}^{n} S_{j} \delta\left(D-D_{j}\right)
$$

or a piece-wise continuous spectrum over many small ranges of $\mathrm{D}$ :

$$
Y\left(b_{i}\right)=\sum_{j=1}^{n} f_{i j} S_{j} \quad \text { where } \quad f_{i j}=\int_{b_{j}}^{D_{j+1}} \exp \left(-b_{i} D\right) d D
$$

The general integral for both of these cases (equation (2.97)) can be written in the following form:

$$
Y_{k}\left(b_{i}\right)=\sum_{j=1}^{n} S_{j} \exp \left(-b_{i} D_{j}\right)+\varepsilon_{k}
$$

where the $S_{j}$ are the unknown fractions at the known variable diffusion coefficients $D_{j}$. This is our assumed model for the diffusion behavior of the brain tissue in the MR-diffusion studies.

Equation (2.100) can also be written in matrix form as

$$
\mathbf{A s}=\mathbf{y}
$$

where $\mathbf{y}$ is an $\mathrm{m}$-vector that contains the results of the $\mathrm{m}$ measurements being considered, $\mathbf{s}$ is an $\mathrm{n}$-vector and $\mathbf{A}$ is an $\mathrm{m} \times \mathrm{n}$ matrix. The elements, $\mathrm{s}_{\mathrm{j}}$, of the vector $\mathbf{s}$ correspond to the signal fractions, $\mathbf{S}_{\mathrm{j}}$ in equation (2.98), and the elements of $\mathbf{A}$ are given by

$$
A_{i j}=e^{-b_{i} D_{j}} .
$$

If the $D_{j}$ values are known, equation (2.101) describes a system of $m$ linear equations in $n$ unknowns. Although the $\mathrm{D}_{\mathrm{j}}$ 's are in fact not known, this will prove to be a convenient formulation of the problem in the following sections. 
This can also be viewed as a least-squares problem since in order to solve equation (2.101) a solution, $\mathbf{s}$, must be found that minimizes the Euclidean norm of $\mathbf{A s}-\mathbf{y}$ denoted as

$$
\|\mathbf{A s}-\mathbf{y}\|=\sqrt{\sum_{i}(\mathrm{As}-\mathrm{y})_{\mathrm{i}}^{2}} .
$$

\subsubsection{Least Squares}

The conventional method of analyzing multi-exponential decay curves uses a least squares analysis to fit the curves to a function with a predetermined number of discrete exponential terms which the experimenter has decided in advance is appropriate for the experimental data set. This is a non-linear optimization method which solves equation (2.100) for the amplitudes, $S_{j}$ at unknown $D_{\mathrm{j}}$. This method normally requires a starting model (i.e. guesses) to begin iterations.

In the non-linear least squares algorithm of Levenberg-Marquardt, $\chi^{2}$ is minimized by iteratively comparing the experimental data with corresponding values generated using the user defined function (and its derivatives with respect to each adjustable parameter) until the best agreement is obtained [147]. The coefficients assigned at the beginning of the program are the initial guesses to be used in the fit. An iteration loop is then entered while monitoring successive values of $\chi^{2}$. Uncertainties are also derived from the square root of the diagonal elements of the covariance matrix which is returned after the fit is completed. This method is often unstable when there are more than two exponential components involved in the decay curves or when any two of the exponential terms are similar. In this case a linear inverse technique based on minimization of the least squares misfit is a better method for fitting multi-exponential decay curves. 


\subsubsection{Non-Negative Least Squares}

Non-Negative Least Squares $(N N L S)$ is a linear inverse technique for solving least squares problems that has been shown to work well for multi-exponential systems [108]. This technique also has the advantage over more commonly used approaches that there is no need to specify beforehand the number of exponential terms in the solution or initial guesses for the fit parameters beyond specifying the limits for the fit parameters to be considered. These limits will normally define the range over which the parameter has physical meaning. It is also not susceptible to converging to a local minimum that is not equal to the true minimization of the Euclidean norm given in equation (2.103).

Consider the system of linear equations

$$
\mathbf{A s}=\mathbf{y}
$$

where $\mathbf{A}$ is an $\mathbf{m} \times \mathbf{n}$ matrix of rank $\mathrm{k}, \mathbf{s}$ is the n-vector of unknowns and $\mathbf{y}$ is an m-vector. The $N N L S$ algorithm computes one component of $\mathrm{y}_{\mathrm{i}}$ at a time by solving the reduced problem

$$
\mathbf{A}_{\mathbf{r}} \mathbf{s}_{\mathbf{r}}=\mathbf{y}
$$

where $\mathbf{A}_{\mathbf{r}}$ is an $\mathrm{m} \times \mathrm{n}$ matrix and $\mathbf{s}_{\mathbf{r}}$ is the trial solution for the given iteration. With each iteration one additional column of $\mathbf{A}$ is introduced into $\mathbf{A}_{\mathbf{r}}$ and the other columns are zeroed. Only those components of $\mathbf{s}$ with a corresponding non-zero column in $\mathbf{A}_{\mathbf{r}}$ are solved for in a particular iteration. The most important part of the algorithm is the way in which the new column to be incorporated into $\mathbf{A}_{\mathbf{r}}$ is chosen for a particular iteration. This choice is done such that the new component introduced into $\mathbf{s}_{\mathrm{r}}$ is positive and the residue function is reduced relative to the previous iteration, thus ensuring that the algorithm converges to a solution where all of the components of $\mathbf{s}$ are non-negative. The algorithm proceeds until all of the columns of $\mathbf{A}$ have 
been considered or until there are no more columns of $\mathbf{A}$ that will lead to an improved solution when included in the algorithm.

The column which is to be added in each iteration is determined by finding the largest component of the dual vector, w, defined as [108]

$$
\mathbf{w}=\mathbf{A}^{\mathrm{T}}(\mathbf{y}-\mathbf{A s})
$$

where $\mathbf{s}$ is the solution obtained in the previous iteration. If $\mathrm{w}_{\ell}$ is the largest component of $\mathbf{w}$ such that the $\ell^{\text {th }}$ column of $\mathbf{A}$ has not yet been incorporated into $\mathbf{A}_{\mathbf{r}}$ and $\mathrm{w}_{\ell}>0$ then the $\ell^{\text {th }}$ column of $\mathbf{A}$ is the new column included in $\mathbf{A}_{\mathbf{r}}$ for the current iteration. The proof that this algorithm chooses the most appropriate column can be found in Chapter 23 of reference 108.

The reduced least squares problem given by equation $(2.104)$ is constructed by the algorithm such that the problem can be readily solved by $\mathbf{Q R}$-decomposition followed by back substitution (see figure 1 in Appendix I). For any $m \times n$ matrix, $\mathbf{A}$, it is possible to construct an orthogonal matrix, $\mathbf{Q}$, such that

$$
\mathbf{A}=\mathbf{Q R}
$$

where the elements of $\mathbf{R}$ below the main diagonal are all zero [108]. After $\mathbf{Q}$ is constructed its transpose can be applied to equation (2.104) to give

$$
\mathbf{Q}^{\mathrm{T}} \mathbf{A s}=\mathbf{R s}=\mathbf{Q}^{\mathrm{T}} \mathbf{y}
$$

As long as $\operatorname{rank}(\mathrm{A})=\mathrm{n} \leq \mathrm{m}$, this system can readily be solved by back substitution. It is important to note that orthogonal matrices preserve the Euclidean norm under multiplication [108]. Thus

$$
\left\|\mathbf{Q}^{\mathrm{T}} \mathbf{A} \mathbf{s}-\mathbf{Q}^{\mathrm{T}} \mathbf{y}\right\|=\left\|\mathbf{Q}^{\mathrm{T}}(\mathbf{A} \mathbf{s}-\mathbf{y})\right\|=\|\mathbf{A} \mathbf{s}-\mathbf{y}\|
$$


The orthogonal matrix $\mathbf{Q}$ is constructed as the product of Householder transformation matrices $[144,108]$. For any non-zero vector $\mathbf{v}$, the Householder transformation matrix, $\mathbf{Q}_{1}$, is defined by $[108,144]$

$$
\mathbf{Q}_{1} \mathbf{v}=-\sigma\|\mathbf{v}\| \mathbf{e}_{1}
$$

where

$$
\mathbf{e}_{1}=\left[\begin{array}{c}
1 \\
0 \\
\cdot \\
\cdot \\
0
\end{array}\right]
$$

and

$$
\sigma= \begin{cases}+1 & \text { if } v \geq 0 \\ -1 & \text { if } v<0\end{cases}
$$

The important thing to note is that the elements of $\mathbf{Q}_{1} \mathbf{v}$ are all zero except for the first element. The orthogonal matrix $\mathbf{Q}_{1}$, can be constructed from $\mathbf{v}$ using the following definition

$$
\mathbf{Q}_{1}=\mathbf{I}_{\mathrm{m}}-\frac{2 \mathbf{u} \mathbf{u}^{\mathbf{T}}}{\|\mathbf{u}\|}
$$

where

$$
\mathbf{u}=\mathbf{v}+\sigma\|\mathbf{v}\| \mathbf{e}_{1}
$$

and $\mathbf{I}_{\mathrm{m}}$ is the $\mathrm{m} \times \mathrm{m}$ identity matrix.

If we let $\mathbf{v}$ be equal to the first column of $\mathbf{A}$ then $\mathbf{Q}_{1} \mathbf{A}$ will have all zeros in the first column below the first row. If we now let $\mathbf{v}$ be equal to the elements of the second column from row 2 to the bottom of the column we can construct an orthogonal matrix $\mathbf{Q}_{2}$ such that $\mathbf{Q}_{1} \mathbf{Q}_{2} \mathbf{A}$ is zero below the main diagonal for the first two columns. If we continue in this way we can 
construct an orthogonal matrix, $\mathbf{Q}=\mathbf{Q}_{\mathrm{n}-1} \ldots \ldots \ldots \mathbf{Q}_{2} \mathbf{Q}_{1}$ such that $\mathbf{Q A}=\mathbf{R}$, where $\mathbf{R}$ is zero below the main diagonal $[144,108]$.

The $N N L S$ algorithm works based on the above mentioned general computational procedure to find the solution vector $\mathbf{s}$ for the constrained least squares problem through minimizing the chisquare misfit. This algorithm converges in a finite number of iterations using non-negativity constraints for the $s_{j}$ and also minimizes the residual value of the linear least squares misfit [108]. $N N L S$ is a linear inverse technique which does not require any initial parameter estimation and it converges smoothly to a solution with all non-negative components. This algorithm has proven to be a very effective technique for the best fitting of multi-exponential decay curves.

The NNLS algorithm solves the general system of equations [108]

$$
\sum_{j=1}^{n} A_{i j} s_{j}-y_{i}=0
$$

by minimizing the least-squares misfit, such that the norm $\|\mathbf{A s}-\mathbf{y}\|$ is minimized, i.e.

$$
\sum_{\mathrm{i}=1}^{\mathrm{m}}\left|\sum_{\mathrm{j}=1}^{\mathrm{n}} \mathrm{A}_{\mathrm{ij}} \mathrm{s}_{\mathrm{j}}-\mathrm{y}_{\mathrm{i}}\right|^{2}=\text { minimum. }
$$

The $\mathrm{A}_{\mathrm{ij}}$ 's are the matrix elements which must be constructed as input for the $N N L S$ algorithm where $A_{i j}=\exp \left(-b_{i} D_{j}\right)$ for a set of $b$-values (measurement parameters) and a large number $\left(\geq 100\right.$ ) of $D_{j}$ diffusion coefficients (trial fit parameters). The set of diffusion coefficients can be arbitrarily selected to be in a reasonable range and can be sampled over logarithmic or linear intervals.

The NNLS algorithm gives the solution with the convergence of equation (2.115) to zero. It solves the equation for the fractions $s_{j}$ corresponding to an unspecified number of exponential 
terms without an initial estimation of the parameters $D_{j}$. The only prior assumption is nonnegativity of the spectral fractions, $s_{j}$. Many of these fractions will be zero in the final solution.

The solution found by $N N L S$ is not unique, but the various possible solutions tend to cluster around the true values of the $\mathrm{D}_{\mathrm{j}}$ coefficients. Further constraints are required to pick the most stable solution. To do this a regularizer must be incorporated into the algorithm. Regularization is discussed in the next section.

\subsubsection{Non-Negative Least Squares with regularization}

The solution to the least squares problem provided by the $N N L S$ algorithm is not unique, particularly when the solution has broadened peaks due to a distribution of diffusion coefficients, for example. In order to obtain a solution which is stable and the best solution, based on some criteria, new constraints have to be incorporated into the $N N L S$ algorithm $[109,110]$. The additional constraints are incorporated as extra rows of the matrix $\mathbf{A}$. The least squares problem that was previously given in equation $(2.115)$ can now be written as $[109,110]$

$$
\sum_{\mathrm{i}=1}^{\mathrm{m}}\left|\sum_{\mathrm{j}=1}^{\mathrm{n}} \mathrm{A}_{\mathrm{ij}} \mathrm{s}_{\mathrm{j}}-\mathrm{y}_{\mathrm{i}}\right|^{2}+\mu \sum_{\mathrm{k}=1}^{\mathrm{K}}\left|\sum_{\mathrm{j}=1}^{\mathrm{n}} \mathrm{R}_{\mathrm{kj}} \mathrm{s}_{\mathrm{j}}-\mathrm{r}_{\mathrm{k}}\right|^{2}=\text { minimum },
$$

where the second term on the right is called the regularizer and the relative strength of the regularizer is determined by $\mu$, the regularization parameter, where $0 \leq \mu \leq 1$. Note that the matrix $\mathrm{R}$ considered in this section has no relationship to the matrix $\mathrm{R}$ discussed in the previous section.

Imposing the extra constraints to minimize along with the chi-square misfit is used to select the 'simplest' members of a large number of possible solutions, protecting the system against 
significant unexpected information such as sudden sharp changes or extra peaks in the spectrum [110]. This is an example of the use of the principle of parsimony which can be defined as choosing the solution spectrum that is the smoothest and has the minimum number of peaks. This tends to protect the system against significant artifacts [110].

The form of the matrix $\mathbf{R}$ and the corresponding vector $\mathbf{r}$ can be varied and specified by the user. The value of the regularization parameter is adjusted iteratively between 0 and 1 trading off with the chi-square misfit until it is close to its expected value, $\mathrm{m}$, which is equal to the number of degrees of freedom (ndf) for the system of equations (see figure 2 of Appendix I). When $\mu=0$, equation (2.116) reduces to equation (2.115) and the basic least-squares solution is obtained. When $\mu>0$, the equality constraints are eliminated and the regularizer imposes new constraints on the problem which have the effect of smoothing the solution. The optimized value of $\mu$ is obtained at the expense of increasing the $\chi^{2}$ misfit, but attaining a smooth spectrum and a more stable solution.

Many different regularizers are possible. The simplest regularizer minimizes the power of the spectrum. This corresponds to setting $K=\mathbf{n}, \mathbf{r}=0$ and $\mathbf{R}=\mathbf{I}_{\mathbf{n}}$ in equation (2.116), where $\mathbf{I}_{\mathrm{n}}$ is the $n \times n$ identity matrix [109]. Then

$$
\|\mathbf{R s}-\mathbf{r}\|^{2} \equiv \sum_{j=1}^{n} s_{j}^{2},
$$

and the solution is now a nice smooth spectrum with a minimum of peaks. This is often more consistent with the real system in character. However, this regularizer tends to underfit the data and the calculated $\chi^{2}$ is normally greater than the ndf, the expected result for the system when $\mu=0$.

There are other possibilities for the regularizer which not only smooth the spectrum but also give a solution which is closer to the true value. Using a regularizer that minimizes the first 
or second order derivatives of $\mathbf{s}$ has been considered by Whittall and MacKay [109] and Provencher [110]. In discrete notation these can be written as

$$
\|\mathbf{R s}-\mathbf{r}\|^{2} \equiv \sum_{j=1}^{n-1}\left|s_{j+1}-s_{j}\right|^{2}
$$

and

$$
\|\mathbf{R s}-\mathbf{r}\|^{2} \equiv \sum_{j=1}^{n-2}\left|s_{j+2}-2 s_{j+1}+s_{j}\right|^{2},
$$

respectively, where $\mathbf{r}=0$. It can be seen that both of these regularizers act to reduce the difference between adjacent points in the solution. More details about implementation of the NNLS algorithm with and without regularization will be given in chapter 5 . The computational code $N N L S$-diff, developed for fitting the diffusion decay curves for this study will also be thoroughly discussed.

\subsubsection{Comparing $N N L S$ with other standard curve fitting methods}

There are some other linear techniques such as Least Distance Programming, LDP, and Linear Programming, LP, with different assumptions and convergence methods which can also be used to fit multi-exponential decay curves.

The LDP method is for problems with fewer data values than unknown spectral amplitudes. Such systems have no unique solution because many spectra satisfy the limited number of inaccurate data points. As discussed by Whittall and MacKay [109], LDP uses a similar approach to fitting as $N N L S$, but from the opposite point of view. The $N N L S$ begins with best-fitting a delta function model and then constructs smoother models when the misfit is allowed to increase and the regularizer is imposed. In contrast, LDP finds the model with the smallest possible power spectrum for some specified misfit governed by a defined parameter, and then constructs less 
smooth models as the parameter decreases. The misfit is forced toward the minimum possible value and should converge to the same result as for the discrete form of $N N L S$ (i.e. without regularization) [109].

Linear Programming (LP) is a different approach to solving a linear system as discussed by Whittall and MacKay [109]. LP finds the solution which satisfies the same constraints as LDP along with minimizing or maximizing the function $\sum_{j=1}^{n} w_{j} s_{j}$, where the $w_{j}$ 's are arbitrary weights. LP solutions tend to have a number of discrete delta functions similar to the NNLS least squares solution. However, the LP method can also construct smooth spectra by including extra constraints into the model. The data analysis with this algorithm tends to provide a good fit to the data but is not as good as other techniques at giving the proper peak positions $[108,109]$.

The method of fitting decay curves with non-linear least squares techniques is used routinely for fitting $\mathrm{T}_{2}$ relaxation and diffusion decay curves. As discussed before, it is well known that this method has the disadvantage of requiring an initial estimation and often converges slowly to a solution (i.e. requires many iterations) [102, 145, 146, 148, 149]. This will be more problematic when the system behavior is assumed to be the sum of an unspecified number of exponential components. Moreover, non-linear methods are inappropriate because of the inherent problem of non-convergence and convergence to local minima. In contrast, the convergence of linear methods such as $N N L S$ has already been proven, no initial estimation is needed and the algorithm is robust in the presence of noise $[109,147]$.

\subsubsection{NNLS literature review}

The NNLS algorithm was first introduced and coded in Fortran by Lawson and Hanson [108]. Provencher [110] discussed the algorithm as a discrete spectrum of coefficients as $\delta$-functions 
and how to improve it to a more stable continuous spectrum by choosing a regularizer to smooth the spectrum. This is the most important part of analyzing distributed noisy data to get a more stable solution with the $N N L S$ algorithm. A general Fortran IV package called CONTIN was developed by Provencher for this purpose [110]. Pfeuffer et. al. used CONTIN for the diffusion data analysis of MR diffusion experiments on rat brain [102].

Whittall and MacKay [108] discussed the advantages of the NNLS algorithm for data analysis of $T_{1}$ and $T_{2}$ relaxation times in MRI including a quantitative interpretation of the parameters. They theoretically compared the other linear inversion approaches with $N N L S$ and then applied the $N N L S$ algorithm for their purposes in $\mathrm{T}_{2}$ relaxation experiments, demonstrating that the NNLS algorithm is the most appropriate method for analyzing the data of an inhomogeneous system like tissue with a multi-exponential model $[109,145,146]$.

\subsection{Literature Review}

Experiments which calculate the ADC in vivo by assuming that the diffusion decay is a monoexponential decay curve have the advantage of describing the diffusion characteristics in tissue by a single decay constant over a wide range of experimental conditions. However, there are other diffusion experiments which demonstrate a significant deviation from mono-exponentiality for the diffusion decay curves in tissue. Early experiments on the diffusion time dependence of the ADC proposed that it was invariant to the diffusion time $[59,150]$. However, further experiments by Horsfield et. al. [115] showed a marked decrease in the ADC for human brain tissue with increasing diffusion time between 40 and $800 \mathrm{~ms}$ and b-values up to about $2200 \mathrm{~s} / \mathrm{mm}^{2}$ which was consistent with restricted diffusion. Subsequently, experiments on adult rat brain in vivo, investigated over a greater range of b-values (up to $1 \times 10^{4} \mathrm{~s} / \mathrm{mm}^{2}$ ), showed that the data could be 
fit to a bi-exponential decay curve with apparent diffusion coefficients for the fast and slow components of $0.824 \times 10^{-3}$ and $0.168 \times 10^{-3} \mathrm{~mm}^{2} / \mathrm{s}$, respectively [99]. However, the relative contribution of the components was opposite to that expected from the volumes of the intracellular and extracellular compartments in rat brain. No diffusion time dependence was observed between 8 and $60 \mathrm{~ms}$. Similar experiments have been performed on the human brain by Kraemer et. al. [101] and have given similar results. Mulkern et. al. reported nonmonoexponential signal attenuation for diffusion in human brain using b-values up to $6000 \mathrm{~s} / \mathrm{mm}^{2}$ and they fit their results to a bi-exponential function $[98,103]$. From more recent experiments, the $\mathrm{ADC}$ of the internal capsule of the white matter was reported to be $1.02 \times 10^{-3}(66 \%) \mathrm{mm}^{2} / \mathrm{s}$ for the fast and $0.11 \times 10^{-3} \mathrm{~mm}^{2} / \mathrm{s}$ for the slow component. The diffusion decay curves for garfish nerves are often fit to three exponentials with $\mathrm{D}$ values of approximately $1.0 \times 10^{-3}, 0.15 \times 10^{-3}, 0.01 \times 10^{-3}$ $\mathrm{mm}^{2} / \mathrm{s}$ with similar relative fractions for all three nerve cell types; bi-exponential fits were not sufficient over a large range of b values. This is an unpublished result of Beaulieu (Ph.D. thesis, 1995) $[34,152]$. Fitting the diffusion decay curves with a multi-exponential function over a large range of $b$ values up to $7 \times 10^{4} \mathrm{~s} / \mathrm{mm}^{2}$ in healthy rat brain was reported by Pfeuffer et. al. [102]. They reported a continuous distribution for the apparent diffusion coefficients resolved into three main components at $0.99 \times 10^{-3}(95 \%), 0.08 \times 10^{-3}(2.8 \%)$ and $0.009 \times 10^{-3}(1.8 \%) \mathrm{mm}^{2} / \mathrm{s}$ [102]. Experiments on excised brain tissue by Assaf and Cohen [100] with large b-values up to $3 \times 10^{4}$ $\mathrm{s} / \mathrm{mm}^{2}$ demonstrated a non-monoexponentiality of the signal attenuation plus a clear diffusion time dependence over the same range of diffusion times as used by Niendorf et. al. [99,100].

Experiments at short diffusion times may also detect the effects of diffusion restriction by sensitizing water molecules of the intracellular space of brain tissue. The Stejskal Tanner sequence can be implemented as a so called $c g$ experiment where the gradient amplitude is kept constant and the b-value is varied by modifying the gradient duration, $\delta$, and thus the diffusion 
time, while keeping the echo time constant. When these experiments are compared with standard $c t$ experiments, in which all timings are held constant and the b-value is varied by changing the gradient amplitude, evidence of restriction is seen in some studies of both healthy and post mortem rat brain for a range of short diffusion times from 1.8 to $6.0 \mathrm{~ms}$ and at longer diffusion times from 4.5 to $11.8 \mathrm{~ms}[125,153,154,155]$. The ADC reported by the standard $c t$ experiment was found to vary between $1.1 \times 10^{-3} \mathrm{~mm}^{2} / \mathrm{s}$ at a diffusion time of $6.0 \mathrm{~ms}$ and $0.89 \times 10^{-3}$ $\mathrm{mm}^{2} / \mathrm{s}$ at $11.8 \mathrm{~ms}$. The authors interpreted these results in terms of changes in the extracellular tortusity. Other experiments in rat brain in-vivo implemented by Pfeuffer et. al. showed a diffusion time dependence of the signal decays in a range of diffusion times between 17 and 63 ms. The apparent intracellular diffusion coefficient was reported to vary between 0.19 and $0.032 \times 10^{-3} \mathrm{~mm}^{2} / \mathrm{s}$, respectively [111]. Assaf et. al. did similar experiments on rat brain to find the displacement distribution in q-space. They also showed the diffusion time dependence of the diffusion signal decays for diffusion times between 4.5 and $72 \mathrm{~ms}$ [156].

Clark and Le Bihan [101,151] measured the ADC of the fast and slow components in human white matter in vivo using three orthogonal diffusion gradient directions to study anisotropy effects. When the gradient was in the readout direction they found $\mathrm{ADC}_{\text {slow }}=0.14 \times 10^{-3}$ $(50 \%)$ and $\mathrm{ADC}_{\mathrm{fast}}=1.12 \times 10^{-3} \mathrm{~mm}^{2} / \mathrm{s}$. For diffusion sensitization in the phase encoding direction they reported $\mathrm{ADC}_{\text {slow }}=0.13 \times 10^{-3}(43 \%), \mathrm{ADC}_{\mathrm{fast}}=1.13 \times 10^{-3} \mathrm{~mm}^{2} / \mathrm{s}$ and when the diffusion gradient was in the slice selection direction they got $\mathrm{ADC}_{\text {slow }}=0.23 \times 10^{-3}(30 \%), \mathrm{ADC}_{\text {fast }}=$ $1.39 \times 10^{-3} \mathrm{~mm}^{2} / \mathrm{s}$. The maximum b-factor used in these experiments was $4 \times 10^{3} \mathrm{~s} / \mathrm{mm}^{2}$. Diffusion anisotropy effects can be fully extracted with diffusion tensor imaging experiments to provide more details on tissue microstructure. The trace of the diffusion tensor, $\operatorname{Tr}(D)=D_{x x}+D_{y y}+D_{z z}$, as defined in section 1.2, is measured in several different studies of brain tissue. Melhem et. al. [33] showed that the average value of $\operatorname{Tr}(\mathrm{D}) / 3$, the so called mean diffusivity, varied between 
$0.83 \times 10^{-3} \mathrm{~mm}^{2} / \mathrm{s}$ in the left centrum semiovale to $1.27 \times 10^{-3} \mathrm{~mm}^{2} / \mathrm{s}$ in the pons for different anatomic locations in the human brain using six b-values between 0 and $800 \mathrm{~s} / \mathrm{mm}^{2}$. Le Bihan et. al. reported mean diffusivities for human brain between $0.64 \times 10^{-3} \mathrm{~mm}^{2} / \mathrm{s}$ for the internal capsule and $0.71 \times 10^{-3} \mathrm{~mm}^{2} / \mathrm{s}$ for pyramidal tracts in human white matter $[12,157]$. 


\section{CHAPTER 3}

\section{Experiments}

The diffusion weighted imaging experiments in this thesis were performed on a Siemens Symphony (Siemens Medical Systems, Erlangen, Germany) clinical magnetic resonance imager with a $1.5 \mathrm{~T}$ static magnetic field and operating under NUMARIS/4 software. The imager is equipped with $x, y$ and $z$ self shielded gradient coils which are capable of switching the gradient amplitude from 0 to $30 \mathrm{mT} / \mathrm{m}$ in $290 \mu \mathrm{s}$ (ramp time) although, in practice, the maximum gradient amplitude is limited to $28.5 \mathrm{mT} / \mathrm{m}$. A $30 \mathrm{~cm}$ diameter RF Circularly Polarized (CP) Head Array was used to detect the RF echo signals from the object voxels. The body coil built into the magnet assembly functioned as the transmitter for the RF excitation signals. A head coil phantom, which consisted of water and a small amount of $\mathrm{NiSO}_{4}\left(1.24 \mathrm{~g} \mathrm{NiSO}_{4}-6 \mathrm{H}_{2} \mathrm{O}\right)$ to reduce $\mathrm{T}_{1}$, and $2.62 \mathrm{~g}$ of $\mathrm{NaCl}$ in a plastic bottle with cylindrical geometry $35 \mathrm{~cm}$ in length and $14 \mathrm{~cm}$ in diameter, was used for verification of the measurements and validation of any new sequence or image reconstruction programming before they were used with human subjects. 
All experiments reported in this thesis were acquired with slices in the transverse orientation with the frequency encoding direction in the right to left direction and the phase encoding in the anterior to posterior direction for subjects in the head first supine orientation.

The work presented in this thesis was approved by The Ottawa Hospital Research Ethics Board. All human subjects were pre-screened for contraindications using the standard MRI questionnaire of The Ottawa Hospital. Informed consent was obtained from all subjects before they were imaged.

The diffusion pulse sequence on Siemens' imager for clinical studies is called ep2d_diff. This sequence performs a diffusion weighted preparation followed by a single shot EPI readout echo train with gradient reversal to acquire the data from which the 2-D MR image is reconstructed. The sequence was developed based on the twice refocused spin echo (TRSE) sequence [124] which has four diffusion sensitizing gradient pulses inserted as explained in section 2.3.5. Figure 2.9 shows typical sequence timing for the measurements reported in this thesis with a $90^{\circ} \mathrm{RF}$ excitation pulse and two refocusing $180^{\circ} \mathrm{RF}$ pulses and diffusion gradients inserted in the $\mathrm{x}$-direction, in this case. Spatial encoding was performed with an EPI echo train, using gradient reversal in the readout $(\mathrm{x})$ direction along with phase encoding gradients applied in the y direction (see also figure 2.5). Slice selection gradients in the $\mathrm{z}$ direction and the required spoiler gradients are also included in the sequence but are not shown in figure 2.9.

To gain access to Siemens' sequence programs on a personal computer we needed to install Siemens' Sequence Development Environment (SDE) which is part of the Integrated Development Environment and Application (IDEA) package provided by Siemens as part of a research agreement. The SDE shell allowed us to develop our own diffusion sequence programs, compile them, create sample protocols of the parameter sets for the measurements, simulate the execution of the sequence, and finally to build the sequence files in the form of dynamic-link 
library (.dll) files and transfer the new sequence to the user environment of the "host" computer of the MR system. The diffusion sequences developed for this thesis were based on Siemens' ep2d-diff.cpp program.

The diffusion experiments in this thesis can be divided in two main categories: diffusion decay studies and diffusion time dependence studies. The other experiments such as noise correction, noise estimation and eddy current effect studies are associated with these two categories.

\subsection{Diffusion Decay Study}

For the diffusion decay study discussed in chapter 5, a TRSE diffusion pulse sequence similar to the one shown in figure 2.9 was used with the diffusion time, $\tau_{\mathrm{D}}$, held constant at about $50 \mathrm{~ms}$ for the experiments. The b-values were changed by adjusting the gradient amplitudes. As shown in the previous chapter, the b-value is obtained by integrating equation (2.74). The resulting expression for $b$ is of the form $b=\gamma^{2} G^{2} \delta^{2} \tau_{D}$. The details of the b-value and $\tau_{D}$ evaluation for this sequence are given in section 6.2.2 and Appendix II.

Sixteen b-values were used in each set of measurements. This was the maximum allowed for the diffusion sequence. For some experiments 96 b-values were used by repeating the measurements six times with sixteen different b-values each time. The large number of b-values was used to obtain higher accuracy for the diffusion decay of water molecules in the white matter of human brain in vivo as well as the verification of the diffusion decay measurements on the water phantom. The maximum b-value for Siemens' diffusion sequence was, by default, 10,000 $\mathrm{s} / \mathrm{mm}^{2}$. We modified this value, within the sequence program, to $12,500 \mathrm{~s} / \mathrm{mm}^{2}$ which corresponds to the maximum allowed gradient strength of $28.5 \mathrm{mT} / \mathrm{m}$. The $96 \mathrm{~b}$-values were 
selected between 0 and $12,500 \mathrm{~s} / \mathrm{mm}^{2}$ and distributed in 6 sets of $16 \mathrm{~b}$-values; the first set was from 0 to $1500 \mathrm{~s} / \mathrm{mm}^{2}$, the second from 1500 to $3000 \mathrm{~s} / \mathrm{mm}^{2}$, the third from 3000 to $4500 \mathrm{~s} / \mathrm{mm}^{2}$, the fourth from 4500 to $6000 \mathrm{~s} / \mathrm{mm}^{2}$ and the fifth set was from 6000 to $7500 \mathrm{~s} / \mathrm{mm}^{2}$, all with an interval of $100 \mathrm{~s} / \mathrm{mm}^{2}$. The sixth set was selected from 7500 to $12500 \mathrm{~s} / \mathrm{mm}^{2}$ with an arbitrary interval of $b$-values. For all of these experiments the diffusion sensitizing gradients were chosen to be in the readout $(\mathrm{x})$ direction.

Many of the protocol parameters for the measurements can be modified interactively via the user interface at the imager's user console. The slice position was selected to be above the ventricles of the human brain in the $\mathrm{z}$ direction (head to foot), the orientation was transverse and the thickness was $10 \mathrm{~mm}$ for this series of experiments. Only one slice was acquired in each experiment. The echo time for all experiments was kept constant at $\mathrm{TE}=200 \mathrm{~ms}$ and the repetition time was $\mathrm{TR}=500 \mathrm{~ms}$ to keep the $\mathrm{T}_{2}$ weighting the same for all points of the diffusion signal decay. The partial Fourier factor was 6/8. The field-of-view (FOV) was selected to be 220 $\mathrm{mm}$ for some experiments and $464 \mathrm{~mm}$ for others. The size of the FOV used for a particular set of experiments will be specified when these measurements are discussed in later chapters. Although the larger FOV had the limitation of degrading the resolution of the image, it had the advantage of improving the SNR per voxel. A matrix size of $128 \times 128$ was used for the reconstruction of the 2D images for all measurements. The maximum number of averages for Siemens' diffusion sequence was set to 32 , but we modified it to allow up to a maximum of 1000 averages. For our measurements we chose to use 50 averages as the best compromise between SNR improvement and keeping the total scan time down to a few minutes per sequence. The total scan time for each sequence with 50 averages and 16 b-values was about 6 minutes. The whole protocol including the 6 sets of b-values, one set of $T_{1}$ weighted spin echo images, with TE=12 ms and TR=800 ms, plus some other supporting sequences took about 40 minutes. The fat suppression button was kept 
ON for all diffusion sequences in order to avoid artifacts related to the chemical shift between fat and water. It should be noted that a "localizer" sequence was also used for each protocol. This sequence generates $T_{1}$ weighted images in 3 planes; the transverse, coronal and sagittal. It normally only takes a few seconds with $\mathrm{TE}=5 \mathrm{~ms}$ and $\mathrm{TR}=20 \mathrm{~ms}$. It is used for positioning the slices for the subsequent sequences.

\subsection{Diffusion Time Dependence Study}

\subsubsection{Primary experiments}

The purpose of the second series of experiments was to study the $\tau_{\mathrm{D}}$ dependence using the measurement of the attenuation of the signal of the water molecules by diffusion in human brain in vivo for short $\tau_{\mathrm{D}}$ 's and b-values which are as high as possible. The diffusion sequence was modified appropriately for this purpose (see section 6.2.3). The $\mathbf{b}$ and $\tau_{\mathrm{D}}$ values were calculated for the new sequence variables using equations presented in section 6.2.1 and Appendix II. The new diffusion sequence was optimized from various possible versions of the sequence developed for the diffusion time dependence study. The optimized sequence could be applied over a range of diffusion times from 6.0 to $53.8 \mathrm{~ms}$ with corresponding maximum b-values between 40 and 2835 $\mathrm{s} / \mathrm{mm}^{2}$. The details about the compromises between these different sequences are discussed in section 6.2.3.

The developed sequence was prepared for $\tau_{\mathrm{D}}$ values of $19.9,25.7,29.7,35.5,44.0$, and 53.8 $\mathrm{ms}$ with $\mathrm{TE}=200 \mathrm{~ms}$ and the diffusion gradient duration, $\delta$, set to $30 \mathrm{~ms}$. The maximum $\mathrm{b}$-value associated with the maximum gradient strength of $28.5 \mathrm{mT} / \mathrm{m}$ for each of these $\tau_{\mathrm{D}}$ 's was calculated to be $1050,1355,1566,1872,2318$ and $2835 \mathrm{~s} / \mathrm{mm}^{2}$, respectively, with the diffusion 
gradient applied along the $\mathrm{x}$-direction (i.e. the frequency encoding direction). These new sequences were first validated using the head coil water phantom. Preliminary experiments were also performed on two healthy human subjects to verify that the new sequences were working properly. Only one slice was imaged and it was positioned above the ventricles of the brain to measure the diffusion decay of the water molecules in white matter. For both the water phantom and the brain imaging, $11 \mathrm{~b}$-values, ranging from zero to the maximum possible $b$-value, were used, the slice thickness was $5 \mathrm{~mm}$, the slice orientation was transverse, FOV $=464 \times 464 \mathrm{~mm}^{2}$, $\mathrm{TE}=200 \mathrm{~ms}, \mathrm{TR}=500 \mathrm{~ms}$, the matrix size was $128 \times 128$ and a partial Fourier factor of $6 / 8$ was used. Fat suppression was turned on for all of these experiments to avoid chemical shift artifacts. For these transverse images phase encoding was applied in the anterior to posterior direction and frequency encoding was left to right. The phantoms and human subjects were always registered in the head first supine orientation. For these preliminary experiments only five $\tau_{\mathrm{D}}$ values were used ( $\tau_{\mathrm{D}}=25.7 \mathrm{~ms}$ was not included) but all six $\tau_{\mathrm{D}}$ 's were used for the actual experiments. The number of averages for the validation studies was 10 but was increased to 50 for the real experiments. The averaging was done in image space (i.e. magnitude averaging) as opposed to in $\mathrm{k}$-space (i.e. complex averaging). The only other difference in the parameter settings between the preliminary experiments and the actual study was that the number of b-values was increased from 11 to 16 for the real experiments. The $16 \mathrm{~b}$-values used are listed in Table 3.1 for each $\tau_{\mathrm{D}}$ value.

The diffusion time dependence measurements of the diffusion of water molecules in human brain in vivo were performed on 8 healthy human subjects; 3 females and 5 males between 23 and 43 years of age. The protocol that was used for these measurements included a localizer, 6 diffusion sequences corresponding to different diffusion times plus Siemens' diffusion sequence as a control. In addition, the $\tau_{\mathrm{D}}=53.8 \mathrm{~ms}$ sequence was repeated with the mode of image reconstruction changed from magnitude averaging to complex averaging. The total scan time was 


\begin{tabular}{|c|c|c|c|c|c|}
\hline \multicolumn{6}{|c|}{$\tau_{\mathrm{D}}(\mathrm{ms})$} \\
\hline 19.9 & 25.7 & 29.7 & 35.5 & 44.0 & 53.8 \\
\hline 0 & 0 & 0 & 0 & 0 & 0 \\
42.0 & 54.2 & 62.6 & 74.9 & 92.7 & 113.4 \\
83.9 & 108.4 & 125.3 & 149.8 & 185.4 & 226.8 \\
125.9 & 162.7 & 187.9 & 224.7 & 278.1 & 340.1 \\
167.8 & 216.9 & 250.5 & 299.6 & 370.8 & 453.5 \\
209.8 & 271.1 & 313.2 & 374.5 & 463.5 & 566.9 \\
251.7 & 325.3 & 375.8 & 449.4 & 556.2 & 680.3 \\
293.7 & 379.5 & 438.5 & 524.3 & 648.9 & 793.7 \\
335.7 & 433.8 & 501.1 & 599.2 & 741.6 & 907.1 \\
377.6 & 488.0 & 563.7 & 674.1 & 834.3 & 1020.5 \\
419.6 & 542.2 & 626.4 & 749.0 & 927.0 & 1133.8 \\
503.5 & 650.6 & 751.6 & 898.8 & 1112.5 & 1360.6 \\
671.3 & 867.5 & 1002.2 & 1198.4 & 1483.3 & 1814.1 \\
839.1 & 1084.4 & 1252.7 & 1498.0 & 1854.1 & 2267.7 \\
1007.0 & 1301.3 & 1503.3 & 1797.6 & 2224.9 & 2721.2 \\
1048.9 & 1355.5 & 1565.9 & 1872.5 & 2317.6 & 2834.6 \\
\hline
\end{tabular}

Table 3.1. The $16 \mathrm{~b}$-values $\left(\mathrm{s} / \mathrm{mm}^{2}\right)$ applied for each diffusion time sequence.

approximately 50 minutes.

The program DImageV3, developed by I. Cameron in IDL version 6, was used to read, display and postprocess the specified set of diffusion weighted DICOM3 images, where DICOM stands for Digital Imaging \& COmmunications in Medicine. Using this program the ROI's were positioned in the white matter of the brain images. The ROI's were rectangular in shape and contained $5 \times 4=20$ pixels. Thus, the voxel size was $18 \times 14.4 \times 5 \mathrm{~mm}^{3}$ based on $3.6 \mathrm{~mm}$ in plane resolution. The ROI's were carefully positioned to avoid N/2 ghosting and Gibbs artifacts. All the data extracted from the diffusion decay for each ROI were analyzed using NNLS-diff which was developed for the data analysis of the diffusion images as a part of this thesis. 


\subsubsection{Supplemental experiments for noise correction and eddy current effects}

Noise bias in MR diffusion studies, particularly for high b-values, can be a significant problem and it must be removed as much as possible to ensure the validity of subsequent analyses. One approach that was considered was complex averaging; signal averaging of the real and imaginary parts of the signal, for which the noise contribution is Gaussian, rather than signal averaging of the magnitude signal. In order to introduce complex averaging it was necessary to modify the programs that perform the image reconstruction for the diffusion sequences. This was done using the Image Calculation Environment (ICE), which is part of the IDEA sequence programming software package provided to the MR research group at The Ottawa Hospital by Siemens under a research agreement. This change involved introducing code to do the complex averaging as well as deactivating the magnitude averaging. Since these are done in different programs within the ICE environment these were two very distinct aspects to the problem. These modifications were implemented for a version of Siemens' diffusion sequence, ep2d-diff, and only affect those programs that are used for the reconstruction of images acquired with this sequence. The sequence timing used for these experiments was the same as for ep2d-diff; it was unaffected by these modifications. More details about the development of these ICEprograms and the complex averaging experiments are discussed in section 4.3.1. Measurements of complex averaging using the developed ICEprograms were performed on the head coil water phantom with the number of averages varied between 10 and 1000 . The most effective number of averages was found to be 50 .

The "eddy current effect" study needed to make sure that the new sequences were still as efficient as Siemens' diffusion sequence in avoiding eddy current effects related to the switching of the diffusion gradients. Eddy currents can cause image distortions. A series of measurements was performed on both a spherical and a cylindrical water phantom to investigate this effect. For 
this study separate measurements were made with the diffusion gradients applied in the readout, phase encoding and slice selection directions. The number of averages was also changed between 5 and 50 . The other parameters for all measurements were kept the same as for the diffusion time dependence protocol discussed in section 3.2.1. 


\section{CHAPTER 4}

\section{Noise Correction}

\subsection{Introduction}

Noise is a problematic source of deterioration in MR-diffusion images, especially when a quantitative analysis is performed based on the magnitude images. The intrinsic noise generated by the imaging electronics hardware and the small random electrical charges within the volume of the object being imaged are added, as unwanted contributions, to the real and imaginary components of the raw data of the MR-diffusion images. Images that are produced from such noise-corrupted signals initially contain both magnitude and phase information although the phase portion of the transformed signal is commonly discarded and only the magnitude image is displayed. This process simplifies subsequent analysis of the image, but can lead to errors in quantitative studies when the signal-to-noise ratio (SNR) is small. In such cases, the received signal for both the real and imaginary components will fluctuate around a low level (e.g. zero) often producing negative values. The magnitude calculation on such signals will rectify all negative values to produce only positive magnitudes, thereby artificially raising the average level 


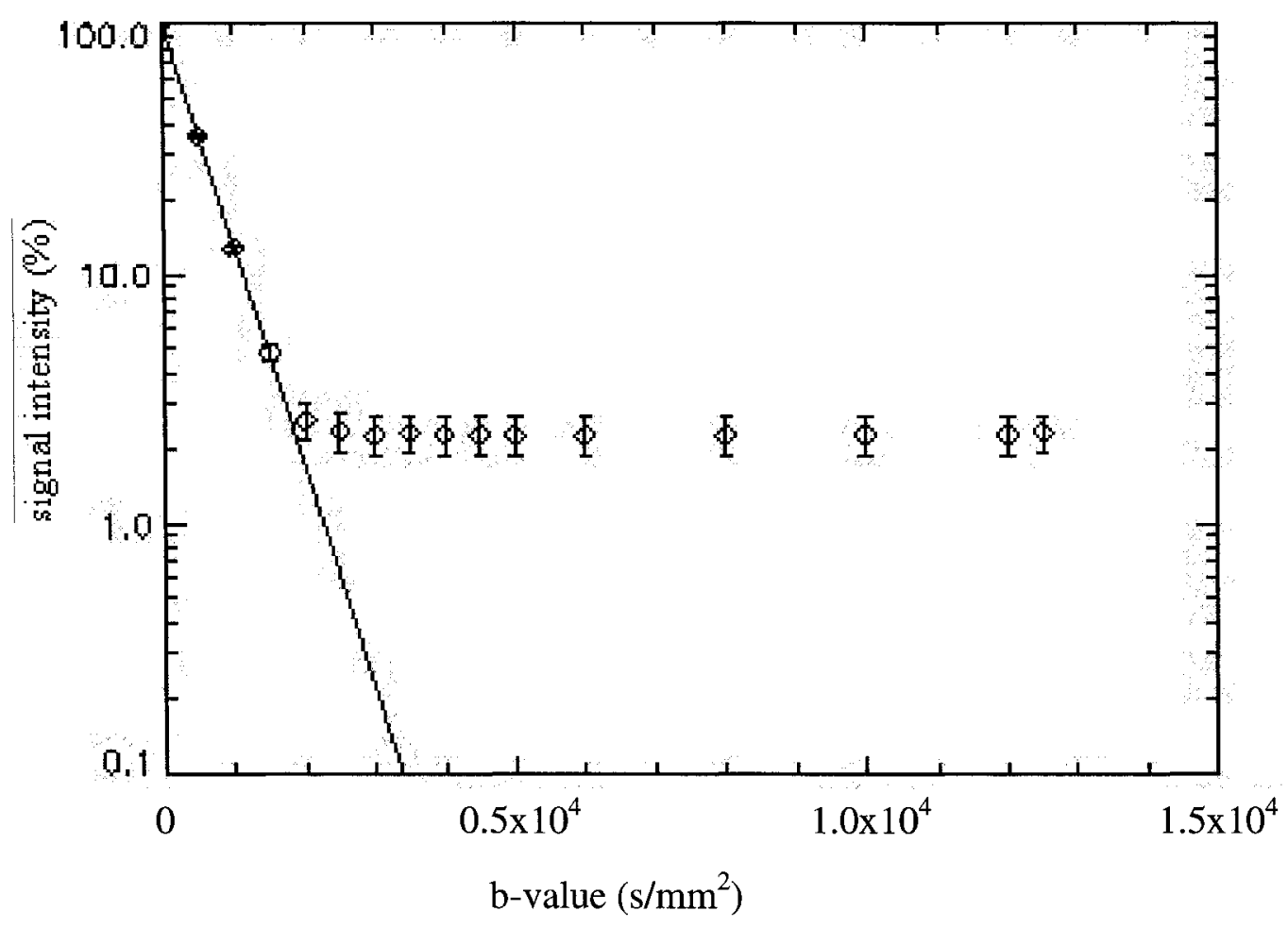

Figure 4.1. Diffusion decay measurements of a water phantom over an extended range of bvalues and fitted with least squares.

of these pixels. The signal thus will be biased by the rectified noise manifesting as a constant positive baseline to the magnitude signal decays, especially when the SNR is low, even when the $\mathrm{SNR}=0$.

A good example system in which to study this effect is the diffusion decay studied over an extended range of $b$-values from zero to very high $b$-values where the signal is expected to approach zero exponentially as is shown in figure 4.1 . This rectified decay curve can be well understood as being due to the change in behavior of the noise from Gaussian (i.e. the high SNR limit for the Rician probability distribution function (PDF)) to non-Gaussian as the signal decays. Although for SNR $\geq 3$ the Rician distribution approaches a Gaussian distribution, the mean of 


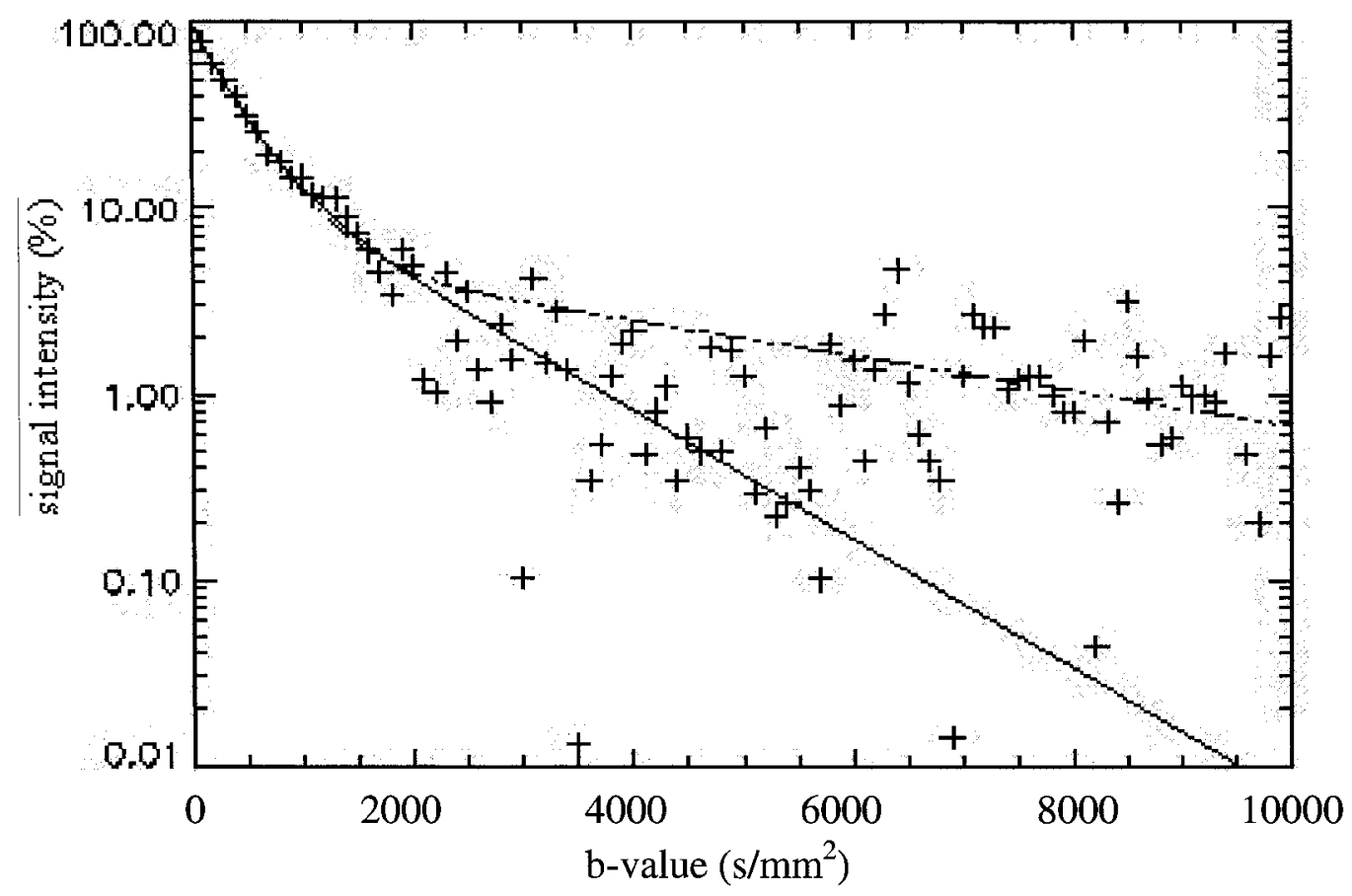

Figure 4.2. A simulated bi-exponential diffusion decay with the Rician noise bias (dashed-line) compared with the actual decay (solid line).

the noise for the magnitude image pixel intensity is not, in general, equal to the "true' signal, but rather it is biased by the noise.

Figure 4.1 demonstrates the noise bias for a water phantom where the diffusion decay is expected to be exponential. For this example, errors associated with the noise bias can easily be avoided by limiting the data acquisition to low b-values where the SNR is high since the decay is known to be exponential. A more illustrative example is presented in figure 4.2 where a simulated bi-exponential diffusion decay is shown. The decay data were calculated from

$$
M(b)=\left[\left(A(b) \cos \varphi+n_{R}\right)^{2}+\left(A(b) \sin \varphi+n_{I}\right)^{2}\right]^{1 / 2}
$$

and 


$$
\mathrm{A}(\mathrm{b})=\mathrm{A}(0)\left[\mathrm{fe}^{-\mathrm{bD}_{1}}+(1-\mathrm{f}) \mathrm{e}^{-\mathrm{bD}_{2}}\right],
$$

where $\varphi$ is the phase angle between the real and imaginary acquisition channels. The noise contributions on the real and imaginary channels, $n_{R}$ and $n_{I}$, respectively, were both chosen at random and independently from a Gaussian distribution with the standard deviation, $\sigma$, arbitrarily set to one and $\mathrm{A}(0)$ is set to 100 for convenience. The simulation was run for $100 \mathrm{~b}$-values and simulates the diffusion decay for a single voxel with no signal averaging. For the example shown in figure 4.2 we chose $f=0.8, D_{1}=3 \times 10^{-3} \mathrm{~mm}^{2} / \mathrm{s}$ and $D_{2}=8 \times 10^{-4} \mathrm{~mm}^{2} / \mathrm{s}$. A least squares fit to this simulated data using a bi-exponential fit function yields values of $\mathrm{f}=0.94, \mathrm{D}_{\mathrm{l}}=2.5 \times 10^{-3} \mathrm{~mm}^{2} / \mathrm{s}$ and $\mathrm{D}_{2}=2.2 \times 10^{-4} \mathrm{~mm}^{2} / \mathrm{s}$. The fit using these parameters is shown in the figure with a dashed line whereas the bi-exponential decay using the correct parameter values is shown as a solid line. It is clear that the noise bias to the data causes significant inaccuracies in the parameter values obtained from fitting the data in this way.

In section 4.2, the theory of Rician noise and its effect on MR signal measurements will be developed. Several schemes have been proposed in the literature for correcting this noise bias [133-142]. Most of these require an accurate value for $\sigma$. In section 4.3, $\sigma$ is evaluated in several different ways and compared with theory for consistency. In section 4.4, several different noise correction schemes from the literature are presented and compared. In section 4.5, a new approach for noise correction is presented which overcomes several of the disadvantages of the existing methods.

\subsection{The Rician Distribution Function and its Moments}

If the real and imaginary data ( $x$ and $y$ ) with mean values of $A_{R}$ and $A_{I}$ respectively, are corrupted by Gaussian, zero mean, stationary noise with standard deviation $\sigma$, then the PDF of the 
magnitude, $M$, can be evaluated as the joint probability function from the two independent Gaussian PDF's as

$$
\mathrm{P}(\mathrm{M} \mid \mathrm{A}, \sigma)=\mathrm{P}\left(\mathrm{x} \mid \mathrm{A}_{\mathrm{R}}, \sigma_{\mathrm{R}}\right) \mathrm{P}\left(\mathrm{y} \mid \mathrm{A}_{\mathrm{I}}, \sigma_{\mathrm{I}}\right)
$$

where $\mathrm{M}$ denotes the noise corrupted pixel magnitude (i.e. the measured pixel intensity) and $\mathrm{A}$ is the "true" magnetic induction signal intensity (i.e. the signal strength that would be obtained in the absence of noise). $\mathrm{M}$ and $\mathrm{A}$ are given by:

$$
\begin{aligned}
& M=\left(x^{2}+y^{2}\right)^{1 / 2}, \\
& A=\left(A_{R}^{2}+A_{I}^{2}\right)^{1 / 2} .
\end{aligned}
$$

In writing equation (4.3) it has been assumed that $P\left(x \mid A_{R}, \sigma_{R}\right)$ and $P\left(y \mid A_{I}, \sigma_{I}\right)$ are independent. We will further assume that $\sigma=\sigma_{R}=\sigma_{I}$. These are common assumptions which are almost always satisfied in MRI.

By substituting in the explicit expressions for the Gaussian PDF's, equation (4.3) can be written as

$$
\mathrm{P}(\mathrm{M} \mid \mathrm{A}, \sigma)=\frac{1}{2 \pi \sigma^{2}} \exp \left\{-\left[\left(\mathrm{x}-\mathrm{A}_{\mathrm{R}}\right)^{2}+\left(\mathrm{y}-\mathrm{A}_{\mathrm{I}}\right)^{2}\right] / 2 \sigma^{2}\right\}
$$

Using the substitutions $x=M \cos \theta, y=M \sin \theta$ and $A_{R}=A \cos \phi, A_{I}=A \sin \phi$ in equation (4.6), $\mathrm{P}(\mathrm{M} \mid \mathrm{A}, \sigma)$ can be expressed in polar coordinates. Since we are interested in only the magnitude of the signal, the phase dependence can be removed by integrating over all $\theta$ values. The resulting PDF is the so called Rician probability distribution function:

$$
\mathrm{P}_{M}(\mathrm{M} \mid \mathrm{A}, \sigma)=\frac{\mathrm{M}}{\sigma^{2}} \exp \left[-\left(\mathrm{M}^{2}+\mathrm{A}^{2}\right) / 2 \sigma^{2}\right] \mathrm{I}_{0}\left(\frac{\mathrm{MA}}{\sigma^{2}}\right)
$$

where $I_{0}(\alpha)=\frac{1}{\pi} \int_{0}^{\pi} \exp (\alpha \cos \theta) \mathrm{d} \theta$ is the zeroth order modified Bessel function of the first kind. The subscripted $M$ on the left hand side of equation (4.7) indicates that $P_{M}(M \mid A, \sigma)$ is the PDF 
for the magnitude of the MR signal. This equation was first derived by Rice [161] in communication systems theory and later discussed by Henkleman [134] who derived closedform expressions in an MR context.

The $v^{\text {th }}$ moment of the Rician PDF is given by

$$
E\left[M^{v}\right]=\int_{0}^{\infty} \frac{M^{v+1}}{\sigma^{2}} \exp \left[-\left(M^{2}+A^{2}\right) / 2 \sigma^{2}\right] I_{0}\left(\frac{M A}{\sigma^{2}}\right) d M,
$$

where $\mathrm{E}[\mathrm{]}$ denotes the expectation operator. The solution of this equation can be expressed in terms of the confluent hypergeometric function ${ }_{1} \mathrm{~F}_{1}\left[-\mathrm{v} / 2 ; 1 ;-\mathrm{A}^{2} / 2 \sigma^{2}\right][27,136,141,142]$. The first two moments of the Rician PDF are given by $[27,141,142]$

$$
\mathrm{E}[\mathrm{M}]=\sigma \sqrt{\frac{\pi}{2}} \mathrm{e}^{-\mathrm{A}^{2} / 4 \sigma^{2}}\left[\left(1+\frac{\mathrm{A}^{2}}{2 \sigma^{2}}\right) \mathrm{I}_{0}\left(\frac{\mathrm{A}^{2}}{4 \sigma^{2}}\right)+\frac{\mathrm{A}^{2}}{2 \sigma^{2}} \mathrm{I}_{1}\left(\frac{\mathrm{A}^{2}}{4 \sigma^{2}}\right)\right]
$$

and

$$
E\left[M^{2}\right]=A^{2}+2 \sigma^{2}
$$

where $I_{1}$ is the first order modified Bessel function of the first kind.

The equation for $\mathrm{E}\left[\mathrm{M}^{2}\right]$ can also be obtained directly as the mean of the magnitude squared when the real and imaginary data are both noisy. If the real and imaginary signals are corrupted by $n_{R}$ and $n_{I}$, respectively, the magnitude of the measured signal can be written as

$$
M=\left[\left(A_{R}+n_{R}\right)^{2}+\left(A_{I}+n_{I}\right)^{2}\right]^{1 / 2} .
$$

At this point the noise is no longer simply additive to the true signal and analysis of such an image must contend with the non-linear contribution of the noise. The power magnitude signal averaged over a region of interest is given by

$$
<\mathrm{M}^{2}>=\mathrm{A}_{\mathrm{R}}^{2}+\mathrm{A}_{\mathrm{I}}^{2}+2 \mathrm{~A}_{\mathrm{R}}<\mathrm{n}_{\mathrm{R}}>+2 \mathrm{~A}_{\mathrm{I}}<\mathrm{n}_{\mathrm{I}}>+<\mathrm{n}_{\mathrm{R}}^{2}>+<\mathrm{n}_{\mathrm{I}}^{2}>
$$


Since the mean value of white noise is zero and $\left\langle n_{R}^{2}\right\rangle=\left\langle n_{I}^{2}\right\rangle=\sigma^{2}$ for a Gaussian PDF, the mean pixel intensity for a power image can be simply evaluated as

$$
<\mathrm{M}^{2}>=\mathrm{A}^{2}+2 \sigma^{2}=\mathrm{E}\left[\mathrm{M}^{2}\right]
$$

which is the same as equation (4.10). Equation (4.13) gives a very simple and convenient way of evaluating $A$ if $\sigma$ is known. On the other hand, when $A=0$ it also provides a nice way to determine a value for $\sigma$. This noise estimator will be written as

$$
\hat{\sigma}=\frac{1}{\sqrt{2}} \sqrt{\left.<\mathrm{M}^{2}\right\rangle}
$$

The expressions given in equations (4.9) and (4.10) are valid for all SNRs, however, equation (4.9) is complicated and not easy to use in practice. Often the high and low SNR limits of $\mathrm{E}[\mathrm{M}]$ are useful. In section 2.4.2 it was shown that

$$
\lim _{A>>\sigma} E[M]=\sqrt{A^{2}+\sigma^{2}}
$$

and

$$
\lim _{\mathrm{A}<<} \mathrm{E}[\mathrm{M}]=\sigma \sqrt{\frac{\pi}{2}}
$$

See also sections 4.2.1 and 4.2.2.

\subsubsection{The low SNR limit of the Rician distribution:} The Rayleigh distribution function and its moments

The Rician distribution, in the limit of small SNR $(\mathrm{A} / \sigma \rightarrow 0)$, tends to a Rayleigh distribution $[133,160]$ :

$$
\mathrm{P}_{\mathrm{M}}(\mathrm{M} \mid \sigma)=\frac{\mathrm{M}}{\sigma^{2}} \exp \left[-\mathrm{M}^{2} / 2 \sigma^{2}\right]
$$

previously given as equation (2.92) and shown in figure 4.3 for $S N R=0$. The Rayleigh 


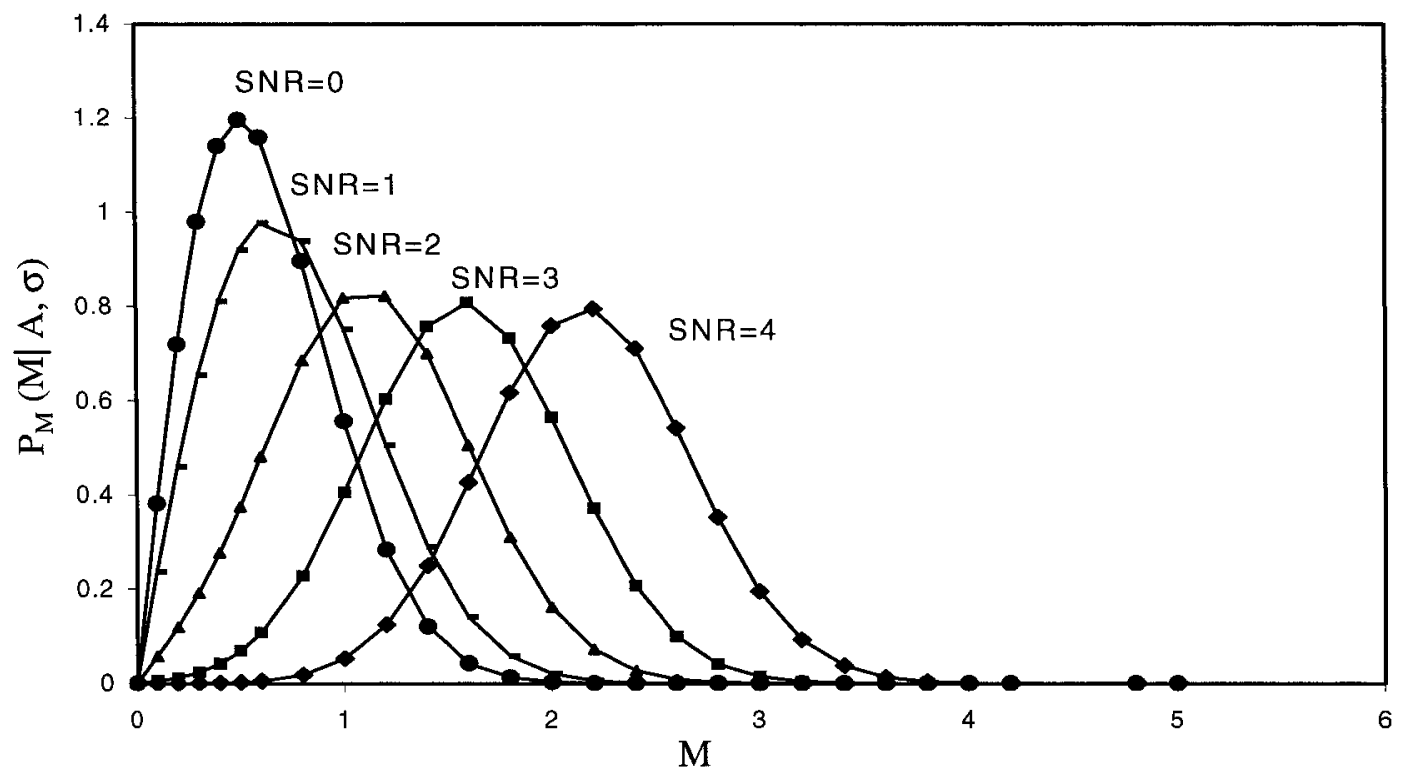

Figure 4.3. The convergence of the Rician distribution to a Gaussian in the high SNR limit and to a Rayleigh distribution at the very low limit of SNR.

distribution function can be more simply obtained by the evaluation of the joint probability function of two Gaussian noise PDFs with a mean of zero, corresponding to the real and imaginary parts of the signal with $A_{R}=A_{I}=0$. The variance, $\sigma^{2}$, of the Gaussian noise distribution, the "true" noise power, can be calculated from a magnitude image when $A=0$ since the mean, $<\mathrm{M}_{\mathrm{bkg}}>$, and variance, $\sigma_{\mathrm{M}}^{2}$, of the Rayleigh distribution function are given by

$$
\mathrm{E}\left[\mathrm{M}_{\mathrm{bkg}}\right]=<\mathrm{M}_{\mathrm{bkg}}>=\int_{0}^{\infty} \frac{\mathrm{M}^{2}}{\sigma^{2}} \exp \left[-\left(\mathrm{M}^{2} / 2 \sigma^{2}\right)\right] \mathrm{dM}=\sigma(\pi / 2)^{1 / 2}
$$

and

$$
\sigma_{\mathrm{M}}^{2}=<\mathrm{M}_{\mathrm{bkg}}>^{2}-<\mathrm{M}_{\mathrm{bkg}}^{2}>=(2-\pi / 2) \sigma^{2},
$$

respectively. $<\mathrm{M}_{\mathrm{bkg}}>$ and $\sigma_{\mathrm{M}}$ can be measured from the background region of a magnitude image. Therefore, $\sigma$ can be estimated from equations (4.18) and (4.19), respectively, as

$$
\hat{\sigma}=<\mathrm{M}_{\mathrm{bkg}}>(\pi / 2)^{-1 / 2}
$$


and

$$
\hat{\sigma}=\sigma_{M}(2-\pi / 2)^{-1 / 2}
$$

Accurate estimation of $\sigma$ is a very important part of the noise correction algorithms discussed in this chapter and will be discussed in detail in section 4.4 .

\subsubsection{The high SNR limit of the Rician distribution}

The Rician distribution for large SNR (i.e. $A / \sigma \geq 3$ ) is the other interesting limit. Gudbjartsson and Patz [133] have shown that the Rician PDF in this limit can be described by a Gaussian PDF with a mean of $\left(\mathrm{A}^{2}+\sigma^{2}\right)^{1 / 2}$ and variance $\sigma^{2}$ expressed as follows:

$$
\mathrm{P}_{M}(\mathrm{M} \mid \mathrm{A}, \sigma) \approx \frac{1}{\sqrt{2 \pi \sigma^{2}}} \exp \left[-\left(\mathrm{M}-\sqrt{\mathrm{A}^{2}+\sigma^{2}}\right)^{2} / 2 \sigma^{2}\right]
$$

As can be seen from the exponent of equation (4.22), the mean of the PDF for the magnitude image intensity in this limit is not equal to the "true" signal intensity, A, and thus results in a bias of the magnitude image data which must be taken into consideration for quantitative studies. This is small for $\mathrm{A} / \sigma \geq 3(\leq 7 \%)$ and becomes negligible in the limit of very large SNR. In figure 4.3, $\mathrm{P}_{\mathrm{M}}(\mathrm{M} \mid \mathrm{A}, \sigma)$ is plotted for $\mathrm{SNR}=\mathrm{A} / \sigma$ of $0,1,2,3$ and 4 to display the convergence of the Rician distribution towards the Gaussian distribution as the SNR increases.

\subsubsection{The noise bias of the Rician distribution}

As shown in the preceding sections, all pixel values from MR magnitude images are biased by the noise to some extent. However, for the purposes of quantifying this effect we will define the noise bias to be equal to the mean of the Rayleigh distribution, $E\left[M_{b k g}\right]=\sigma(\pi / 2)^{1 / 2}$. For simulation 
and experimental studies the noise bias will be calculated as the mean over data points where A can be considered to be zero. This will include data points for b-values larger than an arbitrarily chosen value that is appropriate for the data set being considered. For example, if the noise bias for the two data sets shown in figure 4.4 is calculated as the mean of the data points for $b \geq 10000$, the noise bias for the magnitude and complex averaged data sets is $0.56 \mathrm{~S}(0) \approx \sigma(\pi / 2)^{1 / 2}$ and $0.12 \mathrm{~S}(0)$, respectively.

\subsection{Noise Correction Techniques}

In order to get reliable information from the diffusion decay curves, especially for low SNR data points, noise correction techniques are required. There are several ways in which this can be accomplished, in principle. The most direct is to perform signal averaging on the real and imaginary signals, both of which are corrupted with Gaussian noise. This is performed during image reconstruction. The other noise correction techniques proposed in the literature are employed after image reconstruction has been completed. Henkelman [134] has suggested the use of a lookup table. Miller and Joseph [138] and McGibney and Smith [135] have proposed methods of noise correction based on equation (4.10) for $\mathrm{E}\left[\mathrm{M}^{2}\right]$. Gudbjartsson and Patz [133] have presented a similar but superior technique based on equation (4.15). The pros and cons of all of these techniques will be discussed in the following sections. Other approaches using maximum likelihood theory have also been presented $[27,136,137,139,141,142,166]$ but these will not be considered here. Koay and Basser [184] have recently presented a novel correction scheme. This will also be discussed but not in detail.

Complex averaging will be discussed in section 4.3 .1 with both simulation results and experimental results from phantoms being presented. In section 4.3.2, the various other noise 
correction techniques mentioned above will be explained in more detail. In section 4.3.3, simulation results will be given for both the McGibney and Smith and the Gudbjartsson and Patz noise correction techniques. Both of these techniques require an accurate value for $\sigma$, and it will be assumed in this section that $\sigma$ is known. The presentation of experimental results will be deferred until after section 4.4 where different methods of measuring $\sigma$ are considered.

\subsubsection{Signal averaging of the complex MR signal}

One of the well known methods for increasing the SNR in MR measurements is signal averaging of the image data over the number of measurements. This can be a good estimator of the image signal intensity when the noise is Gaussian since the SNR improves by a factor of $\sqrt{\mathrm{N}}$ when the data are averaged over $\mathrm{N}$ measurements. However, the PDF for the magnitude image data is Rician and only approximates a Gaussian at high SNRs where signal averaging is not required. For low SNR situations signal averaging of the magnitude data will reduce fluctuations about the mean but it will not correct the Rician noise bias (see figure 4.4). Therefore, signal averaging of the complex signal components, where the noise distribution is still Gaussian, was proposed for this study. This will be referred to as complex averaging.

\subsubsection{Complex averaging simulations}

Complex averaging was first tested by simulating image pixel data. The magnitude data of the $i^{\text {th }}$ measurement, $M_{i}$, was calculated from the real $\left(A_{i R}\right)$ and imaginary $\left(A_{i I}\right)$ parts of the pixel signal corrupted by simulated noise values picked at random and independently from a Gaussian PDF with a mean of zero and $\sigma=0.5$ using equation (4.11). The $\mathrm{M}_{\mathrm{i}}$, therefore, correspond to values 


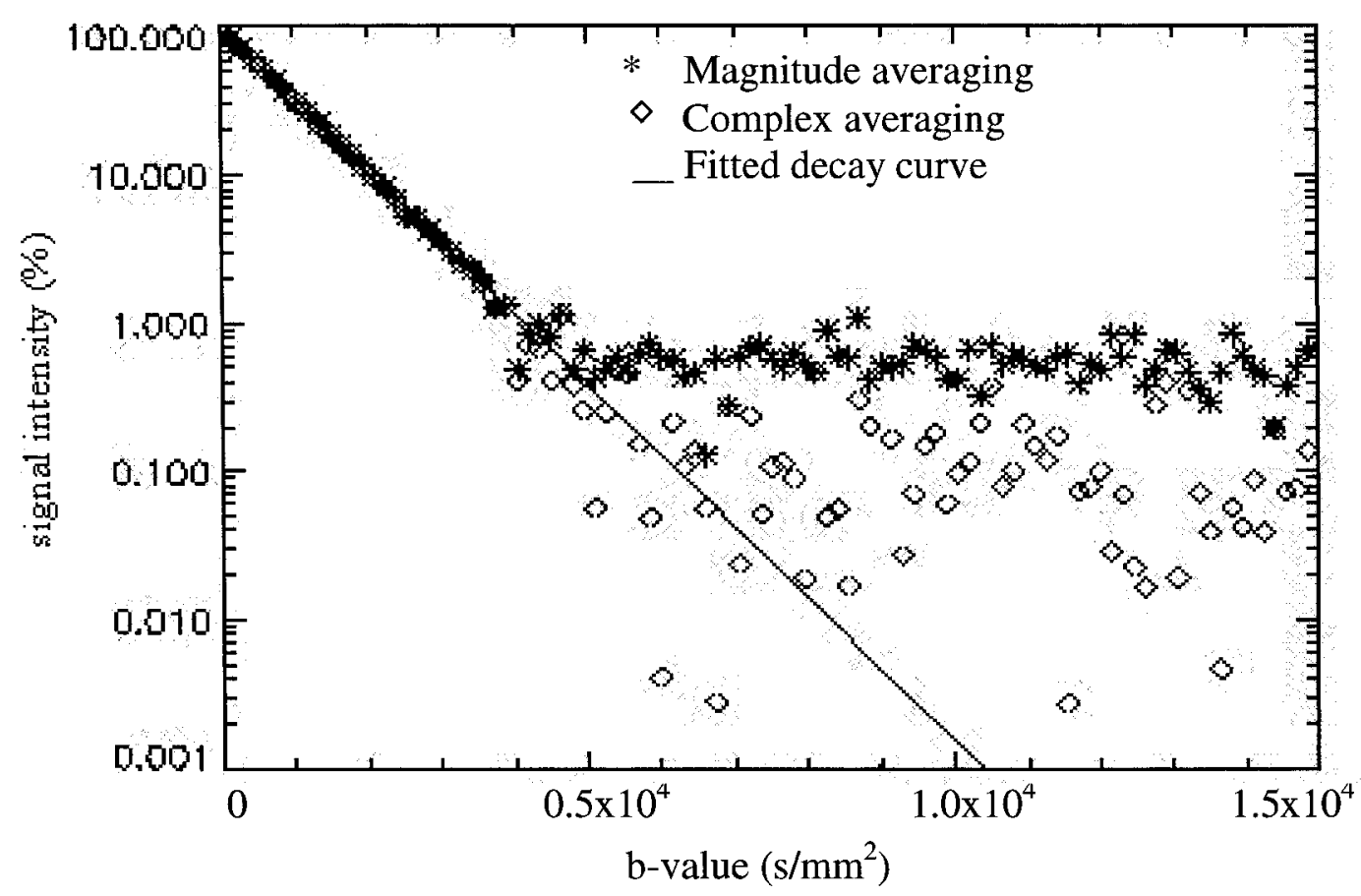

Figure 4.4. A simulated diffusion decay averaged over 10 measurements for complex averaging $(\diamond)$ compared with magnitude averaging $(*)$.

picked at random from a Rician distribution. The mean of the magnitude pixel data over a set of $\mathrm{N}$ measurements was obtained for each pixel as follows:

$$
<\mathrm{M}>=\frac{1}{\mathrm{~N}} \sum_{\mathrm{i}=1}^{\mathrm{N}} \mathrm{M}_{\mathrm{i}}=<\left[\left(\mathrm{A}_{\mathrm{iR}}+\mathrm{n}_{\mathrm{iR}}\right)^{2}+\left(\mathrm{A}_{\mathrm{iI}}+\mathrm{n}_{\mathrm{iI}}\right)^{2}\right]^{1 / 2}>\text {. }
$$

This averaged value is corrupted by a nonlinear contribution from the noise. Since the $\mathrm{M}_{\mathrm{i}}$ are Rician distributed, the PDF for $\langle M>$ will also be Rician but its standard deviation will be $\sqrt{N}$ times smaller than for the PDF for the individual $M_{\mathrm{i}}$.

The mean values of the real and imaginary signals of the pixel, each corrupted with Gaussian noise, were calculated for the $\mathrm{N}$ measurements as follows:

$$
<A_{R}>=\frac{1}{N} \sum_{i=1}^{N}\left(A_{i R}+n_{i R}\right)
$$




$$
<\mathrm{A}_{\mathrm{I}}>=\frac{1}{\mathrm{~N}} \sum_{\mathrm{i}=1}^{\mathrm{N}}\left(\mathrm{A}_{\mathrm{iI}}+\mathrm{n}_{\mathrm{iI}}\right) .
$$

The mean value of the complex averaged pixel magnitude was then calculated as

$$
<\mathrm{M}\rangle_{\mathrm{c}}=\left[<\mathrm{A}_{\mathrm{R}}>^{2}+<\mathrm{A}_{\mathrm{I}}>^{2}\right]^{1 / 2}
$$

The mean magnitude resulting from averaging the complex data, $\langle\mathrm{M}\rangle_{\mathrm{c}}$ was compared with the mean magnitude obtained by averaging of the magnitude data, $\langle\mathrm{M}\rangle$, over $\mathrm{N}=10$ simulated measurements for 100 data points (see figure 4.4). The noise bias of the decay curve for magnitude averaging, computed as the average of the data points for $b \geq 10000 \mathrm{~s} / \mathrm{mm}^{2}$, is $(0.0056 \pm 0.0015) \mathrm{S}(0)$ and the noise bias for complex averaging is $(0.0012 \pm 0.0010) \mathrm{S}(0)$. The noise bias is reduced by a factor of 4.7 when complex averaging is used instead of magnitude averaging, for the same number of measurements. Considering the relatively large fluctuations in the data, it is in satisfactory agreement with the expected result of $\sqrt{\mathrm{N}}=\sqrt{10}=3.16$.

For the case of the magnitude averaging, the low SNR signals that are being averaged together are all biased by the Rician noise. Thus, the averaging removes fluctuations about the noise biased value but does not remove the noise bias. Conversely, the signals being averaged in the complex averaging case contain Gaussian noise. Thus, averaging these signals causes the standard deviation for the real and imaginary signals to decrease by $\sqrt{\mathrm{N}}$ and the SNR to increase by $\sqrt{\mathrm{N}}$. The Rician PDF resulting from these two averaged Gaussian PDF's will have a standard deviation reduced by $\sqrt{\mathrm{N}}$ compared with the case of no averaging. Consequently, the Rician PDF will retain its high SNR Gaussian shape for signals that are $\sqrt{\mathrm{N}}$ smaller than without averaging, thus avoiding the Rician noise bias over a larger range of b-values. The observations presented here are consistent with this explanation. 

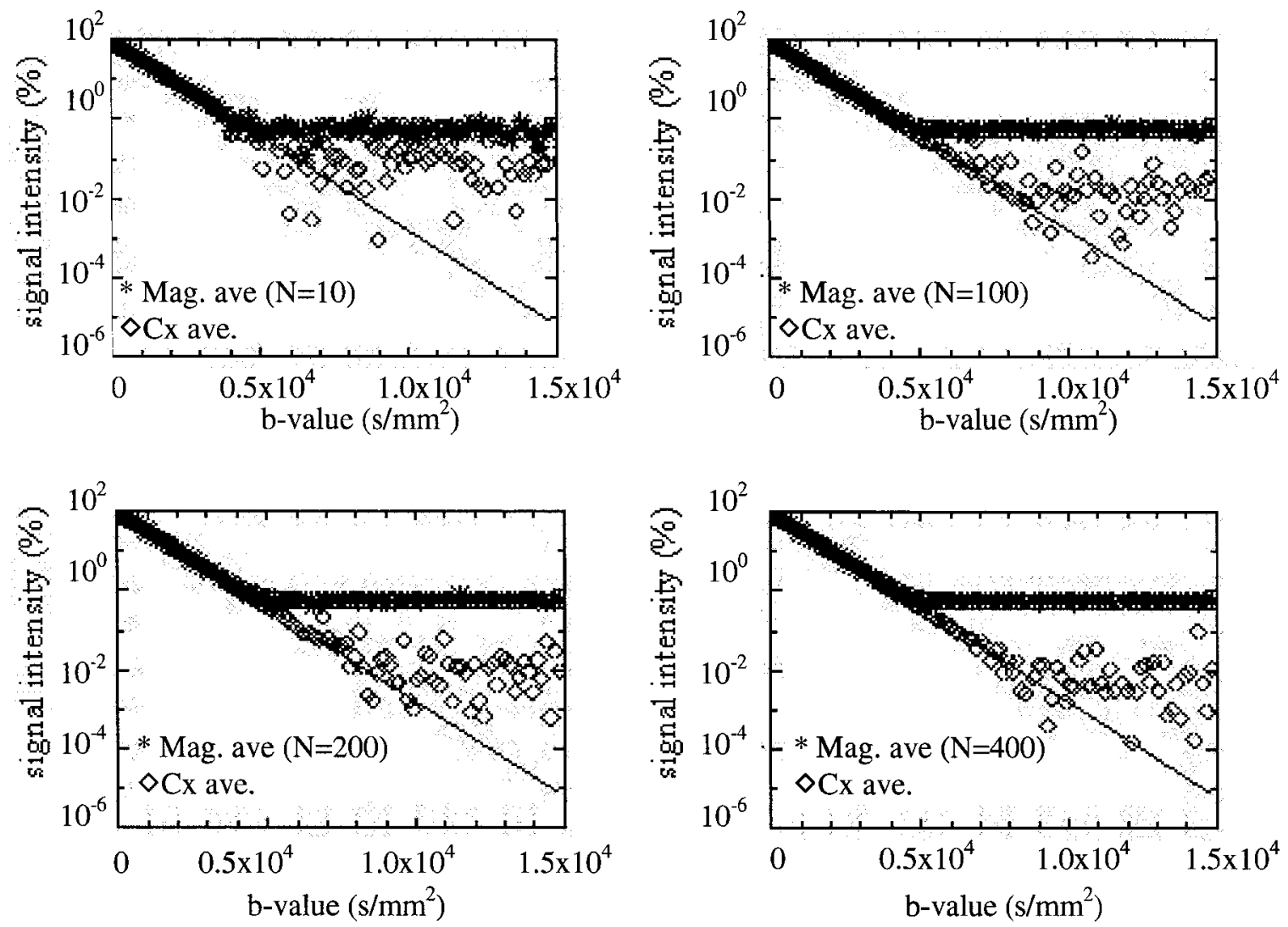

Figure 4.5. Simulated diffusion decays using signal averaging of the magnitude and of the complex data for different amounts of averaging.

To show how the number of measurements for averaging can improve the SNR and consequently may affect how much the Rician PDF resembles a Gaussian PDF for a given situation, complex averaging was studied in terms of the number of averages for the simulated image pixel data. The results of these simulations are displayed in figure 4.5 . The more averaging, the better is the correction for the noise bias, calculated as the mean of the data points for $\mathrm{b} \geq 10000 \mathrm{~s} / \mathrm{mm}^{2}$. While the noise bias for $\mathrm{N}=1,10,100,200$ and 400 magnitude averages was equal to $0.0056 \mathrm{~S}(0)$ for all values of $\mathrm{N}$, the noise bias after complex averaging was respectively $0.0053 \mathrm{~S}(0), 0.0012 \mathrm{~S}(0), 0.00033 \mathrm{~S}(0), 0.00013 \mathrm{~S}(0)$ and $0.00010 \mathrm{~S}(0)$, indicating that the noise bias reduction is proportional to a factor of $\sqrt{\mathrm{N}}$ for complex averaging. The standard deviation 
for the magnitude averaged data for $b \geq 10000 \mathrm{~s} / \mathrm{mm}^{2}$ was $0.004 \mathrm{~S}(0), 0.0015 \mathrm{~S}(0), 0.0005 \mathrm{~S}(0)$, $0.0004 \mathrm{~S}(0), 0.00007 \mathrm{~S}(0)$ for $\mathrm{N}=1,10,100,200$ and 400 , respectively. For complex averaging the corresponding standard deviations were $0.004 \mathrm{~S}(0), 0.001 \mathrm{~S}(0), 0.0007 \mathrm{~S}(0), 0.00013 \mathrm{~S}(0)$ and $0.0001 \mathrm{~S}(0)$. The standard deviations for both magnitude and complex averaging also decrease by a factor of $\sqrt{\mathrm{N}}$ as expected.

These simulations clearly show that complex averaging works to partially remove the Rician noise bias. The degree to which the Rician noise bias is removed depends on $\sqrt{\mathrm{N}}$. This can be an effective method for noise correction as long as only small corrections are required. It becomes impractical for large $\mathrm{N}$.

\subsubsection{Phantom studies of complex averaging}

In order to change the way signal averaging is done for MR measurements on the Siemens MR imager some of the programs in Siemens' Image Calculation Environment (ICE) needed to be modified. There are different ICE programs for different types of pulse sequences. The programs dealing with image reconstruction for diffusion sequences were modified to include averaging of the real and imaginary parts of the image after the signals have been Fourier transformed but before the magnitude of the complex signals is computed and to deactivate magnitude averaging. Further details of the implementation cannot be given without violating non-disclosure agreements with Siemens.

In addition to a basic knowledge of object-oriented programming and the $\mathrm{C}++$ language, coding in ICE requires an understanding of the ICE-functions, basic building blocks and the library of ICE-functors (i.e. function blocks for standard image calculation and reconstruction which can be connected together in a chain). The modified programs were compiled and executed 
on the host computer of the MR image reconstruction environment of the MR system which was a Digital Equipment Corporation (DEC) Alpha computer.

The Siemens head coil water phantom was used for all complex averaging phantom experiments reported here. The number of measurements for averaging was varied between 10 and 1000 . The number of $b$-values used ranged between 3 and 11. The other parameters, which were kept constant, were $\mathrm{TE}=200 \mathrm{~ms}, \mathrm{TR}=500 \mathrm{~ms}$, slice thickness $=7 \mathrm{~mm}$, slice orientation=transverse and the diffusion sensitizing gradient was in the frequency encoding direction $(\mathrm{R} \rightarrow \mathrm{L})$.

The experimental results were not as expected. The standard deviation of the complex averaged noise was a little bit higher $(<10 \%)$ than for the images reconstructed with magnitude averaging for the same amount of averaging. The standard deviation of the noise is expected to be the same for these two cases. This could be related to the noise filtration, which is done on the averaged signal when complex averaging is used instead of on each of the $\mathrm{N}$ signals when magnitude averaging is used. The diffusion decay curves obtained from ROIs using DImageV2 in IDL version 6.0 and averaged over 5 to 100 pixels due to the ROI's size, showed that the rectified decay curves could be corrected by no more than $9 \%$ of the intensities (figure 4.6 ) whereas we expected to see a reduction in the Rician noise bias that is proportional to $\sqrt{\mathrm{N}}$.

It is not clear why these experimental measurements were inconsistent with theoretical predictions. It could possibly be due to different effects of filtering for the two types of averaging as suggested above, or it could be due to some other process within Siemens image calculation environment that we were unaware of and therefore did not take into consideration. The source of the discrepancy remains unexplained.

The results of this section show that complex averaging can, in principle, be a very useful 


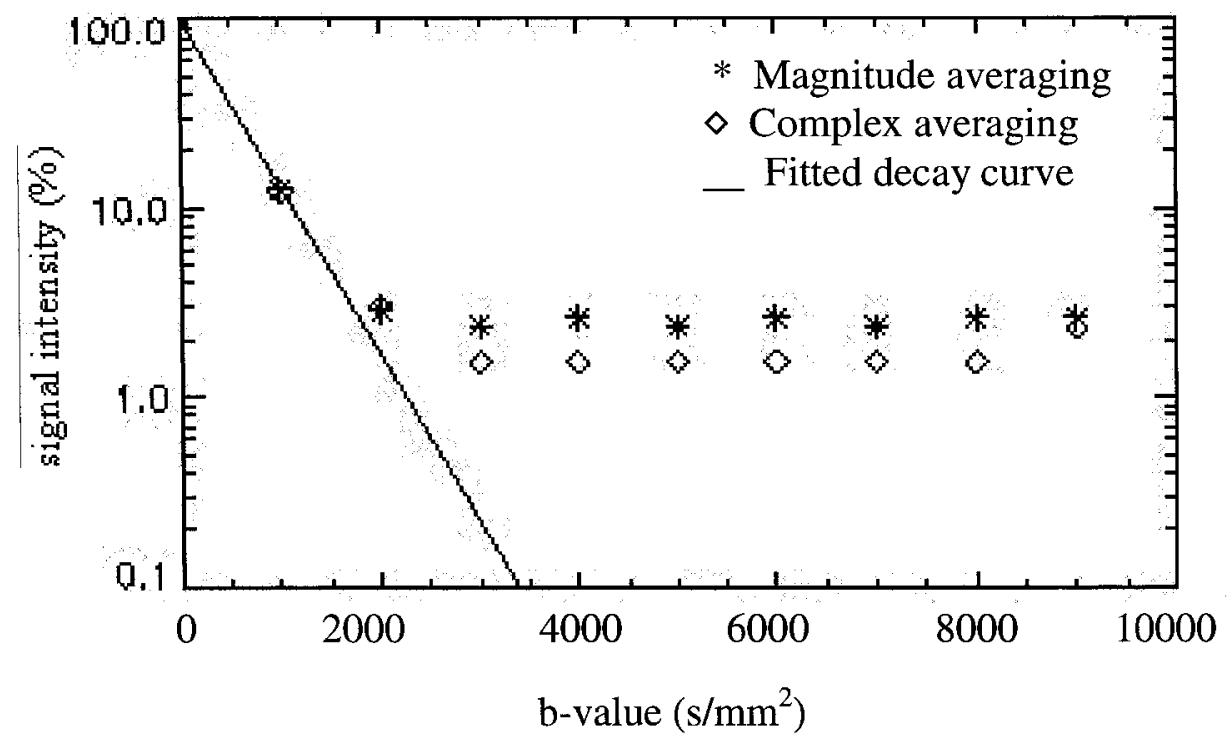

Figure 4.6 The diffusion decay of the water phantom comparing complex averaging with magnitude averaging for $\mathrm{N}=32$ measurements spatially averaged over 50 pixels.

procedure for reducing Rician noise bias, However, when large Rician noise bias corrections are needed the required number of averages is very large and in this situation complex averaging becomes inefficient. For this reason, the post-processing Rician noise bias corrections discussed in the next section are superior to complex averaging.

\subsubsection{Review of post-processing noise correction techniques from the literature}

Several ways of correcting for Rician noise bias after image construction have been proposed in the literature. In 1985, Henkelman [134] showed that the noise bias could be determined by numerically solving the expression for $\mathrm{E}[\mathrm{M}]$ given in equation (4.8) for given values of $\mathrm{A}$ and $\sigma$. 
With this approach he generated a graph of the required correction versus the measured value of $\mathrm{E}[\mathrm{M}]$. This correction for the measured pixel intensity, $\mathrm{M}_{\mathrm{j}}$, can be written as

$$
\hat{\mathrm{A}}_{\mathrm{H}}=\mathbf{M}_{\mathrm{j}}-\Delta \mathrm{M}\left(\mathbf{M}_{\mathrm{j}}\right)
$$

where $\Delta \mathrm{M}\left(\mathrm{M}_{\mathrm{j}}\right)$ is the correction obtained from the graph. In principle, this is a good way to calculate the Rician noise bias but, since it is cumbersome to implement, it has not become popular. This procedure will be referred to in what follows as $H$ noise correction. Two noteworthy characteristics of this correction, which are missing from the other noise correction procedures to be discussed in this section, are (i) the correction increases as $\mathrm{M}_{\mathrm{j}} \rightarrow 0$ and (ii) the Rician distribution of the data points is maintained.

Equations (4.10) and (4.15) have also been used to develop noise correction techniques. From these equations we have

$$
\mathrm{A}=\sqrt{\mathrm{E}\left[\mathrm{M}^{2}\right]-2 \sigma^{2}}
$$

and

$$
A=\sqrt{E^{2}[M]-\sigma^{2}} \quad(\text { for } A>>)
$$

Miller and Joseph [138] suggested that $A^{2}$ could be estimated from equation (4.27) as

$$
\hat{\mathrm{A}}_{\mathrm{MJ}}^{2}=\mathrm{M}_{\mathrm{j}}^{2}-2 \sigma^{2}
$$

They avoided the complication of a square root by analyzing the values of $\hat{\mathrm{A}}_{\mathrm{MJ}}^{2}$ directly, rather than attempting to compute a value of $\hat{\mathrm{A}}$ from $\hat{\mathrm{A}}_{\mathrm{MJ}}^{2}$. McGibney and Smith [135], who also based their noise correction on equation (4.10), proposed

$$
\hat{\mathrm{A}}_{\mathrm{MS}}=\sqrt{\mathrm{M}_{\mathrm{j}}^{2}-2 \sigma^{2}} \text {. }
$$

Using equation (4.15), Gudbjartsson and Patz [133] suggested using

$$
\hat{\mathrm{A}}_{\mathrm{GP}}=\sqrt{\left|\mathrm{M}_{\mathrm{j}}^{2}-\sigma^{2}\right|} \text {. }
$$


These three procedures will be referred to in what follows as MJ, MS and GP noise correction, respectively.

Koay and Basser [184] have recently reported a noise bias correction technique which they claim is an analytically exact correction scheme. By combining equation (4.9) and (4.10) they relate the variance of the Rician PDF to $\sigma^{2}$ via a correction factor, $\xi$. They then derive an expression that relates $\xi$ to the true SNR for the measurement and they present an iterative technique for determining $\xi$. Once $\xi$ is known, $\sigma$ and $\mathrm{A}$ can be evaluated.

This correction scheme appears to be very promising. However, since this approach is very different from the correction method that we propose in this chapter and it was published after the research presented here was completed, the correction scheme of Koay and Basser will not be considered in detail in this chapter. It should be noted, however, that in their paper they assume that the mean and standard deviation of the Rician PDF for the measurement are well known, although they acknowledge that this is not the normal situation. It is not clear how this correction scheme will behave when inaccurate values of these parameters are used.

MJ noise correction is a very nice solution to the problem for some applications but, in general, must be used with caution. It is based on equation (4.10) which is an exact expression; it does not involve approximations and it is valid for all SNRs. However, when considering multiexponential diffusion decays, for example, $\mathbf{M}_{\mathrm{j}}(\mathrm{b})$ will be a sum of exponential terms and the analysis of $\mathrm{M}_{\mathrm{j}}^{2}(\mathrm{~b})$ data will be considerably complicated by the presence of cross terms. The second problem with $\mathrm{MJ}$ noise correction is that for $\mathrm{M}_{\mathrm{j}}$ values that follow a Rician distribution (which is somewhat similar to a Gaussian in shape), the distribution of $\mathrm{M}_{\mathrm{j}}^{2}$ values is far from Gaussian. This is demonstrated in figure 4.7 for $\mathrm{A} / \sigma=0.0$ and 2.0. This can be a problem if least 

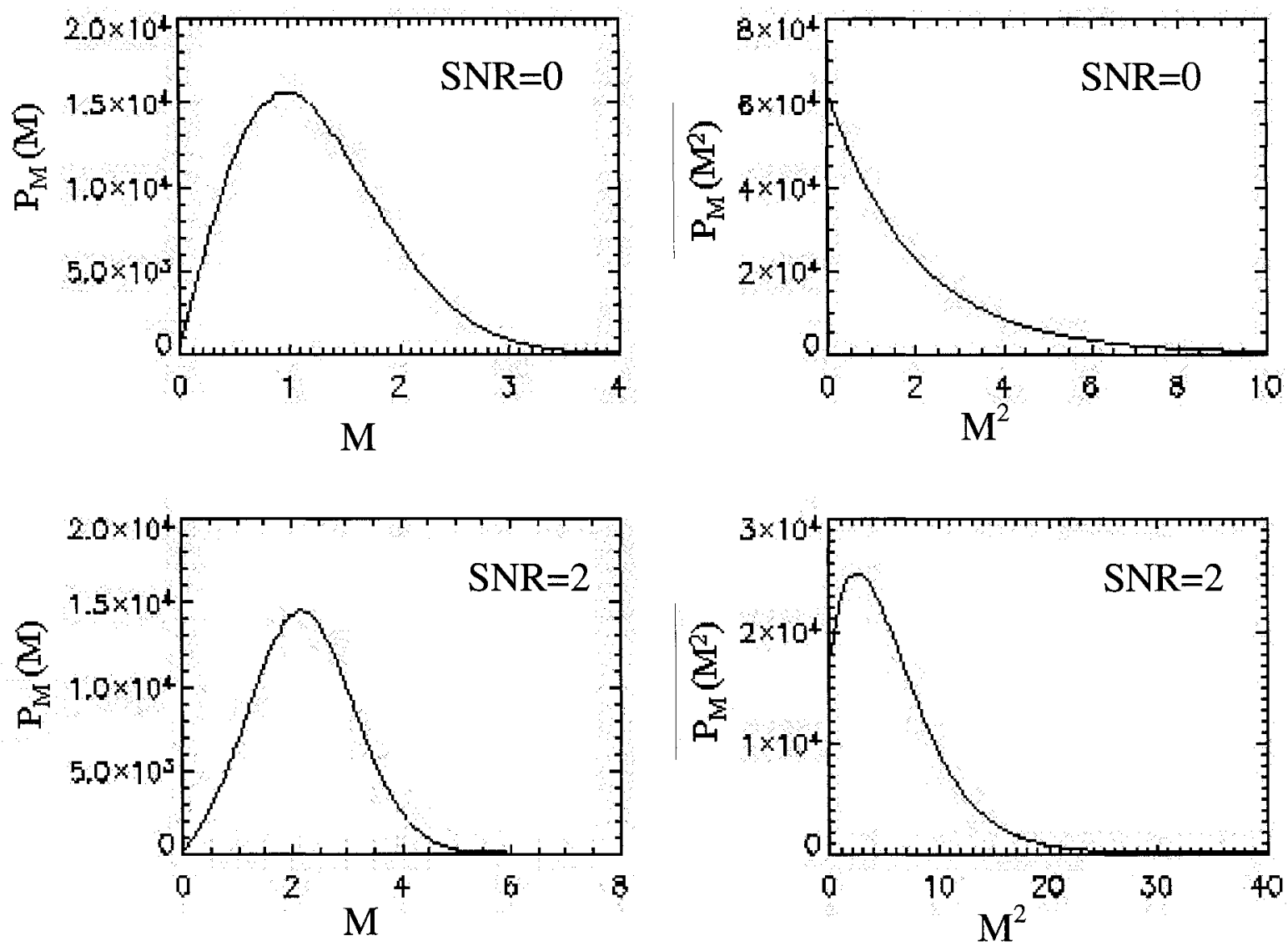

Figure 4.7. The PDFs of the simulated $M_{j}$ (left) and $M_{j}^{2}$ (right) values biased with Rician noise for $\mathrm{SNR}=0.0$ (top) and $\mathrm{SNR}=2.0$ (bottom).

squares procedures, which assume that the data are Gaussian distributed, are used in further analyzing the noise corrected $\mathrm{M}_{\mathrm{j}}^{2}$ data.

The MS and GP noise correction techniques both avoid these problems but they introduce others for small SNRs. Equations (4.27) - (4.31) are valid when the expression under the square root is non-negative. This will always be true for equation (4.27):

$$
\mathrm{A}=\sqrt{\mathrm{E}\left[\mathrm{M}^{2}\right]-2 \sigma^{2}}
$$


but will not always be true for equation (4.30):

$$
\hat{\mathrm{A}}_{\mathrm{MS}}=\sqrt{\mathrm{M}_{\mathrm{j}}^{2}-2 \sigma^{2}} .
$$

In fact, it will be frequently violated when the SNR is small. Similarly, equation (4.28):

$$
\mathrm{A}=\sqrt{\mathrm{E}^{2}[\mathrm{M}]-\sigma^{2}}
$$

is valid when $A \gg>\sigma$ but if $E[M]$ is replaced with $M_{j}$ to get

$$
\hat{\mathrm{A}}=\sqrt{\mathrm{M}_{\mathrm{j}}^{2}-\sigma^{2}},
$$

square roots of negative numbers are again frequently encountered when the SNR is small. Comparing equations (4.30) and (4.32) the latter can be seen to be valid over a larger range of $\mathrm{M}_{\mathrm{j}}$ values (i.e. $M_{j} \geq \sigma$ compared with $M_{j} \geq \sqrt{2} \sigma$ ).

Gudbjartsson and Patz avoid this problem by taking the absolute value of the expression under the square root:

$$
\hat{\mathrm{A}}_{\mathrm{GP}}=\sqrt{\left|\mathrm{M}_{\mathrm{j}}^{2}-\mathrm{n} \sigma^{2}\right|}
$$

with $\mathrm{n}=1$ for GP noise correction. McGibney and Smith did not mention this problem, suggesting that they either ignored these data points or they set them to zero. These two possibilities can be formulated as:

$$
\hat{A}_{M S 1}= \begin{cases}\sqrt{\left|M_{j}^{2}-n \sigma^{2}\right|} & \text { for } M_{j} \geq \sqrt{n} \sigma \\ \text { removed from data set } & \text { for } M_{j}<\sqrt{n} \sigma\end{cases}
$$

and

$$
\hat{A}_{M S 2}= \begin{cases}\sqrt{\left|M_{j}^{2}-n \sigma^{2}\right|} & \text { for } M_{j} \geq \sqrt{n} \sigma \\ 0 & \text { for } M_{j}<\sqrt{n} \sigma\end{cases}
$$

with $n=2$. 
As $M_{j} \rightarrow \sqrt{n} \sigma$ the value of $\hat{A}$ approaches zero suggesting that when $M_{j}<\sqrt{n} \sigma, \hat{A}$ should be negative. Thus, another approach that could be used is

$$
\hat{A}=\left\{\begin{array}{ll}
\sqrt{\left|M_{j}^{2}-n \sigma^{2}\right|} & \text { for } M_{j} \geq \sqrt{n} \sigma \\
-\sqrt{\left|M_{j}^{2}-n \sigma^{2}\right|} & \text { for } M_{j}<\sqrt{n} \sigma
\end{array} .\right.
$$

Simulations were run to generate "corrected" PDFs using equations (4.31) and (4.33) to (4.35) over a range of $\mathrm{SNR}=\mathrm{A} / \sigma$ from 0.0 to 5.0 for both $\mathrm{n}=1$ and $\mathrm{n}=2$. The magnitude data, $\mathrm{M}_{\mathbf{j}}$, were calculated from the real and imaginary parts of the pixel signals corrupted by simulated noise values picked at random from a Gaussian PDF with a mean of zero and $\sigma=1.0$ using equation (4.11). The $\mathrm{M}_{\mathrm{j}}$ therefore correspond to values picked at random from a Rician PDF. In these simulations 500,000 sample points were used.

In figure 4.8, the PDFs for $\hat{\mathrm{A}}_{\mathrm{GP}}$ at several SNRs are shown while in figure 4.9 the PDFs for $\hat{\mathrm{A}}_{\mathrm{GP}}, \hat{\mathrm{A}}_{\mathrm{MS} 2}$ and $\hat{\mathrm{A}}$ are shown for $\mathrm{SNR}=1$. The PDFs for $\hat{\mathrm{A}}_{\mathrm{MS1}}$ and $\hat{\mathrm{A}}_{\mathrm{MS} 2}$ (see figure $4.9 \mathrm{~b}$ ) differ only by a strong peak at $\mathrm{M}=0$ which, for $\mathrm{SNR}=1.0$ corresponds to 130,000 for $\hat{\mathrm{A}}_{\mathrm{MS} 2}$ (not shown) and is approximately equal to zero for $\hat{\mathrm{A}}_{\mathrm{MS} 1}$. The corresponding mean values calculated from these distributions are given in table 4.1. Note that since $\sigma=1.0$ for these simulations, the estimates of A given in table 4.1 should be equal to the SNR value for that row in the table if the correction works properly. For SNR $<3$ none of these corrections are completely satisfactory. The estimates for $\mathrm{A}$, in most cases, get progressively worse as SNR decreases and the distributions of "corrected" data points are far from Gaussian. The corrected mean values are, in most cases, better than the uncorrected ones but there is still considerable room for improvement. Furthermore, least square procedures, which assume that the distribution of data points is 


\begin{tabular}{|c|c|c|c|c|c|c|c|c|c|}
\hline \multirow{2}{*}{$\mathrm{SNR}$} & \multirow{2}{*}{$E\left[\mathrm{M}_{\mathrm{j}}\right]$} & \multicolumn{2}{|c|}{$\mathrm{E}\left[\hat{\mathrm{A}}_{\mathrm{GP}}\right]$} & \multicolumn{2}{c|}{$\mathrm{E}\left[\hat{\mathrm{A}}_{\mathrm{MS} 1}\right]$} & \multicolumn{2}{c|}{$\mathrm{E}\left[\hat{\mathrm{A}}_{\mathrm{MS} 2}\right]$} & \multicolumn{2}{c|}{$\mathrm{E}[\hat{\mathrm{A}}]$} \\
\cline { 3 - 10 } & & $\mathrm{n}=1$ & $\mathrm{n}=2$ & $\mathrm{n}=1$ & $\mathrm{n}=2$ & $\mathrm{n}=1$ & $\mathrm{n}=2$ & $\mathrm{n}=1$ & $\mathrm{n}=2$ \\
\hline 0.0 & 1.253 & 1.036 & 1.115 & 1.254 & 1.255 & 0.760 & 0.461 & 0.485 & -0.192 \\
\hline 0.5 & 1.329 & 1.102 & 1.148 & 1.328 & 1.326 & 0.853 & 0.546 & 0.604 & -0.056 \\
\hline 1.0 & 1.547 & 1.299 & 1.265 & 1.524 & 1.505 & 1.116 & 0.797 & 0.932 & 0.329 \\
\hline 1.5 & 1.873 & 1.616 & 1.495 & 1.804 & 1.751 & 1.507 & 1.190 & 1.397 & 0.884 \\
\hline 2.0 & 2.270 & 2.027 & 1.848 & 2.150 & 2.060 & 1.974 & 1.682 & 1.921 & 1.516 \\
\hline 2.5 & 2.709 & 2.494 & 2.298 & 2.558 & 2.435 & 2.474 & 2.224 & 2.453 & 2.150 \\
\hline 3.0 & 3.170 & 2.988 & 2.804 & 3.014 & 2.873 & 2.981 & 2.777 & 2.975 & 2.750 \\
\hline 5.0 & 5.099 & 4.995 & 4.889 & 4.995 & 4.892 & 4.995 & 4.889 & 4.995 & 4.888 \\
\hline
\end{tabular}

Table 4.1. The estimated mean value of Rician noise corrected PDFs for various approaches over a range of $\mathrm{SNR}$. Note that $\mathrm{SNR}=\mathrm{A} / \sigma=\mathrm{A}$, since $\sigma=1.0$ for these simulations.
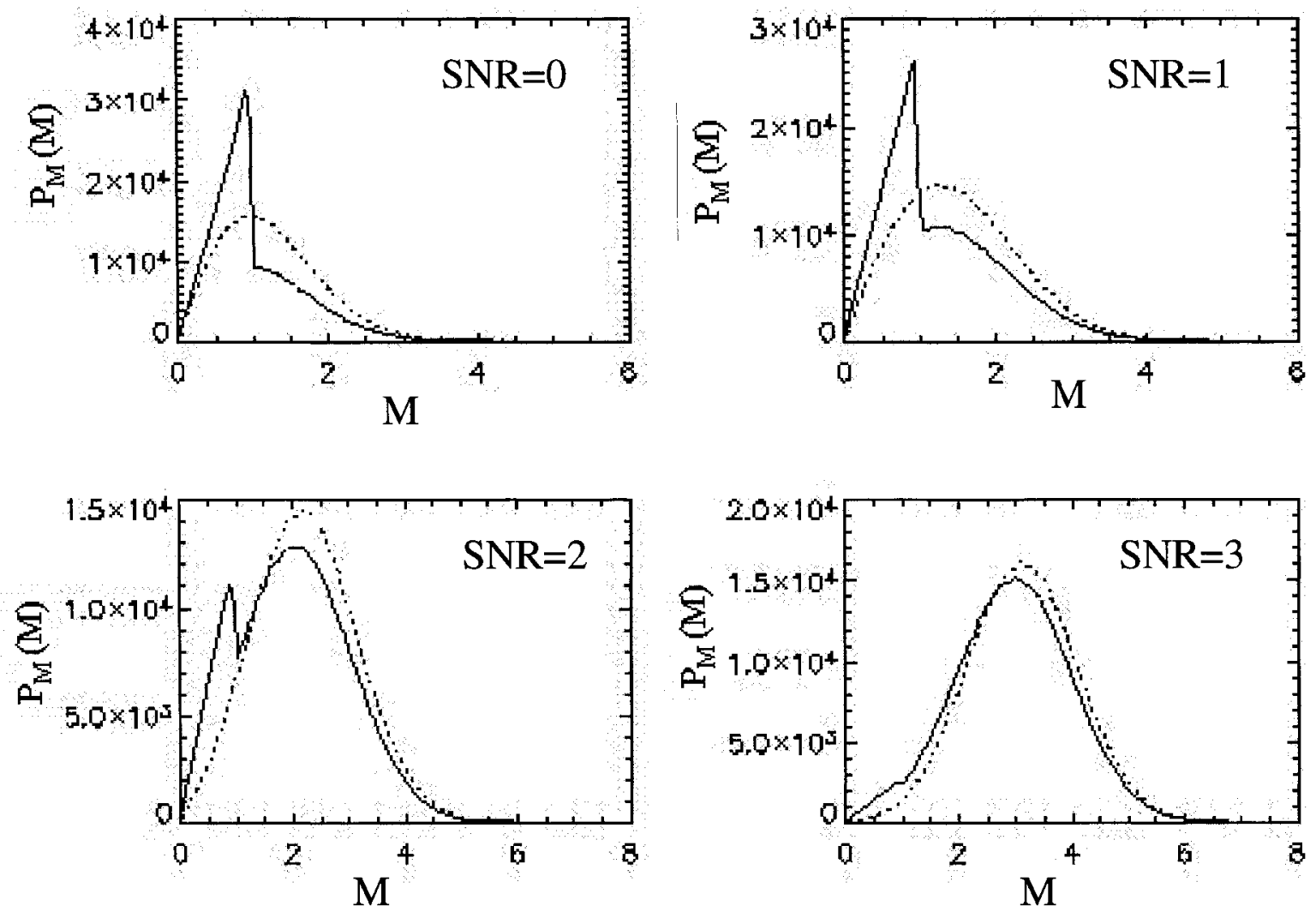

Figure 4.8. The $\hat{\mathrm{A}}_{\mathrm{GP}}$ PDF (solid line) at several SNRs between 0.0 and 3.0 compared to the corresponding Rician noise PDF (dashed line). 

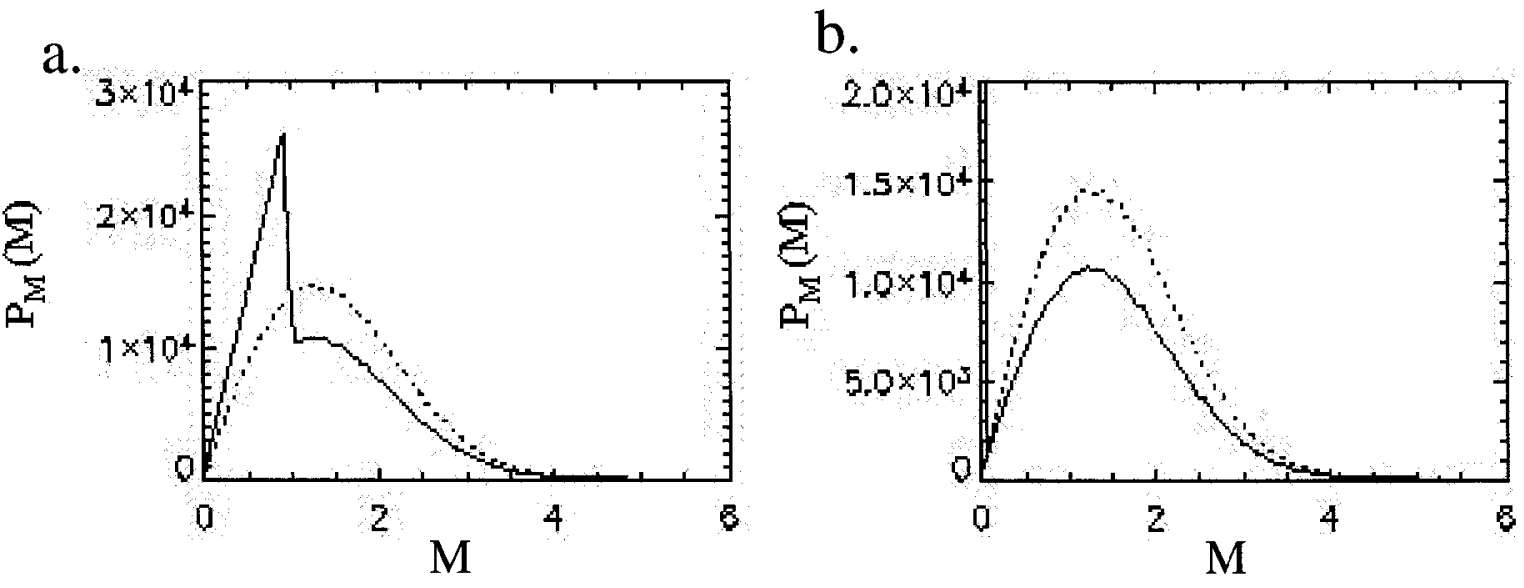

C.

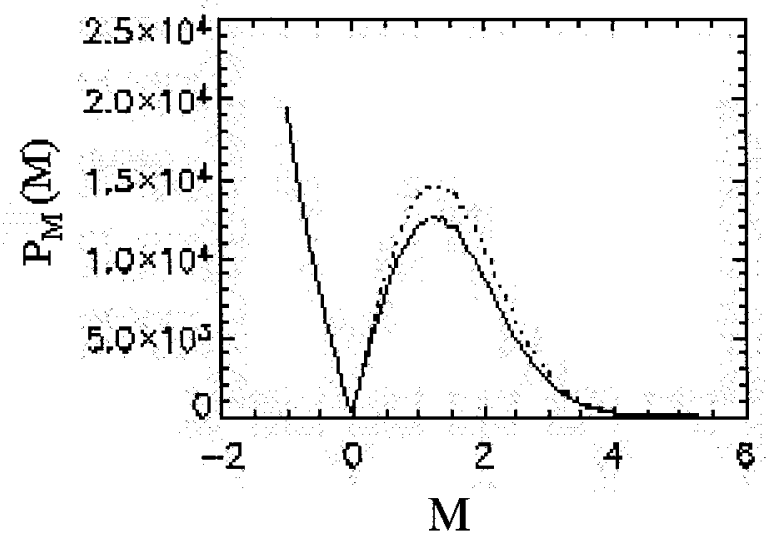

Figure 4.9. The PDFs for the $\hat{\mathrm{A}}_{\mathrm{GP}}, \hat{\mathrm{A}}_{\mathrm{MS} 2}$ and $\hat{\mathrm{A}}$ Rician noise bias correction schemes shown in $\mathrm{a}, \mathrm{b}$ and $\mathrm{c}$, respectively, (solid lines) are simulated for $\mathrm{SNR}=1$ and compared with the corresponding Rician PDF for $\mathrm{M}_{\mathrm{j}}$ (dashed lines).

Gaussian, will not work properly for these distributions of "corrected" data. Of the various possibilities considered here GP noise correction (with $n=1$ ) and MS2 noise correction (with $n=1$ ) are the most satisfactory. For $S N R \geq 3$, it makes very little difference which noise correction method is used. 


\subsubsection{A new Rician noise correction technique}

An ideal noise correction procedure will be conceptually simple, easy to implement and can be applied to each individual data point separately. When applied to a large number of equivalent measurements, $\mathrm{M}_{\mathrm{j}}$, it will yield a distribution of "corrected" data points with a mean of $\mathrm{A}$, the true MR pixel intensity that would be measured in the absence of noise, and will be Gaussian in shape to fulfill the prerequisites for least-squares based post-processing procedures. None of the corrections in the literature fulfill all of these criteria.

One method of noise correction that would come close to meeting these conditions would be to subtract from each data point an amount equal to $\left.\Delta \mathrm{M}=\mathrm{A}-<\mathrm{M}_{\mathrm{j}}\right\rangle$. This would have the effect of shifting the Rician PDF for $M_{j}$ by the correct amount such that the mean over the distribution of corrected data points would equal A, the desired result. This process would also preserve the Rician shape of the PDF which is reasonably close to Gaussian. Unfortunately, in order to calculate $\Delta \mathrm{M}$ in this way, $\mathrm{A}$ must be known. In this section, a new noise correction technique is proposed which is a straight subtraction of a correction term from $M_{j}$ that out performs the other Rician noise correction procedures based on the criteria outlined above.

In the high SNR limit the mean of a Rician PDF is given by equation (4.15):

$$
\bar{M}=\lim _{A>>\sigma} E[M]=\sqrt{A^{2}+\sigma^{2}} \text {. }
$$

Solving for $\mathrm{A}$ and expanding with a binomial expansion gives: 


$$
\begin{aligned}
A & =\bar{M} \sqrt{1-\left(\frac{\sigma}{\bar{M}}\right)^{2}} & \\
& =\bar{M}\left[1-\frac{1}{2}\left(\frac{\sigma}{\bar{M}}\right)^{2}-\frac{1}{8}\left(\frac{\sigma}{\bar{M}}\right)^{4}+\ldots\right] & \text { for } \frac{\bar{M}}{\sigma}>1 \\
& =\bar{M}-\bar{M}\left[\frac{1}{2}\left(\frac{\sigma}{\bar{M}}\right)^{2}+\frac{1}{8}\left(\frac{\sigma}{\bar{M}}\right)^{4}+\ldots\right] & \text { for } \frac{\bar{M}}{\sigma}>1 \\
& =\bar{M}-\Delta M\left(\frac{\sigma}{\bar{M}}\right) . &
\end{aligned}
$$

This equation allows us to determine exactly how much to subtract from $\bar{M}$ to get $A$, and the correction is in terms of $\sigma$ and $\bar{M}$, not $A$. Since this correction is valid for values of $\mathbf{M}_{\mathrm{j}}$ equal to $\bar{M}$, it should be an appropriate correction for all $M_{j}$. Thus, we can write

$$
\hat{\mathrm{A}}_{\mathrm{j}}=\mathrm{M}_{\mathrm{j}}-\Delta \mathrm{M}\left(\frac{\sigma}{\overline{\mathrm{M}}}\right)
$$

Although equation (4.37) is based on equation (4.15), which is strictly speaking only valid in the limit of large SNR, it gives in practice reasonable results for lower SNR as well. This noise correction procedure will be referred to in what follows as $\mathrm{NC1}$ noise correction. Figure 4.10 shows the PDFs for the NC1 noise corrected data compared with the original Rician PDF for selected SNRs. In table 4.2 the values of $\hat{\mathrm{A}}_{\mathrm{NC1}}=\mathrm{E}\left[\hat{\mathrm{A}}_{\mathrm{j}}\right]$ are accurate to better than $1 \%$ for SNR $\geq 2.5$. This compares favorably with the results obtained with GP noise correction. For $\mathrm{SNR}<2.5$, the results are less satisfactory but better than the results for GP noise correction. These results are from simulations similar to those described in the previous section. For these simulations terms in the expansion up to tenth order in $\sigma / \overline{\mathrm{M}}$ (i.e. five terms) were included.

To improve the performance for SNR $\rightarrow 0$ we can make use of equation (4.16):

$$
\lim _{\mathrm{A} \ll \sigma} E[M]=\sigma \sqrt{\frac{\pi}{2}}
$$



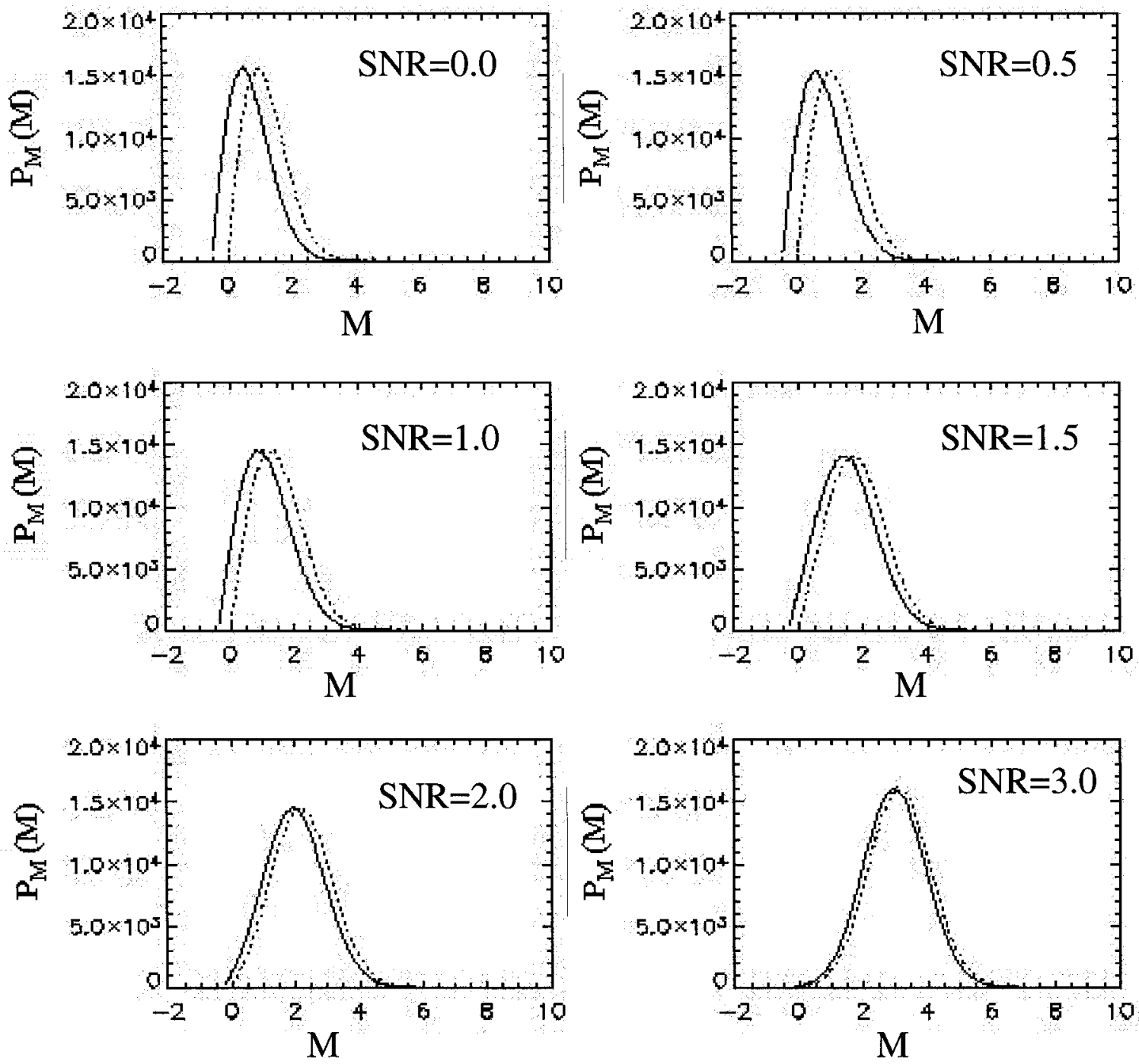

Figure 4.10. PDFs (solid line) when NC1 noise correction is used compared with the original PDFs (dot line) versus SNR.

\begin{tabular}{|l|c|c|c|}
\hline SNR & $\overline{\mathrm{M}}$ & $\hat{\mathrm{A}}_{\mathrm{GP}}$ & $\hat{\mathrm{A}}_{\mathrm{NC} 1}$ \\
\hline 0.0 & 1.253 & 1.036 & 0.759 \\
\hline 0.5 & 1.329 & 1.102 & 0.878 \\
\hline 1.0 & 1.547 & 1.299 & 1.181 \\
\hline 1.5 & 1.873 & 1.616 & 1.584 \\
\hline 2.0 & 2.270 & 2.027 & 2.038 \\
\hline 2.5 & 2.709 & 2.494 & 2.517 \\
\hline 3.0 & 3.170 & 2.988 & 3.008 \\
\hline
\end{tabular}

Table 4.2. The mean value of the PDF when NC1 noise bias correction is used compared to GP noise correction over a range of $\mathrm{SNR}$. Note that $\mathrm{SNR}=\mathrm{A} / \sigma=\mathrm{A}$, since $\sigma=1.0$ for these simulations. 
To obtain the correct value for $\hat{\mathrm{A}}$ as SNR $\rightarrow 0$, equation (4.15) should be modified to

$$
\lim _{\mathrm{A} \rightarrow 0} \overline{\mathrm{M}}=\sqrt{\mathrm{A}^{2}+(\mathrm{n} \sigma)^{2}}
$$

where $n=\sqrt{\frac{\pi}{2}}$. If this is incorporated into the noise correction procedure we get

$$
\begin{aligned}
& \hat{\mathrm{A}}_{j} \approx \mathrm{M}_{j}-\Delta \mathrm{M}\left(\frac{\mathrm{n \sigma}}{\overline{\mathrm{M}}}\right) \\
& \approx \mathrm{M}_{\mathrm{j}}-\overline{\mathrm{M}}\left[\frac{1}{2}\left(\frac{\mathrm{n} \sigma}{\overline{\mathrm{M}}}\right)^{2}+\frac{1}{8}\left(\frac{\mathrm{n} \sigma}{\overline{\mathrm{M}}}\right)^{4}+\ldots\right] \quad \begin{cases}\mathrm{n}=\sqrt{\frac{\pi}{2}} & \text { for } \frac{\overline{\mathrm{M}}}{\sigma}<1.5 \\
n=1 & \text { for } \frac{\overline{\mathrm{M}}}{\sigma} \geq 1.5\end{cases}
\end{aligned}
$$

The value of $\bar{M} / \sigma=1.5$ was determined to be an appropriate transition point for equation (4.39) by trial and error. Noise correction using equation (4.39) will be referred to in what followed as $\mathrm{NC} 2$ noise correction.

In order to implement this correction the convergence of the noise correction equation was investigated to determine how many terms in the expansion are required. The results for $\hat{\mathrm{A}}_{\mathrm{NC2}}=\mathrm{E}\left[\hat{\mathrm{A}}_{\mathrm{j}}\right]$ are summarized in table 4.3 . For $\mathrm{SNR} \geq 1.5$ it converges rapidly and there is no advantage to keeping more than 3 terms in the expansion. For $\mathrm{SNR}<1.5$ the convergence is much slower. Even 8 terms of the expansion is not enough to get full convergence. However, even though including more terms causes the noise correction to continue to improve for $\mathrm{SNR}=0$, it gets worse for $\mathrm{SNR}=0.5$ when more than 5 terms are used. From this it can be seen that the optimal number of terms depends on the data set. In most cases of interest there will be more data points that correspond to $\mathrm{SNR} \geq 0.5$ than $\mathrm{SNR}<0.5$ so it would seem reasonable to use 5 terms, however, in situations with a lot of data points with $\mathrm{SNR}<0.5$ it may be reasonable to include additional terms. 


\begin{tabular}{|c|c|c|c|c|c|c|c|c|c|c|}
\hline \multirow{2}{*}{ SNR } & \multirow{2}{*}{$\bar{M}$} & \multirow{2}{|c|}{$\hat{\mathrm{A}}_{\mathrm{GP}}$} & \multicolumn{9}{|c|}{$\hat{\mathrm{A}}_{\mathrm{NC} 2}$} \\
\cline { 5 - 12 } & & & $\mathrm{m}=1$ & $\mathrm{~m}=2$ & $\mathrm{~m}=3$ & $\mathrm{~m}=4$ & $\mathrm{~m}=5$ & $\mathrm{~m}=6$ & $\mathrm{~m}=7$ & $\mathrm{~m}=8$ \\
\hline 0.0 & 1.253 & 1.036 & 0.627 & 0.470 & 0.392 & 0.343 & 0.308 & 0.283 & 0.262 & 0.246 \\
\hline 0.5 & 1.329 & 1.102 & 0.739 & 0.607 & 0.549 & 0.517 & 0.497 & 0.483 & 0.474 & 0.467 \\
\hline 1.0 & 1.547 & 1.299 & 1.224 & 1.190 & 1.183 & 1.181 & 1.181 & 1.180 & 1.180 & 1.180 \\
\hline 1.5 & 1.873 & 1.616 & 1.606 & 1.587 & 1.584 & 1.584 & 1.584 & 1.584 & 1.584 & 1.584 \\
\hline 2.0 & 2.270 & 2.027 & 2.050 & 2.039 & 2.038 & 2.038 & 2.038 & 2.038 & 2.038 & 2.038 \\
\hline 2.5 & 2.709 & 2.494 & 2.524 & 2.518 & 2.517 & 2.517 & 2.517 & 2.517 & 2.517 & 2.517 \\
\hline 3.0 & 3.170 & 2.988 & 3.012 & 3.008 & 3.008 & 3.008 & 3.008 & 3.008 & 3.008 & 3.008 \\
\hline
\end{tabular}

Table 4.3. Study of $\hat{\mathrm{A}}_{\mathrm{NC} 2}$ as a function of $\mathrm{m}$, the number of correction terms in the expansion, for NC2 noise bias correction using the mean from the original Rician PDF.

This noise bias correction is based on an accurate knowledge of $\sigma$ and $\bar{M}$. The situation with $\sigma$ will be treated in the next section; for now it will be assumed that $\sigma$ is known. $\bar{M}$ is the expectation value of $\mathbf{M}_{\mathrm{j}}$ over a very large number of measurements and, in general, will not be known. For the simulations presented above $\bar{M}$ was calculated from the 500,000 simulated $\mathrm{M}_{\mathrm{j}}$ values chosen at random from the appropriate Rician PDF. When dealing with MR images of a water phantom there will be a large region of equivalent points from which a reasonably accurate estimate for $\overline{\mathrm{M}}$ can be determined but, in general, there will not be a large number of equivalent points from which an accurate value of $\bar{M}$ can be calculated since $A_{j}$ will normally change from pixel to pixel.

For MR images of human anatomy it may be a reasonable approximation to treat neighboring pixels as equivalent. We considered setting $\bar{M}$ equal to the average of nearest neighbor pixels over several layers of nearest neighbors using simulations similar to those described above. One difficulty encountered with these simulations was that there were many "corrected" data points that were less than $-5 \sigma$. When calculating $\overline{\mathrm{M}}$ using only a small number 
of points there is a finite possibility of obtaining $\bar{M} \approx 0$. Since $\bar{M}$ appears in the denominators of the correction terms this results in very large negative correction terms and very large negative "corrected" data points as well. Whenever, this was encountered in the simulations, data points less than $-5 \sigma$ were set equal to $-5 \sigma$ to reduce the effect of these outliers.

The results of the simulations are given in table 4.4 for nearest neighbor averaging that included $3,5,9,16,25,36,100$ and 500,000 points as a function of $\mathrm{m}$, the number of terms in the expansion. The number of points, $\mathrm{k}$, that were less than $-5 \sigma$ for each case is also shown in the table as a percentage. Averaging over one pixel (i.e. setting $\bar{M}=M_{j}$ ) was also investigated but the results were very poor and are not included here.

For SNR $\geq 1.5$ the noise correction term converges very quickly and there is no advantage to keeping more than three terms. However, when $\mathrm{SNR}<1.5$ the situation is more involved. When $\mathrm{SNR}=0.5$, for example, if too few correction terms are included then $\hat{\mathrm{A}}_{\mathrm{NC2}}>0.5$. But as more terms are included $\hat{\mathrm{A}}_{\mathrm{NC} 2}$ converges to a value less than 0.5 . Furthermore, as the number of terms increases, the number of "corrected" data points less than $-5 \sigma$ increases considerably. There appears to be an optimal number of correction terms but this optimum changes with SNR and with the number of pixels, $\mathrm{N}_{\mathrm{Av}}$, included in the average. From table 4.4 it can be determined by inspection that two correction terms should be used when $\mathrm{N}_{\mathrm{AV}}=3$ or 5 , three terms should be used when $\mathrm{N}_{\mathrm{AV}}=9$ or 16 , four terms should be used when $\mathrm{N}_{\mathrm{AV}}=25$ or 36 and five terms should be used when $\mathrm{N}_{\mathrm{AV}}>36$.

If this Rician noise correction scheme is applied to the bi-exponential decay data shown in figure 4.2 using a 9-point average and three terms in the noise correction, the least squares fit parameters are in good agreement with the correct values. The resulting fit is shown in figure 4.11 and the fit parameters are given in table 4.5. 


\begin{tabular}{|c|c|c|c|c|c|c|c|c|c|c|c|}
\hline \multicolumn{12}{|c|}{$N_{A V}=3$} \\
\hline \multirow{2}{*}{ SNR } & \multirow{2}{*}{$\bar{M}$} & \multicolumn{2}{|c|}{$m=1$} & \multicolumn{2}{|c|}{$m=2$} & \multicolumn{2}{|c|}{$m=3$} & \multicolumn{2}{|c|}{$m=4$} & \multicolumn{2}{|c|}{$m=5$} \\
\hline & & $k(\%)$ & $\widehat{A}$ & $k(\%)$ & $\hat{A}$ & $k(\%)$ & $\widehat{A}$ & $k(\%)$ & $\widehat{A}$ & $k(\%)$ & A \\
\hline 0.0 & 1.253 & $5(<<0.1)$ & 0.558 & $2860(0.5)$ & 0.254 & 15351(3) & -0.025 & $33495(6)$. & -0.271 & $52539(11)$ & -0.478 \\
\hline 0.5 & 1.329 & $4(<<0.1)$ & 0.674 & $2071(0.4)$ & 0.417 & $11344(2)$ & 0.190 & $25525(5)$. & -0.011 & $40318(9)$ & -0.182 \\
\hline 1.0 & 1.547 & 0 & 1.191 & $97(<0.1)$ & 1.125 & $903(0.2)$ & 1.084 & $2529(0.5)$ & 1.049 & $4602(0.9)$ & 1.017 \\
\hline 1.5 & 1.873 & 0 & 1.584 & $20(<0.1)$ & 1.550 & $147(<0.1)$ & 1.537 & $466(0.1)$ & 1.527 & $911(0.2)$ & 1.519 \\
\hline 2.0 & 2.270 & 0 & 2.036 & $1(<<0.1)$ & 2.020 & $14(<0.1)$ & 2.016 & $43(<0.1)$ & 2.014 & $98(<0.1)$ & 2.013 \\
\hline 2.5 & 2.709 & 0 & 2.516 & 0 & 2.507 & $1(<<0.1)$ & 2.506 & $3(<<0.1)$ & 2.506 & $7(<<0.1)$ & 2.506 \\
\hline 3.0 & 3.170 & 0 & 3.007 & 0 & 3.002 & 0 & 3.002 & 0 & 3.002 & 0 & 3.002 \\
\hline \multicolumn{12}{|c|}{$N_{A V}=5$} \\
\hline \multirow{2}{*}{ SNR } & \multirow{2}{*}{$\overline{\mathrm{M}}$} & \multicolumn{2}{|c|}{$m=1$} & \multicolumn{2}{|c|}{$m=2$} & \multicolumn{2}{|c|}{$m=3$} & \multicolumn{2}{|c|}{$\mathrm{m}=4$} & \multicolumn{2}{|c|}{$m=5$} \\
\hline & & $k(\%)$ & $\widehat{A}$ & $k(\%)$ & $\widehat{\mathrm{A}}$ & $k(\%)$ & $\widehat{A}$ & $k(\%)$ & $\widehat{A}$ & $k(\%)$ & A \\
\hline 0.0 & 1.253 & 0 & 0.589 & $223(<0.1)$ & 0.359 & $3394(0.7)$ & 0.165 & $11861(2.1)$ & -0.027 & $24114(5)$ & -0.208 \\
\hline 0.5 & 1.329 & 0 & 0.703 & $148(<0.1)$ & 0.511 & $2245(0.5)$ & 0.362 & $7639(1.5)$ & 0.220 & $15834(3)$ & 0.084 \\
\hline 1.0 & 1.547 & 0 & 1.206 & 0 & 1.157 & $38(<0.1)$ & 1.139 & $213(<0.1)$ & 1.126 & $547(0.1)$ & 1.116 \\
\hline 1.5 & 1.873 & 0 & 1.594 & 0 & 1.568 & $5(<<0.1)$ & 1.562 & $14(<0.1)$ & 1.559 & $42(<0.1)$ & 1.558 \\
\hline 2.0 & 2.270 & 0 & 2.042 & 0 & 2.029 & 0 & 2.027 & 0 & 2.026 & 0 & 2.026 \\
\hline 2.5 & 2.709 & 0 & 2.519 & 0 & 2.512 & 0 & 2.511 & 0 & 2.511 & 0 & 2.511 \\
\hline 3.0 & 3.170 & 0 & 3.009 & 0 & 3.005 & 0 & 3.005 & 0 & 3.005 & 0 & 3.005 \\
\hline \multicolumn{12}{|c|}{$N_{A V}=9$} \\
\hline \multirow{2}{*}{ SNR } & \multirow{2}{*}{$\overline{\mathbf{M}}$} & \multicolumn{2}{|c|}{$m=1$} & \multicolumn{2}{|c|}{$m=2$} & \multicolumn{2}{|c|}{$m=3$} & \multicolumn{2}{|c|}{$m=4$} & $\mathrm{~m}=5$ & \\
\hline & & $k(\%)$ & $\widehat{A}$ & $k(\%)$ & $\widehat{A}$ & $k(\%)$ & $\hat{\mathrm{A}}$ & $k(\%)$ & $\widehat{A}$ & $k(\%)$ & $\widehat{A}$ \\
\hline 0.0 & 1.253 & 0 & 0.606 & $2(<<0.1)$ & 0.416 & $185(<0.1)$ & 0.285 & $1701(0.3)$ & 0.162 & $5798(1.2)$ & 0.037 \\
\hline 0.5 & 1.329 & 0 & 0.720 & $1(<<0.1)$ & 0.560 & $97(<0.1)$ & 0.463 & $903(0.2)$ & 0.379 & $2975(0.5)$ & 0.297 \\
\hline 1.0 & 1.547 & 0 & 1.214 & 0 & 1.174 & 0 & 1.162 & $2(<<0.1)$ & 1.158 & $10(<0.1)$ & 1.155 \\
\hline 1.5 & 1.873 & 0 & 1.599 & 0 & 1.577 & 0 & 1.573 & 0 & 1.572 & 0 & 1.572 \\
\hline 2.0 & 2.270 & 0 & 2.046 & 0 & 2.034 & 0 & 2.032 & 0 & 2.032 & 0 & 2.032 \\
\hline 2.5 & 2.709 & 0 & 2.522 & 0 & 2.515 & 0 & 2.514 & 0 & 2.514 & 0 & 2.514 \\
\hline 3.0 & 3.170 & 0 & 3.011 & 0 & 3.006 & 0 & 3.006 & 0 & 3.006 & 0 & 3.006 \\
\hline & & & & & & $\mathrm{N}_{\mathrm{AV}}=16$ & & & & & \\
\hline SNR & $\overline{\mathbf{M}}$ & $\mathrm{m}=$ & & $\mathrm{m}=$ & & $\mathrm{m}=$ & & $\mathrm{m}=$ & & $m=5$ & \\
\hline & & $k(\%)$ & $\widehat{\mathrm{A}}$ & $k(\%)$ & $\widehat{A}$ & $\mathrm{k}(\%)$ & $\widehat{A}$ & $k(\%)$ & $\widehat{A}$ & $k(\%)$ & $\widehat{A}$ \\
\hline 0.0 & 1.253 & 0 & 0.616 & 0 & 0.441 & $3(<<0.1)$ & 0.338 & $62(<0.1)$ & 0.256 & $586(0.1)$ & 0.177 \\
\hline 0.5 & 1.329 & 0 & 0.728 & 0 & 0.582 & 0 & 0.506 & $20(<0.1)$ & 0.451 & $184(<0.1)$ & 0.405 \\
\hline 1.0 & 1.547 & 0 & 1.219 & 0 & 1.181 & 0 & 1.172 & 0 & 1.169 & 0 & 1.168 \\
\hline 1.5 & 1.873 & 0 & 1.602 & 0 & 1.582 & 0 & 1.578 & 0 & 1.578 & 0 & 1.577 \\
\hline 2.0 & 2.270 & 0 & 2.048 & 0 & 2.036 & 0 & 2.035 & 0 & 2.035 & 0 & 2.035 \\
\hline 2.5 & 2.709 & 0 & 2.523 & 0 & 2.516 & 0 & 2.516 & 0 & 2.516 & 0 & 2.516 \\
\hline 3.0 & 3.170 & 0 & 3.011 & 0 & 3.007 & 0 & 3.007 & 0 & 3.007 & 0 & 3.007 \\
\hline
\end{tabular}

Table 4.4. The results of simulations for $\mathrm{NC} 2$ correction versus the number of terms used in the expansion and the number of neighboring pixels included in the average for SNR between 0.0 and 3.0. The percentage of points, $k$, that were less than $-5 \sigma$ is also shown. 


\begin{tabular}{|c|c|c|c|c|c|c|c|c|c|c|c|}
\hline \multicolumn{12}{|c|}{$N_{A V}=25$} \\
\hline \multirow{2}{*}{ SNR } & \multirow{2}{*}{$\overline{\mathbf{M}}$} & \multicolumn{2}{|c|}{$\mathrm{m}=1$} & \multicolumn{2}{|c|}{$m=2$} & \multicolumn{2}{|c|}{$m=3$} & \multicolumn{2}{|c|}{$m=4$} & \multicolumn{2}{|c|}{$m=5$} \\
\hline & & $k(\%)$ & $\widehat{\mathrm{A}}$ & $k(\%)$ & $\overline{\mathrm{A}}$ & $k(\%)$ & $\widehat{\mathrm{A}}$ & $k(\%)$ & $\widehat{\mathrm{A}}$ & $k(\%)$ & $\widehat{A}$ \\
\hline 0.0 & 1.253 & 0 & 0.620 & 0 & 0.452 & 0 & 0.359 & 0 & 0.291 & $21(<0.1)$ & 0.232 \\
\hline 0.5 & 1.329 & 0 & 0.732 & 0 & 0.592 & 0 & 0.523 & 0 & 0.478 & 0 & 0.444 \\
\hline 1.0 & 1.547 & 0 & 1.220 & 0 & 1.185 & 0 & 1.176 & 0 & 1.174 & 0 & 1.173 \\
\hline 1.5 & 1.873 & 0 & 1.604 & 0 & 1.584 & 0 & 1.581 & 0 & 1.580 & 0 & 1.580 \\
\hline 2.0 & 2.270 & 0 & 2.048 & 0 & 2.037 & 0 & 2.036 & 0 & 2.036 & 0 & 2.036 \\
\hline 2.5 & 2.709 & 0 & 2.523 & 0 & 2.517 & 0 & 2.516 & 0 & 2.516 & 0 & 2.516 \\
\hline 3.0 & 3.170 & 0 & 3.012 & 0 & 3.008 & 0 & 3.008 & 0 & 3.008 & 0 & 3.008 \\
\hline \multicolumn{12}{|c|}{$N_{A v}=36$} \\
\hline \multirow{2}{*}{ SNR } & \multirow{2}{*}{$\overline{\mathbf{M}}$} & \multicolumn{2}{|c|}{$m=1$} & \multicolumn{2}{|c|}{$m=2$} & \multicolumn{2}{|c|}{$m=3$} & \multicolumn{2}{|c|}{$\mathrm{m}=4$} & \multicolumn{2}{|c|}{$m=5$} \\
\hline & & $k(\%)$ & $\overline{\mathrm{A}}$ & $k(\%)$ & $\widehat{A}$ & $k(\%)$ & $\widehat{\mathrm{A}}$ & $k(\%)$ & $\widehat{\mathrm{A}}$ & $k(\%)$ & $\widehat{A}$ \\
\hline 0.0 & 1.253 & 0 & 0.622 & 0 & 0.457 & $9(<<0.1)$ & 0.369 & $6(<<0.1)$ & 0.308 & $9(<<0.1)$ & 0.259 \\
\hline 0.5 & 1.329 & 0 & 0.734 & 0 & 0.597 & $9(<<0.1)$ & 0.531 & $4(<<0.1)$ & 0.491 & $6(<<0.1)$ & 0.462 \\
\hline 1.0 & 1.547 & 0 & 1.221 & 0 & 1.186 & $4(<0.1)$ & 1.178 & 0 & 1.176 & 0 & 1.175 \\
\hline 1.5 & 1.873 & 0 & 1.604 & 0 & 1.585 & $3(<<0.1)$ & 1.582 & 0 & 1.581 & 0 & 1.581 \\
\hline 2.0 & 2.270 & 0 & 2.049 & 0 & 2.038 & $2(<<0.1)$ & 2.037 & 0 & 2.037 & 0 & 2.037 \\
\hline 2.5 & 2.709 & 0 & 2.524 & 0 & 2.517 & 0 & 2.517 & 0 & 2.517 & 0 & 2.517 \\
\hline 3.0 & 3.170 & 0 & 3.012 & 0 & 3.008 & 0 & 3.008 & 0 & 3.008 & 0 & 3.008 \\
\hline \multicolumn{12}{|c|}{$N_{A V}=100$} \\
\hline \multirow{2}{*}{ SNR } & \multirow{2}{*}{$\overline{\mathrm{M}}$} & \multicolumn{2}{|c|}{$m=1$} & \multicolumn{2}{|c|}{$m=2$} & \multicolumn{2}{|c|}{$m=3$} & \multicolumn{2}{|c|}{$\mathrm{m}=4$} & $\mathrm{~m}=$ & \\
\hline & & $k(\%)$ & $\widehat{\mathrm{A}}$ & $k(\%)$ & $\widehat{\mathbf{A}}$ & $k(\%)$ & $\widehat{\mathrm{A}}$ & $\mathrm{k}(\%)$ & $\widehat{A}$ & $\mathbf{k}(\%)$ & $\widehat{\mathrm{A}}$ \\
\hline 0.0 & 1.253 & 0 & 0.625 & 0 & 0.465 & $2(<<0.1)$ & 0.383 & $12(<0.1)$ & 0.330 & $19(<0.1)$ & 0.291 \\
\hline 0.5 & 1.329 & 0 & 0.737 & 0 & 0.603 & 0 & 0.542 & $8(<0.1)$ & 0.507 & $17(<0.1)$ & 0.484 \\
\hline 1.0 & 1.547 & 0 & 1.223 & 0 & 1.188 & 0 & 1.181 & 0 & 1.179 & 0 & 1.178 \\
\hline 1.5 & 1.873 & 0 & 1.605 & 0 & 1.586 & 0 & 1.583 & 0 & 1.582 & 0 & 1.582 \\
\hline 2.0 & 2.270 & 0 & 2.049 & 0 & 2.038 & 0 & 2.037 & 0 & 2.037 & 0 & 2.037 \\
\hline 2.5 & 2.709 & 0 & 2.524 & 0 & 2.517 & 0 & 2.517 & 0 & 2.517 & 0 & 2.517 \\
\hline 3.0 & 3.170 & 0 & 3.012 & 0 & 3.008 & 0 & 3.008 & 0 & 3.008 & 0 & 3.008 \\
\hline & & & & & $N_{A}$ & $=500000$ & & & & & \\
\hline SNR & $\overline{\mathbf{M}}$ & & $=1$ & & & & & $m=$ & & $\mathrm{m}=$ & \\
\hline & & $k(\%)$ & $\widehat{\mathrm{A}}$ & $k(\%)$ & $\overline{\mathbf{A}}$ & $k(\%)$ & $\widehat{\mathrm{A}}$ & $k(\%)$ & $\overline{\mathrm{A}}$ & $k(\%)$ & $\widehat{\mathrm{A}}$ \\
\hline 0.0 & 1.253 & 0 & 0.627 & 0 & 0.470 & 0 & 0.392 & 0 & 0.343 & 0 & 0.308 \\
\hline 0.5 & 1.329 & 0 & 0.739 & 0 & 0.607 & 0 & 0.549 & 0 & 0.517 & 0 & 0.497 \\
\hline 1.0 & 1.547 & 0 & 1.224 & 0 & 1.190 & 0 & 1.183 & 0 & 1.181 & 0 & 1.181 \\
\hline 1.5 & 1.873 & 0 & 1.606 & 0 & 1.587 & 0 & 1.584 & 0 & 1.584 & 0 & 1.584 \\
\hline 2.0 & 2.270 & 0 & 2.050 & 0 & 2.039 & 0 & 2.038 & 0 & 2.038 & 0 & 2.038 \\
\hline 2.5 & 2.709 & 0 & 2.524 & 0 & 2.518 & 0 & 2.517 & 0 & 2.517 & 0 & 2.517 \\
\hline 3.0 & 3.170 & 0 & 3.012 & 0 & 3.008 & 0 & 3.008 & 0 & 3.008 & 0 & 3.008 \\
\hline
\end{tabular}

Table 4.4. Cont. 


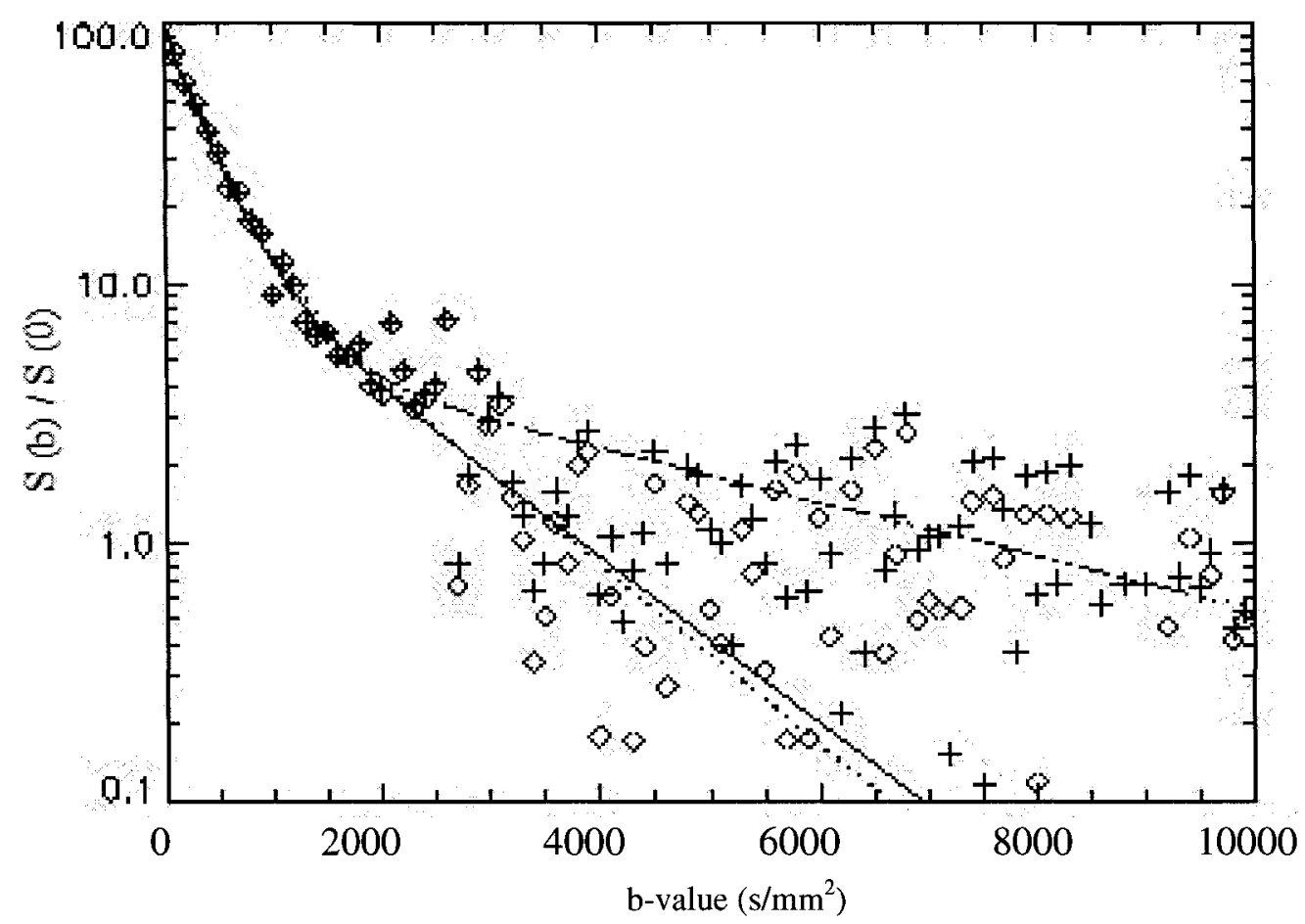

Figure 4.11. Simulated bi-exponential diffusion decay with Rician noise bias $(+)$ and NC2 corrected data $(\diamond)$. The fit to the corrected data (solid line) is in good agreement with the true decay (dotted line) compared with the fit to the Rician biased data (dashed line).

\begin{tabular}{|l|c|c|c|}
\hline & $\mathbf{D}_{\mathbf{1}}\left(\mathrm{mm}^{2} / \mathbf{s}\right)$ & $\mathbf{f}_{1}$ & $\mathbf{D}_{\mathbf{2}}\left(\mathrm{mm}^{2} / \mathbf{s}\right)$ \\
\hline $\begin{array}{l}\text { Input } \\
\text { parameters }\end{array}$ & $3.00 \times 10^{-3}$ & 0.80 & $0.80 \times 10^{-3}$ \\
\hline $\begin{array}{l}\text { Uncorrected } \\
\text { data }\end{array}$ & $(2.50 \pm 0.002) \times 10^{-3}$ & $0.94 \pm 0.01$ & $(0.24 \pm 0.0001) \times 10^{-3}$ \\
\hline $\begin{array}{l}\text { Corrected } \\
\text { data }\end{array}$ & $(3.01 \pm 0.0008) \times 10^{-3}$ & $0.82 \pm 0.009$ & $(0.74 \pm 0.0003) \times 10^{-3}$ \\
\hline
\end{tabular}

Table 4.5. The parameter values of the fit to the simulated decay biased with Rician noise and corrected with NC2 noise correction as shown in figure 4.11. 


\subsection{Noise Estimation from the Image Background}

The post processing noise correction techniques discussed in section 4.3 are all based on the theoretical prediction that the data distribution is Rician and the availability of an accurate value for $\sigma$. In section 4.4.1 the data distribution for $A=0$ is investigated. The rest of this section is devoted to the accurate determination of $\sigma$.

For the experiments reported in this section the head coil was used to measure the diffusion signals, $S(b)$, for 16 b-values including $0,250,500,750,1000,1250,1500,2000,2300,2600$, $3000,3250,3500,4000,4500$, and $5000 \mathrm{~s} / \mathrm{mm}^{2}$. The head coil phantom was first positioned in the head coil and moved to the magnet isocenter. It was left there for $30 \mathrm{~min}$. to make the water completely still and uniformly distributed inside the phantom. The temperature in the magnet bore was then measured to be $22^{\circ} \mathrm{C}$ before the measurements were started. A single transverse slice with $5 \mathrm{~mm}$ thickness was selected in the "head to foot" direction. The phase encoding was chosen to be in the anterior to posterior direction while the diffusion sensitizing gradients as well as the EPI readout gradients were applied in the right to left direction. The measurements were performed with a 128 matrix and a $75 \%$ partial Fourier factor. Thus, the data set was $96 \times 128$ in kspace and reconstructed as $128 \times 128$ images. The images were measured for a FOV=300 mm resulting in $2.3 \mathrm{~mm}$ in plane resolution. The diffusion signal decay was measured once (NEX=1) with the repetition time of $\mathrm{TR}=500 \mathrm{~ms}$ and an echo time of $\mathrm{TE}=200 \mathrm{~ms}$. The image data acquisition was performed with a bandwidth of $752 \mathrm{~Hz} /$ pixel.

For some of the measurements the head coil water phantom was used while for the rest the phantom was removed to provide pure background images. All of the measurements used the parameter settings described above. To ensure that the electronic settings of the imager (e.g. amplifier gain) were the same without the phantom as with the phantom the adjustments were 
first done with the phantom in place. The phantom was then removed without moving the patient table, ensuring that the adjustments were not repeated and the settings remained the same.

\subsubsection{The PDF of the background}

In order to apply the noise correction techniques presented in the previous section to estimate the "true" signal strength, knowledge of the data distribution function is required. To test the hypothesis that it is Rician as expected from theory (see equations (4.7) and (4.17)) a histogram was generated from a measured $M R$ magnitude image corresponding to $A=0$ (i.e. the only contribution to the measured signal was from noise). In this case the distribution should correspond to a Rayleigh distribution. A set of diffusion experiments was performed with the head coil water phantom removed, as described above, to provide pure background images. A histogram of the pixel intensities of the entire image (256x256 pixels) was generated using DImageV3 in IDL version 6.1 (see figure 4.12). Note that DImageV3 interpolates the $128 \times 128$ image matrices to $256 \times 256$ using bilinear interpolation.

The mean and standard deviation were evaluated for the entire image and found to be $<\mathrm{M}\rangle=225 \pm 5$ and $\sigma_{\mathrm{M}}=107 \pm 1$, respectively. From these measurements we find, using equation (4.16) and (4.19), that $\sigma=180 \pm 4$. When the experimental histogram is compared with the Rayleigh distribution for $\sigma=180$ (see figure 4.12) the results are in reasonable agreement, however, it is clear that something else is also involved. It should be noted that the Rayleigh function was scaled by a factor of $256 \times 256$.

Siemens images are filtered using a Hanning filter to avoid truncation artifacts. To see if this is what is causing the differences noted in figure 4.12 we generated a simulated Hanning 


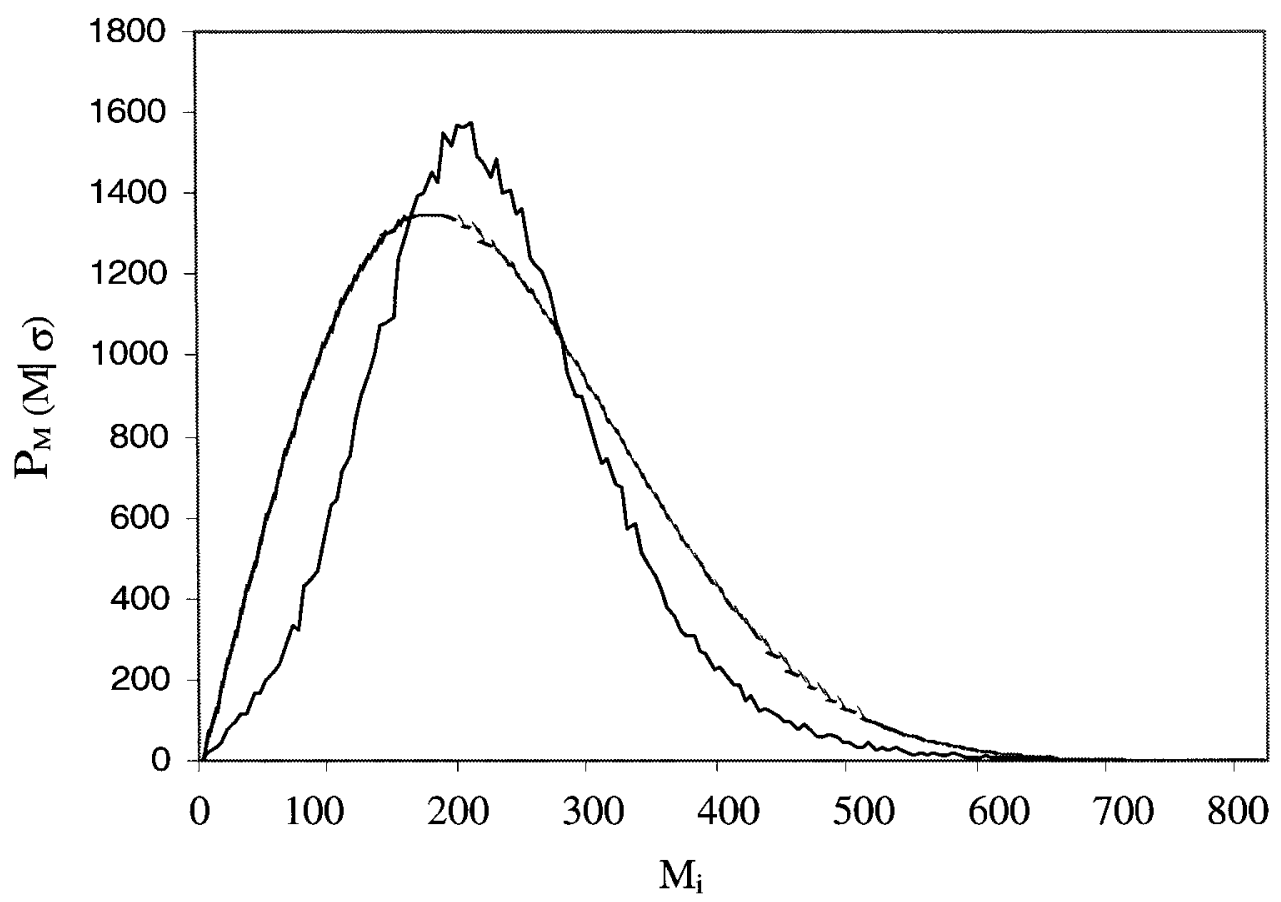

Figure 4.12. The histogram of the magnitude data from the image background of the MR diffusion measurement (solid line) compared with the Rayleigh density function with the same $\sigma$ value (i.e. $\sigma=180$ ).

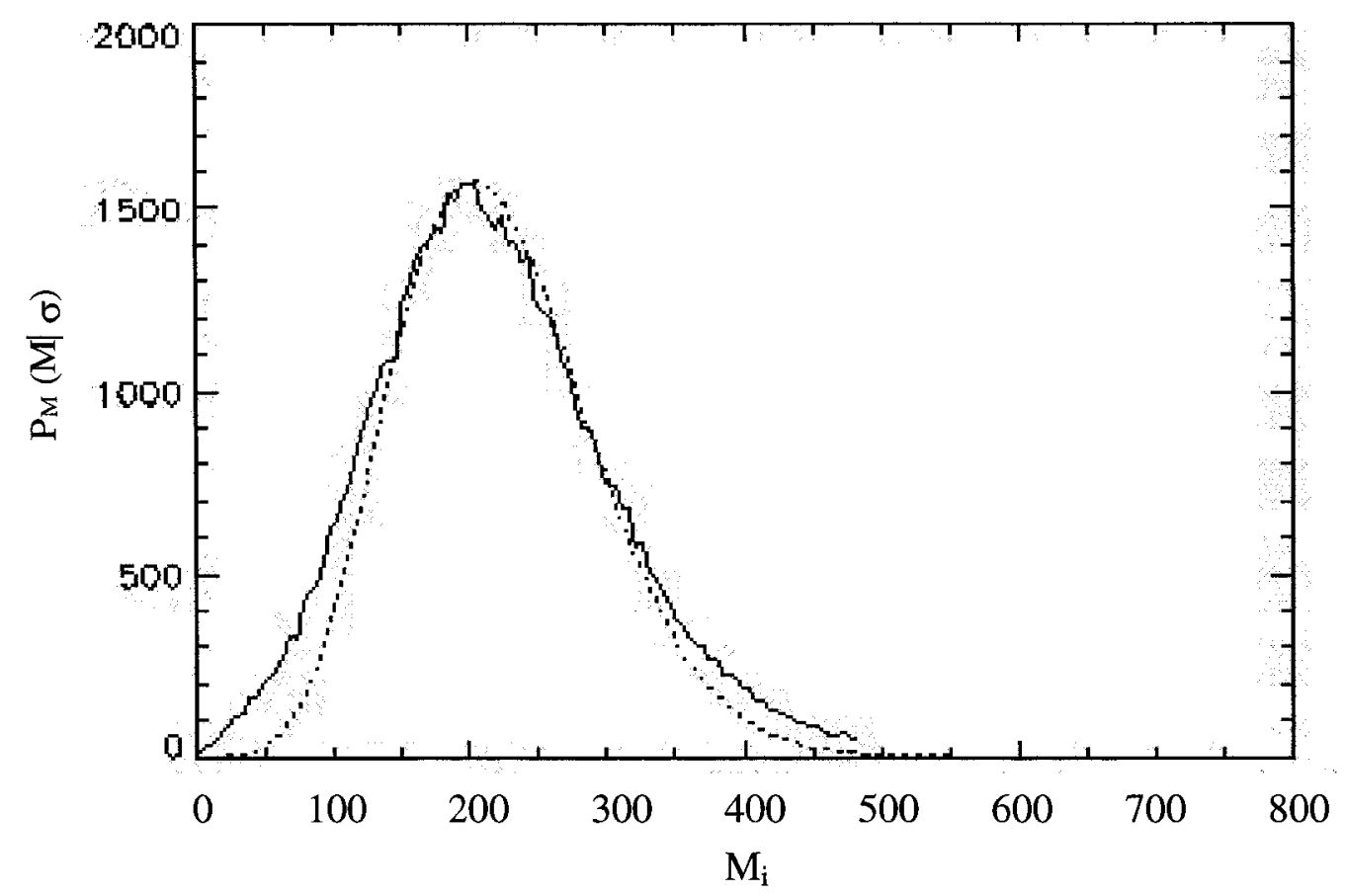

Figure 4.13. The histogram of the magnitude data from the image background of the MR diffusion measurement (solid line) compared with a Hanning filtered Rayleigh density function. 
filtered Rayleigh PDF for $\sigma=180$ to compare with our experimental curve. The Hanning filtered PDF agrees quite well with the measured distribution (see figure 4.13). The remaining differences can likely be attributed to the fact that Siemens uses a different Hanning filter than we used in our simulations. Unfortunately, we do not have access to the details of how Siemens Hanning filtering is done. This is discussed in more detail at the end of section 4.4.3.

This result shows that the noise PDF is a Hanning filtered Rayleigh distribution rather than a straight Rayleigh PDF. The difference is not large but is it significant? The mean and standard deviation of the filtered PDF are 225 and 72, respectively. It is important to note that the mean value is unaffected by the filtering and the standard deviation is reduced by $35 \%$.

It is more difficult to make a similar determination for $\mathrm{A}>0$, however, most of the processes that could potentially distort the distribution from a Rayleigh distribution (e.g. electronic filtering, extraneous rf signals) would be expected to have a similar effect when $A>0$. Thus, it should be reasonable to extrapolate from the results of this section to conclude that the pixel intensities are distributed according to a Hanning filtered Rician distribution for all values of A where the filtering has no effect on the mean of the PDF and a significant effect on the standard deviation.

\subsubsection{Noise estimation from the image background without the phantom}

In order to use any of the noise correction techniques used in this thesis it is necessary to have a reliable value for $\sigma$. Ideally, $\sigma$ should be determined from the measured standard deviation of the actual pixel under consideration. However, this would involve making many measurements of the signal intensity for the pixel of interest and calculating the standard deviation from this data set. This is impractical. Alternatively, the standard deviation could be calculated for a small region of 
interest containing equivalent pixels. This is possible when imaging large water phantoms, however, for in vivo applications adjacent pixels cannot be assumed to be equivalent (i.e. have the same "true" signal intensity). The most practical solution to the problem is to evaluate $\sigma$ from a region of interest in the image background where there is only noise. This introduces the assumption that $\sigma$ is the same for this background region of interest as it is for the pixels of interest. This should be a reasonable assumption in most cases but should be checked experimentally on the imager being used to be sure.

In section 4.2 three expressions were given for estimating $\sigma$ from pixels that contain contributions only from noise (i.e. $\mathrm{A}=0$ ) corresponding to a Rayleigh distribution. These three estimators are:

$$
\begin{aligned}
& \hat{\sigma}\left(<M^{2}>\right)=\frac{1}{\sqrt{2}} \sqrt{\left\langle M^{2}\right\rangle}, \\
& \hat{\sigma}(<M>)=\sqrt{\frac{2}{\pi}}<M>,
\end{aligned}
$$

and

$$
\hat{\sigma}(\operatorname{std}<M>)=\frac{\sigma_{M}}{\sqrt{2-\pi / 2}}
$$

In principle, all of these should give the same result and none is any better than the others. However, if the distribution function differs from Rician or if the region of interest being considered is contaminated by artifacts, these effects can be expected to affect the three estimators differently. Conversely, if the measured values for these estimators are all equal, it gives confidence that this value is the correct value of $\sigma$. If only one estimator was used the risk of using an inaccurate value of $\sigma$ in the noise correction would be significant. Rather than resulting in a more accurate characterization of the diffusion decay it could lead to just the opposite and the user would be unaware. 
The first question that was addressed was whether $\sigma$ changes with position inside the FOV. In particular, is the value of $\sigma$ calculated from an ROI near the center of the FOV, where the subject's brain will be, the same as the value calculated from the outer parts of the FOV which will contain only background signals? To test this a series of experiments was performed with the phantom removed (as discussed earlier in this section) so that the images consisted only of noise. Even though there was no phantom in the imager these measurements were repeated for the $16 \mathrm{~b}$ values given earlier to ensure that $\sigma$ does not change with the strength of the diffusion sensitizing gradient.

Each image was analyzed using 16 square ROIs on a $4 \times 4$ grid positioned uniformly across the FOV. Each ROI was $2500 \mathrm{~mm}^{2}$ in area and contained 2600 pixels. The 16 ROIs are shown in figure 4.14. $\sigma$ was calculated for each ROI using the three estimators introduced above. Selected results from this study are given in table 4.6 and figure 4.15 . No significant change was observed either as a function of position within the FOV or with b-value. In particular, the values of $\sigma$ calculated for the central ROIs $(5,6,9$ and 10$)$ were the same as the values calculated for the rest of the ROIs to within a reasonable experimental error. It is interesting to note that these measurements consistently gave $\hat{\sigma}\left(\operatorname{std}_{<M>}\right)<\hat{\sigma}\left(<M^{2}>\right)<\hat{\sigma}(<M>)$ which is inconsistent with our initial assumption that they should be equal. However, the difference was consistently less than one standard deviation (see the ave and std values given in table 4.6) so the significance of this observation was not pursued further.

The values of $\sigma$ computed using the three estimators were the same to within one standard deviation when averaged over the 16 ROIs. This is additional confirmation that the noise PDF is indeed Rayleigh and there are no significant perturbing influences on the system.

The three $\hat{\sigma}$ values are also shown versus ROI number in figure 4.15 for selected $b$ values. No significant trends are apparent. The average values of $\hat{\sigma}$, averaged over ROI number for each 


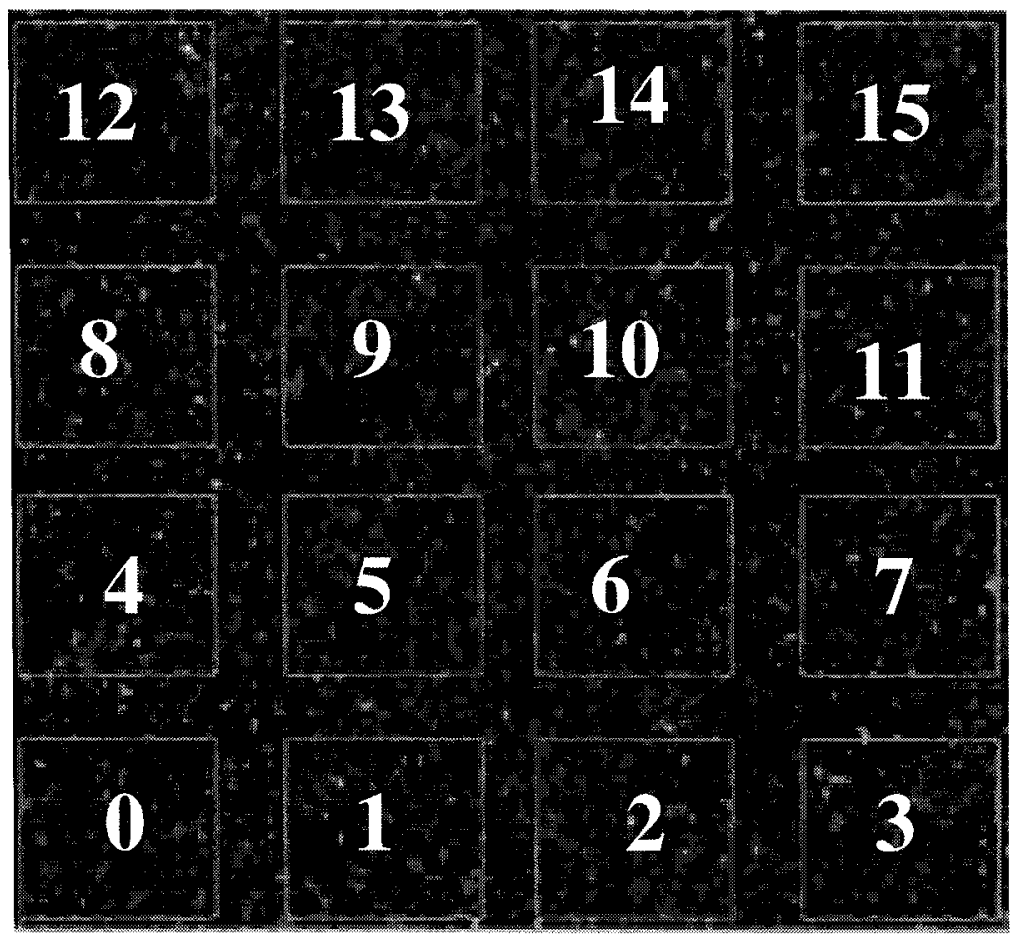

Figure 4.14. The 16 ROIs selected in the background image for MR diffusion measurements to study the noise $\sigma$ estimates.

\begin{tabular}{|cccccccc|}
\hline $\mathrm{ROI}$ & $\mathrm{b}$-value & $\langle\mathrm{M}\rangle$ & $\mathrm{std}_{<\mathrm{M}\rangle}$ & $\left\langle\mathrm{M}^{2}\right\rangle$ & $\hat{\sigma}(<\mathrm{M}\rangle)$ & $\hat{\sigma}\left(\mathrm{std}_{<\mathrm{M}\rangle}\right)$ & $\left.\hat{\sigma}\left(<\mathrm{M}^{2}\right\rangle\right)$ \\
\hline 0 & 0 & 2.841 & 1.516 & 10.369 & 2.267 & 2.313 & 2.277 \\
1 & 0 & 3.099 & 1.546 & 11.994 & 2.473 & 2.360 & 2.449 \\
2 & 0 & 2.977 & 1.515 & 11.157 & 2.375 & 2.312 & 2.362 \\
3 & 0 & 3.049 & 1.571 & 11.763 & 2.433 & 2.398 & 2.425 \\
4 & 0 & 3.152 & 1.655 & 12.676 & 2.515 & 2.526 & 2.518 \\
5 & 0 & 3.052 & 1.557 & 11.738 & 2.435 & 2.377 & 2.423 \\
6 & 0 & 2.887 & 1.526 & 10.662 & 2.304 & 2.329 & 2.309 \\
7 & 0 & 2.970 & 1.531 & 11.163 & 2.370 & 2.337 & 2.363 \\
8 & 0 & 3.221 & 1.596 & 12.919 & 2.570 & 2.436 & 2.542 \\
9 & 0 & 3.091 & 1.543 & 11.937 & 2.466 & 2.356 & 2.443 \\
10 & 0 & 3.150 & 1.693 & 12.791 & 2.514 & 2.585 & 2.529 \\
11 & 0 & 3.000 & 1.555 & 11.418 & 2.394 & 2.374 & 2.389 \\
12 & 0 & 2.935 & 1.612 & 11.210 & 2.341 & 2.461 & 2.367 \\
13 & 0 & 3.074 & 1.533 & 11.797 & 2.452 & 2.340 & 2.429 \\
14 & 0 & 3.094 & 1.559 & 12.005 & 2.469 & 2.380 & 2.450 \\
15 & 0 & 3.150 & 1.562 & 12.358 & 2.513 & 2.384 & 2.486 \\
ave & & 3.046 & 1.567 & 11.747 & 2.431 & 2.392 & 2.422 \\
std & & 0.104 & 0.050 & 0.732 & 0.083 & 0.076 & 0.076 \\
\hline
\end{tabular}

Table 4.6. The noise $\sigma$ estimated with the various estimators for $b=0,2600$ and $5000 \mathrm{~s} / \mathrm{mm}^{2}$ for 16 ROI locations in the image background displayed in figure 4.14. 


\begin{tabular}{|cccccccc|}
\hline ROI & b-value & $\langle\mathrm{M}\rangle$ & $\mathrm{std}_{<\mathrm{M}\rangle}$ & $\left\langle\mathrm{M}^{2}\right\rangle$ & $\hat{\sigma}(<\mathrm{M}\rangle)$ & $\hat{\sigma}\left(\mathrm{std}_{<\mathrm{M}\rangle}\right)$ & $\hat{\sigma}\left(<\mathrm{M}^{2}>\right)$ \\
\hline 0 & 2600 & 3.090 & 1.629 & 12.198 & 2.465 & 2.487 & 2.470 \\
1 & 2600 & 2.984 & 1.519 & 11.208 & 2.381 & 2.318 & 2.367 \\
2 & 2600 & 3.073 & 1.532 & 11.791 & 2.452 & 2.338 & 2.428 \\
3 & 2600 & 3.047 & 1.579 & 11.778 & 2.431 & 2.411 & 2.427 \\
4 & 2600 & 2.961 & 1.498 & 11.011 & 2.362 & 2.287 & 2.346 \\
5 & 2600 & 3.065 & 1.520 & 11.707 & 2.446 & 2.321 & 2.419 \\
6 & 2600 & 3.067 & 1.564 & 11.850 & 2.447 & 2.387 & 2.434 \\
7 & 2600 & 2.974 & 1.491 & 11.066 & 2.373 & 2.276 & 2.352 \\
8 & 2600 & 2.991 & 1.497 & 11.186 & 2.386 & 2.285 & 2.365 \\
9 & 2600 & 3.070 & 1.555 & 11.843 & 2.450 & 2.373 & 2.433 \\
10 & 2600 & 3.081 & 1.579 & 11.988 & 2.458 & 2.411 & 2.448 \\
11 & 2600 & 2.858 & 1.519 & 10.475 & 2.280 & 2.319 & 2.289 \\
12 & 2600 & 3.143 & 1.688 & 12.727 & 2.508 & 2.577 & 2.523 \\
13 & 2600 & 3.105 & 1.495 & 11.878 & 2.478 & 2.282 & 2.437 \\
14 & 2600 & 3.124 & 1.609 & 12.344 & 2.492 & 2.455 & 2.484 \\
15 & 2600 & 3.005 & 1.607 & 11.613 & 2.398 & 2.452 & 2.410 \\
ave & & 3.040 & 1.555 & 11.666 & 2.425 & 2.374 & 2.415 \\
std & & 0.073 & 0.057 & 0.564 & 0.058 & 0.087 & 0.059 \\
\hline
\end{tabular}

\begin{tabular}{|cccccccc|}
\hline $\mathrm{ROI}$ & b-value & $\langle\mathrm{M}\rangle$ & $\mathrm{std}_{<\mathrm{M}\rangle}$ & $\left\langle\mathrm{M}^{2}\right\rangle$ & $\hat{\sigma}(<\mathrm{M}\rangle)$ & $\hat{\sigma}\left(\mathrm{std}_{<\mathrm{M}\rangle}\right)$ & $\left.\hat{\sigma}\left(<\mathrm{M}^{2}\right\rangle\right)$ \\
\hline 0 & 5000 & 3.085 & 1.609 & 12.103 & 2.461 & 2.456 & 2.460 \\
1 & 5000 & 2.968 & 1.492 & 11.037 & 2.368 & 2.278 & 2.349 \\
2 & 5000 & 3.033 & 1.509 & 11.477 & 2.420 & 2.303 & 2.396 \\
3 & 5000 & 2.943 & 1.562 & 11.097 & 2.348 & 2.384 & 2.355 \\
4 & 5000 & 2.937 & 1.546 & 11.014 & 2.343 & 2.360 & 2.347 \\
5 & 5000 & 3.120 & 1.536 & 12.091 & 2.489 & 2.345 & 2.459 \\
6 & 5000 & 3.049 & 1.588 & 11.814 & 2.433 & 2.423 & 2.430 \\
7 & 5000 & 2.951 & 1.532 & 11.056 & 2.355 & 2.339 & 2.351 \\
8 & 5000 & 3.035 & 1.467 & 11.365 & 2.422 & 2.239 & 2.384 \\
9 & 5000 & 2.850 & 1.546 & 10.516 & 2.274 & 2.360 & 2.293 \\
10 & 5000 & 3.127 & 1.604 & 12.351 & 2.495 & 2.449 & 2.485 \\
11 & 5000 & 3.186 & 1.644 & 12.855 & 2.542 & 2.510 & 2.535 \\
12 & 5000 & 3.077 & 1.553 & 11.878 & 2.455 & 2.371 & 2.437 \\
13 & 5000 & 3.161 & 1.617 & 12.604 & 2.522 & 2.468 & 2.510 \\
14 & 5000 & 2.949 & 1.577 & 11.182 & 2.353 & 2.408 & 2.365 \\
15 & 5000 & 3.212 & 1.594 & 12.860 & 2.563 & 2.433 & 2.536 \\
ave & & 3.043 & 1.561 & 11.706 & 2.428 & 2.383 & 2.418 \\
std & & 0.103 & 0.048 & 0.721 & 0.082 & 0.073 & 0.074 \\
\hline
\end{tabular}

Table 4.6. Cont. 
b-value and over the b-values for each ROI are given in table 4.7 and figures 4.16 and 4.17. No significant trends are apparent in these figures either. However, the $\hat{\sigma}$ values for ROI\#15 and to a lesser extent for ROI\#0 are noticeably higher than the rest. This is not likely a significant effect but, to be safe, these regions will be avoided when calculating the $\hat{\sigma}$ values used for noise correction. A linear regression was also performed on the data as a function of b-value. No dependence on b-value was observed. For all three $\sigma$ estimators the slope was very close to zero with two slopes being positive and one being negative (see figure 4.16). A linear regression was not performed on the data as a function of ROI number since ROI number is not a proper ordinate. The ROI numbering is arbitrary and to suggest that $\hat{\sigma}$ is a function of ROI number is meaningless. For these data sets the means were calculated and are displayed on figure 4.17 as lines across the plot.

These measurements show no significant change in $\hat{o}$ with position in the FOV for any of the estimators or b-values studied. This would suggest that use of regions of background in the image can be used to calculate $\sigma$ for pixels at the location of the object in the image (e.g. the brain). The situation is not as obvious as it may seem, however, since the object being imaged may also contribute to the noise (as discussed in section 2.4). In fact, for conductive objects like the human body, the contribution to the noise from the object will be of about the same magnitude as the contribution from the electronics at $1.5 \mathrm{~T}$. However, if $\sigma$ did change with position within the FOV, one would expect this to be a result of the electronic noise rather than the object noise since a spatial dependence in the image corresponds to a frequency modulation of the measured signal and there is no reason to expect the object contribution to the noise to be different for different frequencies within the MR acquisition bandwidth. 

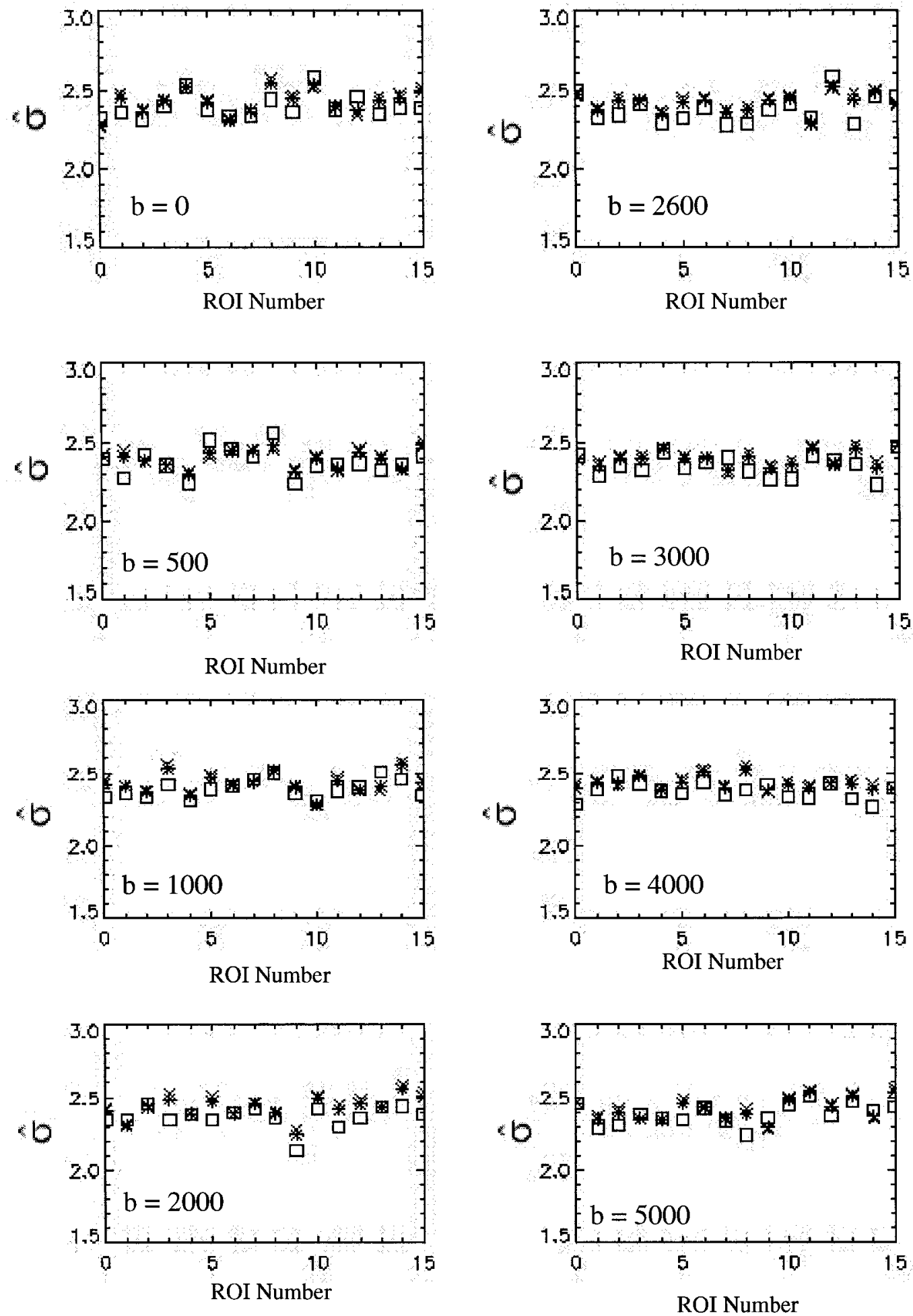

Figure 4.15. Values of the background noise estimates without the phantom are shown vs. ROI location for selected b-values $\left(\times \hat{\sigma}_{<M>}, \square \hat{\sigma}_{s t d<M>}, * \hat{\sigma}_{<M^{2}>}\right)$. The ROI numbering is shown in figure 4.14. The b-values are in units of $\mathrm{s} / \mathrm{mm}^{2}$. 


\begin{tabular}{|c|c|c|c|c|c|c|c|}
\hline $\begin{array}{l}\text { b-value } \\
\left(\mathrm{s} / \mathrm{mm}^{2}\right)\end{array}$ & $\hat{\sigma}(<\mathrm{M}>)$ & $\hat{\sigma}\left(\operatorname{std}_{<M}\right\rangle$ & $\hat{\sigma}\left(<\mathrm{M}^{2}>\right)$ & $\begin{array}{c}\mathrm{ROI} \\
\text { location }\end{array}$ & $\hat{\sigma}(<M>)$ & $\hat{\sigma}\left(\operatorname{std}_{<M>}\right)$ & $\hat{\sigma}\left(<\mathrm{M}^{2}>\right)$ \\
\hline \multicolumn{4}{|c|}{ averaged over $16 \mathrm{ROI}$} & \multicolumn{4}{|c|}{ averaged over b-value } \\
\hline 0 & 2.431 & 2.392 & 2.422 & 0 & 2.450 & 2.372 & 2.433 \\
\hline 250 & 2.401 & 2.337 & 2.387 & 1 & 2.409 & 2.356 & 2.398 \\
\hline 500 & 2.399 & 2.374 & 2.394 & 2 & 2.426 & 2.364 & 2.413 \\
\hline 750 & 2.440 & 2.384 & 2.428 & 3 & 2.400 & 2.349 & 2.389 \\
\hline 1000 & 2.434 & 2.391 & 2.425 & 4 & 2.399 & 2.363 & 2.391 \\
\hline 1250 & 2.376 & 2.367 & 2.374 & 5 & 2.424 & 2.361 & 2.410 \\
\hline 1500 & 2.423 & 2.367 & 2.411 & 6 & 2.409 & 2.381 & 2.403 \\
\hline 2000 & 2.444 & 2.364 & 2.427 & 7 & 2.439 & 2.394 & 2.430 \\
\hline 2300 & 2.427 & 2.387 & 2.418 & 8 & 2.431 & 2.384 & 2.421 \\
\hline 2600 & 2.425 & 2.374 & 2.415 & 9 & 2.401 & 2.343 & 2.389 \\
\hline 3000 & 2.402 & 2.349 & 2.391 & 10 & 2.406 & 2.380 & 2.400 \\
\hline 3250 & 2.455 & 2.421 & 2.448 & 11 & 2.426 & 2.357 & 2.411 \\
\hline 3500 & 2.428 & 2.389 & 2.419 & 12 & 2.412 & 2.391 & 2.408 \\
\hline 4000 & 2.438 & 2.372 & 2.424 & 13 & 2.423 & 2.383 & 2.415 \\
\hline 4500 & 2.396 & 2.325 & 2.381 & 14 & 2.424 & 2.381 & 2.415 \\
\hline 5000 & 2.428 & 2.383 & 2.418 & 15 & 2.469 & 2.416 & 2.458 \\
\hline ave & 2.421 & 2.373 & 2.411 & ave & 2.422 & 2.373 & 2.411 \\
\hline std & 0.020 & 0.023 & 0.021 & std & 0.019 & 0.019 & 0.018 \\
\hline
\end{tabular}

Table 4.7. The noise $\sigma$ estimated from the background of the images without a phantom; a.(left) averaged over 16 ROI locations for each $b$-value, $b$.(right) averaged over $16 \mathrm{~b}$-values for each ROI location.

We would also have checked to see if $\sigma$ is the same at different positions within the FOV with the head coil phantom in place, however, for high SNR situations, subtle variations in the signal across an ROI, due to the presence of small artifacts (e.g. Gibbs ringing) and inherent signal nonuniformity, cause the measured standard deviation to be larger than the true $\sigma$. This makes the comparison of $\sigma$ values from two different regions within the FOV ambiguous when a phantom is used. For this reason we elected to make the measurements without object noise and extrapolate these results to the situation where the object noise exists with the justification given above. 


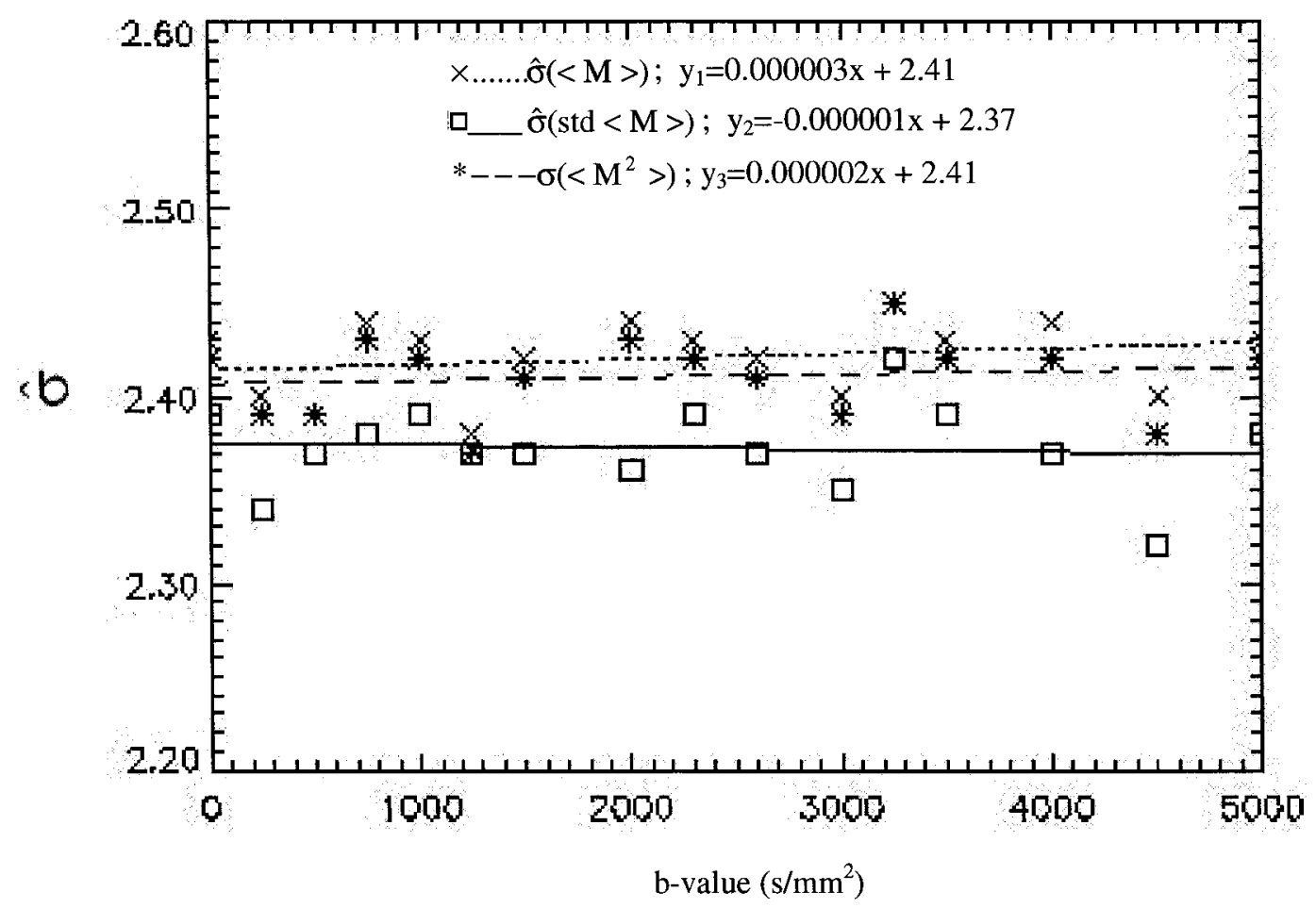

Figure 4.16. The noise $\sigma$ estimates averaged over ROI locations vs. b-value in the background image without phantom.

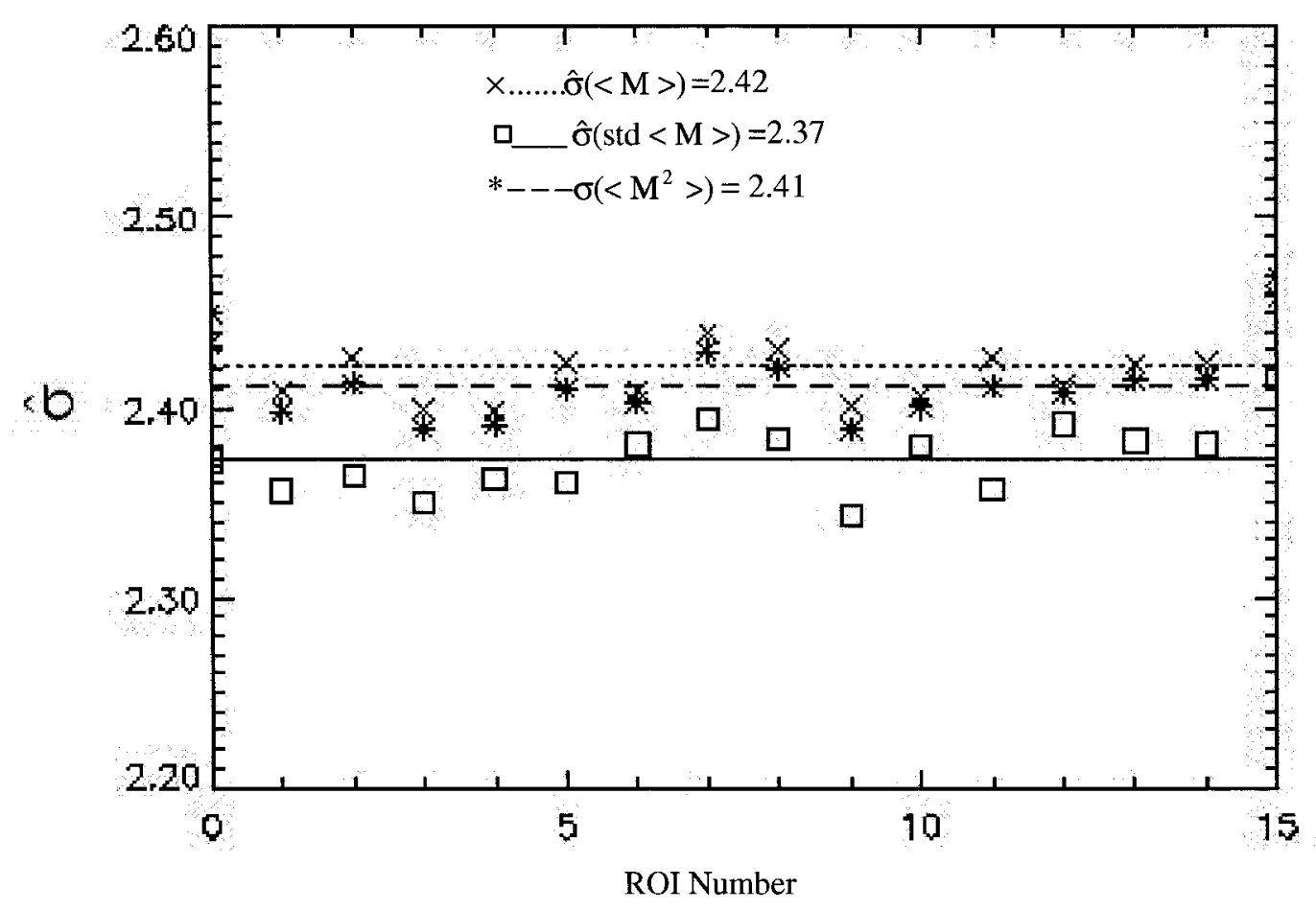

Figure 4.17. The noise $\sigma$ estimates averaged over b-values vs. ROI location in the background image without phantom. 


\subsubsection{Noise estimation from the image background with the phantom}

The next set of experiments was performed using the head coil phantom to see, in the presence of the contribution to the noise from the object being imaged, 1) if the $\hat{\sigma}$ values change with ROI position, 2) if there is a dependence on b-value and 3) if the three $\sigma$ estimators are in agreement with each other. The experimental details are given at the beginning of section 4.4. Each image was analyzed using 8 ROIs positioned along the left and the right sides of the image in the background noise. Since artifacts (e.g. N/2 ghosting) are more likely to appear in the phase encoding direction, which is the vertical direction in the images presented here, ROIs were not placed above or below the image of the phantom. Each ROI was $2500 \mathrm{~mm}^{2}$ in area and contained 2600 pixels. The 8 ROIs are shown in figure 4.18. For each ROI $\hat{\sigma}$ values were calculated using the three estimators introduced above. Selected results from this study are given in table 4.8 and figures 4.19 to 4.21 .

A linear regression was performed on the data as a function of b-value. In all three cases the slope was very close to zero with the slopes being negative (see figure 4.20). A linear regression was not performed on the data as a function of ROI number since this is not a proper ordinate. For these data sets the means were calculated and displayed in figure 4.21 as lines across the plot.

The small dependence of $\hat{\sigma}$ on $\mathrm{b}$ observed in figure 4.20 was initially attributed to the Gibbs ringing artifact which extends well beyond the edge of the phantom and is proportional to the signal amplitude from the phantom. To test this hypothesis the size of the ROIs was reduced to half the original size and positioned as far away from the phantom as possible. The results of this analysis, shown in figure 4.22 , are essentially identical to the original results. This shows that the b-value dependence is not a result of Gibbs artifact. The explanation of this effect remains 


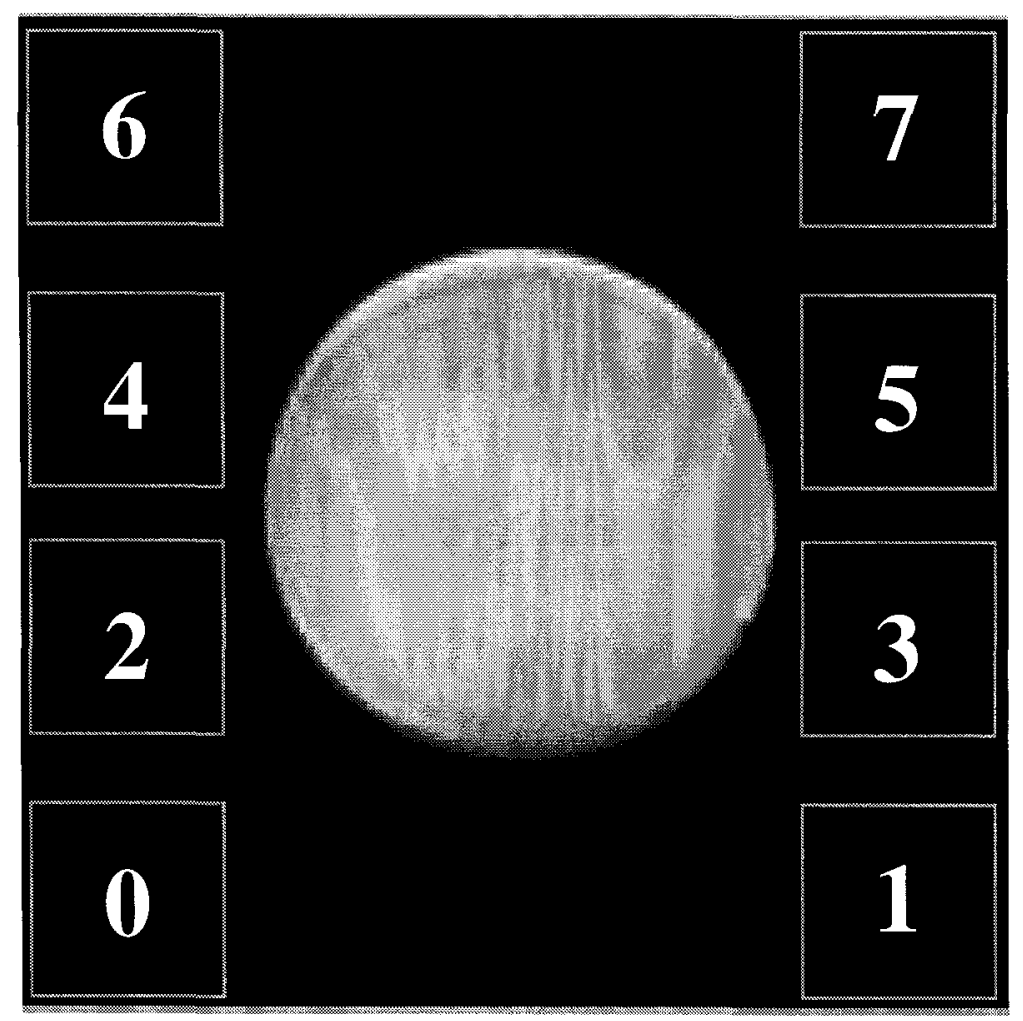

Figure 4.18. The 8 ROIs selected in the image background for MR diffusion measurements of the water phantom to study the noise $\sigma$ estimates.

\begin{tabular}{|cccccccc|}
\hline $\mathrm{ROI}$ & b-value & $\langle\mathrm{M}\rangle$ & $\mathrm{std}_{<\mathrm{M}\rangle}$ & $\left\langle\mathrm{M}^{2}\right\rangle$ & $\hat{\mathrm{\sigma}}(<\mathrm{M}\rangle)$ & $\hat{\sigma}\left(\mathrm{std}_{<\mathrm{M}>}\right)$ & $\hat{\sigma}\left(<\mathrm{M}^{2}>\right)$ \\
\hline 0 & 0 & 5.945 & 2.839 & 43.401 & 4.743 & 4.334 & 4.658 \\
1 & 0 & 6.097 & 2.715 & 44.546 & 4.865 & 4.144 & 4.719 \\
2 & 0 & 6.510 & 3.109 & 52.043 & 5.194 & 4.745 & 5.101 \\
3 & 0 & 6.000 & 2.755 & 43.584 & 4.787 & 4.205 & 4.668 \\
4 & 0 & 6.010 & 2.674 & 43.266 & 4.795 & 4.081 & 4.651 \\
5 & 0 & 6.281 & 2.775 & 47.147 & 5.012 & 4.235 & 4.855 \\
6 & 0 & 6.007 & 2.831 & 44.090 & 4.793 & 4.321 & 4.695 \\
7 & 0 & 6.266 & 2.914 & 47.748 & 4.999 & 4.448 & 4.886 \\
ave & 0 & 6.140 & 2.826 & 45.728 & 4.899 & 4.314 & 4.779 \\
std & 0 & 0.195 & 0.137 & 3.073 & 0.156 & 0.209 & 0.158 \\
\hline
\end{tabular}

Table 4.8. The noise $\sigma$ estimated with the various estimators vs. b-value for 0 to $5000 \mathrm{~s} / \mathrm{mm}^{2}$ in 8 ROI locations in the image background with the phantom displayed in figure 4.18. 


\begin{tabular}{|c|c|c|c|c|c|c|c|}
\hline $\mathrm{ROI}$ & b-value & $<\mathrm{M}\rangle$ & $\mathrm{std}_{<\mathrm{M}>}$ & $\left.<\mathrm{M}^{2}\right\rangle$ & $\hat{\sigma}(<M>)$ & $\hat{\sigma}\left(\operatorname{std}_{<M>}\right)$ & $\hat{\sigma}\left(<M^{2}>\right)$ \\
\hline 0 & 500 & 5.979 & 2.839 & 43.806 & 4.771 & 4.333 & 4.680 \\
\hline 1 & 500 & 6.258 & 2.859 & 47.334 & 4.993 & 4.363 & 4.865 \\
\hline 2 & 500 & 6.039 & 2.827 & 44.454 & 4.818 & 4.315 & 4.715 \\
\hline 3 & 500 & 6.128 & 2.788 & 45.323 & 4.890 & 4.255 & 4.760 \\
\hline 4 & 500 & 6.124 & 2.711 & 44.850 & 4.886 & 4.138 & 4.735 \\
\hline 5 & 500 & 6.172 & 2.771 & 45.771 & 4.925 & 4.230 & 4.784 \\
\hline 6 & 500 & 5.969 & 2.734 & 43.102 & 4.763 & 4.173 & 4.642 \\
\hline 7 & 500 & 6.191 & 2.998 & 47.315 & 4.940 & 4.576 & 4.864 \\
\hline ave & 500 & 6.108 & 2.816 & 45.244 & 4.873 & 4.298 & 4.756 \\
\hline std & 500 & 0.103 & 0.090 & 1.529 & 0.083 & 0.137 & 0.080 \\
\hline $\mathrm{ROI}$ & b-value & $\langle\mathrm{M}\rangle$ & $\operatorname{std}_{<M>}$ & $<\mathrm{M}^{2}>$ & $\hat{\sigma}(<M>)$ & $\hat{\sigma}\left(\operatorname{std}_{<M>}\right)$ & $\hat{\sigma}\left(<\mathrm{M}^{2}>\right)$ \\
\hline 0 & 1000 & 6.205 & 2.778 & 46.217 & 4.951 & 4.241 & 4.807 \\
\hline 1 & 1000 & 6.175 & 2.719 & 45.522 & 4.927 & 4.151 & 4.771 \\
\hline 2 & 1000 & 5.990 & 2.700 & 43.172 & 4.780 & 4.121 & 4.646 \\
\hline 3 & 1000 & 6.128 & 2.869 & 45.783 & 4.890 & 4.379 & 4.785 \\
\hline 4 & 1000 & 6.072 & 2.639 & 43.838 & 4.845 & 4.029 & 4.682 \\
\hline 5 & 1000 & 5.971 & 2.737 & 43.146 & 4.764 & 4.178 & 4.645 \\
\hline 6 & 1000 & 5.734 & 2.788 & 40.648 & 4.575 & 4.256 & 4.508 \\
\hline 7 & 1000 & 6.194 & 2.956 & 47.093 & 4.942 & 4.511 & 4.852 \\
\hline ave & 1000 & 6.059 & 2.773 & 44.427 & 4.834 & 4.233 & 4.712 \\
\hline std & 1000 & 0.159 & 0.100 & 2.111 & 0.127 & 0.153 & 0.113 \\
\hline ROI & b-value & $\langle\mathrm{M}\rangle$ & $\operatorname{std}_{<M>}$ & $\left\langle\mathrm{M}^{2}\right\rangle$ & $\hat{\sigma}(<M>)$ & $\hat{\sigma}\left(\operatorname{std}_{<M>}\right)$ & $\hat{\sigma}\left(<M^{2}>\right)$ \\
\hline 0 & 3000 & 5.953 & 2.702 & 42.733 & 4.750 & 4.124 & 4.622 \\
\hline 1 & 3000 & 6.404 & 2.859 & 49.184 & 5.110 & 4.365 & 4.959 \\
\hline 2 & 3000 & 5.864 & 2.600 & 41.142 & 4.679 & 3.969 & 4.536 \\
\hline 3 & 3000 & 5.929 & 2.610 & 41.962 & 4.730 & 3.985 & 4.581 \\
\hline 4 & 3000 & 6.176 & 2.583 & 44.815 & 4.928 & 3.943 & 4.734 \\
\hline 5 & 3000 & 6.095 & 2.886 & 45.482 & 4.863 & 4.406 & 4.769 \\
\hline 6 & 3000 & 6.151 & 2.743 & 45.352 & 4.908 & 4.187 & 4.762 \\
\hline 7 & 3000 & 6.002 & 2.935 & 44.631 & 4.789 & 4.480 & 4.724 \\
\hline ave & 3000 & 6.072 & 2.740 & 44.412 & 4.845 & 4.182 & 4.711 \\
\hline std & 3000 & 0.174 & 0.139 & 2.522 & 0.138 & 0.213 & 0.133 \\
\hline $\mathrm{ROI}$ & b-value & $\langle\mathrm{M}\rangle$ & $\mathrm{std}_{<\mathrm{M}>}$ & $\left.<\mathrm{M}^{2}\right\rangle$ & $\hat{\sigma}(<\mathrm{M}>)$ & $\hat{\sigma}\left(\operatorname{std}_{<M>}\right)$ & $\hat{\sigma}\left(<M^{2}>\right)$ \\
\hline 0 & 5000 & 5.937 & 2.603 & 42.024 & 4.737 & 3.974 & 4.584 \\
\hline 1 & 5000 & 5.910 & 2.626 & 41.819 & 4.715 & 4.009 & 4.573 \\
\hline 2 & 5000 & 6.009 & 2.672 & 43.244 & 4.794 & 4.079 & 4.650 \\
\hline 3 & 5000 & 5.733 & 2.703 & 40.171 & 4.574 & 4.126 & 4.482 \\
\hline 4 & 5000 & 6.040 & 2.795 & 44.291 & 4.819 & 4.266 & 4.706 \\
\hline 5 & 5000 & 6.236 & 2.848 & 46.995 & 4.976 & 4.347 & 4.847 \\
\hline 6 & 5000 & 5.794 & 2.711 & 40.918 & 4.623 & 4.138 & 4.523 \\
\hline 7 & 5000 & 6.068 & 2.823 & 44.790 & 4.842 & 4.309 & 4.732 \\
\hline ave & 5000 & 5.966 & 2.723 & 43.031 & 4.760 & 4.156 & 4.637 \\
\hline std & 5000 & 0.160 & 0.091 & 2.254 & 0.127 & 0.138 & 0.121 \\
\hline
\end{tabular}

Table 4.8. Cont. 

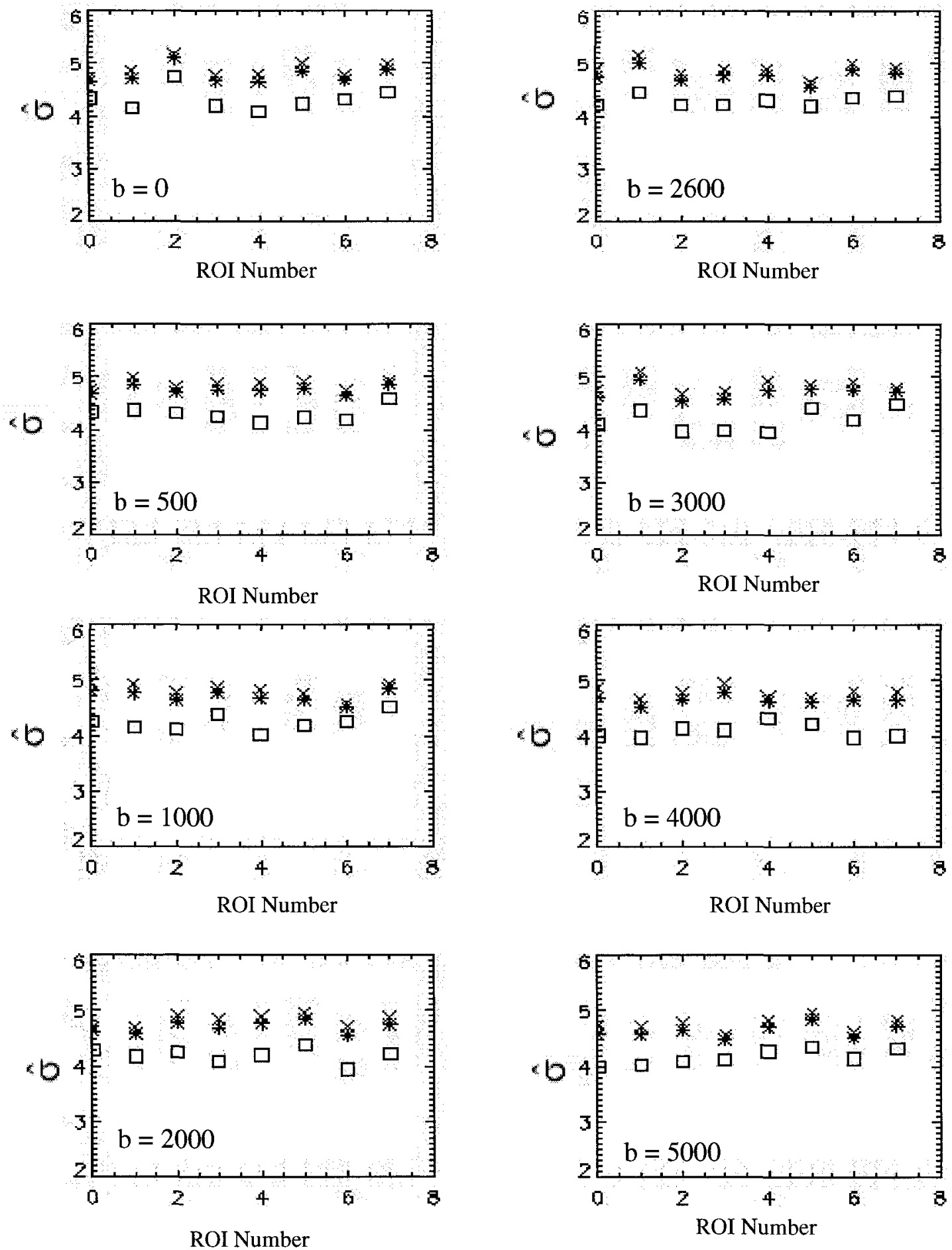

Figure 4.19. Values of the background noise estimates with the water phantom are shown vs.

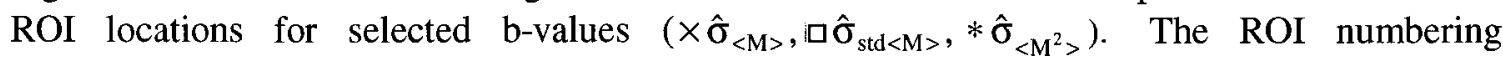
convention is shown in figure 4.18. The b-values are in units of $\mathrm{s} / \mathrm{mm}^{2}$. 


\begin{tabular}{|c|c|c|c|c|c|c|c|}
\hline $\begin{array}{l}\text { b-value } \\
\left(\mathrm{s} / \mathrm{mm}^{2}\right)\end{array}$ & $\hat{\sigma}(<\mathrm{M}>)$ & $\hat{\sigma}\left(\operatorname{std}_{<M}\right.$ & $\hat{\sigma}\left(<M^{2}>\right)$ & $\begin{array}{l}\mathrm{ROI} \\
\text { location }\end{array}$ & \multicolumn{2}{|c|}{$\hat{\sigma}(<M>) \quad \hat{\sigma}\left(s_{t d}<M\right.$} & $\hat{\sigma}\left(<\mathrm{M}^{2}>\right.$ \\
\hline \multicolumn{4}{|c|}{ averaged over $8 \mathrm{ROI}$} & \multicolumn{4}{|c|}{ averaged over 16 b-values } \\
\hline 0 & 4.899 & 4.314 & 4.779 & 0 & 4.797 & 4.190 & 4.673 \\
\hline 250 & 4.877 & 4.258 & 4.752 & 1 & 4.860 & 4.176 & 4.722 \\
\hline 500 & 4.873 & 4.298 & 4.756 & 2 & 4.830 & 4.246 & 4.711 \\
\hline 750 & 4.876 & 4.326 & 4.763 & 3 & 4.826 & 4.192 & 4.697 \\
\hline 1000 & 4.834 & 4.233 & 4.712 & 4 & 4.857 & 4.189 & 4.722 \\
\hline 1250 & 4.835 & 4.158 & 4.698 & 5 & 4.850 & 4.206 & 4.720 \\
\hline 1500 & 4.827 & 4.164 & 4.693 & 6 & 4.829 & 4.197 & 4.701 \\
\hline 2000 & 4.832 & 4.190 & 4.701 & 7 & 4.885 & 4.303 & 4.767 \\
\hline 2300 & 4.871 & 4.114 & 4.719 & ave & 4.842 & 4.212 & 4.714 \\
\hline 2600 & 4.909 & 4.293 & 4.783 & std & 0.027 & 0.042 & 0.027 \\
\hline 3000 & 4.845 & 4.182 & 4.711 & & & & \\
\hline 3250 & 4.820 & 4.265 & 4.707 & & & & \\
\hline 3500 & 4.742 & 4.115 & 4.615 & & & & \\
\hline 4000 & 4.788 & 4.089 & 4.647 & & & & \\
\hline 4500 & 4.882 & 4.244 & 4.752 & & & & \\
\hline 5000 & 4.760 & 4.156 & 4.637 & & & & \\
\hline ave & 4.842 & 4.212 & 4.714 & & & & \\
\hline std & 0.048 & 0.076 & 0.050 & & & & \\
\hline
\end{tabular}

Table 4.9. The noise $\sigma$ estimated in the image background of the water phantom; a.(left) averaged over 8 ROI locations for each b-value, b.(right) averaged over $16 \mathrm{~b}$-values for each ROI location

unknown, however, since the difference in the $\hat{\sigma}$ values at $b=0$ and $5000 \mathrm{~s} / \mathrm{mm}^{2}$ is only about $3 \%$ it is not a large effect and should have, at most, a very minor effect on our results.

From table 4.9 and figures $4.19-4.21$ it can be seen that consistently $\hat{\sigma}\left(\operatorname{std}_{<M}\right)<\hat{\sigma}\left(<M^{2}>\right)<\hat{\sigma}(<M>)$ as was noted for the experiments performed without the phantom. We also found that $\hat{\sigma}(<M>)$ and $\hat{\sigma}\left(<M^{2}>\right)$ were within one standard deviation of each other, consistent with the no phantom experiments. However, when the phantom is used $\hat{\sigma}\left(\operatorname{std}_{<M>}\right)$ is found to be much more than one standard deviation lower than $\hat{\sigma}(<M>)$. This was unexpected and is inconsistent with the theory. It suggests that the PDF in this case is not a 


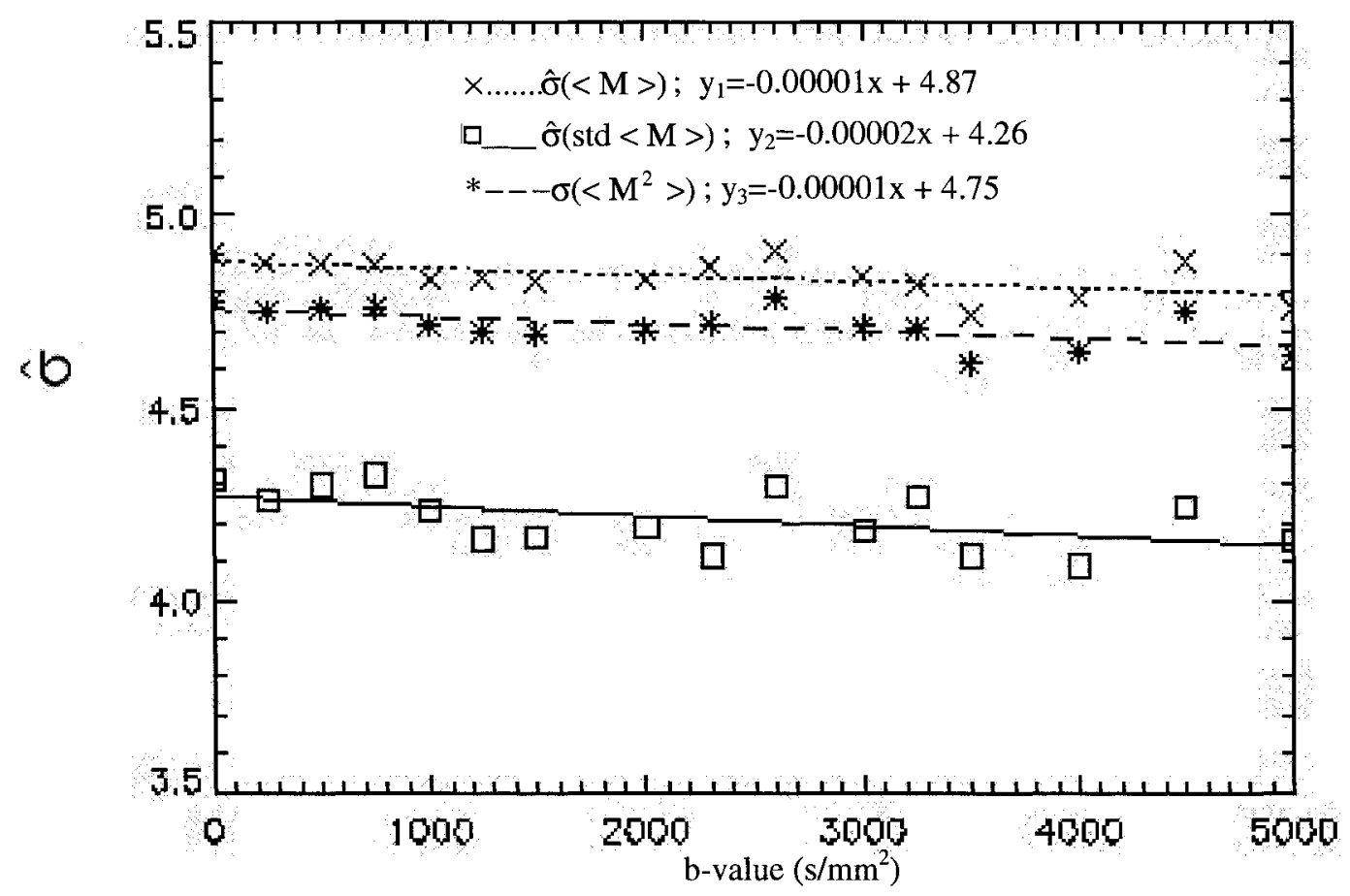

Figure 4.20. The noise $\sigma$ estimates vs. $b$-value in the image background of the water phantom over the 8 larger ROIs. A small dependence on $b$ is apparent.

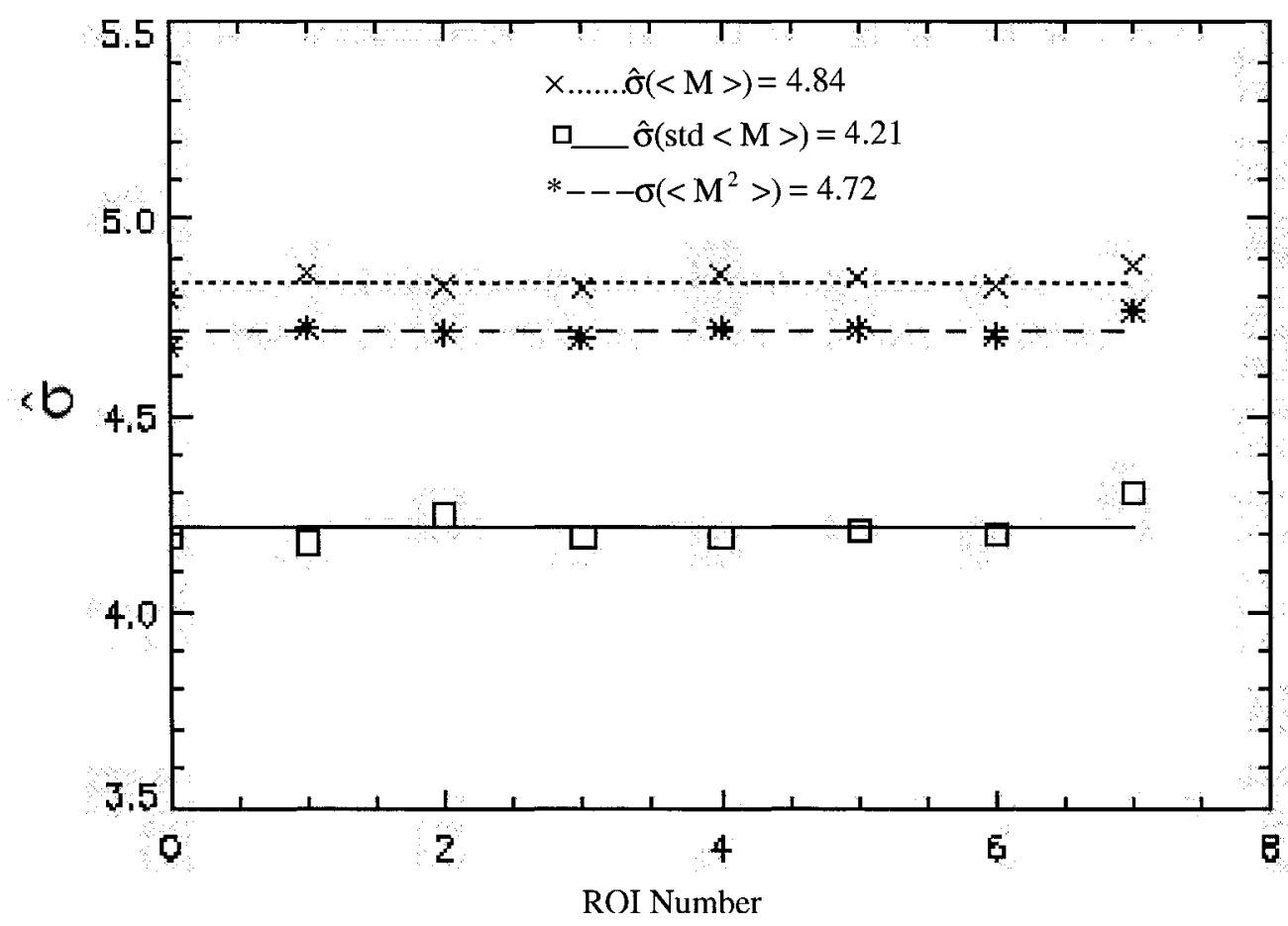

Figure 4.21. The noise $\sigma$ estimates averaged over b-values vs. ROI location in the image background of the water phantom. 


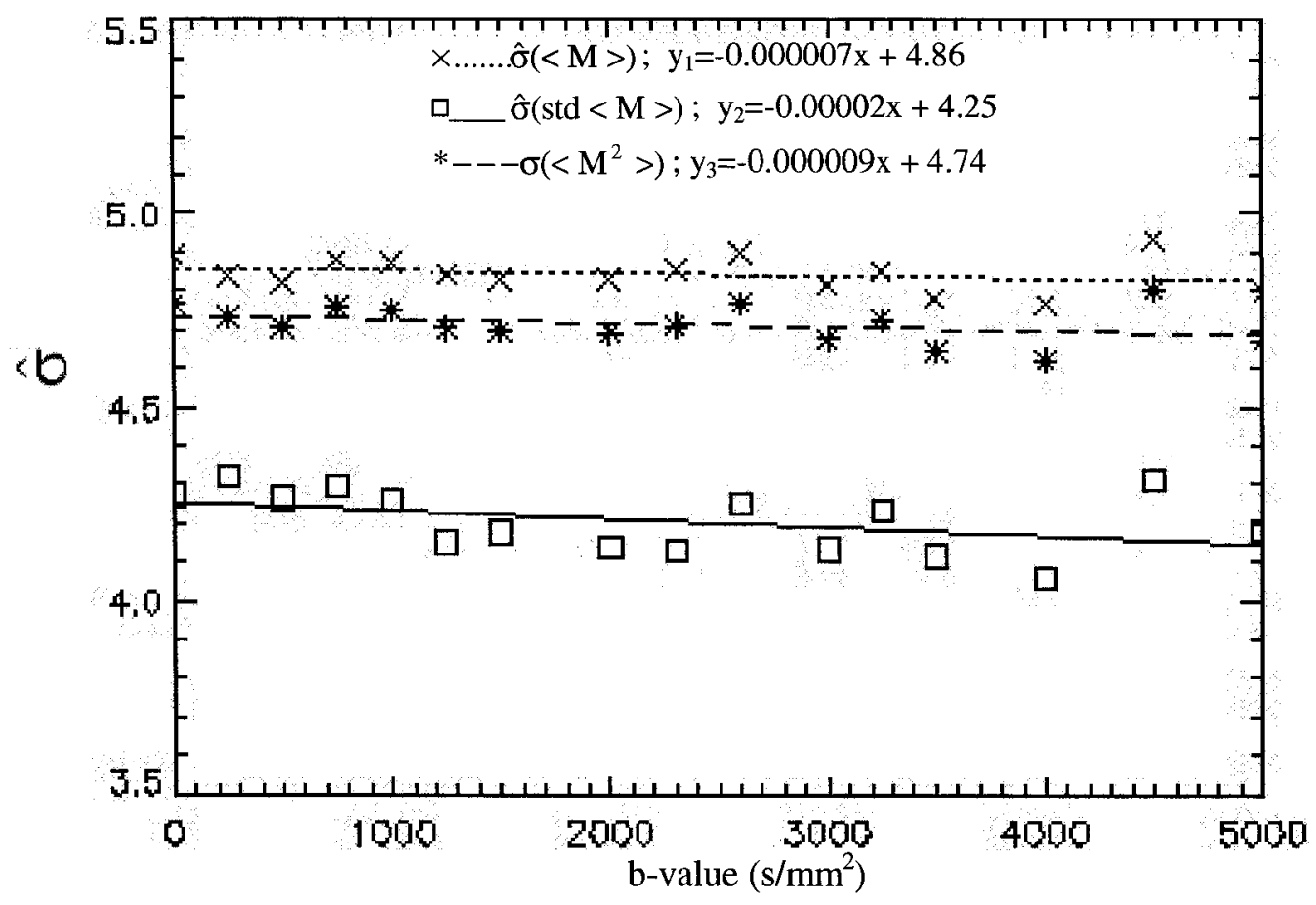

Figure 4.22. The noise $\sigma$ estimates vs. $b$-value in the image background of the water phantom over the 8 smaller ROI locations as discussed in the text.

Rayleigh PDF since, if it were, the theory says that $\hat{\sigma}(<M>)$ would be equal to both $\hat{\sigma}\left(\operatorname{std}_{<M>}\right)$ and $\hat{\sigma}\left(<M^{2}>\right)$.

In the process of reconstructing the images Siemens applies a Hanning filter to the data. The exact details of how Siemens performs this filtering are not available to us however it can be expected to have a significant effect on the standard deviation of the distribution of pixel intensities while having a minimal effect on the mean. A typical Hanning filter in k-space would be defined by [158]

$$
\mathrm{H}(\mathrm{k})=\frac{1}{2}\left[1+\cos \left(\frac{2 \pi \mathrm{k}}{2 \mathrm{k}_{\max }}\right)\right]=\cos ^{2}\left(\frac{\pi \mathrm{k}}{2 \mathrm{k}_{\max }}\right)
$$

By Fourier transforming this expression we see that it corresponds to a weighted nearest neighbor average of pixels in the image [158]: 


$$
\mathrm{FT}\{\mathrm{H}(\mathrm{k})\}=\frac{1}{2} \delta(\mathrm{x})+\frac{1}{4} \delta\left(\mathrm{x}-\frac{1}{2 \mathrm{k}_{\max }}\right)+\frac{1}{4} \delta\left(\mathrm{x}+\frac{1}{2 \mathrm{k}_{\max }}\right)
$$

To see the effect of this Hanning filter on the Rayleigh PDF a simulation similar to those discussed earlier was performed and equation (4.41) was used to filter the simulated pixel intensities. For the purposes of this simulation $\hat{\sigma}(<M>)=4.8$ (see table 4.9 ) was considered to be correct.

From the results shown in figure 4.23 it is clear that the Hanning filter causes the distribution of pixel intensities to narrow but has little effect on the position of the peak. In fact, the means before and after filtering are both equal to 6.02 while the standard deviation of the unfiltered Rayleigh PDF is 3.14, which agrees with equation (4.21), and the standard deviation for the filtered distribution is 1.93 . If these two standard deviation values are used to calculate values for $\hat{\sigma}$ we get values of 4.80 and 2.95 , respectively. Thus, the correct value for $\hat{\sigma}$ is obtained when we use the standard deviation from the Rayleigh PDF and a considerably smaller value when the filtered distribution is used. This agrees qualitatively with what we observe experimentally but the value from these simulations is considerably smaller than the experimental value. The results are consistent with a Hanning filtered data set, however, it appears that the filtering in our simulation is too strong.

Suppose that the bandwidth of the acquisition is not wide enough to allow the highest frequencies in the image space. This means that adjacent pixels are not independent. For example, a pixel that has the highest possible positive value will have adjacent pixels which are more likely to be positive than negative. If we model this by setting each nearest neighbor pixel value to be the average of its two nearest neighbors, equation (4.41) can be rewritten as 


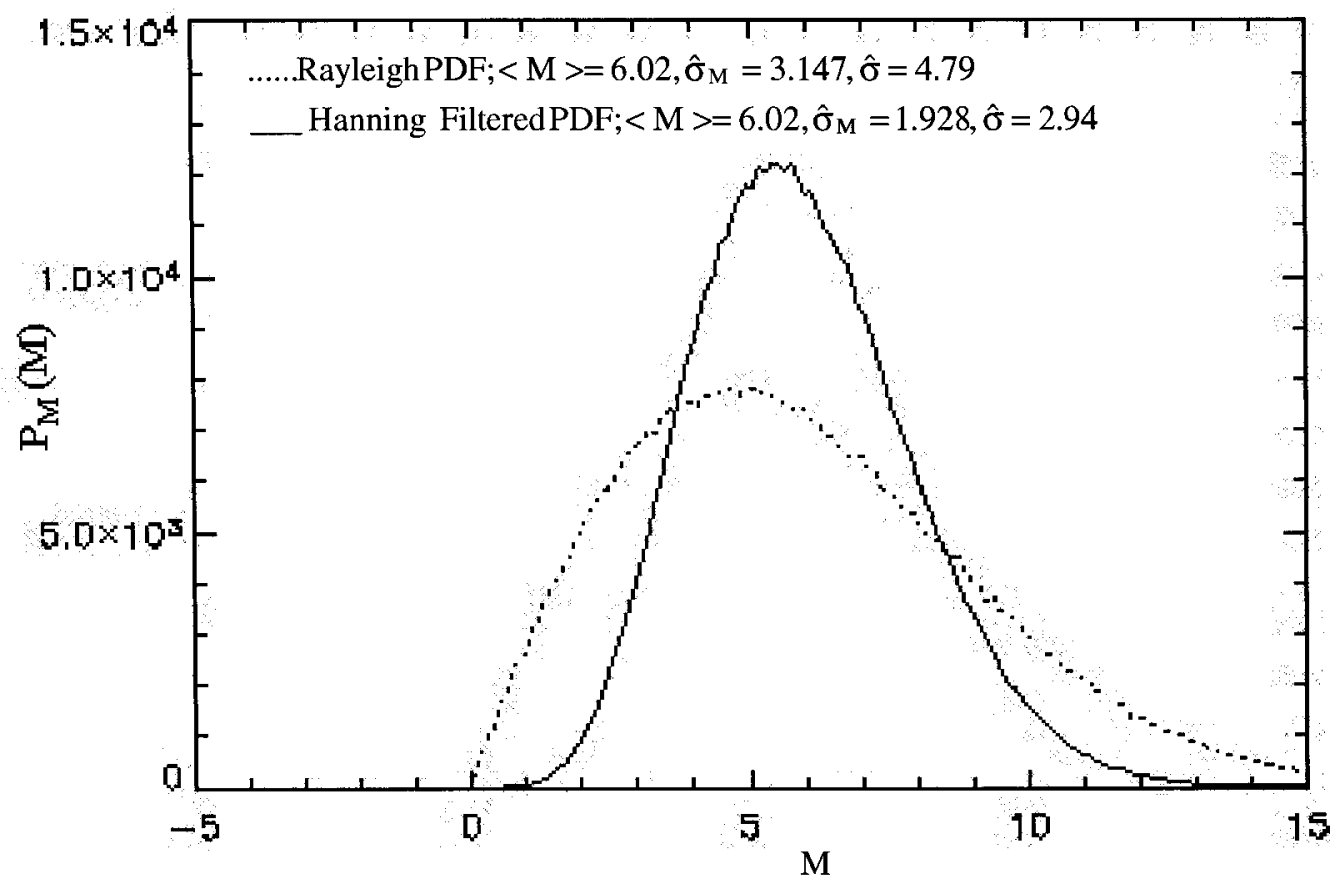

Figure 4.23. Simulated Rayleigh PDF (dot line) filtered by Hanning filtration (solid line) using equation (4.41).

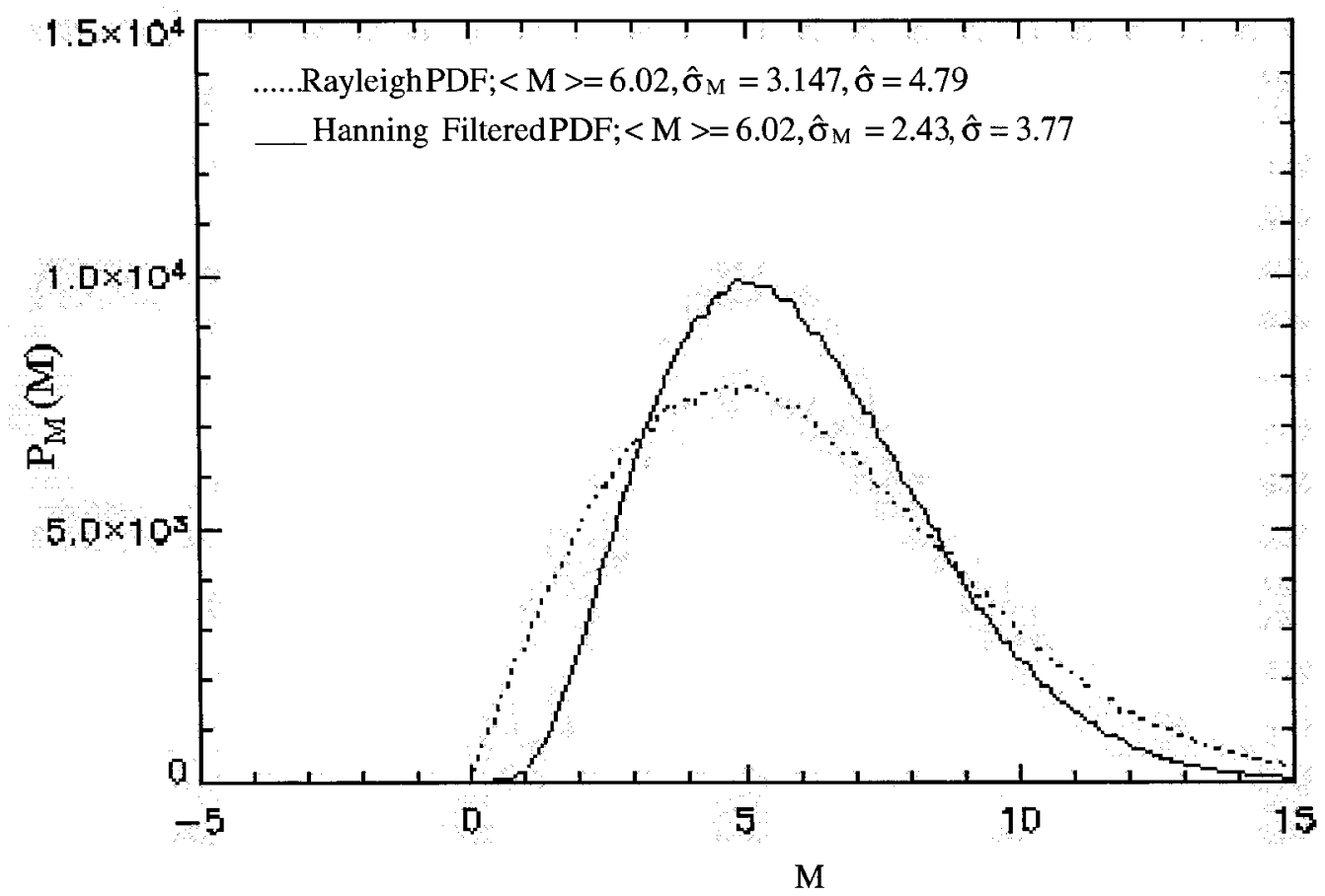

Figure 4.24. Simulated Rayleigh PDF (dot line) filtered by Hanning filtration (solid line) using equation (4.42). 


$$
\begin{aligned}
\mathrm{FT}\{\mathrm{H}(\mathrm{k})\} & =\frac{1}{2} \delta(\mathrm{x})+\frac{1}{4} \delta(\mathrm{x}-\Delta \mathrm{x})+\frac{1}{4} \delta(\mathrm{x}+\Delta \mathrm{x}) \\
= & \frac{1}{2} \delta(\mathrm{x})+\frac{1}{4}\left[\frac{\delta(\mathrm{x})+\delta(\mathrm{x}-2 \Delta)}{2}\right]+\frac{1}{4}\left[\frac{\delta(\mathrm{x})+\delta(\mathrm{x}+2 \Delta)}{2}\right] \\
= & \frac{3}{4} \delta(\mathrm{x})+\frac{1}{8} \delta(\mathrm{x}-2 \Delta)+\frac{1}{8} \delta(\mathrm{x}+2 \Delta)
\end{aligned}
$$

The resulting distribution, filtered using equation (4.42), is shown in figure 4.24 . If this filter is used in the simulations instead of the one defined by equation (4.41) the standard deviation of the filtered distribution becomes 2.43 and the value of $\hat{\sigma}\left(\operatorname{std}_{<M>}\right)$ calculated from this is 3.77 which is closer to the experimental value (see table 4.9 where the average value for $\hat{\sigma}\left(\operatorname{std}_{<M>}\right)$ is 4.212). The mean of the PDF with this filtering still remained the same $(<M>=6.02)$. This proves that the standard deviation of the Rician PDF is more sensitive than its mean value to processes such as Hanning filtration or others which can affect $\hat{\sigma}$ estimation.

From this analysis we can conclude that the $\sigma$ estimators calculated using $<M>$ and $\left\langle\mathrm{M}^{2}\right\rangle$ are more reliable than the estimator calculated from $s t d_{<\mathrm{M}}>$ since the width of the distribution of pixel intensity values is more likely than $<M>$ and $\left\langle\mathrm{M}^{2}>\right.$ to be affected by such things as filtering and bandwidth limiting. It should also be noted that when this analysis using a Hanning filter is applied to the results without a phantom it gives inconsistent results suggesting that the filtering was different for these two sets of experiments. This small discrepancy remains unexplained.

\subsection{Validation of the Noise Bias Correction Procedure}

The diffusion decay is a very nice system for testing noise correction since the SNR can easily be adjusted by incrementing the b-value. Furthermore, for a water phantom the diffusion decay is an exponential decay with a well known slope. If the noise correction scheme works properly, the 
corrected data points will follow a straight line on a plot of $\ln [S(b)]$ vs. $b$, or at least have a Gaussian or nearly Gaussian scatter about a straight line. In this section we will discuss diffusion decay measurements as tests of how well the noise correction procedures are performing.

\subsubsection{Signal strength estimation from the phantom image}

In order to properly use the noise estimator to correct the diffusion signal decay for Rician noise bias, the selection of the ROI for the object in the image to measure the signal strength, $\mathrm{S}(\mathrm{b})$, is as important as the selection of the ROI in the background of the image for noise estimation. The signals in the ROIs can be influenced by unwanted phase encoding due to artifacts caused by, for example, motion, N/2 ghosting, or by Gibbs ringing. Thus, the resulting nonuniformity of these regions could affect the evaluated mean signal and its variance giving over or under estimated values for $\mathbf{S}(b)$.

The ROI size and its position were optimized using an image of the water phantom in our study by measuring the mean pixel intensity for the diffusion decay measurements with $b=0$ and the standard deviation of the mean inside the phantom image. 16 circular ROIs centered with the phantom were selected to cover a full range of diameters between $5 \mathrm{~mm}$ and $131 \mathrm{~mm}$ including 15 to 13751 pixels, respectively (see figure 4.25 and table 4.10 ). The mean values and their standard deviations are plotted against the ROI index in figures 4.26 and 4.27 to precisely detect the variations of these two values to find the optimal ROI size. The mean value of the pixel intensities and its standard deviation in these figures are normalized to their respective maximum values.

The most consistency for the mean is observed between $\mathrm{ROI}(0)$ and $\mathrm{ROI}(8)$ in the first measurement with less than $1 \%$ variation (figure 4.26). The data from the second measurement, 


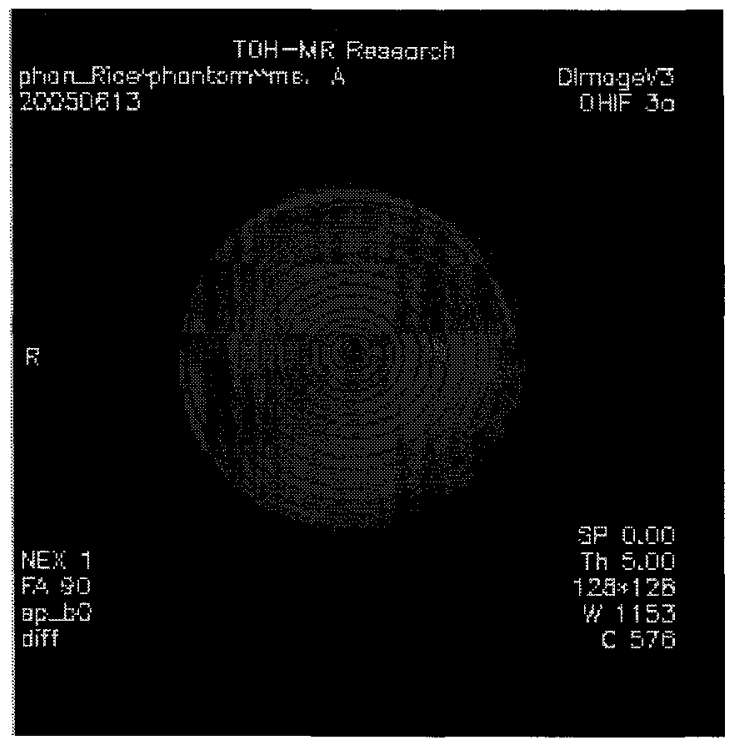

Figure 4.25. The 16 circular ROIs selected in the phantom image of the MR diffusion measurements to study the signal and noise uniformity vs. ROI size.

\begin{tabular}{|c|c|c|cc|cc|}
\hline ROI & diameter & \# of pixels & \multicolumn{2}{|c|}{$<\mathrm{M}>$} & \multicolumn{2}{|c|}{ std } \\
\cline { 4 - 7 } & $(\mathrm{mm})$ & & expt \#1 & expt \#2 & expt \#1 & expt \#2 \\
\hline 0 & 4.8 & 15 & 457.33 & 453.00 & 2.3 & 4.0 \\
1 & 5.9 & 41 & 452.46 & 452.92 & 3.8 & 4.4 \\
2 & 8.9 & 61 & 454.42 & 452.97 & 4.6 & 3.9 \\
3 & 15.0 & 207 & 456.40 & 452.56 & 4.3 & 5.4 \\
4 & 22.9 & 465 & 456.04 & 453.58 & 4.9 & 6.4 \\
5 & 29.9 & 765 & 455.31 & 453.27 & 5.5 & 6.4 \\
6 & 37.9 & 1209 & 454.58 & 453.30 & 5.9 & 6.7 \\
7 & 49.0 & 1979 & 454.23 & 453.00 & 6.9 & 7.4 \\
8 & 57.0 & 2675 & 453.99 & 452.68 & 7.7 & 8.2 \\
9 & 67.8 & 3757 & 453.14 & 451.93 & 8.8 & 9.1 \\
10 & 81.8 & 5433 & 451.44 & 450.35 & 10.3 & 10.4 \\
11 & 92.0 & 6821 & 449.66 & 448.72 & 11.4 & 11.7 \\
12 & 102.8 & 8531 & 447.17 & 446.31 & 13.1 & 13.4 \\
13 & 114.0 & 10441 & 443.36 & 442.55 & 15.7 & 15.8 \\
14 & 123.8 & 12313 & 440.36 & 439.74 & 18.9 & 18.9 \\
15 & 130.8 & 13751 & 429.34 & 428.94 & 60.6 & 60.5 \\
\hline
\end{tabular}

Table 4.10. The mean values and their standard deviations vs. the ROI index. The diameter of the ROI and the number of pixels in the ROI are also given in the table. 

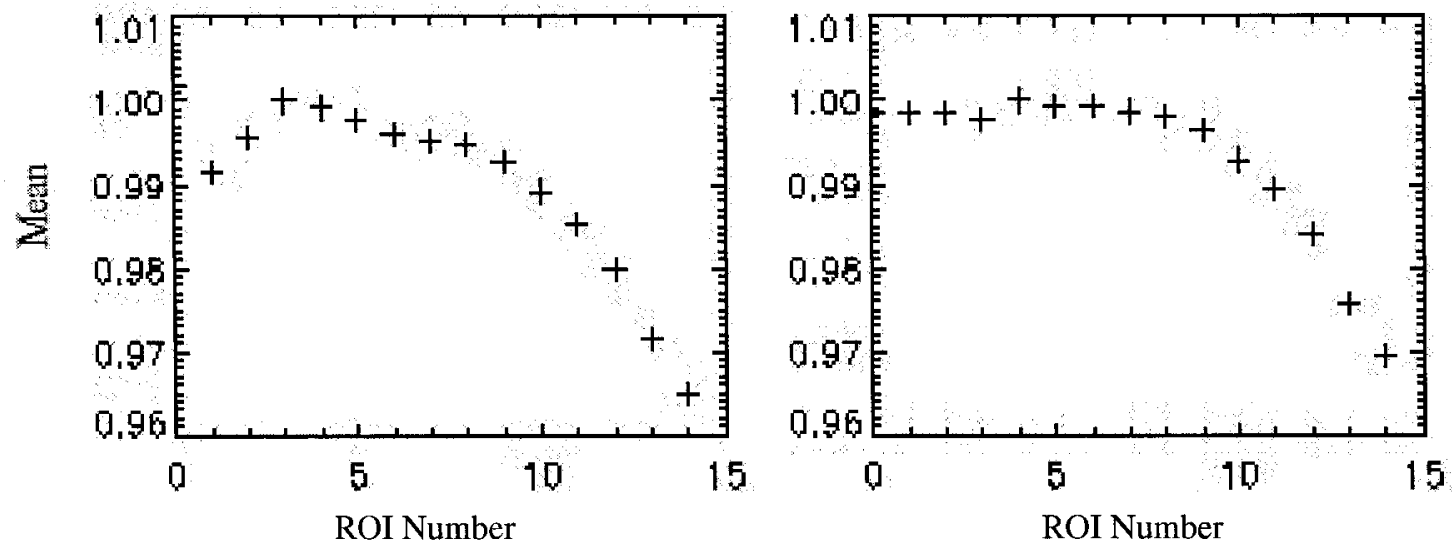

Figure 4.26. The mean values vs. the ROI index for the $1^{\text {st }}$ (left) and the $2^{\text {nd }}$ (right) set of measurements on the water phantom. The mean values are normalized to the maximum mean signal for each experiment.
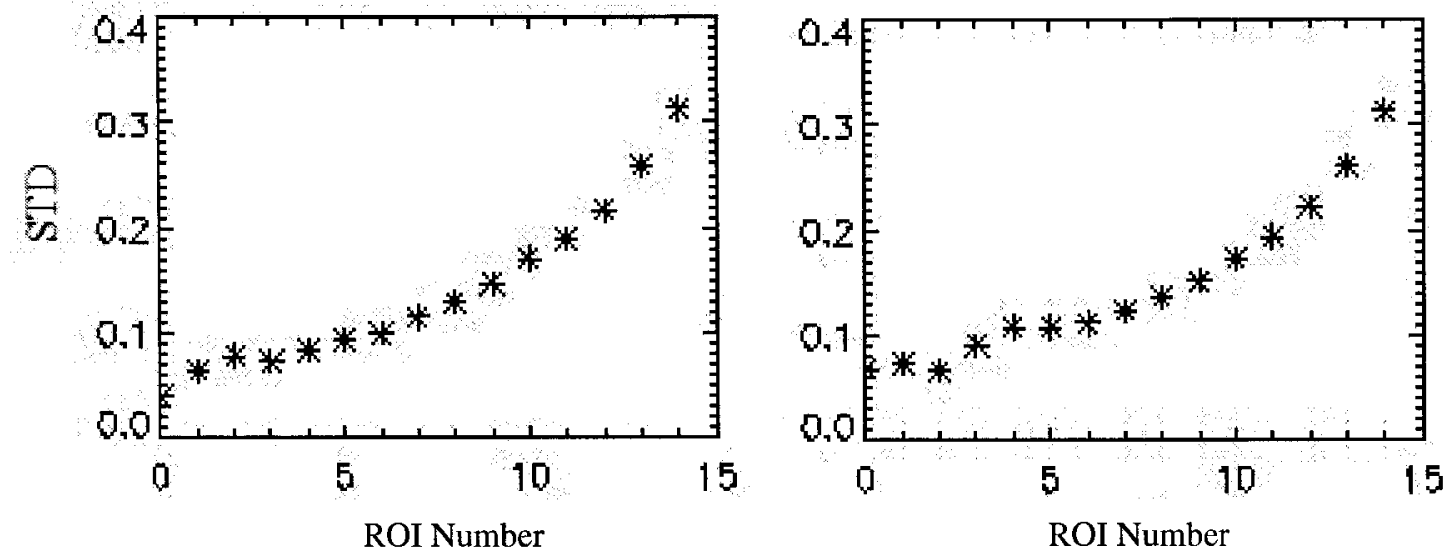

Figure 4.27. The std of the mean vs. the ROI index for the $1^{\text {st }}$ (left) and the $2^{\text {nd }}$ (right) set of measurements on the water phantom. The std values are normalized to the maximum std for each experiment.

which was performed right after the first one, showed more consistency for the mean values even up to ROI(10). The selection of the optimal ROI size should also consider the variation of the standard deviation of the mean versus ROI size. The plots of std vs. ROI size (figure 4.27) show that the standard deviation drastically rises for large ROI and small ROIs suffer from poor statistics. This is clearly observed for the ROIs smaller than ROI(3) in the first set of 


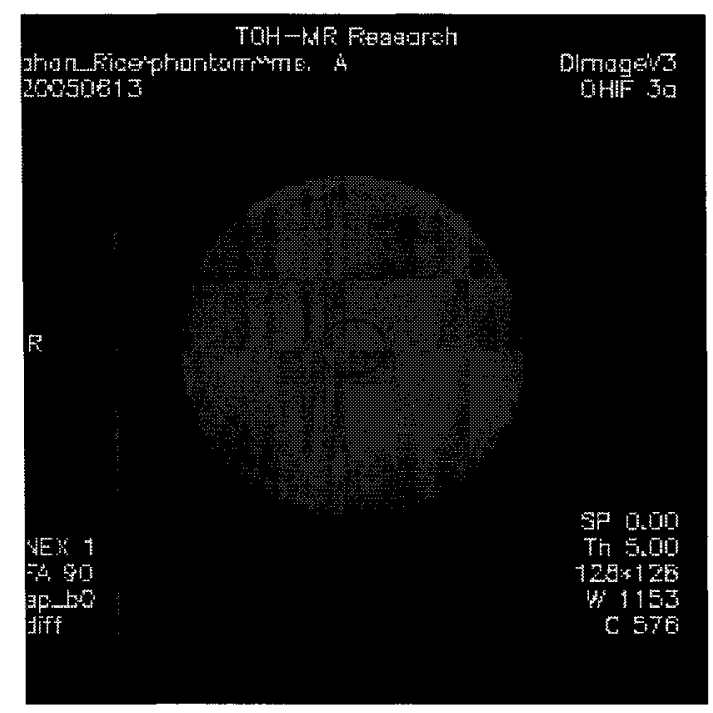

Figure 4.28. The optimum circular ROI size with 460 pixels in the phantom image of the MR diffusion measurement with the ROI selected in the background.

measurements and ROI(4) in the second set of measurements. From these measurements it can be seen that ROIs from ROI(4) to ROI(7) give the best results. ROI(4) with 460 pixels, was chosen for the signal measurements in the Rician noise tests discussed in the next section.

\subsubsection{Noise bias correction testing using the head coil phantom}

Using the optimized ROI size from section 4.5.1 and the ROI in the background of the image shown in figure 4.28 , we applied $\mathrm{NC} 2$ noise correction to diffusion data from the head coil phantom. The results are shown in figures $4.29-4.31$ on two different scales. The experimental details of these measurements are given in chapter 3 .

In order to compare the effect of the $\sigma$ estimators on the Rician noise bias correction, the diffusion decay for the optimal ROI size was corrected using all three estimators. A single diffusion coefficient of $\mathrm{D}=2.0 \times 10^{-3} \mathrm{~mm}^{2} / \mathrm{s}$ is always evaluated by the analysis of the signal decay. This is not surprising since the fit is heavily weighted by the larger data values at small b- 
a.
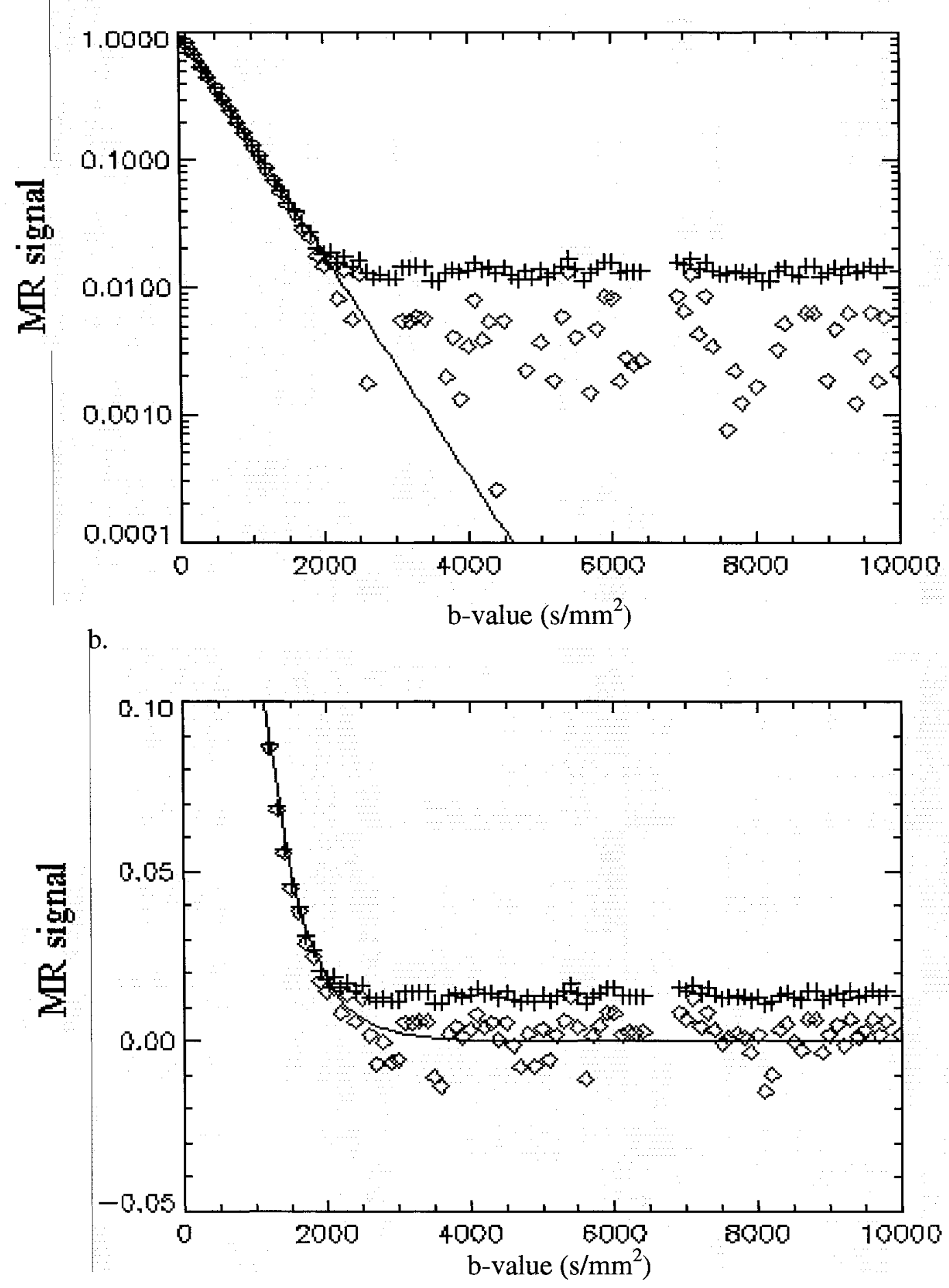

Figure 4.29 The diffusion MR signal for a water phantom biased with Rician noise $(+)$ and corrected $(\diamond)$ with $\mathrm{NC} 2$ noise correction using $\hat{\sigma}(<\mathrm{M}>)$ a. on a logarithmic scale fit with least squares (solid line), b. on a linear scale. 


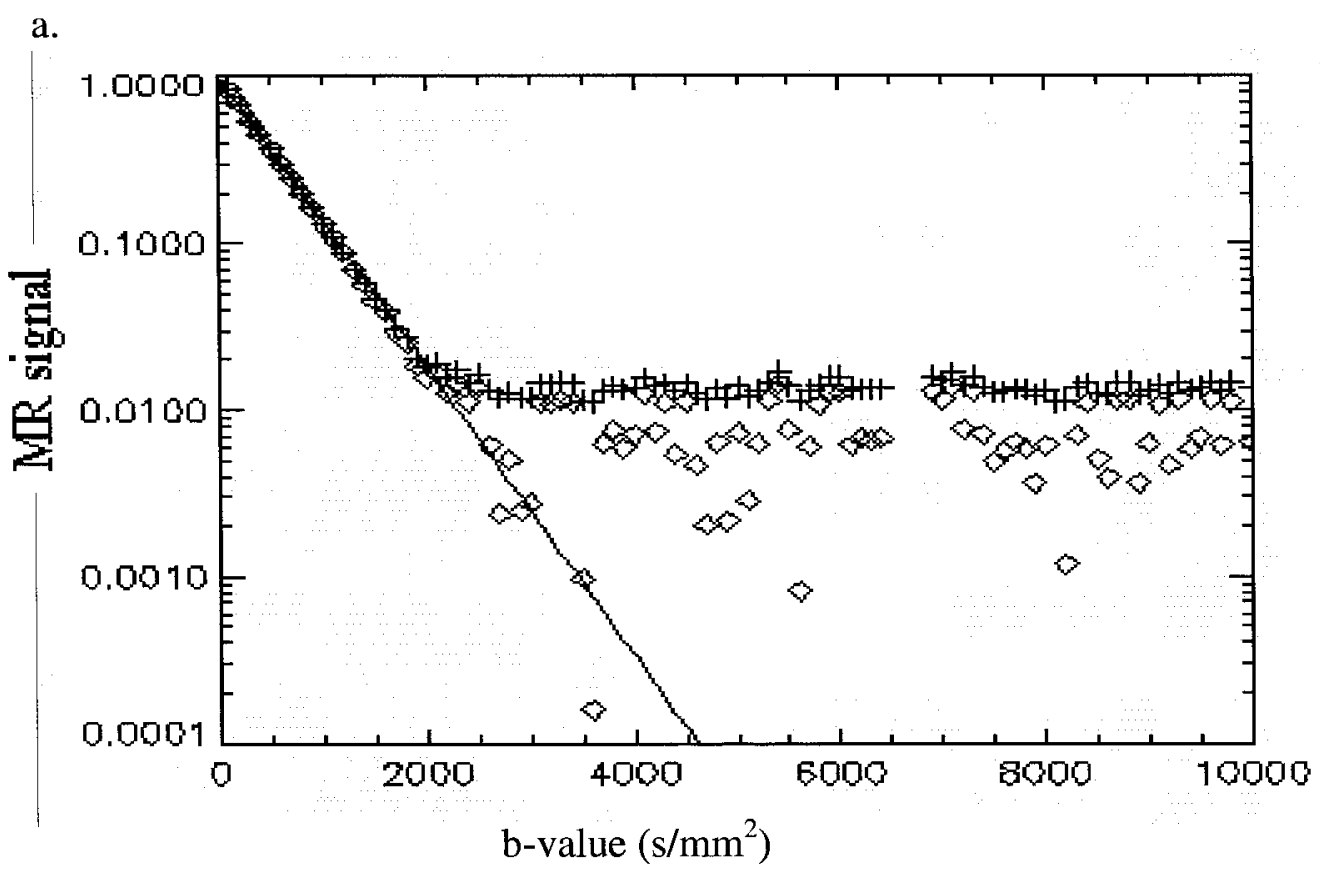

b.

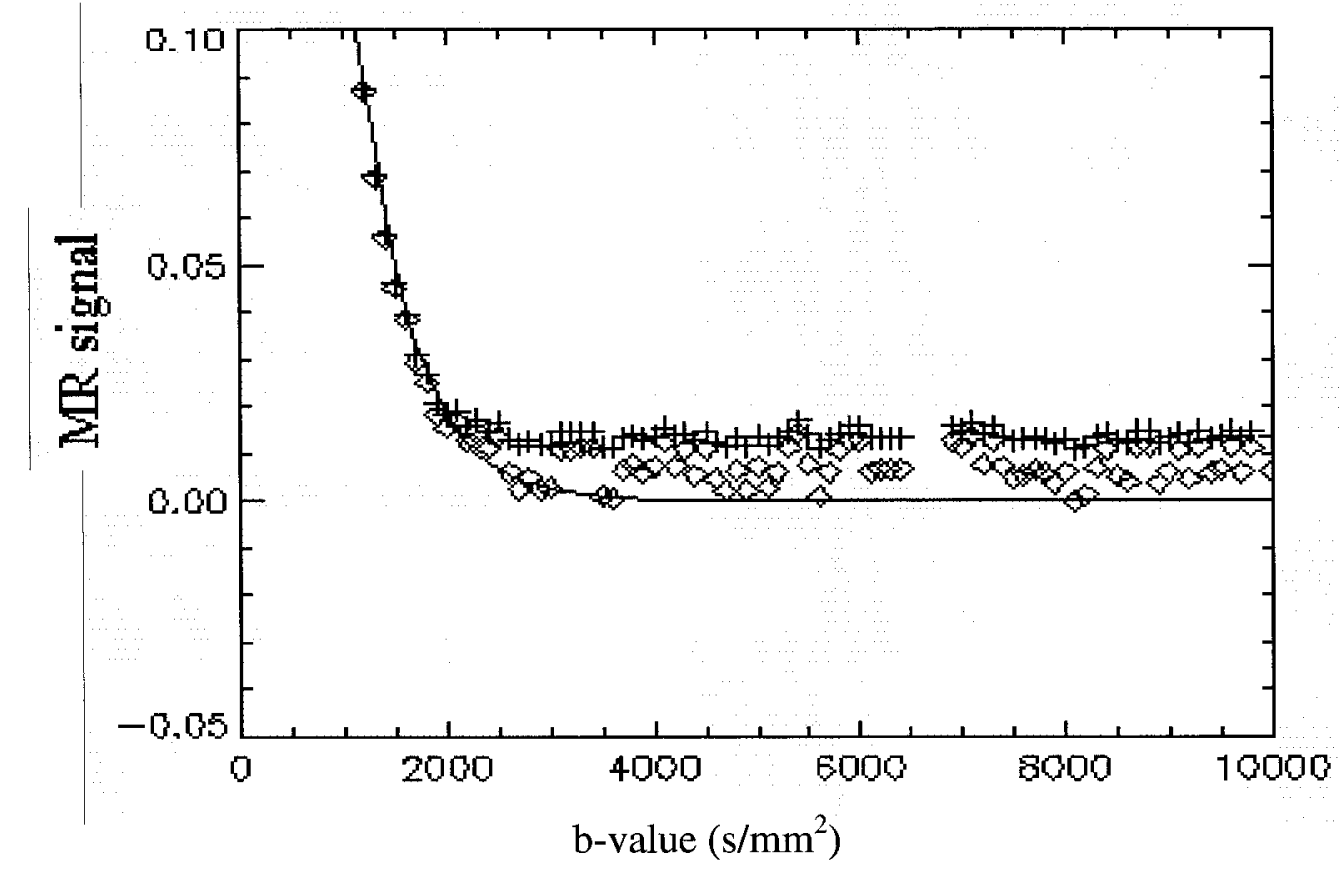

Figure 4.30 The diffusion MR signal for a water phantom biased with Rician noise $(+)$ and corrected $(\Leftrightarrow)$ with $\mathrm{NC} 2$ noise correction using $\hat{\sigma}(\operatorname{std}<\mathrm{M}>$ ) a. on a logarithmic scale fit with least squares (solid line), b. on a linear scale. 


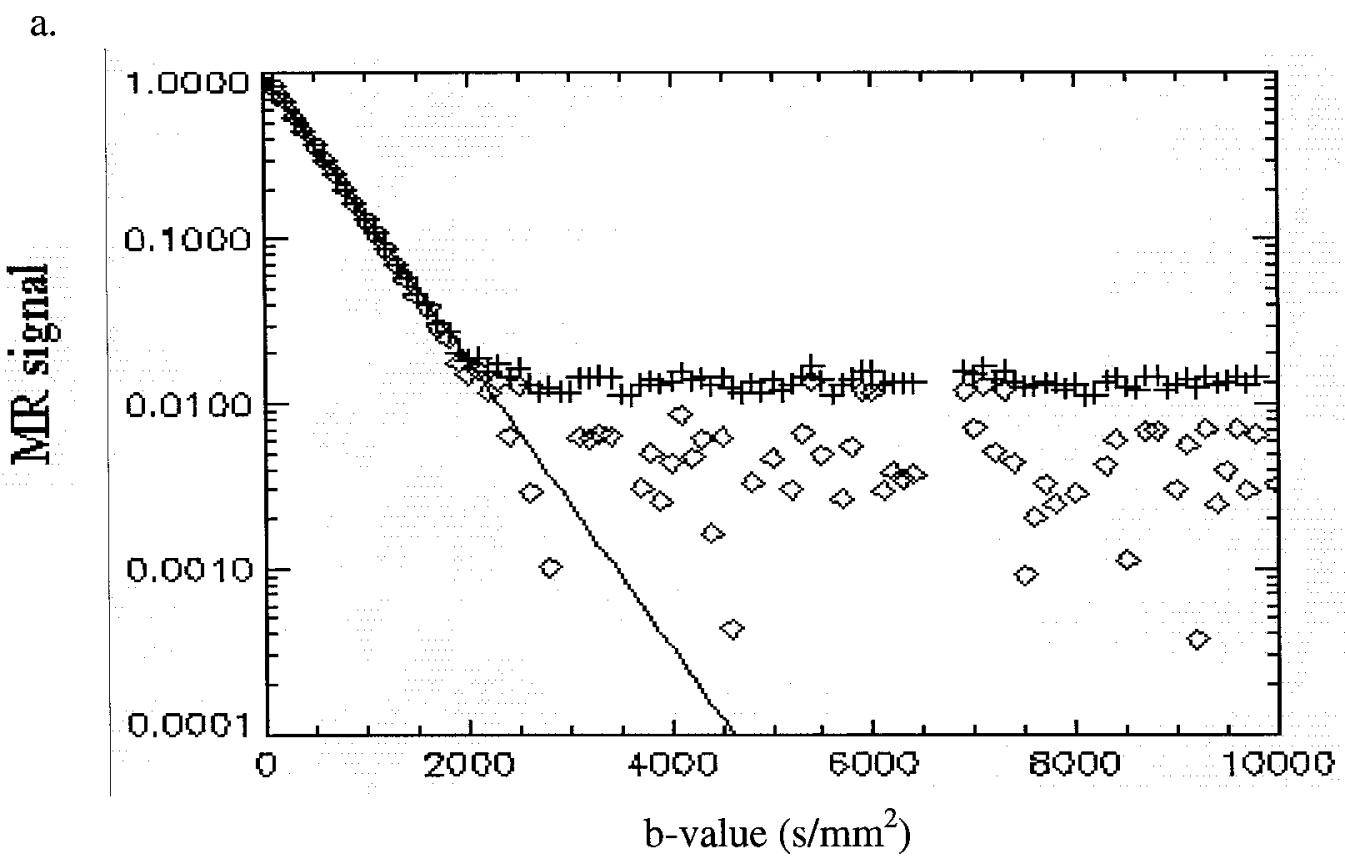

b.

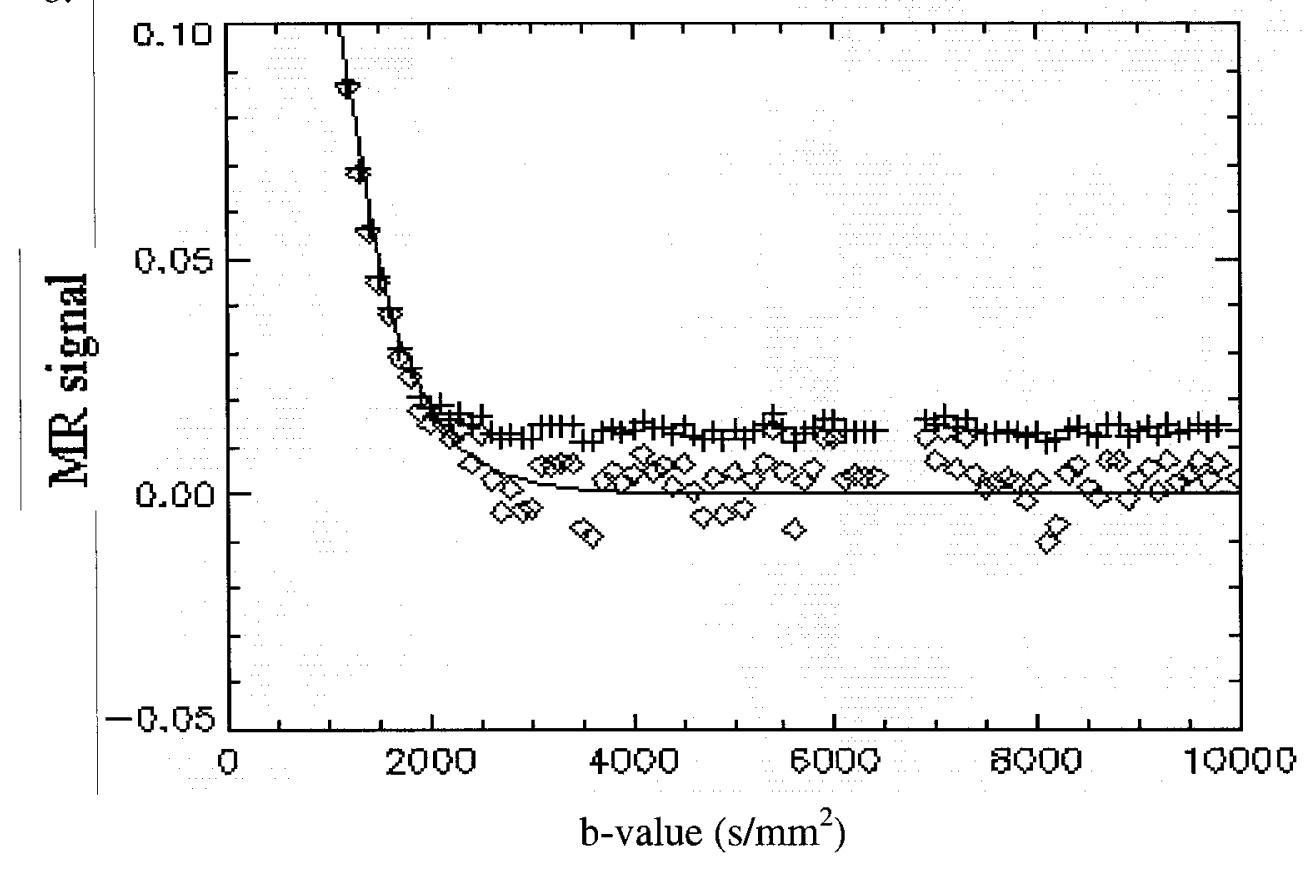

Figure 4.31 The diffusion MR signal for a water phantom biased with Rician noise (+) and corrected $(\mid \vartheta)$ with NC2 noise correction using $\left.\hat{\sigma}\left(<\mathrm{M}^{2}\right\rangle\right)$ a. on a logarithmic scale fit with least squares (solid line), b. on a linear scale. 
values. There is, however, a significant difference in the removal of the noise bias from the decay when $\hat{\sigma}($ std $<M>)$ is used as opposed to the other two estimators, $\hat{\sigma}(<M>)$ and $\hat{\sigma}\left(<\mathrm{M}^{2}>\right)$, (see figures 4.29-4.31). If the Rician noise bias is calculated as the average over the data points from $b=5000$ to $10000 \mathrm{~s} / \mathrm{mm}^{2}$ the uncorrected Rician noise bias is $0.014 \mathrm{~S}(0)$. Using NC2 noise correction with 8 terms in the expansion and $\sigma$ set equal to $\hat{\sigma}(<M>), \hat{\sigma}(\operatorname{std}<M>)$ and $\hat{\sigma}\left(<\mathrm{M}^{2}>\right)$ the Rician noise bias reduced to $0.0022 \mathrm{~S}(0), 0.0075 \mathrm{~S}(0)$ and $0.0037(\mathrm{~S} 0)$, respectively. Since the ideal result for the bias after noise correction is 0.0 , it can be seen that $\hat{\sigma}(<M>)$ gives the best result since it is closer to zero.

\subsection{Discussion of the Noise Correction}

Detection of the "true" information from the MR diffusion signal decay in the magnitude form requires the removal of the Rician noise bias from the decay. The Rician noise shows its most dominance on the decay when the SNR is low causing incorrect decay parameters such as the diffusion coefficient and/or its contribution to the decay to be obtained from the analysis. Several noise correction techniques were studied with simulated image data and measured MR diffusion data to determine the best method for correction of the Rician noise bias of the diffusion signal decays for heterogeneous systems like tissue.

Complex averaging was shown, using simulations, to be a very useful technique for reducing the Rician noise bias as long as the required number of averages is small enough that the total length of the exam does not become unreasonably long. These simulations demonstrated that the standard deviation of the averaged noise decreases and this reduction is proportional to $\sqrt{\mathrm{N}}$ for both magnitude and complex averaging. But more importantly, the Rician noise bias was shown to decrease by a factor of $\sqrt{\mathrm{N}}$ for complex averaging while it did not change with the 
number of averages for magnitude averaging. Attempts to demonstrate these effects experimentally were only partially successful.

When an ROI analysis is performed on complex averaged images, the average over the pixels in the ROI is a magnitude average, since the pixel values are obtained from the magnitude image. If this averaging over the ROI was done to the real and imaginary data following the 2D Fourier transform but before the magnitude and phase of the signal are computed this should have a similar effect on the Rician noise bias as the complex averaging simulations presented here. Unfortunately, we did not have access to the necessary parts of ICE to test this suggestion.

Various post-processing noise correction procedures from the literature were considered. All of them performed well for $\mathrm{SNR} \geq 3$ but none of them were acceptable for $\mathrm{SNR}<3$. An ideal noise correction procedure will be conceptually simple, easy to implement and can be applied to each individual data point separately. When applied to a large number of equivalent measurements it will yield a distribution of corrected data points with a mean of $\mathrm{A}$, the true MR pixel intensity that would be measured in the absence of noise, and will be Gaussian in shape to fulfill the prerequisites for least-squares based post-processing procedures. None of the corrections proposed in the literature fulfill all of these criteria but a new correction technique proposed in this chapter and called NC2 comes close to fulfilling these criteria for an ideal Rician noise bias correction technique. It was also demonstrated that least squares fitting of a Rician noise biased bi-exponential signal decay gives the wrong values for the decay parameters. The least squares fit to the same data after NC2 noise correction gave the correct values for the decay parameters.

All of the post-processing noise correction procedures considered here, including the NC2 technique, rely on having an accurate value of $\sigma$, the standard deviation of the Gaussian noise on the real and imaginary signals. Ideally, $\sigma$ should be determined from the measured standard 
deviation of the actual pixel under consideration. However, this would involve making many measurements of the signal intensity for the pixel of interest and calculating the standard deviation for this data set. This is impractical. Alternatively, $\sigma$ can be evaluated from an ROI at some other location within the FOV, as long as $\sigma$ is the same for all locations within the FOV. This was checked and found to be true for our imager. This allows us to determine $\sigma$ from an ROI in the background of the image and to apply it to the signal for pixels at the centre of the image.

Estimation of $\sigma$ was obtained from the measured mean, standard deviation and second moment for an ROI in the image background where A can be expected to be zero and these estimates were compared. In principle, they should all give the correct value of $\sigma$ but, since they may be influenced differently by systematic errors, the three were compared to see which gives the most accurate and consistent results. MR diffusion measurements of the image background were made in the presence and in the absence of the water phantom to make these comparisons.

As expected, the measured $\hat{\sigma}$ values from the image background with and without the phantom were different due to the various sources of noise such as the electronic noise, and the noise from the conducting subjects (e.g. phantom or human body) causing both dielectric and inductive loss. For a typical MR measurement at $1.5 \mathrm{~T}$ about half of the noise is generated by the receiving coil depending on its resistance and its temperature. Conducting samples such as water phantoms and human subjects couple with the $B_{1}$ magnetic field (RF), causing an increase in the effective series resistance due to dielectric and inductive effects and this causes the noise detected by the electronics to increase. Therefore, the noise estimated from images acquired with no phantom are expected to be much less than the noise from the background of images of conducting samples which induce extra noise. This is consistent with our observations.

In addition to electronic noise and induced noise due to sample losses, systematic errors such as Gibbs ringing, N/2 ghosting and motion artifacts can also influence the $\sigma$ estimates. Care 
must be taken to select uniform ROI's in the image background with no signal and no artifacts such as motion artifacts, N/2 ghosting or Gibbs ringing.

The measurements of $\hat{\sigma}$ without the phantom showed no dependence on ROI position or bvalues and the three estimators were in agreement with each other to within 1.6\% (see table 4.11). The relative values of $\left\langle\mathrm{M}>, \sigma_{\mathrm{M}}\right.$ and $\left\langle\mathrm{M}^{2}\right\rangle$ are also consistent with each other and with theory (see table 4.12).

The measurements of $\hat{\sigma}$ with the water phantom showed no dependence on ROI position and only a very small dependence on b-value; the $\hat{\sigma}$ values varied by about $3 \%$ over the range of b-values studied. The values of $\hat{\sigma}(<M>)$ and $\hat{\sigma}\left(<M^{2}>\right)$ were in agreement to within $3 \%$ (see table 4.11). The relative values of $\langle\mathrm{M}\rangle$ and $\left\langle\mathrm{M}^{2}\right\rangle$ are also consistent with each other and with theory (see table 4.12). However, $\hat{\sigma}(\operatorname{std}<M>$ ) was significantly smaller (13.9\%) than $\hat{\sigma}(<M>)$. This was shown to be caused, most likely, by the effect of Hanning filtering on the width of the measured distribution.

From these comparisons it appears that $\hat{\sigma}(<M>)$ and $\hat{\sigma}\left(<M^{2}>\right)$ are both reliable estimates of $\sigma$ but that $\hat{\sigma}(\operatorname{std}<M>)$ is not, probably due to the effect of the Hanning filter. It is useful to note that using $\hat{\sigma}$ values in the $\mathrm{NC} 2$ noise bias correction algorithm that differ by about $3 \%$ (i.e. $\hat{\sigma}(<M>)$ and $\left.\hat{\sigma}\left(<M^{2}>\right)\right)$ gives very similar noise bias correction.

The NC2 noise bias correction procedure introduced in this chapter was tested with all three estimators. Diffusion decays were measured for the head coil water phantom. Various ROI sizes were tested to find the optimal size such that it had enough pixels to make the results statistically significant but not large enough that signal variance due to artifacts was introduced. It can be seen by a comparison of figures $4.29-4.31$ that the noise bias correction is poor when $\hat{\sigma}(\operatorname{std}<M>)$ is used but it is very good when either $\hat{\sigma}(<M>)$ or $\hat{\sigma}\left(<M^{2}>\right)$ is used 


\begin{tabular}{|c|c|c|c|c|c|c|}
\hline \multirow{2}{*}{$\%$ difference } & \multicolumn{3}{|c|}{ with phantom } & \multicolumn{3}{c|}{ without phantom } \\
\cline { 2 - 7 } & $\hat{\sigma}(<\mathrm{M}>)$ & $\hat{\sigma}\left(\mathrm{std}_{<\mathrm{M}>}\right)$ & $\hat{\sigma}\left(<\mathrm{M}^{2}>\right)$ & $\hat{\sigma}(<\mathrm{M}>)$ & $\hat{\sigma}\left(\mathrm{std}_{<\mathrm{M}>}\right)$ & $\hat{\sigma}\left(<\mathrm{M}^{2}>\right)$ \\
\cline { 2 - 7 } & $4.84 \pm 0.04$ & $4.21 \pm 0.08$ & $4.71 \pm 0.05$ & $2.42 \pm 0.02$ & $2.37 \pm 0.019$ & $2.41 \pm 0.018$ \\
\hline $\begin{array}{c}\hat{\sigma}(<\mathrm{M}>)- \\
\hat{\sigma}\left(\mathrm{std}_{<\mathrm{M}>}\right)\end{array}$ & \multicolumn{3}{|c|}{$13.9 \%$} & \multicolumn{3}{|c|}{$2.00 \%$} \\
\hline $\begin{array}{l}\hat{\sigma}(<\mathrm{M}>)- \\
\hat{\sigma}\left(<\mathrm{M}^{2}>\right)\end{array}$ & \multicolumn{3}{|c|}{$2.7 \%$} & \multicolumn{3}{|c|}{$0.42 \%$} \\
\hline
\end{tabular}

Table 4.11. Comparison of the three estimators averaged over both ROI locations and b-values.

\begin{tabular}{|c|c|c|c|}
\hline & Theory & \multicolumn{2}{|c|}{ experiments } \\
\cline { 2 - 4 } & & with phantom & without phantom \\
\hline$\langle\mathrm{M}\rangle / \sigma_{\mathrm{M}}$ & 1.91 & $2.2 \pm 0.023$ & $1.95 \pm 0.01$ \\
\hline$\langle\mathrm{M}\rangle /\left\langle\mathrm{M}^{2}\right\rangle$ & 0.89 & $0.91 \pm 0.002$ & $0.89 \pm 0.001$ \\
\hline
\end{tabular}

Table 4.12. Comparison of the Rician theoretical ratio with the measured ratio with and without the phantom.

with $\hat{\sigma}(<M>)$ giving slightly better results for this set of measurements.

\subsection{Conclusion}

Removing the Rician noise bias from the signal decays is an important requirement for analyzing diffusion data from images and getting reliable information from the decays. A new Rician noise bias correction algorithm developed in this study and applied in image post processing showed significant advantages both theoretically and experimentally over conventional methods and complex averaging. This was most significant in situations with $\mathrm{SNR}<1.0$. The noise correction 
procedure requires that $\sigma$ of the noise be accurately estimated from the image background. We showed that the noise estimators using the mean and the second moment of the background are more reliable than using the variance of the mean. The precise selection of the ROI location and the ROI size in the background as well as in the image are also important in order to have accurate parameter estimates of the signal diffusion decay. This study showed that $\hat{\sigma}(<M>)$ and $\hat{\sigma}\left(<\mathrm{M}^{2}>\right)$ determined from the background of the image can both be used in the NC2 algorithm to effectively correct the diffusion signal decay for the Rician noise bias. 


\section{CHAPTER 5}

\section{Diffusion Decay Measurements in Human Brain in vivo and Analysis with $N N L S$}

\subsection{Introduction}

Clinical measurements of the apparent diffusion coefficient in vivo assume that the diffusion decay is mono-exponential and 2 or 3 data points over a small range of b-values up to 1000 $\mathrm{s} / \mathrm{mm}^{2}$ is considered to be adequate for characterizing the decay. This has the advantage of describing the diffusion characteristics with a single decay constant over a wide range of experimental conditions. However, a great number of studies of the MR diffusion properties of tissues indicate that the diffusion decay is poorly represented by a single exponential decay in tissues $[98,99,103]$. When the MR diffusion measurements are sampled with more b-values over an extended b-value range, much larger than typically used in clinical practice, the brain water signal decay in vivo no longer displays a mono-exponential signal decay but rather the decay appears to be a multi-exponential decay. The precise diffusion decay behavior in tissue and its 
explanation are still not well understood. The decay can be affected by many things including different tissue environments and exchange among them, anisotropy and so on. A quantitative study of the multi-exponential diffusion decay of tissue such as brain tissue must satisfy two important criteria: accurate data measurement and reliable data analysis which is appropriate for the data being considered. Therefore, for the measurement:

- A sufficient number of b-values must be sampled to adequately characterize the observed diffusion decay.

- The echo time must be kept constant and short enough that the SNR remains high.

- Eddy current effects and ghosting must be avoided as much as possible in the diffusion decay measurements since they can distort the data.

For the data analysis:

- The ROI's must be selected very carefully to avoid N/2 ghosting and Gibbs artifacts.

- Rician noise bias correction based on accurate noise estimation must be applied in the post processing.

- An appropriate curve fitting method for multi-exponential diffusion decay with an unknown number of components should be used to characterize the diffusion information in tissue.

We established these criteria for our diffusion experiments in order to allow us to obtain an accurate knowledge of diffusion characteristics in brain tissue.

\subsection{Materials and Methods}

A protocol was developed using Siemens' diffusion-EPI pulse sequence (ep2d-diff) with a constant diffusion time of $\tau_{\mathrm{D}}=50 \mathrm{~ms}$ to do MR diffusion measurements on the $1.5 \mathrm{~T}$ MR scanner. 

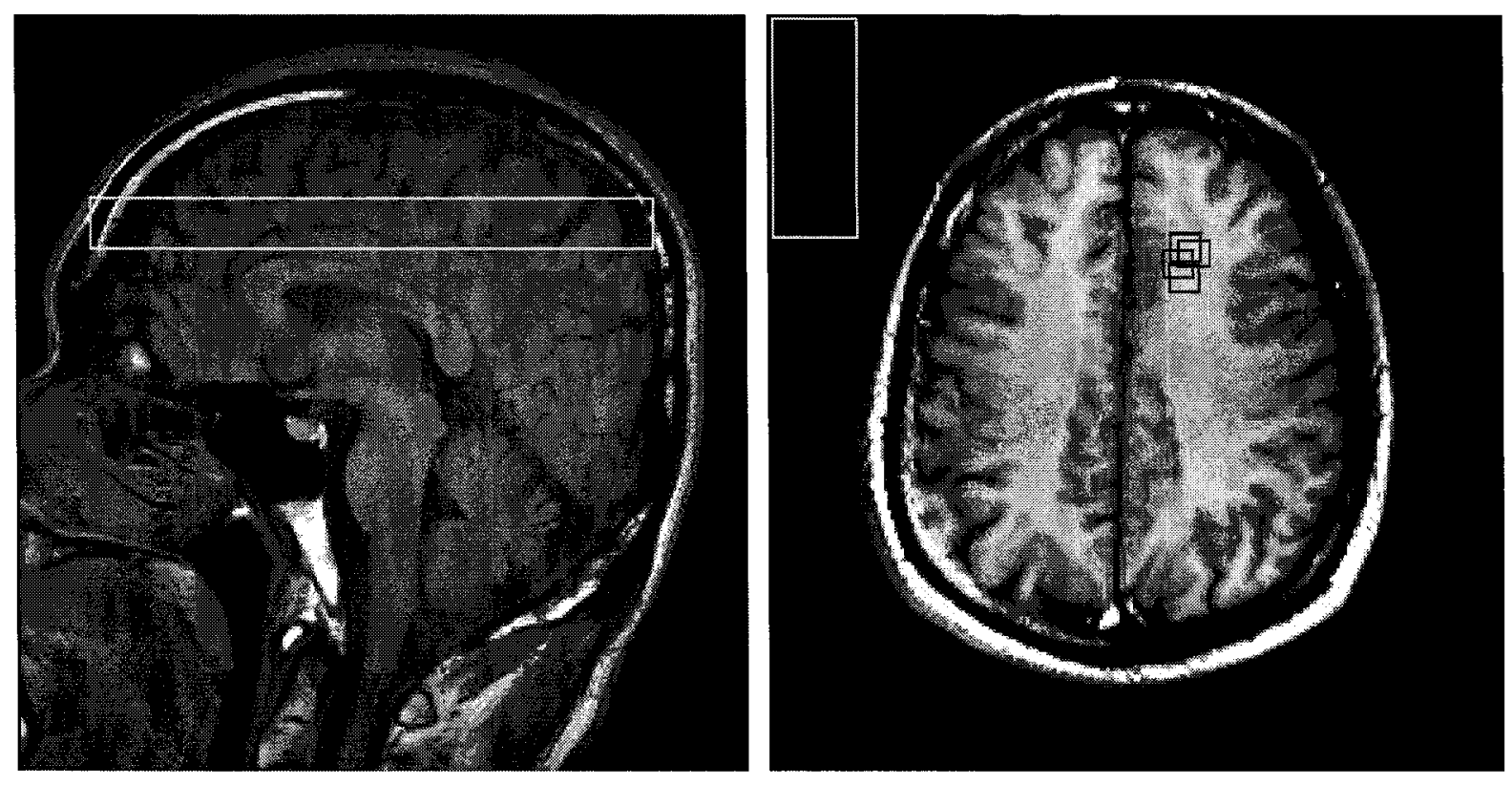

Figure 5.1. MR images showing the region of the brain studied. Left: this represents the slice selected above the ventricles. Right: the ROI's selected in the white matter and in the background noise.

Using this protocol, data points were measured for 96 b-values to study the diffusion decay of water molecules in a single slice of the human brain. The pulse sequence was first modified to allow up to 1000 averages to find the optimum number of averages for our experiments. We used 50 signal averages as the optimum number of measurements as a compromise between increased scan time and a better SNR. The $96 \mathrm{~b}$-values were chosen from zero to $12,500 \mathrm{~s} / \mathrm{mm}^{2}$ associated with the maximum possible amplitude for the diffusion sensitizing gradient of $28.5 \mathrm{mT} / \mathrm{m}$ which was applied in the readout direction (i.e. left to right in the images presented in this study). Since the maximum number of $b$-values that could be measured with this sequence was fixed at 16 , the sequence was repeated 6 times and the b-values are distributed over 6 sets of 16 values each with an interval of $100 \mathrm{~s} / \mathrm{mm}^{2}$ in the first 5 sets and a random interval in the last set up to the maximum b-value. The echo time, TE, was kept constant at $200 \mathrm{~ms}$ along with a repetition time 
of $\mathrm{TR}=500 \mathrm{~ms}$. The slice was selected in the transverse plane with a thickness of $10 \mathrm{~mm}$. A field of view (FOV) of $464 \mathrm{~mm}$ was selected in order to improve the SNR per voxel, at the expense of resolution, and to keep any N/2 ghosting artifacts away from the image area. The images were sampled with a matrix size of $128 \times 128$.

A typical image of the selected transverse slice is displayed in figure 5.1 for a human volunteer. The position of the $10 \mathrm{~mm}$ thick slice is superior to the ventricles at the level of the centrum semiovale. The ROIs were positioned in the left hemisphere in an area of white matter superior and lateral to the genu of the corpus callosum. The axonal orientation within these ROIs should therefore be predominantly inferior to superior and the experiments reported here, which are sensitized to diffusion in the left to right direction, are expected to correspond to measurements of diffusion perpendicular to the axon fibers for most of the axons in the ROIs analyzed. The position of the ROIs was also chosen very carefully to avoid artifacts such as N/2 ghosting and Gibbs ringing as much as possible. The size of the ROIs was 5 pixels by 4 pixels which corresponds to a volume of $18 \times 14 \times 10=2520 \mathrm{~mm}^{3}$. This should be large enough to provide statistically significant mean and standard deviation values of the pixel intensity for the ROIs.

Rician noise correction was applied to the data to remove the Rician noise bias. Since the minimum SNR for these experiments was greater than 3.0 the results from chapter 4 show that all of the correction schemes considered there perform equally well (see section 4.3.2). The method of McGibney and Smith as given by equation (4.33) with $\mathrm{n}=2$ was used for the Rician noise bias correction for the brain diffusion decay presented here. The positioning of the ROI in the background for the determination of $\hat{\sigma}$ was done carefully to avoid the effects of artifacts. The location of this ROI was similar to that shown in figure 5.1. 


\subsection{Data Analysis with $N N L S$}

To analyze the measured diffusion decays an extension of the $N N L S$ algorithm (see section 2.5.2) that includes regularization (see section 2.5.3) was used. Such solutions are composed of distributions of diffusion coefficients $\left(D_{a}\right)$ with smoothly varying amplitudes. The regularizer allows the algorithm to perform more robustly for noisy data at the expense of less than $3 \%$ of the minimum $\chi^{2}$ of the discrete $N N L S$ solution. The diffusion coefficient axis was partitioned into 150 values logarithmically spaced at equal intervals (in $\log$ space) between $1.0 \times 10^{-5}$ and $0.1 \mathrm{~mm}^{2} / \mathrm{s}$. The computational code, $N N L S$-diff, was developed for the diffusion data analysis based on the block diagrams given in Appendix I.

As introduced in chapter 2 , the $N N L S$ algorithm basically solves a general system of equations like

$$
\sum_{j=1}^{n} A_{i j} s_{j}-y_{i}=0
$$

by minimizing the least-squares misfit, (i.e. $\sum_{i=1}^{m}\left|\sum_{j=1}^{n} A_{i j} s_{j}-y_{i}\right|^{2}=$ minimum) subject to the constraints that $s_{j} \geq 0$ for all $s_{j}$ where $y_{i}$ is the measured data value for $b_{i}$. The vector $\mathbf{y}$ is an input for the $N N L S$ algorithm as well as the matrix $\mathbf{A}$. The matrix elements, $\mathrm{A}_{\mathrm{ij}}$, are equal to $\exp \left(-\mathrm{b}_{\mathrm{i}} \cdot \mathrm{D}_{\mathrm{j}}\right)$ for a given set of $b$-values and a large number of diffusion coefficients, $D_{j}$. The diffusion coefficients, which are known input parameters to the code, are arbitrarily selected over a reasonable range and sampled in logarithmic intervals. The $N N L S$ algorithm determines which combination of $D_{j}$ values generates the best fit and sets the corresponding $s_{j}$ values accordingly. The $s_{j}$ are set to zero for all $D_{j}$ values that are not part of the solution. For example, if the best fit corresponds to a bi-exponential with two equally weighted exponential terms for $D_{k}$ and $D_{l}$ then 
the $N N L S$ algorithm sets $\mathrm{s}_{\mathrm{k}}=\mathrm{s}_{l}=0.5$ and $\mathrm{s}_{\mathrm{j}}=0$ for all $\mathrm{j} \neq \mathrm{k}, l$. Even though a very large number of diffusion coefficients are considered, only $\mathrm{D}_{\mathrm{k}}$ and $\mathrm{D}_{l}$ appear in the final solution.

Additional subroutines for specific applications were also coded into NNLS-diff in order to construct the A matrix, generate noisy data by Monte Carlo simulation for testing the algorithm before its use with unknown measured data, and to calculate statistics such as chi-square, the mean value and the variance of the data for each peak in the spectrum. One of the most important subroutines of $N N L S$-diff is for developing the regularizer in order to smooth the spectrum of the diffusion coefficients when analyzing noisy diffusion decay curves. The flow chart of this code based on NNLS with regularization is shown in figure 2 of Appendix I. The program NNLS-diff was developed in Microsoft Visual C++ (version 6.0 ). It consists of about 1200 lines of code and the execution time is approximately $5 \mathrm{~s}$ on IBM PC with an NT4 operating system for an $\mathrm{m} \times \mathrm{n}=100 \times 100 \mathrm{~A}$ matrix.

\subsection{Using $N N L S$ for the Analysis of the Simulated Data}

The code was first tested for simulated data with Monte Carlo generated random Gaussian noise, $\varepsilon_{\mathrm{i}}$, added to the signal so that it would be similar to measured MR data. The simulated decay data were computed for an inhomogeneous system based on the discrete version of equation (2.97) given in equation (2.100) and rewritten here as follows:

$$
\mathrm{Y}_{\mathrm{k}}\left(\mathrm{b}_{\mathrm{i}}\right)=\sum_{\mathrm{j}=1}^{\mathrm{n}} \mathrm{S}_{\mathrm{j}} \exp \left(-\mathrm{b}_{\mathrm{i}} \mathrm{D}_{\mathrm{j}}\right)+\varepsilon_{\mathrm{k}}(0, \sigma) \quad \mathrm{k}=1,2, \ldots, \mathrm{m}
$$

The Gaussian noise is considered to be uncorrelated, with zero mean and standard deviation $\sigma$.

These computations were performed for two different cases: discrete spectra and piece-wise continuous spectra consisting of several distributions of diffusion coefficients (figures 5.2 and 5.3). By constructing the matrix elements of $\mathrm{A}_{\mathrm{ij}}$ for the $N N L S$ algorithm appropriately, good 

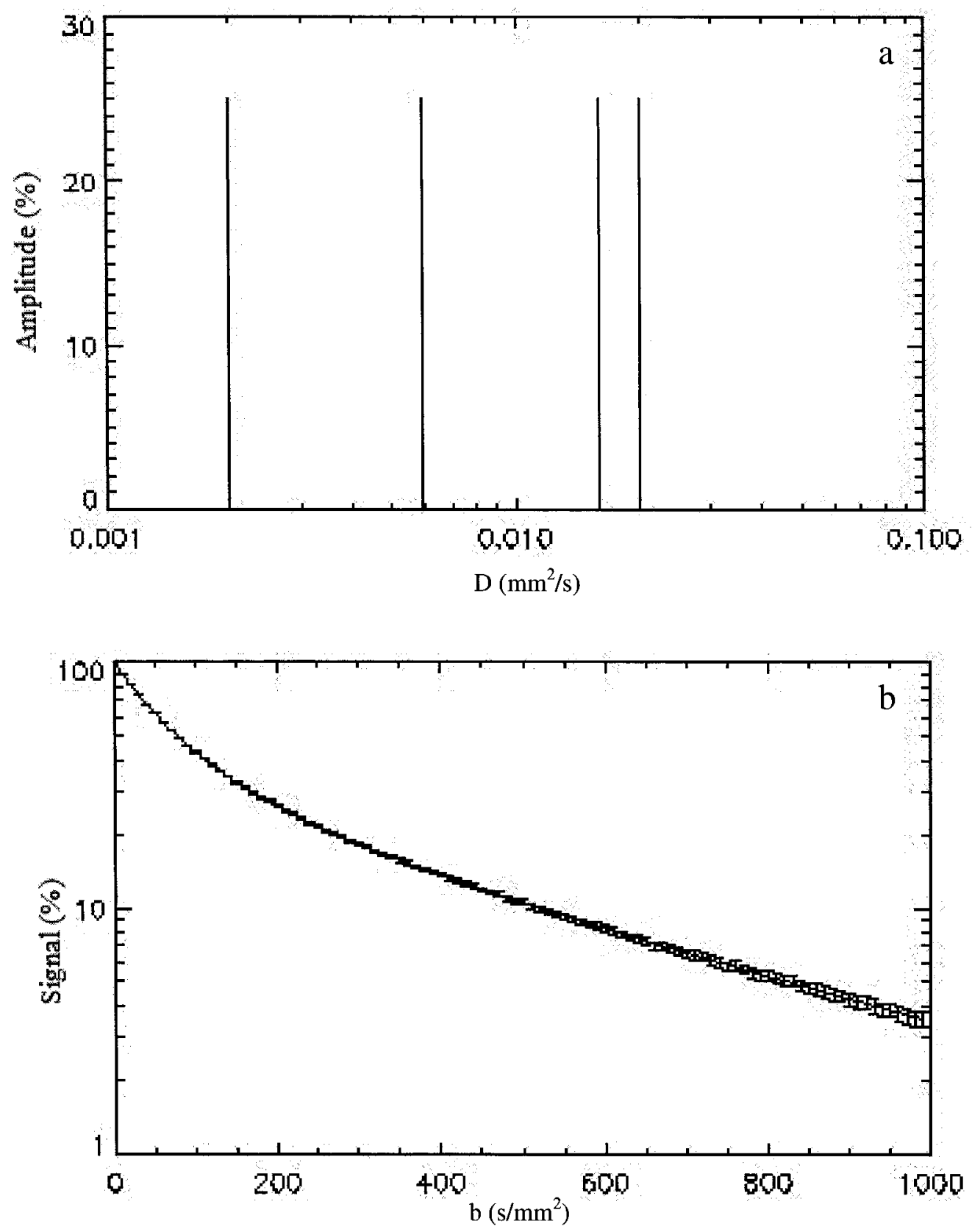

Figure 5.2 a. Assumed discrete spectrum of diffusion coefficients, b. Generated diffusion decay curve with simulated noisy data computed using the diffusion coefficient distribution shown in a. 

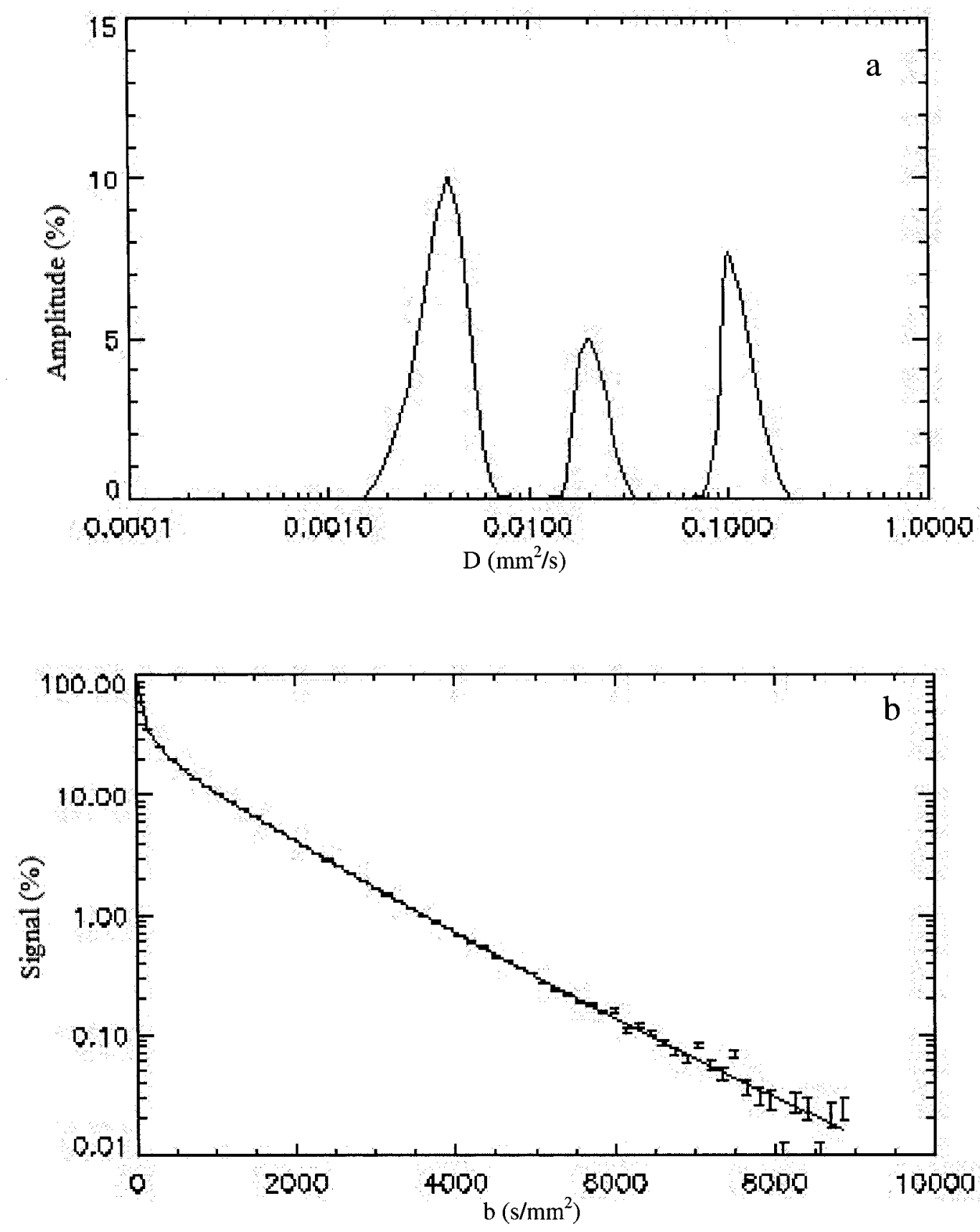

Figure 5.3 a. Assumed continuous spectrum of diffusion coefficients, b. Generated diffusion decay curve with simulated noisy data computed using the diffusion coefficient distribution shown in a. 
results for both classes can be obtained. The discrete version of the $N N L S$ algorithm gives a solution with the convergence of equation (5.1) to zero. The only prior assumption is nonnegativity of the spectral fractions, $\mathrm{s}_{\mathrm{j}}$. The results in figure $5.4 \mathrm{a}$ show that the $N N L S$ solution is compatible with the assumed discrete spectrum (figure 5.4b) used to generate the data (see also figures $5.2 \mathrm{a}, \mathrm{b})$. For the discrete $N N L S$ spectrum, the real amplitude of each peak is equal to the sum of the two smaller peaks clustered around the real D value which corresponds to the weighted average of these two $D_{j}$ 's.

$\chi^{2}$ is found to be about 80 for the 100 generated data points which is the number of degrees of freedom (ndf) and therefore the expected value of $\chi^{2}$. The assumed standard deviation for creating the added noise to the data is $\sigma=0.5 \%$ of the maximum signal strength which is also applied for the evaluation of chi-square by the following equation:

$$
\chi^{2}=\sum_{\mathrm{i}=1}^{\mathrm{m}}\left(\mathrm{y}_{\mathrm{i}}^{\mathrm{c}}-\mathrm{y}_{\mathrm{i}}\right)^{2} / \sigma^{2},
$$

where $\mathrm{y}_{\mathrm{i}}^{\mathrm{c}}$ are the data constructed from the NNLS results.

For a continuous spectrum, which is normally assumed to be more like a real system, the integral of the true model is equal to the integral of the solution provided by the discrete form of $N N L S$ (i.e. with no regularization). This means that there is a very good fit in terms of the chisquare calculation. However, the results show a completely different spectrum compared with the real constructed spectrum. As can be seen from figure 5.5, the solution produced by $N N L S$ for the diffusion decay given in figure 5.3b, which was generated from the piece-wise continuous spectrum shown in figure 5.3a, is a sum of a few delta functions and it doesn't really resemble the true system very well. The $\chi^{2}$ value obtained in this case was 79.02 and the ndf was 100 . 

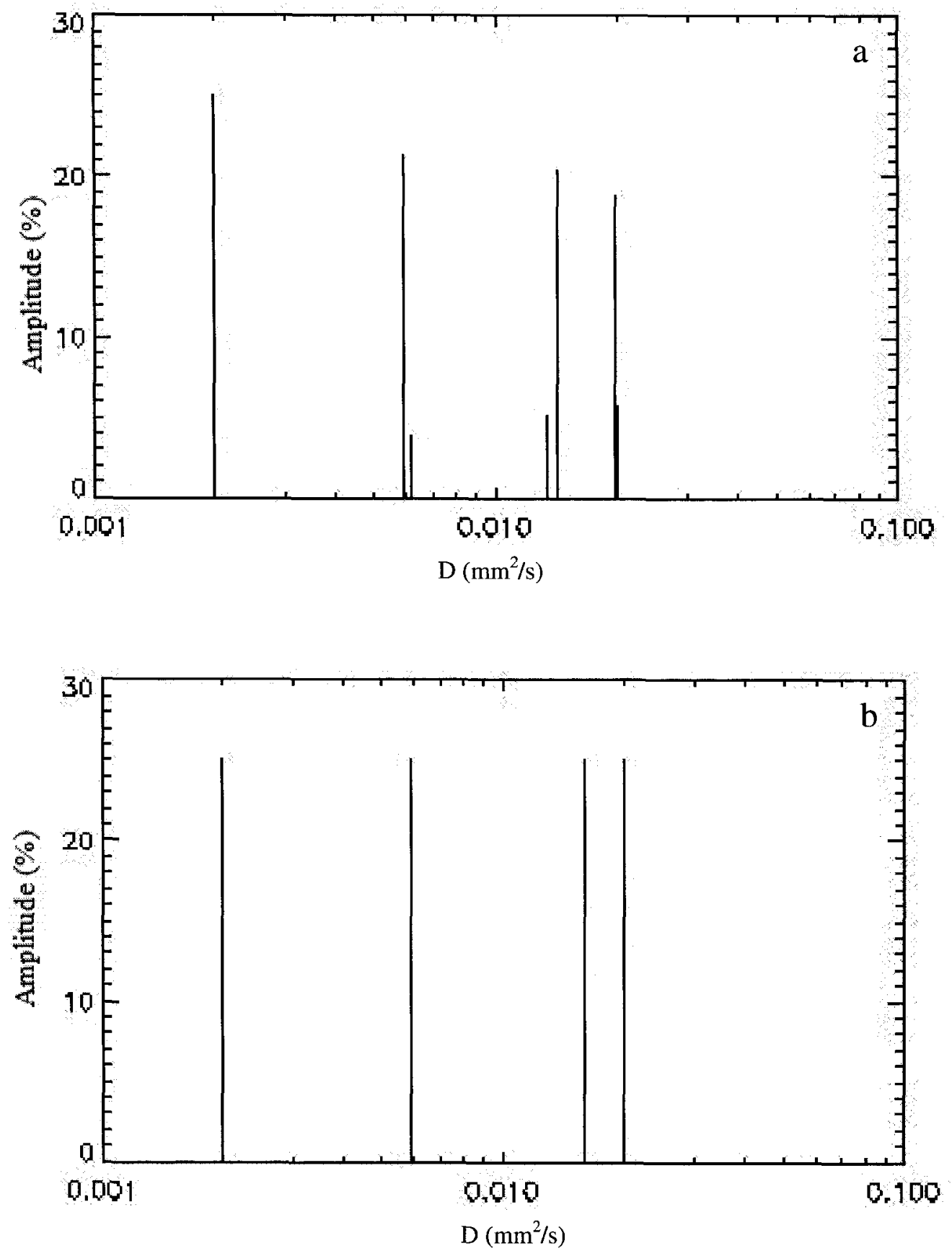

Figure 5.4 a. Discrete spectrum of diffusion coefficients obtained using NNLS analysis of simulated diffusion data, $b$. True discrete spectrum. 
For the adaptation of $N N L S$ to solve the problem of the continuous model, which may be a more realistic model for the particular system under study, extra constraints are required to find the solution which is both the most accurate and the most stable. In other words the solution, $\mathbf{s}$, must satisfy the condition

$$
\sum_{i=1}^{m}\left|\sum_{j=1}^{n} A_{i j} s_{j}-y_{i}\right|^{2}+\mu \sum_{k=1}^{K}\left|\sum_{j=1}^{n} R_{k j} s_{j}-r_{k}\right|^{2}=\text { minimum }
$$

where the second term on the right is called the regularizer and the relative strength of the two terms is determined by $\mu$, the regularization parameter as defined in section 2.5.3. These constraints thus minimize the extra terms, $\|\mathbf{R s}-\mathbf{r}\|^{2}$, along with the chi-square misfit and change the ordinary constrained least-squares solution. The additional constraints are incorporated as rows of the matrix $\mathbf{A}$.

Imposing the extra constraints to minimize along with the chi square misfit is used to select the 'simplest' members of a large number of possible solutions, protecting the system against significant unexpected information such as sudden sharp changes or extra peaks in the spectrum. This is an example of the principle of parsimony which can be defined as choosing the solution spectrum that is the smoothest with the minimum number of peaks. This tends to prevent the system of solutions from significant artifacts [110].

The form of $\mathbf{R}$ and $\mathbf{r}$ can be specified by the user (see below). The value of the regularization parameter is adjusted iteratively between 0 and 1 trading off with the chi-square misfit until it is close to $\mathrm{m}$, which is equal to the ndf for the system of equations. This is the expected value of $\chi^{2}$. When $\mu=0$, equation (5.4) reduces to equation (5.1) and the basic leastsquares solution with the minimum $\chi^{2}$ is obtained. When $\mu>0$, the equality constraints are eliminated and the regularizer imposes smoothness to the solution. The optimized value of $\mu$ is 
obtained at the expense of increasing the $\chi^{2}$ misfit, but it provides a smooth spectrum and a more stable solution.

Three regularizers were defined in section 2.5.3. By applying the simplest regularizer, minimization of the power of the spectrum (i.e. the matrix $\mathbf{R}$ is set to the identity matrix and $\mathbf{r}=0$ ) the solution will be a smooth spectrum with the minimum number of peaks. As can be seen in figure 5.6, a continuous spectrum with 3 peaks is determined by $N N L S$ with this regularizer. This is in better agreement with the real model than the solution of $N N L S$ without the regularizer shown in figure 5.5, however, the agreement is still not as good as we would like. The solution with this regularizer underfits the data such that $\chi^{2}=112>\chi_{\min }^{2}=79$, where $\chi_{\min }^{2}$ is the $\chi^{2}$ value obtained for $\mu=0$.

To compute the regularizer from the first and second derivatives of the spectrum, the matrix R should be formed in the computational code respectively as follows $[109,110]$ :

and

$$
\mathbf{R}_{1 \mathrm{st}}=\left[\begin{array}{ccccc}
1 & & & & 0 \\
-1 & 1 & & & \\
& -1 & 1 & & \\
& & \ddots & \ddots & \\
0 & & & -1 & 1
\end{array}\right]
$$

$$
\mathbf{R}_{2 \text { nd }}=\left[\begin{array}{cccccc}
1 & & & & & 0 \\
-2 & 1 & & & & \\
1 & -2 & 1 & & & \\
& 1 & -2 & 1 & & \\
& & \ddots & \ddots & \ddots & \\
0 & & & 1 & -2 & 1
\end{array}\right]
$$




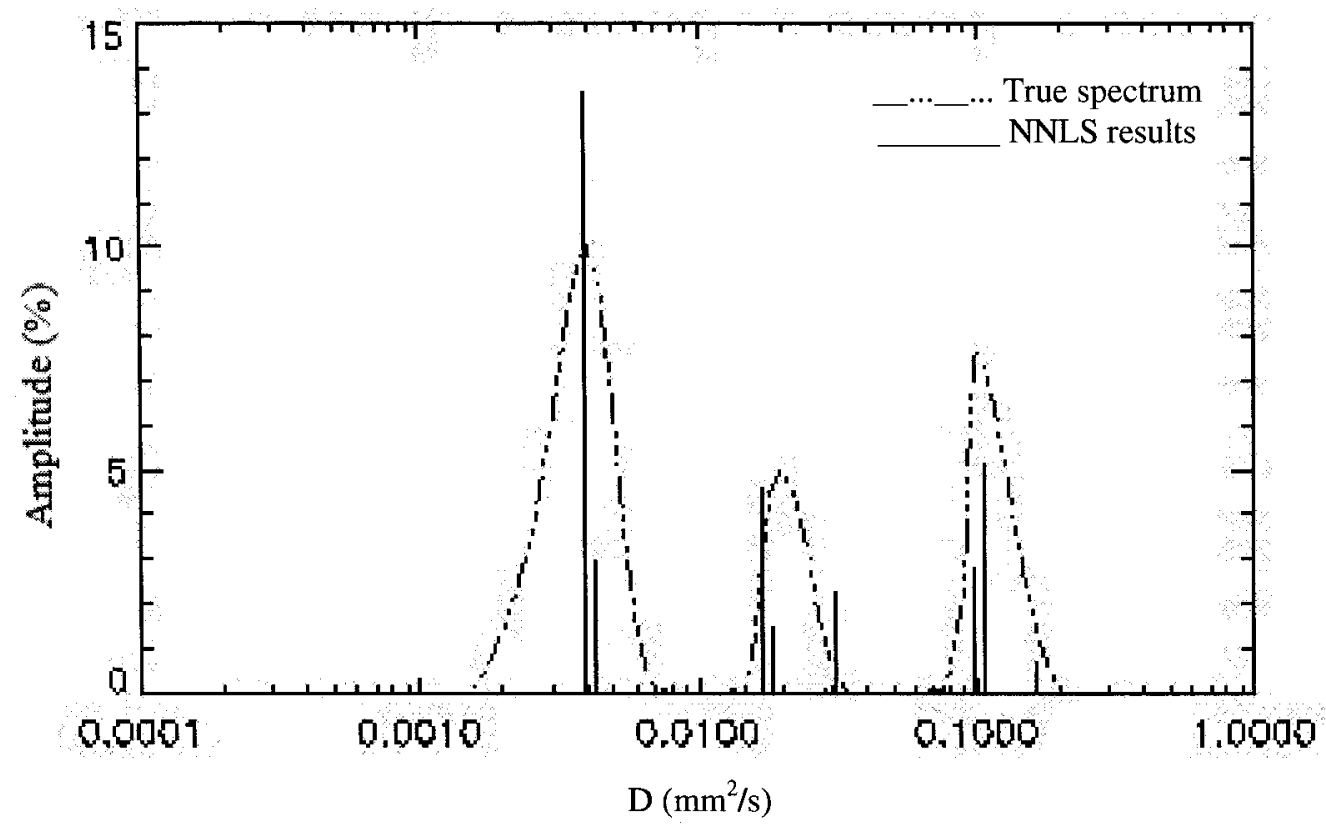

Figure 5.5. The spectrum of diffusion coefficients found using the discrete form of $N N L S$ for a continuous distribution of diffusion coefficients. The $N N L S$ results are divided by three for easier comparison with the true spectrum.

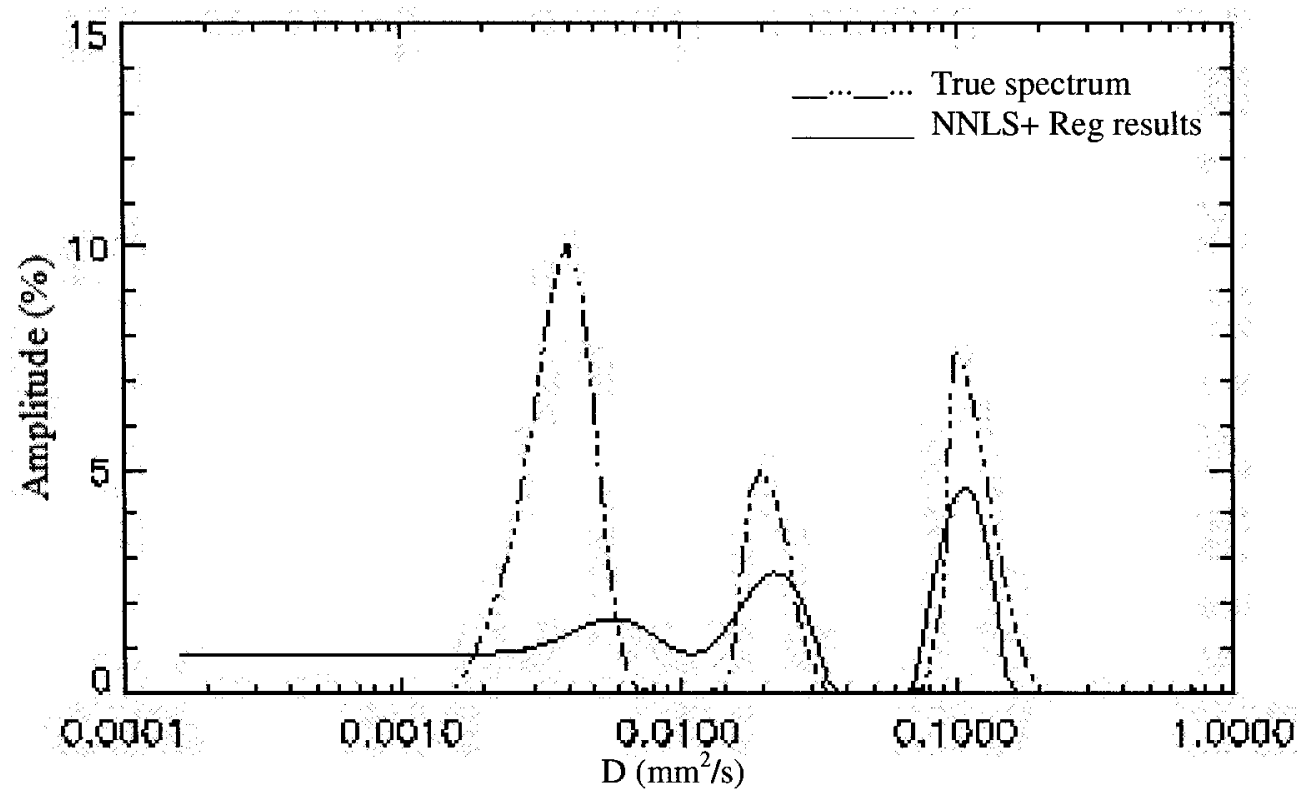

Figure 5.6. The spectrum of diffusion coefficients obtained using $N N L S$ with a regularizer to minimize the power of the spectrum. 
It was not clear from the literature which regularizer is the best one to use. Provencher [110] seems to favour minimization of the second derivative of $\mathbf{s}$ while Whittall and MacKay [109] suggest that minimization of the first derivative of $\mathbf{s}$ is preferable. As can be seen from equations (5.5) and (5.6) both act to reduce the difference between adjacent points in the solution. These two regularizers and minimization of the power spectrum are all considered here to determine which performs the best for the diffusion decays reported in this thesis.

Figure 5.7 shows a comparison of these three regularizers. It shows that the spectrum which is least consistent with the assumed continuous model is the regularizer that minimizes the power of the spectrum. For this case the $\chi^{2}$ value was 112 which is much higher than the expected value of $\chi^{2}=79$ which is obtained when $\mu=0$. For the other two regularizers, the first and the second order derivatives of the spectrum, the $\chi^{2}$ values were 82 and 80 , respectively, which is considerably better than the $\chi^{2}$ value for the power spectrum regularizer. Both the first and second derivative regularizers give results that are in much better agreement with the true model in the overall shape than for the power spectrum regularizer. This can also be seen from the position of the peak maxima. In all three cases the total integral of the spectrum is equal to that of the true model. The results of these analyses for $N N L S$ with the three regularizers compared to the true model are summarized in table 5.1. It should be pointed out that the values of $\langle\mathrm{D}\rangle$, the expected value of $\mathrm{D}$ for peak $\mathrm{k}$, given in the table were computed as the weighted average over peak $k$ of the spectrum rather than the mean value of $D$ or the value of $D$ at the peak maxima. The fractional contribution $<\mathrm{s}>$ of peak $\mathrm{k}$ to the total diffusion decay is calculated as the mean over the peak, i.e. $<s>=\frac{1}{n_{k}} \sum_{j=1}^{n_{k}} s_{j}$ where $n_{k}$ are the number of points in the spectrum for peak $k$. For each peak $\left\langle\mathrm{D}>,\left\langle\mathrm{s}>\right.\right.$ and their standard deviations $\sigma_{\mathrm{D}}$ and $\sigma_{\mathrm{s}}$, as well as $\chi^{2}$ for the total spectrum, are calculated in the statistical part of $N N L S$-diff (see figure 2 of Appendix I). 


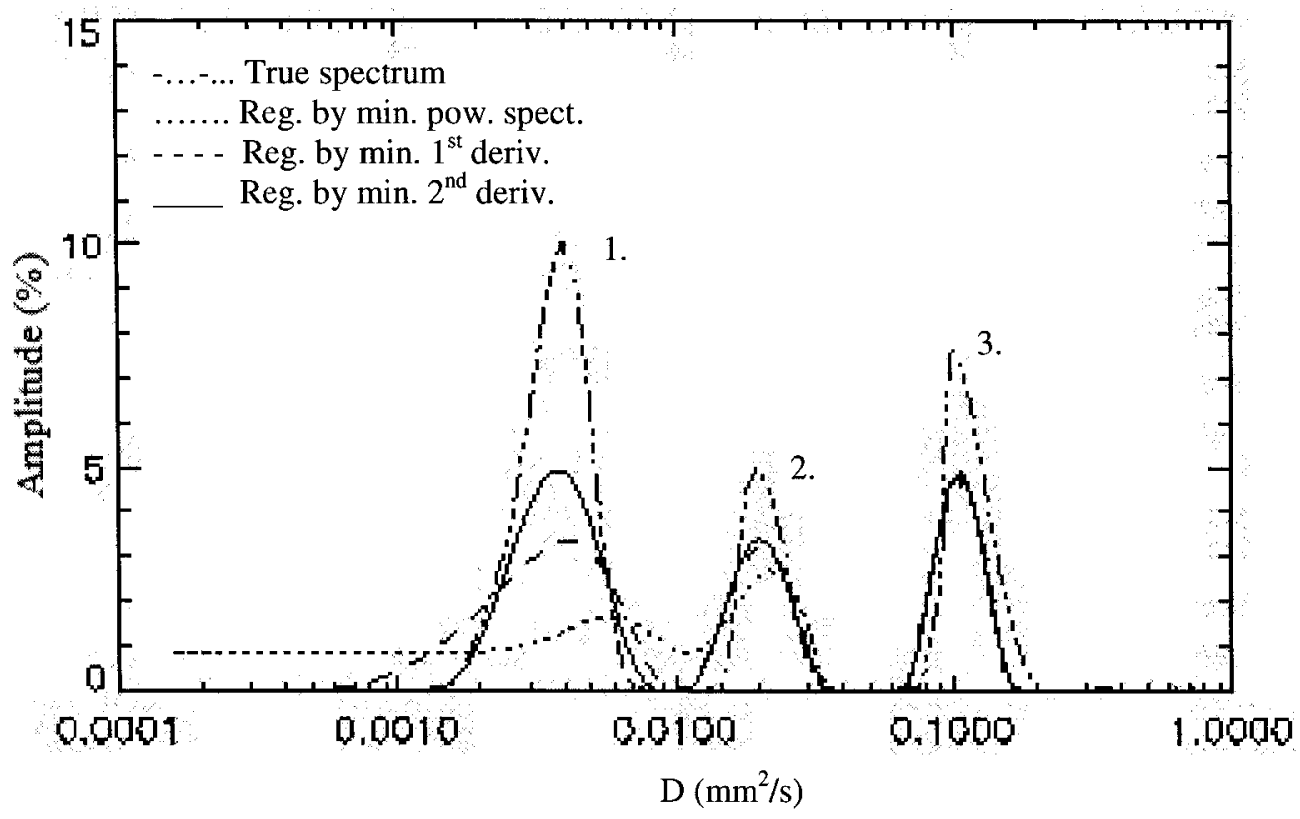

Figure 5.7. Spectrum of the diffusion coefficients obtained using NNLS compared with the true solution for three forms of the regualrizer.

\begin{tabular}{|c|c|c|c|c|c|c|c|c|c|c|c|}
\hline & & & \multicolumn{3}{|c|}{ Peak 1} & \multicolumn{3}{|c|}{ Peak 2} & \multicolumn{3}{|c|}{ Peak 3} \\
\hline & $\begin{array}{c}\text { Spectrum } \\
\text { Integral }\end{array}$ & $\chi^{2}$ & $\begin{array}{c}\text { Weighted } \\
\text { Mean } \\
<\mathrm{D}>\left(\sigma_{D}\right) \\
\mathrm{mm}^{2} / \mathrm{s}\end{array}$ & $\begin{array}{l}\mathrm{FWHM} \\
\mathrm{mm}^{2} / \mathrm{s}\end{array}$ & $\begin{array}{c}\text { Frac } \\
\text { Cont \% } \\
<s> \\
\left(\sigma_{s}\right) \\
\end{array}$ & $\begin{array}{c}\text { Weighted } \\
\text { Mean } \\
<D>\left(\sigma_{D}\right) \\
\mathrm{mm}^{2} / \mathrm{s}\end{array}$ & $\begin{array}{l}\mathrm{FWHM} \\
\mathrm{mm}^{2} / \mathrm{s}\end{array}$ & $\begin{array}{c}\text { Frac } \\
\text { Cont \% } \\
<s> \\
\left(\sigma_{s}\right) \\
\end{array}$ & $\begin{array}{c}\text { Weighted } \\
\text { Mean } \\
<D>\left(\sigma_{D}\right) \\
\mathrm{mm}^{2} / \mathrm{s} \\
\end{array}$ & $\begin{array}{l}\mathrm{FWHM} \\
\mathrm{mm}^{2} / \mathrm{s}\end{array}$ & $\begin{array}{c}\text { Frac } \\
\text { Cont \% } \\
<s> \\
\left(\sigma_{s}\right) \\
\end{array}$ \\
\hline $\begin{array}{c}\text { True } \\
\text { Spectrum }\end{array}$ & 140.68 & & $\begin{array}{c}0.004 \\
(0.0003) \\
\end{array}$ & 0.004 & $\begin{array}{l}50.4 \\
(3.6) \\
\end{array}$ & $\begin{array}{c}0.021 \\
(0.0017)\end{array}$ & 0.010 & $\begin{array}{l}22.8 \\
(1.9) \\
\end{array}$ & $\begin{array}{c}0.107 \\
(0.0012)\end{array}$ & 0.05 & $\begin{array}{l}26.8 \\
(2.8) \\
\end{array}$ \\
\hline NNLS & 141.08 & 79.02 & $\begin{array}{r}0.0043 \\
(0.005)\end{array}$ & NA & $\begin{array}{c}49.0 \\
(22.0)\end{array}$ & $\begin{array}{c}\text { 1) } 0.019 \\
(0.02) \\
\text { 2) } 0.031 \\
(0.005)\end{array}$ & NA & $\begin{array}{l}\text { 1) } 18.1 \\
(6.7) \\
\text { 2) } 6.9 \\
(4.7)\end{array}$ & $\begin{array}{l}\text { 1) } 0.107 \\
(0.12) \\
\text { 2) } 0.166 \\
\text { (NA) }\end{array}$ & NA & $\begin{array}{l}\text { 1) } 23.9 \\
(5) \\
\text { 2) } 2.1 \\
\text { (NA) }\end{array}$ \\
\hline $\begin{array}{c}\text { NNLS } \\
\text { (Pow_Reg) }\end{array}$ & 138.33 & 112.68 & $\begin{array}{c}0.0030 \\
(0.0001)\end{array}$ & 0.006 & $\begin{array}{l}50.9 \\
(0.2)\end{array}$ & $\begin{array}{c}0.021 \\
(0.0010)\end{array}$ & 0.015 & $\begin{array}{c}22.4 \\
(0.87)\end{array}$ & $\begin{array}{c}0.107 \\
(0.0075)\end{array}$ & 0.05 & $\begin{array}{l}26.6 \\
(1.6)\end{array}$ \\
\hline $\begin{array}{c}\text { NNLS } \\
\text { (1stD_Reg) }\end{array}$ & 140.13 & 82.40 & $\begin{array}{c}0.0035 \\
(0.0001)\end{array}$ & 0.006 & $\begin{array}{l}49.9 \\
(1.2)\end{array}$ & $\begin{array}{c}0.021 \\
(0.0012)\end{array}$ & 0.012 & $\begin{array}{l}23.3 \\
(1.2)\end{array}$ & $\begin{array}{c}0.107 \\
(0.0075)\end{array}$ & 0.05 & $\begin{array}{l}26.8 \\
(1.8)\end{array}$ \\
\hline $\begin{array}{c}\text { NNLS } \\
\text { (2ndD_Reg) }\end{array}$ & 140.62 & 80.30 & $\begin{array}{c}0.0038 \\
(0.0001)\end{array}$ & 0.0045 & $\begin{array}{l}49.6 \\
(1.8)\end{array}$ & $\begin{array}{c}0.021 \\
(0.0012)\end{array}$ & 0.011 & $\begin{array}{l}23.6 \\
(1.3)\end{array}$ & $\begin{array}{c}0.107 \\
(0.072)\end{array}$ & 0.05 & $\begin{array}{l}26.8 \\
(1.9)\end{array}$ \\
\hline
\end{tabular}

Table 5.1. The statistics of the simulated data of a continuous spectrum analyzed by NNLS with the three regularizers ( $\mathrm{ndf}=100, \mu=0.005, \sigma=0.5 \%$ ). The $N N L S$ analysis in discrete mode gives a spectrum with nine peaks (see figure 5.5). Using the weighted average of the adjacent double peaks the spectrum is reduced to five $\langle D\rangle$ values as shown in the table. 
The full width at half maximum (FWHM) indicates how noisy the D parameters are after adjusting the regularizer by trading off with the $\chi^{2}$ misfit. FWHM can be used for an interpretation of the relative inhomogeneity of one system compared with another when the spectra from the different systems being compared were analyzed using the same criteria for the regularizer adjustment. This is what Whittall et. al. [146] have done for their study of the $\mathrm{T}_{2}$ relaxation time distribution in different areas of human brain.

Larger values of $\mu$ give wider and shorter peaks while increasing $\chi^{2}$. The properly adjusted value of $0 \leq \mu \leq 1$ gives the optimum $\chi^{2}$ which depends on the data for the system under study. Our simulation showed that by adjusting $\mu$ to 0.005 , the optimum $\chi^{2}$ after regularization does not exceed 3\% difference when compared with $\chi_{\min }^{2}$ (when $\mu=0$ ) for our simulated data set. The best fit and the most stable solution is gained by minimizing the second-order derivative of the spectrum along with the chi-square misfit. From figure 5.7 and table 5.1 it is clear that the maximum amplitude of the peaks, the intervals between them and their FWHM have the most consistency with those of the true spectrum for the second derivative regularization.

\subsection{The Diffusion Measurements for a Water Phantom and Analysis by $N N L S$}

The data analysis of the water phantom was the first analysis of measured data by NNLS-diff. This was done to test the code for experimental data with a known diffusion coefficient. The cylindrical head coil water phantom was at room temperature $\left(22^{\circ} \mathrm{C}\right)$ when the MR-diffusion images were acquired using the ep2d-diff pulse sequence. The water phantom also contained a small amount of $\mathrm{NiSO}_{4}$ (see chapter 3 ) to reduce $\mathrm{T}_{1}$ for convenience but not enough to affect the measured diffusion coefficient. Three measurements were performed. In the first experiment 11 
b-values between 0 and $10,000 \mathrm{~s} / \mathrm{mm}^{2}$ with an interval of $1000 \mathrm{~s} / \mathrm{mm}^{2}$ were used. In the second experiment $16 \mathrm{~b}$-values between 0 and $12,500 \mathrm{~s} / \mathrm{mm}^{2}$ with smaller intervals were used. In the third experiment 96 b-values were used with the same protocol that was used to study the diffusion decay for human brain (see sections 5.2 and 3.1). The measured data were corrected for Rician noise bias using the NC2 approach as given by equation (4.39) in section 4.3.3.

The data analysis by $N N L S$ always resulted in one component (see figure 5.8a) when the discrete version of the code (i.e. no regularizer) was used or one peak in the spectrum with a mean value for the diffusion coefficient of $\mathrm{D}=(2.07 \pm 0.03) \times 10^{-3} \mathrm{~mm}^{2} / \mathrm{s}$ when the continuous version of $N N L S$ was used with the second derivative regularizer. The curves were very well fitted with the expected value of $\chi^{2}=3.8,2.2$ and 6.9 for ndf $=11,16$ and 96 , respectively, with $\sigma_{\chi^{2}}=0.6 \%$ of the maximum measured signals from the data analysis (see equation (5.3)). Figure $5.8 \mathrm{~b}$ shows the diffusion decay curves fitted with the NNLS-diff result for the water phantom measurements. The measured value of the diffusion coefficient of water at $22^{\circ} \mathrm{C}$ from this study is the expected result and is in good agreement with the literature value [99]. Mills [183] measured the free diffusion coefficient of water from $1-45^{\circ} \mathrm{C}$. By interpolating his results to $21^{\circ} \mathrm{C}$ we find $\mathrm{D}=(2.077 \pm 0.025) \times 10^{-3} \mathrm{~mm}^{2} / \mathrm{s}$, in good agreement with the value reported here. The diffusion coefficient of water measured by others $[102,115]$ at $19^{\circ} \mathrm{C}$ was reported to be $1.97 \times 10^{-3}$ to $1.98 \times 10^{-3} \mathrm{~mm}^{2} / \mathrm{s}$ which is also consistent with our results, considering the temperature.

As mentioned before, the incorporation of a regularizer into the least squares problem mathematically causes smoothing of the spectrum. However, a continuous spectrum with one (or more) peak with a finite width for a homogeneous system like a water phantom cannot have any physical meaning. As can be seen in figure 5.8a, the spectrum of diffusion coefficients for water has only one peak even with regularization. This could indicate the physical situation of an exact 

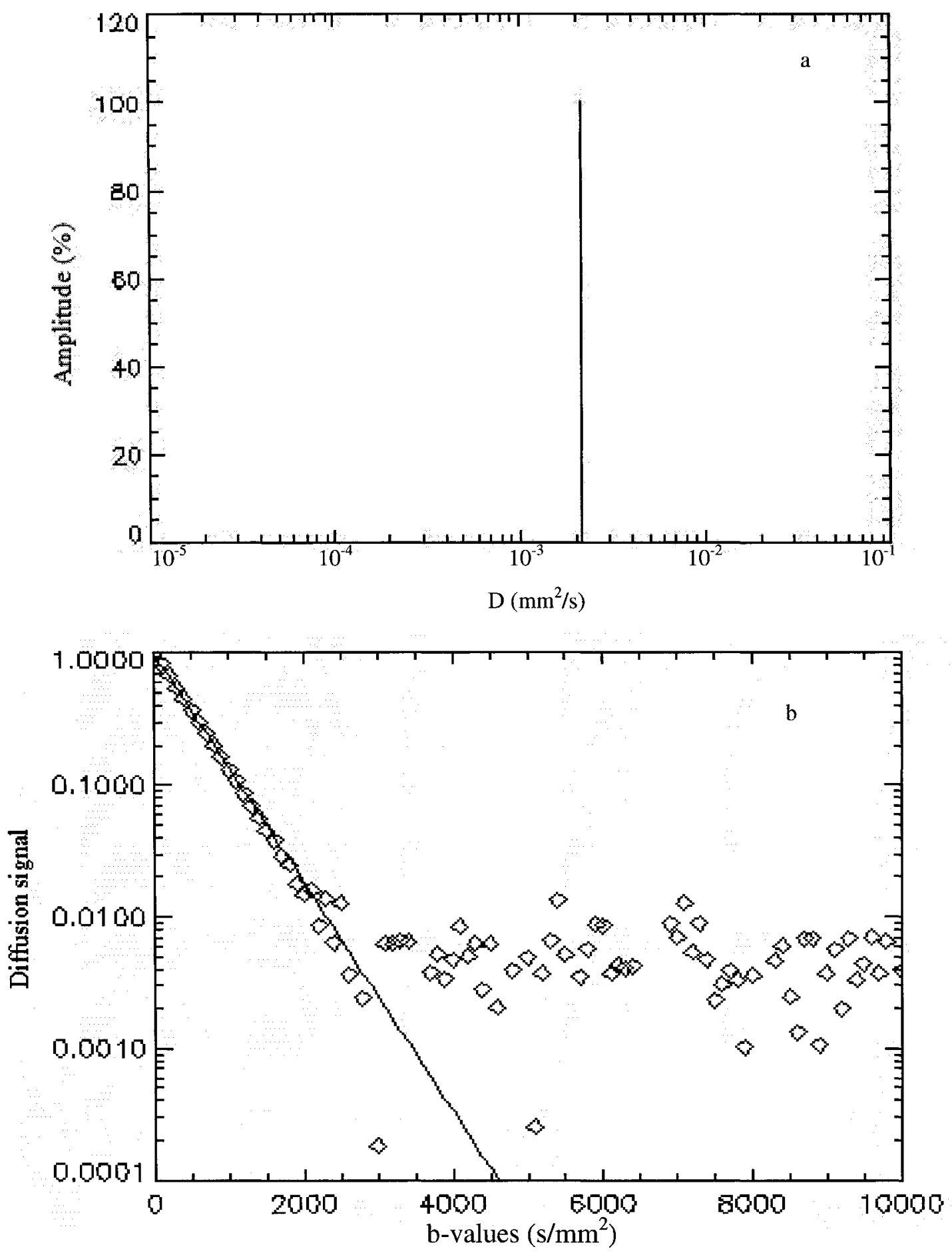

Figure 5.8 a. The spectrum for the diffusion coefficient of the water phantom using NNLS analysis with and without regularizer, which are the same for pure water. $b$. The diffusion decay with $96 \mathrm{~b}$-values for the water phantom after noise correction. 
homogeneity of our water phantom. It is important to note that the main reason for this indication by the $N N L S$ analysis was the proper adjustment of the regularizer based on the criteria that we empirically approved and set for finding the optimum $\chi^{2}$ misfit. Based on the simulations in section 5.4 and our trials in analysis, we set specifically the condition

$$
1 \% \leq\left(\chi^{2}-\chi_{\min }^{2}\right) / \chi_{\min }^{2} \leq 3 \%
$$

into the $N N L S$-diff code to properly adjust the regularizer parameter, $0 \leq \mu \leq 1$, and to be able to more reliably relate the spectrum resulting from $N N L S$ analysis with regularization to the physical system. This condition was also used for our analysis of MR diffusion decays for human brain.

For the diffusion data analysis of the water phantom, the regularizer parameter was adjusted to $\mu=0.001$ to satisfy the above condition. However, these criteria also depend on the ndf for the measurements. For example, while $\mu$ was adjusted to $\mu=0.001$ to satisfy this condition in the experiment with $16 \mathrm{~b}$-values to show the homogeneity of the system by one peak, for the water phantom experiment with 96 b-values it was possible to accept a $10 \%$ difference in $\chi^{2}$ with the regularizer parameter adjusted to $\mu=0.005$ in order for the solution to reduce to a single peak. In contrast, the analysis for the experiment with $11 \mathrm{~b}$-values showed this physical situation with the $\%$ difference in $\chi^{2}=2 \%$ and $\mu=0.0005$. In all cases the mean value of $\mathrm{D}$ and the total content of the peak (i.e. the water signal) were the same. For consistency and to obtain reliable information about the homogeneity of the system from the FWHM of the peaks we set the criteria to be from $1 \%$ to $3 \%$ as given by equation (5.7) for the analysis of data from human brain. 


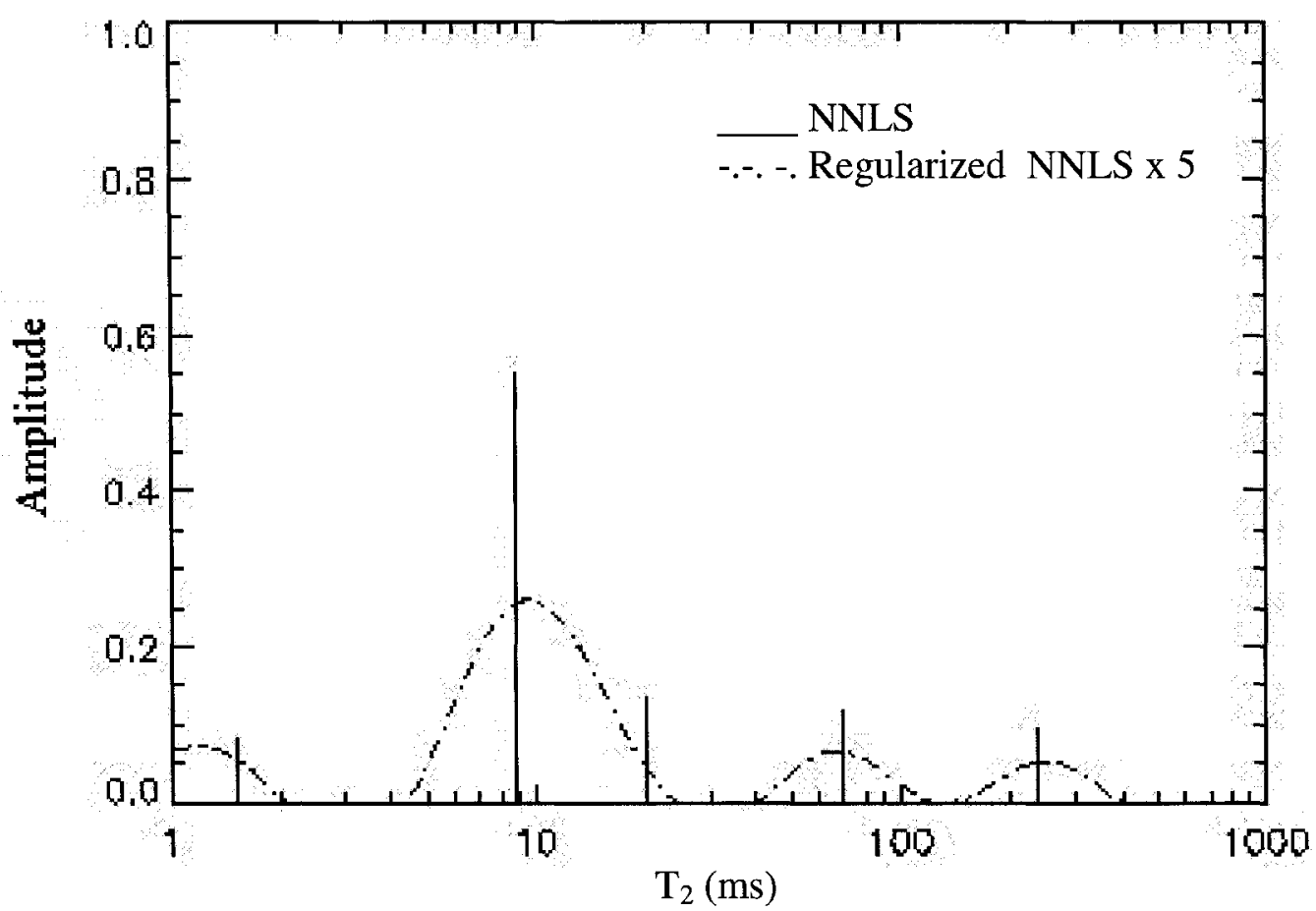

Figure 5.9. Typical spectra of $\mathrm{T}_{2}$ relaxation time distributions for a CPMG experiment on a $0.85 \%$ EYP emulsion using $N N L S$ showing the four main peaks. The discrete results are shown as solid lines and the results using the regularizer, which are multiplied by 5 in the figure, are drawn with a dashed line.

\subsection{Using $N N L S$ for $\mathrm{T}_{2}$ Relaxation Analysis in HXe Experiments}

As another test of our implementation of the $N N L S$ algorithm we used it to analyze $\mathrm{T}_{2}$ relaxation data which was kindly provided by Dr. Giles Santyr and his group from the Carleton Magnetic Resonance Facility (CMRF) at Carleton University. The first use of the NNLS algorithm in MR by Whittall et. al. was also a study of $T_{2}$ relaxation [108]. To use this code for $T_{2}$ analysis it was necessary to change the kernel of equation (2.97) and consequently to change the diffusion exponential components, $\exp \left(-\mathrm{b}_{\mathrm{i}} \mathrm{D}_{\mathrm{j}}\right)$, to $\mathrm{T}_{2}$ relaxation exponential terms, $\exp \left(-\mathrm{t}_{\mathrm{i}} / \mathrm{T}_{2 \mathrm{j}}\right)$, where 
the $t_{i}$ are the echo time intervals, nTE. We analyzed the $T_{2}$ decay of Hyperpolarized ${ }^{129} \mathrm{Xe}(\mathrm{HXe})$ dissolved in PerFluoro-Octyl Bromide (PFOB) emulsions studied by Wallace et. al. [149]. The purpose of the authors was to compare $\mathrm{T}_{2}$ relaxation of HXe dissolved in two PFOB emulsions containing two different droplet sizes $(1.4 \mu \mathrm{m}$ and $4.7 \mu \mathrm{m}$ diameters). The ultimate goal was to develop a theoretical compartmental framework to optimize the design of perfluorocarbon emulsions of HXe which may lead to a better way of delivering HXe to particular tissues and ultimately to find a more sensitive and precise way of measuring blood flow in breast cancer [149].

The ${ }^{129} \mathrm{Xe}$ gas was hyperpolarized by Dr. Albert Cross and the PFOB emulsions were prepared by Dr. Julia Wallace. She measured the $\mathrm{T}_{2}$ relaxation data at CMRF as well. The theoretical calculation with which the $N N L S$ results were compared was done by Mihai Gherase. The HXe $\mathrm{T}_{2}$ measurements were made using a CPMG (Carr, Purcell, Meiboom and Gill) sequence; a sequence with a cascade of $180^{\circ} \mathrm{RF}$ pulses to read the echo signals due to $T_{2}$ relaxation attenuation. The echo train consisted of 5000 echoes in increments of $0.4 \mathrm{~ms}$ from 0.4 to $2000 \mathrm{~ms}$. The signals were measured at $1.89 \mathrm{~T}$ using a Magnex (Abingdon, UK) $30 \mathrm{~cm}$ bore magnet after inserting the sample into a $1 \mathrm{~cm}$ diameter solenoid coil tuned to the ${ }^{129} \mathrm{Xe}$ resonance frequency $(22.18 \mathrm{MHz})$. Of the 5000 measured echoes, 1000 were used for the analysis, the rest were in the noise. The 1000 data points for the PFOB emulsions with $0.85 \%$ EYP $(4.7 \mu \mathrm{m}$ diameter droplets), and the 300 data points for the emulsions with $4 \%$ DPOC (1.4 $\mu \mathrm{m}$ diameter droplets) were analyzed with the extended $N N L S$ algorithm for the $T_{2}$ analysis using the data range of $n=150$ logarithmically scaled between 1 and $1000 \mathrm{~ms}$. EYP and DPOC are different kind of phospholipids. A typical spectrum of $\mathrm{T}_{2}$ relaxation data for the $0.85 \%$ EYP sample is shown in figure 5.9. The data for both emulsions were very well fitted to a multi-exponential decay curve by $N N L S$. The results were much better than the fit from a bi-exponential regression 


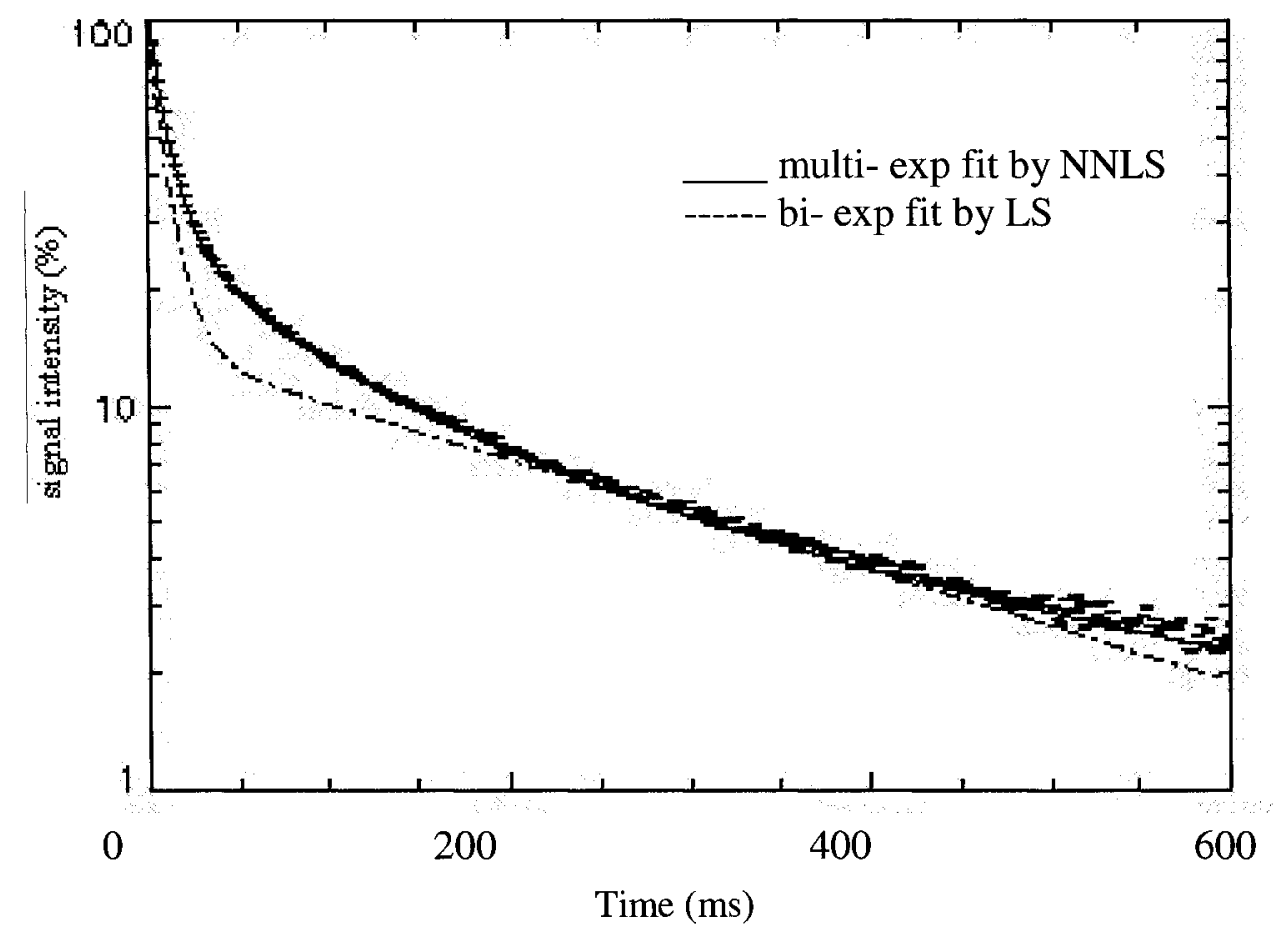

Figure 5.10. Comparison of the multi-exponential fit to the $T_{2}$ relaxation measurements for the EYP emulsion using the $N N L S$ method (solid line) versus the bi-exponential fit using non-linear least squares (dashed line).

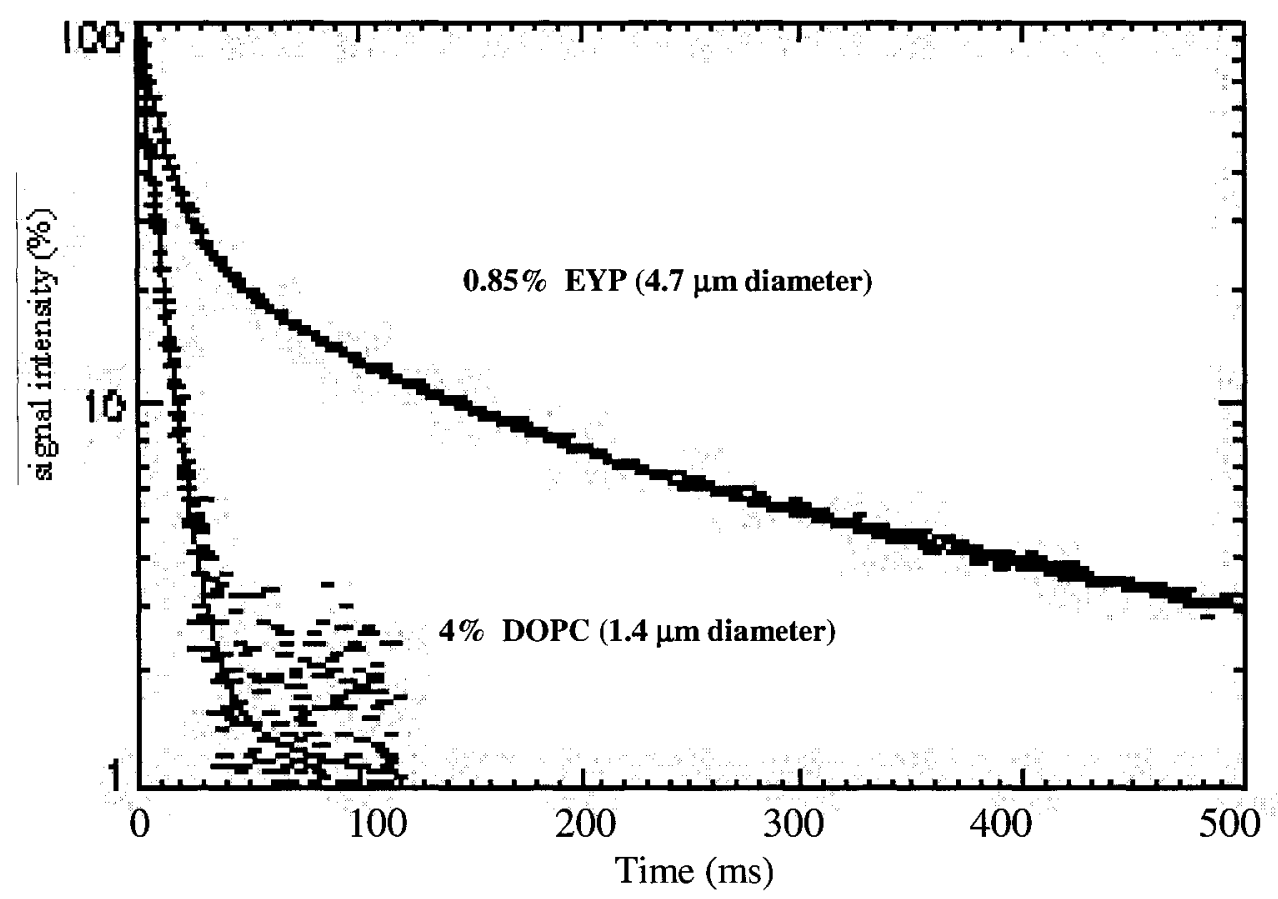

Figure 5.11. A typical best fit of $\mathrm{T}_{2}$ relaxation for the two emulsions by multi-exponential analysis using the $N N L S$ algorithm. 


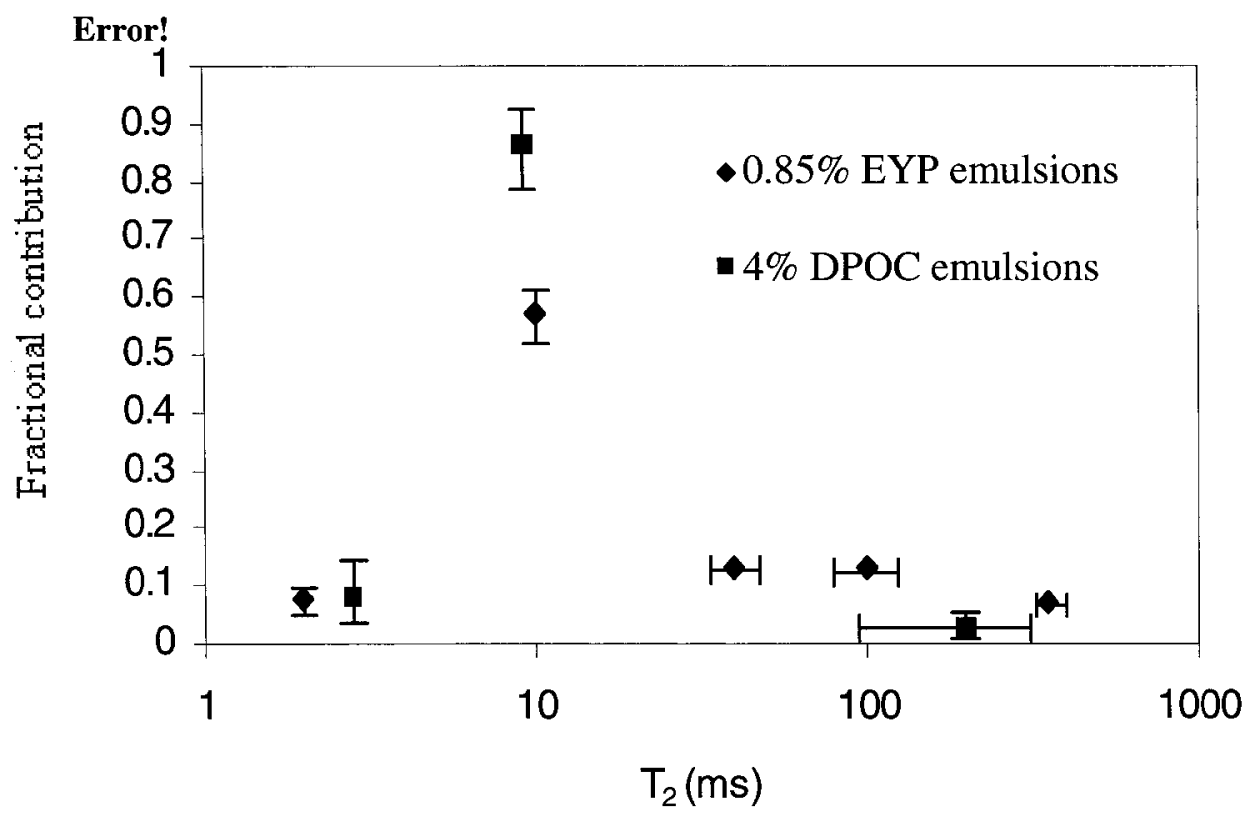

Figure 5.12. The $N N L S$ results for the two emulsions of short and long $\mathrm{T}_{2}$ relaxation in 4 experiments.

obtained using more conventional least squares analysis. Typical measured CPMG decay curves for the two emulsions of PFOB, $0.85 \%$ EYP and 4\% DPOC, are displayed in figures 5.10 and 5.11. The smaller droplet emulsion showed more than $95 \%$ contribution to $\mathrm{T}_{2}$ relaxation times of less than $10 \mathrm{~ms}$, whereas for the larger droplet emulsion, the longer $T_{2}$ component contributed more than $30 \%$ to the corresponding decay. The decay of the larger droplet emulsions is much slower than for the smaller emulsion droplets, as expected [149].

Figure 5.12 represents the proportion of short and long $T_{2}$ components with their error bars for 4 experiments and more than 40 data sets. The results were consistent with fast (smaller droplets) and intermediate (larger droplets) exchange of HXe between the droplets and the surrounding aqueous environment. From the authors' study, the multi-exponential behavior of the $\mathrm{T}_{2}$ decay of the PFOB emulsions is quite consistent with the predictions of chemical exchange 
theory [149]. Based on this theory, the CPMG curves for a single size droplet that is not exchanging would decay bi-exponentially.

The NNLS algorithm provided a multi-exponential solution for this complex emulsion system which gave a very good fit to the experimental data and agreed well with theoretical predictions. This gave us added confidence that the $N N L S$ algorithm was working properly and that it would be a good procedure to use for analyzing diffusion decays for human brain.

\subsection{The Data Analysis of the Diffusion Decay in Human Brain by NNLS-diff}

When the analysis of the diffusion decay for brain tissue was started we did not want to make any assumptions as to the number of components or what their diffusion coefficients might be. We used the $N N L S$ algorithm and began by considering $\mathrm{n}=150$ values logarithmically spaced at equal intervals (in log space) between $1 \times 10^{-5}$ and $0.1 \mathrm{~mm}^{2} / \mathrm{s}$ to cover the full range of possible diffusion coefficients. The stability of the results was confirmed by repeating the analysis 15 times all with 150 data points with small changes to the data interval, on a logarithmic scale, such that the data range being considered changed from $\left(1 \times 10^{-5}-0.01\right) \mathrm{mm}^{2} / \mathrm{s}$ to $\left(1 \times 10^{-5}-5.3\right) \mathrm{mm}^{2} / \mathrm{s}$. No major inconsistencies were observed in the results of the different analyses. NNLS was used both with the second derivative regularizer and with no regularizer and the results were consistent. Figure 5.13 shows the spectrum of diffusion coefficients for the diffusion decay of human brain tissue measured with 96 b-values. The mean $D_{a}$ value for each peak and its contribution to the decay along with their standard deviations were evaluated by NNLS-diff as part of the data analysis and the results are given in table 5.2.

The diffusion decay is very well fitted with the $N N L S$ results. For the discrete version of $N N L S$ we obtained $\chi_{\min }^{2}=91(\mu=0)<\mathrm{ndf}=96$ with $\sigma_{\chi^{2}}=0.68 \%$ of the maximum signal (figure 


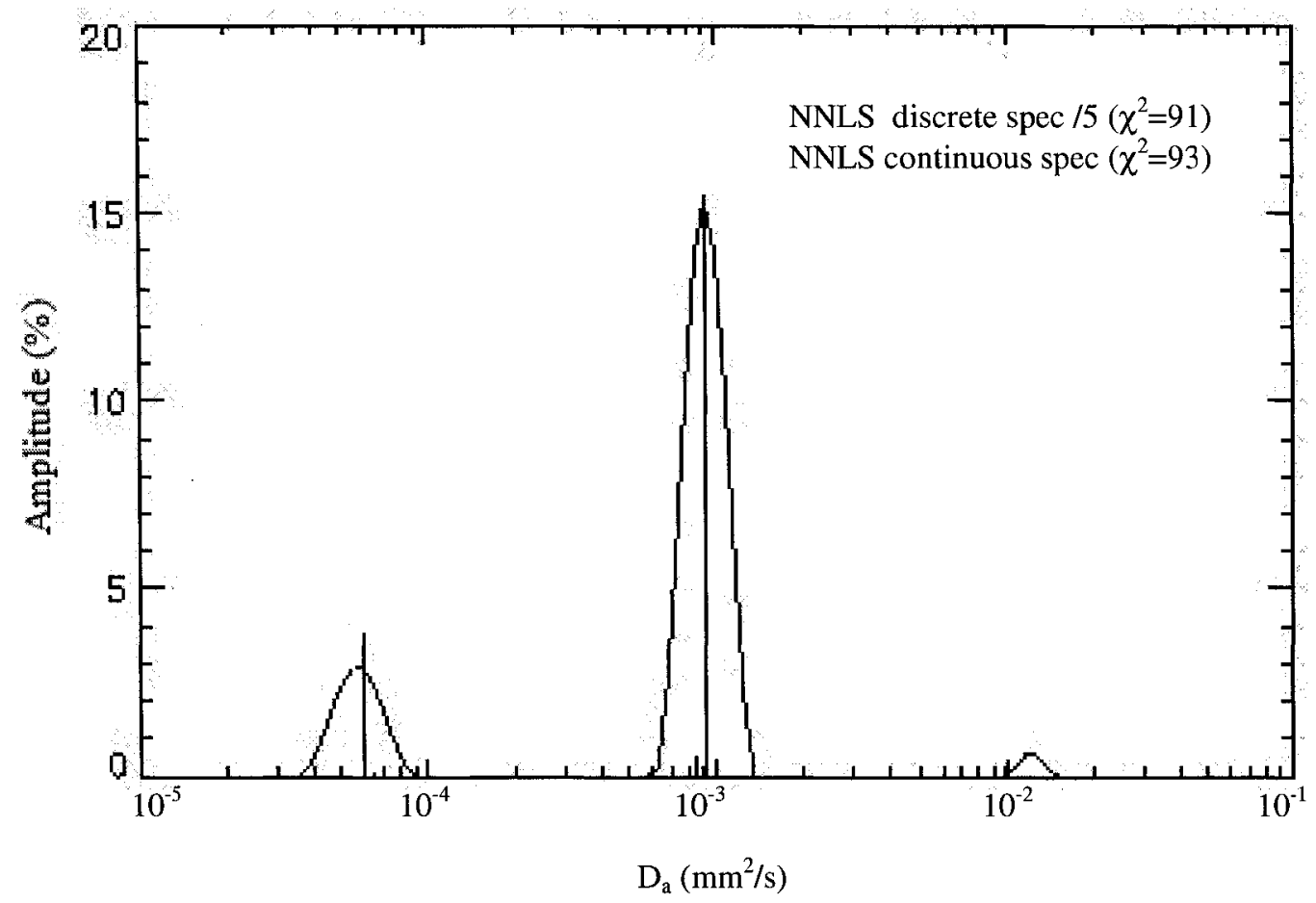

Figure 5.13. The spectrum of diffusion coefficients for human brain tissue observed by MR diffusion measurements in vivo. The data fractions were normalized to 100 for the continuous spectrum and to 20 for the discrete results. The mean values for each component and their contribution to the net decay are $D_{1}=0.06 \times 10^{-3}(19 \%) \mathrm{mm}^{2} / \mathrm{s}, D_{2}=0.93 \times 10^{-3}(80 \%) \mathrm{mm}^{2} / \mathrm{s}$ and $\mathrm{D}_{3}=1.2 \times 10^{-2}(1 \%) \mathrm{mm}^{2} / \mathrm{s}$.

5.13). When regularization was included in the $N N L S$ algorithm the regularizer parameter for this data analysis was adjusted to $\mu=0.1$ to give a smooth spectrum (figures 5.13 and 5.14) subject to the condition defined in equation (5.7) (i.e. $1 \% \leq\left(\chi^{2}-\chi_{\min }^{2}\right) / \chi_{\min }^{2} \leq 3 \%$ ). The $\chi^{2}$ misfit for this version of $N N L S$ was evaluated to be $\chi^{2}=93$ which is $2.2 \%$ higher than the minimum $\chi^{2}$ and falls within a reliable range for interpretation of the results from the continuous spectrum.

Three diffusion coefficients were observed for most (14 of 15) of the data analysis trials with $N N L S$ when the full data range was covered. The highest diffusion coefficient has a very small contribution of about $1 \%$ to the decay and its magnitude, $1.2 \times 10^{-2} \mathrm{~mm}^{2} / \mathrm{s}$, is larger than the 


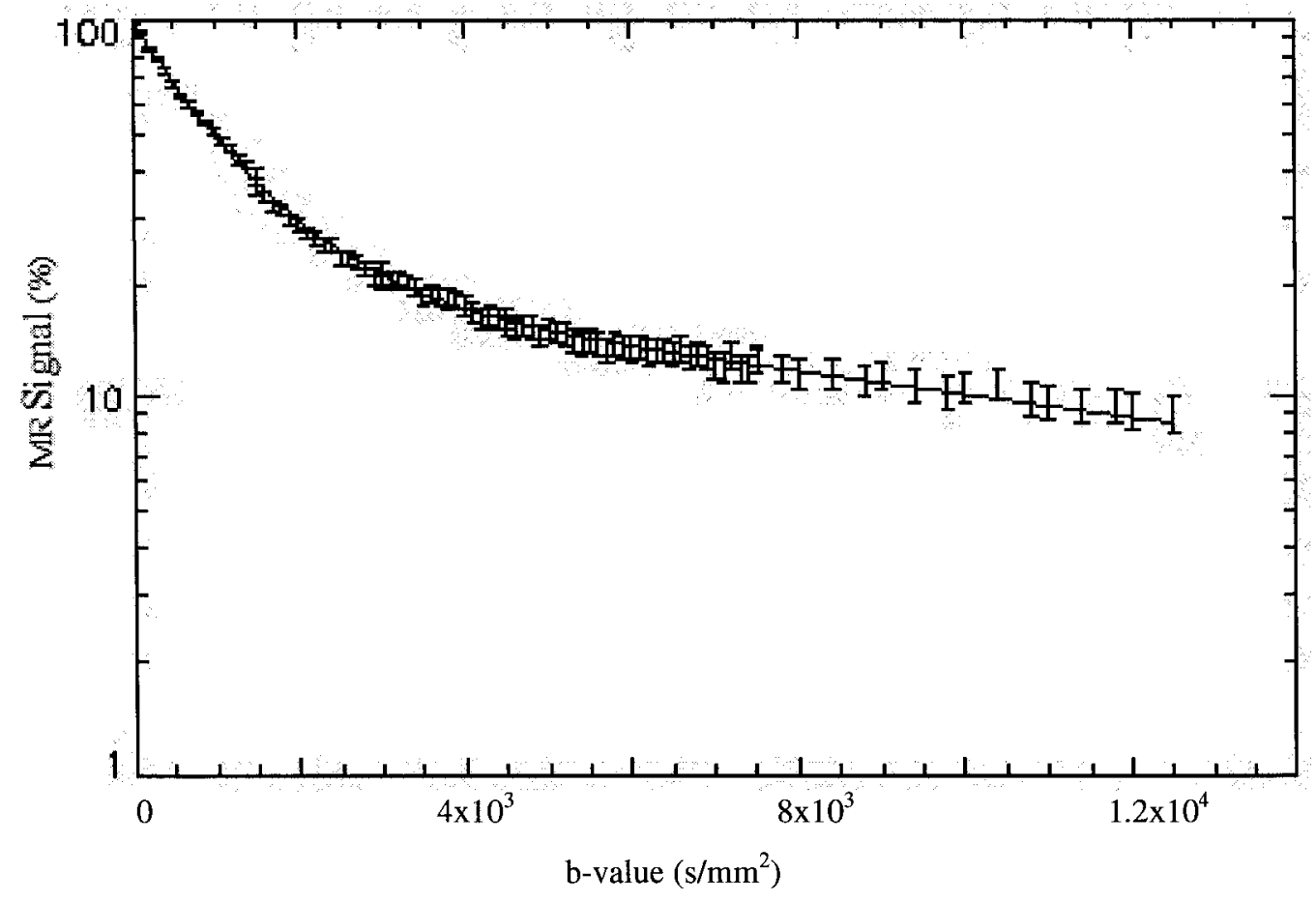

Figure 5.14. The diffusion decay curve of human brain tissue fitted with NNLS-diff results as a tri-exponential decay with diffusion coefficients of $\mathrm{D}_{1}=0.06 \times 10^{-3}(19 \%), \mathrm{D}_{2}=0.93 \times 10^{-3}(80 \%)$ and $\mathrm{D}_{3}=1.2 \times 10^{-2}(1 \%) \mathrm{mm}^{2} / \mathrm{s}$.

\begin{tabular}{|c|c|c|c|c|}
\hline \multicolumn{2}{|c|}{$\mathrm{D}_{\mathrm{a}}\left(\mathrm{mm}^{2} / \mathrm{s}\right)$} & \multicolumn{2}{c|}{$f(\%)$} & FWHM \\
\hline $\mathrm{D}_{1}$ & $(0.0576 \pm 0.002) \times 10^{-3}$ & $\mathrm{f}_{1}$ & $18.6 \pm 0.1$ & $0.028 \times 10^{-3}$ \\
\hline $\mathrm{D}_{2}$ & $(0.93 \pm 0.003) \times 10^{-3}$ & $\mathrm{f}_{2}$ & $80.5 \pm 0.08$ & $0.39 \times 10^{-3}$ \\
\hline $\mathrm{D}_{3}$ & $(1.2 \pm 0.02) \times 10^{-2}$ & $\mathrm{f}_{3}$ & $0.85 \pm 0.05$ & $0.1 \times 10^{-2}$ \\
\hline
\end{tabular}

Table 5.2. The quantitative results of diffusion measurements on human brain tissue. The FWHM represents the variance of the peaks in the continuous spectrum while the $\mathrm{D}_{\mathrm{a}}$ are the mean values (i.e. weighted average) of the diffusion coefficients for each peak; $\left(\sigma_{\chi^{2}}=0.68 \%, \chi_{\min }^{2}=91, \chi^{2}=93, \mu=0.1\right.$ and $\left.\mathrm{ndf}=96\right)$.

diffusion coefficient of pure water at body temperature, which is $3.0 \times 10^{-3} \mathrm{~mm}^{2} / \mathrm{s}$ [179]. As such, it is likely not diffusion at all but rather a manifestation of blood perfusion in the tissue $[9,172]$. Since this is probably not a diffusion component it was decided to omit this small component 
from subsequent analyses by reducing the range of test values for $N N L S$ to consider. The reduced range was reduced to $5 \times 10^{-5}$ to $1.5 \times 10^{-3} \mathrm{~mm}^{2} / \mathrm{s}$ with $\mathrm{n}=95$. This analysis gave a fast diffusion coefficient at $0.96 \times 10^{-3} \mathrm{~mm}^{2} / \mathrm{s}(79.3 \%)$ and a slower one at $0.07 \times 10^{-3} \mathrm{~mm}^{2} / \mathrm{s}(20.4 \%)$. This is the diffusion coefficient range used for the results presented in chapter 6 .

\subsection{Discussion}

The multi-exponential behavior of the diffusion signal decay in brain tissue observed in this study and others suggests that the current clinical practice of sampling only a few b-values under 1500 $\mathrm{s} / \mathrm{mm}^{2}$ largely limits the true information about the diffusion characteristics of human brain that can be obtained with these measurements. More information would be clearly accessed by extending the number of $b$-values measured and the b-value range in order to accurately measure the slowly diffusing component. The clinical utility of the information obtained using the conventional approach for the diagnosis of neurological disorders such as stroke, tumor and multiple sclerosis will be discussed and compared with the better understanding of this multicomponent diffusion behavior in brain that is provided when the diffusion decay is measured more completely. The multi-exponential diffusion decays observed in this study are basically consistent with observations in many different biological samples such as those reported by Pfeuffer et. al. [111] in rat brain in vivo, Stanisz et. al. [106] in bovine optic nerve and Mulkern et. al. [103] in human brain in vivo. Mulkern et. al. measured the diffusion signal decay for bvalues up to $6000 \mathrm{~s} / \mathrm{mm}^{2}$ and fitted the data with a bi-exponential curve. They reported fast and slow components of $1.02(66 \%) \mu \mathrm{m}^{2} / \mathrm{ms}$ and $0.11 \mu \mathrm{m}^{2} / \mathrm{ms}$, respectively, for water in the internal capsule of the white matter of human brain in vivo. Conturo et. al. [162] have theoretically shown that the use of higher b-values, and consequently higher diffusion sensitizing gradients, improves 
the accuracy of the diffusion coefficient obtained from the analysis when noise on the experimental data points is considered. Their results were derived by error propagation.

Our study of human brain in vivo was performed using an extended range of b-values up to $\mathrm{b}=12,5000 \mathrm{~s} / \mathrm{mm}^{2}$ and the maximum possible diffusion gradient taking care to deal with the Rician noise bias properly to improve the accuracy of our findings for the diffusion information of the brain tissue. Our results showed a clear deviation from mono-exponentiality, which was completely consistent with reports of others in the literature. One possible explanation for this effect is a compartmentalization of the water into various environments within the brain tissue such as the intracellular, extracellular and intravascular spaces where each of these components is considered to have its own intrinsic diffusion properties. It is normally assumed that the intracellular and extracellular environments have slow and fast intrinsic diffusion coefficients, respectively, and the intravascular space has an ultra fast pseudo diffusion coefficient due to the perfusion of the blood.

Pfeuffer et. al. [102] observed four discrete exponential components for rat brain in vivo. The diffusion decay was characterized with two main components, $D_{1}$ and $D_{2}$, equal to 0.3 $\mu \mathrm{m}^{2} / \mathrm{ms}$ and $1.1 \mu \mathrm{m}^{2} / \mathrm{ms}$ and together their contribution was between 80 and $95 \%$ of the full decay curve. The two slowly decaying components of $\mathrm{D}_{3}$ and $\mathrm{D}_{4}$ were of the order of 0.1 and $0.01 \mu \mathrm{m}^{2} / \mathrm{ms}$, respectively. The range of b-values used in these experiments was very large; up to $70,000 \mathrm{~s} / \mathrm{mm}^{2}$. It is significant to note that we also observed four discrete components in our multi-exponential analysis using $N N L S$-diff before the Rician noise correction algorithm was applied. We obtained diffusion coefficients equal to $0.01 \times 10^{-3}(6 \%), 0.09 \times 10^{-3}(13 \%), 0.93 \times 10^{-3}$ $(79 \%)$ and $10.1 \times 10^{-3}(1 \%) \mathrm{mm}^{2} / \mathrm{s}$. However, after applying the Rician noise correction algorithm the first two components were combined into one component equal to $0.06 \times 10^{-3}(19 \%) \mathrm{mm}^{2} / \mathrm{s}$. This was true for both the discrete version of $N N L S$ as well as for NNLS with second derivative 
regularizer. Thus, it seems likely that groups reporting a fourth diffusion component may not have dealt with the effects of the Rician noise bias properly. The third component observed in our work appears to be a real diffusion contribution and not an artifactual component related to the noise bias.

Although we measured the signal decay over a range of b-values starting from $100 \mathrm{~s} / \mathrm{mm}^{2}$ in order to avoid the effect of perfusing blood, it is still possible that the third component is due to intravascular blood flow in the capillaries. The observed contribution of this diffusion coefficient $(\approx 1 \%)$ is lower than the values normally reported in the literature $(4 \%)$ using the IVIM (IntraVoxel Incoherent Motion) model $[163,164]$ for the contribution of perfusion to the diffusion decay. However, the capillary density in the deep white matter where our ROI is located is lower than for other regions of the brain so it is not surprising that it would have a smaller effect than usual in this location.

Water in the cerebrospinal fluid (CSF) has a higher $D_{a}$ than intracellular and interstitial water, mainly because there are fewer barriers to the diffusion of water in CSF. This would suggest that CSF should contribute a very large diffusion coefficient. However, displacement of water molecules in the CSF that might lead to attenuation of the MR signal in a diffusion experiment is more likely associated with pulsations in the CSF due to changes in blood flow in the brain during the cardiac cycle. As blood is pumped into the brain, pressure is induced on the CSF causing it to be displaced. In this case the contribution of the CSF to the diffusion decay would correspond to a very short diffusion component. CSF is not expected to be present in the ROI considered here but if there were a small amount, this could also be an explanation for observed results. Thus, the fastest component of the signal attenuation could possibly arise from water in CSF. 
When making the diffusion measurements caution must be used to avoid contributions from artifacts in the images. Diffusion weighted single shot EPI was used to make the measurements since it has very low sensitivity to bulk motion, blood flow and pulsations which cause ghost artifacts and unwanted modulation to the signals in the phase encoding direction. This is an important advantage of EPI over other diffusion weighted sequences. EPI, however, can also be the source of other artifacts such as N/2 ghosting. The selection of a large FOV allowed us to choose the ROIs for the images such that the signal information could be determined without being influenced by $\mathrm{N} / 2$ ghosting. Extra care was taken to choose ROIs which avoided Gibbs artifacts as well. Diffusion measurements are also often affected by eddy currents, however, the TRSE sequence used for our measurements is specifically designed to minimize these effects.

As with many other studies reported in the literature, it is possible to associate the slow and fast diffusion coefficients from our results with the intracellular and extracellular environments of the brain tissue, respectively. However, signal fractions are inconsistent with the volume fraction of these compartments in the tissue; in fact, they appear to be reversed. This discrepancy was also observed by Niendorf et. al. [154] for healthy rat brain.

According to some authors, this discrepancy might be related to differences in $\mathrm{T}_{2}$ relaxation times for intracellular and extracellular water. That means that the signal arising from the region having a shorter $T_{2}$ (such as the intracellular space) should be more attenuated for a given $T_{2}$ weighting than that related to the region with the longer $T_{2}$ (such as the extracellular space). However, this suggestion has not been confirmed either by experiments with variable TE and constant $\tau_{D}$ or by $T_{2}$ measurements using CPMG sequences $[165,99,102,103,146]$. There are several studies in the literature $[146,165,166]$ of $T_{2}$ measurements employing the CPMG sequence to measure the $T_{2}$ relaxation distribution of human brain tissue. These studies show that 
the intracellular and extracellular $T_{2}$ relaxation times form a single peak between 50 and $150 \mathrm{~ms}$ that varies in width, but is not separated into two components. They also report another peak between 10 and $50 \mathrm{~ms}$ assigned to water in the myelin sheath.

Anisotropy effects due to the various orientations of axonal fiber tracts is another source of concern in diffusion measurements when only one diffusion gradient direction is used. The locations of the selected ROIs in the white matter were carefully chosen for these analyses to minimize the effects of anisotropy since the orientation of the axonal fibers at this location is expected to be perpendicular to the direction of the diffusion sensitizing gradient $(R \rightarrow L)$ used in our measurements for a very high percentage of the axons in the ROI. The multi-component analysis of these regions would show more components associated with the additional fiber orientations along with information on the different compartments involved in the region if this assumption was incorrect. This will be discussed in more detail in chapter 7 .

\subsection{Conclusions}

The tri-exponential diffusion results reported here, with diffusion coefficient components separated by an order of magnitude, show that the "true" description of diffusion in a heterogeneous system such as brain tissue cannot be explained in terms of a single weighted average diffusion coefficient as is implicitly assumed in many clinical diffusion measurements.

Factors affecting the accuracy of a diffusion measurement and its analysis were an important focus of the work presented in this chapter. Employing an extended range of b-values, up to the highest possible diffusion sensitizing gradient, on human brain in vivo was shown to be remarkably useful to obtain diffusion information with the highest possible accuracy. 
Multi-exponential analysis that gave four discrete diffusion components for our diffusion data was shown to be incorrect due to the effects of Rician noise bias. However, when the analysis was repeated with regularization the spectrum was stabilized to the main three peaks. The noise corrected diffusion decay, when analyzed either with the discrete or with the continuous multi-exponential model, was shown to contain three components. Nonetheless, complicating effects such as a distribution of cell size and type and the permeability of membranes to water, did not allow us to simply interpret our tri-exponential components in terms of three tissue compartments with distinct intrinsic diffusion coefficients.

The most important goal of this chapter was to determine the number of exponential components in the diffusion decay for the selected region of interest and to identify factors that can influence this. By using a large number of b-values over an extended range and by using Rician noise bias correction we were able to show using $N N L S$ analysis that the decay has two principle components and an additional very small contribution. Care was taken when choosing the ROI location to pick a region where anisotropy affects should be minimized. Thus, the components cannot be explained in terms of anisotropy of the axonal fibers.

Although it is often assumed that the diffusion decay has two principle components, consistent with our results for the selected ROI, this will not always be the case and should not be assumed. ROIs in different locations may well have additional components. Fitting diffusion data with more than two components to a bi-exponential model will produce incorrect information about the diffusion. This problem is avoided by using $N N L S$ analysis.

The ROI location for this work was chosen to avoid anisotropy effects to simplify the interpretation of the results. However, this analysis should also be extended to other ROI locations with different fiber orientations. Correlations with physiological parameters such as tissue perfusion, energy status and extracellular volume would also be desirable. 


\section{CHAPTER 6}

\section{Diffusion Time Dependence}

\subsection{Introduction}

Brain tissue is capable of dramatic but reversible changes in the apparent diffusion coefficient, $D_{a}$, depending upon the pathological state of the tissue. The challenge of MR diffusion is to find explanations for the changes in $\mathrm{D}_{\mathrm{a}}$ during ischemia or other pathologies, the changes in $\mathrm{D}_{\mathrm{a}}$ due to maturation and the mechanisms responsible for anisotropic diffusion. Many experimental and theoretical studies have been made to unravel the underlying mechanisms of the diffusion behavior and its change in brain tissue due to ischemia. The $D_{a}$ reduction within minutes of an ischemic insult is assumed to be mainly because of the shift of extracellular water to the intracellular space. It has also been proposed that changes in cell membrane permeability may cause the observed decrease in $\mathrm{D}_{\mathrm{a}}$. Modeling of in vivo diffusion experiments can help to determine physiological boundary conditions for testing analytical models. It can offer new insight into the processes that affect diffusion weighted measurements and it can contribute to a mechanistic understanding of the behavior of the brain tissue system. Many properties of brain 
tissue can be examined with these experiments in order to better understand the complex interaction between intracellular and extracellular compartments. These may include such things as intracellular and extracellular diffusion coefficients, the volume fractions of these compartments, cell membrane permeability, compartmental exchange times, and extracellular tortuosity. However, changes in the extracellular volume fraction and the extracellular tortuosity, as measured by iontopherosis (i.e. examining the concentration of ions introduced into the extracellular space as a function of time), have not been sufficient to determine the absolute value of the $D_{a}$ and/or its decrease during ischemia. Tissue parameters such as the intracellular and extracellular diffusion coefficients, the exchange time and intracellular diffusion restriction have to be measured simultaneously in order to understand the complex environment affecting diffusion in brain tissue.

Based on diffusion theory, as outlined in chapter 2, Einstein's equation for free diffusion in three dimensions is

$$
<\mathrm{r}^{2}>=6 \mathrm{D} \tau_{\mathrm{D}}
$$

showing that the mean squared displacement, $\left\langle\mathrm{r}^{2}\right\rangle$, of freely diffusible particles changes linearly with time, $\tau_{D}$, where the free diffusion coefficient, $D$, is time independent. MR diffusion measurements in pure liquids show this type of behavior where the mean squared displacement varies linearly with time.

However, for diffusion in a confined space where the motion of the particles is affected by the restricting geometry, the displacement will, in general, be a complicated non-linear function of the diffusion time, $\tau_{\mathrm{D}}$, the diffusion coefficient, $\mathrm{D}$, and the size and shape of the restricting geometry. Consequently, if the boundary effects are not properly accounted for and the data are analyzed using the model for free diffusion, the apparent diffusion coefficient, $D_{a}$, which is often referred to as the ADC, will be measured instead of the true diffusion coefficient for the system. 
In a pure liquid like water, the true diffusion coefficient corresponds to the bulk diffusion coefficient. The situation is, however, more complex in a macromolecular solution (e.g. cell cytoplasm, protein solution, etc.) where the water molecule has to move around larger "obstructing" molecules like proteins or organelles in brain tissue as well as interacting with protein hydration shells. These effects cause the true diffusion coefficient for the system to be an average bulk diffusion coefficient which is affected by all of the interactions that affect the diffusion of the molecule. The situation is further complicated by the effects of exchange through cell membranes. Diffusion of water molecules in brain tissue, which is mainly composed of intracellular and extracellular compartments partitioned by semi-permeable cell membranes, with an additional very small contribution from the vascular sub-region, can be pictured as diffusive particles confined to a sphere (see figure 2.6) with a finite probability of moving through the spherical barrier. This simple model is useful for discussing restricted diffusion and exchange. The exchange of the water molecule from the intracellular to the extracellular space which happens during the diffusion time, is related to its mean life time or residence time in the intracellular compartment.

In the short time limit, where $\tau_{\mathrm{D}}$ is much shorter than the mean life time in the compartment, such that the diffusing particle has not diffused far enough to feel the effect of the cell membrane as a boundary (on average), the system will behave as two distinct nonexchanging compartments with two independent diffusion coefficients. The diffusion coefficients are thus time independent for this case.

For the very long time limit, where a complete exchange between intracellular and extracellular compartments has occurred, the diffusion coefficient can be determined as the weighted average of the intracellular and extracellular diffusion coefficients. As discussed in section 2.2.3 for the diffusion of a particle confined to the inside of a sphere, the maximum 
distance that the confined water molecule can travel is limited by the boundaries, and thus the measured displacement and diffusion coefficient become independent of $\tau_{\mathrm{D}}$ in this limit as well. The diffusion decay for long diffusion times, however, is sensitive to the shape and dimensions of the restricting geometry.

In the intermediate time limit, the exchange regime, the behavior of the system is generally a more complicated weighted average of the intracellular and extracellular diffusion coefficients. Some of the particles feel the effects of restriction and the diffusion behavior measured within this time limit will be a non-linear function of $\tau_{D}$; the mean squared displacement will also not vary linearly with $\tau_{\mathrm{D}}$. There are a few analytical models, such as those given by Karger, that deal with restricted diffusion and exchange for a system in the intermediate exchange time limit [127]. These models will be discussed in section 6.5 and in chapter 7 where the experimental results will be interpreted in terms of restriction and exchange. The concept of restricted diffusion has been successfully applied to cell experiments $[15,113,148,156,170,175,181]$. It has also been adapted to study rat brain tissue using 'diffusion time dependence' imaging experiments on healthy rat brain in vivo $[99,102,111,125,126,153,154,155]$.

In this study, the $\tau_{\mathrm{D}}$ dependence of water molecules in MR diffusion imaging experiments is measured for healthy human brain in vivo over a range of diffusion times in the intermediate exchange limit for large b-values in order to examine the intracellular restricted diffusion and exchange behavior from the intracellular to the extracellular space. 

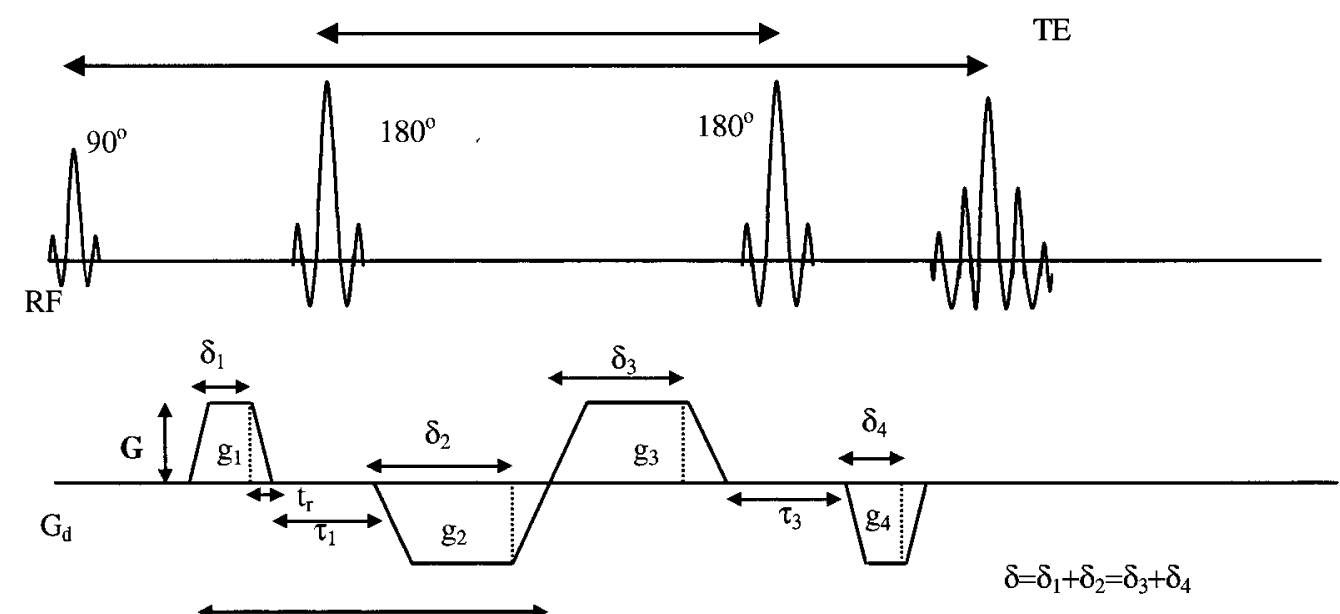

$\Delta$

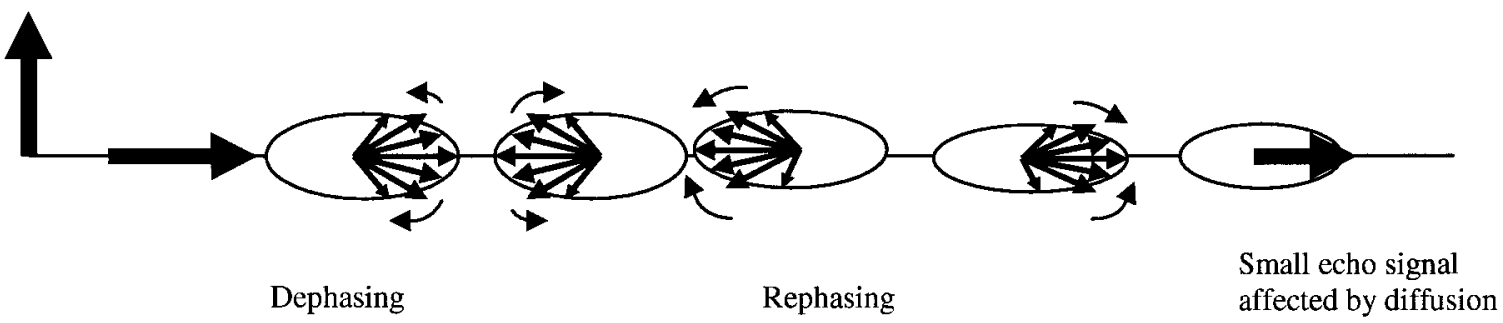

Figure 6.1. The TRSE diffusion pulse sequence with two pairs of bipolar diffusion gradients. The first two gradients dephase the spins, the second two gradients cause the spins to rephase again and the signal is attenuated due to diffusion of the water molecules.

\subsection{Materials and Methods}

\subsubsection{The TRSE sequence}

The TRSE sequence $[124,132]$ was originally designed to provide better image quality for diffusion weighted images compared with the conventional Pulsed Gradient Spin Echo (PGSE) sequence of Stejskal-Tanner (see also section 2.3.4). The PGSE diffusion sequence (see figure

2.8) combined with an echo planar readout typically induces large eddy currents and 
consequently time dependent magnetic field gradients which cause spatial distortions of the image. The TRSE sequence has been shown to reduce these distortions by adding another RF refocusing pulse to the spin echo sequence and splitting the field gradients into shorter pulses of alternating polarity (see figure 6.1) $[124,132]$. As shown in figure 6.1 , the two bipolar field gradients of length $\delta_{1}+\delta_{2}$ and $\delta_{3}+\delta_{4}$ are each split by RF refocusing pulses. Siemens' diffusion weighted sequence, which they call ep2d-diff, is a TRSE sequence with $\tau_{\mathrm{D}} \approx 50 \mathrm{~ms}$. To allow us to do this diffusion time dependence study we had to create our own version of ep2d-diff using Siemens sequence programming software so that $\tau_{\mathrm{D}}$ could be adjusted.

With the TRSE sequence the $90^{\circ} \mathrm{RF}$ pulse is initially applied to tip the magnetization into the XY plane. All of the spins are in phase immediately following the pulse. The signal then begins to decay due to $T_{2}$ relaxation. The first two diffusion sensitizing gradients $g_{1}=G$ and $g_{2}=$ $-G$, with equal amplitude but opposite polarity and unequal durations $\delta_{1}$ and $\delta_{2}$, respectively, dephase the net signal of the spins. The first refocusing $180^{\circ} \mathrm{RF}$ pulse is applied between these two gradients. The other two gradients $g_{3}=-G$ and $g_{4}=G$, which also have the same amplitude but opposite polarity and unequal durations $\delta_{3}$ and $\delta_{4}$, respectively, along with the second $180^{\circ} \mathrm{RF}$ pulse are applied to rephase the spins. The area under the diffusion sensitizing gradients of durations $\delta_{1}+\delta_{2}$ and $\delta_{3}+\delta_{4}$ must be equal to complete the rephasing of the spins.

Since the on and off field gradient ramps produce equal and opposite eddy currents, the shorter the time between these on and off transitions, the less the residual fields due to the eddy currents decay during the gradient pulse and the more complete the cancellation of the eddy current effect by these two ramps. The TRSE sequence also gives the possibility of a shorter TE for the same b-value compared with the Stejskal-Tanner sequence [124]. This makes the TRSE sequence a more efficient sequence since the measured signal is not affected by $T_{2}$ relaxation as much and therefore has a larger SNR. The diffusion gradients can be applied in the readout, the 
phase encoding, the slice selection direction or at any oblique or double oblique angle with the amplitude increasing in steps up to the highest value of $28.5 \mathrm{mT} / \mathrm{m}$ in 1 direction or $49.4 \mathrm{mT} / \mathrm{m}$ for all 3 directions (i.e. $28.5 \vec{i}+28.5 \vec{j}+28.5 \vec{k}$ ). This gives a maximum b-value of about 12,500 $\mathrm{s} / \mathrm{mm}^{2}$ for 1 direction and $38,000 \mathrm{~s} / \mathrm{mm}^{2}$ for 3 directions. The maximum b-value for the sequence is directly related with the echo time for the sequence. The two $180^{\circ} \mathrm{RF}$ pulses are delivered during the $\tau_{1}$ and $\tau_{3}$ time periods.

The sequence timing for the TRSE sequence (see figure 6.2) should satisfy the following conditions $[124,132]$ :

$$
\begin{gathered}
\delta_{1}+\delta_{2}+\delta_{3}+\delta_{4}=\mathrm{T}, \\
\delta_{1}+\delta_{2}=\delta_{3}+\delta_{4}, \\
-\delta_{1}+\delta_{2}+\delta_{3}=\delta_{4}+\mathrm{t}_{\mathrm{s}},
\end{gathered}
$$

where $T$ is the total duration of the diffusion gradients and $t_{s}$ is the time after the end of the fourth diffusion gradient at which the echo occurs. From these expressions it can easily be shown that

$$
\begin{aligned}
& \delta_{2}=\frac{\mathrm{T}}{2}-\delta_{1}, \\
& \delta_{3}=\frac{\mathrm{t}_{\mathrm{s}}}{2}+\delta_{1},
\end{aligned}
$$

and

$$
\delta_{4}=\frac{\mathrm{T}-\mathrm{t}_{\mathrm{s}}}{2}-\delta_{1}
$$

These can also be related to the echo time, TE, by considering the full sequence timing. For example, if it is assumed that $\tau_{1}$ and $\tau_{3}$ are both very small compared with the gradient pulse durations and that the ramp times are negligible, then $\mathrm{TE}=\mathrm{T}+\mathrm{t}_{\mathrm{s}}$. 
In ep2d-diff, Siemens' TRSE sequence, spoiler gradients of amplitude $16.5 \mathrm{mT} / \mathrm{m}$ and duration $1160 \mu$ s are included immediately after each $180^{\circ}$ pulse to dephase the small FID that will be generated by these pulses since it is difficult to generate a perfect $180^{\circ}$ pulse. The dephasing effects of these spoiler gradients on the spins contributing to the echo at TE are cancelled out by identical gradients inserted immediately before the $180^{\circ}$ pulses. The contribution of this pair of spoiler gradients to $b$ will also be negligible. These spoiler gradients are retained in the various sequence modifications presented in this chapter but are not considered explicitly in what follows.

The timing of the TRSE sequence, as presented by Heid [132] and implemented by Siemens in ep2d-diff, is optimized to reduce eddy current effects as much as possible by imposing the additional constraint that

$$
\delta_{1}=\frac{1}{\lambda_{0}} \ln \left(\frac{1+\cosh \left(\frac{\lambda_{0} \mathrm{~T}}{2}\right)}{\mathrm{e}^{\lambda_{0} t_{\mathrm{s}} / 2}+\mathrm{e}^{-\lambda_{0} \mathrm{~T} / 2}}\right)
$$

where $\lambda_{0}$ is the effective exponential time constant for the decay of the eddy currents. As shown in section 6.2.3, eddy currents were not a major concern for the present study so we did not impose this constraint for the short $\tau_{\mathrm{D}}$ sequences developed for this study. This allowed us more freedom in designing these sequences. The b-values calculated by the sequence code were no longer correct and had to be re-calculated offline using the equation developed in section 6.2.2.

\subsubsection{The $b$-factor and $\tau_{\mathrm{D}}$ evaluation for the TRSE sequence}

The diffusion decay is a plot of the MR signal intensity as a function of the b-factor for the sequence used. The calculation of the b-factor for the TRSE sequence is not as simple as for the 


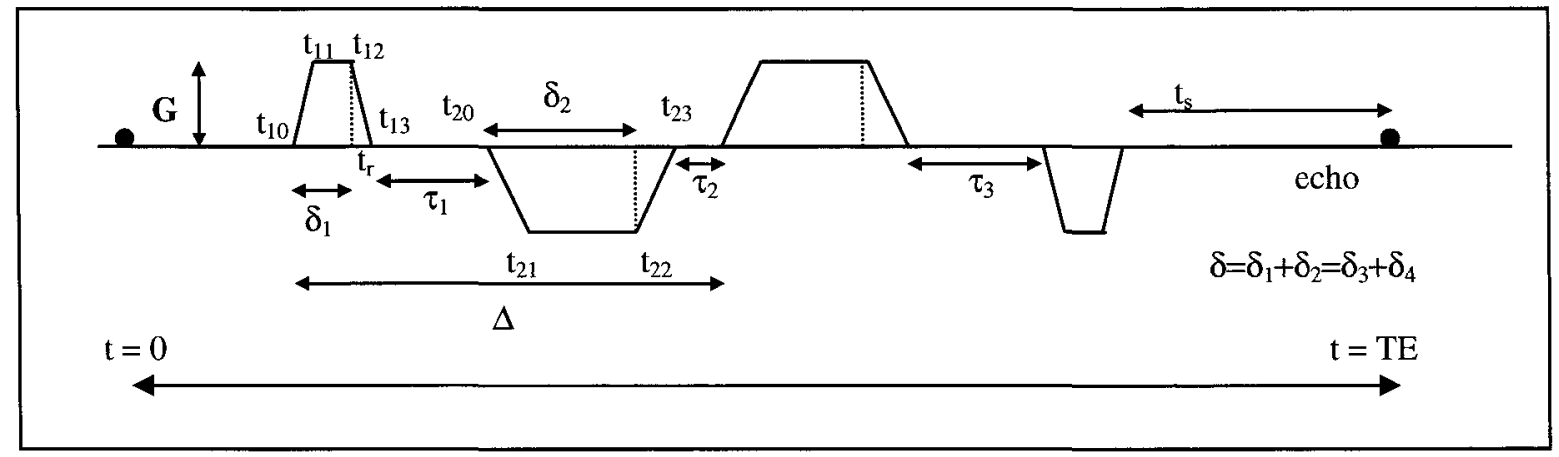

Figure 6.2. The TRSE sequence timing for the b-value and $\tau_{\mathrm{D}}$ evaluation.

PGSE sequence. The b-factor for the TRSE sequence was analytically evaluated by the integration of equation (2.74) as follows:

$$
\mathbf{b}=\gamma^{2} \int_{0}^{\mathrm{TE}} \mathrm{F}^{2}(\mathrm{t}) \mathrm{dt}=\gamma^{2} \int_{0}^{\mathrm{TE}}\left(\int_{0}^{\mathrm{t}} \mathbf{g}\left(\mathrm{t}^{\prime}\right) \mathrm{dt^{ \prime }}\right)^{2} \mathrm{dt} .
$$

Sequence timing diagrams for the TRSE sequence are shown in figures 6.1 and 6.2. The on and off ramps are both of duration $t_{\mathrm{r}}$ for all four gradient pulses and the ramps are linear. The duration of the $i^{\text {th }}$ gradient pulse, which is denoted $\delta_{i}$, includes the ramp on time but not the ramp off time (e.g. $\left.\delta_{1}=\mathrm{t}_{\mathrm{r}}+\left(\mathrm{t}_{12}-\mathrm{t}_{11}\right)\right)$. The $180^{\circ} \mathrm{RF}$ pulses are applied in the $\tau_{1}$ and $\tau_{3}$ time periods. No gradient or RF pulses are applied during the time period labeled $\tau_{2}$. The effect of the $180^{\circ} \mathrm{RF}$ pulses is to change the phase angle of the spins from $\phi$ to $-\phi$. This effect causes a gradient pulse of amplitude $\mathrm{G}$ and duration $\delta$ following the $180^{\circ}$ pulse to have the same effect that a gradient pulse of amplitude $-\mathrm{G}$ and duration $\delta$ would have without the $180^{\circ}$ pulse as shown in figure 6.1 (see also section 2.3). This effect will be incorporated into what we will call the effective gradient amplitudes which, for the TRSE sequence shown in figure 6.1, are given by $g_{1}=g_{2}=-g_{3}=-g_{4}=G$. 
The integral in equation (6.5) is most easily solved by breaking the outer integral into the sum of integrals over which the gradient amplitude is zero, a non-zero constant or a linear function of time (i.e. the ramps). Thus, the integral can be expanded as:

$$
\begin{aligned}
\frac{b}{\gamma^{2}} & =\int_{t_{10}}^{t_{13}} F^{2}(t) d t+\int_{t_{13}}^{t_{20}} F^{2}(t) d t+\int_{t_{20}}^{t_{23}} F^{2}(t) d t+\int_{t_{23}}^{t_{30}} F^{2}(t) d t+\int_{t_{30}}^{t_{33}} F^{2}(t) d t+\int_{t_{33}}^{t_{40}} F^{2}(t) d t+\int_{t_{40}}^{t_{43}} F^{2}(t) d t+\int_{t_{43}}^{T E} F^{2}(t) d t \\
& =I_{\delta_{1}}+I_{\tau_{1}}+I_{\delta_{2}}+I_{\tau_{2}}+I_{\delta_{3}}+I_{\tau_{3}}+I_{\delta_{4}}+I_{\tau_{4}}
\end{aligned}
$$

These integrations are carried out in detail in Appendix II. The resulting expression for $b$ is:

$$
\frac{b}{\gamma^{2}}=\sum_{i=1}^{4}\left\{\frac{G^{2}}{60}\left[20 \delta_{i}^{3}+30 \delta_{i}^{2} t_{r}-5 \delta_{i} t_{r}^{2}+t_{r}^{3}\right]+\operatorname{Mom}_{i-1} \operatorname{Mom}_{i}\left(\delta_{i}+t_{r}\right)+\operatorname{Mom}_{i} \operatorname{Mom}_{i} \tau_{i}\right\},
$$

where $\operatorname{Mom}_{\mathrm{i}}=\sum_{0}^{\mathrm{i}} \mathrm{g}_{\mathrm{i}} \delta_{\mathrm{i}}$ and the effective gradient amplitudes are $\mathrm{g}_{0}=0$ and $\mathrm{g}_{1}=\mathrm{g}_{2}=-\mathrm{g}_{3}=-\mathrm{g}_{4}=\mathrm{G}$.

If we also set $\delta_{1}=\delta_{4}$ and $\delta_{2}=\delta_{3}$, which is true for the TRSE sequences being considered here, then it can be shown that (see Appendix II).

$$
\frac{\mathrm{b}}{\gamma^{2} \mathrm{G}^{2} \delta^{2}}=\left(\Delta-\tau_{1}\right)-\frac{\delta}{3}+\left[\frac{\delta_{1}^{2}}{\delta^{2}}\left(\tau_{1}+\tau_{3}\right)+\mathrm{t}_{\mathrm{r}}\left(\frac{\mathrm{t}_{\mathrm{r}}^{2}}{\delta^{2}}-\frac{\mathrm{t}_{\mathrm{r}}}{6 \delta}+\frac{2 \delta_{1}^{2}}{\delta^{2}}-1\right)\right] \text {, }
$$

where

$$
\delta=\delta_{1}+\delta_{2}
$$

and

$$
\Delta=\delta_{1}+\delta_{2}+2 \mathrm{t}_{\mathrm{r}}+\tau_{1}+\tau_{2}
$$

(see figure 6.1).

The diffusion time, $\tau_{\mathrm{D}}$, can be defined from equation (6.8), by analogy with the PGSE sequence of Stejskal and Tanner (see section 2.3.3) as

$$
\tau_{\mathrm{D}}=\frac{\mathrm{b}}{\gamma^{2} \mathrm{G}^{2} \delta^{2}}=\left(\Delta-\tau_{1}\right)-\frac{\delta}{3}+\left[\frac{\delta_{1}^{2}}{\delta^{2}}\left(\tau_{1}+\tau_{3}\right)+\mathrm{t}_{\mathrm{r}}\left(\frac{\mathrm{t}_{\mathrm{r}}^{2}}{\delta^{2}}-\frac{\mathrm{t}_{\mathrm{r}}}{6 \delta}+\frac{2 \delta_{1}^{2}}{\delta^{2}}-1\right)\right] .
$$


When $t_{1}=0$ and $\tau_{1}=\tau_{3}=0$, the TRSE sequence reduces to a form of the PGSE sequence and equation (6.9) reduces to equation (2.85), as it should.

The diffusion time and b-factor were also evaluated for the variations of the TRSE sequence shown in figures $6.3 \mathrm{~b}$ and $6.3 \mathrm{c}$. Equation (6.7) is valid for these cases except that the summation is over two terms instead of four and the effective gradient amplitudes are $g_{1}=-g_{2}=G$. For these cases equation (6.7) gives

$$
\tau_{D}=\frac{b}{\gamma^{2} G^{2} \delta^{2}}=\Delta-\frac{1}{3} \delta-\frac{t_{r}^{2}}{6 \delta}\left[1-\frac{t_{r}}{5 \delta^{2}}\right] .
$$

The maximum possible $b$-value for the Siemens sequence was calculated to be 12,500 $\mathrm{s} / \mathrm{mm}^{2}$ for a gradient amplitude of $28.5 \mathrm{mT} / \mathrm{m}$ in one direction using equation (6.7). The diffusion time was evaluated for the Siemens sequence from equation (6.9) to be $\tau_{D} \cong 50 \mathrm{~ms}$. The echo time for this sequence is $\mathrm{TE}=200 \mathrm{~ms}$.

\subsubsection{Optimizing the diffusion pulse sequence for short $\tau_{\mathrm{D}}$ and high $b$-values}

The TRSE sequence was modified to obtain the shortest possible $\tau_{D}$ with the highest available bvalue for human subjects. The main difficulty with this is that the maximum possible b-value decreases with decreasing $\tau_{\mathrm{D}}$. In order to find the best compromise between maximizing the $b$ value and reducing $\tau_{D}$, we developed the sequence in the various forms shown in figure 6.3 to find the most appropriate sequence for our study. For all of the sequence variations considered here the position of the RF pulses, and therefore TE, was kept the same to avoid introducing $\mathrm{T}_{2}$ relaxation effects into our results.

The first possibility was considered to use the same basic formation as the original TRSE sequence where the gradient amplitude of all four gradients was kept the same. The duration of 
the gradients was varied such that the diffusion time evaluated using equation (6.9) would change due to these variations. The two refocusing RF pulses are located at specific times in the sequence based on the desired echo time. Thus, by deciding to keep TE constant we could only change $\tau_{D}$ by adjusting the diffusion gradient durations or their start times. Consequently, the range over which $\tau_{\mathrm{D}}$ could be adjusted was limited for this sequence. The maximum possible b-value was $12,500 \mathrm{~s} / \mathrm{mm}^{2}$ (for one direction) with TE=200 ms, $\delta_{1}=22480 \mu \mathrm{s}, \delta_{2}=45930 \mu \mathrm{s}, \delta_{3}=48610$ $\mu \mathrm{s}, \delta_{4}=19800 \mu \mathrm{s}, \tau_{1}=\tau_{3}=4010 \mu \mathrm{s}$ and $\tau_{2}=0$. By the variation of $\delta=\delta_{1}+\delta_{2}=\delta_{3}+\delta_{4}$ between 6 and $70 \mathrm{~ms}$ and a corresponding adjustment of $\tau_{2}$, the diffusion time, $\tau_{\mathrm{D}}$, was changed between 46 to $85 \mathrm{~ms}$.

In order to have more flexibility with $\tau_{\mathrm{D}}$ variation to shorter values for the same $\mathrm{TE}$ of 200 $\mathrm{ms}$, two of the diffusion gradients were nulled to remove contributions to $b$ and $\tau_{\mathrm{D}}$ from times following the second refocusing RF pulse. To do this, we had to keep either the first and third gradients (named the $\mathrm{g}_{1} \mathrm{~g}_{3}$ sequence) or the second and third gradients (named the $\mathrm{g}_{2} \mathrm{~g}_{3}$ sequence) and set the other gradient amplitudes to zero (see figures 6.3). By investigating the two new forms of the sequence, we found that the $\mathrm{g}_{2} \mathrm{~g}_{3}$ sequence is the optimum pulse sequence for our $\tau_{\mathrm{D}}$ dependence study.

We started from the shortest possible duration of the diffusion gradient, $\delta=\delta_{1}=\delta_{3}=1000$ $\mu$ s for the $\mathrm{g}_{1} \mathrm{~g}_{3}$ sequence or $\delta=\delta_{2}=\delta_{3}=1000 \mu$ s for the $\mathrm{g}_{2} \mathrm{~g}_{3}$ sequence. Although there was the advantage of gaining the shortest diffusion time of $5000 \mu$ s for $g_{1} g_{3}$ and $600 \mu$ s for $\mathrm{g}_{2} \mathrm{~g}_{3}$, the maximum possible b-values were too low to be able to obtain appropriate information from the experiments. Therefore, we decided to increase $\delta$. With $\delta$ set to between 10 and $19 \mathrm{~ms}, \tau_{\mathrm{D}}$ for the $\mathrm{g}_{1} \mathrm{~g}_{3}$ sequence could be varied from 8 to $88 \mathrm{~ms}$ (evaluated by equation $(6.10)$ ) with the maximum 

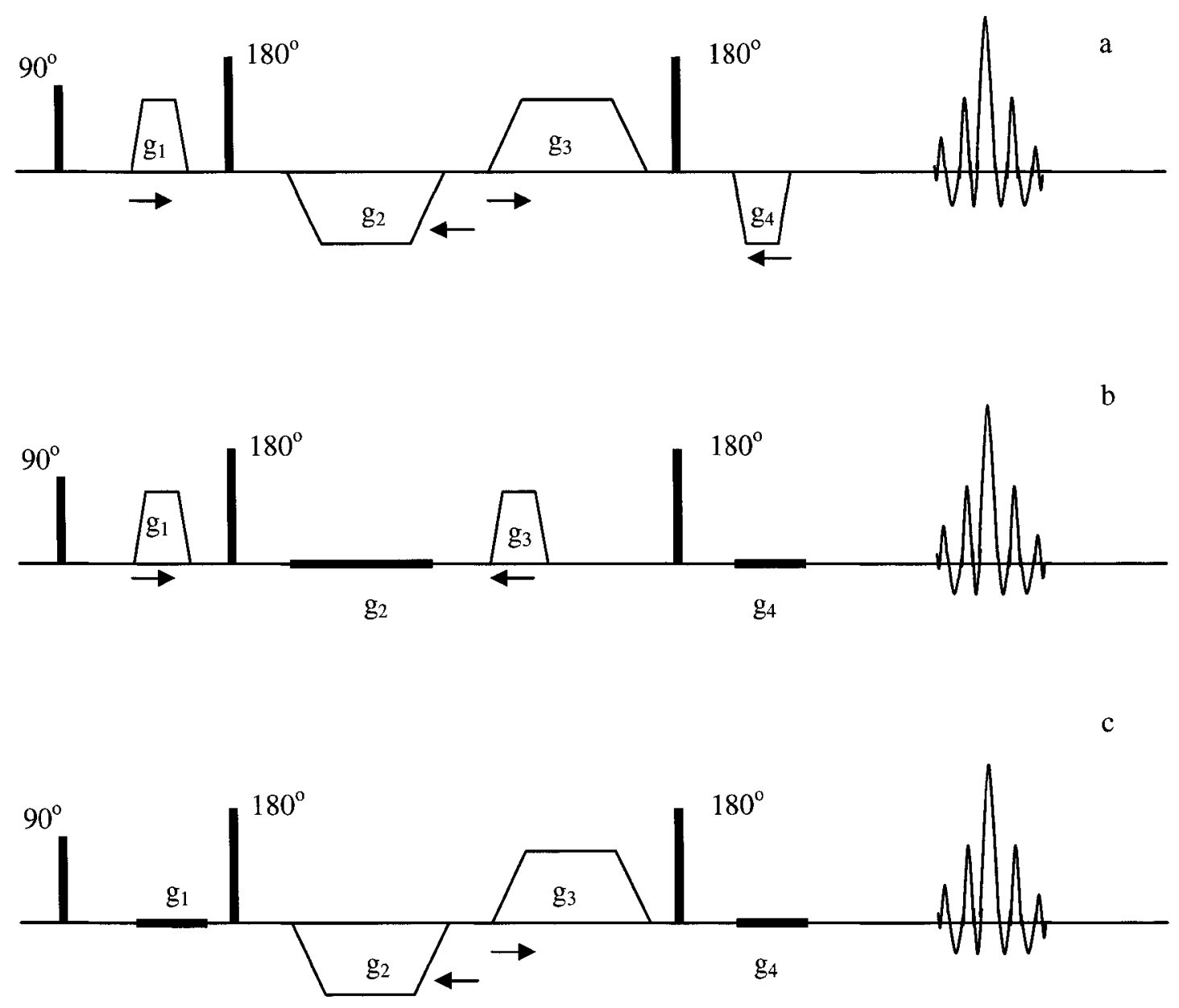

Figure 6.3. a. The TRSE sequence developed based on keeping two pairs of bipolar diffusion gradients. This sequence is limited to diffusion times, $\tau_{\mathrm{D}}$, between 46 and $85 \mathrm{~ms}$ with maximum b-values up to $12,500 \mathrm{~s} / \mathrm{mm}^{2}$. b. The TRSE sequence with one pair of bipolar gradients $\left(\mathrm{g}_{1}\right.$ and $\left.\mathrm{g}_{3}\right)$. This sequence, which we call the $\mathrm{g}_{1} \mathrm{~g}_{3}$ sequence, can have $\tau_{\mathrm{D}}$ values between 8 and $88 \mathrm{~ms}$ with $\delta$ between 10 and $19 \mathrm{~ms}$ and the maximum b-values between 11 and $2600 \mathrm{~s} / \mathrm{mm}^{2}$, respectively. c. Another TRSE sequence with one set of bipolar gradients $\left(g_{2}\right.$ and $\left.g_{3}\right)$. For this sequence, which we call the $\mathrm{g}_{2} \mathrm{~g}_{3}$ sequence, $\tau_{\mathrm{D}}$ can be varied between 6.0 and $53.8 \mathrm{~ms}$ with $\delta$ between 10 and $30 \mathrm{~ms}$ and maximum b-values between 40 and $2835 \mathrm{~s} / \mathrm{mm}^{2}$, respectively.

b-values ranging from 11 to $2600 \mathrm{~s} / \mathrm{mm}^{2}$ for the different $\tau_{\mathrm{D}}$ 's, respectively. However, $\tau_{\mathrm{D}}$ for the $\mathrm{g}_{2} \mathrm{~g}_{3}$ sequence showed more flexibility for shorter diffusion times. By changing $\delta$ to between 10 

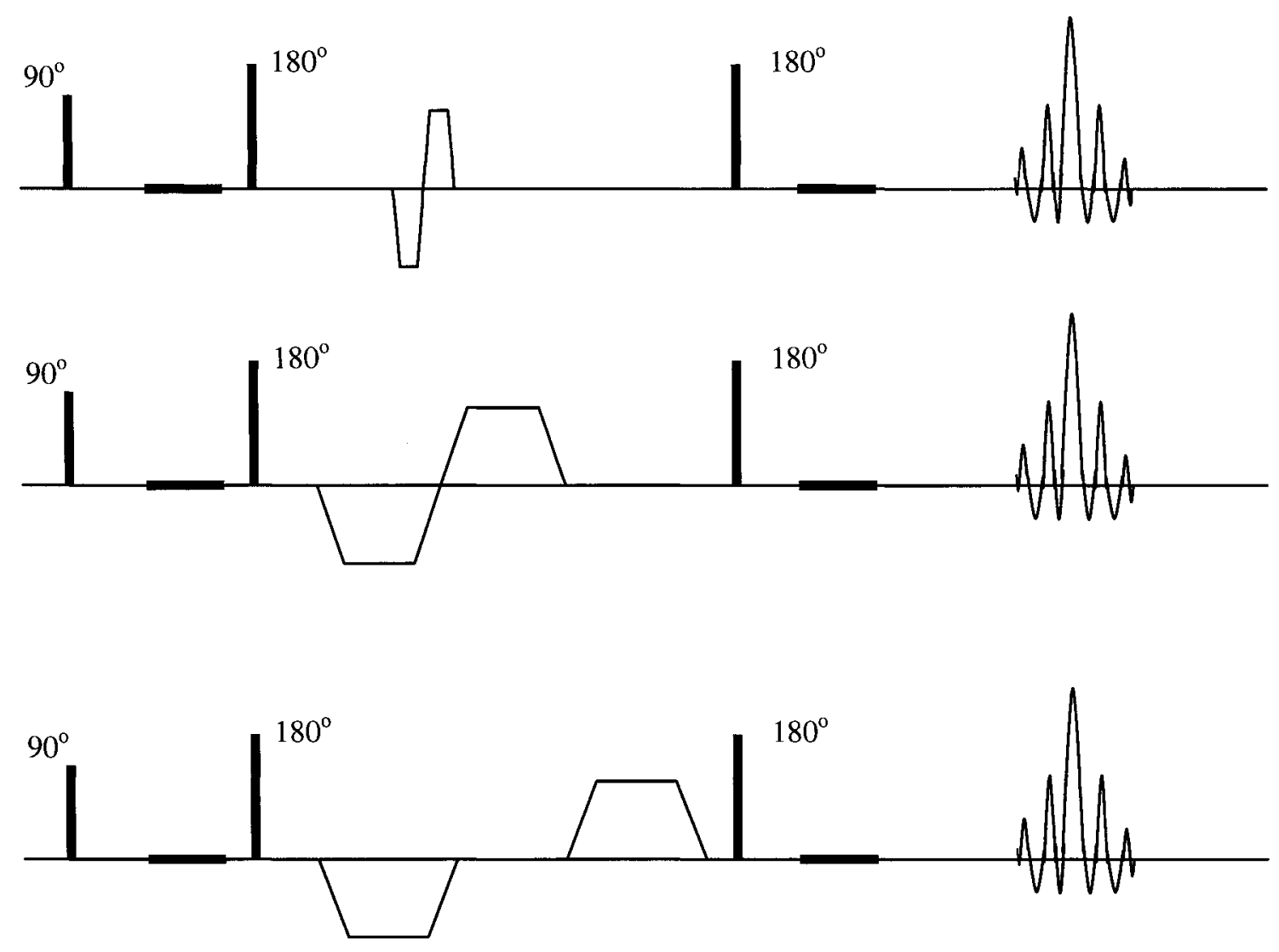

Figure 6.4. The typical $\mathrm{g}_{2} \mathrm{~g}_{3}$ diffusion sequence for diffusion times of 6,20 and $54 \mathrm{~ms}$ from top to bottom, respectively.

and $30 \mathrm{~ms}$ for this sequence, $\tau_{\mathrm{D}}$ could be varied between 6.0 and $53.8 \mathrm{~ms}$ giving the maximum possible b-value between 40 and $2835 \mathrm{~s} / \mathrm{mm}^{2}$, respectively. This $\mathrm{g}_{2} \mathrm{~g}_{3}$ sequence thus was chosen as the best compromise for the requirements of the diffusion time dependence study in the short time limit. Figure 6.4 shows a few typical $g_{2} g_{3}$ sequence configurations for $\tau_{D}=6$ ms with $\delta=10$ ms and for $\tau_{D}=20$ and $54 \mathrm{~ms}$ with $\delta=30 \mathrm{~ms}$. 
Another reason for choosing this sequence for this study, was that by keeping the same bipolarity of the diffusion gradients, the eddy current nulling effects of the original sequence were maintained. This issue was investigated for a series of diffusion measurements on a water phantom employing the diffusion gradients in the frequency encoding, the phase encoding and the slice selection direction for the $\mathrm{g}_{2} \mathrm{~g}_{3}$ sequence over the full range of diffusion times. Siemens' sequence was used as the control case. A very slight edge magnification was observed in a few of the images from $\mathrm{g}_{2} \mathrm{~g}_{3}$ for high $\mathrm{b}$-values. It should be noted that this slight edge magnification observed in the water phantom images was not observable in human brain images.

For the evaluation of these slight magnifications, we subtracted the $b=0$ image from the image corresponding to the maximum b-value for each sequence. Figure 6.5 shows typical water phantom subtraction images for the sequence with $\tau_{D}=53.8 \mathrm{~ms}$ which was the worst case when compared with the Siemens sequence. The part of the image magnified at the edge is shown by the arrow. Figures 6.6 show typical phantom images after subtraction for all of the developed sequences as well as for the Siemens sequence. No matter which diffusion gradient direction was used, these edge enhancements, when seen, were always observed in the phase encoding direction.

The unwanted edge magnification is barely observable, as shown by the arrow in figures 6.6 on the image for the $\tau_{\mathrm{D}}=53.8 \mathrm{~ms}$ sequence. We measured the signal magnitude at the edge of the subtracted images obtained for each diffusion time variant of the pulse sequence pixel by pixel for 10 to 20 pixels in the vicinity of the observed edge magnification and averaged over these pixels. The means and standard deviations for these calculations are shown in table 6.1. As seen in figure 6.7, the edge magnification, even in the worst case, is under the noise level.

When the direction of the diffusion gradient is changed the nature of the image distortion is 
$\mathrm{b}=0 \mathrm{~s} / \mathrm{mm}^{2}$

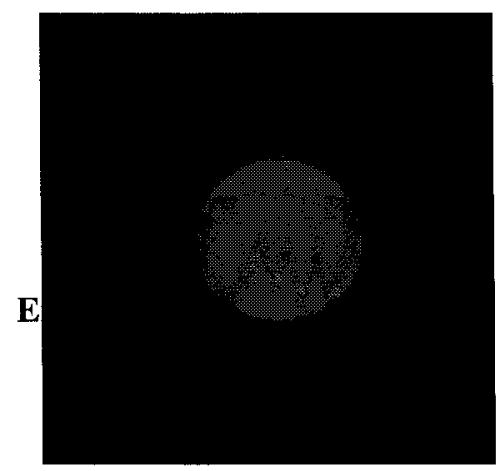

$\mathrm{b}=0 \mathrm{~s} / \mathrm{mm}^{2}$

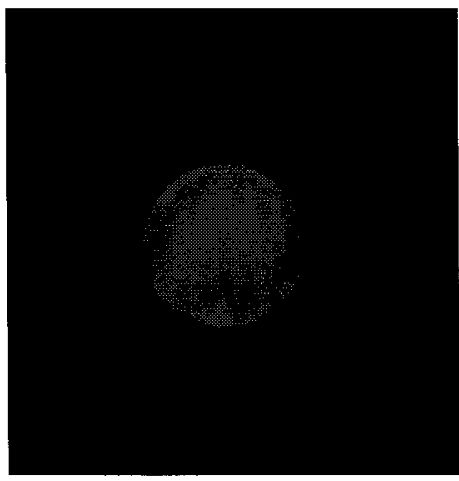

$\mathrm{b}=1500 \mathrm{~s} / \mathrm{mm}^{2}$

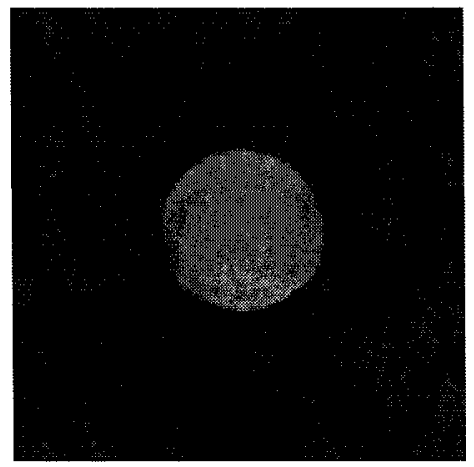

$\mathrm{b}=1500 \mathrm{~s} / \mathrm{mm}^{2}$

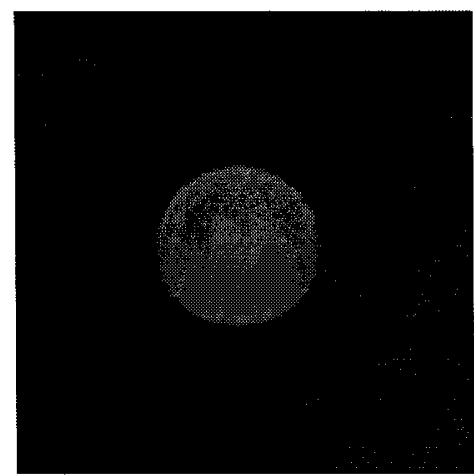

$\mathrm{b}=2000 \mathrm{~s} / \mathrm{mm}^{2}$

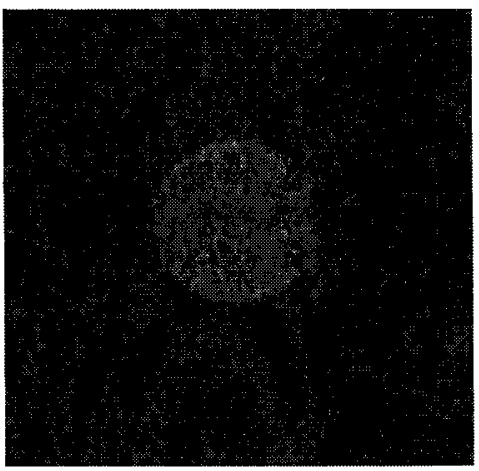

$\mathrm{b}=2000 \mathrm{~s} / \mathrm{mm}^{2}$

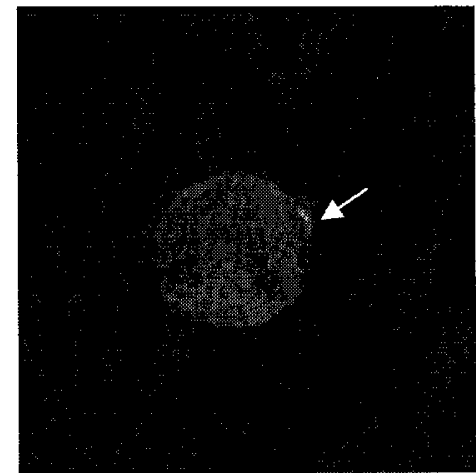

Figure 6.5. Water phantom images measured with Siemens' sequence (upper images) compared with the $\mathrm{g}_{2} \mathrm{~g}_{3}$ sequence for $\tau_{\mathrm{D}}=53.8 \mathrm{~ms}$ (lower images) showing a very small amount of edge magnification which is indicated by the arrow.

expected to change. For diffusion gradients in the frequency encoding, phase encoding or slice selection direction the image distortion is expected to be a shear, a magnification (or demagnification) or a translation, respectively. This suggests that the observed artifacts are not due to eddy currents. Furthermore, because they are very small in magnitude (of the order of the noise) the performance of the imager for these measurements is likely near the limit of the expected reproducibility of the equipment. In any case, these effects will not affect the results presented here, particularly since we use a region of interest analysis rather than a pixel by pixel procedure. 

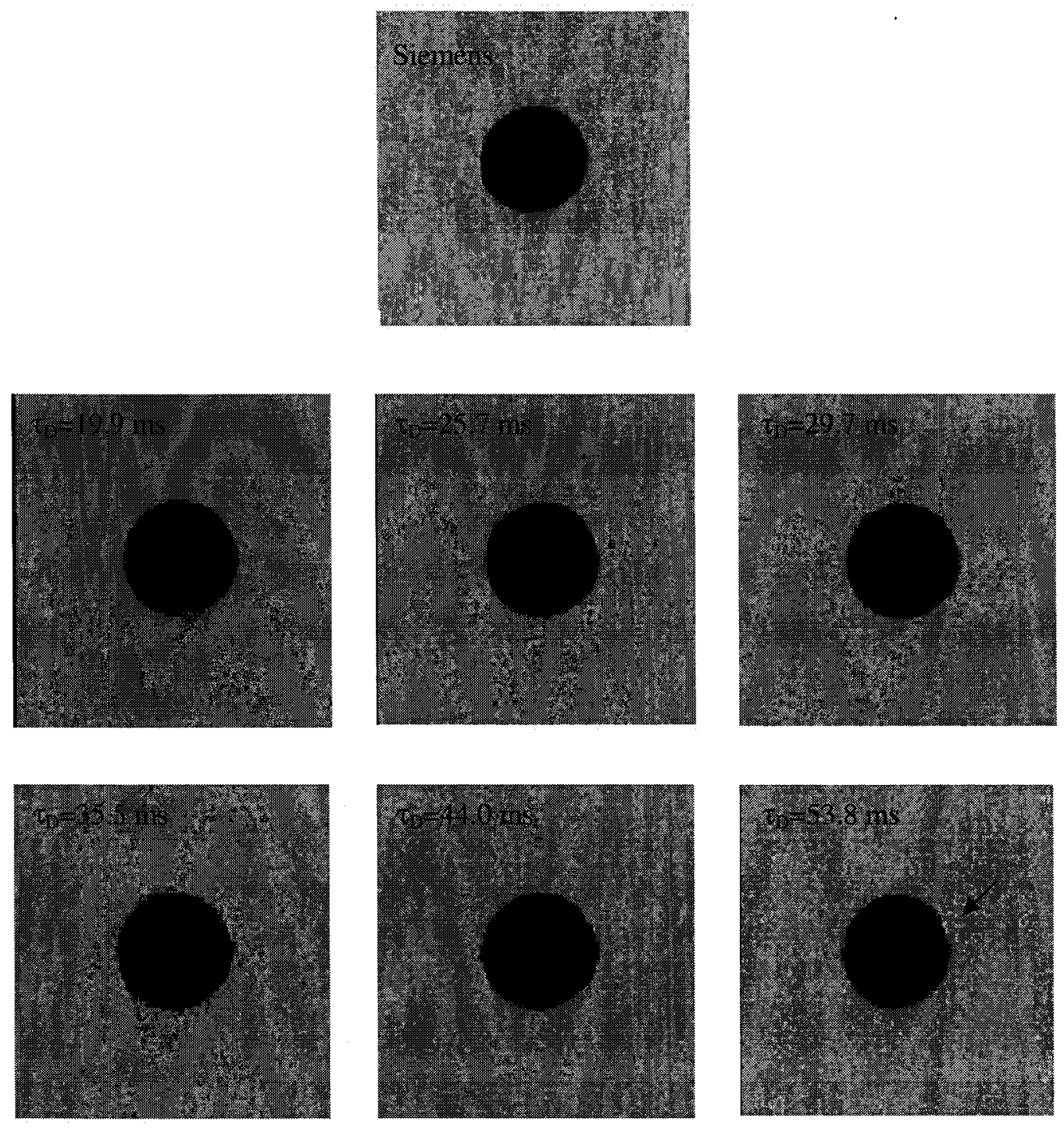

Figure 6.6. The subtracted water phantom images of the $b=0$ images from the corresponding maximum b-value images to detect any edge magnification due to eddy current effects. The top image is for the Siemens sequence and the rest are for the $\mathrm{g}_{2} \mathrm{~g}_{3}$ sequence for diffusion times between 19.9 and $53.8 \mathrm{~ms}$ respectively, from the upper left to the lower right. 


\begin{tabular}{|c|c|c|c|c|c|c|}
\hline Diffusion sequence & $\begin{array}{c}\mathrm{b} \text {-difference } \\
\left(\mathrm{s} / \mathrm{mm}^{2}\right)\end{array}$ & $\begin{array}{c}\mathrm{b}-\mathrm{max} \\
\left(\mathrm{s} / \mathrm{mm}^{2}\right)\end{array}$ & edge signal & noise & $\begin{array}{c}\text { edge signal } \\
\text { normalized to noise }\end{array}$ & $\sigma(+/-)$ \\
\hline & & & & & & \\
td54 & $\mathrm{b}(0)-\mathrm{b}(2835)$ & 2835 & 138.1 & 149 & 0.92 & 0.09 \\
td44 & $\mathrm{b}(0)-\mathrm{b}(2318)$ & 2318 & 134 & 149 & 0.8 & 0.08 \\
Siemens & $\mathrm{b}(0)-\mathrm{b}(2000)$ & 2000 & 90.5 & 183 & 0.49 & 0.05 \\
td35 & $\mathrm{b}(0)-\mathrm{b}(1872)$ & 1872 & 63.1 & 150 & 0.42 & 0.06 \\
td30 & $\mathrm{b}(0)-\mathrm{b}(1566)$ & 1566 & 48.9 & 110 & 0.44 & 0.1 \\
td25 & $\mathrm{b}(0)-\mathrm{b}(1355)$ & 1355 & 39 & 80 & 0.49 & 0.06 \\
td20 & $\mathrm{b}(0)-\mathrm{b}(1050)$ & 1050 & 18 & 38 & 0.47 & 0.08 \\
\hline
\end{tabular}

Table 6.1. The results of eddy current detection for the $g_{2} g_{3}$ sequences. $\sigma$ is the error (standard deviation) on the edge signal normalized to noise.

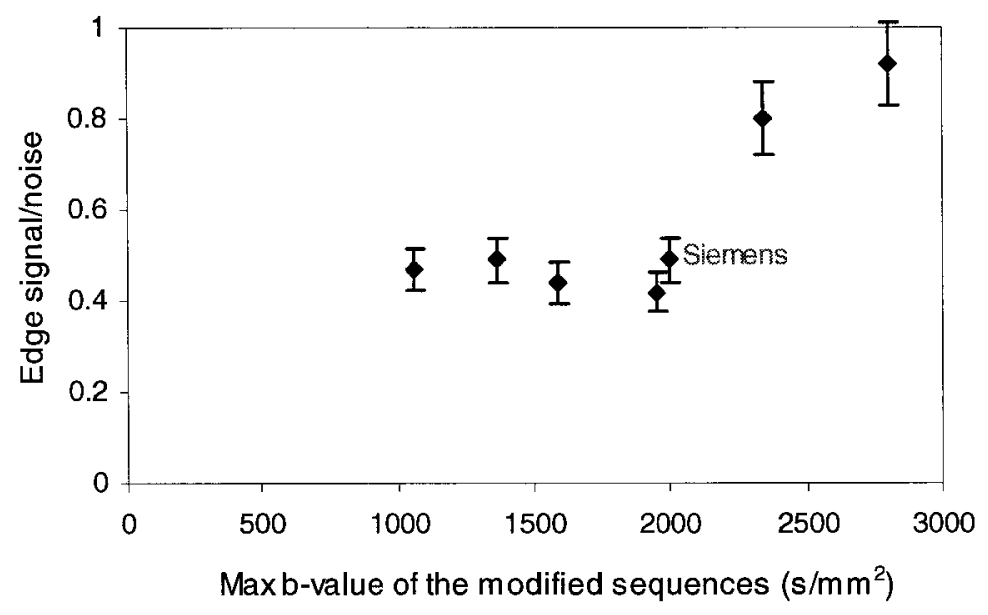

Figure 6.7. The results of the normalized edge magnification detected in the images taken with the $\mathrm{g}_{2} \mathrm{~g}_{3}$ sequences for the different diffusion times as well as Siemens' sequence for the investigation of possible eddy current effects. 


\subsection{Experimental Procedure and Sequence Validation}

The $\mathrm{g}_{2} \mathrm{~g}_{3}$ sequence for six different diffusion times with the diffusion gradient duration constant at $\delta=30 \mathrm{~ms}$ and the echo time constant at $\mathrm{TE}=200 \mathrm{~ms}$ was employed for the diffusion time dependence experiments. The $\tau_{\mathrm{D}}$ was varied from 19.9 to $53.8 \mathrm{~ms}$ with the respective maximum b-values of $1050 \mathrm{~s} / \mathrm{mm}^{2}$ to $2835 \mathrm{~s} / \mathrm{mm}^{2}$. The diffusion gradients were applied in the frequency encoding (right to left) direction for $16 \mathrm{~b}$-values up to the maximum possible value for the diffusion time being used. See section 3.2.1 for the details of the parameter settings for these measurements.

The $\mathrm{g}_{2} \mathrm{~g}_{3}$ sequences were first validated by diffusion measurements with the water phantom at room temperature. The diffusion coefficient evaluated from each measurement for different ROI's with FOV $=464 \mathrm{~mm}$ all gave $\mathrm{D}=2.0 \times 10^{-3} \mathrm{~mm}^{2} / \mathrm{s}$ which is the expected value for water at room temperature [183].

The initial experiments on humans were performed on 2 healthy volunteers using 5 sequences with various diffusion times, 10 magnitude averages and $11 \mathrm{~b}$-values up to 10,000 $\mathrm{s} / \mathrm{mm}^{2}$. The results were analyzed by non-linear least squares. These experiments were done to validate the $\mathrm{g}_{2} \mathrm{~g}_{3}$ sequences on human subjects as well as to examine the restricted diffusion behavior of brain tissue primarily by analyzing various ROI's in white matter. Based on these results it was decided that the sequences were working properly but that the number of averages should be increased from 10 to 50 , that one more diffusion time $\left(\tau_{\mathrm{D}}=25.7 \mathrm{~ms}\right)$ should also be included and that the number of $b$-values should be increased from 11 to 16 . Otherwise, the actual experiments in this study were performed with the same settings as the validation trials just discussed. See section 3.2.1 for further experimental details.

The real experiments were continued with 8 different healthy human subjects with 5 males 
$\tau_{\mathrm{D}}=19.9 \mathrm{~ms}$

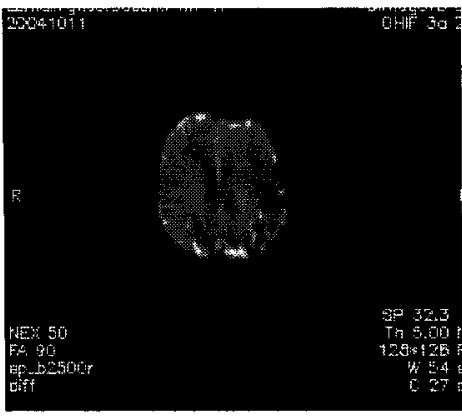

$\tau_{\mathrm{D}}=35.5 \mathrm{~ms}$

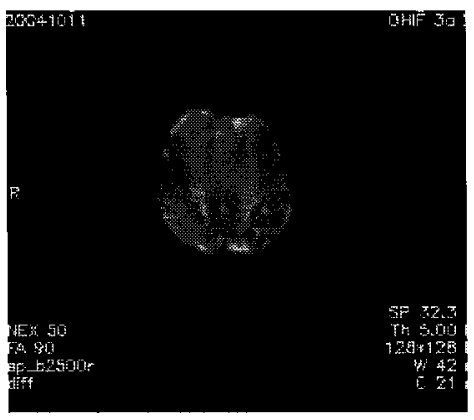

$\tau_{\mathrm{D}}=25.7 \mathrm{~ms}$

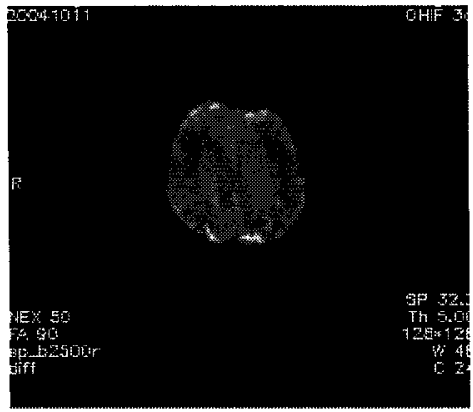

$\tau_{\mathrm{D}}=44.0 \mathrm{~ms}$

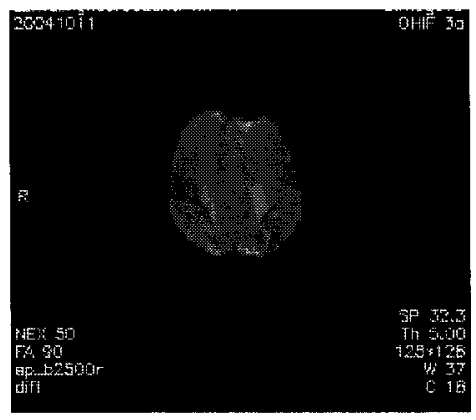

$\tau_{\mathrm{D}}=29.7 \mathrm{~ms}$

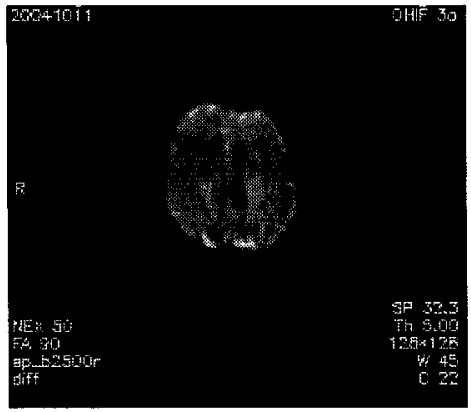

$\tau_{\mathrm{D}}=53.8 \mathrm{~ms}$

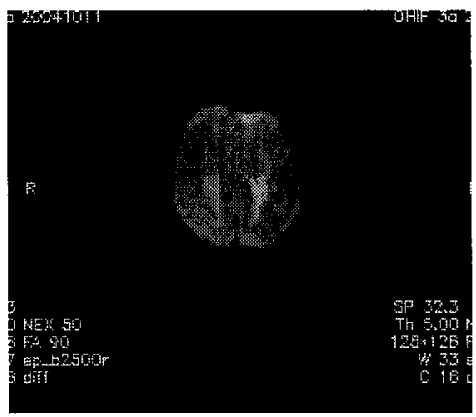

Figure $6.8 \mathrm{a}$-f. Typical images of human brain obtained using the $\mathrm{g}_{2} \mathrm{~g}_{3}$ sequences for $\tau_{\mathrm{D}}=19.9$ to $53.8 \mathrm{~ms}$ and their respective b-values associated with a $13 \mathrm{mT} / \mathrm{m}$ diffusion sensitizing gradient strength.

and 3 females in an age range of 23 to 44 years old. As mentioned in section 3.2.1, the protocol for these measurements included 6 sequences with different diffusion times (see table 6.2) plus Siemens' diffusion sequence as a control with the number of averages (magnitude averaging) set to 50 for these sequences. In addition, the $\tau_{D}=53.8 \mathrm{~ms}$ sequence was repeated with 50 averages of the complex valued signals in k-space (complex averaging). The total scan time for each subject was about 50 minutes. Typical human brain images for the sequences with diffusion times of 19.9 to $53.8 \mathrm{~ms}$ for the b-value associated with $13 \mathrm{mT} / \mathrm{m}$ diffusion gradients as well as the maximum b-value (i.e. the noisiest image) for each diffusion time are shown in figures 6.8 a-f and 6.9 a-f, respectively. 
$\tau_{\mathrm{D}}=19.9 \mathrm{~ms}$

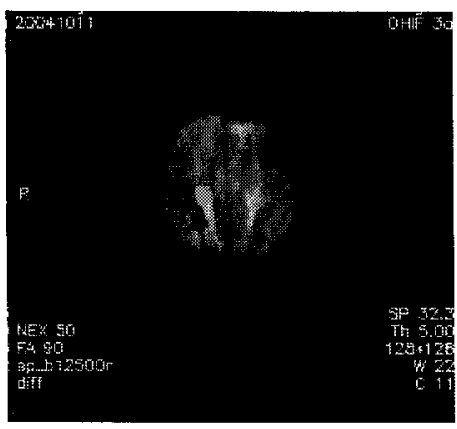

$\tau_{\mathrm{D}}=35.5 \mathrm{~ms}$

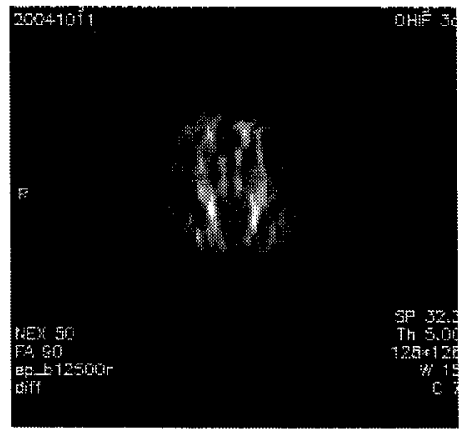

$\tau_{\mathrm{D}}=25.7 \mathrm{~ms}$

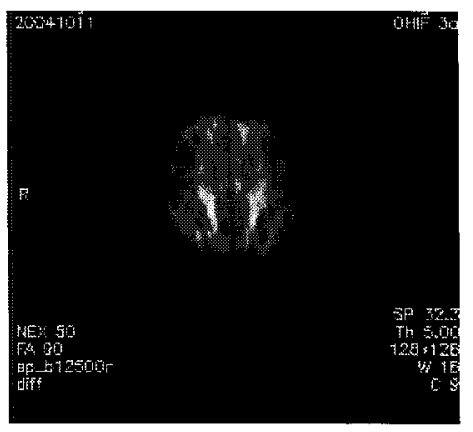

$\tau_{\mathrm{D}}=44.0 \mathrm{~ms}$

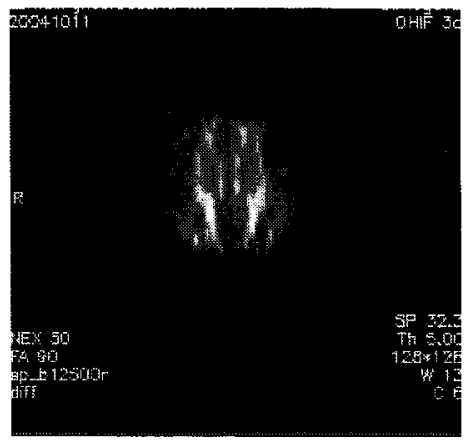

$\tau_{\mathrm{D}}=29.7 \mathrm{~ms}$

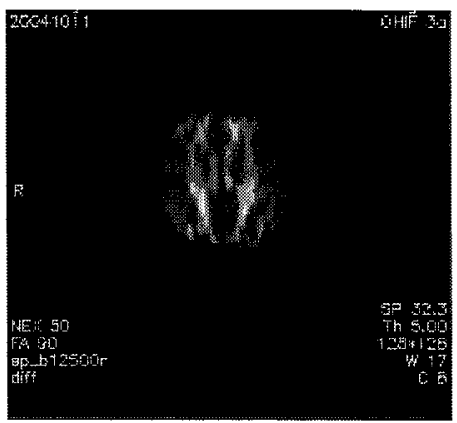

$\tau_{\mathrm{D}}=53.8 \mathrm{~ms}$

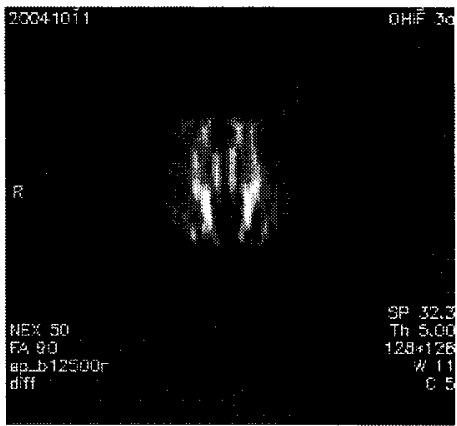

Figure 6.9 a-f. Typical images of human brain obtained using the $\mathrm{g}_{2} \mathrm{~g}_{3}$ sequences for $\tau_{\mathrm{D}}=19.9$ to $53.8 \mathrm{~ms}$ and their respective maximum b-values associated with the $28.5 \mathrm{mT} / \mathrm{m}$ diffusion sensitizing gradient strength.

\section{Error!}

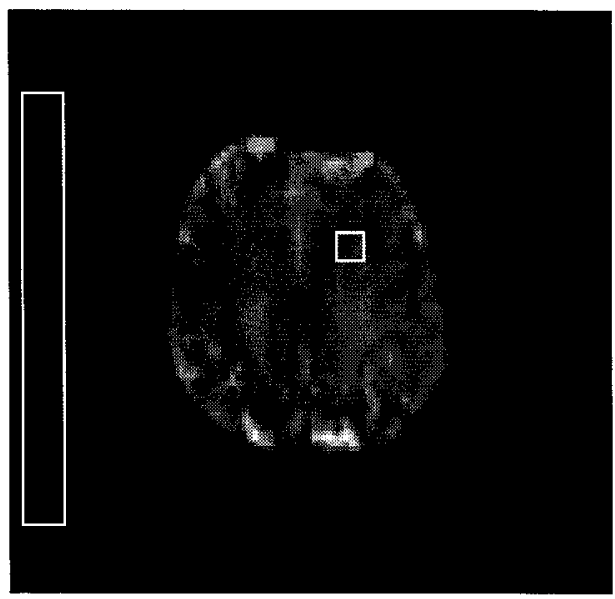

Figure 6.10. A typical human brain image for the $\mathrm{g}_{2} \mathrm{~g}_{3}$ sequence of the slice selected above the ventricles. The ROI's were selected in the white matter of the images and the background for the Rician noise bias correction. The size of the ROI in the image was $4 \times 5$ pixels, which corresponds to a volume of $18 \times 14 \times 5 \mathrm{~mm}^{3}$. 
A Rician noise correction algorithm was applied in the image post processing using the program DimageV3 written in IDL version 6.0 to unbias the magnitude signals from the ROI's before the diffusion decays were analyzed to evaluate the diffusion coefficients. Since the signalto-noise ratio was greater than 3.0 for all of the diffusion times and b-values discussed in this chapter, the results of chapter 4 show that all of the Rician noise bias correction algorithms considered there perform equally well. The Rician noise bias correction algorithms used here was McGibney and Smith's (see equation (4.33)). The standard deviation of the Gaussian noise distribution for the Rician noise bias correction algorithm was estimated by choosing a large ROI in the background of the magnitude image (see figure 6.10). The ROI's for both the signals on the object area of the image and the background were chosen with care to avoid possible N/2 ghosting artifacts in the phase encoding direction and Gibbs artifacts. The ROI's selected in the white matter of the brain images included $5 \times 4=20$ pixels in the axial plane and corresponded to a volume of $18 \times 14 \times 5 \mathrm{~mm}^{3}$.

The signal decays were all analyzed using the computational analysis code $N N L S$-diff. In the preliminary analyses we used $\mathrm{N}=150$ diffusion components logarithmically spaced at equal intervals (in $\log$ space) between $1 \times 10^{-5}$ and $3 \times 10^{-1}$. Although all of our measurements were performed with the full care of having the same physical and environmental conditions, there were still dispersions of the results either related to the selection of the ROI size and/or position in the white matter or to the non-uniqueness of the solution to the best fit (see figure 6.11). However, the solution generally converged to two strong components: one around $0.9 \times 10^{-3} \mathrm{~mm}^{2} / \mathrm{s}$ and a small number of dispersed diffusion coefficients around $0.07 \times 10^{-3} \mathrm{~mm}^{2} / \mathrm{s}$. Two more components were observed for $D_{a} \sim 0.01 \mathrm{~mm}^{2} / \mathrm{s}$ in the first trials but they corresponded to a very small fraction of the total decay and were not well resolved. For these initial analyses the ROI's were $21 \times 20=420$ pixels in size. As an attempt to reduce the dispersion of the results we decided 


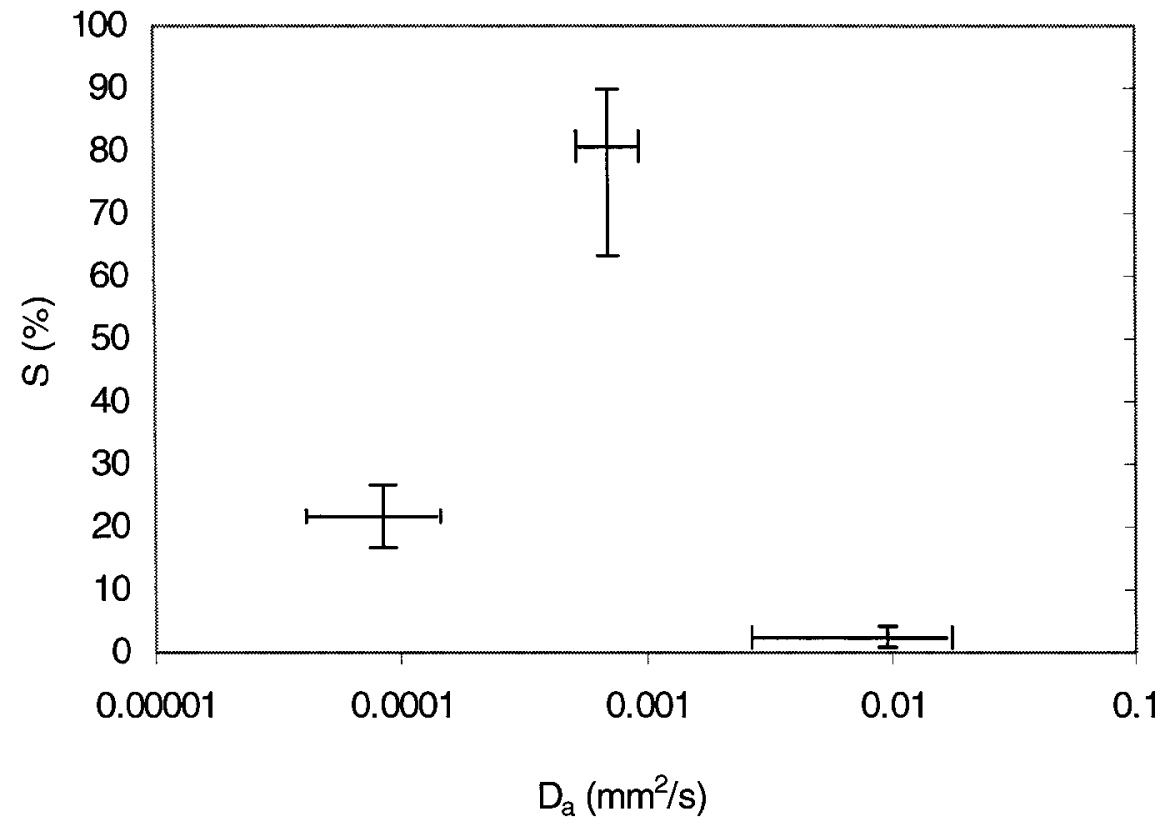

Figure 6.11. The results of diffusion of water molecules in human brain tissue analyzed by $N N L S$ diff using the wide range of 150 diffusion data of the first trials in 8 to 10 subjects and 6 diffusion times.

to choose smaller ROI's ( 20 to 50 pixels) and average over a number of ROI's around our intended position in the white matter (upper right in figure 6.10). We also realized that if we reduced the range of diffusion coefficients investigated with $N N L S$ we would reduce the dispersion on the two principle components. Based on the initial $N N L S$ results, diffusion components greater than $2 \times 10^{-3} \mathrm{~mm}^{2} / \mathrm{s}$ contribute about $1 \%$ or less to the diffusion decay. By restricting the $N N L S$ analysis to equal logarithmically spaced diffusion coefficients between $1 \times 10^{-}$ ${ }^{5}$ and $2 \times 10^{-3} \mathrm{~mm}^{2} / \mathrm{s}$ we were able to reduce the dispersion of the two diffusion components within this range. If the extra small components are truly part of the decay then ignoring them, in principle, introduces inaccuracy to the values determined for the two main peaks, however, since the third and fourth components are so small, any inaccuracy introduced by ignoring them in the analysis should be negligible. After all of the experimental data sets were analyzed using the full 
range of diffusion coefficients, they were all analyzed a second time using the reduced range mentioned above. This modified analysis procedure allowed us to more easily compare the diffusion components determined for the different diffusion times since the analysis always yielded two main diffusion coefficients around the values mentioned above. The signal decays from the ROI's selected in the images for the 6 diffusion time sequences were all analyzed over the same data range.

From this second round of analysis we found that the fraction of the signal that contributes to the slow diffusion coefficient, $D_{s}$, covered a range of $14 \%$ to $29 \%$. Since the signal decay fraction is not expected to change very much from subject to subject and since the average signal fraction for each $\tau_{D}$ value did not show a dependence on $\tau_{D}$ (see table 6.3), it was decided to set the fraction corresponding to $D_{s}$ to the midpoint of the observed range, $21.7 \pm 2 \%$. This constraint was incorporated into the analysis procedure and the data were analyzed again.

\subsection{Results}

The data analysis of the water phantom performed with NNLS-diff for all diffusion times always resulted in one diffusion coefficient very close to $2.05 \times 10^{-3} \mathrm{~mm}^{2} / \mathrm{s}$ (see table 6.2 ) as expected for water molecules [183]. The signal decays all fit well to a mono-exponential curve with no diffusion time dependence as expected for free diffusion of particles in a homogenous system based on Einstein's diffusion equation (equation (2.51)). The diffusion signal decays of the water phantom for these sequences are plotted in figure 6.12 .

The results of the signal decay analysis for the ROI's selected in the white matter of 8 human subjects are tabulated for each subject at each diffusion time in tables $6.3 \mathrm{a}$ to $6.3 \mathrm{f}$. The average of the diffusion components and their contribution to the signal decay over the 8 human 
subjects for each $\tau_{\mathrm{D}}$ are also given in these tables. There are a few data points that are outliers well away from the mean value for each parameter which increases the standard deviation of the mean. We decided to reject these data points by imposing the criterion that only the data points within one standard deviation of the mean (i.e. $\pm \sigma$ ) for each diffusion parameter would be considered in the analysis. With this objective criterion, most of the data points $(\sim 75 \%)$ are accepted but the standard deviation is reduced (see table 6.4).

Typical diffusion decays for a typical human subject are shown in figure 6.13 for all six diffusion times. A small $\tau_{\mathrm{D}}$ dependence is observed in the slow diffusion coefficients. The decay slopes start to deviate from each other while the decay is falling down to the slow component contributions at the highest b-values. The different decay lengths are due to different maximum bvalues for each diffusion time.

Typical spectra of NNLS-diff analyzed human brain tissue diffusion decays are demonstrated in figure 6.14 for different diffusion times. These spectra show that the fast diffusion components are in good agreement for all diffusion times with a mean value of $0.98 \times 10^{-3} \mathrm{~mm}^{2} / \mathrm{s}$. The slow diffusion components, however, are peaked at slightly different values due to the diffusion time variance.

Diffusion decays generated from the $N N L S$ fit parameters averaged over 8 human subjects are plotted in figure 6.15 for all 6 diffusion times. The variation of the signal decay with $\tau_{\mathrm{D}}$ is better seen in these curves. A significant change in $D_{s}$ can be seen between $\tau_{D}=20-25 \mathrm{~ms}$ and 30-54 ms. A smaller change can also be seen in the diffusion decays for $\tau_{\mathrm{D}}>30 \mathrm{~ms}$. These decays are also very consistent with the $96 \mathrm{~b}$-value decay curve presented in chapter 5 .

Figure 6.16 shows $D_{s}$ and $D_{f}$ averaged over 8 subjects plotted as a function of $\tau_{D}$. $D_{f}$ is independent of $\tau_{D}$ but $D_{s}$ shows a clear dependence on $\tau_{D}$. These results will be discussed in terms of brain tissue structure and water exchange in the next section and in more detail in chapter 7. 


\begin{tabular}{|ccccc|}
\hline $\begin{array}{c}\text { Diffusion sequence } \\
(\mathrm{ms})\end{array}$ & $\begin{array}{c}\mathrm{b} \text {-max } \\
\left(\mathrm{s} / \mathrm{mm}^{2}\right)\end{array}$ & $\begin{array}{c}\mathrm{D} \\
\left(10^{-3} \mathrm{~mm}^{2} / \mathrm{s}\right)\end{array}$ & $\begin{array}{c}\sigma_{\mathrm{D}} \\
\left(10^{-3} \mathrm{~mm}^{2} / \mathrm{s}\right)\end{array}$ & $\chi^{2}$ \\
\hline & & & & \\
td54(53.8) & 2800 & 2.05 & 0.008 & 1.6 \\
Siemens(50) & 12500 & 2.04 & 0.006 & 15 \\
td44(44.0) & 2345 & 2.06 & 0.007 & 6 \\
td35(35.5) & 1948 & 2.05 & 0.006 & 1.1 \\
td30(29.7) & 1588 & 2.05 & 0.008 & 1.2 \\
td25(25.7) & 1371 & 2.05 & 0.007 & 1.2 \\
td20(19.9) & 1061 & 2.05 & 0.006 & 1.2 \\
\hline
\end{tabular}

Table 6.2. The results of diffusion for the water phantom using the $g_{2} g_{3}$ sequences analyzed with $N N L S$-diff after noise bias correction.

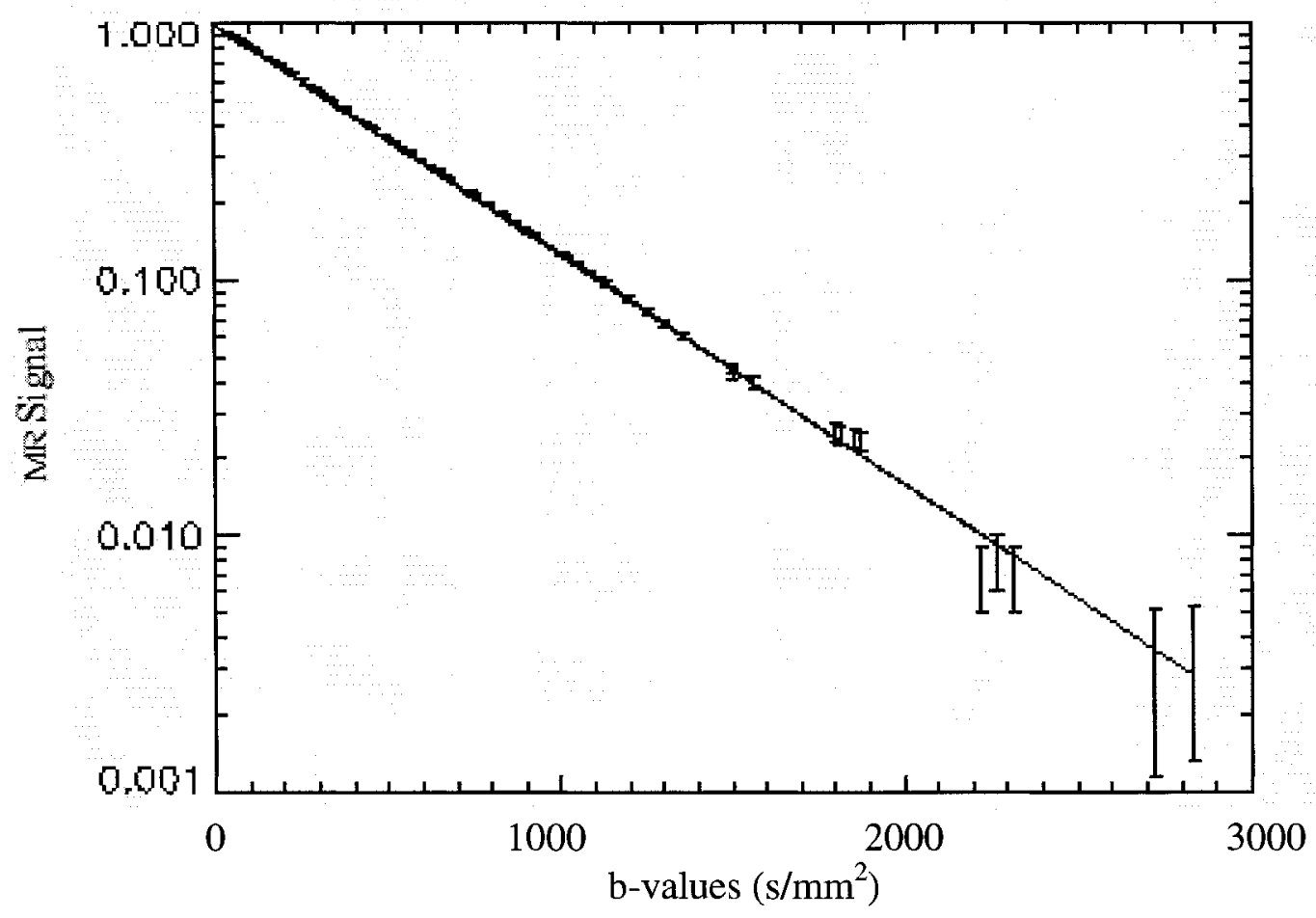

Figure 6.12. The diffusion decays for the water phantom measured with the $\mathrm{g}_{2} \mathrm{~g}_{3}$ sequences for $\tau_{\mathrm{D}}=19.9$ to $53.8 \mathrm{~ms}$ each measured with $16 \mathrm{~b}$-values up to the maximum possible b-value and analyzed using both $N N L S$-diff and the regular Least Squares after Rician noise bias correction. 


\begin{tabular}{|cccccc|}
\hline \multicolumn{5}{|c|}{$\tau_{D}=19.9 \mathrm{~ms}$} \\
\hline subject\# & $\mathrm{f}_{\mathrm{s}}(\%)$ & $\mathrm{D}_{\mathrm{s}}\left(10^{-3} \mathrm{~mm}^{2} / \mathrm{s}\right)$ & $\mathrm{f}_{\mathrm{f}}(\%)$ & $\mathrm{D}_{\mathrm{f}}\left(10^{-3} \mathrm{~mm}^{2} / \mathrm{s}\right)$ & $\chi^{2}$ \\
\hline 1 & 19.8 & 0.15 & 80 & 1.5 & 1.7 \\
2 & 22 & 0.095 & 77.1 & 0.88 & 2.5 \\
3 & 19.8 & 0.10 & 80 & 1.2 & 1.6 \\
4 & 16.7 & 0.15 & 82 & 0.84 & 1.2 \\
5 & 22.2 & 0.10 & 78.5 & 1.0 & 1.5 \\
6 & 20.8 & 0.094 & 77 & 0.98 & 1.5 \\
7 & 18.6 & 0.15 & 81.2 & 1.1 & 1.4 \\
8 & 19.9 & 0.10 & 79.2 & 0.95 & 2.2 \\
ave. & 20.0 & 0.12 & 79.4 & 1.1 & \\
std & 1.8 & 0.027 & 1.8 & 0.21 & \\
\hline
\end{tabular}

\begin{tabular}{|cccccc|}
\hline \multicolumn{6}{|c|}{$\tau_{\mathrm{D}}=25.7 \mathrm{~ms}$} \\
\hline subject\# & $\mathrm{f}_{\mathrm{s}}(\%)$ & $\mathrm{D}_{\mathrm{s}}\left(10^{-3} \mathrm{~mm}^{2} / \mathrm{s}\right)$ & $\mathrm{f}_{\mathrm{f}}(\%)$ & $\mathrm{D}_{\mathrm{f}}\left(10^{-3} \mathrm{~mm}^{2} / \mathrm{s}\right)$ & $\chi^{2}$ \\
\hline 1 & 20.1 & 0.1 & 78 & 1.3 & 1.0 \\
2 & 21.8 & 0.09 & 77 & 0.83 & 3.5 \\
3 & 19.3 & 0.11 & 80.7 & 1.04 & 2.5 \\
4 & 21.9 & 0.067 & 77.2 & 1.0 & 2.8 \\
5 & 20.9 & 0.1 & 79.6 & 1.0 & 2.8 \\
6 & 19.6 & 0.1 & 77.4 & 0.94 & 4.8 \\
7 & 18 & 0.15 & 81.9 & 1.1 & 1.6 \\
8 & 19.6 & 0.095 & 79.2 & 1.5 & 1.1 \\
ave. & 20.2 & 0.10 & 78.9 & 1.1 & \\
std & 1.3 & 0.023 & 1.8 & 0.22 & \\
\hline
\end{tabular}

\begin{tabular}{|cccccc|}
\hline \multicolumn{5}{|c|}{$\tau_{\mathrm{D}}=29.7 \mathrm{~ms}$} \\
\hline subject\# & $\mathrm{f}_{\mathrm{s}}(\%)$ & $\mathrm{D}_{\mathrm{s}}\left(10^{-3} \mathrm{~mm}^{2} / \mathrm{s}\right)$ & $\mathrm{f}_{\mathrm{f}}(\%)$ & $\mathrm{D}_{\mathrm{f}}\left(10^{-3} \mathrm{~mm}^{2} / \mathrm{s}\right)$ & $\chi^{2}$ \\
\hline 1 & 23.2 & 0.06 & 76.3 & 0.78 & 1.2 \\
2 & 20.3 & 0.052 & 77.9 & 0.80 & 5.1 \\
3 & 20.1 & 0.1 & 84.5 & 0.97 & 9.1 \\
4 & 19.6 & 0.062 & 79.3 & 0.96 & 1.9 \\
5 & 20.0 & 0.068 & 77.2 & 1.0 & 4 \\
6 & 20.9 & 0.05 & 76.5 & 0.76 & 5.2 \\
7 & 20.1 & 0.1 & 78.6 & 1.2 & 3.8 \\
8 & 20.7 & 0.08 & 77.1 & 1.2 & 4 \\
ave. & 20.6 & 0.072 & 78.4 & 0.96 & \\
std & 1.1 & 0.02 & 2.7 & 0.18 & \\
\hline
\end{tabular}

Table $6.3 \mathrm{a}-\mathrm{f}$. The results of diffusion in human brain tissue for the diffusion times between 19.9 and $53.8 \mathrm{~ms}$ from top to the bottom respectively of 8 human subjects analyzed using $N N L S$-diff. The standard deviation of the parameters for individual subject is too small to be reported. 


\begin{tabular}{|cccccc|}
\hline \multicolumn{6}{|c|}{$\tau_{D}=35.5 \mathrm{~ms}$} \\
\hline subject\# & $\mathrm{f}_{s}(\%)$ & $\mathrm{D}_{s}\left(10^{-3} \mathrm{~mm}^{2} / \mathrm{s}\right)$ & $\mathrm{f}_{\mathrm{f}}(\%)$ & $\mathrm{D}_{\mathrm{f}}\left(10^{-3} \mathrm{~mm}^{2} / \mathrm{s}\right)$ & $\chi^{2}$ \\
\hline 1 & 23.3 & 0.05 & 75.7 & 0.76 & 2.6 \\
2 & 20.0 & 0.055 & 77.5 & 0.84 & 4.8 \\
3 & 22.9 & 0.081 & 81.5 & 1.0 & 10.7 \\
4 & 19.8 & 0.067 & 78.4 & 1.0 & 3.5 \\
5 & 19.2 & 0.062 & 79.9 & 0.95 & 3.7 \\
6 & 16.0 & 0.061 & 83 & 0.94 & 8.3 \\
7 & 22.5 & 0.1 & 77.6 & 0.6 & 4.4 \\
8 & 9.8 & 0.063 & 89 & 0.98 & 5.8 \\
ave. & 19.2 & 0.067 & 80.3 & 0.88 & \\
std & 4.5 & 0.016 & 4.2 & 0.14 & \\
\hline
\end{tabular}

\begin{tabular}{|cccccc|}
\hline \multicolumn{6}{|c|}{$\tau_{\mathrm{D}}=44.0 \mathrm{~ms}$} \\
\hline subject\# & $\mathrm{f}_{\mathrm{s}}(\%)$ & $\mathrm{D}_{\mathrm{s}}\left(10^{-3} \mathrm{~mm}^{2} / \mathrm{s}\right)$ & $\mathrm{f}_{\mathrm{f}}(\%)$ & $\mathrm{D}_{\mathrm{f}}\left(10^{-3} \mathrm{~mm}^{2} / \mathrm{s}\right)$ & $\chi^{2}$ \\
\hline 1 & 23.6 & 0.05 & 74.1 & 0.76 & 5.1 \\
2 & 20.2 & 0.06 & 76.2 & 0.93 & 7.7 \\
3 & 20.6 & 0.061 & 82.5 & 0.93 & 9.3 \\
4 & 20.9 & 0.05 & 76.8 & 0.76 & 5.3 \\
5 & 19.7 & 0.06 & 77.8 & 0.93 & 8 \\
6 & 15.6 & 0.09 & 81.5 & 1.4 & 9.7 \\
7 & 20.3 & 0.045 & 81.1 & 0.68 & 4.3 \\
8 & 20.8 & 0.1 & 75.8 & 0.93 & 4.5 \\
ave. & 20.2 & 0.065 & 78.2 & 0.92 & \\
std & 2.2 & 0.02 & 3.1 & 0.22 & \\
\hline
\end{tabular}

\begin{tabular}{|cccccc|}
\hline \multicolumn{6}{|c|}{$\tau_{D}=53.8 \mathrm{~ms}$} \\
\hline subject\# & $\mathrm{f}_{\mathrm{s}}(\%)$ & $\mathrm{D}_{\mathrm{s}}\left(10^{-3} \mathrm{~mm}^{2} / \mathrm{s}\right)$ & $\mathrm{f}_{\mathrm{f}}(\%)$ & $\mathrm{D}_{\mathrm{f}}\left(10^{-3} \mathrm{~mm}^{2} / \mathrm{s}\right)$ & $\chi^{2}$ \\
\hline 1 & 23.9 & 0.051 & 77 & 1.0 & 3.1 \\
2 & 23.5 & 0.05 & 75.5 & 0.76 & 11 \\
3 & 20.7 & 0.06 & 82.7 & 0.92 & 6 \\
4 & 20.3 & 0.055 & 78.7 & 0.84 & 1.2 \\
5 & 19.4 & 0.06 & 79.7 & 0.93 & 6 \\
6 & 20.9 & 0.1 & 75.9 & 1.5 & 9.3 \\
7 & 20.2 & 0.062 & 77 & 0.96 & 4.5 \\
8 & 25 & 0.05 & 73.6 & 1.2 & 3.9 \\
ave. & 21.7 & 0.061 & 77.5 & 1.0 & \\
std & 2.1 & 0.016 & 2.8 & 0.23 & \\
\hline
\end{tabular}

Table $6.3 \mathrm{a}-\mathrm{f}$. Cont. 


\begin{tabular}{|c|c|c|c|c|c|}
\hline $\begin{array}{c}\text { diffusion time } \\
\tau_{\mathrm{D}}(\mathrm{ms})\end{array}$ & $\begin{array}{c}\text { max. bvalue } \\
\left(\mathrm{s} / \mathrm{mm}^{2}\right)\end{array}$ & $\begin{array}{c}\mathrm{f}_{\mathrm{s}} \pm \mathrm{std} \\
(\%)\end{array}$ & $\begin{array}{c}\mathrm{D}_{\mathrm{s}} \pm \mathrm{std} \\
\left(10^{-3} \mathrm{~mm}^{2} / \mathrm{s}\right)\end{array}$ & $\begin{array}{c}\mathrm{f}_{\mathrm{f}} \pm \mathrm{std} \\
(\%)\end{array}$ & $\begin{array}{c}\mathrm{D}_{\mathrm{f}} \pm \mathrm{std} \\
\left(10^{-3} \mathrm{~mm}^{2} / \mathrm{s}\right)\end{array}$ \\
\hline 19.9 & 1050 & $20.1 \pm 0.5$ & $0.1 \pm 0.003$ & $79.8 \pm 1.0$ & $1.0 \pm 0.1$ \\
25.7 & 1355 & $19.9 \pm 0.6$ & $0.1 \pm 0.007$ & $78.7 \pm 1.4$ & $1.1 \pm 0.1$ \\
29.7 & 1566 & $20.2 \pm 0.4$ & $0.068 \pm 0.009$ & $77.6 \pm 1.1$ & $1.0 \pm 0.2$ \\
35.5 & 1872 & $20.5 \pm 2.6$ & $0.065 \pm 0.009$ & $79.7 \pm 2.2$ & $0.9 \pm 0.09$ \\
44.0 & 2318 & $20.4 \pm 0.4$ & $0.056 \pm 0.006$ & $77.6 \pm 2.3$ & $0.9 \pm 0.09$ \\
53.8 & 2835 & $21.1 \pm 1.4$ & $0.055 \pm 0.005$ & $77.3 \pm 1.6$ & $1.0 \pm 0.1$ \\
\hline
\end{tabular}

Table 6.4 The diffusion data of human brain tissue vs. diffusion time after removing the points which exceed $\pm \sigma$ of the averaged data over 8 human subjects. 


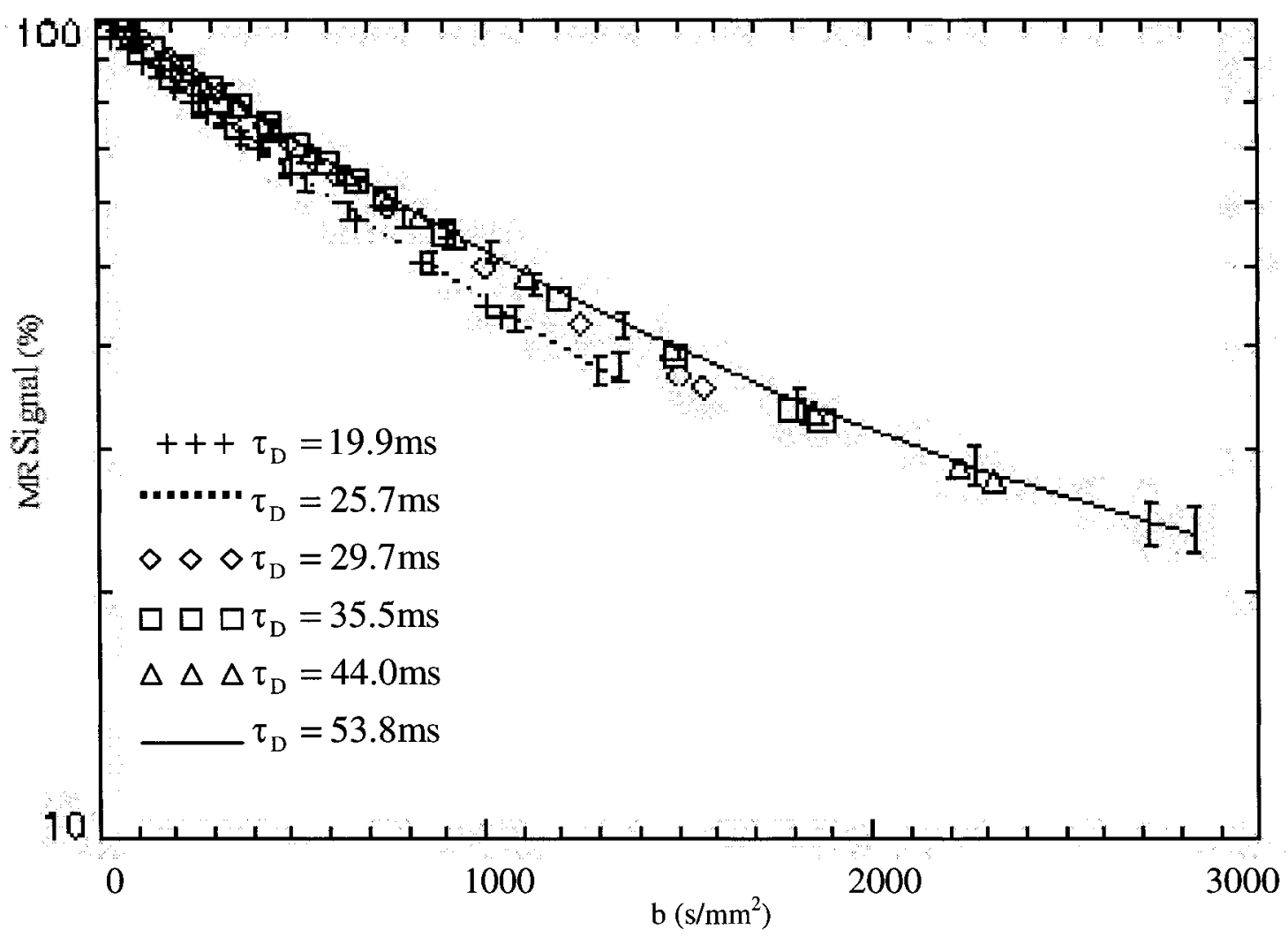

Figure 6.13. Typical diffusion decays for human brain tissue vs. $\tau_{D}$ for one subject from $\tau_{D}=19.9$ $\mathrm{ms}$ (bottom curve) which has the highest slope and the shortest length, to $\tau_{\mathrm{D}}=53.8 \mathrm{~ms}$ (top curve) with the lowest slope and the largest length due to their maximum possible b-values. The data are all very well fitted with their corresponding curves, however, to avoid confusion, the fitted curves were presented just for two of them $\left(\tau_{D}=25.7 \mathrm{~ms}\right.$ and $\left.53.8 \mathrm{~ms}\right)$. 


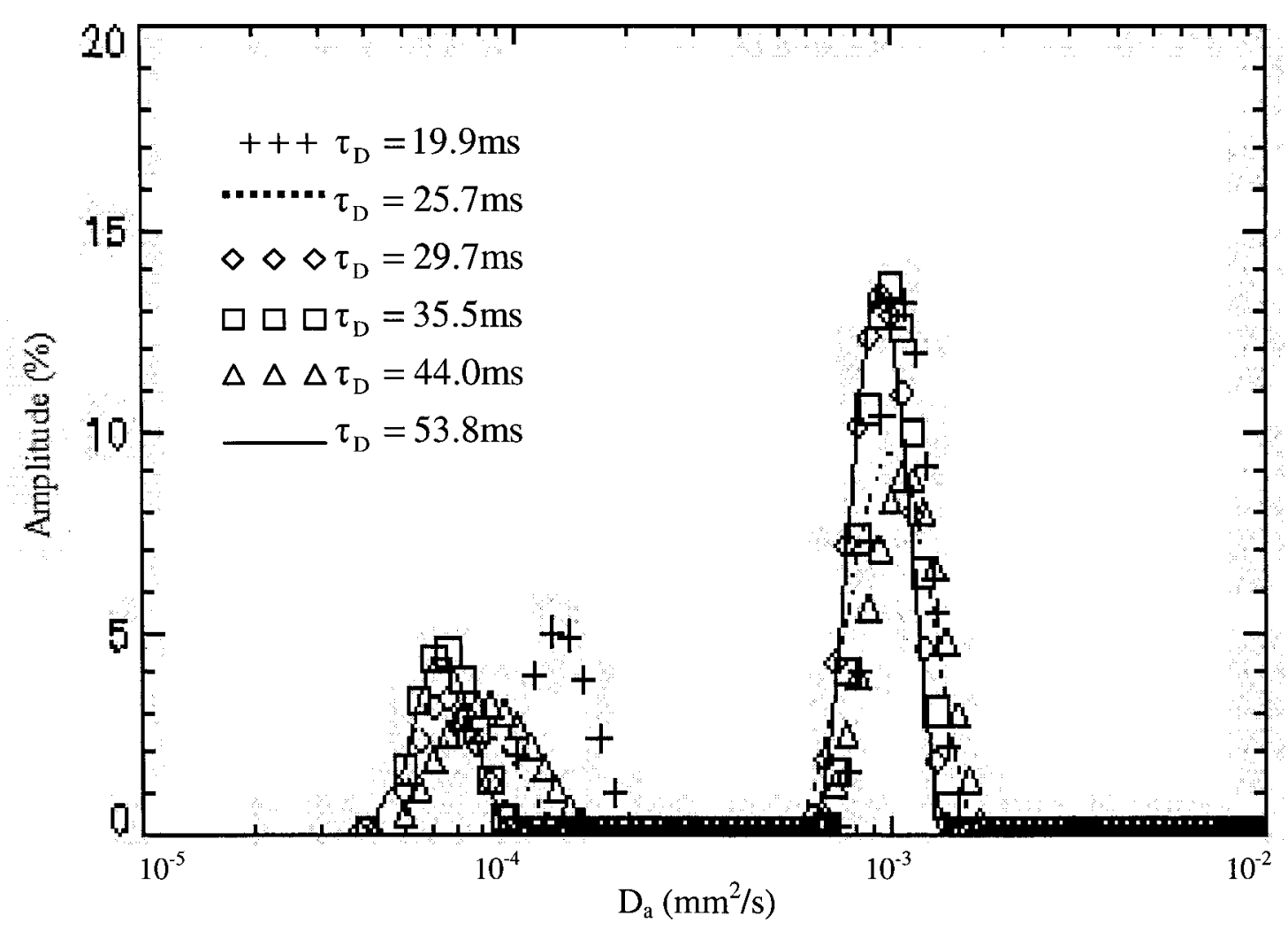

Figure 6.14. Typical diffusion spectra of human brain tissue vs. $\tau_{D}$. 


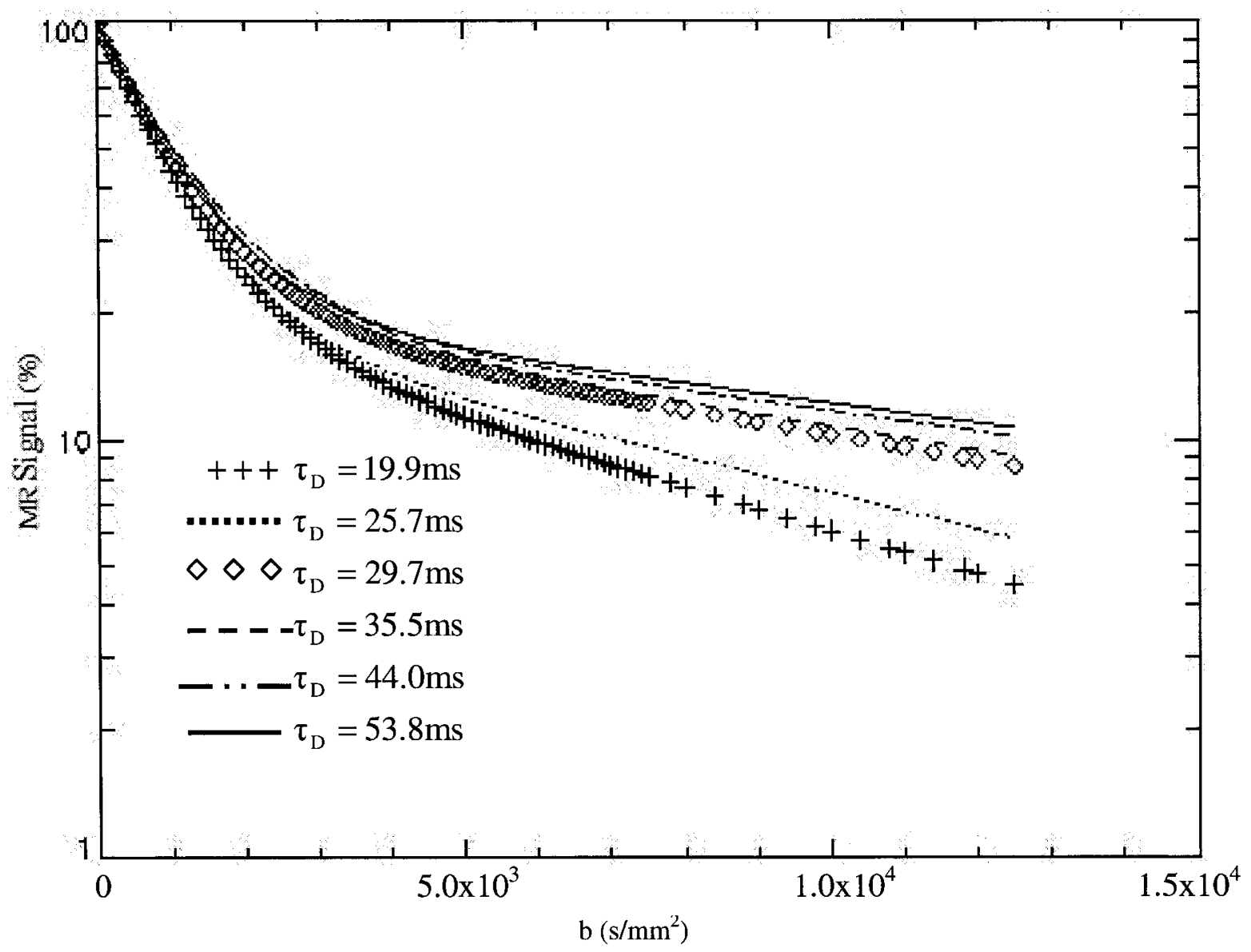

Figure 6.15. The diffusion decays for human brain tissue averaged over 8 human subjects vs. $\tau_{\mathrm{D}}$. All data were Rician noise bias corrected and analyzed by $N N L S$-diff multi-exponential analysis. 


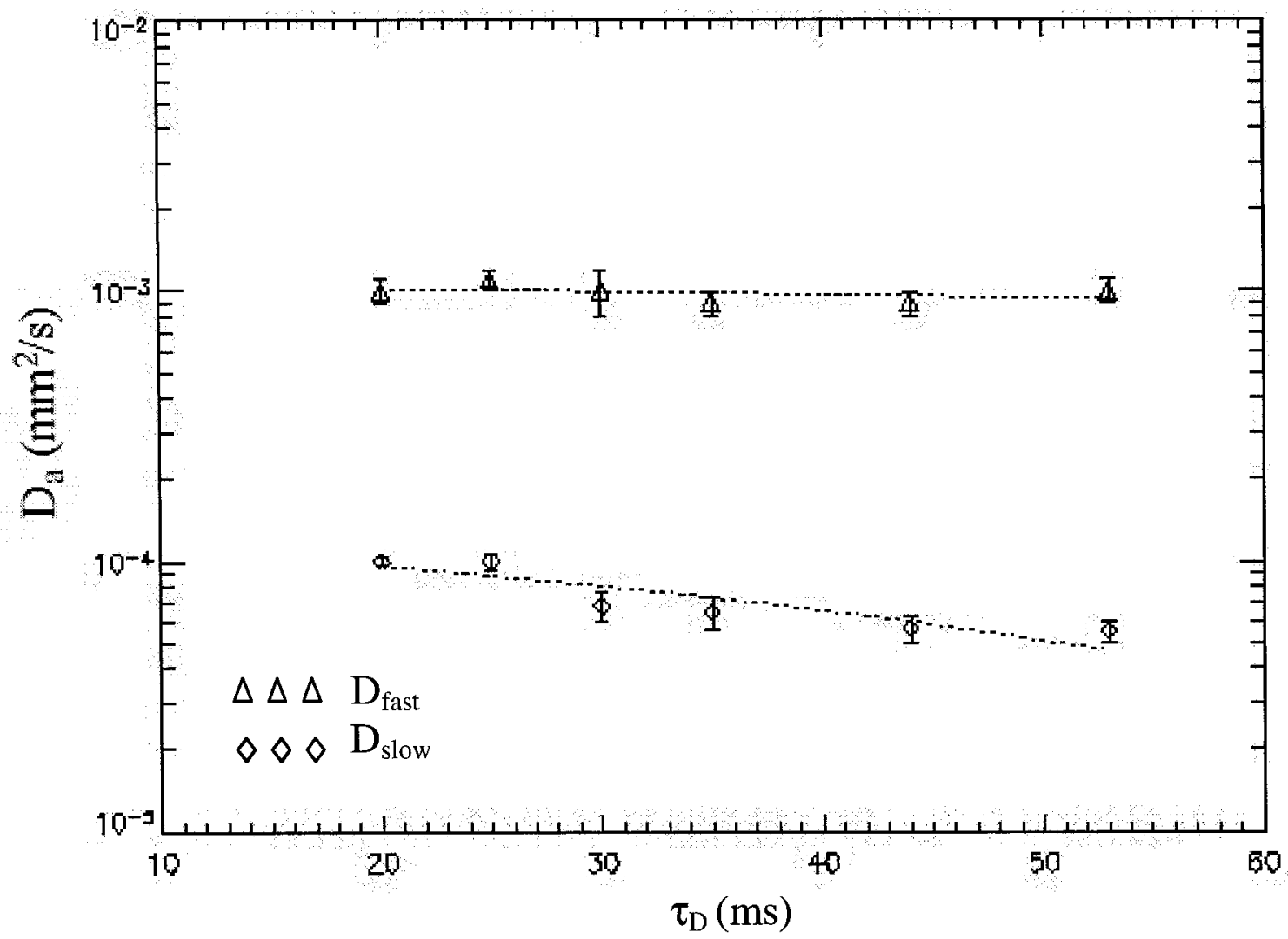

Figure 6.16. The variation of the slow and the fast diffusion coefficients for human brain tissue vs. diffusion time. The data were fitted with linear regression. 


\subsection{Interpretation of the $\tau_{\mathrm{D}}$ Dependence}

The observed $\tau_{\mathrm{D}}$ dependence of the diffusion decays and the spectrum of diffusion components for human brain tissue by multi-exponential analysis are consistent with the diffusion theory for a restricting geometry discussed in chapters 2 and 7 . The true interpretation of these effects requires consideration of brain tissue structure and function as well as sources of experimental error that could possibly also induce these variations. As mentioned before, intracellular diffusion is generally considered to be more restricted than extracellular diffusion in white matter. However, this remains somewhat controversial since fractional contributions from the measured diffusion components do not agree with the volume fractions of the intracellular and extracellular spaces in human brain. Even so, it is reasonable to assign the slow diffusion component, $D_{s}$, to the intracellular compartment and $\mathrm{D}_{\mathrm{f}}$, which is determined from the steep slope at low b-values, to the extracellular compartment. As can be seen from figure 6.15, the variation between the curves is not observed for small b-values $\left(b<1000 \mathrm{~s} / \mathrm{mm}^{2}\right)$ where the slow and the fast diffusion components both contribute to the decay. Restricted diffusion becomes more evident at high bvalues where the decay curves are more sensitive to the intracellular water molecules. The restriction manifests as a difference in signal attenuation of the diffusion measurements for the same b-value at different diffusion times. From this study, $D_{s}$ varies from $0.12 \times 10^{-3}$ to $0.061 \times 10^{-3}$ $\mathrm{mm}^{2} / \mathrm{s}$ for $\tau_{\mathrm{D}}$ values between 19.9 and $53.8 \mathrm{~ms}$ with a consistent trend as seen in table 6.3 and figure 6.16. Although the variations are small and some of them are within one standard deviation of the mean calculated for eight subjects, the difference between the $D_{s}$ values at $\tau_{D}=19.9 \mathrm{~ms}$ and $\tau_{\mathrm{D}}=53.8 \mathrm{~ms}$ is significant and considerably larger than the noise level (i.e. the standard deviation).

The experimental procedure that we introduced here is superior to others in the literature since the measured decays were corrected for Rician noise bias and the $N N L S$ algorithm was used 
to perform the analysis. Rician noise bias can have a significant effect on the decays for large bvalues. This correction was particularly helpful for the experiments at $\tau_{D}=44.0 \mathrm{~ms}$ and $\tau_{D}=53.8$ ms where the maximum b-value was greater than $2000 \mathrm{~s} / \mathrm{mm}^{2}$. Further technical improvements that would allow the use of higher gradient strengths (i.e. higher b-values) would improve this study by allowing us to study these effects over an extended range for the experimental parameters.

The observed variation of the diffusion signal decays with $\tau_{\mathrm{D}}$ could be due to the exchange of water molecules across the cell membrane in the intermediate exchange limit as expected from Karger exchange theory. For the range of diffusion times used in these measurements, the molecular exchange between the intracellular and extracellular compartments is not expected to be fast enough that a mono-exponential decay with a diffusion coefficient which is the average of the diffusion coefficients for these compartments will be obtained. However, it is also not expected to be slow enough that a bi-exponential decay with two distinct terms due to the true intracellular and extracellular diffusion coefficients will be measured. Based on Karger theory [127], intermediate exchange behavior is expected to lead to a bi-exponential decay with parameters which are related to both the intracellular and extracellular compartments such that the measured diffusion coefficients are not the "true" diffusion coefficients for these compartments but rather they are "apparent" diffusion coefficients. The apparent diffusion coefficients may also be dependent on other parameters such as residence time in the intracellular compartment, volume fractions and so on. The diffusion signal decay according to this theory, for intermediate diffusion times, is expressed in terms of the apparent diffusion coefficients $\left(D_{i}^{\prime}, D_{e}^{\prime}\right)$ and the fractional contributions $\left(f_{i}^{\prime}, f_{e}^{\prime}\right)$ of the two spaces after exchange $[98,99,103$, 127]: 


$$
\frac{S(b)}{S_{0}}=f_{e}^{\prime} \exp \left(-b D_{e}^{\prime}\right)+f_{i}^{\prime} \exp \left(-b D_{i}^{\prime}\right)
$$

where

$$
\begin{aligned}
D_{e(i)}^{\prime}=\frac{1}{2}\left\{\left[D_{e}+D_{i}+\frac{1}{(\gamma g \delta)^{2}}\left(\frac{1}{\tau_{e}}+\frac{1}{\tau_{i}}\right)\right] \pm\left[\left[D_{i}-D_{e}+\frac{1}{(\gamma g \delta)^{2}}\left(\frac{1}{\tau_{i}}-\frac{1}{\tau_{e}}\right)\right]^{2}+\frac{4}{(\gamma g \delta)^{4} \tau_{e} \tau_{i}}\right]^{1 / 2}\right\} \\
f_{i}^{\prime}=\frac{1}{D_{i}^{\prime}-D_{e}^{\prime}}\left(f_{e} D_{e}+f_{i} D_{i}-D_{e}^{\prime}\right) \\
f_{e}^{\prime}=1-f_{i}^{\prime}
\end{aligned}
$$

and the plus and minus signs go with $D_{i}^{\prime}$ and $D_{e}^{\prime}$, respectively. The $f_{i}$ and $f_{e}$ are the volume fractions of the intracellular and extracellular water and $\mathrm{D}_{\mathrm{i}}$, defined as the true intracellular diffusion coefficient, is considered to be smaller than $D_{e}$, the true extracellular diffusion coefficient, due to the higher protein concentration and viscosity in the cytosol. $\tau_{\mathrm{i}}$ and $\tau_{\mathrm{e}}$ are defined as residence times or exchange times of the intracellular and extracellular environments, respectively. There are several analytical and numerical models, such as Tanner's model, which can be used to calculate the apparent diffusion coefficients of the intracellular water vs. diffusion times with some assumptions and then the results can be used with the Karger theory to model the diffusion decay behavior of brain tissue. In the presence of exchange, the bi-exponential fits to the signal decays are not expected to directly reflect the intrinsic volume fractions and the "true" diffusion coefficients of each compartment. These measured parameters will, however, depend on the gradient strength and diffusion time used for the measurement, as can be seen from the results of this study. Our results will be discussed in more detail in terms of restricted diffusion and exchange theory in chapter 7 .

In the more general case, the diffusion signal decay can be fit by three exponential terms as already discussed. As mentioned in chapter 5 , the additional contribution could be related to 
perfusion in the intravascular compartment. However, to interpret this more responsibly, we would require a more complicated model with exchange between the three environments by the extension of Karger's formulation. A consequence of such a complex model is that there would be nine free parameters that must be considered.

The diffusion decay experiments discussed in chapter 5, known as "ct" experiments, in which all timings are held constant and the b-value is varied by changing the gradient strength, suggest that the concept of intracellular restriction with exchange may hold for brain tissue. This is due to the observed deviation from mono-exponential behavior and also the discrepancy of the contributions of the slow and the fast components to the decay with volume fractions of the intracellular and extracellular spaces, respectively. Experiments where the diffusion time (i.e. $\Delta$ ) is varied with a constant gradient for high b-values not only can indicate the restricted diffusion but may also lead to an estimation of the exchange time, from the measured signal decays vs. diffusion times. However, this evaluation from in vivo experiment needs further modeling and experimentation for conclusive interpretations. This is discussed further in chapter 7 .

As can be seen from the variation of the diffusion decays with $\tau_{\mathrm{D}}$, the signal attenuation becomes more $\tau_{D}$ independent for $\tau_{D}>30 \mathrm{~ms}$. In this limit, more diffusive water molecules in the restricted space feel the effects of the boundary causing the net displacement to be dependent on the shape and dimensions of the restricting geometry. Diffusion, at this point, may be influenced by anisotropy of the restricting boundaries and the observed signal attenuation may be more orientation dependent than in the shorter time limit that we used for diffusion sensitizing of the restricted intracellular water. 


\subsection{Conclusion}

The time dependent diffusion behavior of water in human brain tissue in vivo along with the nonmono-exponential diffusion attenuation showed consistency with theoretical works of others and the experimental studies on the rat brain and cell cultures $[102,106,111,110,114]$. Considering the complex effects of the structure of brain tissue and its cell size distributions, a simple assignment of the multi-exponential components to the tissue compartments and their intrinsic diffusion constants is not straightforward. The concept of diffusion restriction and exchange is, however, useful to assign the variation of the slow diffusion component with diffusion time to the intracellular water molecules in brain tissue.

More complicated models which incorporate all of the effects of exchange, restricted diffusion, anisotropy and compartmentalization of the signal behavior should be considered for a more complete interpretation. The precise measurement of water displacement using q-space MR imaging with much higher gradients would be useful future experiments to help provide a complete explanation of water diffusion and restriction in human brain tissue, similar to the results previously reported for rat brain [102]. 


\section{CHAPTER 7}

\section{Discussion and Conclusions}

\subsection{Brain Tissue Structure}

The diffusion of water molecules in human brain in vivo is dependent upon the microscopic brain tissue structure. Brain tissue consists of two main types of cells, neurons and glia, with the latter being more numerous than the former. A human brain has about 10 billion neurons and may contain a trillion glial cells.

Neuronal cells consist of a cell body, one primary projection called an axon and many secondary projections called dendrites. A typical neuron has dendritic connections with at least 1000 other neurons forming the basis of the complicated signaling system of the brain. The functioning of the nervous system is dependent upon the anatomical arrangement and the interconnectivity of the neuronal cells. The neuronal membrane, like all cell membranes, is primarily a phospholipid bilayer but it also contains many different proteins which have various functions. For example, some of these proteins serve as ion channels allowing the passage of the 
positive ions of sodium and potassium $\left(\mathrm{Na}^{+}\right.$and $\left.\mathrm{K}^{+}\right)$. The membranes of the cell and its organelles as well as the proteins and nucleic acids (e.g. DNA) can affect the diffusive movements of the water molecules inside cells. The cell body of the neuron is usually found in the cerebral cortex (i.e. the gray matter). The axons originate at the cell body and extend to other parts of the cerebral cortex or to peripheral nerves along well defined routes or tracts. The directional orientation of axon tracts gives rise to the diffusion anisotropy effect.

White matter is made up of axons surrounded by myelin sheaths, which serve a protective role for the axons as well as improving the speed of conduction of neuronal action potentials. Myelin is actually the cell membrane of oligodendrocyte glial cells, the predominant neuroglia of the white matter, which wrap themselves around the axons. A single oligodendrocyte can extend its membranous myelin sheath to wrap around 50 or more axons. Conversely, a single axon will typically be supplied with myelin from many oligodendrocyte cells.

Astrocytes, another type of glial cell, are the predominant neuroglia of the gray matter. They mediate metabolic exchange between neurons and blood capillaries and provide structural support.

The main contribution to the MR signal ( $>95 \%)$ comes from water inside the axons (intracellular), inside the myelin and in the interstitial space. The other part of the signal, about $4 \%$ of the total signal, is from water in brain capillaries which is pushed by the slow pulsatile flow of the blood (see figure 7.1). Brain capillary wall cells bind together very tightly to form a blood brain barrier. Only some lipid soluble molecules, small molecules such as $\mathrm{H}_{2} \mathrm{O}$ or $\mathrm{O}_{2}$ and actively transported molecules such as glucose can enter the brain from the bloodstream across the blood brain barrier. The tissue examined in the experiments reported in this thesis essentially consists of the intravascular, interstitial and intracellular environments. The $\mathrm{T}_{2}$ of myelin water is too short $(\sim 10 \mathrm{~ms})$ [146] to be detected in the experiments reported here. Water can exchange 
between these spaces which are separated by semi-permeable barriers. Thus, the measured diffusion will, in general, be a weighted average of the diffusion in these three compartments. Another more complicated effect of the tissue structure on diffusion is due to "obstructing" molecules (e.g. proteins) as well as interactions with protein hydration shells. These effects will reduce the mobility of water molecules in the brain and the diffusion coefficient measured with diffusion weighted MRI experiments.

The water molecules in the intracellular and interstitial spaces will normally have different diffusion coefficients which depend on the levels of hindrance and restriction the water molecules experience in these regions. The complex interaction between these two compartments is affected by many different properties of the tissue including the intracellular and extracellular diffusion coefficients $\left(D_{i}\right.$ and $\left.D_{e}\right)$, the respective volume fractions $\left(f_{i}\right.$ and $\left.f_{e}\right)$, cell membrane permeability, intracellular to extracellular and extracellular to intracellular exchange rates for water molecules $\left(\mathrm{k}_{\mathrm{ie}}\right.$ and $\mathrm{k}_{\mathrm{ei}}$ ), and the extracellular tortuosity factor, $\lambda$ (defined as the square root of the ratio of the free diffusion coefficient to the restricted diffusion coefficient for the restricted extracellular space). These physiological parameters have been measured in many cell culture experiments using iontopherosis and other techniques $[73-80,170,174,179,180,182,113,123]$ either to test analytical models of diffusion or to offer new insight into the processes that affect diffusion weighted experiments. These experiments have also shown good correlation between pathological conditions and changes of the diffusion parameters. For instance, the dramatic decrease in $\mathrm{D}_{\mathrm{a}}$ of about $40 \%$ that occurs within minutes of the onset of global ischemia followed by a continuous gradual decline, which is attributed to a drop in temperature (ATP depletion), was shown to be possibly related to changes in cell volume (due to cell swelling), extracellular tortuosity and the intracellular to extracellular exchange rate. 
MR diffusion experiments are capable of examining the diffusion characteristics of brain tissue as well as their changes under controlled pathological conditions. In this study we measured diffusion decays at high b-values and short diffusion times for human subjects which allowed us to properly characterize the restricted diffusion of the intracellular water molecules in white matter of healthy human brain. Thus, we can reasonably discuss our results in terms of the multi-compartment model of brain white matter. In all of our modeling in this discussion, we simply consider the cells to be axons of uniform size (as opposed to a Gaussian distribution of cell sizes, for example), with square cross-section and the same orientation (i.e. the diffusion of water molecules in both the intracellular and extracellular spaces is considered to be influenced in the same way by anisotropy due to the axon tract orientation).

\subsection{Diffusion Decay Interpretation}

The results of our experiments show that the water diffusion signal decay for brain tissue deviates, as expected, from mono-exponential behavior (which is commonly assumed to be the case when generating ADC maps clinically) when high b-values are employed. Using the extended range of b-values along with Rician noise bias correction techniques and multicomponent analysis, three diffusion coefficients were always detected for the signal decays from the regions of interest used in this study. As reported before, the curvature of the brain diffusion decay curves generally becomes noticeable for b-values above $2000 \mathrm{~s} / \mathrm{mm}^{2}$ regardless of whether or not anisotropic diffusion effects are present [98]. However, sampling only a few b-values under $1500 \mathrm{~s} / \mathrm{mm}^{2}$, which is the current clinical practice, largely limits the information obtained to a single diffusion component. More information can clearly be accessed from the diffusion signal decay for slower diffusion by extending the b-value range. The use and interpretation of 
the additional information that will result from a better understanding of multi-component diffusion decay behavior is expected to lead to better clinical diagnosis of neurological disorders.

The tri-exponential behavior of the diffusion decay observed in this study is fundamentally different from that seen by Mulkern et. al. [98, 103] who reported bi-exponential diffusion behavior. However, by using numerous b-values up to a maximum of $12,500 \mathrm{~s} / \mathrm{mm}^{2}$ (compared with $6000 \mathrm{~s} / \mathrm{mm}^{2}$ used by Mulkern et. al. [103]), systematic Rician noise bias correction and by finding the diffusion components with $N N L S$ multi-component analysis, which does not require the number of components to be specified or an initial estimation of the fit parameters, we have improved the experimental procedure to allow us to get more reliable diffusion information from the signal decays than previously reported.

\subsubsection{Compartmentalization of tissue structure}

One possible explanation of the tri-exponential behavior of the diffusion signal decay measured in our experiments is compartmentalization of water into intracellular, interstitial and intravascular spaces. This is due to the presumption that these environments have slow, fast and ultra fast intrinsic diffusion coefficients, respectively.

Some cell culture studies have shown previously that the intracellular diffusion coefficient, $D_{i}$, is smaller than the extracellular diffusion coefficient, $D_{e}$, for red blood cells in suspension by using paramagnetic doping, $T_{2}$ relaxation and diffusion experiments $[176,177]$. As convincingly demonstrated by their studies, the slow diffusion component, $D_{s}$, of the system they examined was most reasonably associated with the intracellular water compartment. The interpretation of multi-component diffusion decays based on intracellular and extracellular compartments has also been used in diffusion measurements of perfused cancer cell preparations [113,114,175]. An 
extensive analysis based on this interpretation was also developed by Pfeuffer et. al. [102,111] with the Karger two site exchange theory considering restricted diffusion with semi-permeable barriers. It should be noted that for better understanding of the tri-exponential behavior of the diffusion decay, an analytical model with three-site exchange similar to what was proposed by Stanisz et. al. [106], would probably be more representative of brain tissue. However, since the contribution of the third component is less than $2 \%$ of the total signal, we can reasonably ignore this component and use the simpler two-site exchange Karger theory which has fewer free parameters to fit.

The observation of decreased ADC in acute stroke is quantitatively consistent with the prediction of a volume averaged fast exchange model using literature values for the intracellular to extracellular volume ratio in healthy and ischemic adult brain tissue, where the change in this ratio is attributed to cell swelling in ischemia. The bi-exponential diffusion decay behavior in the rat brain experiments of Niendorf et. al. [99] was also reasonably explained in terms of intracellular and extracellular compartments. They reported an $80 \%$ volume fraction for the fast ADC component of the healthy adult rat brain compared with a $90 \%$ volume fraction for brain in immature rats. This is consistent with a larger extracellular space in immature brain. They also reported a reduced volume fraction from $80 \%$ for healthy rat brain to $69 \%$ postmortem. The good correlation between changes of the intracellular volume fraction and changes of the slow diffusion component fraction observed during recovery from excitotoxic brain edema was also shown using electrical conductivity measurement in both rat and cat experiments [73-80]. All of these observations as well as our experimental results are consistent with this compartmentalization interpretation for the slow and fast diffusion components. 


\subsubsection{Intracellular restricted diffusion}

Assuming that the two main diffusion coefficients can be associated with the intracellular and extracellular water compartments, it is reasonable to assume that the diffusion of water molecules within the cells can be associated with the slow diffusion coefficient as a consequence of interactions with intracellular organelles and membranes.

Many analytical theories [111-115, 98,123] have been developed to predict the results of diffusion experiments for restricted diffusion. To review all these theories and to interpret our results based on them is far beyond the scope of this thesis. Some of them were previously reviewed in M.Sc. and Ph.D. theses from the MR lab at The Ottawa Hospital [84,173]. In our study, we use Tanner-planar theory which was developed for impermeable parallel planes separated by a distance a (see the equation (2.82)) to model the inherent intracellular restricted diffusion, ignoring the effects of exchange at this stage. In this theory, the duration of the diffusion sensitizing gradient, $\delta$, is considered to be small enough that the average displacement that occurs during this time interval is much smaller than that which will eventually occur during the larger time interval $\Delta$, which is equal to the diffusion time when $\delta$ is small (see the StejskalTanner diffusion sequence in chapter 2). This condition is much easier to fulfill for rat brain experiments where very high gradient amplitudes can be used. For human studies, such as the ones reported here, this condition is often poorly satisfied. In this situation, correction terms for $\tau_{\mathrm{D}}$, such as those calculated in section 6.2.2 and appendix II, must be used (see also section 2.3.3).

Using the Tanner formula, we evaluated the diffusion coefficient of restricted water molecules confined to a box of square cross-section with the length of a side, a, equal to $5 \mu \mathrm{m}$ to represent the mean axonal cross-sectional area for the human brain. We also used the experimental parameters from our diffusion decay study (e.g. $\tau_{\mathrm{D}}=50 \mathrm{~ms}, \mathrm{~g}=28.5 \mathrm{mT} / \mathrm{m}$ and $\delta=30$ 
$\mathrm{ms}$ ) and the summation in equation (2.82) was evaluated for $\mathrm{n}=1$ term. As previously mentioned, the idea of setting our experimental parameters to correspond to a very high b-value, associated with the highest possible value of $(\gamma \delta \mathrm{g})^{2}$, was the correlation of this parameter to the reciprocal of the mean squared displacement of the diffusing water molecules. Our presumption is that on this scale we are able to sensitize the confined water molecules inside the cells which still have not experienced the cell membrane because of short displacement where, as justified above, it is assumed that the intracellular diffusion coefficient can be associated with the slow diffusion coefficient of the measured decay.

The primary diffusion coefficient for human brain cells used in many studies in the literature (i.e. $D_{i}=1 \times 10^{-3} \mathrm{~mm}^{2} / \mathrm{s}$ ) was applied in the Tanner formula as the free diffusion coefficient. With these settings the diffusion coefficient for restricted intracellular diffusion for this model of human axons was found to be $D_{i}^{a}=0.044 \times 10^{-3} \mathrm{~mm}^{2} / \mathrm{s}$ from the initial slope of the signal attenuation with the limit of $b \rightarrow 0$ (see equation (2.83)). The same experimental parameters were used for a range of cell size from $\mathrm{a}=2$ to $10 \mu \mathrm{m}$ (table 7.1). The diffusion coefficients calculated with the Tanner formula for restricted intracellular water, for a cell size of $\mathrm{a}=5 \mu \mathrm{m}$ and $\mathrm{a}=6 \mu \mathrm{m}$ are in agreement with the slow diffusion coefficient measured in our experiment, $\mathrm{D}_{\mathrm{s}}=0.06 \times 10^{-3} \mathrm{~mm}^{2} / \mathrm{s}$.

\subsubsection{Extracellular diffusion}

Although the accurate determination of $D_{i}$ and $D_{e}$ is essential for the verification of most analytical models and thus to be compared with the diffusion components measured with diffusion weighted MR imaging (DWI) experiments, it is by its nature difficult, particularly the determination of $D_{e}$. Since water exchanges between the two compartments, it may not be reliable 


\begin{tabular}{|c|c|c|c|c|c|c|}
\hline $\mathrm{a}(\mu \mathrm{m})$ & 2 & 4 & 5 & 6 & 8 & 10 \\
\hline $\mathrm{D}_{\mathrm{i}}^{\mathrm{a}}\left(10^{-3} \mathrm{~mm}^{2} / \mathrm{s}\right)$ & 0.0067 & 0.028 & 0.044 & 0.065 & 0.13 & 0.23 \\
\hline
\end{tabular}

Table 7.1. The evaluated intracellular restricted diffusion coefficient vs. cell size for the Tannerplanar equation with appropriate experimental settings for the experimental data reported in this thesis (e.g. $\tau_{D}=50 \mathrm{~ms}$ with the maximum b-value $=12,500 \mathrm{~s} / \mathrm{mm}^{2}$ ).

to derive a value of $D_{e}$ based on the diffusion coefficient of a particle that remains in one compartment. Like some other researchers $[102,123,170]$, we have simply taken the value of water at body temperature to represent the unhindered value of $\mathrm{D}_{\mathrm{e}}$. Water diffusion in the interstitial space is obviously not free, but hindered by the cell walls. The particles have to use longer diffusion pathways around neurons and glial cells, which can be described by a decreased diffusion coefficient for restricted extracellular diffusion $[17,18,178,179,180]$ :

$$
D_{\mathrm{e}}^{\mathrm{a}}=\frac{\mathrm{D}_{\mathrm{e}}}{\lambda^{2}}
$$

where $\lambda$ is called the extracellular tortuosity factor and normally $\lambda \geq 1$. The concept of a tortuosity factor was first introduced by Nicholson and Phillips in the theory of porous media $[17,180]$.

Once the slow diffusion component of our measurements is associated with the intracellular water molecules, it is natural to associate the fast diffusion component with the extracellular water. By setting the free extracellular diffusion coefficient, $D_{e}$, equal to the free diffusion coefficient of water at $37^{\circ} \mathrm{C}$ (i.e. $\mathrm{D}_{\mathrm{e}}=3 \times 10^{-3} \mathrm{~mm}^{2} / \mathrm{s}$ ), and the fast diffusion component of our measurements as the restricted extracellular diffusion coefficient, we were able to calculate a tortuosity factor from equation (7.1) of 1.8. This is larger than the value of $1.5-1.6$ reported by others for healthy brain $[170,182,123]$. The measurement of the tortuosity factor is generally done using iontopherosis, by examining the motion of ions introduced into the extracellular space as a 
function of time. The process thus corresponds to Fickian diffusion in the presence of a concentration gradient. The tortuosity factor for rat brain is about 1.6 for adults; 1.5 for newborn and 2.0 for postmortem [123]. Experiments on human brain cells, histologically verified to be normal tissue, have given $\lambda=1.55 \pm .03$ for adults [170]. By setting $\lambda=1.6$ in equation (7.1), we calculated the restricted extracellular diffusion coefficient to be $D_{\mathrm{e}}^{\mathrm{a}}=1.17 \times 10^{-3} \mathrm{~mm}^{2} / \mathrm{s}$ which is not unreasonably different from the value of $D_{\mathrm{f}}=0.9 \times 10^{-3} \mathrm{~mm}^{2} / \mathrm{s}$ that we obtained experimentally for the fast diffusion component.

By using these analytical formulas (equations (2.82) and (7.1)), we were able to calculate both $\mathrm{D}_{\mathrm{i}}^{\mathrm{a}}$ and $\mathrm{D}_{\mathrm{e}}^{\mathrm{a}}$ by assuming that the diffusion is restricted to the intracellular and extracellular spaces, respectively. As yet, molecular transport between these two compartments has not been taken into account. The theory of exchange between two compartments was first proposed for MR experiments by Zimmerman and Brittin [171] for spin relaxation behavior $\left(\mathrm{T}_{1}\right.$ and $\left.\mathrm{T}_{2}\right)$, and then extended by Karger [127] to molecular diffusion in a two compartment system. Based on exchange theory it is likely that water exchange between compartments will affect the measured diffusion coefficients and the relative contribution of the fast and slow diffusion components to the water signal decay in DWI experiments $[98,99,103]$. This will be considered in the next section.

\subsubsection{Exchange between compartments}

According to the above discussion, one can assign the slow and the fast diffusion components to the intracellular and extracellular compartments, respectively. However, the relative water contributions are largely opposite to that which would be expected on the basis of the known 


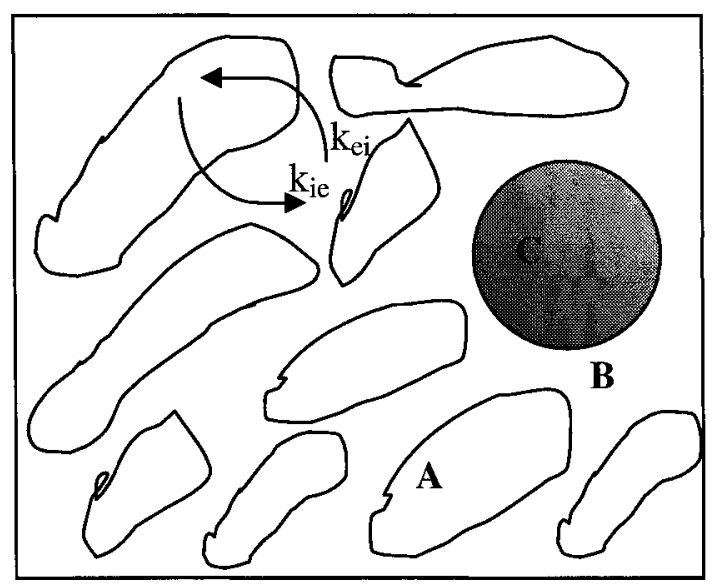

Figure 7.1. A schematic diagram showing the regions of the brain and water exchange between the two main compartments; $\mathrm{A}$ is the intracellular space, $\mathrm{B}$ is the interstitial space, and $\mathrm{C}$ is a capillary.

intracellular and extracellular volume fractions in brain tissue $[98,99,103,123]$. Even when the possibility of water exchange between compartments is considered, the interpretation of the results in terms of intracellular and extracellular water compartmentalization is problematic.

Theoretically, the effect on the diffusion decay of water exchange in a two-site system can be demonstrated with the Karger formulation (equations (6.11) to (6.14)), where the apparent diffusion coefficients of the intracellular and extracellular compartments after exchange between the two spaces, $D_{i}^{\prime}$ and $D_{e}^{\prime}$, and their relative fractions, $f_{i}^{\prime}$ and $f_{e}^{\prime}$, can be written in terms of the exchange rates as follows:

$$
\begin{aligned}
D_{\mathrm{e}(\mathrm{i})}^{\prime}=\frac{1}{2}\left\{\left[\mathrm{D}_{\mathrm{e}}+\mathrm{D}_{\mathrm{i}}+\frac{1}{(\gamma g \delta)^{2}}\right.\right. & \left.\left.\left(\mathrm{k}_{\mathrm{ei}}+\mathrm{k}_{\mathrm{ie}}\right)\right] \pm\left[\left[\mathrm{D}_{\mathrm{i}}-\mathrm{D}_{\mathrm{e}}+\frac{1}{(\gamma \mathrm{g} \delta)^{2}}\left(\mathrm{k}_{\mathrm{ie}}-\mathrm{k}_{\mathrm{ei}}\right)\right]^{2}+\mathrm{k}_{\mathrm{ei}} \mathrm{k}_{\mathrm{ie}} \frac{4}{(\gamma g \delta)^{4}}\right]^{1 / 2}\right\}, \\
\mathrm{f}_{\mathrm{i}}^{\prime} & =\frac{1}{\mathrm{D}_{\mathrm{i}}^{\prime}-\mathrm{D}_{\mathrm{e}}^{\prime}}\left(\mathrm{f}_{\mathrm{e}} \mathrm{D}_{\mathrm{e}}+\mathrm{f}_{\mathrm{i}} \mathrm{D}_{\mathrm{i}}-\mathrm{D}_{\mathrm{e}}^{\prime}\right), \\
\mathrm{f}_{\mathrm{e}}^{\prime} & =1-\mathrm{f}_{\mathrm{i}}^{\prime} .
\end{aligned}
$$

where the plus and minus signs go with $\mathrm{D}_{\mathrm{i}}^{\prime}$ and $\mathrm{D}_{\mathrm{e}}^{\prime}$, respectively. The $\mathrm{k}_{\mathrm{ie}}=\frac{1}{\tau_{\mathrm{i}}}, \mathrm{k}_{\mathrm{ei}}=\frac{1}{\tau_{\mathrm{e}}}$ are the 
exchange rates from the intracellular to extracellular and extracellular to intracellular compartments, respectively, which are constrained by the steady state relationship $\mathrm{f}_{\mathrm{i}}^{\prime} \mathrm{k}_{\mathrm{ie}}=\mathrm{f}_{\mathrm{e}}^{\prime} \mathrm{k}_{\mathrm{ei}}$. $\tau_{\mathrm{i}}$ and $\tau_{\mathrm{e}}$ are the residence times in the intracellular and extracellular compartments, respectively. Figure 7.1 shows schematically the exchange of the diffusing molecules inside the cell with those outside the cell and vise versa with the rates of $k_{i e}$ and $k_{e i}$, respectively. Only in the absence of exchange, $\mathrm{k}_{\mathrm{ie}}=\mathrm{k}_{\mathrm{e}}=0$, will the parameters extracted experimentally from bi-exponential fits of diffusion signal decays give the true intrinsic volume fractions and diffusion coefficients of each compartment. In the more complicated case due to exchange, the measured parameters will correspond to the apparent ones (i.e. primed values) rather than the intrinsic parameters (i.e. the unprimed values). It should be noted that the term "apparent diffusion coefficient" in exchange theory has a very different meaning from the apparent diffusion coefficient, $D_{a}$, that we have considered up to now. The apparent parameters in exchange theory will be indicated with a prime.

We used our experimental settings with the Karger equation to generate diffusion decay curves for various exchange rates between the two environments. The intrinsic parameters of $D_{i}=1 \times 10^{-3} \mathrm{~mm}^{2} / \mathrm{s}$ and $D_{e}=3 \times 10^{-3} \mathrm{~mm}^{2} / \mathrm{s}$ along with $\mathrm{a}=5 \mu \mathrm{m}, \tau_{\mathrm{D}}=50 \mathrm{~ms}$ and $\lambda=1.6$ were first used to estimate the restricted diffusion coefficients for the intracellular and extracellular spaces, respectively, using the Tanner-planar formula (equation (2.82)) and equation (7.1) as discussed in sections 7.2.2 and 7.2.3. The resulting values, $\mathrm{D}_{\mathrm{i}}^{\mathrm{a}}$ and $\mathrm{D}_{\mathrm{e}}^{\mathrm{a}}$, were then substituted into the Karger formula (see equation (7.2)), for $D_{i}$ and $D_{e}$ with their relative initial volume fractions set to $f_{i}=0.8$ and $f_{e}=0.2$, respectively. The $D_{i}^{\prime}$ and $D_{e}^{\prime}$ as well as $f_{i}^{\prime}$ and $f_{e}^{\prime}$ values were evaluated using this equation for exchange rate, between 0 and $70 \mathrm{~s}^{-1}$ and the results are tabulated in table 7.2a. Most of the exchange rates considered for these calculations were extracted from the literature for cell culture or rat brain experiments $[98,111,123,115,170,174]$. These calculations were repeated for 
the initial fractions of $\mathrm{f}_{\mathrm{i}}=0.2$ and $\mathrm{f}_{\mathrm{e}}=0.8$ which were the measured fractions from our experiments for the slow and the fast diffusion components, respectively. Table 7.2b shows the results of these calculations.

As depicted in the figures $7.2 \mathrm{a}$ and $7.2 \mathrm{~b}$, both calculations show that the decays with no exchange (solid line) and slow exchange (dashed line), associated with an intracellular residence time of $\tau_{\mathrm{i}}=610 \mathrm{~ms}$ as reported by Duong et. al. [174], do not affect the diffusion parameters appreciably. On the other hand, fast exchange associated with the exchange time of $\tau_{i}=15 \mathrm{~ms}$ (dots) reported for rat brain [111] and $17 \mathrm{~ms}$ (dash-dots) for human erythrocytes (red blood cells) [123], can drastically affect the parameters including the diffusion coefficients and their relative fractions. This behavior is consistent with diffusion over sufficiently large paths during the diffusion time (i.e. $\tau_{\mathrm{i}}<<\tau_{\mathrm{D}}$ ), where the diffusion coefficient can be determined as the weighted average of the diffusion coefficients for the two sub regions [99, 127]. In fast exchange the diffusion induced signal attenuation shows a mono-exponential dependence on diffusion weighting and appears to be a quasi homogenous system. The diffusion decays for $\tau_{\mathrm{i}}=50 \mathrm{~ms}(\diamond)$ reported for glial cells [123] and $\tau_{\mathrm{i}}=100 \mathrm{~ms}\left(^{*}\right)$ also reported for red blood cells [98] are plotted in figure 7.2 and correspond to intermediate exchange.

Pfeuffer et. al. [102,111] derived the apparent diffusion coefficient of the slow component assigned to the restricted intracellular diffusion coefficient, and the intercept using large b-values between 8000 and $10000 \mathrm{~s} / \mathrm{mm}^{2}$ from the analytical diffusion decays of the Karger exchange theory to quantitatively analyze the rat brain model system. Using this procedure, the apparent intracellular fractions were estimated to be between 18 and $30 \%$ for the simulated signals over the same range of exchange rates as we have considered. These numbers are comparable with the range of the slow diffusion contributions to the experimental signal decays that we have reported. 


\begin{tabular}{|c|c|c|c|c|c|}
\hline $\mathrm{k}_{\mathrm{ie}}\left(\mathrm{s}^{-1}\right)$ & $\tau_{\mathrm{i}}(\mathrm{ms})$ & $\begin{array}{c}\mathrm{D}_{\mathrm{i}}^{\prime}\left(\mathrm{mm}^{2} / \mathrm{s}\right) \\
\times 10^{-3}\end{array}$ & $\begin{array}{c}\mathrm{D}_{\mathrm{e}}^{\prime}\left(\mathrm{mm}^{2} / \mathrm{s}\right) \\
\times 10^{-3}\end{array}$ & $\mathrm{f}_{\mathrm{i}}^{\prime}$ & $\mathrm{f}_{\mathrm{e}}^{\prime}$ \\
\hline 0 & inf. & 0.044 & 1.17 & 0.8 & 0.2 \\
1.6 & 610 & 0.05 & 1.2 & 0.809 & 0.191 \\
10 & 100 & 0.079 & 1.3 & 0.848 & 0.152 \\
20 & 50 & 0.1 & 1.5 & 0.883 & 0.117 \\
30 & 33 & 0.13 & 1.6 & 0.908 & 0.092 \\
40 & 25 & 0.14 & 1.8 & 0.927 & 0.073 \\
50 & 20 & 0.15 & 2.1 & 0.940 & 0.060 \\
58 & 17.2 & 0.16 & 2.2 & 0.949 & 0.051 \\
60 & 16.7 & 0.17 & 2.2 & 0.951 & 0.049 \\
66 & 15 & 0.17 & 2.4 & 0.956 & 0.044 \\
70 & 14 & 0.17 & 2.4 & 0.959 & 0.041 \\
\hline
\end{tabular}

Table 7.2a. The results of Karger exchange theory with $\mathrm{f}_{\mathrm{i}}=0.8, \mathrm{D}_{\mathrm{i}}=0.001 \mathrm{~mm}^{2} / \mathrm{s}$ and $\mathrm{D}_{\mathrm{e}}=0.003$ $\mathrm{mm}^{2} / \mathrm{s}$. Restricted diffusion coefficients were calculated from $D_{i}$ and $D_{e}$ for intracellular and extracellular diffusion, respectively, using the Tanner formula and equation (7.1). These values were then used in Karger's exchange theory (equation (7.2)).

\begin{tabular}{|c|c|c|c|c|c|}
\hline $\mathrm{k}_{\mathrm{ie}}\left(\mathrm{s}^{-1}\right)$ & $\tau_{\mathrm{i}}(\mathrm{ms})$ & $\begin{array}{c}\mathrm{D}_{\mathrm{i}}^{\prime}\left(\mathrm{mm}^{2} / \mathrm{s}\right) \\
\times 10^{-3}\end{array}$ & $\begin{array}{c}\mathrm{D}_{\mathrm{e}}^{\prime}\left(\mathrm{mm}^{2} / \mathrm{s}\right) \\
\times 10^{-3}\end{array}$ & $\mathrm{f}_{\mathrm{i}}^{\prime}$ & $\mathrm{f}_{\mathrm{e}}^{\prime}$ \\
\hline 0 & inf. & 0.044 & 1.17 & 0.2 & 0.8 \\
1.6 & 610 & 0.05 & 1.17 & 0.202 & 0.798 \\
10 & 100 & 0.083 & 1.18 & 0.215 & 0.785 \\
20 & 50 & 0.12 & 1.19 & 0.231 & 0.769 \\
30 & 33 & 0.16 & 1.2 & 0.248 & 0.752 \\
40 & 25 & 0.2 & 1.2 & 0.266 & 0.734 \\
50 & 20 & 0.23 & 1.2 & 0.286 & 0.714 \\
58 & 17.2 & 0.26 & 1.2 & 0.303 & 0.697 \\
60 & 16.7 & 0.27 & 1.2 & 0.307 & 0.693 \\
66 & 15 & 0.29 & 1.3 & 0.321 & 0.679 \\
70 & 14 & 0.3 & 1.3 & 0.33 & 0.67 \\
\hline
\end{tabular}

Table 7.2b. The results of Karger exchange theory with the same settings as for table $7.2 \mathrm{a}$ but with the intracellular fraction set to $\mathrm{f}_{\mathrm{i}}=0.2$. 
a.

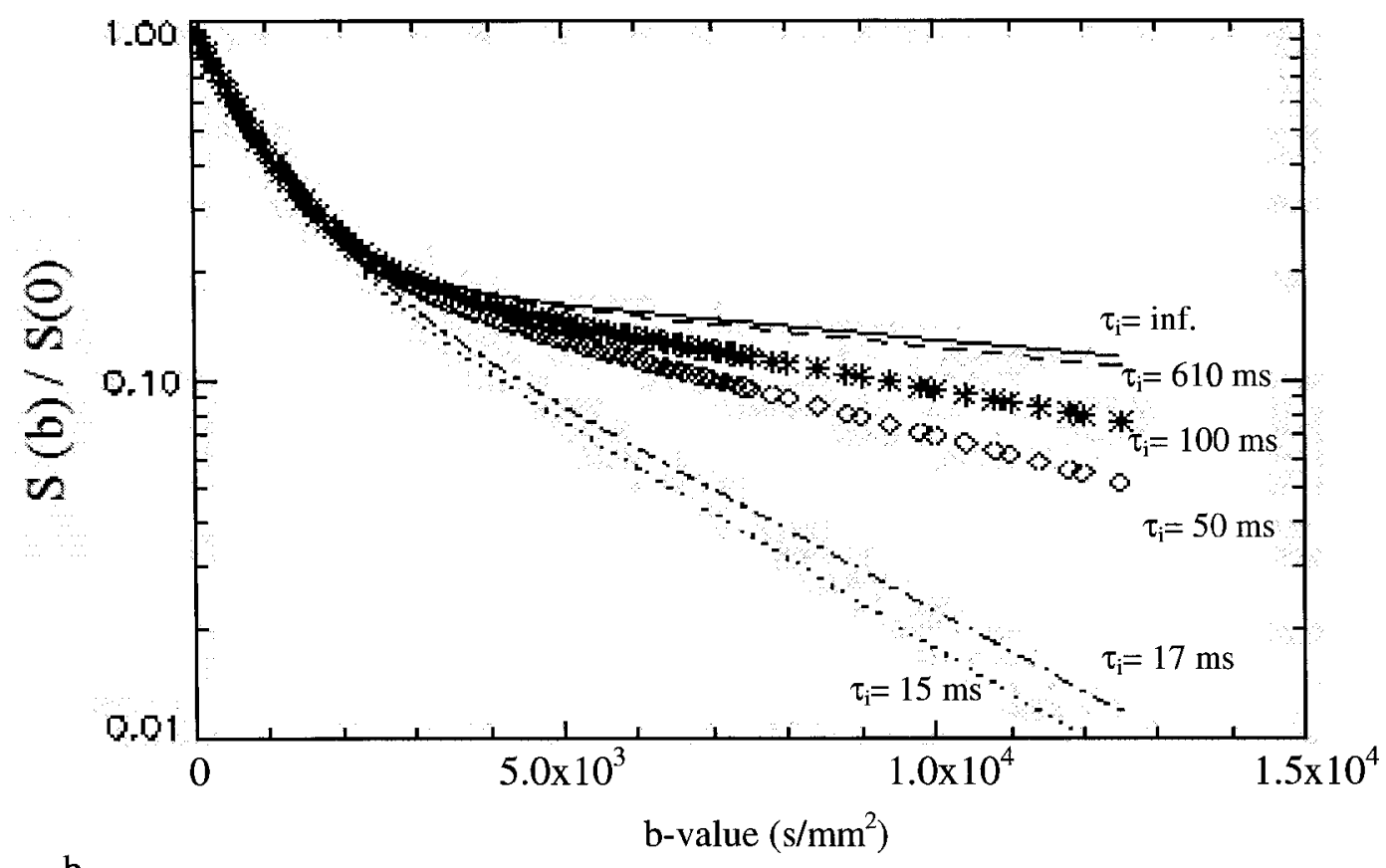

b.

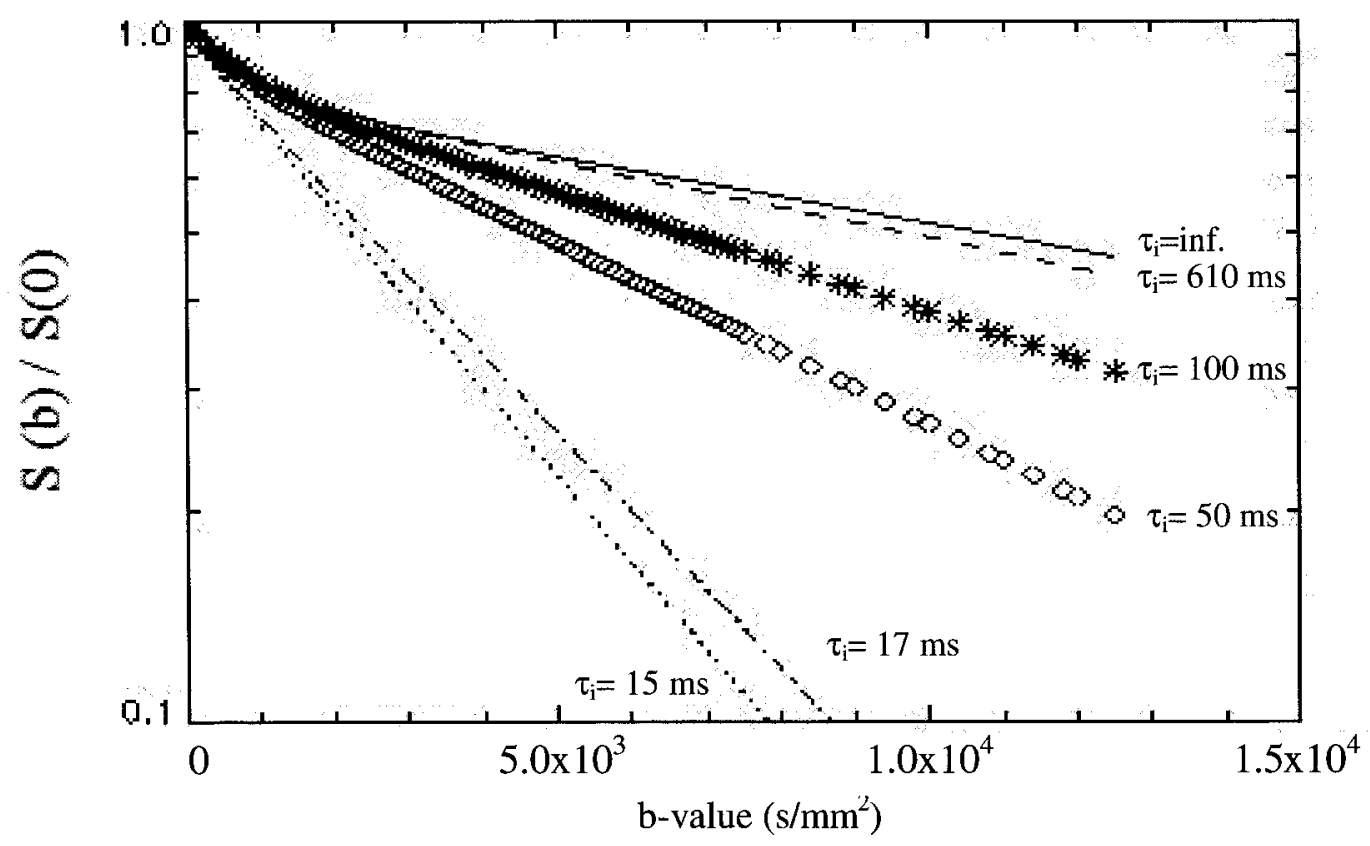

Figure 7.2 The calculated diffusion signal decays using the Tanner-planar and Karger exchange theory for a healthy human model $\left(D_{i}=0.001 \mathrm{~mm}^{2} / \mathrm{s}, D_{e}=0.003 \mathrm{~mm}^{2} / \mathrm{s}, \lambda=1.6\right)$ vs. intracellular residence time with an initial intracellular fraction of a. (top) $f_{i}=0.2$ and $b$. (bottom) $f_{i}=0.8$. 
In the rest of the discussion we used our measured diffusion fractions in the analytical models (i.e. $\mathrm{f}_{\mathrm{i}}=0.2$ and $\mathrm{f}_{\mathrm{e}}=0.8$ ) to better compare the experimental results with theory.

\subsection{5 $\mathrm{T}_{2}$ relaxation, anisotropy, and blood flow effects}

A more heavily $\mathrm{T}_{2}$-weighted intracellular water pool compared with the extracellular pool could also explain the discrepancy between the actual volume fraction and the measured apparent water contribution to the slow component, particularly for long TE such as was used in our experiments ( $\mathrm{TE}=200 \mathrm{~ms}$ ). However, there is still no evidence in the literature of well separating $\mathrm{T}_{2}$ relaxation components corresponding to intracellular and extracellular compartments [146, 165] for healthy brain tissue. Thus, the two diffusion components are likely unaffected by the $T_{2}$ relaxation behavior. Although the use of relatively long TE values causes the MR signal to be attenuated due to $T_{2}$ relaxation causing the diffusion decays to have a lower $S N R$, a $T_{2}$ dependence is generally excluded from our experimental diffusion decays by keeping the echo time constant in all diffusion measurements.

A multi-exponential water diffusion decay in brain tissue could also be caused by different fiber tract orientations within the ROI. This anisotropic behavior can be detected by applying the diffusion sensitizing gradients in different directions. As previously shown $[98,103]$, biexponential behavior is not restricted to brain tissue in which anisotropic diffusion decay behavior is obvious (e.g. internal capsule). It also occurs in regions such as the cortex where the anisotropy is very low.

In our analysis of diffusion in brain tissue, we placed the ROIs in locations where the axon orientations are known to be predominantly in the inferior to superior direction. For our experiments this means that the majority of the axons are perpendicular to the diffusion 
sensitizing gradient direction used in the measurements, consistent with one orientation for all of the axons in the ROI and isotropic behavior. Taking advantage of multi-component analysis would also allow us to detect the effect of anisotropy by finding additional components contributing to the diffusion decays due to the distribution of axon orientations in the studied regions. However, the three components observed for the diffusion decays after Rician noise bias correction are less than what would be expected if the anisotropy effects existed along with compartmentalization. To assign the slow and the fast components of the diffusion decay resulting from our analysis to parallel and perpendicular diffusion coefficients is somewhat unreasonable since there is no evidence to support this proposition. It should also be noted that the anisotropy effect is mostly removed at shorter diffusion times, as previously discussed by Gates [84] in his Ph.D. thesis.

The ultra fast component $\left(D_{a}=1 \times 10^{-2} \mathrm{~mm}^{2} / \mathrm{s}\right)$, which has the smallest water contribution $(<2 \%)$, can be explained by blood perfusion. This component is expected when b-values less than $100 \mathrm{~s} / \mathrm{mm}^{2}$ are included in the protocol. By interpretation of the diffusion decay behavior based on compartmentalization of brain tissue it is possible to assign this ultra fast component to the intravascular water molecules because of the low volume fraction $(<4 \%)$ and relative high pseudo diffusion coefficient for intravascular water in human white matter. This effect was first demonstrated by Le Bihan et. al. [172] where the rapidly and slowly decaying components are interpreted as arising from intravascular perfusion and extravascular diffusion, respectively.

\subsection{Rician Noise Effects on the Diffusion Decay}

Using the NNLS multi-component method for the analysis of the diffusion decays gave us the capability of determining the number of the components of the unknown system, human brain 
tissue, rather than assuming a set number of components as most previous studies have done. With this analysis method, we first detected four discrete (weighted average) components in the spectrum (see figure 7.3a). A similar four component diffusion decay was previously reported by Pfeuffer et. al. [102,111] for their rat brain studies. This observation might have misguided us in our quantitative analysis of the multi-exponential diffusion decay considering that after the Rician noise bias was removed from diffusion decays the number of peaks reduced to three components that can be interpreted quite naturally in terms of the intracellular, interstitial and intravascular compartments.

In this study, we investigated the MR diffusion decay properly including correcting the data for the effects of Rician noise bias particularly at high b-values. Systematic errors, noise and contributions to the measured signal from different artifacts was also studied. Several correction methods were proposed and tested both theoretically and experimentally. The most consistent method was then applied to all diffusion decays. The diffusion information was extracted from the measured decays after applying the Rician noise correction algorithm as discussed in the previous chapters. As an example, diffusion analysis after Rician noise bias correction shows a three component discrete spectrum as shown in figure $7.3 \mathrm{~b}$, compared with the four component diffusion spectrum without noise bias correction shown in figure $7.3 \mathrm{a}$.

We then conclude that reliable diffusion parameters, including the diffusion coefficients and their relative fractions, can only be obtained by first removing the Rician noise bias from the diffusion signal decays. 


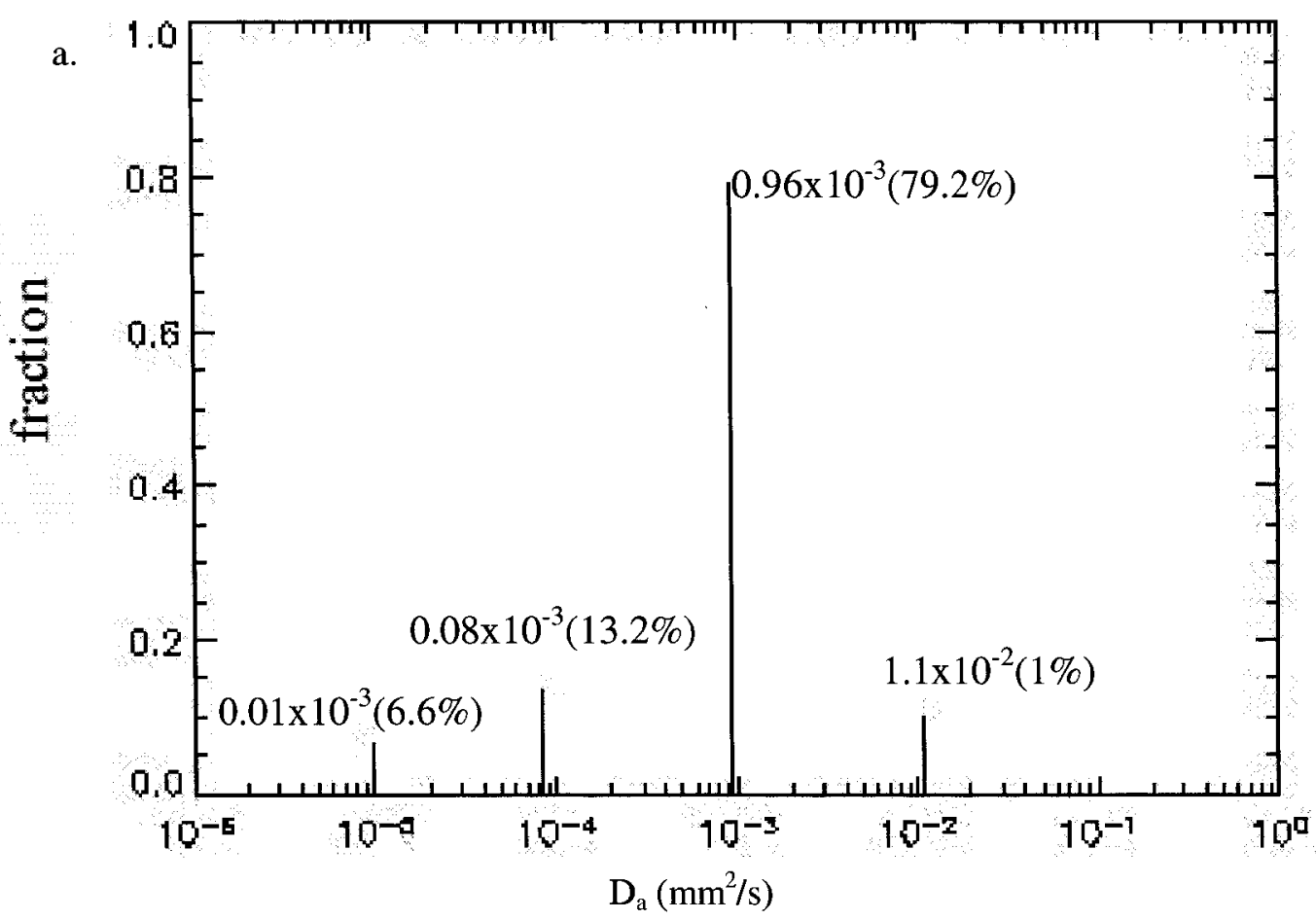

b.

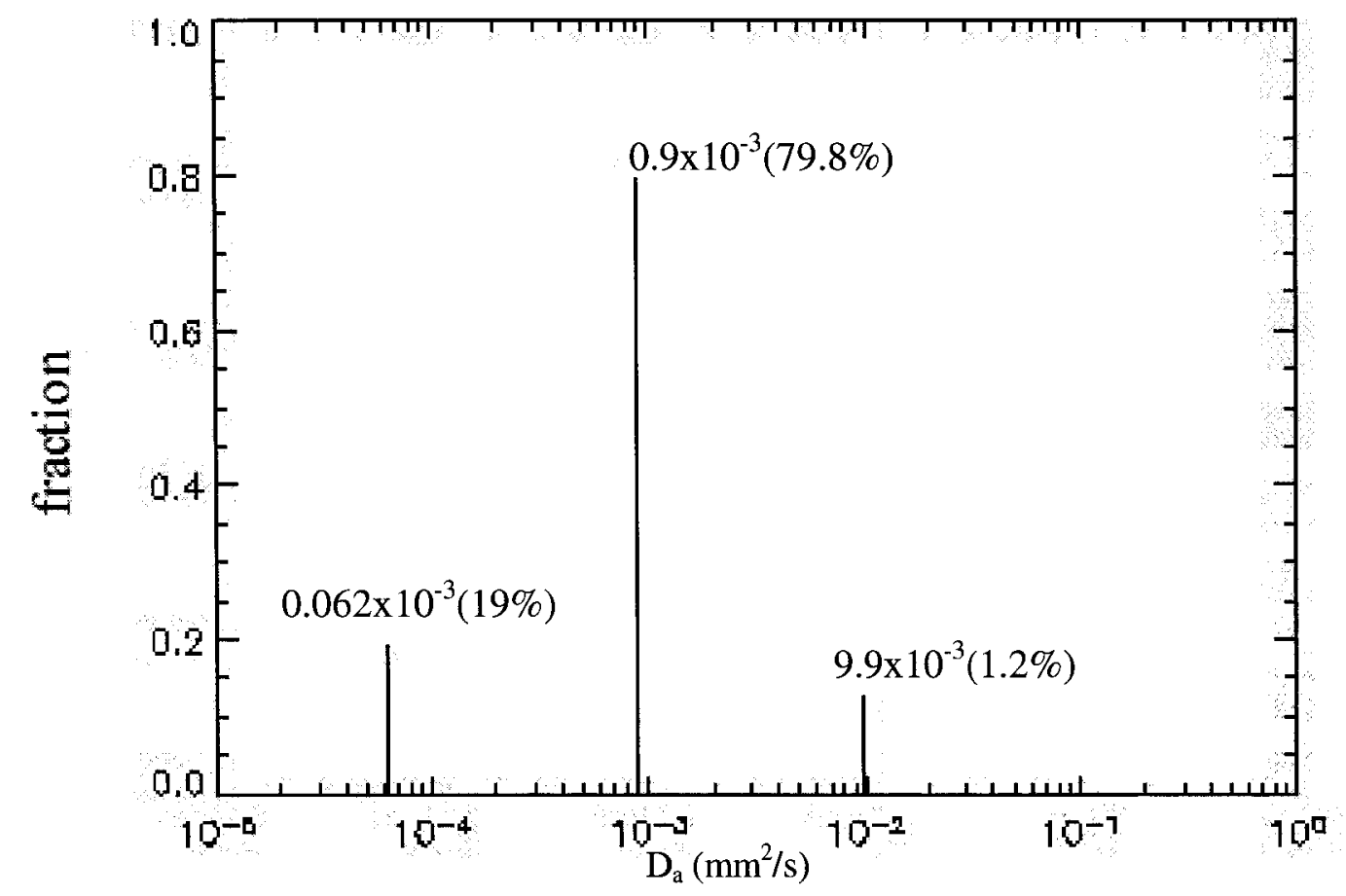

Figure 7.3 The discrete spectrum of diffusion coefficients obtained using NNLS-diff analysis of the experimental diffusion signal decays for human brain a. before Rician noise bias correction and $b$. after Rician noise bias correction. The amplitude of the line corresponding to the largest $D_{a}$ value in the spectrum was multiplied by 10 to make it more visible. 


\subsection{Diffusion Time Dependence Interpretation}

As discussed in the diffusion theory of section 2.2, restricted diffusion can be detected from the dependence on diffusion time of the diffusion coefficients for the molecules that interact with the cell walls. This effect can also be studied numerically using Monte Carlo simulations or with analytical models as has been done in a great number of studies [111-115, 84,173,123]. Tannerplanar theory is used here to calculate the $\tau_{D}$ dependence of the restricted diffusion coefficients of diffusing molecules confined to a square cross-section area with a, the length of a side, set to 5 $\mu \mathrm{m}$, to be consistent with the mean axon size for humans. With the dimensionless reduced time, $\xi$, which was defined in equation (2.53) to be $\xi=\frac{D \tau_{D}}{R^{2}}$, it is possible to use these simulations to demonstrate the $\xi$ dependence of the normalized restricted diffusion coefficient, $D_{a} / D$, where $D$ is the free diffusion coefficient of water at $37^{\circ} \mathrm{C}$. As depicted in figure 7.4 , the restricted diffusion coefficient is particularly sensitive to $\xi$ for $\xi=4 \times 10^{-8}$ to $4 \times 10^{-5}$. This corresponds, for our study, to diffusion times in the range of $0.1 \mathrm{~ms}$ to $100 \mathrm{~ms}$. A more complete study of this was previously performed by Olariu [173] in her M.Sc. thesis using Monte Carlo simulations and several analytical models. Her study showed that the dependence of the restricted diffusion coefficient on $\xi$ is independent of the actual shape of the cell. This is important since the shape of human body cells is not square, circular or any other regular geometric shape. Olariu's analysis has also shown that the results for a Gaussian distribution of cell sizes are quite similar to that of the mean value.

In our experiments we have used a range of intermediate diffusion times limited to $\tau_{\mathrm{D}}=19.9$ to $60 \mathrm{~ms}$. The corresponding reduced times of $8 \times 10^{-6}$ and $2.8 \times 10^{-5}$ are demonstrated by vertical lines in figure 7.4. 


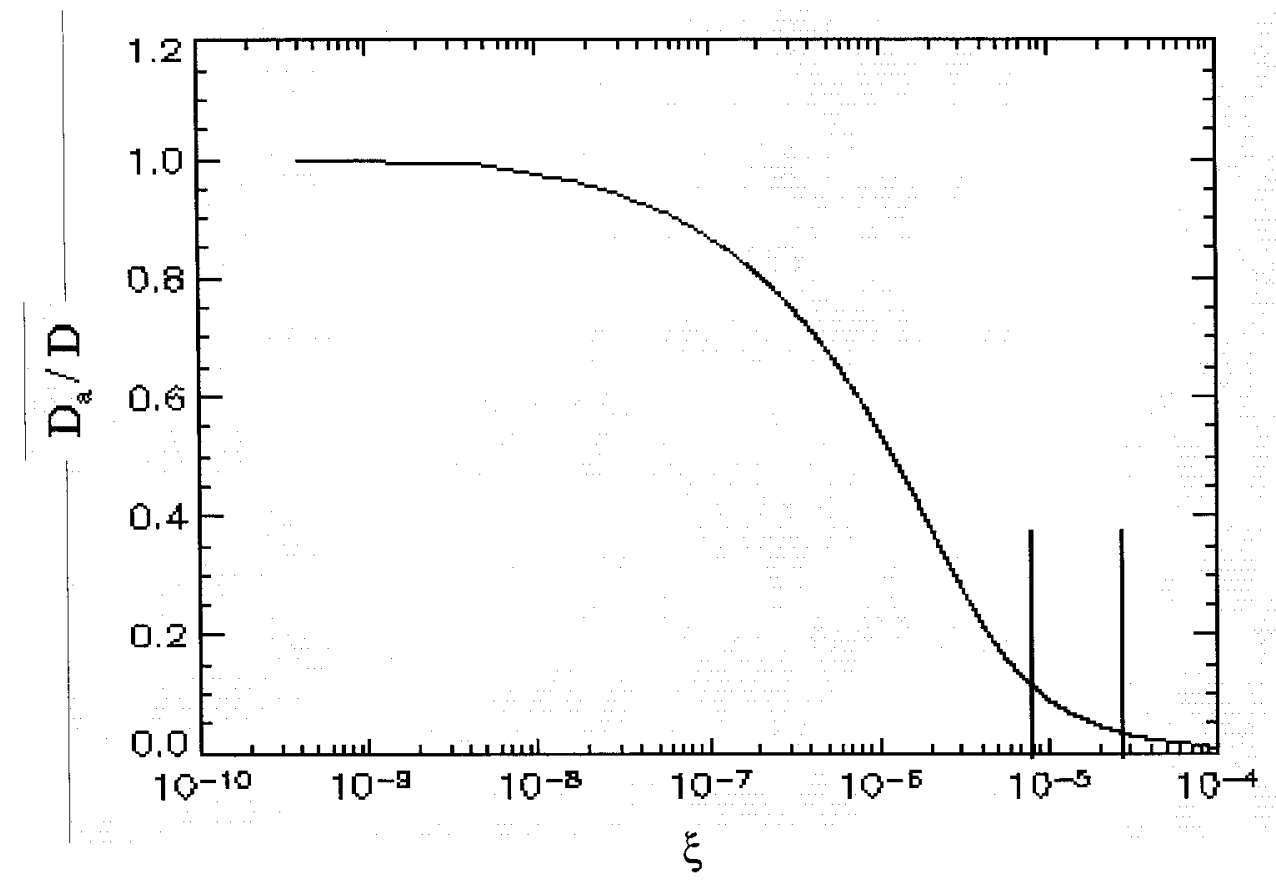

Figure 7.4 The normalized diffusion coefficient for restricted diffusion calculated from the Tanner-Planar formula with the box length $\mathrm{a}=5 \mu \mathrm{m}$ vs. the dimensionless reduced time, $\xi$. The vertical lines correspond to $\tau_{D}=19.9$ and $60 \mathrm{~ms}$, indicating the range of diffusion times considered here.

\subsubsection{The intracellular diffusion restriction}

By using the Tanner-planar formulation to model diffusion in human axons, we calculated diffusion coefficients for restricted diffusion as a function of $\tau_{\mathrm{D}}$ over a range of $\tau_{\mathrm{D}}$ values from 10 to $100 \mathrm{~ms}$ which was appropriate for our experiments. The results are tabulated in table 7.3 and depicted in figure 7.5 for various sizes of the square cross-sectional area with the length of a side varied between 2 and $10 \mu \mathrm{m}$, consistent with the range of cell sizes. The experimental results are demonstrated by stars in figure 7.5. Figure 7.6 shows the experimental results for the slow 


\begin{tabular}{|c|c|c|c|c|c|c|}
\hline \multirow{2}{*}{$\tau_{\mathrm{D}}(\mathrm{ms})$} & \multicolumn{6}{|c|}{$\mathrm{a}(\mu \mathrm{m})$} \\
\cline { 2 - 7 } & 2 & 4 & 5 & 6 & 8 & 10 \\
\hline 10 & 0.0336 & 0.138 & 0.215 & 0.326 & 0.62 & 0.98 \\
15 & 0.0224 & 0.0921 & 0.146 & 0.218 & 0.42 & 0.733 \\
20 & 0.0168 & 0.069 & 0.11 & 0.163 & 0.315 & 0.567 \\
25 & 0.0134 & 0.0552 & 0.0882 & 0.131 & 0.252 & 0.457 \\
30 & 0.0112 & 0.046 & 0.0735 & 0.109 & 0.21 & 0.381 \\
35 & 0.0096 & 0.0395 & 0.063 & 0.0933 & 0.18 & 0.327 \\
40 & 0.0084 & 0.0345 & 0.0551 & 0.0816 & 0.158 & 0.286 \\
45 & 0.00747 & 0.0307 & 0.049 & 0.0725 & 0.14 & 0.254 \\
50 & 0.00672 & 0.0276 & 0.0441 & 0.0653 & 0.126 & 0.229 \\
55 & 0.00611 & 0.0251 & 0.0401 & 0.0593 & 0.115 & 0.208 \\
60 & 0.0056 & 0.023 & 0.0367 & 0.0544 & 0.105 & 0.191 \\
65 & 0.00517 & 0.0212 & 0.0339 & 0.0502 & 0.097 & 0.176 \\
70 & 0.0048 & 0.0197 & 0.0315 & 0.0466 & 0.0901 & 0.163 \\
75 & 0.00448 & 0.0184 & 0.0294 & 0.0435 & 0.0841 & 0.152 \\
80 & 0.0042 & 0.0173 & 0.0276 & 0.0408 & 0.0788 & 0.143 \\
85 & 0.00395 & 0.0162 & 0.0259 & 0.0384 & 0.0742 & 0.135 \\
90 & 0.00374 & 0.0153 & 0.0245 & 0.0363 & 0.0701 & 0.127 \\
95 & 0.00354 & 0.0145 & 0.0232 & 0.0344 & 0.0664 & 0.12 \\
100 & 0.00336 & 0.0138 & 0.022 & 0.0326 & 0.0631 & 0.114 \\
\hline
\end{tabular}

Table 7.3. $\mathrm{D}_{\mathrm{a}}\left(10^{-3} \mathrm{~mm}^{2} / \mathrm{s}\right)$ values calculated using the Tanner-planar equation vs. diffusion time for restricted diffusion in a square box over lengths, a, consistent with the range of axon size for human brain.

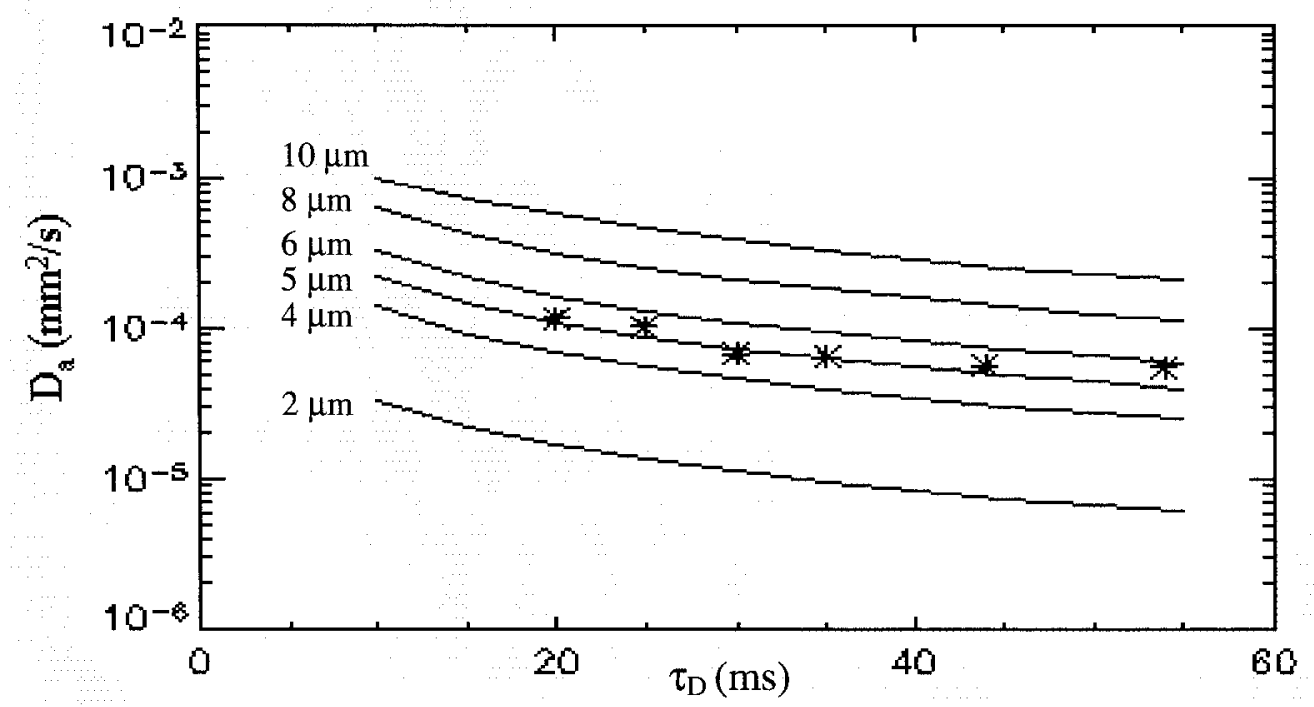

Figure 7.5. $\mathrm{D}_{\mathrm{a}}\left(\mathrm{mm}^{2} / \mathrm{s}\right)$ calculated using the Tanner-planar equation vs. diffusion time for restricted diffusion in a square box over lengths, a, consistent with the range of axon size for human brain. The stars show the experimental results of the diffusion time dependence for human brain averaged over 8 healthy subjects. The box size, a, for each of the calculated curves is indicated on the graph. 


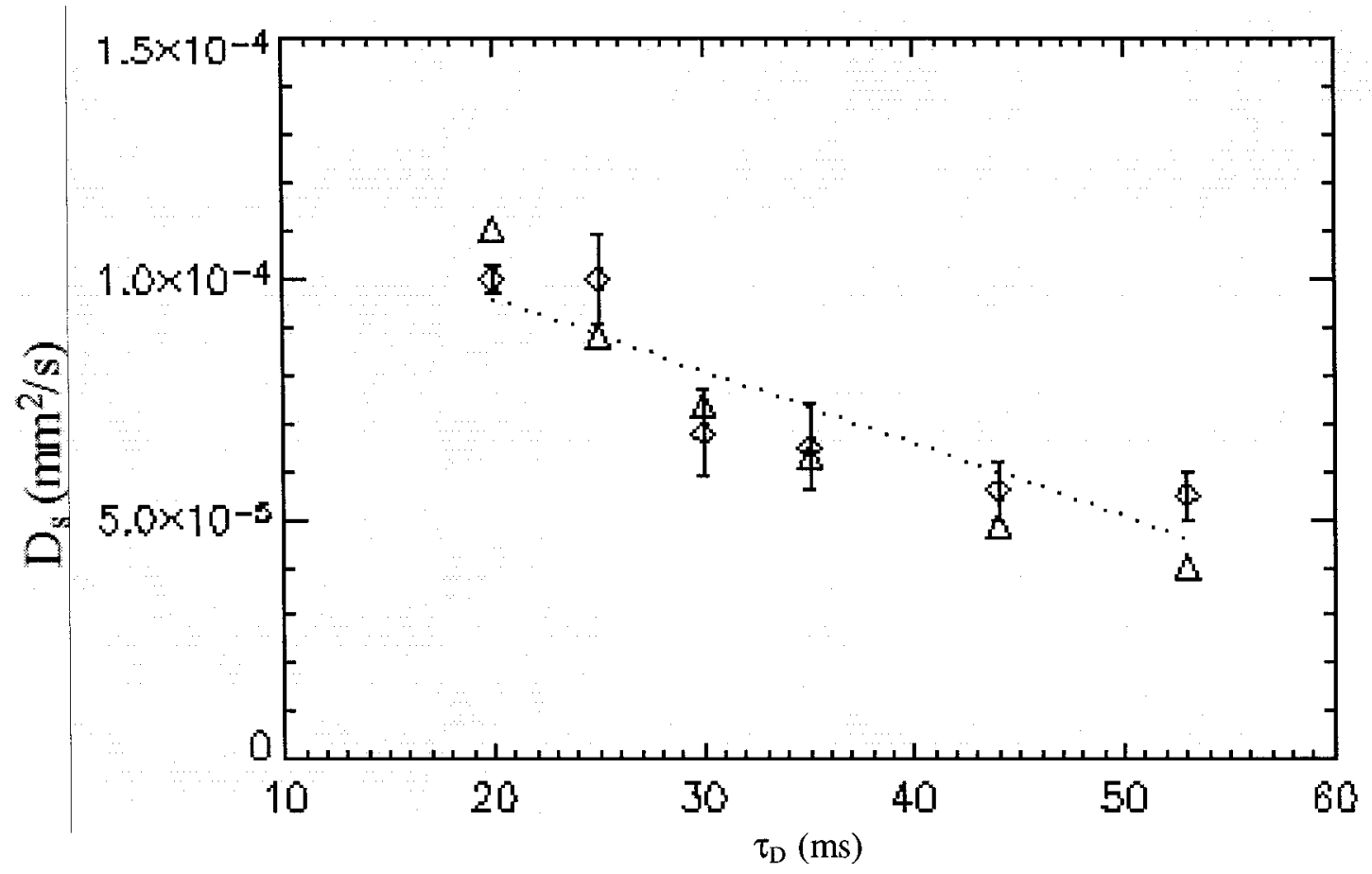

Figure 7.6. The apparent slow diffusion coefficient of the diffusion time dependence experiments on human brain averaged over 8 healthy volunteers. The experimental data (diagonal) are compared with the results from the Tanner-planar equation (triangle) for restricted diffusion in square box assuming an axon size, $\mathrm{a}=5 \mu \mathrm{m}$ for human brain.

diffusion coefficient of the diffusion signal decays, $D_{s}$, averaged over 8 healthy subjects as a function of $\tau_{\mathrm{D}}$ on a linear scale. The variation of $D_{\mathrm{s}}$ with $\tau_{\mathrm{D}}$ seen in figure 7.6 is evidence of restriction. A slightly stronger $\tau_{\mathrm{D}}$ dependence is observed when $\tau_{\mathrm{D}}$ is between 20 and $30 \mathrm{~ms}$ compared with $\tau_{D}=30$ to $60 \mathrm{~ms}$. This is consistent with the behavior of $D_{a} / D$ vs. $\xi$ (see figure 7.4 ) calculated with the Tanner-planar equation for $\xi$ values between the two vertical lines in figure 7.4 . 


\subsubsection{The two-site diffusion exchange theory}

Equation (7.2) for exchange of diffusing molecules between compartments in a two compartment system is used here to simulate the diffusion decays by setting the extracellular diffusion coefficient equal to $\mathrm{D}_{\mathrm{e}}^{\mathrm{a}}$ calculated from equation (7.1) and the intracellular diffusion coefficient equal to $D_{i}^{a}$ which was calculated using the Tanner-planar formula (equation (2.82)). The intrinsic values of $D_{e}$ and $D_{i}$ were used as the input values for equations (7.1) and (2.82), respectively, as explained in the previous sections, along with the experimental settings corresponding to the maximum value of $(\gamma g \delta)^{2}$ (i.e. the maximum b-value). The calculated diffusion decays with the two-site exchange are plotted in figures 7.7 and 7.8 for diffusion times between $5 \mathrm{~ms}$ and $60 \mathrm{~ms}$. Diffusion decays were generated for the exchange rate of $\mathrm{k}_{\mathrm{i}}=10 \mathrm{~s}^{-1}$, consistent with the exchange time of $\tau_{\mathrm{i}}=100 \mathrm{~ms}$, and for the exchange rate of $\mathrm{k}_{\mathrm{i}}=58 \mathrm{~s}^{-1}$, consistent with the exchange time of $\tau_{\mathrm{i}}=17 \mathrm{~ms}$, which is the value reported in the literature for red blood cells $[98,123]$. In both calculations, we set the intracellular fraction, $\mathrm{f}_{\mathrm{i}}$, equal to the measured slow diffusion fraction (0.2) from our experiments and others [98, 99,102,103,111]. Although the experimental results (figure 7.9) do not quantitatively agree exactly with the computed diffusion decays (figure $7.8 \mathrm{~b}$ ), the trend of the diffusion time dependent decays observed in our experiments on human brain is consistent with the results of our human model based on the combined Tanner and Karger theories. Our results are in particularly good agreement with theory for $\tau_{\mathrm{i}}=100 \mathrm{~ms}$, suggesting that the exchange time for water moving from inside axons to the interstitial compartment is on the order of $100 \mathrm{~ms}$. The restriction behavior is observed in the results as a difference in signal attenuation for the same b-value at different diffusion times. 


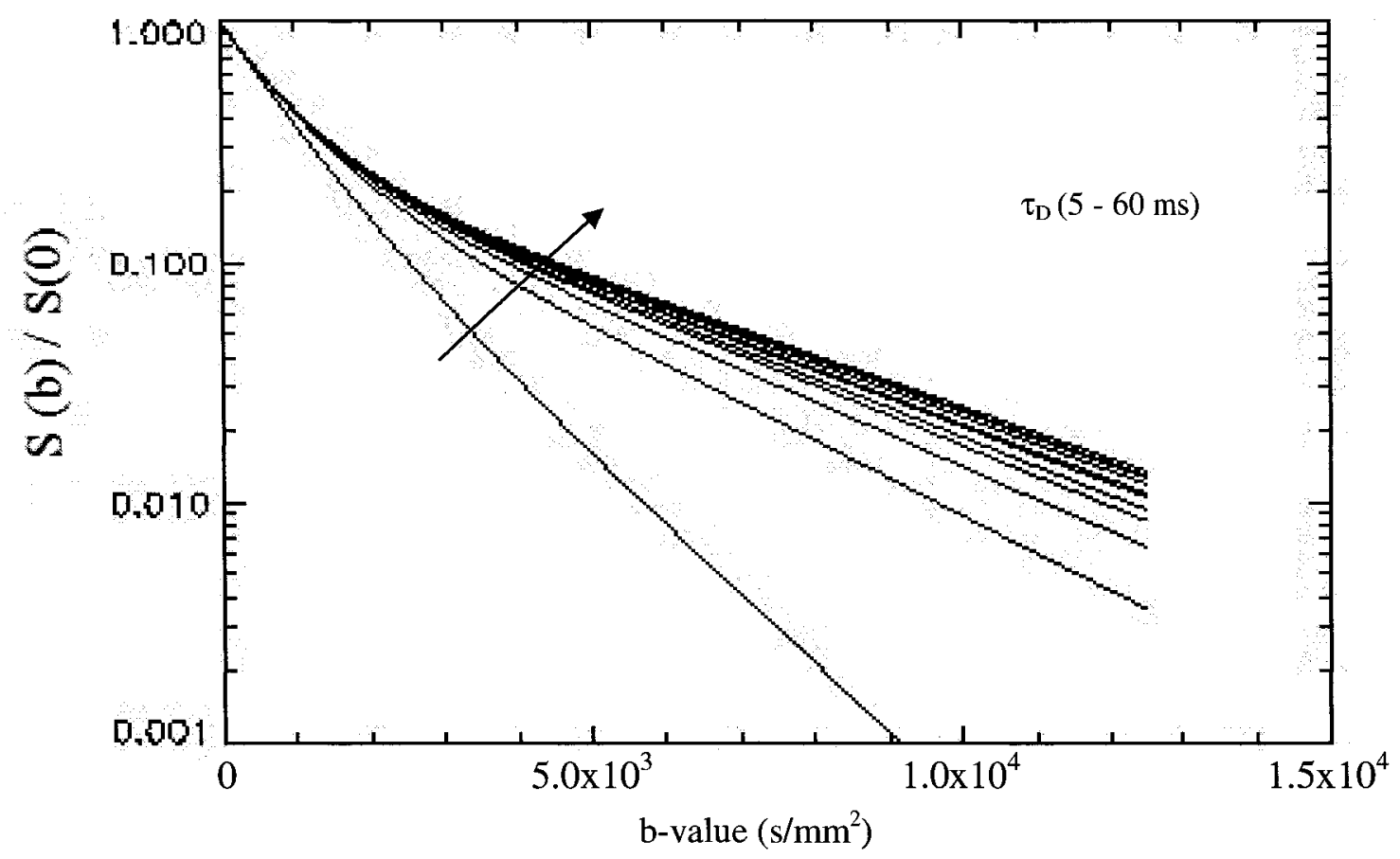

Figure 7.7. Diffusion decays computed using Tanner and Karger theory for $\tau_{\mathrm{i}}=17 \mathrm{~ms}$. The arrow indicates the direction of increasing $\tau_{\mathrm{D}}$.

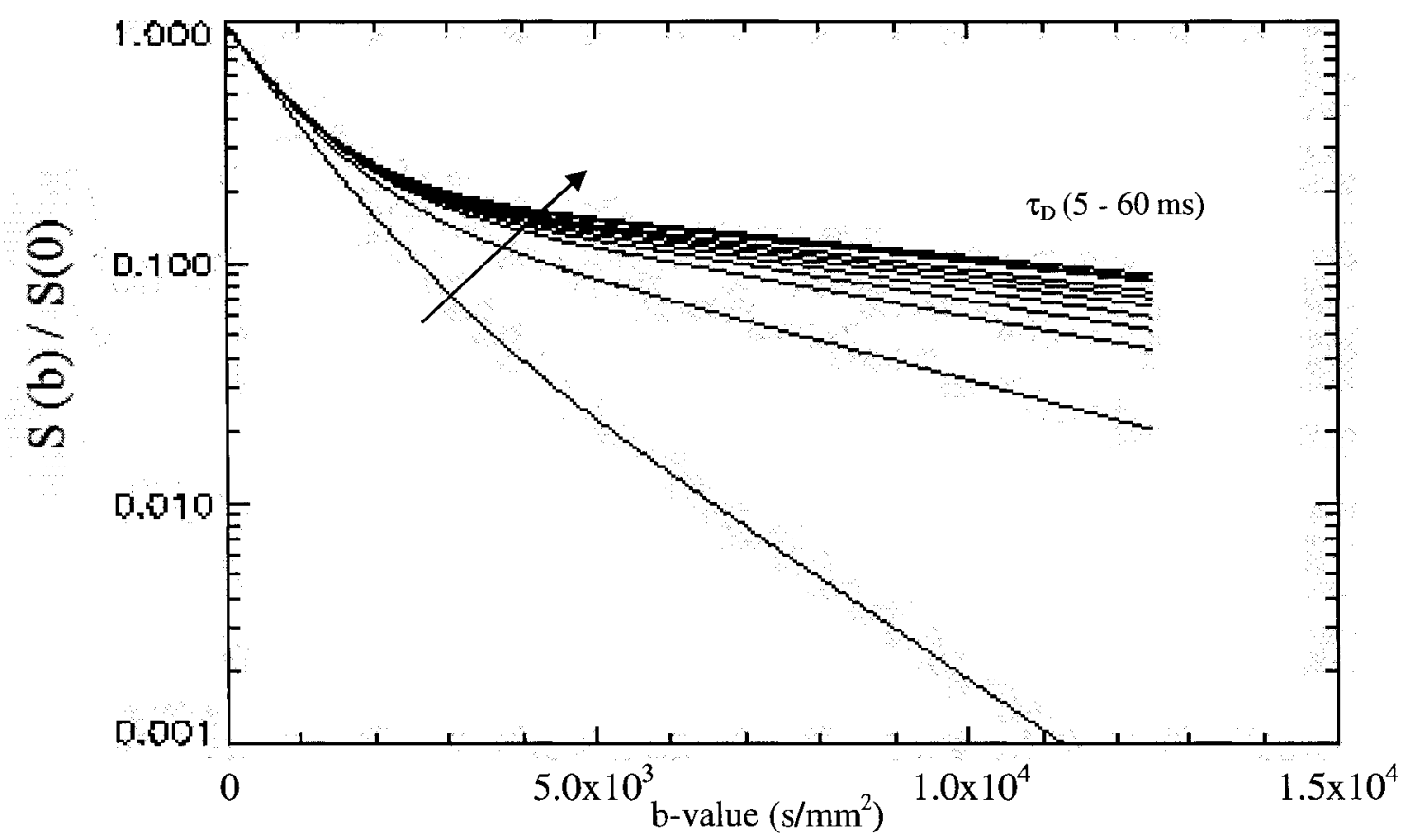

Figure 7.8.a. Diffusion decays computed using Tanner and Karger theory for $\tau_{\mathrm{i}}=100 \mathrm{~ms}$. The arrow indicates the direction of increasing $\tau_{\mathrm{D}}$. 


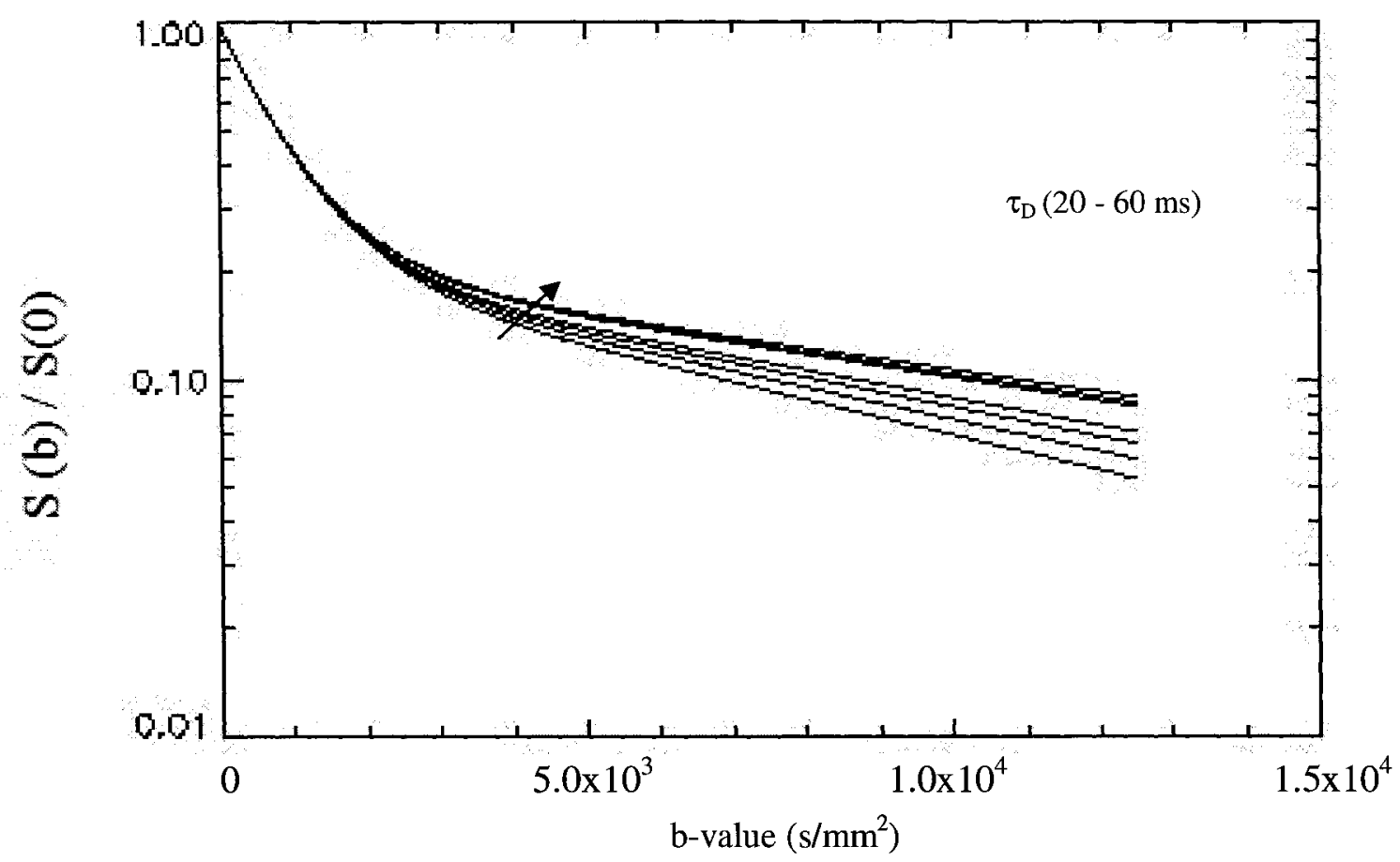

Figure 7.8.b. Diffusion decays computed using Tanner and Karger theory for $\tau_{\mathrm{i}}=100 \mathrm{~ms}$. The arrow indicates the direction of increasing $\tau_{\mathrm{D}}$.

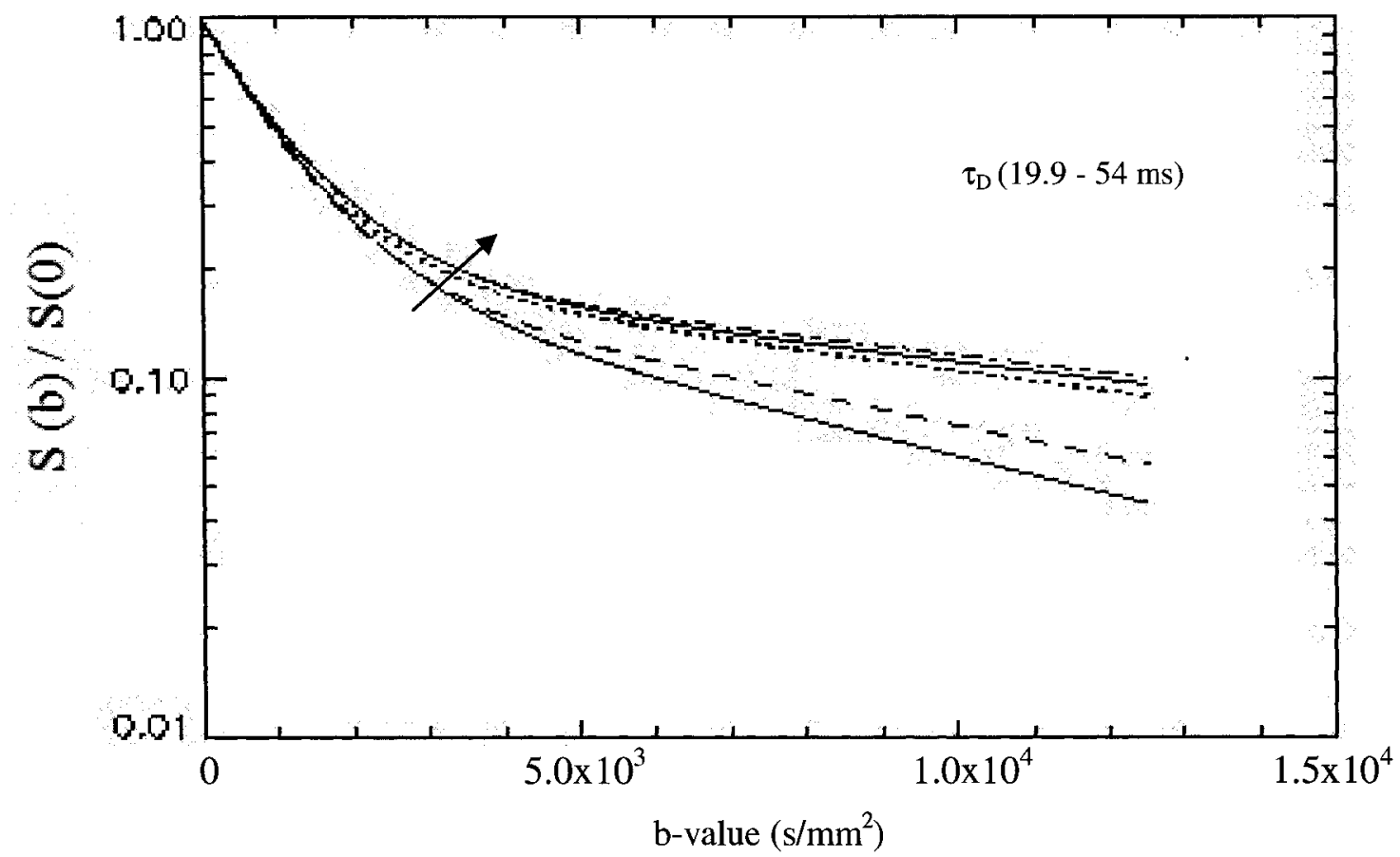

Figure 7.9. The experimental diffusion decays from the diffusion time dependence study on human brain in vivo averaged over 8 volunteers. The arrow indicates the direction of increasing $\tau_{\mathrm{D}}$. 
To interpret the results more responsibly based on the two site diffusion exchange theory, a more detailed model with more consistent morphological parameters for human brain is required. Various pathological situations with more reliable parameters for these situations should also be examined.

To improve the experimental results, it would be necessary to improve the MR system by employing higher gradient strengths with shorter durations. Besides, for the estimation of the exchange time as well as the molecular net displacement, it might be more appropriate if the diffusion time dependence of the signals was studied as a function of $(\gamma g \delta)^{2}$ (i.e. q-space measurements) instead of b-values, as performed in most cell and animal studies [102, $111,114,156,181]$

\subsection{Conclusions}

In this thesis, diffusion information for human brain tissue is reliably reported based on a multiexponential analysis and the removal of the Rician noise bias from the MR-diffusion signal decay. Our study represents what we believe to be quantitatively accurate diffusion parameters. This was made possible by the use of very large b-values along with a new procedure for Rician noise bias correction. The behavior of the data is consistent with restricted diffusion. The applied high b-values have been purposely chosen to sensitize the diffusion of water molecules inside the cells. The tri-exponential diffusion decay behavior is consistent with the compartmentalization of the spins into the intracellular, interstitial and intravascular spaces. These components were detected by taking advantage of the multi-component analysis method, and the Rician noise bias correction procedure developed for this study. 
These developed procedures have allowed us to observe a diffusion time dependence for the slow diffusion coefficient of healthy human brain in vivo which has not been reported previously. The behavior of $D_{s}$ observed in this study is consistent with the expected behavior for the diffusion coefficient of water molecules inside the cell (i.e. restricted diffusion).

We also did a rough evaluation of the exchange time between the two compartments in a two compartment system, and found that the exchange time for water moving from inside the axon to the interstitial space is of the order of $100 \mathrm{~ms}$.

As previously mentioned, to better understand the diffusion time dependence behavior in human brain tissue, a complete analysis of various regions of brain tissue for healthy subjects and patients with disorders along with more detailed analytical and numerical modeling on the basis of the morphology and the pathologies of the system is required. 


\section{Appendix I}

\section{The NNLS Flow Charts}




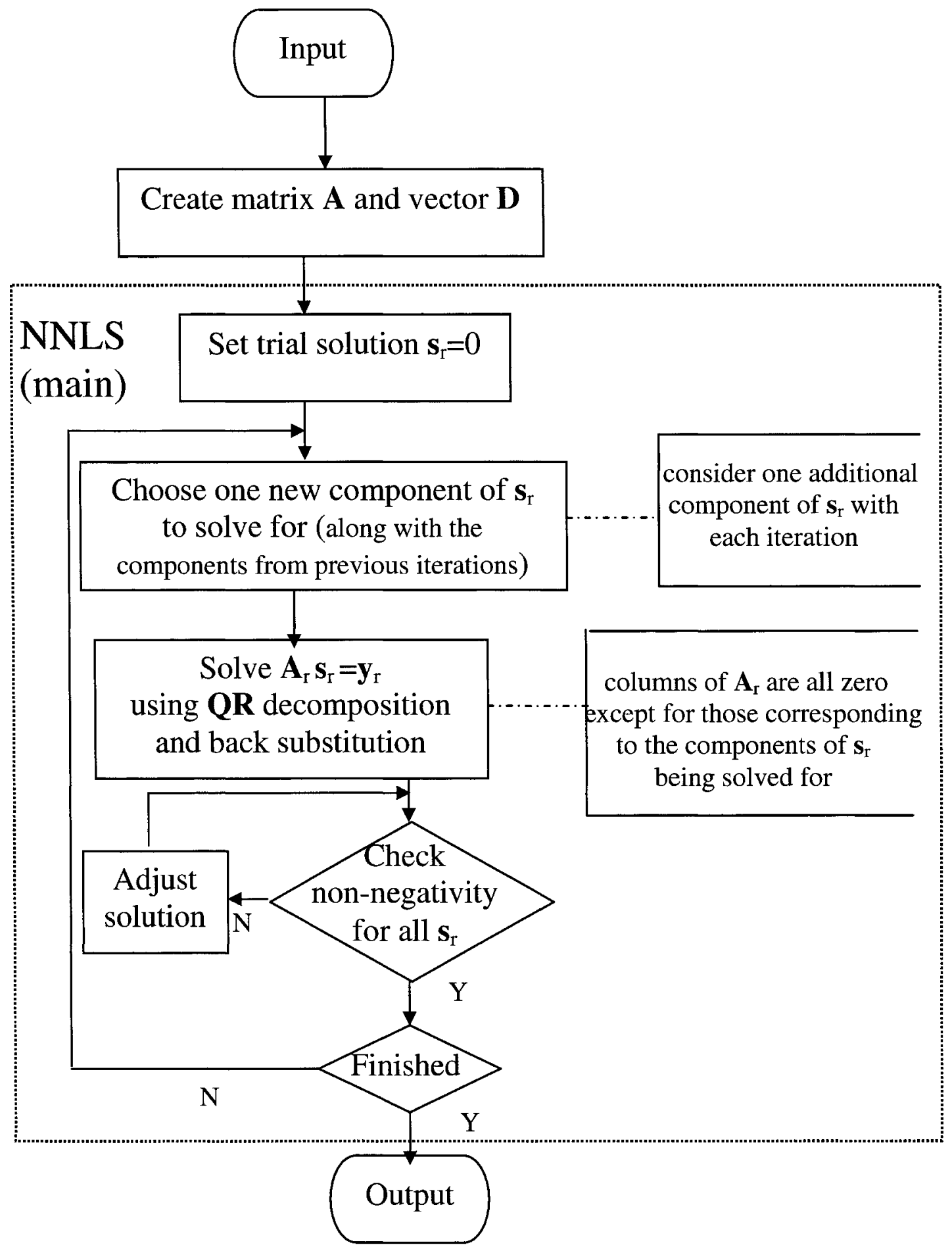

Figure I.1. Flow chart for the $N N L S$ algorithm without regularization. 


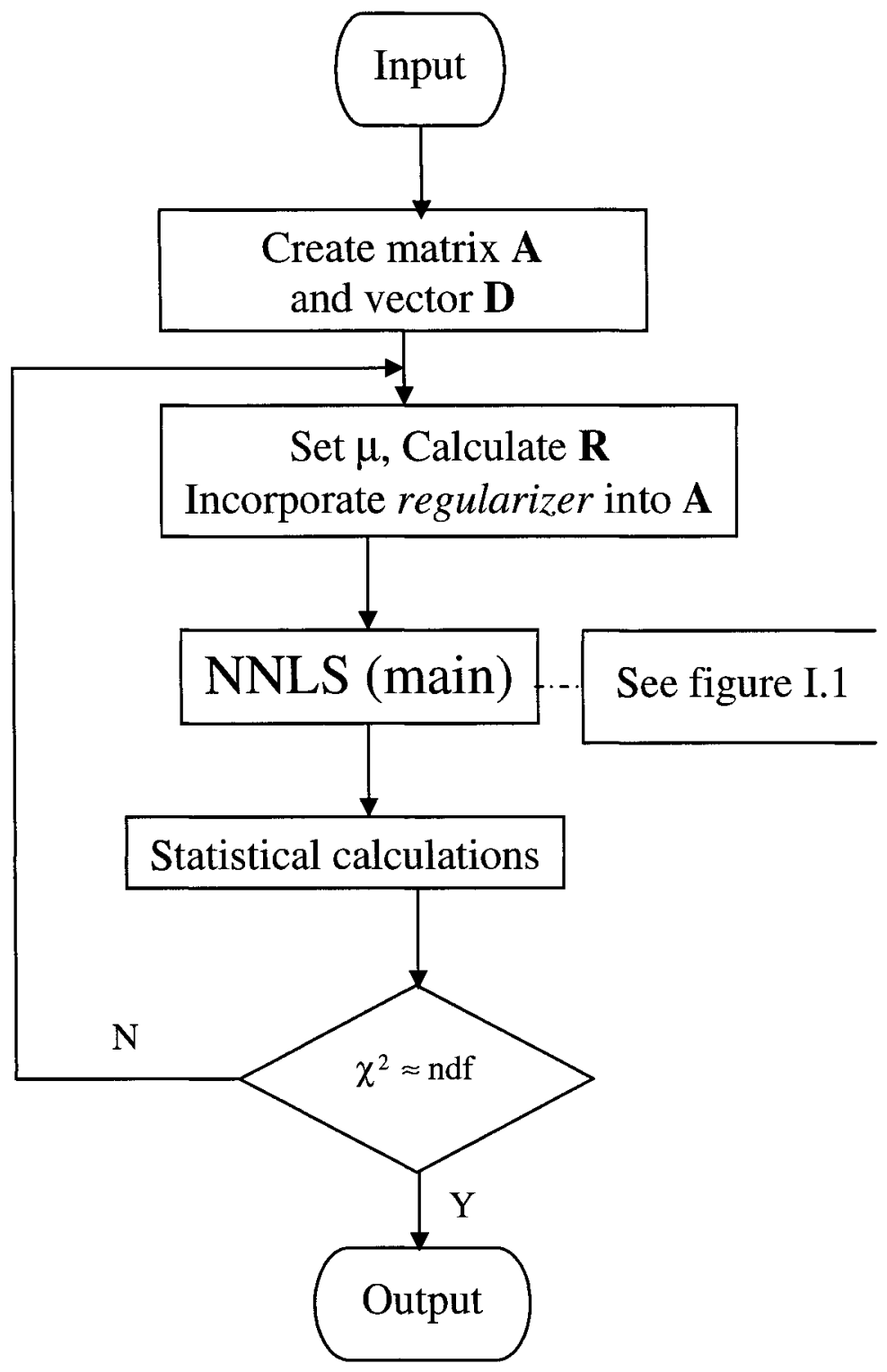

Figure I.2. Flow chart for $N N L S$-diff based on the $N N L S$ algorithm with regularization. 


\section{Appendix II}

\section{The b-factor and $\tau_{\mathrm{D}}$ for the TRSE Sequence}

\section{II.1 b-value Calculation}

The b-factor for the TRSE sequence was analytically evaluated by the integration of equation (2.74):

$$
\mathrm{b}=\int_{0}^{\mathrm{TE}} \mathrm{F}^{2}(\mathrm{t}) \mathrm{dt}=\gamma^{2} \int_{0}^{\mathrm{TE}}\left(\int_{0}^{\mathrm{t}} \mathbf{g}\left(\mathrm{t}^{\prime}\right) \mathrm{dt^{ \prime }}\right)^{2} \mathrm{dt}
$$

The sequence diagram for the TRSE sequence is given in figure II.1 (see also figures 6.1 to 6.3). The on and off ramps are both of duration $t_{r}$ for all four gradient pulses and the ramps are linear. The duration of the $i^{\text {th }}$ gradient pulse will be denoted $\delta_{i}$ which includes the ramp on time but not the ramp off time (i.e. $\delta_{1}=\mathrm{t}_{\mathrm{r}}+\left(\mathrm{t}_{12}-\mathrm{t}_{11}\right)$ ). The $180^{\circ} \mathrm{RF}$ pulses are applied in the $\tau_{1}$ and $\tau_{3}$ time periods. No gradient or RF pulses are applied during the time period labeled $\tau_{2}$. The effect of the $180^{\circ} \mathrm{RF}$ pulses is to change the phase angle of the spins from $\phi$ to $-\phi$. This effect causes a gradient pulse of amplitude $\mathrm{G}$ and duration $\delta$ following the $180^{\circ} \mathrm{RF}$ pulse to have the same effect 


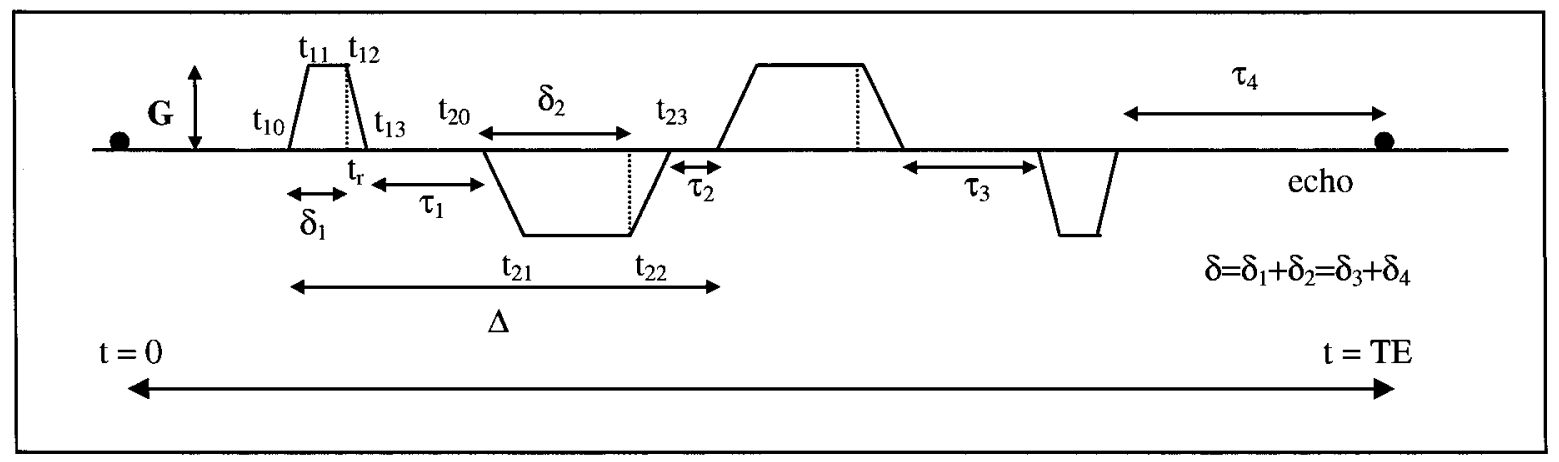

Figure II.1. The TRSE sequence timing for b-value and diffusion time evaluation.

that a gradient pulse of amplitude $-\mathrm{G}$ and duration $\delta$ would have without the $180^{\circ}$ RF pulse as shown in figure 6.1 (see also section 2.3). This effect will be incorporated into what we will call the effective gradient amplitudes which, for the TRSE sequence shown in figure 6.1, are $\mathrm{g}_{1}=\mathrm{g}_{2}=-\mathrm{g}_{3}=-\mathrm{g}_{4}=\mathrm{G}$.

The integral can be expanded as:

$$
\begin{aligned}
\frac{b}{\gamma^{2}} & =\int_{t_{10}}^{t_{13}} F^{2}(t) d t+\int_{t_{13}}^{t_{20}} F^{2}(t) d t+\int_{t_{20}}^{t_{23}} F^{2}(t) d t+\int_{t_{23}}^{t_{30}} F^{2}(t) d t+\int_{t_{30}}^{t_{33}} F^{2}(t) d t+\int_{t_{33}}^{t_{40}} F^{2}(t) d t+\int_{t_{40}}^{t_{43}} F^{2}(t) d t+\int_{t_{43}}^{T E} F^{2}(t) d t \\
& =I_{\delta_{1}}+I_{\tau_{1}}+I_{\delta_{2}}+I_{\tau_{2}}+I_{\delta_{3}}+I_{\tau_{3}}+I_{\delta_{4}}+I_{\tau_{4}} .
\end{aligned}
$$

a) Evaluation of $I_{\delta 1}$

$$
\begin{aligned}
I_{\delta_{1}} & =I_{10}+I_{11}+I_{12} \\
I_{10} & =\int_{t_{10}}^{t_{11}}\left(\int_{t_{10}}^{t} g\left(t^{\prime}\right) d t^{\prime}\right)^{2} d t=\int_{t_{10}}^{t_{11}} d t\left[\int_{t_{10}}^{t} d t^{\prime} \frac{G}{t_{r}}\left(t^{\prime}-t_{10}\right)\right]^{2}=\int_{t_{10}}^{t_{11}}\left(\frac{G}{t_{r}}\right)^{2} d t\left[\int_{t_{10}}^{t} d t^{\prime}\left(t^{\prime}-t_{10}\right)\right]^{2}=\int_{t_{10}}^{t_{11}}\left(\frac{G}{t_{r}}\right)^{2} d t\left[\frac{1}{2}\left(t-t_{10}\right)^{2}\right]^{2} \\
& =\frac{1}{4}\left(\frac{G}{t_{r}}\right)^{2} \int_{t_{10}}^{t_{11}} d t\left(t-t_{10}\right)^{4}=\frac{1}{4}\left(\frac{G}{t_{r}}\right)^{2} \frac{1}{5}\left(t_{11}-t_{10}\right)^{5}=\frac{1}{20}\left(\frac{G}{t_{r}}\right)^{2} t_{r}^{5}=\frac{1}{20} G^{2} t_{r}^{3}
\end{aligned}
$$




$$
\begin{aligned}
I_{11} & =\int_{t_{11}}^{t_{12}}\left(\int_{t_{10}}^{t} g\left(t^{\prime}\right) d t^{\prime}\right)^{2} d t=\int_{t_{11}}^{t_{12}}\left\{\int_{t_{10}}^{t_{11}} g\left(t^{\prime}\right) d t^{\prime}+\int_{t_{11}}^{t} g\left(t^{\prime}\right) d t^{\prime}\right\}^{2} d t=\int_{t_{11}}^{t_{12}} d t\left\{\left[\frac{1}{2} G t_{r}+G\left(t-t_{11}\right)\right\}^{2}\right. \\
& =\int_{t_{11}}^{t_{12}} d t\left\{\left[\frac{1}{2} G t_{r}+G\left(t-t_{11}\right)\right\}^{2}=\int_{t_{11}}^{t_{12}} G^{2} d t\left\{\frac{1}{4}\left(t_{r}\right)^{2}+\left(t-t_{11}\right)^{2}+t_{r}\left(t-t_{11}\right)\right\}\right. \\
& =G^{2}\left\{\frac{1}{4} t_{r}^{2}\left(\delta_{1}-t_{r}\right)+\frac{1}{3}\left(\delta_{1}-t_{r}\right)^{3}+\frac{1}{2} t_{r}\left(\delta_{1}-t_{r}\right)^{2}\right\} \\
& =G^{2}\left\{\frac{1}{4}\left(t_{r}^{2} \delta_{1}-t_{r}^{3}\right)+\frac{1}{3}\left(\delta_{1}^{3}-t_{r}^{3}-3 \delta_{1}^{2} t_{r}+3 \delta_{1} t_{r}^{2}\right)+\frac{1}{2}\left(t_{r} \delta_{1}^{2}+t_{r}^{3}-2 \delta_{1} t_{r}^{2}\right)\right\} \\
& =G^{2}\left(\frac{1}{3} \delta_{1}^{3}-\frac{1}{12} t_{r}^{3}-\frac{1}{2} \delta_{1}^{2} t_{r}+\frac{1}{4} \delta_{1} t_{r}^{2}\right) \\
I_{12} & =\int_{t_{12}}^{t_{13}}\left(\int_{t_{10}}^{t} g\left(t^{\prime}\right) d t^{\prime}\right)^{2} d t=\int_{t_{12}}^{t_{13}}\left\{\int_{t_{10}}^{t_{11}} g\left(t^{\prime}\right) d t^{\prime}+\int_{t_{11}}^{t_{12}} g\left(t^{\prime}\right) d t^{\prime}+\int_{t_{12}}^{t} g\left(t^{\prime}\right) d t^{\prime}\right\}^{2} d t \\
& =\int_{t_{12}}^{t_{13}} d t\left\{\frac{1}{2} G t_{r}+G\left(t_{12}-t_{11}\right)+\int_{t_{12}}^{t}-\frac{G}{t_{r}}\left(t^{\prime}-t_{13}\right) d t^{\prime}\right\}^{2} \\
& =\int_{t_{12}}^{t_{13}} d t\left[\frac{1}{2} G t_{r}+G\left(t_{12}-t_{11}\right)-\frac{G}{2 t_{r}}\left(t-t_{13}\right)^{2}+\frac{G}{2 t_{r}}\left(t_{12}-t_{13}\right)^{2}\right]^{2} \\
& =\int_{t_{12}}^{t_{13}} d t G^{2}\left[\frac{1}{2} t_{r}+\left(\delta_{1}-t_{r}\right)-\frac{1}{2 t}\left(t-t_{13}\right)^{2}+\frac{1}{2 t_{r}}\left(t_{r}\right)^{2}\right]^{2} \\
& =\int_{t_{12}}^{t_{13}} d t G^{2}\left[\delta_{1}^{2}+\frac{1}{4 t_{r}^{2}}\left(t-t_{13}\right)^{4}-\frac{\delta_{1}}{t_{r}}\left(t-t_{13}\right)^{2}\right]=G^{2}\left[\delta_{1}^{2} t_{r}+\frac{1}{20} t_{r}^{3}-\frac{\delta_{1}}{3} t_{r}^{2}\right] \\
&
\end{aligned}
$$

where $t_{12}-t_{11}=\delta_{1}-t_{r}$ and $t_{11}-t_{10}=t_{13}-t_{12}=t_{r}$.

Therefore, the contribution of the first gradient can be summarized as follows:

$$
\begin{aligned}
\mathrm{I}_{\delta 1} & =\frac{1}{20} \mathrm{G}^{2} \mathrm{t}_{\mathrm{r}}^{3}+\mathrm{G}^{2}\left(\frac{1}{3} \delta_{1}^{3}-\frac{1}{12} \mathrm{t}_{\mathrm{r}}^{3}-\frac{1}{2} \delta_{1}^{2} \mathrm{t}_{\mathrm{r}}+\frac{1}{4} \delta_{1} \mathrm{t}_{\mathrm{r}}^{2}\right)+\mathrm{G}^{2}\left[\delta_{1}^{2} \mathrm{t}_{\mathrm{r}}+\frac{1}{20} \mathrm{t}_{\mathrm{r}}^{3}-\frac{1}{3} \delta_{1} \mathrm{t}_{\mathrm{r}}^{2}\right] \\
& =\mathrm{G}^{2}\left[\frac{1}{3} \delta_{1}^{3}+\frac{1}{2} \delta_{1}^{2} \mathrm{t}_{\mathrm{r}}-\frac{1}{12} \delta_{1} \mathrm{t}_{\mathrm{r}}^{2}+\frac{1}{60} \mathrm{t}_{\mathrm{r}}^{3}\right] \\
\therefore \mathrm{I}_{\delta 1} & =\frac{1}{60} \mathrm{G}^{2}\left[20 \delta_{1}^{3}+30 \delta_{1}^{2} \mathrm{t}_{\mathrm{r}}-5 \delta_{1} \mathrm{t}_{\mathrm{r}}^{2}+\mathrm{t}_{\mathrm{r}}^{3}\right] .
\end{aligned}
$$

This is the contribution of the first gradient to the b-factor for the sequence. 
b) Evaluation of $\mathrm{I}_{\tau 1}$

$$
\begin{aligned}
I_{\tau 1} & =\int_{t_{13}}^{t_{20}}\left(\int_{t_{10}}^{t} g\left(t^{\prime}\right) d t^{\prime}\right)^{2} d t=\int_{t_{13}}^{t_{20}}\left\{\int_{t_{10}}^{t_{11}} g\left(t^{\prime}\right) d t^{\prime}+\int_{t_{11}}^{t_{12}} g\left(t^{\prime}\right) d t^{\prime}+\int_{t_{12}}^{t_{13}} g\left(t^{\prime}\right) d t^{\prime}+\int_{t_{13}}^{t} g\left(t^{\prime}\right) d t^{\prime}\right\}^{2} d t \\
& =\int_{t_{13}}^{t_{20}} d t\left[\frac{1}{2} G t_{r}+G\left(\delta_{1}-t_{r}\right)+\frac{1}{2} G t_{r}+0\right]^{2}=\int_{t_{13}}^{t_{20}} d t\left[G \delta_{1}\right]^{2}=\left(t_{20}-t_{13}\right)\left[G \delta_{1}\right]^{2}=G^{2} \delta_{1}^{2} \tau_{1} .
\end{aligned}
$$

It is also sometimes convenient to write this in terms of the gradient moments (i.e. the area under the diffusion gradients). For the evaluation of $I_{\tau 1}$ and $I_{\delta 2}$ the gradient moment is defined as $\mathrm{Mom}_{1}=\mathrm{G} \delta_{1}$.

$$
\therefore \mathrm{I}_{\tau 1}=\operatorname{Mom}_{1} \operatorname{Mom}_{1} \tau_{1}
$$

\section{c) Evaluation of $\mathrm{I}_{\delta 2}$}

The contribution to $\mathrm{b}$ from the second gradient, $\mathrm{I}_{\delta 2}$, is evaluated as follows:

$$
\begin{aligned}
I_{\delta 2} & =I_{20}+I_{21}+I_{22} \\
I_{20} & =\int_{t_{20}}^{t_{21}}\left(\int_{t_{10}}^{t} g\left(t^{\prime}\right) d t^{\prime}\right)^{2} d t=\int_{t_{20}}^{t_{21}} d t\left\{\int_{t_{10}}^{t_{11}} g\left(t^{\prime}\right) d t^{\prime}+\int_{t_{11}}^{t_{12}} g\left(t^{\prime}\right) d t^{\prime}+\int_{t_{12}}^{t_{13}} g\left(t^{\prime}\right) d t^{\prime}+\int_{t_{13}}^{t_{20}} g\left(t^{\prime}\right) d t^{\prime}+\int_{t_{20}}^{t} g\left(t^{\prime}\right) d t^{\prime}\right\}^{2} \\
& =\int_{t_{20}}^{t_{22}} d t\left[\frac{1}{2} G t_{r}+G\left(\delta_{1}-t_{r}\right)+\frac{1}{2} G t_{r}+0+\int_{t_{20}}^{t} d t^{\prime} \frac{G}{t_{r}}\left(t^{\prime}-t_{20}\right)\right]^{2} \\
& =\int_{t_{20}}^{t_{21}} d t\left[G \delta_{1}+\frac{G}{2 t_{r}}\left(t-t_{20}\right)^{2}\right]^{2}=\int_{t_{20}}^{t_{22}} d t G^{2}\left[\delta_{1}^{2}+\frac{1}{4 t_{r}^{2}}\left(t-t_{20}\right)^{4}+\frac{\delta_{1}}{t_{r}}\left(t-t_{20}\right)^{2}\right] \\
& =G^{2}\left[\delta_{1}^{2} t_{r}+\frac{1}{5} \frac{1}{4 t_{r}^{2}}\left(t_{r}\right)^{5}+\frac{1}{3} \frac{\delta_{1}}{t_{r}}\left(t_{r}\right)^{3}=G^{2}\left[\frac{1}{20} t_{r}^{3}+\frac{1}{3} \delta_{1} t_{r}^{2}+\delta_{1}^{2} t_{r}\right] .\right. \\
I_{21} & =\int_{t_{21}}^{t_{22}} d t\left[\frac{1}{2} G t_{r}+G\left(\delta_{1}-t_{r}\right)+\frac{1}{2} G t_{r}+0+\frac{1}{2} G t_{r}+\int_{t_{21}}^{t} G d t^{\prime}\right]^{2} \\
& =\int_{t_{21}}^{t_{22}} G^{2} d t\left[\delta_{1}+\frac{1}{2} t_{r}+\left(t-t_{21}\right)\right]^{2}=\int_{t_{21}}^{t_{22}} G^{2} d t\left[\delta_{1}^{2}+\frac{1}{4}\left(t_{r}\right)^{2}+\left(t-t_{21}\right)^{2}+2 \delta_{1}\left(t-t_{21}\right)+t_{r}\left(t-t_{21}\right)+\delta_{1} t_{r}\right]
\end{aligned}
$$




$$
\begin{aligned}
& =G^{2}\left[\delta_{1}^{2}\left(t_{22}-t_{21}\right)+\frac{1}{4} t_{r}^{2}\left(t_{22}-t_{21}\right)+\frac{1}{3}\left(t_{22}-t_{21}\right)^{3}+\delta_{1}\left(t_{22}-t_{21}\right)^{2}+\frac{1}{2} t_{r}\left(t_{22}-t_{21}\right)^{2}+\delta_{1} t_{r}\left(t_{22}-t_{21}\right)\right] \\
& =G^{2}\left[\delta_{1}^{2}\left(\delta_{2}-t_{r}\right)+\frac{1}{4} t_{r}^{2}\left(\delta_{2}-t_{r}\right)+\frac{1}{3}\left(\delta_{2}-t_{r}\right)^{3}+\delta_{1}\left(\delta_{2}-t_{r}\right)^{2}+\frac{1}{2} t_{r}\left(\delta_{2}-t_{r}\right)^{2}+\delta_{1} t_{r}\left(\delta_{2}-t_{r}\right)\right] \\
& =G^{2}\left[\delta_{1}^{2} \delta_{2}-\delta_{1}^{2} t_{r}+\frac{1}{4} \delta_{2} t_{r}^{2}-\frac{1}{4} t_{r}^{3}+\frac{1}{3} \delta_{2}^{3}-\delta_{2}^{2} t_{r}+t_{r}^{2} \delta_{2}-\frac{1}{3} t_{r}^{3}+\delta_{1} \delta_{2}^{2}+\delta_{1} t_{r}^{2}-2 \delta_{1} \delta_{2} t_{r}+\frac{1}{2} t_{r} \delta_{2}^{2}\right. \\
& \left.+\frac{1}{2} t_{r}^{3}-t_{r}^{2} \delta_{2}+\delta_{1} t_{r} \delta_{2}-t_{r}^{2} \delta_{1}\right] \\
& =G^{2}\left[\frac{1}{3} \delta_{2}^{3}-\frac{1}{2} t_{r} \delta_{2}^{2}+\frac{1}{4} t_{r}^{2} \delta_{2}-\frac{1}{12} t_{r}^{3}-\delta_{1} \delta_{2} t_{r}+\delta_{1} \delta_{2}^{2}-\delta_{1}^{2} t_{r}+\delta_{2} \delta_{1}^{2}\right],
\end{aligned}
$$

where $t_{22}-t_{21}=\delta_{2}-t_{r}$ and $t_{21}-t_{20}=t_{23}-t_{22}=t_{r}$.

$$
\begin{aligned}
\mathrm{I}_{22} & =\int_{\mathrm{t}_{22}}^{\mathrm{t}_{23}} \mathrm{dt}\left\{\frac{1}{2} \mathrm{Gt} \mathrm{t}_{\mathrm{r}}+\mathrm{G}\left(\delta_{1}-\mathrm{t}_{\mathrm{r}}\right)+\frac{1}{2} \mathrm{Gt}_{\mathrm{r}}+0+\frac{1}{2} \mathrm{Gt} \mathrm{t}_{\mathrm{r}}+\mathrm{G}\left(\delta_{2}-\mathrm{t}_{\mathrm{r}}\right)+\int_{\mathrm{t}_{22}}^{\mathrm{t}}-\frac{\mathrm{G}}{\mathrm{t}_{\mathrm{r}}}\left(\mathrm{t}^{\prime}-\mathrm{t}_{23}\right) \mathrm{dt}^{\prime}\right\}^{2} \\
& =\int_{\mathrm{t}_{22}}^{\mathrm{t}_{23}} \mathrm{dtG} \mathrm{G}^{2}\left[\delta_{1}+\delta_{2}-\frac{1}{2 \mathrm{t}_{\mathrm{r}}}\left(\mathrm{t}-\mathrm{t}_{23}\right)^{2}\right]^{2} \\
& =\int_{\mathrm{t}_{22}}^{\mathrm{t}_{23}} \mathrm{dtG} \mathrm{G}^{2}\left[\delta_{1}^{2}+\delta_{2}^{2}+\frac{1}{4 \mathrm{t}_{\mathrm{r}}^{2}}\left(\mathrm{t}-\mathrm{t}_{23}\right)^{4}-\frac{\delta_{1}}{\mathrm{t}_{\mathrm{r}}}\left(\mathrm{t}-\mathrm{t}_{23}\right)^{2}-\frac{\delta_{2}}{\mathrm{t}_{\mathrm{r}}}\left(\mathrm{t}-\mathrm{t}_{23}\right)^{2}+2 \delta_{1} \delta_{2}\right] \\
& =\mathrm{G}^{2}\left[\delta_{1}^{2} \mathrm{t}_{\mathrm{r}}+\delta_{2}^{2} \mathrm{t}_{\mathrm{r}}+\frac{1}{4 \mathrm{t}_{\mathrm{r}}^{2}} \frac{1}{5} \mathrm{t}_{\mathrm{r}}^{5}-\frac{1}{3} \frac{\delta_{1}}{\mathrm{t}_{\mathrm{r}}} \mathrm{t}_{\mathrm{r}}^{3}-\frac{1}{3} \frac{\delta_{2}}{\mathrm{t}_{\mathrm{r}}} \mathrm{t}_{\mathrm{r}}^{3}+2 \delta_{1} \delta_{2} \mathrm{t}_{\mathrm{r}}\right] \\
& =\mathrm{G}^{2}\left[\delta_{1}^{2} \mathrm{t}_{\mathrm{r}}+\delta_{2}^{2} \mathrm{t}_{\mathrm{r}}+\frac{1}{20} \mathrm{t}_{\mathrm{r}}^{3}-\frac{1}{3} \delta_{1} \mathrm{t}_{\mathrm{r}}^{2}-\frac{1}{3} \delta_{2} \mathrm{t}_{\mathrm{r}}^{2}+2 \delta_{1} \delta_{2} \mathrm{t}_{\mathrm{r}}\right] .
\end{aligned}
$$

Therefore, the contribution of the second gradient, $\mathrm{I}_{\delta 2}$ will be as follows:

$$
\begin{aligned}
& \mathrm{I}_{\delta 2}=\mathrm{G}^{2}\left[\frac{1}{20} \mathrm{t}_{\mathrm{r}}{ }^{3}+\frac{1}{3} \delta_{1} \mathrm{t}_{\mathrm{r}}{ }^{2}+\delta_{1}{ }^{2} \mathrm{t}_{\mathrm{r}}+\frac{1}{3} \delta_{2}{ }^{3}-\frac{1}{2} \delta_{2}{ }^{2} \mathrm{t}_{\mathrm{r}}+\frac{1}{4} \delta_{2} \mathrm{t}_{\mathrm{r}}{ }^{2}-\frac{1}{12} \mathrm{t}_{\mathrm{r}}{ }^{3}-\delta_{1} \delta_{2} \mathrm{t}_{\mathrm{r}}+\delta_{1}{ }^{2} \delta_{2}-\delta_{1}{ }^{2} \mathrm{t}_{\mathrm{r}}+\delta_{1} \delta_{2}{ }^{2}\right. \\
& \left.+\delta_{1}^{2} t_{r}+\delta_{2}^{2} t_{r}+\frac{1}{20} t_{r}^{3}-\frac{1}{3} \delta_{1} t_{r}^{2}-\frac{1}{3} \delta_{2} t_{r}^{2}+2 \delta_{1} \delta_{2} t_{r}\right] \\
& =G^{2}\left[\frac{1}{3} \delta_{2}^{3}+\frac{1}{2} \delta_{2}^{2} t_{r}-\frac{1}{12} \delta_{2} t_{r}^{2}+\frac{1}{60} t_{r}^{3}\right]+G^{2}\left[\delta_{1} \delta_{2} t_{r}+\delta_{1}^{2} t_{r}+\delta_{1}^{2} \delta_{2}+\delta_{1} \delta_{2}^{2}\right] \\
& \therefore \quad \mathrm{I}_{\delta 2}=\frac{1}{60} \mathrm{G}^{2}\left[20 \delta_{2}^{3}+30 \delta_{2}^{2} \mathrm{t}_{\mathrm{r}}-5 \delta_{2} \mathrm{t}_{\mathrm{r}}^{2}+\mathrm{t}_{\mathrm{r}}^{3}\right]+\operatorname{Mom}_{1}\left(\operatorname{Mom}_{1}+\mathrm{G} \delta_{2}\right)\left(\delta_{2}+\mathrm{t}_{\mathrm{r}}\right)
\end{aligned}
$$




\section{d) Evaluation of $\mathrm{I}_{\tau 2}$}

For the blank time between the second gradient, $g_{2}$, and the third gradient, $g_{3}$, the integration gives:

$$
\begin{aligned}
& \mathrm{I}_{\tau_{2}}=\int_{\mathrm{t}_{23}}^{\mathrm{t}_{30}} \mathrm{dt}\left[\mathrm{G} \delta_{1}+\mathrm{G} \delta_{2}\right]^{2}=\mathrm{G}^{2}\left[\delta_{1}^{2}+\delta_{2}^{2}+2 \delta_{1} \delta_{2}\right]\left(\mathrm{t}_{30}-\mathrm{t}_{23}\right) \\
& \therefore \mathrm{I}_{\tau_{2}}=\operatorname{Mom}_{2} \operatorname{Mom}_{2} \tau_{2},
\end{aligned}
$$

where $\mathrm{Mom}_{2}=\mathrm{G} \delta_{1}+\mathrm{G} \delta_{2}$.

\section{e) Evaluation of the other contributions to $b$}

Following the same rules for the other integrals for the contributions from the rest of the parts of the b-factor, it is possible to write all of the gradient contributions in terms of the gradient moments. Thus, in general, the b-factor for the ep2d-diff sequence can be evaluated by adding the contributions of all parts of the sequence as given below:

$$
\begin{aligned}
& \mathrm{I}_{\delta 1}=\frac{1}{60} \mathrm{G}^{2}\left[20 \delta_{1}{ }^{3}+30 \delta_{1}{ }^{2} \mathrm{t}_{\mathrm{r}}-5 \delta_{1} \mathrm{t}_{\mathrm{r}}{ }^{2}+\mathrm{t}_{\mathrm{r}}{ }^{3}\right]+\operatorname{Mom}_{0}\left(\operatorname{Mom}_{0}+\mathrm{g}_{1} \delta_{1}\right)\left(\delta_{1}+\mathrm{t}_{\mathrm{r}}\right), \\
& \mathrm{I}_{\tau 1}=\operatorname{Mom}_{1} \operatorname{Mom}_{1} \tau_{1}, \\
& \mathrm{I}_{\delta 2}=\frac{1}{60} \mathrm{G}^{2}\left[20 \delta_{2}^{3}+30 \delta_{2}{ }^{2} \mathrm{t}_{\mathrm{r}}-5 \delta_{2} \mathrm{t}_{\mathrm{r}}{ }^{2}+\mathrm{t}_{\mathrm{r}}^{3}\right]+\operatorname{Mom}_{1}\left(\operatorname{Mom}_{1}+\mathrm{g}_{2} \delta_{2}\right)\left(\delta_{2}+\mathrm{t}_{\mathrm{r}}\right), \\
& \mathrm{I}_{\delta 3}=\operatorname{Mom}_{2} \operatorname{Mom}_{2} \tau_{2}, \\
& \mathrm{I}_{\tau 3}=\operatorname{Mom}_{3} \mathrm{Mom}_{3} \tau_{3}, \\
& \mathrm{I}_{\delta 4}=\frac{1}{60} \mathrm{G}^{2}\left[20 \delta_{3}{ }^{3}+30 \delta_{3}{ }^{3} \mathrm{t}_{\mathrm{r}}-5 \delta_{3} \mathrm{t}_{\mathrm{r}}{ }^{2}+30 \mathrm{t}_{\mathrm{r}}{ }^{3} \mathrm{t}_{\mathrm{r}}-5 \delta_{4} \mathrm{t}_{\mathrm{r}}{ }^{2}+\mathrm{tr}_{\mathrm{r}}{ }^{3}\right]+\operatorname{Mom}_{2}\left(\operatorname{Mom}_{2}+\mathrm{gom}_{3} \delta_{3}\right)\left(\delta_{3}+\mathrm{t}_{\mathrm{r}}\right), \\
& \mathrm{I}_{\tau 4}=\operatorname{Mom}_{4} \operatorname{Mom}_{4} \tau_{4},
\end{aligned}
$$


where $g_{1,2}=+G$ and $g_{3,4}=-G$ are the effective gradient amplitudes, Mom $3=G \delta_{1}+G \delta_{2}-G \delta_{3}$ and $\mathrm{Mom}_{4}=\mathrm{G} \delta_{1}+\mathrm{G} \delta_{2}-\mathrm{G} \delta_{3}-\mathrm{G} \delta_{4}=0$ since $\delta_{1}+\delta_{2}=\delta_{3}+\delta_{4}$.

\section{f) Evaluation of $b$}

Combining the results from the previous sections we find that

$\frac{b}{\gamma^{2}}=\sum_{i=1}^{4}\left\{\frac{1}{60} G^{2}\left[20 \delta_{i}^{3}+30 \delta_{i}^{2} t_{r}-5 \delta_{i} t_{r}^{2}+t_{r}^{3}\right]+\operatorname{Mom}_{i-1} \operatorname{Mom}_{i}\left(\delta_{i}+t_{r}\right)+\operatorname{Mom}_{i} \operatorname{Mom}_{i} \tau_{i}\right\}$,

where the effective gradient amplitudes are $\mathrm{g}_{1}=\mathrm{g}_{2}=-\mathrm{g}_{3}=-\mathrm{g}_{4}=\mathrm{G}$ and $\mathrm{Mom}_{0}=0$.

\section{II.2 Diffusion Time Calculation}

In order to evaluate the diffusion time, $\tau_{\mathrm{D}}$, for the TRSE sequence, we need to further simplify and rearrange the expression for $b$ given in the previous section. To do this we will make use of the conditions $\mathrm{g}_{1}=\mathrm{g}_{2}=-\mathrm{g}_{3}=-\mathrm{g}_{4}=\mathrm{G}, \delta_{1}=\delta_{4}$ and $\delta_{2}=\delta_{4}$ in the sequence which are true for all of the diffusion sequences considered in this thesis. For this case it is easily shown that

$$
\begin{aligned}
& \operatorname{Mom}_{1}=\operatorname{Mom}_{3}=G \delta_{1}, \\
& \operatorname{Mom}_{2}=\mathrm{G}\left(\delta_{1}+\delta_{2}\right), \\
& \operatorname{Mom}_{4}=0
\end{aligned}
$$

Thus, b can be written as

$$
\begin{aligned}
& \frac{\mathrm{b}}{\gamma^{2} \mathrm{G}^{2}}=\frac{2}{3}\left(\delta_{1}^{3}+\delta_{2}^{3}\right)+\left(\delta_{1}^{2}+\delta_{2}^{2}\right) \mathrm{t}_{\mathrm{r}}-\frac{1}{6}\left(\delta_{1}+\delta_{2}\right) \mathrm{t}_{\mathrm{r}}^{2}+\mathrm{t}_{\mathrm{r}}^{3}+\delta_{1}^{2} \tau_{1}+\delta_{1}\left(\delta_{1}+\delta_{2}\right)\left(\delta_{2}+\mathrm{t}_{\mathrm{r}}\right) \\
& +\left(\delta_{1}+\delta_{2}\right)^{2} \tau_{2}+\left(\delta_{1}+\delta_{2}\right) \delta_{1}\left(\delta_{2}+\mathrm{t}_{\mathrm{r}}\right)+\delta_{1}^{2} \tau_{3} .
\end{aligned}
$$

If we now define

$$
\delta=\delta_{1}+\delta_{2}
$$

and 


$$
\Delta=\delta_{1}+\delta_{2}+2 \mathrm{t}_{\mathrm{r}}+\tau_{1}+\tau_{2}
$$

(see figure II.1) and note that

$$
\delta_{1}^{2}+\delta_{2}^{2}=\left(\delta_{1}+\delta_{2}\right)^{2}-2 \delta_{1} \delta_{2}
$$

and

$$
\delta_{1}^{3}+\delta_{2}^{3}=\left(\delta_{1}+\delta_{2}\right)^{3}-3 \delta_{1}^{2} \delta_{2}-3 \delta_{1} \delta_{2}^{2},
$$

b can be written as

$$
\begin{aligned}
\frac{\mathrm{b}}{\gamma^{2} \mathrm{G}^{2}}= & \frac{2}{3}\left[\delta^{3}-3 \delta_{1}^{2} \delta_{2}-3 \delta_{1} \delta_{2}^{2}\right]+\left[\delta^{2}-2 \delta_{1} \delta_{2}\right] \mathrm{t}_{\mathrm{r}}-\frac{1}{6} \delta \mathrm{t}_{\mathrm{r}}^{2}+\mathrm{t}_{\mathrm{r}}^{3}+\delta_{1}^{2}\left(\tau_{1}+\tau_{3}\right) \\
& +2 \delta_{1}^{2} \delta_{2}+2 \delta_{1} \delta_{2}^{2}+2 \delta_{1} \delta_{2} \mathrm{t}_{\mathrm{r}}+2 \delta_{1}^{2} \mathrm{t}_{\mathrm{r}}+\delta^{2} \tau_{2} \\
= & \frac{2}{3} \delta^{3}+\delta^{2}\left(\tau_{2}+\mathrm{t}_{\mathrm{r}}\right)-\frac{1}{6} \delta \mathrm{t}_{\mathrm{r}}^{2}+\mathrm{t}_{\mathrm{r}}^{3}+\delta_{1}^{2}\left(\tau_{1}+\tau_{3}\right)+2 \delta_{1}^{2} \mathrm{t}_{\mathrm{r}} \\
= & \frac{2}{3} \delta^{3}+\delta^{2}\left(\Delta-\delta-\mathrm{t}_{\mathrm{r}}-\tau_{1}\right)-\frac{1}{6} \delta \mathrm{t}_{\mathrm{r}}^{2}+\mathrm{t}_{\mathrm{r}}^{3}+\delta_{1}^{2}\left(\tau_{1}+\tau_{3}\right)+2 \delta_{1}^{2} \mathrm{t}_{\mathrm{r}} \\
\therefore & \frac{\mathrm{b}}{\gamma^{2} \mathrm{G}^{2} \delta^{2}}=\left(\Delta-\tau_{1}\right)-\frac{\delta}{3}+\left[\frac{\delta_{1}^{2}}{\delta^{2}}\left(\tau_{1}+\tau_{3}\right)+\mathrm{t}_{\mathrm{r}}\left(\frac{\mathrm{t}_{\mathrm{r}}^{2}}{\delta^{2}}-\frac{\mathrm{t}_{\mathrm{r}}}{6 \delta}+2 \frac{\delta_{1}^{2}}{\delta}-1\right)\right] .
\end{aligned}
$$

The diffusion time for the Stejskal Tanner diffusion sequence is defined in section 2.3 .3 by

$$
\tau_{\mathrm{D}}=\frac{\mathrm{b}}{\gamma^{2} \mathrm{G}^{2} \delta^{2}}=\Delta-\frac{\delta}{3}
$$

By analogy, we define the diffusion time for the TRSE sequence considered here to be

$$
\tau_{\mathrm{D}}=\frac{\mathrm{b}}{\gamma^{2} \mathrm{G}^{2} \delta^{2}}=\left(\Delta-\tau_{1}\right)-\frac{\delta}{3}+\left[\frac{\delta_{1}^{2}}{\delta^{2}}\left(\tau_{1}+\tau_{3}\right)+\mathrm{t}_{\mathrm{r}}\left(\frac{\mathrm{t}_{\mathrm{r}}^{2}}{\delta^{2}}-\frac{\mathrm{t}_{\mathrm{r}}}{6 \delta}+2 \frac{\delta_{1}^{2}}{\delta}-1\right)\right] .
$$

When $\mathrm{t}_{\mathrm{r}}=0$ and $\tau_{1}=\tau_{3}=0$, the TRSE sequence reduces to a form of the PGSE sequence. In this limit equation (II.14) readily reduces to equation II.13, as it should. 


\section{References}

1) Bloch F., Hansen W.W., Packard M.; Nuclear Induction, Phys. Rev. 69, 127, 460-474 (1946).

2) Purcel E.U., Torrey H.C., Pound R.V.; Resonance absorption by nuclear magnetic moments in a solid, Phys. Rev., 69, 37, 681-689 (1946).

3) Lauterbur P.C.; Image Formation by Induced Local Interactions: Examples Employing Nuclear Magnetic Resonance, Nature, 242, 190-191 (1973).

4) Mansfield P., Grannell P.K.; NMR diffraction in solids, J. Phys. C. 6, L422-L426 (1973).

5) Morris P.G.; Nuclear Magnetic Resonance Imaging in Medicine and Biology, Oxford University Press, New York, 1986.

6) Hahn E.L.; Spin Echoes, Phys. Rev. 80, 580-594 (1950).

7) Carr H.Y., Purcell E.M.; Effects of Diffusion on Free Precession in Nuclear Magnetic Resonance Experiments, Phys. Rev. 94, 630-638 (1954).

8) Tanner J.E., Stejskal E.O.; Restricted Self-Diffusion of Protons in Colloidal Systems by the Pulsed-Gradient, Spin-Echo Method, J. Chem. Phys., 49, No.4, 1768- 1777 (1968).

9) Le Bihan D., Breton E., Syrota A.; In-vivo self-diffusion magnetic resonance imaging, Proc., ISMRM $4^{\text {th }}$ Meeting, Berkely, California, 1238-39 (1985).

10) Stejskal E.O. and Tanner J.E.; Spin Diffusion Measurements: Spin Echoes in the Presence of a Time-Dependent Field Gradient, J. Chem. Phys. ,42,1,288-292 (1965).

11) Price W.S.; Pulsed-Field Gradient Nuclear Magnetic Resonance as a Tool for Studying Translational Diffusion: Part 1. Basic Theory, John Wiley \& Sons, New York (1997).

12) Le Bihan D., Mangin J.F, Poupon C., Clark C.A., Pappata S., Molko N., Chabrita H.; Diffusion Tensor Imaging: Concepts and Applications, J. Magn. Reson. Imag., 13, 534$536(2001)$.

13) Moseley M.E., Cohen Y., Mintorovitch J., Chileuitt L., Shimizu H., Kucharczyk J., Wendland M.F., and Weinstein P.R.; Early Detection of Regional Cerebral Ischemia in Cats: Comparison of Diffusion and $\mathrm{T}_{2}$ Weighted MRI and Spectroscopy, Mag. Reson. Med., 14, 330-346 (1990)

14) Moseley M.E., Butts, K. Yenari M.A., Marks M., de Crespigny A.; Clinical aspects of DWI, NMR Biomed., 8 , 387-396 (1995). 
15) Anderson A.W., Zhong J., Petroff O.A.C., Szafer A., Ransom B.R., Prichard J.W., Gore J.C.; Effects of osmotically driven cell volume changes on diffusion-weighted imaging of the rats optic nerve, Magn. Reson. Med., 35, 162-167 (1996).

16) van der Toorn A., Sykova E., Dijkhuizen R.M., Vorisek I., Vargova L., Skobisova E., van Lookeren Campagne M., Reese T., Nicolay K.; Dynamic changes in water ADC, energy metabolism, extracellular space volume, and tortuosity in neonatal rat brain during global ischemia., Magn. Reson. Med., 36, 52-60 (1996).

17) Lehmenkuhler A., Sykova E., Svoboda J., Zilles K., Nicholson C.: Extra cellular space parameters in the rat neocortex and subcortical white matter during postnatal development determined by diffusion analysis, Neuroscience, 55, 339-351 (1993).

18) Vorisek I., Sykova E.: Ischemia-induced changes in the extra cellular space diffusion parameters, $\mathrm{K}^{+}$, and $\mathrm{pH}$ in the developing rat cortex and corpus callosum, J. Cereb. Blood Flow Metab., 17, 191-203 (1997).

19) Hajnal J.V., Doran M., Hall A.S., Collins A.G., Oatridge A., Pennock J.M., Young I.R., Bydder G.M.; MR imaging of anisotropically restricted diffusion of water diffusion weighted MR imaging of anisotropic water diffusion in cat central nervous system., Radiology ,176, 439-445 (1990).

20) Moseley M.E., Kucharczyk J., Asgari H.S., Norman D.; Anisotropy in diffusionweighted MRI, Magn. Reson. Med., 19, 321- 326 (1991).

21) Howe F.A., Filler A.G., Bell B.A., Griffiths J.R.; magnetic resonance neurolography, Magn. Reson. Med., 28, 328-338 (1992).

22) Sotak C.H., Li L.; MR imaging of anisotropic and restricted diffusion by simultaneous use of spin and stimulated echoes, Magn. Reson., Med., 26, 174- 183 (1992).

23) King M.D., van Bruggen N., Ahier R.G., Cremer J.E., Hajnal J.V., Williams S.R., Doran M.; Diffusion-weighted imaging of kainic acid lesions in the rat brain, Magn. Reson. Med. , 20, 158- 164 (1991).

24) Basser P.G.; Inferring Microstructural Features and the Physiological State of Tissues from Diffusion-Wieghted Images, NMR in Biomed., 8, 333-344 (1995).

25) Bammer R., Burak A., Moseley M.E.; In-vivo MR tractrography using diffusion imaging, European J. Radiology, 45, 223-234 (2003).

26) Pierpaoli C., and Basser P.J.; Toward a Quantitative Assessment of Diffusion Anisotropy, Mag. Reson. Med., 36, 893- 906 (1996). 
27) Sijbers J.; Signal and noise estimation from magnetic resonance images, $\mathrm{Ph} . \mathrm{D}$. thesis, University of Antwerp (CDE), Belgium (1998).

28) Warach S., Gaa J., Siewert B., Weilpolski P., Edelman R.R.; Acute human stroke studied by whole brain echo planar diffusion weighted magnetic resonance imaging, Ann Neurol, 7, 231- 241 (1995)

29) Contour T.E., Mackinstry R.C., Robinson B.H.; Encoding of anisotropic diffusion with tetrahedral gradients: a general mathematical diffusion formalism and experiments results. Magn. Reson. Med., 35, 399-412 (1996)

30) Mori S., van Zijl P.C.M.; Diffusion weighted by the trace of the diffusion tensor within a single scan, Magn. Reson. Med., 33, $41-52$ (1995).

31) Chun T., Ulug A.M., van Zijl P.C.M.; Single shot diffusion weighted trace imaging on a clinical scanner, Magn. Reson. Med., 40, 622- 628 (1998).

32) Hsu E.W., Mori S.; Analytical expressions for the NMR apparent diffusion coefficients in an anisotropic system and a simplified method for determining fiber orientation, Magn. Reson. Med., 34, 194- 200 (1995).

33) Melhem E.R., Itoh R., Jons L., Barker P.B.; Diffusion tensor MR imaging of the brain: effect of diffusion weighting on trace and anisotropy measurements, American Journal of Neuroradiology, 21, 1813- 1820 (2000).

34) Beaulieu C.,Fenrich F.R., Allen P.S.; Multicomponent water proton transverse relaxation and T2 discriminated water diffusion in myelinated and non-myelinated nerve, Magn. Reson. Imag. 16, 1201-1210 (1998).

35) Basser P.J., Mattielo J., Turner R., Le Bihan D.; Diffusion tensor echo-planar imaging of human brain, Proceeding of the ISMRM, p.584 (1993).

36) Basser P.J., Mattielo J., Le Bihan D.; Estimation of the effective self-diffusion tensor from the NMR spin echo, J. Magn. Reson., 103, 247-257 (1994).

37) Basser P.J., Mattielo J., Le Bihan D.; MR diffusion tensor spectroscopy and imaging, Biophys. J., 66, 259- 267 (1994).

38) Melhem E.R., Mori S., Mukundan G., Kraut M.A., Pomper M.G., van Zijl P.C.M.; Diffusion tensor MR imaging of the brain and white matter tractrography, American Journal of Roentgenology, 178, 3-16 (2002).

39) Neil J.J., Shiran S.I., Mckinstry R.C., Schefft G.L., Snyder A.Z., Almli C.R., Akbudak E., Aronovitz J.A., Miller J.P., Lee B.C.P, Conturo T.E.; Normal brain in human 
newborns: apparent diffusion coefficient and diffusion anisotropy measured by using diffusion tensor, Radiology, 209, 57-66 (1998).

40) Huppi P.S., Maier S.E., Peled S., Zientara G.P., Barnes P.D., Jolesz F.A., Volp J.J.; Microstructural development of human newborn cerebral white matter assessed by diffusion tensor magnetic resonance imaging, Pediatr. Res., 44, 583- 590 (1998).

41) Barrati C., Barnett A.S., Pierpaoli C.; Comparative MR imaging study of brain maturation in kittens with $T_{1}, T_{2}$, and the trace of the diffusion tensor, Radiology, 210, 133-142, (1999).

42) Bammer R., Agustin M., Strasser-Fuchs S., Seifert T., Kapeller P., Stoll- berger R Ebner F, Hartung HP, Fazekas F.; Magnetic resonance diffusion tensor imaging for characterizing diffusion and focal white matter abnormalities in multiple sclerosis, Magn Reson. Med., 44, 583- 591 (2000).

43) O. Ciccarelli, D.J. Werring, C.A.M. Wheeler-Kingshott, G.J. Barker, G.J.M. Parker, A.J. Thompson, and D.H. Miller, Investigation of MS normal-appearing brain using diffusion tensor MRI with clinical correlations, Neurology, 56, 926- 933 (2001).

44) Fillip M., Cercignani M., Inglese M., Horsfield M.A., Comi G.; Diffusion tensor magnetic resonance imaging in multiple sclerosis, Neurology, 56, 304- 311 (2001).

45) E. V. Sullivan; E. Adalsteinsson; M. Hedehus; C. Ju; M. Moseley; K.O. Lim; A. Pfefferbaum; Equivalent disruption of regional white matter microstructure in aging healthy men and women, Neuroreport, 12, 99-104 (2001).

46) Foong J., Maier M. Clark C.A., Barker G.J., Miller D.H., Ron M.A.; Neuropathological abnormalities of the corpus callosum in schizophrenia : a diffusion tensor imaging study, J. Neurol Neurosurg Psychiatry, 68, 242- 244 (2000).

47) Rose S.E., Chen F., Chalk J.B., et.al.; Loss of connectivity in Alzheimer's disease: an evaluation of white matter tract integrity with color-coded MR diffusion tensor imaging, J. Neurol. Neurosurg Psychiatry, 69, 528- 530 (2000).

48) Eriksson S.H., Rugg-Gunn F.J., Symms M.R., Barker G.J., Duncan J.S.; Diffusion tensor imaging in patients with epilepsy and malformations of cortical development, Brain, 124, 627- 636 (2001).

49) Krabbe K, Gideon P, Wagn P, Hansen U, Thomsen C, Madsen F. ; MR diffusion imaging of human intracranial tumors, Neuroradiology, 39, $483-489$ (1997). 
50) H Chabriat, K Vahedi, CA Clark, C.Poupon, A. Ducros ans C. Denier, D. Le Bihan, MG Bousser; Decreased hemispheric water mobility in hemiplegics migraine related to mutation of CACNAIA gene, Neurorology, 54, 510- 512 (2000).

51) Schaefer P.W., Buonamo F.S., Gonzales R.G. Schwamm L.H.; Diffusion-weighted imaging discriminates between cytotoxic and vasogenic edema in a patient with eclapmsia, Stroke, 28, 1082- 1085 (1997).

52) Westin C.F., Maier S.E., Mamata H., Nabavi A., Jolesz F.A., Kikinis R.; Processing and visualization for diffusion tensor MRI, Medical Image Analysis, 6, 93- 108 (2002).

53) Damon B.M., Ding Z., Anderson A.W., Freyer A.S., Gore J.C.; Validation of diffusion tensor MRI-based muscle fiber tracking Magn. Reson. Med., 48, 97- 104 (2002).

54) Melhem E.R., Barker B.P., Raymond G.V., Moser H.W.; X-linked adrenoleukodystrophy :review of genetic, clinical, and MR imaging characteristics, American Journal of Roentgenology, 173, 1575-1583 (1999).

55) Melhem E.R., Hoon A.H., Ferrucci J.T. III, et.al.; Brain MR imaging in peri ventricular leukomalacia: relationship between lateral ventricular volume and severity of cognitive and motor impairment, Radiology, 214, 199- 204 (2002).

56) Fitz C.R.; Holoprosencelaphaly and related entities, Neuroradiology, 25, 225-238 (1983).

57) Moseley M.E., Kucharczyk J., Mitorovitch J., Cohen Y., Kurhanewicz J., Derugin N., Asgari H., Norman D.; Diffusion weighted MR imaging of acue stroke: correlation with $\mathrm{T}_{2}$ weighted and magnetic susceptibility enhanced MR imaging in cats; American Journal of Neuroradiology, 11, 423-432 (1990).

58) Moseley M., Cohen Y., Kucharczyk J., Asgari H., Wendland M.F., Tsuruda J., Norman D.; Diffuison weighted MR imaging of anisotropic water diffusion in cat central nervous system, Radiology, 176, 439- 445 (1990).

59) Van Gelderen P., de Vleeschouwer M.H.M., DesPres D., Pekar J., van Zijl P.C.M., Moonen C.T.W.; Water diffusion and acute stroke, Magn. Reson. Med., 31, 154-163 (1994).

60) Yongbi M.N., Haung N.C., Branch C.A., Helpern J.A.; The application of diffusion weighted line scanning for the rapid assessment of water ADC changes in stroke at high magnetic fields, NMR Biemed, 10, 79- 86 (1997). 
61) Moonen C.T.W., Pekar J., de Vleeschouuwer M.H.M., Despres, D.; Restricted and anisotropic displacement of water in healthy cat brain and in stroke studied by NMR diffusion imaging, Magn. Reson. Med., 19, 327- 332 (1991).

62) Maier S.E., Gudbjartsson H., Patz S., Hsu L., Lovblad K.O., Edelman R.R., Warach S., Jolesz F.A.; Line scan diffusion imaging: Characterization in healthy subjects and stroke patients, American Journal of Roentgenology, 171, 85- 93 (1998).

63) Werring D.J., Clark C.A., Barker G.J., Thompson A.J., Miller D.H.; Diffusion tensor imaging of lesions and normal appearing white matter in multiple sclerosis, Neurology, 52, 1626- 1632 (1999).

64) Sugahara T., Korogi Y., Kochi M., Lkushima I., Shigematu Y., Hirai T., Okuda T., Liang L., Ge Y., Komohara Y., Ushio Y., Takahashi M.; Usefulness of diffusion weighted MRI with echo planar technique in the evaluation of cellularity in glomias, J. Magn. Reson. Imaging, 9, 53-60 (1999).

65) Wieshmann U.C., Clark C.A., Symms M.R., Barker G.J., Birnie K.D., Shorvon S.D.; Water diffusion in the human hippocampus in epilepsy, Magn. Reson. Imaging, 17, 2936 (1999).

66) Bahn M.M., Kido D.K., Lin W., Pearlman A.L.; Brain magnetic resonance diffusion abnormalities in Creutzfeld-Jakob disease, Arch Neurol, 54, 1411 - 1415 (1997).

67) Lim K.O., Hedehus M., Moseley M., de Crespigny A., Sullivan E.V., Pfefferbaum A.; Compromised white matter tract integrity in schizophrenia inferred from diffusion tensor imaging, Arch. Gen. Psychiatry, 56, 367- 374 (1999).

68) Werring D.J., Clark C.A., Barker G.J., Miller D.H., Parker G.J.M., Brammer M.J., Bullmore E.T., Giampietro V.P. Thompson A.J.; The structural and functional mechanisms of motor recovery: complementary use of diffusion tensor and functional magnetic resonance imaging in traumatic injury of the internal capsule. J. Neurol. Neurosurg. Psych., 65, 863- 869 (1998).

69) Busza A.L., Allen K.L., King M.D., van Bruggen N., Williams S.R., Gadian D.G.; Diffusion weighted imaging studies of cerebral ischemia in gerbils, Stroke, 23, 1604, 1304-1311 (1992).

70) Larsson H.B.W., Thomsen C., Frederiksen J., Stubgaard M., Henriksen O.; In-vivo magnetic resonance diffusion measurement in the brain of patients with multiple sclerosis, Magn. Reson. Imaging, 10, 7, 1717-1723 (1992). 
71) Ford J.C., Hackney D.B., Alsop D.C., Jara H., Joseph P.M., Hand C.M., Black P.; MRI characterization of diffusion coefficients in a spinal cord injury model, Magn. Reson. Med., 31, 488- 494 (1994).

72) Nevo U., Hauben E., Yoles E., Agranov E., Akselrod S., Schwartz M., Neeman M.; Diffusion anisotropy MRI for quantitative assessment of recovery in injured rat spinal cord, Magn. Reson. Med., 45, 1 - 9 (2001).

73) Davis D., Ulatowski J., leff S., Izuta M., Mori S., Shugu D., van Zijl P.C.M.; Rapid monitoring of changes in water diffusion coefficients during reversible ischemia in cat and rat brain, Magn. Reson. Med., 31, 454- 460 (1994).

74) Dijkhuizen R.M., Berkelbach van der, Sprenkel J.W., Tulleken C.A.F., Nicolay K.; Correlation between hemodynamics and water diffusion changes in a rat model of acute focal ischemia, Brain Res., 750, 161- 170 (1997).

75) Knight R.A., Ordidge R.J., Helpern J.A., Chopp M., Rodolosi L.C., Peck D.; Temporal evolution of ischemic damage in rat brain measured by proton nuclear magnetic resonance imaging, Stroke, 22, 802- 808 (1991).

76) Helpern J.A., Dereski M.O., Knight R.A., Ordidge R.J., Chopp M., Qing Z.X.; Histopathologcal correlation of nuclear magnetic resonance imaging parameters in experimental cerebral ischemia, Magn. Reson. Imaging, 11, 241 - 246 (1993).

77) Zhong J., Petroff O.A.C., Prichard J.W., Gore J.C.; Changes in water diffusion and relaxation properties of rat cerebrum during status epilepticus, Magn. Reson. Med., 30, 241- 246 (1993).

78) Hansen A.J., Olsen C.E.; Brain extra cellular space during spreading depression and ischemia, Acta. Physiol. Scand., 108, 355- 365 (1980).

79) Grander-Medwin A.R., van Bruggen N., Williams S.R., Ahier R.G.; Magnetic Resonance imaging of spreading depression in anaesthized rat, J. Cereb. Blood Flow Metab., 14, 711 (1994).

80) Latour L.L., Hasegawa Y., Formato J.E., Fisher M., Sotak C.H.; Spreading waves of decreased diffusion coefficient after cortical stimulation in the rat brain, Magn. Reson. Med., 32, 189- 198 (1994).

81) Busch E., Gyngell M.L., Eis M., Hoehn-Berlage M., Hossmann K.A.; Potassium induced cortical spreading depressions during focal cerebral ischemia in rats; contribution to 
lesion growth assessed by diffusion weighted NMR and biochemical imaging, NMR Biomed., 8, 59- 64 (1995).

82) Cruz A., Green B.G.; Thermal stimulation of taste, Nature, 403, 889- 892 (2000).

83) Meyer J.W., Makris N., Bates J.F., Caviness V.S., Kennedy D.N.; MRI based topographic parcellation of human cerebral white matter I. Technical foundations, Neuroimage, 9, 1-17 (1999).

84) Gates L.; A study of water mobility in human tissue in vivo: Apparent diffusion coefficients in brain and leg muscle, Ph.D thesis, Carleton University, Dept. of Phys. (1997).

85) Morvan D.; In vivo measurement of diffusion and pseudo-diffusion in skeletal muscle at rest and after exercise, Magn. Reson. Imaging, 13, $193-199$ (1997).

86) Baur A., Stabler A., Bruning R.; Diffusion weighted MR imaging of bone marrow: differentiation of being versus pathologic compression fractures, Radiology, 207, 349356 (1998).

87) Akansel G., Haughton V.M., Papke R.A., Censky S.; Diffusion into human intervertebral disks studied with MR and gadoteriodol, American Journal of Neuroradiology, 18, 443445 (1997).

88) Englander S.A., Ulug A.M., Brem R., Glockson J.D., van Zijl P.C.M.; Diffusion imaging of human breast, NMR Biomed. , 10, 348- 352 (1997).

89) Maier C.F., Nikolov H.N., Chu K.C., Chronik B.A., Rutt B.K.; Practical design of a highstrength breast gradient coil, Magn. Reson. Med., 39, 392- 401 (1998).

90) Namimoto T., Yamashita Y., Sumi S., Tang Y., Takahashi M.; Focal liver masses: characterization with diffusion-weighted echo planar MR imaging, Radiology, 204, 739744 (1997).

91) Yamada I., Aung W., Himeno Y., Nakagawa T., Shibuya H.; Diffusion coefficients in abdominal organs and hepatic lesions: evaluation with intravoxel incoherent motion echo planar MR imaging, Radiology, 210, 17- 623 (1999).

92) Scollan D.F., Holmes A., Winslow R., Forder J.; Histological validation of myocardial microstructure obtained from diffusion tensor magnetic resonance imaging, Am. J. Physiol. Heart Circ. Phys., 44, H2308- H2318 (1998). 
93) Guo A.C., Petrella J.R., Kurtzberg J., Provenzale J.M.; Evaluation of white matter anisotropy in Krabbe disease with diffusion tensor MR imaging: initial experience, Radiology, 218, 809-815 (2001).

94) Ito R., Melhem E.R., Mori S., Eichr F.S., Raymond G.V., Moser H.W.; Diffusion tensor brain MR imaging in X-linked cerebral adrenoleukodystrophy, Neurology, 56, 544- 547 (2001).

95) Pomara N., Crandall D.T., Choi S.J., Johnson G., Lim K.O.; White matter abnormalities in HIV-1 infection: diffusion tensor imaging study, Psychiatry Res. 106, 15-24 (2001).

96) Schwartz R.B., Mulkern R.V., Gudbjartsson H., Jolesz F.; Diffusion weighted MR imaging in hypertensive encephalopathy: clues to pathogenesis, American Journal of Neuroradiology, 19, 859- 862 (1998).

97) Jones D.K., Lythgoe D., Horsfield M.A., Simmons A., Williams S.C., Markus H.S.; Characterization of white matter damage in ischemic leukoaraiosis with diffusion tensor MRI, Stroke, 30, 393- 397 (1999).

98) Mulkern RV, Gudbjartsson H, Westin C-F, Zengingonul HP, Gartner W, Guttmann CRG, Robertson R, Kyriakos W, Schwartz R, Holtzman D, Jolesz FA, Maier SE.; Multicomponent apparent diffusion coefficients in human brain, NMR in Biomed., 12, 51- 62 (1999).

99) Niendorf T., Dijkhuizen R., Norris D.G., van Lookeren, Campagne M., Nicolay K.; Biexponential diffusion attenuation in various states of brain tissue: implications for diffusion weighted imaging, Magn. Reson. Med., 36, 847- 857 (1996).

100) Assaf Y., Cohen Y.; Non-mono-exponential attenuation of water and $\mathrm{N}$-acetyl asparate signals due to diffusion in brain tissue, J. Magn. Reson., 131, 69-85 (1998).

101) Kraemer F., Darquie A., Clark C.A., Le Bihan D.; Separation of two diffusion compartments in the human brain, Proc. $7^{\text {th }}$ Meeting ISMRM, Philadelphia, p.1808 (1999).

102) Pfeuffer J., Provencher S.W., Gruetter R.; Water diffusion in rat brain in vivo at very large b values is multicompartmental, Magma, 8, 98- 108 (1999).

103) Mulkern, RV; Zengingonul, HP; Robertson, RL; Bogner, P; Zou, KH; Gudbjartsson, H; Guttmann, CRG; Holtzman, D; Kyriakos, W; Jolesz, FA; Maier, SE.; Multi-component apparent diffusion coefficients in human brain: Relationship to spin-lattice relaxation, Magn. Reson. Med., 44, 292-300 (2000). 
104) Latour L.L., Svoboda K., Mitra P.P., Sotak C.H.; Time dependent diffusion of water in a biological model system, Proc. Natl. Acad. Sci. USA, 91, 1229-1233 (1994).

105) Szafer A., Zhong J., Gore J.C.; Theoretical model for water diffusion in tissues, Magn. Reson. Med., 33, 697-712 (1994).

106) Stanisz G.J., Szafer A., Wright G.A., Henkelman R.M.; An analytical model of restricted diffusion in bovine optic nerve, Magn. Reson. Med., 37, 103-111 (1997).

107) Lipinski H.G.; Monte Carlo simulation of extra cellular diffusion in brain tissues, Phys. Med. Biol., 35, 441- 447 (1990).

108) Lawson C.L. and Hanson R.J.; Solving least squares problems, Prentice-Hall, Englewood Cliffs, New Jersey, 1974.

109) Whittall K.P., MacKay R.L.; Quantitative interpretation of NMR relaxation data, J. Magn. Reson., 84, 134- 152 (1989).

110) Provencher S.W.; A constrained regularization method for inverting data represented by linear algebra or integral equations, Computer physics communications, 27, 231-227 (1982).

111) Pfeuffer J., Dreher W., Sykova E., Leibfritz D.; Water signal attenuation in diffusion weighted ${ }^{1} \mathrm{H}$ NMR experiments during cerebral ischemia: influence of intracellular restrictions, extra cellular tortusity, and exchange, Magn. Reson. Imaging, 16, No.9, 1023- 1032 (1998).

112) Stanisz G.J., Szafer A., Wright G.A., Henkelman R.M.; An analytical model of restricted diffusion in bovine optic nerve, Magn. Reson. Med., 37, 103- 111 (1997).

113) Pilatus U., Shim H., Artemov D., Davis D., van Zijl P.C.M., Glickson J.; Intracellular volume and apparent diffusion constants perfused cancer cell cultures as measured by NMR, Magn. Reson. Med., 37, 825- 832 (1997).

114) Pfeuffer J., Flogel U., Dreher W., Leibfritz D.; Restricted diffusion and exchange of intracellular water: Theoretical modeling and diffusion time dependence of ${ }^{1} \mathrm{H}$ NMR measurements on perfused glial cells, NMR Biomed., 11, 19- 31 (1998).

115) Horsfield M.A., Barker G.J., Ian McDonald W.; Self diffusion in CNS tissue by volume selective proton NMR, Magn. Reson. Med., 31, 637- 644 (1994).

116) Dixon R.L., Ekstrand K.E.; The Physics of Proton NMR, Med. Phys., 9 (6), 807-818, (1982). 
117) Stephan R.; NMR in Medicine: The instrumentation and clinical applications, American Inst. of Phys., NY (1985).

118) Hoult D.I., Richard R.E.; The signal-to-noise ratio of the nuclear magnetic resonance experiment, J. Magn. Reson., 24, $71-85$ (1976).

119) Hoult D.I., Lauterbur P.C.; The sensitivity of the Zeugmatographic experiment involving human samples, J. Magn. Reson., 34, 425- 433 (1979).

120) H. Bruder, H. Fischer, H.E. Reinfelder, F. Schmitt; Image Reconstruction for Echo Planar Imaging with Nonequidistant k-Space Sampling, Magn. Reson. Med, 23, 311-323 (1992).

121) Bronskill M.J., Sprawls P.; The Physics of MRI, AAPM summer school proceedings, No.21 (1992).

122) Gasiorowicz S.; Quantum physics, John Wiley \& Sons, $3^{\text {rd }}$ Ed. (1995).

123) Norris D.G.; The effects of microscopic tissue parameters on the diffusion weighted magnetic resonance imaging experiment, NMR in Biomed., 14, 77- 93 (2001).

124) Reese T.G., Heid O., Weisskoff R.M., Wedeen V.J.; Reduction of eddy current induced distortion in diffusion MRI using a twice refocused spin echo, Magn. Reson. Med., 49, 177- 182 (2003).

125) Norris D.G., Niendorf T.; Interpretation of DW-NMR data: dependence on experimental conditions, NMR in Biomed., 8, 280-288 (1995).

126) Norris D.G.; Implications of bulk motion for diffusion weighted imaging experiments: Effects - Mechanisms - Solutions, J. Magn. Reson. Imaging, 13, 486- 495 (2001).

127) Karger J., Pfeifer H., Heink W.; Principles and application of self diffusion measurements by nuclear magnetic resonance, Adv. Magn. Res., 12, 1- 89 (1988).

128) Calamante F., Porter D.A., Gadian D.G., Connelly A.; Correction for eddy current induced $\mathrm{B}_{0}$ shifts in diffusion weighted echo planar imaging, Magn. Reson. Med., 41, 95- 102 (1999).

129) Crank J.; The Mathematics of Diffusion, $2^{\text {nd }}$ edition, Clarendon Press, Oxford, (1975).

130) Haselgrove J.C., Moor J.R.; Correction for distortion of echo planar images used to calculate the apparent diffusion coefficient, MRM , 36, 960- 964 (1996).

131) Horsfield MA.; Mapping eddy current induced fields for the correction of diffusion weighted echo planar images, Magn. Reson. Imaging, 17, No.9, 1335- 1345 (1999). 
132) Heid O.; Eddy current nulled diffusion weighting, Proceedings ISMRM $8^{\text {th }}$, p.799, (2000).

133) Gudbjartsson H., Patz S.; The Rician distribution of noisy MRI data, MRM, 34, 910914 (1995).

134) Henkleman R.M.; Measurement of signal intensities in the presence of noise in MR images, Med. Phys., 12 (2), 232-233, 1985.

135) McGibney G., Smith M.R.; An unbiased signal-to-noise ratio measure for magnetic resonance images, Med. Phys., 20 (4), 1077-1078, 1993.

136) Sijbers J., den Dekker A.J., Scheunders P., Van Dyck D.; Maximum-Likelihood estimation of Rician distribution parameters, IEEE Trans. Med. Imag., Vol 17, No.3, 357 361 (1998).

137) Bonny J.M., Renou J.P., Zanca M.; Optimal measurement of magnitude and the phase from MR data, JMR, B, 113, 136- 144 (1996).

138) Miller A.J., Joseph P.M.; The use of power images to perform quantitative analysis on low SNR MR images, Magn. Reson. Imaging, Vol. 11, 1051- 1056 (1993).

139) Bonny J.M., Boespflug-Tanguly O., Zanca M., Renou J.P.; Muti ־exponential analysis of magnitude MR images using a quantitative multispectral edge-preserving filter, Journal of Mag. Reson., 161, 25-34 (2003).

140) Bernstein M.A., Thomasson D.M., Perman W.H.; Improved detectability in low signalto-noise ratio magnetic resonance images by means of a phase-corrected real reconstruction, Med. Phys., 16 (5), 813-817, 1989.

141) Sijbers J., den Dekker A.J., Van Audekerke J., Verhoye M., Van Dyck D.; Estimation of the noise in magnitude MR images, Magn. Reson. Imag., Vol. 16, No. 1, 87 - 90 (1998).

142) Sijbers J., den Dekker A.J.; Maximum likelihood estimation of signal amplitude and noise variance from MR data, Magn. Reson. Med., Vol. 51, No. 3, 586- 594 (2003).

143) Chen C.N., Hoult D.I.; Biomedical Magnetic Resonance Technology, NY, (1989).

144) Pemberton M., Gates K.; Notes of Linear Algebra, Math2000, Dept. of Mathematics, Univ. of Queensland, (2001).

145) Graham S.J., Stanchev P.L., Bronskill M.J.; Criteria for Analysis of Multicomponent Tissue T2 Relaxation Data, Magn. Reson. Med., 35:370-378 (1996). 
146) Whittall K.P., MacKay A.L., Graeb D.A., Nugent R.A., Li D.K.B., Paty D.W.; In Vivo Measurement of $\mathrm{T}_{2}$ Distributions and Water Contents in Normal Human Brain”, Magn. Reson. Med., 37:34-43 (1997).

147) Vetterling W. T.; Numerical recipes example book, University Press, Cambridge (1992).

148) Smouha E. and Neeman M.; Compartmentation of intracellular water in multicellular tumor spheroids: Diffusion and relaxation NMR, MRM, 46, 68- 77 (2001)

149) Wallace J, Gherase M., Bernas L., Nezamzadeh M., Cameron I., Cross A., Santyr G.; Multiexponential analysis of CPMG $\mathrm{T}_{2}$ decay curves for ${ }^{129} \mathrm{Xe}$ dissolved in perfluorooctylbromide emulsions: implications for hyperpolarized Xenon agent development, ISMRM Proc., Toronto, p. 1378 (2003).

150) Le Bihan D., Turner R., Douck P.; Is water diffusion restricted in human brain white matter? An echo planar NMR imaging study., Neuroreport, 4, 887- 890 (1993).

151) Clark C.A., Le Bihan D.; Water diffusion compartmentation and anisotropy at high b-values in the human brain, Magn. Reson. Med., 44, 852- 859 (2000).

152) Beaulieu $C$.; The basis of anisotropic water diffusion in the nervous system- a technical review, NMR in Biomed., 15, 435- 455 (2002).

153) Norris D.G.,Niendorf T., Hohen_Berlage M., Kohno K., Schneider E.J., Hainz P., Hropot M., Leibfritz D.; the incidence of apparent restriction diffusion in three different models of cerebral infarction., Magn. Reson. Imag., 12,1175- 1182 (1994).

154) Niendorf T., Norris D.G., Leibfritz D.; Detection of apparent restriction diffusion in healthy rat brain at short diffusion times, Magn. Reson. Med., 32, 672- 677 (1994).

155) Norris D.G., Niendorf T., Leibfritz D.; Healthy and infarcted brain tissues studied at short diffusion times: the origins of apparent diffusion coefficient. NMR Biomed.,7, 304310 (1994).

156) Assaf Y., Ben-Bashat D., Chapman J., Peled S., Biton I.E., Kafir M., Segev Y., Hendler T., Korczyn A.D., Graif M., Cohen Y.I.; High b-value q-space analyzed diffusion weighted MRI: Application to Multiple Sclerosis, Magn. Reson. Med., 47, 115-126 (2002)

157) Pierpaoli C., Jezzard P., Basser P.J., Barnett A., DiChiro G.; Diffusion tensor MR imaging of the human brain, Radiology, 201, 637-648, (1996).

158) Haacke E.M.; Magnetic Resonance Imaging, Physical principles and sequence design, WILEY, (1999). 
159) M. Abromowitz and A. Stegun; Handbook of Mathematical Functions, Dover, New York, 1972.

160) Papoulis A; Probability, Random Variables, and Stochastic Processes, McGraw-Hill Inc., New York, (2000).

161) Rice S.O.; Mathematical analysis of random noise, Bell System Tech. J. 23, 282 (1944).

162) Conturo TE, McKinstry RC, Aronovitz JA, Neil JJ.; Diffusion MRI: Precision, Accuracy and Flow Effects, NMR in BioMed., 8, 307-332 (1995).

163) King MD, van Bruggen N, Busza AL, Houseman J, Williams SR, Gadian DG. ; Perfusion and diffusion MR imaging ,Mag. Reson. Med.,24, 288-301 (1992).

164) MacFall JR, Maki JH, Johnson GA, Hedlund L, Benveniste H, Copher G.; Diffusion / microcirculation MRI in the rat brain. Mag. Reson. Med., 19, 305- 310 (1991).

165) Skinner MG, Whittall KP, MacKay AL.; Effect of variable echo spacing in multi-echo sequences for resolving long T2 components in multiple sclerosis, Proc. ISMRM, (2001).

166) White S.; Volume Estimation from Three-dimensional Hyperpolarized ${ }^{129} \mathrm{Xe}$ Magnetic Resonance Images, M.Sc. thesis, Carleton University, Dept. of Phys., 2004.

167) Zhi-Pei Liang and P.C. Lauterbur; Principles of Magnetic Resonance Imaging, a signal processing perspective, IEEE Press, New York, (2000).

168) Abragam A.; The Principles of Nuclear Magnetism, Oxford University, London, 1961.

169) Dmitriy A.Y., A.L. Sukstanskii, J.C. Leawoods, D.S. Gierada, G.L. Bretthorst, S.S. Lefrak, J.D. Cooper, M.S. Conradi.; Quantitative in vivo assessment of lung microstructure at the alveolar level with hyperpolarized ${ }^{3} \mathrm{He}$ diffusion MRI, Proc of Natl Acad of Sci, USA, Vol.99, No.5, 3111-3116 (2002).

170) Vargova L, Homola A, Zamecnik J, Tichy M, Benes V, Sykova E.; Diffusion parameters of the extracellular space in human gliomas, Glia, 42, 77- 88, 2003.

171) J.R. Zimmerman and Brittin; J.Phys. Chem., 61, 1228 (1957).

172) Le Bihan D, Breton E, Lallemand D, Aubin ML; Separation of diffusion and perfusion in intravoxel incoherent motion MR imaging, Radiology 168, 497-505 (1988).

173) Olariu E.; Monte Carlo studies of the magnetic resonance diffusion decays, M.Sc. thesis, Carleton University, Dept. of Phys., 2003.

174) T.Q. Duong, C.S. Springer, C.H. Sotak, G.L. Bretthorst, G. Vetek, I. Palyka, J.J.H. Ackerman and J.J. Neil.; Evaluation of equilibrium transcytolemmal water exchange in intact rat brain, Proc. $6^{\text {th }}$ ISMRM, Sydney, p. 208 (1998). 
175) PCM van Zijl, CTW Moonen, P Faustino, J Pekar, O Kaplan and JS Cohen ; Complete separation of intracellular and extracellular information in NMR spectra of perfused cells by diffusion weighted spectroscopy. Proc Natl Acad Sci, USA, 88, 3228-3232 (1991).

176) Stanisz GJ, Henkleman RM; Integrated analysis of diffusion and relaxation of water in blood, Mag Reson Med, 40, 79-88 (1998).

177) Stanisz GJ, Li JG, Wright GA, Henkelman RM; Water dynamics in human blood via combined measurements of $T_{2}$ relaxation and diffusion in the presence of gadolinium, Magn Reson Med, 39, 223-233 (1998).

178) Le Bihan,; Molecular diffusion, tissue microdynamics and microstructure, NMR Biomed, 8, 375- 386 (1995).

179) Vorisek I., Sykova E.; Ischemia-induced changes in the extracellular space diffusion parameters, $\mathrm{K}+$ and $\mathrm{pH}$ in the developing rat cortex and corpus callosum, J. Cereb. Blood Flow Metab, 17, 191-203 (1997).

180) Nicholson C., Philips J.M.; Ion diffusion modified by tortuosity and volume fraction in the extracellular micro-environment of the rat cerebellum, J.Physiol., 321, 225-257 (1981).

181) Assaf Y., Freidlin R.Z., Basser P.J.; The measurement of the axon diameter distribution in white matter using diffusion MR methods, Proc. ISMRM, 2005.

182) Sykova E.; Diffusion properties of the brain in health and disease, Neurochemistry Int., 45, 453-466 (2004).

183) Mills; Self diffusion in normal and heavy water in the range $1-45^{\circ}$, J. Phys. Chem., 77 : 685-688 (1973).

184) Koay C.G., Basser J.B.; Quatitatively exact correction scheme for signal extraction from noisy magnitude MR signals, J. Magn. Reson. 179, 317-322 (2006).

185) Nezamzadeh M., Cameron I.; Diffusion studies of human brain tissue in-vivo by MRI, COMP, McMaster Univ., Hamilton, Canada, 2005.

186) Nezamzadeh M., Cameron I.; A new Rician noise correction, $14^{\text {th }}$ ISMRM, Seattle, Washington, USA, p. 710, 2006.

187) Nezamzadeh M., Cameron I.; Rician noise corrected and multi-component analyzed diffusion signal decays at high b-values, $14^{\text {th }}$ ISMRM, Seattle, Washington, USA, p. 346, 2006. 